\title{
A COMPLETE PROOF OF THE POINCARÉ AND GEOMETRIZATION CONJECTURES - APPLICATION OF THE HAMILTON-PERELMAN THEORY OF THE RICCI FLOW*
}

\author{
HUAI-DONG $\mathrm{CAO}^{\dagger}$ AND XI-PING ZHU
}

\begin{abstract}
In this paper, we give a complete proof of the Poincaré and the geometrization conjectures. This work depends on the accumulative works of many geometric analysts in the past thirty years. This proof should be considered as the crowning achievement of the Hamilton-Perelman theory of Ricci flow.
\end{abstract}

Key words. Ricci flow, Ricci flow with surgery, Hamilton-Perelman theory, Poincaré Conjecture, geometrization of 3-manifolds

AMS subject classifications. $53 \mathrm{C} 21,53 \mathrm{C} 44$

\section{CONTENTS}

$\begin{array}{lr}\text { Introduction } & 167\end{array}$

1 Evolution Equations $\quad 172$

1.1 The Ricci Flow . . . . . . . . . . . . . . . . . . . . 172

1.2 Short-time Existence and Uniqueness . . . . . . . . . . . . . . . 177

1.3 Evolution of Curvatures . . . . . . . . . . . . . . . . . . . . . . . . . . . . . . . . . . . . . . . . . .

1.4 Derivative Estimates . . . . . . . . . . . . . . . . . 190

1.5 Variational Structure and Dynamic Property . . . . . . . . . . 199

2 Maximum Principle and Li-Yau-Hamilton Inequalities 210

2.1 Preserving Positive Curvature . . . . . . . . . . . . . . . . 210

2.2 Strong Maximum Principle . . . . . . . . . . . . . . . . . 213

2.3 Advanced Maximum Principle for Tensors . . . . . . . . . . . . . . . 217

2.4 Hamilton-Ivey Curvature Pinching Estimate . . . . . . . . . . . . . . 223

2.5 Li-Yau-Hamilton Estimates . . . . . . . . . . . . . . . . 226

2.6 Perelman's Estimate for Conjugate Heat Equations . . . . . . . . . . . 234

3 Perelman's Reduced Volume 239

3.1 Riemannian Formalism in Potentially Infinite Dimensions . . . . . . . 239

3.2 Comparison Theorems for Perelman's Reduced Volume . . . . . . . . . 243

3.3 No Local Collapsing Theorem I . . . . . . . . . . . . . . . . . . . . 255

3.4 No Local Collapsing Theorem II . . . . . . . . . . . . . . . . . . . . 261

4 Formation of Singularities $\quad \mathbf{2 6 7}$

4.1 Cheeger Type Compactness . . . . . . . . . . . . . . . . . 267

4.2 Injectivity Radius Estimates . . . . . . . . . . . . . . . . . . 286

4.3 Limiting Singularity Models . . . . . . . . . . . . . . . . . . 291

4.4 Ricci Solitons . . . . . . . . . . . . . . . . . . 302

\footnotetext{
*Received December 12, 2005; accepted for publication April 16, 2006.

$\dagger$ Department of Mathematics, Lehigh University, Bethlehem, PA 18015, USA (huc2@lehigh.edu).

${ }_{\ddagger}^{\ddagger}$ Department of Mathematics, Zhongshan University, Guangzhou 510275, P. R. China (stszxp@ zsu.edu.cn).
} 
5 Long Time Behaviors $\quad 307$

5.1 The Ricci Flow on Two-manifolds . . . . . . . . . . . . . . 308

5.2 Differentiable Sphere Theorems in 3-D and 4-D . . . . . . . . . . 321

5.3 Nonsingular Solutions on Three-manifolds . . . . . . . . . . . . 336

6 Ancient $\kappa$-solutions 357

6.1 Preliminaries . . . . . . . . . . . . . . . . . . . . 357

6.2 Asymptotic Shrinking Solitons . . . . . . . . . . . . . . . . . . . 364

6.3 Curvature Estimates via Volume Growth . . . . . . . . . . . . . . . 373

6.4 Ancient $\kappa$-solutions on Three-manifolds . . . . . . . . . . . . . . . 384

7 Ricci Flow on Three-manifolds $\quad 398$

7.1 Canonical Neighborhood Structures _ . . . . . . . . . . . . . . 398

7.2 Curvature Estimates for Smooth Solutions . . . . . . . . . . . . . . . . 405

7.3 Ricci Flow with Surgery . . . . . . . . . . . . . . . . . . . . . 413

7.4 Justification of the Canonical Neighborhood Assumptions . . . . . . . 432

7.5 Curvature Estimates for Surgically Modified Solutions . . . . . . . . . 452

7.6 Long Time Behavior . . . . . . . . . . . . . . . . . . . . . 468

7.7 Geometrization of Three-manifolds . . . . . . . . . . . . . . . 481

$\begin{array}{ll}\text { References } & 486\end{array}$

$\begin{array}{ll}\text { Index } & 491\end{array}$ 
Introduction. In this paper, we shall present the Hamilton-Perelman theory of Ricci flow. Based on it, we shall give the first written account of a complete proof of the Poincaré conjecture and the geometrization conjecture of Thurston. While the complete work is an accumulated efforts of many geometric analysts, the major contributors are unquestionably Hamilton and Perelman.

An important problem in differential geometry is to find a canonical metric on a given manifold. In turn, the existence of a canonical metric often has profound topological implications. A good example is the classical uniformization theorem in two dimensions which, on one hand, provides a complete topological classification for compact surfaces, and on the other hand shows that every compact surface has a canonical geometric structure: a metric of constant curvature.

How to formulate and generalize this two-dimensional result to three and higher dimensional manifolds has been one of the most important and challenging topics in modern mathematics. In 1977, W. Thurston [122], based on ideas about Riemann surfaces, Haken's work and Mostow's rigidity theorem, etc, formulated a geometrization conjecture for three-manifolds which, roughly speaking, states that every compact orientable three-manifold has a canonical decomposition into pieces, each of which admits a canonical geometric structure. In particular, Thurston's conjecture contains, as a special case, the Poincaré conjecture: A closed three-manifold with trivial fundamental group is necessarily homeomorphic to the 3 -sphere $\mathbb{S}^{3}$. In the past thirty years, many mathematicians have contributed to the understanding of this conjecture of Thurston. While Thurston's theory is based on beautiful combination of techniques from geometry and topology, there has been a powerful development of geometric analysis in the past thirty years, lead by S.-T. Yau, R. Schoen, C. Taubes, K. Uhlenbeck, and S. Donaldson, on the construction of canonical geometric structures based on nonlinear PDEs (see, e.g., Yau's survey papers $[129,130]$ ). Such canonical geometric structures include Kähler-Einstein metrics, constant scalar curvature metrics, and self-dual metrics, among others. However, the most important contribution for geometric analysis on three-manifolds is due to Hamilton.

In 1982, Hamilton [58] introduced the Ricci flow

$$
\frac{\partial g_{i j}}{\partial t}=-2 R_{i j}
$$

to study compact three-manifolds with positive Ricci curvature. The Ricci flow, which evolves a Riemannian metric by its Ricci curvature, is a natural analogue of the heat equation for metrics. As a consequence, the curvature tensors evolve by a system of diffusion equations which tends to distribute the curvature uniformly over the manifold. Hence, one expects that the initial metric should be improved and evolve into a canonical metric, thereby leading to a better understanding of the topology of the underlying manifold. In the celebrated paper [58], Hamilton showed that on a compact three-manifold with an initial metric having positive Ricci curvature, the Ricci flow converges, after rescaling to keep constant volume, to a metric of positive constant sectional curvature, proving the manifold is diffeomorphic to the three-sphere $\mathbb{S}^{3}$ or a quotient of the three-sphere $\mathbb{S}^{3}$ by a linear group of isometries. Shortly after, Yau suggested that the Ricci flow should be the best way to prove the structure theorem for general three-manifolds. In the past two decades, Hamilton proved many important and remarkable theorems for the Ricci flow, and laid the foundation for the program to approach the Poincaré conjecture and Thurston's geometrization conjecture via the Ricci flow. 
The basic idea of Hamilton's program can be briefly described as follows. For any given compact three-manifold, one endows it with an arbitrary (but can be suitably normalized by scaling) initial Riemannian metric on the manifold and then studies the behavior of the solution to the Ricci flow. If the Ricci flow develops singularities, then one tries to find out the structures of singularities so that one can perform (geometric) surgery by cutting off the singularities, and then continue the Ricci flow after the surgery. If the Ricci flow develops singularities again, one repeats the process of performing surgery and continuing the Ricci flow. If one can prove there are only a finite number of surgeries during any finite time interval and if the long-time behavior of solutions of the Ricci flow with surgery is well understood, then one would recognize the topological structure of the initial manifold.

Thus Hamilton's program, when carried out successfully, will give a proof of the Poincaré conjecture and Thurston's geometrization conjecture. However, there were obstacles, most notably the verification of the so called "Little Loop Lemma" conjectured by Hamilton [63] (see also [17]) which is a certain local injectivity radius estimate, and the verification of the discreteness of surgery times. In the fall of 2002 and the spring of 2003, Perelman [103, 104] brought in fresh new ideas to figure out important steps to overcome the main obstacles that remained in the program of Hamilton. (Indeed, in page 3 of [103], Perelman said "the implementation of Hamilton program would imply the geometrization conjecture for closed three-manifolds" and "In this paper we carry out some details of Hamilton program".) Perelman's breakthrough on the Ricci flow excited the entire mathematics community. His work has since been examined to see whether the proof of the Poincaré conjecture and geometrization program, based on the combination of Hamilton's fundamental ideas and Perelman's new ideas, holds together. The present paper grew out of such an effort.

Now we describe the three main parts of Hamilton's program in more detail.

\section{(i) Determine the structures of singularities}

Given any compact three-manifold $M$ with an arbitrary Riemannian metric, one evolves the metric by the Ricci flow. Then, as Hamilton showed in [58], the solution $g(t)$ to the Ricci flow exists for a short time and is unique (also see Theorem 1.2.1). In fact, Hamilton [58] showed that the solution $g(t)$ will exist on a maximal time interval $[0, T)$, where either $T=\infty$, or $0<T<\infty$ and the curvature becomes unbounded as $t$ tends to $T$. We call such a solution $g(t)$ a maximal solution of the Ricci flow. If $T<\infty$ and the curvature becomes unbounded as $t$ tends to $T$, we say the maximal solution develops singularities as $t$ tends to $T$ and $T$ is the singular time.

In the early 1990s, Hamilton systematically developed methods to understand the structure of singularities. In [61], based on suggestion by Yau, he proved the fundamental Li-Yau [82] type differential Harnack estimate (the Li-Yau-Hamilton estimate) for the Ricci flow with nonnegative curvature operator in all dimensions. With the help of Shi's interior derivative estimate [114], he [62] established a compactness theorem for smooth solutions to the Ricci flow with uniformly bounded curvatures and uniformly bounded injectivity radii at the marked points. By imposing an injectivity radius condition, he rescaled the solution to show that each singularity is asymptotic to one of the three types of singularity models [63]. In [63] he discovered (also independently by Ivey [73]) an amazing curvature pinching estimate for the Ricci flow on three-manifolds. This pinching estimate implies that any three-dimensional singularity model must have nonnegative curvature. Thus in dimension three, one only needs to obtain a complete classification for nonnegatively curved singularity models. 
For Type I singularities in dimension three, Hamilton [63] established an isoperimetric ratio estimate to verify the injectivity radius condition and obtained spherical or necklike structures for any Type I singularity model. Based on the Li-Yau-Hamilton estimate, he showed that any Type II singularity model with nonnegative curvature is either a steady Ricci soliton with positive sectional curvature or the product of the so called cigar soliton with the real line [66]. (Characterization for nonnegatively curved Type III models was obtained in [30].) Furthermore, he developed a dimension reduction argument to understand the geometry of steady Ricci solitons [63]. In the three-dimensional case, he showed that each steady Ricci soliton with positive curvature has some necklike structure. Hence Hamilton had basically obtained a canonical neighborhood structure at points where the curvature is comparable to the maximal curvature for solutions to the three-dimensional Ricci flow.

However two obstacles remained: (a) the verification of the imposed injectivity radius condition in general; and (b) the possibility of forming a singularity modelled on the product of the cigar soliton with a real line which could not be removed by surgery. The recent spectacular work of Perelman [103] removed these obstacles by establishing a local injectivity radius estimate, which is valid for the Ricci flow on compact manifolds in all dimensions. More precisely, Perelman proved two versions of "no local collapsing" property (Theorem 3.3.3 and Theorem 3.3.2), one with an entropy functional he introduced in [103], which is monotone under the Ricci flow, and the other with a space-time distance function obtained by path integral, analogous to what Li-Yau did in [82], which gives rise to a monotone volume-type (called reduced volume by Perelman) estimate. By combining Perelman's no local collapsing theorem $\mathrm{I}^{\prime}$ (Theorem 3.3.3) with the injectivity radius estimate of Cheng-Li-Yau (Theorem 4.2.2), one immediately obtains the desired injectivity radius estimate, or the Little Loop Lemma (Theorem 4.2.4) conjectured by Hamilton.

Furthermore, Perelman [103] developed a refined rescaling argument (by considering local limits and weak limits in Alexandrov spaces) for singularities of the Ricci flow on three-manifolds to obtain a uniform and global version of the canonical neighborhood structure theorem. We would like to point out that our proof of the singularity structure theorem (Theorem 7.1.1) is different from that of Perelman in two aspects: (1) we avoid using his crucial estimate in Claim 2 in Section 12.1 of [103]; (2) we give a new approach to extend the limit backward in time to an ancient solution. These differences are due to the difficulties in understanding Perelman's arguments at these points.

\section{(ii) Geometric surgeries and the discreteness of surgery times}

After obtaining the canonical neighborhoods (consisting of spherical, necklike and caplike regions) for the singularities, one would like to perform geometric surgery and then continue the Ricci flow. In [64], Hamilton initiated such a surgery procedure for the Ricci flow on four-manifolds with positive isotropic curvature and presented a concrete method for performing the geometric surgery. His surgery procedures can be roughly described as follows: cutting the neck-like regions, gluing back caps, and removing the spherical regions. As will be seen in Section 7.3 of this paper, Hamilton's geometric surgery method also works for the Ricci flow on compact orientable threemanifolds.

Now an important challenge is to prevent surgery times from accumulating and make sure one performs only a finite number of surgeries on each finite time interval. The problem is that, when one performs the surgeries with a given accuracy at each surgery time, it is possible that the errors may add up to a certain amount which 
could cause the surgery times to accumulate. To prevent this from happening, as time goes on, successive surgeries must be performed with increasing accuracy. In [104], Perelman introduced some brilliant ideas which allow one to find "fine" necks, glue "fine" caps, and use rescaling to prove that the surgery times are discrete.

When using the rescaling argument for surgically modified solutions of the Ricci flow, one encounters the difficulty of how to apply Hamilton's compactness theorem (Theorem 4.1.5), which works only for smooth solutions. The idea to overcome this difficulty consists of two parts. The first part, due to Perelman [104], is to choose the cutoff radius in neck-like regions small enough to push the surgical regions far away in space. The second part, due to the authors and Chen-Zhu [34], is to show that the surgically modified solutions are smooth on some uniform (small) time intervals (on compact subsets) so that Hamilton's compactness theorem can still be applied. To do so, we establish three time-extension results (see Step 2 in the proof of Proposition 7.4.1.). Perhaps, this second part is more crucial. Without it, Shi's interior derivative estimate (Theorem 1.4.2) may not applicable, and hence one cannot be certain that Hamilton's compactness theorem holds when only having the uniform $C^{0}$ bound on curvatures. We remark that in our proof of this second part, as can be seen in the proof of Proposition 7.4.1, we require a deep comprehension of the prolongation of the gluing "fine" caps for which we will use the recent uniqueness theorem of BingLong Chen and the second author [33] for solutions of the Ricci flow on noncompact manifolds.

Once surgeries are known to be discrete in time, one can complete the classification, started by Schoen-Yau [109, 110], for compact orientable three-manifolds with positive scalar curvature. More importantly, for simply connected three-manifolds, if one can show that solutions to the Ricci flow with surgery become extinct in finite time, then the Poincaré conjecture would follow. Such a finite extinction time result was proposed by Perelman [105], and a proof also appears in Colding-Minicozzi [42]. Thus, the combination of Theorem 7.4.3 (i) and the finite extinction time result provides a complete proof to the Poincaré conjecture.

\section{(iii) The long-time behavior of surgically modified solutions.}

To approach the structure theorem for general three-manifolds, one still needs to analyze the long-time behavior of surgically modified solutions to the Ricci flow. In [65], Hamilton studied the long time behavior of the Ricci flow on compact threemanifolds for a special class of (smooth) solutions, the so called nonsingular solutions. These are the solutions that, after rescaling to keep constant volume, have (uniformly) bounded curvature for all time. Hamilton [65] proved that any three-dimensional nonsingular solution either collapses or subsequently converges to a metric of constant curvature on the compact manifold or, at large time, admits a thick-thin decomposition where the thick part consists of a finite number of hyperbolic pieces and the thin part collapses. Moreover, by adapting Schoen-Yau's minimal surface arguments in [110] and using a result of Meeks-Yau [86], Hamilton showed that the boundary of hyperbolic pieces are incompressible tori. Consequently, when combined with the collapsing results of Cheeger-Gromov [24, 25], this shows that any nonsingular solution to the Ricci flow is geometrizable in the sense of Thurston [122]. Even though the nonsingular assumption seems very restrictive and there are few conditions known so far which can guarantee a solution to be nonsingular, nevertheless the ideas and arguments of Hamilton's work [65] are extremely important.

In [104], Perelman modified Hamilton's arguments to analyze the long-time be- 
havior of arbitrary smooth solutions to the Ricci flow and solutions with surgery to the Ricci flow in dimension three. Perelman also argued that the proof of Thurston's geometrization conjecture could be based on a thick-thin decomposition, but he could only show the thin part will only have a (local) lower bound on the sectional curvature. For the thick part, based on the Li-Yau-Hamilton estimate, Perelman [104] established a crucial elliptic type estimate, which allowed him to conclude that the thick part consists of hyperbolic pieces. For the thin part, he announced in [104] a new collapsing result which states that if a three-manifold collapses with (local) lower bound on the sectional curvature, then it is a graph manifold. Assuming this new collapsing result, Perelman [104] claimed that the solutions to the Ricci flow with surgery have the same long-time behavior as nonsingular solutions in Hamilton's work, a conclusion which would imply a proof of Thurston's geometrization conjecture. Although the proof of this new collapsing result promised by Perelman in [104] is still not available in literature, Shioya-Yamaguchi [118] has published a proof of the collapsing result in the special case when the manifold is closed. In the last section of this paper (see Theorem 7.7.1), we will provide a proof of Thurston's geometrization conjecture by only using Shioya-Yamaguchi's collapsing result. In particular, this gives another proof of the Poincaré conjecture.

We would like to point out that Perelman [104] did not quite give an explicit statement of the thick-thin decomposition for surgical solutions. When we were trying to write down an explicit statement, we needed to add a restriction on the relation between the accuracy parameter $\varepsilon$ and the collapsing parameter $w$. Nevertheless, we are still able to obtain a weaker version of the thick-thin decomposition (Theorem 7.6.3) that is sufficient to deduce the geometrization result.

In this paper, we shall give complete and detailed proofs of what we outlined above, especially of Perelman's work in his second paper [104] in which many key ideas of the proofs are sketched or outlined but complete details of the proofs are often missing. As we pointed out before, we have to substitute several key arguments of Perelman by new approaches based on our study, because we were unable to comprehend these original arguments of Perelman which are essential to the completion of the geometrization program.

Our paper is aimed at both graduate students and researchers who want to learn Hamilton's Ricci flow and to understand the Hamilton-Perelman theory and its application to the geometrization of three-manifolds. For this purpose, we have made the paper to be essentially self-contained so that the proof of the geometrization is accessible to those who are familiar with basics of Riemannian geometry and elliptic and parabolic partial differential equations. The reader may find some original papers, particularly those of Hamilton's on the Ricci flow, before the appearance of Perelman's preprints in the book "Collected Papers on Ricci Flow" [17]. For introductory materials to the Hamilton-Perelman theory of Ricci flow, we also refer the reader to the recent book by B. Chow and D. Knopf [39] and the forthcoming book by B. Chow, P. $\mathrm{Lu}$ and $\mathrm{L}$. Ni [41]. We remark that there have also appeared several sets of notes on Perelman's work, including the one written by B. Kleiner and J. Lott [78], which cover part of the materials that are needed for the geometrization program. There also have appeared several survey articles by Cao-Chow [16], Milnor [91], Anderson [4] and Morgan [95] for the geometrization of three-manifolds via the Ricci flow.

We are very grateful to Professor S.-T. Yau, who suggested us to write this paper based on our notes, for introducing us to the wonderland of the Ricci flow. His vision and strong belief in the Ricci flow encouraged us to persevere. We also thank 
him for his many suggestions and constant encouragement. Without him, it would be impossible for us to finish this paper. We are enormously indebted to Professor Richard Hamilton for creating the Ricci flow and developing the entire program to approach the geometrization of three-manifolds. His work on the Ricci flow and other geometric flows has influenced on virtually everyone in the field. The first author especially would like to thank Professor Hamilton for teaching him so much about the subject over the past twenty years, and for his constant encouragement and friendship.

We are indebted to Dr. Bing-Long Chen, who contributed a great deal in the process of writing this paper. We benefited a lot from constant discussions with him on the subjects of geometric flows and geometric analysis. He also contributed many ideas in various proofs in the paper. We would like to thank Ms. Huiling Gu, a Ph.D student of the second author, for spending many months of going through the entire paper and checking the proofs. Without both of them, it would take much longer time for us to finish this paper.

The first author would like to express his gratitude to the John Simon Guggenheim Memorial Foundation, the National Science Foundation (grants DMS-0354621 and DMS-0506084), and the Outstanding Overseas Young Scholar Fund of Chinese National Science Foundation for their support for the research in this paper. He also would like to thank Tsinghua University in Beijing for its hospitality and support while he was working there. The second author wishes to thank his wife, Danlin Liu, for her understanding and support over all these years. The second author is also indebted to the National Science Foundation of China for the support in his work on geometric flows, some of which has been incorporated in this paper. The last part of the work in this paper was done and the material in Chapter 3, Chapter 6 and Chapter 7 was presented while the second author was visiting the Harvard Mathematics Department in the fall semester of 2005 and the early spring semester of 2006 . He wants to especially thank Professor Shing-Tung Yau, Professor Cliff Taubes and Professor Daniel W. Stroock for the enlightening comments and encouragement during the lectures. Also he gratefully acknowledges the hospitality and the financial support of Harvard University.

1. Evolution Equations. In this chapter, we introduce Hamilton's Ricci flow and derive evolution equations of curvatures. The short time existence and uniqueness theorem of the Ricci flow on a compact manifold is proved in Section 1.2. In Section 1.4, we prove Shi's local derivative estimate, which plays an important role in the Ricci flow. Perelman's two functionals and their monotonicity properties are discussed in Section 1.5.

1.1. The Ricci Flow. Let $M$ be an $n$-dimensional complete Riemannian manifold with the Riemannian metric $g_{i j}$. The Levi-Civita connection is given by the Christoffel symbols

$$
\Gamma_{i j}^{k}=\frac{1}{2} g^{k l}\left(\frac{\partial g_{j l}}{\partial x^{i}}+\frac{\partial g_{i l}}{\partial x^{j}}-\frac{\partial g_{i j}}{\partial x^{l}}\right)
$$

where $g^{i j}$ is the inverse of $g_{i j}$. The summation convention of summing over repeated indices is used here and throughout the book. The Riemannian curvature tensor is given by

$$
R_{i j l}^{k}=\frac{\partial \Gamma_{j l}^{k}}{\partial x^{i}}-\frac{\partial \Gamma_{i l}^{k}}{\partial x^{j}}+\Gamma_{i p}^{k} \Gamma_{j l}^{p}-\Gamma_{j p}^{k} \Gamma_{i l}^{p} .
$$


We lower the index to the third position, so that

$$
R_{i j k l}=g_{k p} R_{i j l}^{p}
$$

The curvature tensor $R_{i j k l}$ is anti-symmetric in the pairs $i, j$ and $k, l$ and symmetric in their interchange:

$$
R_{i j k l}=-R_{j i k l}=-R_{i j l k}=R_{k l i j} .
$$

Also the first Bianchi identity holds

$$
R_{i j k l}+R_{j k i l}+R_{k i j l}=0 .
$$

The Ricci tensor is the contraction

$$
R_{i k}=g^{j l} R_{i j k l},
$$

and the scalar curvature is

$$
R=g^{i j} R_{i j}
$$

We denote the covariant derivative of a vector field $v=v^{j} \frac{\partial}{\partial x^{j}}$ by

$$
\nabla_{i} v^{j}=\frac{\partial v^{j}}{\partial x^{i}}+\Gamma_{i k}^{j} v^{k}
$$

and of a 1-form by

$$
\nabla_{i} v_{j}=\frac{\partial v_{j}}{\partial x^{i}}-\Gamma_{i j}^{k} v_{k} .
$$

These definitions extend uniquely to tensors so as to preserve the product rule and contractions. For the exchange of two covariant derivatives, we have

$$
\begin{aligned}
\nabla_{i} \nabla_{j} v^{l}-\nabla_{j} \nabla_{i} v^{l} & =R_{i j k}^{l} v^{k}, \\
\nabla_{i} \nabla_{j} v_{k}-\nabla_{j} \nabla_{i} v_{k} & =R_{i j k l} g^{l m} v_{m},
\end{aligned}
$$

and similar formulas for more complicated tensors. The second Bianchi identity is given by

$$
\nabla_{m} R_{i j k l}+\nabla_{i} R_{j m k l}+\nabla_{j} R_{m i k l}=0 .
$$

For any tensor $T=T_{j k}^{i}$ we define its length by

$$
\left|T_{j k}^{i}\right|^{2}=g_{i l} g^{j m} g^{k p} T_{j k}^{i} T_{m p}^{l},
$$

and we define its Laplacian by

$$
\Delta T_{j k}^{i}=g^{p q} \nabla_{p} \nabla_{q} T_{j k}^{i},
$$

the trace of the second iterated covariant derivatives. Similar definitions hold for more general tensors.

The Ricci flow of Hamilton [58] is the evolution equation

$$
\frac{\partial g_{i j}}{\partial t}=-2 R_{i j}
$$


for a family of Riemannian metrics $g_{i j}(t)$ on $M$. It is a nonlinear system of second order partial differential equations on metrics.

In order to get a feel for the Ricci flow (1.1.5) we first present some examples of specific solutions.

(1) Einstein metrics

A Riemannian metric $g_{i j}$ is called Einstein if

$$
R_{i j}=\lambda g_{i j}
$$

for some constant $\lambda$. A smooth manifold $M$ with an Einstein metric is called an Einstein manifold.

If the initial metric is Ricci flat, so that $R_{i j}=0$, then clearly the metric does not change under (1.1.5). Hence any Ricci flat metric is a stationary solution of the Ricci flow. This happens, for example, on a flat torus or on any $K 3$-surface with a Calabi-Yau metric.

If the initial metric is Einstein with positive scalar curvature, then the metric will shrink under the Ricci flow by a time-dependent factor. Indeed, since the initial metric is Einstein, we have

$$
R_{i j}(x, 0)=\lambda g_{i j}(x, 0), \quad \forall x \in M
$$

and some $\lambda>0$. Let

$$
g_{i j}(x, t)=\rho^{2}(t) g_{i j}(x, 0)
$$

From the definition of the Ricci tensor, one sees that

$$
R_{i j}(x, t)=R_{i j}(x, 0)=\lambda g_{i j}(x, 0) .
$$

Thus the equation (1.1.5) corresponds to

$$
\frac{\partial\left(\rho^{2}(t) g_{i j}(x, 0)\right)}{\partial t}=-2 \lambda g_{i j}(x, 0) .
$$

This gives the ODE

$$
\frac{d \rho}{d t}=-\frac{\lambda}{\rho}
$$

whose solution is given by

$$
\rho^{2}(t)=1-2 \lambda t
$$

Thus the evolving metric $g_{i j}(x, t)$ shrinks homothetically to a point as $t \rightarrow T=1 / 2 \lambda$. Note that as $t \rightarrow T$, the scalar curvature becomes infinite like $1 /(T-t)$.

By contrast, if the initial metric is an Einstein metric of negative scalar curvature, the metric will expand homothetically for all times. Indeed if

$$
R_{i j}(x, 0)=-\lambda g_{i j}(x, 0)
$$

with $\lambda>0$ and

$$
g_{i j}(x, t)=\rho^{2}(t) g_{i j}(x, 0) .
$$


Then $\rho(t)$ satisfies the ODE

$$
\frac{d \rho}{d t}=\frac{\lambda}{\rho}
$$

with the solution

$$
\rho^{2}(t)=1+2 \lambda t
$$

Hence the evolving metric $g_{i j}(x, t)=\rho^{2}(t) g_{i j}(x, 0)$ exists and expands homothetically for all times, and the curvature will fall back to zero like $-1 / t$. Note that now the evolving metric $g_{i j}(x, t)$ only goes back in time to $-1 / 2 \lambda$, when the metric explodes out of a single point in a "big bang".

(2) Ricci Solitons

We will call a solution to an evolution equation which moves under a oneparameter subgroup of the symmetry group of the equation a steady soliton. The symmetry group of the Ricci flow contains the full diffeomorphism group. Thus a solution to the Ricci flow (1.1.5) which moves by a one-parameter group of diffeomorphisms $\varphi_{t}$ is called a steady Ricci soliton.

If $\varphi_{t}$ is a one-parameter group of diffeomorphisms generated by a vector field $V$ on $M$, then the Ricci soliton is given by

$$
g_{i j}(x, t)=\varphi_{t}^{*} g_{i j}(x, 0)
$$

which implies that the Ricci term -2Ric on the RHS of (1.1.5) is equal to the Lie derivative $\mathcal{L}_{V} g$ of the evolving metric $g$. In particular, the initial metric $g_{i j}(x, 0)$ satisfies the following steady Ricci soliton equation

$$
2 R_{i j}+g_{i k} \nabla_{j} V^{k}+g_{j k} \nabla_{i} V^{k}=0 .
$$

If the vector field $V$ is the gradient of a function $f$ then the soliton is called a steady gradient Ricci soliton. Thus

$$
R_{i j}+\nabla_{i} \nabla_{j} f=0, \quad \text { or } \quad R i c+\nabla^{2} f=0,
$$

is the steady gradient Ricci soliton equation.

Conversely, it is clear that a metric $g_{i j}$ satisfying (1.1.10) generates a steady gradient Ricci soliton $g_{i j}(t)$ given by (1.1.8). For this reason we also often call such a metric $g_{i j}$ a steady gradient Ricci soliton and do not necessarily distinguish it with the solution $g_{i j}(t)$ it generates.

More generally, we can consider a solution to the Ricci flow (1.1.5) which moves by diffeomorphisms and also shrinks or expands by a (time-dependent) factor at the same time. Such a solution is called a homothetically shrinking or homothetically expanding Ricci soliton. The equation for a homothetic Ricci soliton is

$$
2 R_{i j}+g_{i k} \nabla_{j} V^{k}+g_{j k} \nabla_{i} V^{k}-2 \lambda g_{i j}=0,
$$

or for a homothetic gradient Ricci soliton,

$$
R_{i j}+\nabla_{i} \nabla_{j} f-\lambda g_{i j}=0,
$$

where $\lambda$ is the homothetic constant. For $\lambda>0$ the soliton is shrinking, for $\lambda<0$ it is expanding. The case $\lambda=0$ is a steady Ricci soliton, the case $V=0$ (or $f$ being 
a constant function) is an Einstein metric. Thus Ricci solitons can be considered as natural extensions of Einstein metrics. In fact, the following result states that there are no nontrivial gradient steady or expanding Ricci solitons on any compact manifold.

We remark that if the underlying manifold $M$ is a complex manifold and the initial metric is Kähler, then it is well known (see, e.g., $[62,11]$ ) that the solution metric to the Ricci flow (1.1.5) remains Kähler. For this reason, the Ricci flow on a Kähler manifold is called the Kähler-Ricci flow. A (steady, or shrinking, or expanding) Ricci soliton to the Kähler-Ricci flow is called a (steady, or shrinking, or expanding repectively) Kähler-Ricci soliton.

Proposition 1.1.1. On a compact $n$-dimensional manifold $M$, a gradient steady or expanding Ricci soliton is necessarily an Einstein metric.

Proof. We shall only prove the steady case and leave the expanding case as an exercise. Our argument here follows that of Hamilton [63].

Let $g_{i j}$ be a complete steady gradient Ricci soliton on a manifold $M$ so that

$$
R_{i j}+\nabla_{i} \nabla_{j} f=0 .
$$

Taking the trace, we get

$$
R+\Delta f=0
$$

Also, taking the covariant derivatives of the Ricci soliton equation, we have

$$
\nabla_{i} \nabla_{j} \nabla_{k} f-\nabla_{j} \nabla_{i} \nabla_{k} f=\nabla_{j} R_{i k}-\nabla_{i} R_{j k} .
$$

On the other hand, by using the commutating formula (1.1.3), we otain

$$
\nabla_{i} \nabla_{j} \nabla_{k} f-\nabla_{j} \nabla_{i} \nabla_{k} f=R_{i j k l} \nabla_{l} f .
$$

Thus

$$
\nabla_{i} R_{j k}-\nabla_{j} R_{i k}+R_{i j k l} \nabla_{l} f=0 .
$$

Taking the trace on $j$ and $k$, and using the contracted second Bianchi identity

$$
\nabla_{j} R_{i j}=\frac{1}{2} \nabla_{i} R
$$

we get

$$
\nabla_{i} R-2 R_{i j} \nabla_{j} f=0
$$

Then

$$
\nabla_{i}\left(|\nabla f|^{2}+R\right)=2 \nabla_{j} f\left(\nabla_{i} \nabla_{j} f+R_{i j}\right)=0 .
$$

Therefore

$$
R+|\nabla f|^{2}=C
$$

for some constant $C$. 
Taking the difference of (1.1.13) and (1.1.15), we get

$$
\Delta f-|\nabla f|^{2}=-C \text {. }
$$

We claim $C=0$ when $M$ is compact. Indeed, this follows either from

$$
0=-\int_{M} \Delta\left(e^{-f}\right) d V=\int_{M}\left(\Delta f-|\nabla f|^{2}\right) e^{-f} d V
$$

or from considering (1.1.16) at both the maximum point and minimum point of $f$. Then, by integrating (1.1.16) we obtain

$$
\int_{M}|\nabla f|^{2} d V=0
$$

Therefore $f$ is a constant and $g_{i j}$ is Ricci flat.

REMARK 1.1.2. By contrast, there do exist nontrivial compact gradient shrinking Ricci solitons (see Koiso [80], Cao [13] and Wang-Zhu [127] ). Also, there exist complete noncompact steady gradient Ricci solitons that are not Ricci flat. In two dimensions Hamilton [60] wrote down the first such example on $\mathbb{R}^{2}$, called the cigar soliton, where the metric is given by

$$
d s^{2}=\frac{d x^{2}+d y^{2}}{1+x^{2}+y^{2}}
$$

and the vector field is radial, given by $V=-\partial / \partial r=-(x \partial / \partial x+y \partial / \partial y)$. This metric has positive curvature and is asymptotic to a cylinder of finite circumference $2 \pi$ at $\infty$. Higher dimensional examples were found by Robert Bryant [10] on $\mathbb{R}^{n}$ in the Riemannian case, and by the first author [13] on $\mathbb{C}^{n}$ in the Kähler case. These examples are complete, rotationally symmetric, of positive curvature and found by solving certain nonlinear ODE (system). Noncompact expanding solitons were also constructed by the first author [13]. More recently, Feldman, Ilmanen and Knopf [46] constructed new examples of noncompact shrinking and expanding Kähler-Ricci solitons.

1.2. Short-time Existence and Uniqueness. In this section we establish the short-time existence and uniqueness result for the Ricci flow (1.1.5) on a compact $n$ dimensional manifold $M$. We will see that the Ricci flow is a system of second order nonlinear weakly parabolic partial differential equations. In fact, the degeneracy of the system is caused by the diffeomorphism group of $M$ which acts as the gauge group of the Ricci flow. For any diffeomorphism $\varphi$ of $M$, we have $\operatorname{Ric}\left(\varphi^{*}(g)\right)=\varphi^{*}(\operatorname{Ric}(g))$. Thus, if $g(t)$ is a solution to the Ricci flow (1.1.5), so is $\varphi^{*}(g(t))$.

Because the Ricci flow (1.1.5) is only weakly parabolic, even the existence and uniqueness result on a compact manifold does not follow from standard PDE theory. The short-time existence and uniqueness result in the compact case is first proved by Hamilton [58] using the Nash-Moser implicit function theorem. Shortly after Denis De Turck [43] gave a much simpler proof using the gauge fixing idea which we will present here.

In the noncompact case, the short-time existence was established by Shi [114] in 1989, but the uniqueness result has been proved only very recently by Bing-Long Chen and the second author. These results will be presented at the end of this section. 
Let $M$ be a compact $n$-dimensional Riemannian manifold. The Ricci flow equation is a second order nonlinear partial differential system

$$
\frac{\partial}{\partial t} g_{i j}=E\left(g_{i j}\right)
$$

for a family of Riemannian metrics $g_{i j}(\cdot, t)$ on $M$, where

$$
\begin{aligned}
E\left(g_{i j}\right)= & -2 R_{i j} \\
= & -2\left(\frac{\partial}{\partial x^{k}} \Gamma_{i j}^{k}-\frac{\partial}{\partial x^{i}} \Gamma_{k j}^{k}+\Gamma_{k p}^{k} \Gamma_{i j}^{p}-\Gamma_{i p}^{k} \Gamma_{k j}^{p}\right) \\
= & \frac{\partial}{\partial x^{i}}\left\{g^{k l} \frac{\partial}{\partial x^{j}} g_{k l}\right\}-\frac{\partial}{\partial x^{k}}\left\{g^{k l}\left(\frac{\partial}{\partial x^{i}} g_{j l}+\frac{\partial}{\partial x^{j}} g_{i l}-\frac{\partial}{\partial x^{l}} g_{i j}\right)\right\} \\
& +2 \Gamma_{i p}^{k} \Gamma_{k j}^{p}-2 \Gamma_{k p}^{k} \Gamma_{i j}^{p} .
\end{aligned}
$$

The linearization of this system is

$$
\frac{\partial \tilde{g}_{i j}}{\partial t}=D E\left(g_{i j}\right) \tilde{g}_{i j}
$$

where $\tilde{g}_{i j}$ is the variation in $g_{i j}$ and $D E$ is the derivative of $E$ given by

$$
\begin{aligned}
D E\left(g_{i j}\right) \tilde{g}_{i j}= & g^{k l}\left\{\frac{\partial^{2} \tilde{g}_{k l}}{\partial x^{i} \partial x^{j}}-\frac{\partial^{2} \tilde{g}_{j l}}{\partial x^{i} \partial x^{k}}-\frac{\partial^{2} \tilde{g}_{i l}}{\partial x^{j} \partial x^{k}}+\frac{\partial^{2} \tilde{g}_{i j}}{\partial x^{k} \partial x^{l}}\right\} \\
& + \text { (lower order terms). }
\end{aligned}
$$

We now compute the symbol of $D E$. This is to take the highest order derivatives and replace $\frac{\partial}{\partial x^{i}}$ by the Fourier transform variable $\zeta_{i}$. The symbol of the linear differential operator $D E\left(g_{i j}\right)$ in the direction $\zeta=\left(\zeta_{1}, \ldots, \zeta_{n}\right)$ is

$$
\sigma D E\left(g_{i j}\right)(\zeta) \tilde{g}_{i j}=g^{k l}\left(\zeta_{i} \zeta_{j} \tilde{g}_{k l}+\zeta_{k} \zeta_{l} \tilde{g}_{i j}-\zeta_{i} \zeta_{k} \tilde{g}_{j l}-\zeta_{j} \zeta_{k} \tilde{g}_{i l}\right) .
$$

To see what the symbol does, we can always assume $\zeta$ has length 1 and choose coordinates at a point such that

$$
\left\{\begin{array}{l}
g_{i j}=\delta_{i j}, \\
\zeta=(1,0, \ldots, 0) .
\end{array}\right.
$$

Then

$$
\begin{aligned}
\left(\sigma D E\left(g_{i j}\right)(\zeta)\right)\left(\tilde{g}_{i j}\right)= & \tilde{g}_{i j}+\delta_{i 1} \delta_{j 1}\left(\tilde{g}_{11}+\cdots+\tilde{g}_{n n}\right) \\
& -\delta_{i 1} \tilde{g}_{1 j}-\delta_{j 1} \tilde{g}_{1 i}
\end{aligned}
$$

i.e.,

$$
\begin{aligned}
& {\left[\sigma D E\left(g_{i j}\right)(\zeta)\left(\tilde{g}_{i j}\right)\right]_{11}=\tilde{g}_{22}+\cdots+\tilde{g}_{n n},} \\
& {\left[\sigma D E\left(g_{i j}\right)(\zeta)\left(\tilde{g}_{i j}\right)\right]_{1 k}=0, \quad \text { if } k \neq 1,} \\
& {\left[\sigma D E\left(g_{i j}\right)(\zeta)\left(\tilde{g}_{i j}\right)\right]_{k l}=\tilde{g}_{k l}, \quad \text { if } k \neq 1, l \neq 1 .}
\end{aligned}
$$


In particular

$$
\left(\tilde{g}_{i j}\right)=\left(\begin{array}{cccc}
* & * & \cdots & * \\
* & 0 & \cdots & 0 \\
\vdots & \vdots & \ddots & \vdots \\
* & 0 & \cdots & 0
\end{array}\right)
$$

are zero eigenvectors of the symbol.

The presence of the zero eigenvalue shows that the system can not be strictly parabolic. Therefore, instead of considering the system (1.2.1) (or the Ricci flow equation (1.1.5)) we will follow a trick of De Turck[43] to consider a modified evolution equation, which turns out to be strictly parabolic, so that we can apply the standard theory of parabolic equations.

Suppose $\hat{g}_{i j}(x, t)$ is a solution of the Ricci flow (1.1.5), and $\varphi_{t}: M \rightarrow M$ is a family of diffeomorphisms of $M$. Let

$$
g_{i j}(x, t)=\varphi_{t}^{*} \hat{g}_{i j}(x, t)
$$

be the pull-back metrics. We now want to find the evolution equation for the metrics $g_{i j}(x, t)$.

Denote by

$$
y(x, t)=\varphi_{t}(x)=\left\{y^{1}(x, t), y^{2}(x, t), \ldots, y^{n}(x, t)\right\}
$$

in local coordinates. Then

$$
g_{i j}(x, t)=\frac{\partial y^{\alpha}}{\partial x^{i}} \frac{\partial y^{\beta}}{\partial x^{j}} \hat{g}_{\alpha \beta}(y, t)
$$

and

$$
\begin{aligned}
\frac{\partial}{\partial t} g_{i j}(x, t)= & \frac{\partial}{\partial t}\left[\frac{\partial y^{\alpha}}{\partial x^{i}} \frac{\partial y^{\beta}}{\partial x^{j}} \hat{g}_{\alpha \beta}(y, t)\right] \\
= & \frac{\partial y^{\alpha}}{\partial x^{i}} \frac{\partial y^{\beta}}{\partial x^{j}} \frac{\partial}{\partial t} \hat{g}_{\alpha \beta}(y, t)+\frac{\partial}{\partial x^{i}}\left(\frac{\partial y^{\alpha}}{\partial t}\right) \frac{\partial y^{\beta}}{\partial x^{j}} \hat{g}_{\alpha \beta}(y, t) \\
& +\frac{\partial y^{\alpha}}{\partial x^{i}} \frac{\partial}{\partial x^{j}}\left(\frac{\partial y^{\beta}}{\partial t}\right) \hat{g}_{\alpha \beta}(y, t) .
\end{aligned}
$$

Let us choose a normal coordinate $\left\{x^{i}\right\}$ around a fixed point $p \in M$ such that $\frac{\partial g_{i j}}{\partial x^{k}}=0$ at $p$. Since

$$
\frac{\partial}{\partial t} \hat{g}_{\alpha \beta}(y, t)=-2 \hat{R}_{\alpha \beta}(y, t)+\frac{\partial \hat{g}_{\alpha \beta}}{\partial y^{\gamma}} \frac{\partial y^{\gamma}}{\partial t}
$$


we have in the normal coordinate,

$$
\begin{aligned}
\frac{\partial}{\partial t} g_{i j}(x, t) & -2 \frac{\partial y^{\alpha}}{\partial x^{i}} \frac{\partial y^{\beta}}{\partial x^{j}} \hat{R}_{\alpha \beta}(y, t)+\frac{\partial y^{\alpha}}{\partial x^{i}} \frac{\partial y^{\beta}}{\partial x^{j}} \frac{\partial \hat{g}_{\alpha \beta}}{\partial y^{\gamma}} \frac{\partial y^{\gamma}}{\partial t} \\
& +\frac{\partial}{\partial x^{i}}\left(\frac{\partial y^{\alpha}}{\partial t}\right) \frac{\partial y^{\beta}}{\partial x^{j}} \hat{g}_{\alpha \beta}(y, t)+\frac{\partial}{\partial x^{j}}\left(\frac{\partial y^{\beta}}{\partial t}\right) \frac{\partial y^{\alpha}}{\partial x^{i}} \hat{g}_{\alpha \beta}(y, t) \\
= & -2 R_{i j}(x, t)+\frac{\partial y^{\alpha}}{\partial x^{i}} \frac{\partial y^{\beta}}{\partial x^{j}} \frac{\partial \hat{g}_{\alpha \beta}}{\partial y^{\gamma}} \frac{\partial y^{\gamma}}{\partial t}+\frac{\partial}{\partial x^{i}}\left(\frac{\partial y^{\alpha}}{\partial t}\right) \frac{\partial x^{k}}{\partial y^{\alpha}} g_{j k} \\
& +\frac{\partial}{\partial x^{j}}\left(\frac{\partial y^{\beta}}{\partial t}\right) \frac{\partial x^{k}}{\partial y^{\beta}} g_{i k} \\
= & -2 R_{i j}(x, t)+\frac{\partial y^{\alpha}}{\partial x^{i}} \frac{\partial y^{\beta}}{\partial x^{j}} \frac{\partial \hat{g}_{\alpha \beta}}{\partial y^{\gamma}} \frac{\partial y^{\gamma}}{\partial t}+\frac{\partial}{\partial x^{i}}\left(\frac{\partial y^{\alpha}}{\partial t} \frac{\partial x^{k}}{\partial y^{\alpha}} g_{j k}\right) \\
& +\frac{\partial}{\partial x^{j}}\left(\frac{\partial y^{\beta}}{\partial t} \frac{\partial x^{k}}{\partial y^{\beta}} g_{i k}\right)-\frac{\partial y^{\alpha}}{\partial t} \frac{\partial}{\partial x^{i}}\left(\frac{\partial x^{k}}{\partial y^{\alpha}}\right) g_{j k}-\frac{\partial y^{\beta}}{\partial t} \frac{\partial}{\partial x^{j}}\left(\frac{\partial x^{k}}{\partial y^{\beta}}\right) g_{i k} .
\end{aligned}
$$

The second term on the RHS gives, in the normal coordinate,

$$
\begin{aligned}
\frac{\partial y^{\alpha}}{\partial x^{i}} \frac{\partial y^{\beta}}{\partial x^{j}} \frac{\partial y^{\gamma}}{\partial t} \frac{\partial \hat{g}_{\alpha \beta}}{\partial y^{\gamma}} & =\frac{\partial y^{\alpha}}{\partial x^{i}} \frac{\partial y^{\beta}}{\partial x^{j}} \frac{\partial y^{\gamma}}{\partial t} g_{k l} \frac{\partial}{\partial y^{\gamma}}\left(\frac{\partial x^{k}}{\partial y^{\alpha}} \frac{\partial x^{l}}{\partial y^{\beta}}\right) \\
& =\frac{\partial y^{\alpha}}{\partial x^{i}} \frac{\partial y^{\gamma}}{\partial t} \frac{\partial}{\partial y^{\gamma}}\left(\frac{\partial x^{k}}{\partial y^{\alpha}}\right) g_{j k}+\frac{\partial y^{\beta}}{\partial x^{j}} \frac{\partial y^{\gamma}}{\partial t} \frac{\partial}{\partial y^{\gamma}}\left(\frac{\partial x^{k}}{\partial y^{\beta}}\right) g_{i k} \\
& =\frac{\partial y^{\alpha}}{\partial t} \frac{\partial^{2} x^{k}}{\partial y^{\alpha} \partial y^{\beta}} \frac{\partial y^{\beta}}{\partial x^{i}} g_{j k}+\frac{\partial y^{\beta}}{\partial t} \frac{\partial^{2} x^{k}}{\partial y^{\alpha} \partial y^{\beta}} \frac{\partial y^{\alpha}}{\partial x^{j}} g_{i k} \\
& =\frac{\partial y^{\alpha}}{\partial t} \frac{\partial}{\partial x^{i}}\left(\frac{\partial x^{k}}{\partial y^{\alpha}}\right) g_{j k}+\frac{\partial y^{\beta}}{\partial t} \frac{\partial}{\partial x^{j}}\left(\frac{\partial x^{k}}{\partial y^{\beta}}\right) g_{i k}
\end{aligned}
$$

So we get

$$
\begin{aligned}
& \frac{\partial}{\partial t} g_{i j}(x, t) \\
& =-2 R_{i j}(x, t)+\nabla_{i}\left(\frac{\partial y^{\alpha}}{\partial t} \frac{\partial x^{k}}{\partial y^{\alpha}} g_{j k}\right)+\nabla_{j}\left(\frac{\partial y^{\beta}}{\partial t} \frac{\partial x^{k}}{\partial y^{\beta}} g_{i k}\right) .
\end{aligned}
$$

If we define $y(x, t)=\varphi_{t}(x)$ by the equations

$$
\left\{\begin{array}{l}
\frac{\partial y^{\alpha}}{\partial t}=\frac{\partial y^{\alpha}}{\partial x^{k}} g^{j l}\left(\Gamma_{j l}^{k}-\stackrel{o}{\Gamma}_{j l}^{k}\right), \\
y^{\alpha}(x, 0)=x^{\alpha}
\end{array}\right.
$$

and $V_{i}=g_{i k} g^{j l}\left(\Gamma_{j l}^{k}-\stackrel{o}{\Gamma}_{j l}^{k}\right)$, we get the following evolution equation for the pull-back metric

$$
\left\{\begin{array}{l}
\frac{\partial}{\partial t} g_{i j}(x, t)=-2 R_{i j}(x, t)+\nabla_{i} V_{j}+\nabla_{j} V_{i}, \\
g_{i j}(x, 0)=\stackrel{o}{g_{i j}}(x),
\end{array}\right.
$$


where $\stackrel{o}{g}_{i j}(x)$ is the initial metric and $\stackrel{o}{\Gamma}_{j l}^{k}$ is the connection of the initial metric.

Lemma 1.2.1. The modified evolution equation (1.2.5) is a strictly parabolic system.

Proof. The RHS of the equation (1.2.5) is given by

$$
\begin{aligned}
- & 2 R_{i j}(x, t)+\nabla_{i} V_{j}+\nabla_{j} V_{i} \\
= & \frac{\partial}{\partial x^{i}}\left\{g^{k l} \frac{\partial g_{k l}}{\partial x^{j}}\right\}-\frac{\partial}{\partial x^{k}}\left\{g^{k l}\left(\frac{\partial g_{j l}}{\partial x^{i}}+\frac{\partial g_{i l}}{\partial x^{j}}-\frac{\partial g_{i j}}{\partial x^{l}}\right)\right\} \\
& +g_{j k} g^{p q} \frac{\partial}{\partial x^{i}}\left\{\frac{1}{2} g^{k l}\left(\frac{\partial g_{p l}}{\partial x^{q}}+\frac{\partial g_{q l}}{\partial x^{p}}-\frac{\partial g_{p q}}{\partial x^{l}}\right)\right\} \\
& +g_{i k} g^{p q} \frac{\partial}{\partial x^{j}}\left\{\frac{1}{2} g^{k l}\left(\frac{\partial g_{p l}}{\partial x^{q}}+\frac{\partial g_{q l}}{\partial x^{p}}-\frac{\partial g_{p q}}{\partial x^{l}}\right)\right\} \\
& +(\text { lower order terms }) \\
= & g^{k l}\left\{\frac{\partial^{2} g_{k l}}{\partial x^{i} \partial x^{j}}-\frac{\partial^{2} g_{j l}}{\partial x^{i} \partial x^{k}}-\frac{\partial^{2} g_{i l}}{\partial x^{j} \partial x^{k}}+\frac{\partial^{2} g_{i j}}{\partial x^{k} \partial x^{l}}\right\} \\
& +\frac{1}{2} g^{p q}\left\{\frac{\partial^{2} g_{p j}}{\partial x^{i} \partial x^{q}}+\frac{\partial^{2} g_{q j}}{\partial x^{i} \partial x^{p}}-\frac{\partial^{2} g_{p q}}{\partial x^{i} \partial x^{j}}\right\} \\
& +\frac{1}{2} g^{p q}\left\{\frac{\partial^{2} g_{p i}}{\partial x^{j} \partial x^{q}}+\frac{\partial^{2} g_{q i}}{\partial x^{j} \partial x^{p}}-\frac{\partial^{2} g_{p q}}{\partial x^{i} \partial x^{j}}\right\} \\
& +(\text { lower order terms }) \\
= & g^{k l} \frac{\partial^{2} g_{i j}}{\partial x^{k} \partial x^{l}}+\text { (lower order terms). }
\end{aligned}
$$

Thus its symbol is $\left(g^{k l} \zeta_{k} \zeta_{l}\right) \tilde{g}_{i j}$. Hence the equation in (1.2.5) is strictly parabolic. $\square$

Now since the equation (1.2.5) is strictly parabolic and the manifold $M$ is compact, it follows from the standard theory of parabolic equations (see for example [81]) that (1.2.5) has a solution for a short time. From the solution of (1.2.5) we can obtain a solution of the Ricci flow from (1.2.4) and (1.2.2). This shows existence. Now we argue the uniqueness of the solution. Since

$$
\Gamma_{j l}^{k}=\frac{\partial y^{\alpha}}{\partial x^{j}} \frac{\partial y^{\beta}}{\partial x^{l}} \frac{\partial x^{k}}{\partial y^{\gamma}} \hat{\Gamma}_{\alpha \beta}^{\gamma}+\frac{\partial x^{k}}{\partial y^{\alpha}} \frac{\partial^{2} y^{\alpha}}{\partial x^{j} \partial x^{l}}
$$

the initial value problem (1.2.4) can be written as

$$
\left\{\begin{array}{l}
\frac{\partial y^{\alpha}}{\partial t}=g^{j l}\left(\frac{\partial^{2} y^{\alpha}}{\partial x^{j} \partial x^{l}}-\stackrel{o}{\Gamma}_{j l}^{k} \frac{\partial y^{\alpha}}{\partial x^{k}}+\hat{\Gamma}_{\gamma \beta}^{\alpha} \frac{\partial y^{\beta}}{\partial x^{j}} \frac{\partial y^{\gamma}}{\partial x^{l}}\right), \\
y^{\alpha}(x, 0)=x^{\alpha}
\end{array}\right.
$$

This is clearly a strictly parabolic system. For any two solutions $\hat{g}_{i j}^{(1)}(\cdot, t)$ and $\hat{g}_{i j}^{(2)}(\cdot, t)$ of the Ricci flow (1.1.5) with the same initial data, we can solve the initial value problem (1.2.6) (or equivalently, (1.2.4)) to get two families $\varphi_{t}^{(1)}$ and $\varphi_{t}^{(2)}$ of diffeomorphisms of $M$. Thus we get two solutions, $g_{i j}^{(1)}(\cdot, t)=\left(\varphi_{t}^{(1)}\right)^{*} \hat{g}_{i j}^{(1)}(\cdot, t)$ and $g_{i j}^{(2)}(\cdot, t)=\left(\varphi_{t}^{(2)}\right)^{*} \hat{g}_{i j}^{(2)}(\cdot, t)$, to the modified evolution equation (1.2.5) with the same 
initial metric. The uniqueness result for the strictly parabolic equation (1.2.5) implies that $g_{i j}^{(1)}=g_{i j}^{(2)}$. Then by equation (1.2.4) and the standard uniqueness result of ODE systems, the corresponding solutions $\varphi_{t}^{(1)}$ and $\varphi_{t}^{(2)}$ of (1.2.4) (or equivalently (1.2.6)) must agree. Consequently the metrics $\hat{g}_{i j}^{(1)}$ and $\hat{g}_{i j}^{(2)}$ must agree also. Thus we have proved the following result.

Theorem 1.2.2 (Hamilton [58], De Turck [43]). Let $\left(M, g_{i j}(x)\right)$ be a compact Riemannian manifold. Then there exists a constant $T>0$ such that the initial value problem

$$
\left\{\begin{array}{l}
\frac{\partial}{\partial t} g_{i j}(x, t)=-2 R_{i j}(x, t) \\
g_{i j}(x, 0)=g_{i j}(x)
\end{array}\right.
$$

has a unique smooth solution $g_{i j}(x, t)$ on $M \times[0, T)$.

The case of a noncompact manifold is much more complicated and involves a huge amount of techniques from the theory of partial differential equations. Here we will only state the existence and uniqueness results and refer the reader to the cited references for the proofs.

The following existence result was obtained by Shi [114] in his thesis published in 1989.

TheOREm 1.2.3 (Shi [114]). Let $\left(M, g_{i j}(x)\right)$ be a complete noncompact Riemannian manifold of dimension $n$ with bounded curvature. Then there exists a constant $T>0$ such that the initial value problem

$$
\left\{\begin{array}{l}
\frac{\partial}{\partial t} g_{i j}(x, t)=-2 R_{i j}(x, t) \\
g_{i j}(x, 0)=g_{i j}(x)
\end{array}\right.
$$

has a smooth solution $g_{i j}(x, t)$ on $M \times[0, T]$ with uniformly bounded curvature.

The Ricci flow is a heat type equation. It is well-known that the uniqueness of a heat equation on a complete noncompact manifold is not always held if there are no further restrictions on the growth of the solutions. For example, the heat equation on Euclidean space with zero initial data has a nontrivial solution which grows faster than $\exp \left(a|x|^{2}\right)$ for any $a>0$ whenever $t>0$. This implies that even for the standard linear heat equation on Euclidean space, in order to ensure the uniqueness one can only allow the solution to grow at most as $\exp \left(C|x|^{2}\right)$ for some constant $C>0$. Note that on a Kähler manifold, the Ricci curvature is given by $R_{\alpha \bar{\beta}}=-\frac{\partial^{2}}{\partial z^{\alpha} \partial \bar{z}^{\beta}} \log \operatorname{det}\left(g_{\gamma \bar{\delta}}\right)$. So the reasonable growth rate for the uniqueness of the Ricci flow to hold is that the solution has bounded curvature. Thus the following uniqueness result of Bing-Long Chen and the second author [33] is essentially the best one can hope for.

Theorem 1.2.4 (Chen-Zhu [33]). Let $\left(M, \hat{g}_{i j}\right)$ be a complete noncompact Riemannian manifold of dimension $n$ with bounded curvature. Let $g_{i j}(x, t)$ and $\bar{g}_{i j}(x, t)$ be two solutions, defined on $M \times[0, T]$, to the Ricci flow (1.1.5) with $\hat{g}_{i j}$ as initial data and with bounded curvatures. Then $g_{i j}(x, t) \equiv \bar{g}_{i j}(x, t)$ on $M \times[0, T]$. 
1.3. Evolution of Curvatures. The Ricci flow is an evolution equation on the metric. The evolution for the metric implies a nonlinear heat equation for the Riemannian curvature tensor $R_{i j k l}$ which we will now derive.

Proposition 1.3.1 (Hamilton [58]). Under the Ricci flow (1.1.5), the curvature tensor satisfies the evolution equation

$$
\begin{aligned}
\frac{\partial}{\partial t} R_{i j k l}= & \Delta R_{i j k l}+2\left(B_{i j k l}-B_{i j l k}-B_{i l j k}+B_{i k j l}\right) \\
& -g^{p q}\left(R_{p j k l} R_{q i}+R_{i p k l} R_{q j}+R_{i j p l} R_{q k}+R_{i j k p} R_{q l}\right)
\end{aligned}
$$

where $B_{i j k l}=g^{p r} g^{q s} R_{p i q j} R_{r k s l}$ and $\Delta$ is the Laplacian with respect to the evolving metric.

Proof. Choose $\left\{x^{1}, \ldots, x^{m}\right\}$ to be a normal coordinate system at a fixed point. At this point, we compute

$$
\begin{aligned}
\frac{\partial}{\partial t} \Gamma_{j l}^{h} & =\frac{1}{2} g^{h m}\left\{\frac{\partial}{\partial x^{j}}\left(\frac{\partial}{\partial t} g_{l m}\right)+\frac{\partial}{\partial x^{l}}\left(\frac{\partial}{\partial t} g_{j m}\right)-\frac{\partial}{\partial x^{m}}\left(\frac{\partial}{\partial t} g_{j l}\right)\right\} \\
& =\frac{1}{2} g^{h m}\left(\nabla_{j}\left(-2 R_{l m}\right)+\nabla_{l}\left(-2 R_{j m}\right)-\nabla_{m}\left(-2 R_{j l}\right)\right), \\
\frac{\partial}{\partial t} R_{i j l}^{h} & =\frac{\partial}{\partial x^{i}}\left(\frac{\partial}{\partial t} \Gamma_{j l}^{h}\right)-\frac{\partial}{\partial x^{j}}\left(\frac{\partial}{\partial t} \Gamma_{i l}^{h}\right), \\
\frac{\partial}{\partial t} R_{i j k l} & =g_{h k} \frac{\partial}{\partial t} R_{i j l}^{h}+\frac{\partial g_{h k}}{\partial t} R_{i j l}^{h} .
\end{aligned}
$$

Combining these identities we get

$$
\begin{aligned}
\frac{\partial}{\partial t} R_{i j k l}= & g_{h k}\left[\left(\frac{1}{2} \nabla_{i}\left[g^{h m}\left(\nabla_{j}\left(-2 R_{l m}\right)+\nabla_{l}\left(-2 R_{j m}\right)-\nabla_{m}\left(-2 R_{j l}\right)\right)\right]\right)\right. \\
& \left.-\left(\frac{1}{2} \nabla_{j}\left[g^{h m}\left(\nabla_{i}\left(-2 R_{l m}\right)+\nabla_{l}\left(-2 R_{i m}\right)-\nabla_{m}\left(-2 R_{i l}\right)\right)\right]\right)\right] \\
& -2 R_{h k} R_{i j l}^{h} \\
= & \nabla_{i} \nabla_{k} R_{j l}-\nabla_{i} \nabla_{l} R_{j k}-\nabla_{j} \nabla_{k} R_{i l}+\nabla_{j} \nabla_{l} R_{i k} \\
& -R_{i j l p} g^{p q} R_{q k}-R_{i j k p} g^{p q} R_{q l}-2 R_{i j p l} g^{p q} R_{q k} \\
= & \nabla_{i} \nabla_{k} R_{j l}-\nabla_{i} \nabla_{l} R_{j k}-\nabla_{j} \nabla_{k} R_{i l}+\nabla_{j} \nabla_{l} R_{i k} \\
& -g^{p q}\left(R_{i j k p} R_{q l}+R_{i j p l} R_{q k}\right) .
\end{aligned}
$$

Here we have used the exchanging formula (1.1.3).

Now it remains to check the following identity, which is analogous to the Simon's identity in extrinsic geometry,

$$
\begin{aligned}
& \Delta R_{i j k l}+2\left(B_{i j k l}-B_{i j l k}-B_{i l j k}+B_{i k j l}\right) \\
& =\nabla_{i} \nabla_{k} R_{j l}-\nabla_{i} \nabla_{l} R_{j k}-\nabla_{j} \nabla_{k} R_{i l}+\nabla_{j} \nabla_{l} R_{i k} \\
& \quad+g^{p q}\left(R_{p j k l} R_{q i}+R_{i p k l} R_{q j}\right) .
\end{aligned}
$$

Indeed, from the second Bianchi identity (1.1.4), we have

$$
\begin{aligned}
\Delta R_{i j k l} & =g^{p q} \nabla_{p} \nabla_{q} R_{i j k l} \\
& =g^{p q} \nabla_{p} \nabla_{i} R_{q j k l}-g^{p q} \nabla_{p} \nabla_{j} R_{q i k l} .
\end{aligned}
$$


Let us examine the first term on the RHS. By using the exchanging formula (1.1.3) and the first Bianchi identity (1.1.1), we have

$$
\begin{aligned}
& g^{p q} \nabla_{p} \nabla_{i} R_{q j k l}-g^{p q} \nabla_{i} \nabla_{p} R_{q j k l} \\
& =g^{p q} g^{m n}\left(R_{p i q m} R_{n j k l}+R_{p i j m} R_{q n k l}+R_{p i k m} R_{q j n l}+R_{p i l m} R_{q j k n}\right) \\
& =R_{i m} g^{m n} R_{n j k l}+g^{p q} g^{m n} R_{p i m j}\left(R_{q k l n}+R_{q l n k}\right) \\
& \quad+g^{p q} g^{m n} R_{p i k m} R_{q j n l}+g^{p q} g^{m n} R_{p i l m} R_{q j k n} \\
& =R_{i m} g^{m n} R_{n j k l}-B_{i j k l}+B_{i j l k}-B_{i k j l}+B_{i l j k},
\end{aligned}
$$

while using the contracted second Bianchi identity

$$
g^{p q} \nabla_{p} R_{q j k l}=\nabla_{k} R_{j l}-\nabla_{l} R_{j k},
$$

we have

$$
g^{p q} \nabla_{i} \nabla_{p} R_{q j k l}=\nabla_{i} \nabla_{k} R_{j l}-\nabla_{i} \nabla_{l} R_{j k} .
$$

Thus

$$
\begin{aligned}
& g^{p q} \nabla_{p} \nabla_{i} R_{q j k l} \\
& \quad=\nabla_{i} \nabla_{k} R_{j l}-\nabla_{i} \nabla_{l} R_{j k}-\left(B_{i j k l}-B_{i j l k}-B_{i l j k}+B_{i k j l}\right)+g^{p q} R_{p j k l} R_{q i} .
\end{aligned}
$$

Therefore we obtain

$$
\begin{aligned}
\Delta & R_{i j k l} \\
= & g^{p q} \nabla_{p} \nabla_{i} R_{q j k l}-g^{p q} \nabla_{p} \nabla_{j} R_{q i k l} \\
= & \nabla_{i} \nabla_{k} R_{j l}-\nabla_{i} \nabla_{l} R_{j k}-\left(B_{i j k l}-B_{i j l k}-B_{i l j k}+B_{i k j l}\right)+g^{p q} R_{p j k l} R_{q i} \\
& -\nabla_{j} \nabla_{k} R_{i l}+\nabla_{j} \nabla_{l} R_{i k}+\left(B_{j i k l}-B_{j i l k}-B_{j l i k}+B_{j k i l}\right)-g^{p q} R_{p i k l} R_{q j} \\
= & \nabla_{i} \nabla_{k} R_{j l}-\nabla_{i} \nabla_{l} R_{j k}-\nabla_{j} \nabla_{k} R_{i l}+\nabla_{j} \nabla_{l} R_{i k} \\
& +g^{p q}\left(R_{p j k l} R_{q i}+R_{i p k l} R_{q j}\right)-2\left(B_{i j k l}-B_{i j l k}-B_{i l j k}+B_{i k j l}\right)
\end{aligned}
$$

as desired, where in the last step we used the symmetries

$$
B_{i j k l}=B_{k l i j}=B_{j i l k} .
$$

CoROllary 1.3.2. The Ricci curvature satisfies the evolution equation

$$
\frac{\partial}{\partial t} R_{i k}=\Delta R_{i k}+2 g^{p r} g^{q s} R_{p i q k} R_{r s}-2 g^{p q} R_{p i} R_{q k} .
$$

Proof.

$$
\begin{aligned}
\frac{\partial}{\partial t} R_{i k}= & g^{j l} \frac{\partial}{\partial t} R_{i j k l}+\left(\frac{\partial}{\partial t} g^{j l}\right) R_{i j k l} \\
= & g^{j l}\left[\Delta R_{i j k l}+2\left(B_{i j k l}-B_{i j l k}-B_{i l j k}+B_{i k j l}\right)\right. \\
& \left.-g^{p q}\left(R_{p j k l} R_{q i}+R_{i p k l} R_{q j}+R_{i j p l} R_{q k}+R_{i j k p} R_{q l}\right)\right] \\
& -g^{j p}\left(\frac{\partial}{\partial t} g_{p q}\right) g^{q l} R_{i j k l} \\
= & \Delta R_{i k}+2 g^{j l}\left(B_{i j k l}-2 B_{i j l k}\right)+2 g^{p r} g^{q s} R_{p i q k} R_{r s} \\
& -2 g^{p q} R_{p k} R_{q i} .
\end{aligned}
$$


We claim that $g^{j l}\left(B_{i j k l}-2 B_{i j l k}\right)=0$. Indeed by using the first Bianchi identity, we have

$$
\begin{aligned}
g^{j l} B_{i j k l} & =g^{j l} g^{p r} g^{q s} R_{p i q j} R_{r k s l} \\
& =g^{j l} g^{p r} g^{q s} R_{p q i j} R_{r s k l} \\
& =g^{j l} g^{p r} g^{q s}\left(R_{p i q j}-R_{p j q i}\right)\left(R_{r k s l}-R_{r l s k}\right) \\
& =2 g^{j l}\left(B_{i j k l}-B_{i j l k}\right)
\end{aligned}
$$

as desired.

Thus we obtain

$$
\frac{\partial}{\partial t} R_{i k}=\Delta R_{i k}+2 g^{p r} g^{q s} R_{p i q k} R_{r s}-2 g^{p q} R_{p i} R_{q k} .
$$

COROLlaRY 1.3.3. The scalar curvature satisfies the evolution equation

$$
\frac{\partial R}{\partial t}=\Delta R+2|\operatorname{Ric}|^{2}
$$

Proof.

$$
\begin{aligned}
\frac{\partial R}{\partial t} & =g^{i k} \frac{\partial R_{i k}}{\partial t}+\left(-g^{i p} \frac{\partial g_{p q}}{\partial t} g^{q k}\right) R_{i k} \\
& =g^{i k}\left(\Delta R_{i k}+2 g^{p r} g^{q s} R_{p i q k} R_{r s}-2 g^{p q} R_{p i} R_{q k}\right)+2 R_{p q} R_{i k} g^{i p} g^{q k} \\
& =\Delta R+2|\operatorname{Ric}|^{2} .
\end{aligned}
$$

To simplify the evolution equations of curvatures, we will represent the curvature tensors in an orthonormal frame and evolve the frame so that it remains orthonormal. More precisely, let us pick an abstract vector bundle $V$ over $M$ isomorphic to the tangent bundle $T M$. Locally, the frame $F=\left\{F_{1}, \ldots, F_{a}, \ldots, F_{n}\right\}$ of $V$ is given by $F_{a}=F_{a}^{i} \frac{\partial}{\partial x^{i}}$ with the isomorphism $\left\{F_{a}^{i}\right\}$. Choose $\left\{F_{a}^{i}\right\}$ at $t=0$ such that $F=\left\{F_{1}, \ldots, F_{a}, \ldots, F_{n}\right\}$ is an orthonormal frame at $t=0$, and evolve $\left\{F_{a}^{i}\right\}$ by the equation

$$
\frac{\partial}{\partial t} F_{a}^{i}=g^{i j} R_{j k} F_{a}^{k}
$$

Then the frame $F=\left\{F_{1}, \ldots, F_{a}, \ldots, F_{n}\right\}$ will remain orthonormal for all times since the pull back metric on $V$

$$
h_{a b}=g_{i j} F_{a}^{i} F_{b}^{j}
$$

remains constant in time. In the following we will use indices $a, b, \ldots$ on a tensor to denote its components in the evolving orthonormal frame. In this frame we have the 
following:

$$
\begin{aligned}
R_{a b c d} & =F_{a}^{i} F_{b}^{j} F_{c}^{k} F_{d}^{l} R_{i j k l}, \\
\Gamma_{j b}^{a} & =F_{i}^{a} \frac{\partial F_{b}^{i}}{\partial x^{j}}+\Gamma_{j k}^{i} F_{i}^{a} F_{b}^{k}, \quad\left(\left(F_{i}^{a}\right)=\left(F_{a}^{i}\right)^{-1}\right) \\
\nabla_{i} V^{a} & =\frac{\partial}{\partial x^{i}} V^{a}+\Gamma_{i b}^{a} V^{b}, \\
\nabla_{b} V^{a} & =F_{b}^{i} \nabla_{i} V^{a},
\end{aligned}
$$

where $\Gamma_{j b}^{a}$ is the metric connection of the vector bundle $V$ with the metric $h_{a b}$. Indeed, by direct computations,

$$
\begin{aligned}
\nabla_{i} F_{b}^{j} & =\frac{\partial F_{b}^{j}}{\partial x^{i}}+F_{b}^{k} \Gamma_{i k}^{j}-F_{c}^{j} \Gamma_{i b}^{c} \\
& =\frac{\partial F_{b}^{j}}{\partial x^{i}}+F_{b}^{k} \Gamma_{i k}^{j}-F_{c}^{j}\left(F_{k}^{c} \frac{\partial F_{b}^{k}}{\partial x^{i}}+\Gamma_{i k}^{l} F_{l}^{c} F_{b}^{k}\right) \\
& =0, \\
\nabla_{i} h_{a b} & =\nabla_{i}\left(g_{i j} F_{a}^{i} F_{b}^{j}\right)=0 .
\end{aligned}
$$

So

$$
\nabla_{a} V_{b}=F_{a}^{i} F_{b}^{j} \nabla_{i} V^{j}
$$

and

$$
\begin{aligned}
\Delta R_{a b c d} & =\nabla_{l} \nabla_{l} R_{a b c d} \\
& =g^{i j} \nabla_{i} \nabla_{j} R_{a b c d} \\
& =g^{i j} F_{a}^{k} F_{b}^{l} F_{c}^{m} F_{d}^{n} \nabla_{i} \nabla_{j} R_{k l m n} .
\end{aligned}
$$

In an orthonormal frame $F=\left\{F_{1}, \ldots, F_{a}, \ldots, F_{n}\right\}$, the evolution equations of curvature tensors become

$$
\begin{aligned}
\frac{\partial}{\partial t} R_{a b c d} & =\Delta R_{a b c d}+2\left(B_{a b c d}-B_{a b d c}-B_{a d b c}+B_{a c b d}\right) \\
\frac{\partial}{\partial t} R_{a b} & =\Delta R_{a b}+2 R_{a c b d} R_{c d} \\
\frac{\partial}{\partial t} R & =\Delta R+2|\operatorname{Ric}|^{2}
\end{aligned}
$$

where $B_{a b c d}=R_{a e b f} R_{c e d f}$.

Equation (1.3.4) is a reaction-diffusion equation. We can understand the quadratic terms of this equation better if we think of the curvature tensor $R_{a b c d}$ as a symmetric bilinear form on the two-forms $\Lambda^{2}(V)$ given by the formula

$$
R m(\varphi, \psi)=R_{a b c d} \varphi_{a b} \psi_{c d}, \quad \text { for } \varphi, \psi \in \Lambda^{2}(V) .
$$

A two-form $\varphi \in \Lambda^{2}(V)$ can be regarded as an element of the Lie algebra $s o(n)$ (i.e. the skew-symmetric matrix $\left.\left(\varphi_{a b}\right)_{n \times n}\right)$, where the metric on $\Lambda^{2}(V)$ is given by

$$
\langle\varphi, \psi\rangle=\varphi_{a b} \psi_{a b}
$$


and the Lie bracket is given by

$$
[\varphi, \psi]_{a b}=\varphi_{a c} \psi_{b c}-\psi_{a c} \varphi_{b c}
$$

Choose an orthonormal basis of $\Lambda^{2}(V)$

$$
\Phi=\left\{\varphi^{1}, \ldots, \varphi^{\alpha}, \ldots, \varphi^{\frac{n(n-1)}{2}}\right\}
$$

where $\varphi^{\alpha}=\left\{\varphi_{a b}^{\alpha}\right\}$. The Lie bracket is given by

$$
\left[\varphi^{\alpha}, \varphi^{\beta}\right]=C_{\gamma}^{\alpha \beta} \varphi^{\gamma}
$$

where $C^{\alpha \beta \gamma}=C_{\sigma}^{\alpha \beta} \delta^{\sigma \gamma}=\left\langle\left[\varphi^{\alpha}, \varphi^{\beta}\right], \varphi^{\gamma}\right\rangle$ are the Lie structure constants.

Write $R_{a b c d}=M_{\alpha \beta} \varphi_{a b}^{\alpha} \varphi_{c d}^{\beta}$. We now claim that the first part of the quadratic terms in (1.3.4) is given by

$$
2\left(B_{a b c d}-B_{a b d c}\right)=M_{\alpha \gamma} M_{\beta \gamma} \varphi_{a b}^{\alpha} \varphi_{c d}^{\beta} .
$$

Indeed, by the first Bianchi identity,

$$
\begin{aligned}
B_{a b c d}-B_{a b d c} & =R_{a e b f} R_{c e d f}-R_{a e b f} R_{d e c f} \\
& =R_{a e b f}\left(-R_{c e f d}-R_{c f d e}\right) \\
& =R_{a e b f} R_{c d e f}
\end{aligned}
$$

On the other hand,

$$
\begin{aligned}
R_{a e b f} R_{c d e f} & =\left(-R_{a b f e}-R_{a f e b}\right) R_{c d e f} \\
& =R_{a b e f} R_{c d e f}-R_{a f e b} R_{c d e f} \\
& =R_{a b e f} R_{c d e f}-R_{a f b e} R_{c d f e}
\end{aligned}
$$

which implies $R_{a e b f} R_{c d e f}=\frac{1}{2} R_{a b e f} R_{c d e f}$. Thus we obtain

$$
2\left(B_{a b c d}-B_{a b d c}\right)=R_{a b e f} R_{c d e f}=M_{\alpha \gamma} M_{\beta \gamma} \varphi_{a b}^{\alpha} \varphi_{c d}^{\beta} .
$$

We next consider the last part of the quadratic terms:

$$
\begin{aligned}
& 2\left(B_{a c b d}-B_{a d b c}\right) \\
& =2\left(R_{a e c f} R_{b e d f}-R_{a e d f} R_{b e c f}\right) \\
& =2\left(M_{\gamma \delta} \varphi_{a e}^{\gamma} \varphi_{c f}^{\delta} M_{\eta \theta} \varphi_{b e}^{\eta} \varphi_{d f}^{\theta}-M_{\gamma \theta} \varphi_{a e}^{\gamma} \varphi_{d f}^{\theta} M_{\eta \delta} \varphi_{b e}^{\eta} \varphi_{c f}^{\delta}\right) \\
& =2\left[M_{\gamma \delta}\left(\varphi_{a e}^{\eta} \varphi_{b e}^{\gamma}+C_{\alpha}^{\gamma \eta} \varphi_{a b}^{\alpha}\right) \varphi_{c f}^{\delta} M_{\eta \theta} \varphi_{d f}^{\theta}-M_{\eta \theta} \varphi_{a e}^{\eta} \varphi_{d f}^{\theta} M_{\gamma \delta} \varphi_{b e}^{\gamma} \varphi_{c f}^{\delta}\right] \\
& =2 M_{\gamma \delta} \varphi_{c f}^{\delta} M_{\eta \theta} \varphi_{d f}^{\theta} C_{\alpha}^{\gamma \eta} \varphi_{a b}^{\alpha} .
\end{aligned}
$$

But

$$
\begin{aligned}
& M_{\gamma \delta} \varphi_{c f}^{\delta} M_{\eta \theta} \varphi_{d f}^{\theta} C_{\alpha}^{\gamma \eta} \varphi_{a b}^{\alpha} \\
& =M_{\gamma \delta} M_{\eta \theta} C_{\alpha}^{\gamma \eta} \varphi_{a b}^{\alpha}\left[\varphi_{c f}^{\theta} \varphi_{d f}^{\delta}+C_{\beta}^{\delta \theta} \varphi_{c d}^{\beta}\right] \\
& =-M_{\eta \theta} M_{\gamma \delta} C_{\alpha}^{\gamma \eta} \varphi_{a b}^{\alpha} \varphi_{c f}^{\delta} \varphi_{d f}^{\theta}+M_{\gamma \delta} M_{\eta \theta} C_{\alpha}^{\gamma \eta} C_{\beta}^{\delta \theta} \varphi_{a b}^{\alpha} \varphi_{c d}^{\beta} \\
& =-M_{\eta \theta} M_{\gamma \delta} C_{\alpha}^{\gamma \eta} \varphi_{a b}^{\alpha} \varphi_{c f}^{\delta} \varphi_{d f}^{\theta}+\left(C_{\alpha}^{\gamma \eta} C_{\beta}^{\delta \theta} M_{\gamma \delta} M_{\eta \theta}\right) \varphi_{a b}^{\alpha} \varphi_{c d}^{\beta}
\end{aligned}
$$


which implies

$$
M_{\gamma \delta} \varphi_{c f}^{\delta} M_{\eta \theta} \varphi_{d f}^{\theta} C_{\alpha}^{\gamma \eta} \varphi_{a b}^{\alpha}=\frac{1}{2}\left(C_{\alpha}^{\gamma \eta} C_{\beta}^{\delta \theta} M_{\gamma \delta} M_{\eta \theta}\right) \varphi_{a b}^{\alpha} \varphi_{c d}^{\beta}
$$

Then we have

$$
2\left(B_{a c b d}-B_{a d b c}\right)=\left(C_{\alpha}^{\gamma \eta} C_{\beta}^{\delta \theta} M_{\gamma \delta} M_{\eta \theta}\right) \varphi_{a b}^{\alpha} \varphi_{c d}^{\beta} .
$$

Therefore, combining (1.3.7) and (1.3.8), we can reformulate the curvature evolution equation (1.3.4) as follows.

Proposition 1.3.4 (Hamilton [59]). Let $R_{a b c d}=M_{\alpha \beta} \varphi_{a b}^{\alpha} \varphi_{c d}^{\beta}$. Then under the Ricci flow (1.1.5), $M_{\alpha \beta}$ satisfies the evolution equation

$$
\frac{\partial M_{\alpha \beta}}{\partial t}=\Delta M_{\alpha \beta}+M_{\alpha \beta}^{2}+M_{\alpha \beta}^{\#}
$$

where $M_{\alpha \beta}^{2}=M_{\alpha \gamma} M_{\beta \gamma}$ is the operator square and $M_{\alpha \beta}^{\#}=\left(C_{\alpha}^{\gamma \eta} C_{\beta}^{\delta \theta} M_{\gamma \delta} M_{\eta \theta}\right)$ is the Lie algebra square.

Let us now consider the operator $M_{\alpha \beta}^{\#}$ in dimensions 3 and 4 in more detail.

In dimension 3 , let $\omega_{1}, \omega_{2}, \omega_{3}$ be a positively oriented orthonormal basis for oneforms. Then

$$
\varphi^{1}=\sqrt{2} \omega_{1} \wedge \omega_{2}, \quad \varphi^{2}=\sqrt{2} \omega_{2} \wedge \omega_{3}, \quad \varphi^{3}=\sqrt{2} \omega_{3} \wedge \omega_{1}
$$

form an orthonormal basis for two-forms $\Lambda^{2}$. Write $\varphi^{\alpha}=\left\{\varphi_{a b}^{\alpha}\right\}, \alpha=1,2,3$, as

$$
\begin{gathered}
\left(\varphi_{a b}^{1}\right)=\left(\begin{array}{ccc}
0 & \frac{\sqrt{2}}{2} & 0 \\
-\frac{\sqrt{2}}{2} & 0 & 0 \\
0 & 0 & 0
\end{array}\right), \quad\left(\varphi_{a b}^{2}\right)=\left(\begin{array}{ccc}
0 & 0 & 0 \\
0 & 0 & \frac{\sqrt{2}}{2} \\
0 & -\frac{\sqrt{2}}{2} & 0
\end{array}\right), \\
\left(\varphi_{a b}^{3}\right)=\left(\begin{array}{ccc}
0 & 0 & -\frac{\sqrt{2}}{2} \\
0 & 0 & 0 \\
\frac{\sqrt{2}}{2} & 0 & 0
\end{array}\right),
\end{gathered}
$$

then

$$
\begin{aligned}
{\left[\varphi^{1}, \varphi^{2}\right] } & =\left(\begin{array}{ccc}
0 & \frac{\sqrt{2}}{2} & 0 \\
-\frac{\sqrt{2}}{2} & 0 & 0 \\
0 & 0 & 0
\end{array}\right)\left(\begin{array}{ccc}
0 & 0 & 0 \\
0 & 0 & -\frac{\sqrt{2}}{2} \\
0 & \frac{\sqrt{2}}{2} & 0
\end{array}\right)-\left(\begin{array}{ccc}
0 & 0 & 0 \\
0 & 0 & \frac{\sqrt{2}}{2} \\
0 & -\frac{\sqrt{2}}{2} & 0
\end{array}\right)\left(\begin{array}{ccc}
0 & -\frac{\sqrt{2}}{2} & 0 \\
\frac{\sqrt{2}}{2} & 0 & 0 \\
0 & 0 & 0
\end{array}\right) \\
& =\left(\begin{array}{ccc}
0 & 0 & -\frac{1}{2} \\
0 & 0 & 0 \\
\frac{1}{2} & 0 & 0
\end{array}\right) \\
& =\frac{\sqrt{2}}{2} \varphi^{3} .
\end{aligned}
$$

So $C^{123}=\left\langle\left[\varphi^{1}, \varphi^{2}\right], \varphi^{3}\right\rangle=\frac{\sqrt{2}}{2}$, in particular

$$
C^{\alpha \beta \gamma}= \begin{cases} \pm \frac{\sqrt{2}}{2}, & \text { if } \alpha \neq \beta \neq \gamma \\ 0, & \text { otherwise. }\end{cases}
$$


Hence the matrix $M^{\#}=\left(M_{\alpha \beta}^{\#}\right)$ is just the adjoint matrix of $M=\left(M_{\alpha \beta}\right)$ :

$$
M^{\#}=\operatorname{det} M \cdot{ }^{t} M^{-1} .
$$

In dimension 4, we can use the Hodge star operator to decompose the space of two-forms $\Lambda^{2}$ as

$$
\Lambda^{2}=\Lambda_{+}^{2} \oplus \Lambda_{-}^{2}
$$

where $\Lambda_{+}^{2}$ (resp. $\Lambda_{-}^{2}$ ) is the eigenspace of the star operator with eigenvalue +1 (resp. -1 ). Let $\omega_{1}, \omega_{2}, \omega_{3}, \omega_{4}$ be a positively oriented orthonormal basis for one-forms. A basis for $\Lambda_{+}^{2}$ is then given by

$$
\varphi^{1}=\omega_{1} \wedge \omega_{2}+\omega_{3} \wedge \omega_{4}, \quad \varphi^{2}=\omega_{1} \wedge \omega_{3}+\omega_{4} \wedge \omega_{2}, \quad \varphi^{3}=\omega_{1} \wedge \omega_{4}+\omega_{2} \wedge \omega_{3},
$$

while a basis for $\Lambda_{-}^{2}$ is given by

$$
\psi^{1}=\omega_{1} \wedge \omega_{2}-\omega_{3} \wedge \omega_{4}, \quad \psi^{2}=\omega_{1} \wedge \omega_{3}-\omega_{4} \wedge \omega_{2}, \quad \psi^{3}=\omega_{1} \wedge \omega_{4}-\omega_{2} \wedge \omega_{3} .
$$

In particular, $\left\{\varphi^{1}, \varphi^{2}, \varphi^{3}, \psi^{1}, \psi^{2}, \psi^{3}\right\}$ forms an orthonormal basis for the space of two-forms $\Lambda^{2}$. By using this basis we obtain a block decomposition of the curvature operator matrix $M$ as

$$
M=\left(M_{\alpha \beta}\right)=\left(\begin{array}{cc}
A & B \\
{ }^{t} B & C
\end{array}\right) .
$$

Here $A, B$ and $C$ are $3 \times 3$ matrices with $A$ and $C$ being symmetric. Then we can write each element of the basis as a skew-symmetric $4 \times 4$ matrix and compute as above to get

$$
M^{\#}=\left(M_{\alpha \beta}^{\#}\right)=2\left(\begin{array}{cc}
A^{\#} & B^{\#} \\
{ }^{t} B^{\#} & C^{\#}
\end{array}\right),
$$

where $A^{\#}, B^{\#}, C^{\#}$ are the adjoint of $3 \times 3$ submatrices as before.

For later applications in Chapter 5 , we now give some computations for the entries of the matrices $A, C$ and $B$ as follows. First for the matrices $A$ and $C$, we have

$$
\begin{aligned}
& A_{11}=R m\left(\varphi^{1}, \varphi^{1}\right)=R_{1212}+R_{3434}+2 R_{1234} \\
& A_{22}=R m\left(\varphi^{2}, \varphi^{2}\right)=R_{1313}+R_{4242}+2 R_{1342} \\
& A_{33}=\operatorname{Rm}\left(\varphi^{3}, \varphi^{3}\right)=R_{1414}+R_{2323}+2 R_{1423}
\end{aligned}
$$

and

$$
\begin{aligned}
& C_{11}=R m\left(\psi^{1}, \psi^{1}\right)=R_{1212}+R_{3434}-2 R_{1234} \\
& C_{22}=R m\left(\psi^{2}, \psi^{2}\right)=R_{1313}+R_{4242}-2 R_{1342} \\
& C_{33}=R m\left(\psi^{3}, \psi^{3}\right)=R_{1414}+R_{2323}-2 R_{1423} .
\end{aligned}
$$


By the Bianchi identity

$$
R_{1234}+R_{1342}+R_{1423}=0
$$

so we have

$$
\operatorname{tr} A=\operatorname{tr} C=\frac{1}{2} R
$$

Next for the entries of the matrix $B$, we have

$$
\begin{aligned}
& B_{11}=R m\left(\varphi^{1}, \psi^{1}\right)=R_{1212}-R_{3434} \\
& B_{22}=R m\left(\varphi^{2}, \psi^{2}\right)=R_{1313}-R_{4242} \\
& B_{33}=R m\left(\varphi^{3}, \psi^{3}\right)=R_{1414}-R_{2323}
\end{aligned}
$$

and

$$
B_{12}=R m\left(\varphi^{1}, \psi^{2}\right)=R_{1213}+R_{3413}-R_{1242}-R_{3442} \quad \text { etc. }
$$

Thus the entries of $B$ can be written as

$$
\begin{aligned}
& B_{11}=\frac{1}{2}\left(R_{11}+R_{22}-R_{33}-R_{44}\right) \\
& B_{22}=\frac{1}{2}\left(R_{11}+R_{33}-R_{44}-R_{22}\right) \\
& B_{33}=\frac{1}{2}\left(R_{11}+R_{44}-R_{22}-R_{33}\right)
\end{aligned}
$$

and

$$
B_{12}=R_{23}-R_{14} \quad \text { etc. }
$$

If we choose the frame $\left\{\omega_{1}, \omega_{2}, \omega_{3}, \omega_{4}\right\}$ so that the Ricci tensor is diagonal, then the matrix $B$ is also diagonal. In particular, the matrix $B$ is identically zero when the four-manifold is Einstein.

1.4. Derivative Estimates. In the previous section we have seen that the curvatures satisfy nonlinear heat equations with quadratic growth terms. The parabolic nature will give us a bound on the derivatives of the curvatures at any time $t>0$ in terms of a bound of the curvatures.

We begin with the global version of the derivative estimates.

Theorem 1.4.1 (Shi [114]). There exist constants $C_{m}, m=1,2, \ldots$, such that if the curvature of a complete solution to Ricci flow is bounded by

$$
\left|R_{i j k l}\right| \leq M
$$

up to time $t$ with $0<t \leq \frac{1}{M}$, then the covariant derivative of the curvature is bounded by

$$
\left|\nabla R_{i j k l}\right| \leq C_{1} M / \sqrt{t}
$$


and the $m^{\text {th }}$ covariant derivative of the curvature is bounded by

$$
\left|\nabla^{m} R_{i j k l}\right| \leq C_{m} M / t^{\frac{m}{2}} .
$$

Here the norms are taken with respect to the evolving metric.

Proof. We shall only give the proof for the compact case. The noncompact case can be deduced from the next local derivative estimate theorem. Let us denote the curvature tensor by $R m$ and denote by $A * B$ any tensor product of two tensors $A$ and $B$ when we do not need the precise expression. We have from Proposition 1.3.1 that

$$
\frac{\partial}{\partial t} R m=\Delta R m+R m * R m
$$

Since

$$
\begin{aligned}
\frac{\partial}{\partial t} \Gamma_{j k}^{i} & =\frac{1}{2} g^{i l}\left\{\nabla_{j} \frac{\partial g_{k l}}{\partial t}+\nabla_{k} \frac{\partial g_{j l}}{\partial t}-\nabla_{l} \frac{\partial g_{j k}}{\partial t}\right\} \\
& =\nabla R m
\end{aligned}
$$

it follows that

$$
\frac{\partial}{\partial t}(\nabla R m)=\Delta(\nabla R m)+R m *(\nabla R m) .
$$

Thus

$$
\begin{aligned}
\frac{\partial}{\partial t}|R m|^{2} & \leq \Delta|R m|^{2}-2|\nabla R m|^{2}+C|R m|^{3}, \\
\frac{\partial}{\partial t}|\nabla R m|^{2} & \leq \Delta|\nabla R m|^{2}-2\left|\nabla^{2} R m\right|^{2}+C|R m| \cdot|\nabla R m|^{2},
\end{aligned}
$$

for some constant $C$ depending only on the dimension $n$.

Let $A>0$ be a constant (to be determined) and set

$$
F=t|\nabla R m|^{2}+A|R m|^{2} .
$$

We compute

$$
\begin{aligned}
\frac{\partial F}{\partial t} & =|\nabla R m|^{2}+t \frac{\partial}{\partial t}|\nabla R m|^{2}+A \frac{\partial}{\partial t}|R m|^{2} \\
& \leq \Delta\left(t|\nabla R m|^{2}+A|R m|^{2}\right)+|\nabla R m|^{2}(1+t C|R m|-2 A)+C A|R m|^{3} .
\end{aligned}
$$

Taking $A \geq C+1$, we get

$$
\frac{\partial F}{\partial t} \leq \Delta F+\bar{C} M^{3}
$$

for some constant $\bar{C}$ depending only on the dimension $n$. We then obtain

$$
F \leq F(0)+\bar{C} M^{3} t \leq(A+\bar{C}) M^{2},
$$

and then

$$
|\nabla R m|^{2} \leq(A+\bar{C}) M^{2} / t
$$


The general case follows in the same way. If we have bounds

$$
\left|\nabla^{k} R m\right| \leq C_{k} M / t^{\frac{k}{2}}
$$

we know from (1.4.1) and (1.4.2) that

$$
\frac{\partial}{\partial t}\left|\nabla^{k} R m\right|^{2} \leq \Delta\left|\nabla^{k} R m\right|^{2}-2\left|\nabla^{k+1} R m\right|^{2}+\frac{C M^{3}}{t^{k}},
$$

and

$$
\frac{\partial}{\partial t}\left|\nabla^{k+1} R m\right|^{2} \leq \Delta\left|\nabla^{k+1} R m\right|^{2}-2\left|\nabla^{k+2} R m\right|^{2}+C M\left|\nabla^{k+1} R m\right|^{2}+\frac{C M^{3}}{t^{k+1}} .
$$

Let $A_{k}>0$ be a constant (to be determined) and set

$$
F_{k}=t^{k+2}\left|\nabla^{k+1} R m\right|^{2}+A_{k} t^{k+1}\left|\nabla^{k} R m\right|^{2} .
$$

Then

$$
\begin{aligned}
\frac{\partial}{\partial t} F_{k}= & (k+2) t^{k+1}\left|\nabla^{k+1} R m\right|^{2}+t^{k+2} \frac{\partial}{\partial t}\left|\nabla^{k+1} R m\right|^{2} \\
& +A_{k}(k+1) t^{k}\left|\nabla^{k} R m\right|^{2}+A_{k} t^{k+1} \frac{\partial}{\partial t}\left|\nabla^{k} R m\right|^{2} \\
\leq & (k+2) t^{k+1}\left|\nabla^{k+1} R m\right|^{2} \\
& +t^{k+2}\left[\Delta\left|\nabla^{k+1} R m\right|^{2}-2\left|\nabla^{k+2} R m\right|^{2}+C M\left|\nabla^{k+1} R m\right|^{2}+\frac{C M^{3}}{t^{k+1}}\right] \\
& +A_{k}(k+1) t^{k}\left|\nabla^{k} R m\right|^{2} \\
& +A_{k} t^{k+1}\left[\Delta\left|\nabla^{k} R m\right|^{2}-2\left|\nabla^{k+1} R m\right|^{2}+\frac{C M^{3}}{t^{k}}\right] \\
\leq & \Delta F_{k}+C_{k+1} M^{2}
\end{aligned}
$$

for some positive constant $C_{k+1}$, by choosing $A_{k}$ large enough. This implies that

$$
\left|\nabla^{k+1} R m\right| \leq \frac{C_{k+1} M}{t^{\frac{k+1}{2}}}
$$

The above derivative estimate is a somewhat standard Bernstein estimate in PDEs. By using a cutoff argument, we will derive the following local version, which is called Shi's derivative estimate. The following proof is adapted from Hamilton [63].

Theorem 1.4.2 (Shi [114]). There exist positive constants $\theta, C_{k}, k=1,2, \ldots$, depending only on the dimension with the following property. Suppose that the curvature of a solution to the Ricci flow is bounded

$$
|R m| \leq M, \quad \text { on } U \times\left[0, \frac{\theta}{M}\right]
$$

where $U$ is an open set of the manifold. Assume that the closed ball $B_{0}(p, r)$, centered at $p$ of radius $r$ with respect to the metric at $t=0$, is contained in $U$ and the time $t \leq \theta / M$. Then we can estimate the covariant derivatives of the curvature at $(p, t)$ by

$$
|\nabla R m(p, t)|^{2} \leq C_{1} M^{2}\left(\frac{1}{r^{2}}+\frac{1}{t}+M\right),
$$


and the $k^{\text {th }}$ covariant derivative of the curvature at $(p, t)$ by

$$
\left|\nabla^{k} R m(p, t)\right|^{2} \leq C_{k} M^{2}\left(\frac{1}{r^{2 k}}+\frac{1}{t^{k}}+M^{k}\right) .
$$

Proof. Without loss of generality, we may assume $r \leq \theta / \sqrt{M}$ and the exponential map at $p$ at time $t=0$ is injective on the ball of radius $r$ (by passing to a local cover if necessary, and pulling back the local solution of the Ricci flow to the ball of radius $r$ in the tangent space at $p$ at time $t=0$ ).

Recall

$$
\begin{aligned}
\frac{\partial}{\partial t}|R m|^{2} & \leq \Delta|R m|^{2}-2|\nabla R m|^{2}+C|R m|^{3} \\
\frac{\partial}{\partial t}|\nabla R m|^{2} & \leq \Delta|\nabla R m|^{2}-2\left|\nabla^{2} R m\right|^{2}+C|R m| \cdot|\nabla R m|^{2} .
\end{aligned}
$$

Define

$$
S=\left(B M^{2}+|R m|^{2}\right)|\nabla R m|^{2}
$$

where $B$ is a positive constant to be determined. By choosing $B \geq C^{2} / 4$ and using the Cauchy inequality, we have

$$
\begin{aligned}
\frac{\partial}{\partial t} S \leq & \Delta S-2 B M^{2}\left|\nabla^{2} R m\right|^{2}-2|\nabla R m|^{4} \\
& +C M|\nabla R m|^{2} \cdot\left|\nabla^{2} R m\right|+C B M^{3}|\nabla R m|^{2} \\
\leq & \Delta S-|\nabla R m|^{4}+C B^{2} M^{6} \\
\leq & \Delta S-\frac{S^{2}}{(B+1)^{2} M^{4}}+C B^{2} M^{6} .
\end{aligned}
$$

If we take

$$
F=b\left(B M^{2}+|R m|^{2}\right)|\nabla R m|^{2} / M^{4}=b S / M^{4},
$$

and $b \leq \min \left\{1 /(B+1)^{2}, 1 / C B^{2}\right\}$, we get

$$
\frac{\partial F}{\partial t} \leq \Delta F-F^{2}+M^{2}
$$

We now want to choose a cutoff function $\varphi$ with the support in the ball $B_{0}(p, r)$ such that at $t=0$,

$$
\varphi(p)=r, \quad 0 \leq \varphi \leq A r
$$

and

$$
|\nabla \varphi| \leq A, \quad\left|\nabla^{2} \varphi\right| \leq \frac{A}{r}
$$

for some positive constant $A$ depending only on the dimension. Indeed, let $g$ : $(-\infty,+\infty) \rightarrow[0,+\infty)$ be a smooth, nonnegative function satisfying

$$
g(u)= \begin{cases}1, & u \in\left(-\frac{1}{2}, \frac{1}{2}\right), \\ 0, & \text { outside }(-1,1)\end{cases}
$$


Set

$$
\varphi=r g\left(\frac{s^{2}}{r^{2}}\right)
$$

where $s$ is the geodesic distance function from $p$ with respect to the metric at $t=0$. Then

$$
\nabla \varphi=\frac{1}{r} g^{\prime}\left(\frac{s^{2}}{r^{2}}\right) \cdot 2 s \nabla s
$$

and hence

$$
|\nabla \varphi| \leq 2 C_{1}
$$

Also,

$$
\nabla^{2} \varphi=\frac{1}{r} g^{\prime \prime}\left(\frac{s^{2}}{r^{2}}\right) \frac{1}{r^{2}} 4 s^{2} \nabla s \cdot \nabla s+\frac{1}{r} g^{\prime}\left(\frac{s^{2}}{r^{2}}\right) 2 \nabla s \cdot \nabla s+\frac{1}{r} g^{\prime}\left(\frac{s^{2}}{r^{2}}\right) \cdot 2 s \nabla^{2} s .
$$

Thus, by using the standard Hessian comparison,

$$
\begin{aligned}
\left|\nabla^{2} \varphi\right| & \leq \frac{C_{1}}{r}+\frac{C_{1}}{r}\left|s \nabla^{2} s\right| \\
& \leq \frac{C_{1}}{r}\left(1+s\left(\frac{C_{2}}{s}+\sqrt{M}\right)\right) \\
& \leq \frac{C_{3}}{r} .
\end{aligned}
$$

Here $C_{1}, C_{2}$ and $C_{3}$ are positive constants depending only on the dimension.

Now extend $\varphi$ to $U \times\left[0, \frac{\theta}{M}\right]$ by letting $\varphi$ to be zero outside $B_{0}(p, r)$ and independent of time. Introduce the barrier function

$$
H=\frac{(12+4 \sqrt{n}) A^{2}}{\varphi^{2}}+\frac{1}{t}+M
$$

which is defined and smooth on the set $\{\varphi>0\} \times(0, T]$.

As the metric evolves, we will still have $0 \leq \varphi \leq A r$ (since $\varphi$ is independent of time $t$ ); but $|\nabla \varphi|^{2}$ and $\varphi\left|\nabla^{2} \varphi\right|$ may increase. By continuity it will be a while before they double.

Claim 1. As long as

$$
|\nabla \varphi|^{2} \leq 2 A^{2}, \quad \varphi\left|\nabla^{2} \varphi\right| \leq 2 A^{2},
$$

we have

$$
\frac{\partial H}{\partial t}>\Delta H-H^{2}+M^{2}
$$

Indeed, by the definition of $H$, we have

$$
\begin{aligned}
& H^{2}>\frac{(12+4 \sqrt{n})^{2} A^{4}}{\varphi^{4}}+\frac{1}{t^{2}}+M^{2}, \\
& \frac{\partial H}{\partial t}=-\frac{1}{t^{2}},
\end{aligned}
$$


and

$$
\begin{aligned}
\Delta H & =(12+4 \sqrt{n}) A^{2} \Delta\left(\frac{1}{\varphi^{2}}\right) \\
& =(12+4 \sqrt{n}) A^{2}\left(\frac{6|\nabla \varphi|^{2}-2 \varphi \Delta \varphi}{\varphi^{4}}\right) \\
& \leq(12+4 \sqrt{n}) A^{2}\left(\frac{12 A^{2}+4 \sqrt{n} A^{2}}{\varphi^{4}}\right) \\
& =\frac{(12+4 \sqrt{n})^{2} A^{4}}{\varphi^{4}} .
\end{aligned}
$$

Therefore,

$$
H^{2}>\Delta H-\frac{\partial H}{\partial t}+M^{2}
$$

Claim 2. If the constant $\theta>0$ is small enough compared to $b, B$ and $A$, then we have the following property: as long as $r \leq \theta / \sqrt{M}, t \leq \theta / M$ and $F \leq H$, we will have

$$
|\nabla \varphi|^{2} \leq 2 A^{2} \quad \text { and } \quad \varphi\left|\nabla^{2} \varphi\right| \leq 2 A^{2} .
$$

Indeed, by considering the evolution of $\nabla \varphi$, we have

$$
\begin{aligned}
\frac{\partial}{\partial t} \nabla_{a} \varphi & =\frac{\partial}{\partial t}\left(F_{a}^{i} \nabla_{i} \varphi\right) \\
& =F_{a}^{i} \nabla_{i}\left(\frac{\partial \varphi}{\partial t}\right)+\nabla_{i} \varphi R_{k}^{i} F_{a}^{k} \\
& =R_{a b} \nabla_{b} \varphi
\end{aligned}
$$

which implies

$$
\frac{\partial}{\partial t}|\nabla \varphi|^{2} \leq C M|\nabla \varphi|^{2}
$$

and then

$$
|\nabla \varphi|^{2} \leq A^{2} e^{C M t} \leq 2 A^{2},
$$

provided $t \leq \theta / M$ with $\theta \leq \log 2 / C$.

By considering the evolution of $\nabla^{2} \varphi$, we have

$$
\begin{aligned}
\frac{\partial}{\partial t}\left(\nabla_{a} \nabla_{b} \varphi\right)= & \frac{\partial}{\partial t}\left(F_{a}^{i} F_{b}^{j} \nabla_{i} \nabla_{j} \varphi\right) \\
= & \frac{\partial}{\partial t}\left(F_{a}^{i} F_{b}^{j}\left(\frac{\partial^{2} \varphi}{\partial x^{i} \partial x^{j}}-\Gamma_{i j}^{k} \frac{\partial \varphi}{\partial x^{k}}\right)\right) \\
= & \nabla_{a} \nabla_{b}\left(\frac{\partial \varphi}{\partial t}\right)+R_{a c} \nabla_{b} \nabla_{c} \varphi+R_{b c} \nabla_{a} \nabla_{c} \varphi \\
& +\left(\nabla_{c} R_{a b}-\nabla_{a} R_{b c}-\nabla_{b} R_{a c}\right) \nabla_{c} \varphi
\end{aligned}
$$


which implies

$$
\frac{\partial}{\partial t}\left|\nabla^{2} \varphi\right| \leq C|R m| \cdot\left|\nabla^{2} \varphi\right|+C|\nabla R m| \cdot|\nabla \varphi| .
$$

By assumption $F \leq H$, we have

$$
|\nabla R m|^{2} \leq \frac{2 M^{2}}{b B}\left(\frac{(12+4 \sqrt{n}) A^{2}}{\varphi^{2}}+\frac{1}{t}\right), \quad \text { for } \quad t \leq \theta / M .
$$

Thus by noting $\varphi$ independent of $t$ and $\varphi \leq A r$, we get from (1.4.5) and (1.4.6) that

$$
\frac{\partial}{\partial t}\left(\varphi\left|\nabla^{2} \varphi\right|\right) \leq C M\left(\varphi\left|\nabla^{2} \varphi\right|+1+\frac{r}{\sqrt{t}}\right)
$$

which implies

$$
\begin{aligned}
\varphi\left|\nabla^{2} \varphi\right| & \leq e^{C M t}\left[\left.\left(\varphi\left|\nabla^{2} \varphi\right|\right)\right|_{t=0}+C M \int_{0}^{t}\left(1+\frac{r}{\sqrt{t}}\right) d t\right] \\
& \leq e^{C M t}\left[A^{2}+C M(t+2 r \sqrt{t})\right] \\
& \leq 2 A^{2}
\end{aligned}
$$

provided $r \leq \theta / \sqrt{M}$, and $t \leq \theta / M$ with $\theta$ small enough. Therefore we have obtained Claim 2.

The combination of Claim 1 and Claim 2 gives us

$$
\frac{\partial H}{\partial t}>\Delta H-H^{2}+M^{2}
$$

as long as $r \leq \theta / \sqrt{M}, \quad t \leq \theta / M$ and $F \leq H$. And (1.4.3) tells us

$$
\frac{\partial F}{\partial t} \leq \Delta F-F^{2}+M^{2}
$$

Then the standard maximum principle immediately gives the estimate

$$
|\nabla R m|^{2} \leq C M^{2}\left(\frac{1}{\varphi^{2}}+\frac{1}{t}+M\right) \quad \text { on }\{\varphi>0\} \times\left(0, \frac{\theta}{M}\right],
$$

which implies the first order derivative estimate.

The higher order derivative estimates can be obtained in the same way by induction. Suppose we have the bounds

$$
\left|\nabla^{k} R m\right|^{2} \leq C_{k} M^{2}\left(\frac{1}{r^{2 k}}+\frac{1}{t^{k}}+M^{k}\right) .
$$

As before, by (1.4.1) and (1.4.2), we have

$$
\frac{\partial}{\partial t}\left|\nabla^{k} R m\right|^{2} \leq \Delta\left|\nabla^{k} R m\right|^{2}-2\left|\nabla^{k+1} R m\right|^{2}+C M^{3}\left(\frac{1}{r^{2 k}}+\frac{1}{t^{k}}+M^{k}\right),
$$

and

$$
\begin{aligned}
\frac{\partial}{\partial t}\left|\nabla^{k+1} R m\right|^{2} \leq & \Delta\left|\nabla^{k+1} R m\right|^{2}-2\left|\nabla^{k+2} R m\right|^{2} \\
& +C M\left|\nabla^{k+1} R m\right|^{2}+C M^{3}\left(\frac{1}{r^{2(k+1)}}+\frac{1}{t^{k+1}}+M^{k+1}\right) .
\end{aligned}
$$


Here and in the following we denote by $C$ various positive constants depending only on $C_{k}$ and the dimension.

Define

$$
S_{k}=\left[B_{k} M^{2}\left(\frac{1}{r^{2 k}}+\frac{1}{t^{k}}+M^{k}\right)+\left|\nabla^{k} R m\right|^{2}\right] \cdot\left|\nabla^{k+1} R m\right|^{2}
$$

where $B_{k}$ is a positive constant to be determined. By choosing $B_{k}$ large enough and Cauchy inequality, we have

$$
\begin{aligned}
& \frac{\partial}{\partial t} S_{k} \leq\left[-\frac{k}{t^{k+1}}+\Delta\left|\nabla^{k} R m\right|^{2}-2\left|\nabla^{k+1} R m\right|^{2}\right. \\
& \left.+C M^{3}\left(\frac{1}{r^{2 k}}+\frac{1}{t^{k}}+M^{k}\right)\right] \cdot\left|\nabla^{k+1} R m\right|^{2} \\
& +\left[B_{k} M^{2}\left(\frac{1}{r^{2 k}}+\frac{1}{t^{k}}+M^{k}\right)+\left|\nabla^{k} R m\right|^{2}\right] \\
& \cdot\left[\Delta\left|\nabla^{k+1} R m\right|^{2}-2\left|\nabla^{k+2} R m\right|^{2}+C M\left|\nabla^{k+1} R m\right|^{2}\right. \\
& \left.+C M^{3}\left(\frac{1}{r^{2(k+1)}}+\frac{1}{t^{k+1}}+M^{k+1}\right)\right] \\
& \leq \Delta S_{k}+8\left|\nabla^{k} R m\right| \cdot\left|\nabla^{k+1} R m\right|^{2} \cdot\left|\nabla^{k+2} R m\right|-\frac{k}{t^{k+1}}\left|\nabla^{k+1} R m\right|^{2} \\
& -2\left|\nabla^{k+1} R m\right|^{4}+C M^{3}\left|\nabla^{k+1} R m\right|^{2}\left(\frac{1}{r^{2 k}}+\frac{1}{t^{k}}+M^{k}\right) \\
& -2\left|\nabla^{k+2} R m\right|^{2}\left[B_{k} M^{2}\left(\frac{1}{r^{2 k}}+\frac{1}{t^{k}}+M^{k}\right)+\left|\nabla^{k} R m\right|^{2}\right] \\
& +C M\left|\nabla^{k+1} R m\right|^{2}\left[B_{k} M^{2}\left(\frac{1}{r^{2 k}}+\frac{1}{t^{k}}+M^{k}\right)+\left|\nabla^{k} R m\right|^{2}\right] \\
& +C M^{3}\left(\frac{1}{r^{2(k+1)}}+\frac{1}{t^{k+1}}+M^{k+1}\right) \\
& \cdot\left[B_{k} M^{2}\left(\frac{1}{r^{2 k}}+\frac{1}{t^{k}}+M^{k}\right)+\left|\nabla^{k} R m\right|^{2}\right] \\
& \leq \Delta S_{k}-\left|\nabla^{k+1} R m\right|^{4}+C B_{k}^{2} M^{6}\left(\frac{1}{r^{4 k}}+\frac{1}{t^{2 k}}+M^{2 k}\right) \\
& +C B_{k} M^{5}\left(\frac{1}{r^{2(2 k+1)}}+\frac{1}{t^{2 k+1}}+M^{2 k+1}\right) \\
& \leq \Delta S_{k}-\left|\nabla^{k+1} R m\right|^{4}+C B_{k}^{2} M^{5}\left(\frac{1}{r^{2(2 k+1)}}+\frac{1}{t^{2 k+1}}+M^{2 k+1}\right) \\
& \leq \Delta S_{k}-\frac{S_{k}}{(B+1)^{2} M^{4}\left(\frac{1}{r^{2 k}}+\frac{1}{t^{k}}+M^{k}\right)^{2}} \\
& +C B_{k}^{2} M^{5}\left(\frac{1}{r^{2(2 k+1)}}+\frac{1}{t^{2 k+1}}+M^{2 k+1}\right) \text {. }
\end{aligned}
$$


Let $u=1 / r^{2}+1 / t+M$ and set $F_{k}=b S_{k} / u^{k}$. Then

$$
\begin{aligned}
\frac{\partial F_{k}}{\partial t} & \leq \Delta F_{k}-\frac{F_{k}^{2}}{b\left(B_{k}+1\right)^{2} M^{4} u^{k}}+b C B_{k}^{2} M^{5} u^{k+1}+k F_{k} u \\
& \leq \Delta F_{k}-\frac{F_{k}^{2}}{2 b\left(B_{k}+1\right)^{2} M^{4} u^{k}}+b\left(C+2 k^{2}\right)\left(B_{k}+1\right)^{2} M^{4} u^{k+2} .
\end{aligned}
$$

By choosing $b \leq 1 /\left(2\left(C+2 k^{2}\right)\left(B_{k}+1\right)^{2} M^{4}\right)$, we get

$$
\frac{\partial F_{k}}{\partial t} \leq \Delta F_{k}-\frac{1}{u^{k}} F_{k}^{2}+u^{k+2} .
$$

Introduce

$$
H_{k}=5(k+1)(2(k+1)+1+\sqrt{n}) A^{2} \varphi^{-2(k+1)}+L t^{-(k+1)}+M^{k+1},
$$

where $L \geq k+2$. Then by using Claim 1 and Claim 2, we have

$$
\begin{gathered}
\frac{\partial H_{k}}{\partial t}=-(k+1) L t^{-(k+2)} \\
\Delta H_{k} \leq 20(k+1)^{2}(2(k+1)+1+\sqrt{n}) A^{4} \varphi^{-2(k+2)}
\end{gathered}
$$

and

$$
H_{k}^{2}>25(k+1)^{2}(2(k+1)+1+\sqrt{n}) A^{4} \varphi^{-4(k+1)}+L^{2} t^{-2(k+1)}+M^{2(k+1)} .
$$

These imply

$$
\frac{\partial H_{k}}{\partial t}>\Delta H_{k}-\frac{1}{u^{k}} H_{k}^{2}+u^{k+2}
$$

Then the maximum principle immediately gives the estimate

$$
F_{k} \leq H_{k}
$$

In particular,

$$
\begin{aligned}
& \frac{b}{u^{k}} B_{k} M^{2}\left(\frac{1}{r^{2 k}}+\frac{1}{t^{k}}+M^{k}\right) \cdot\left|\nabla^{k+1} R m\right|^{2} \\
& \leq 5(k+1)(2(k+1)+1+\sqrt{n}) A^{2} \varphi^{-2(k+1)}+L t^{-(k+1)}+M^{k+1} .
\end{aligned}
$$

So by the definition of $u$ and the choosing of $b$, we obtain the desired estimate

$$
\left|\nabla^{k+1} R m\right|^{2} \leq C_{k+1} M^{2}\left(\frac{1}{r^{2(k+1)}}+\frac{1}{t^{k+1}}+M^{k+1}\right) .
$$

Therefore we have completed the proof of the theorem. 
1.5. Variational Structure and Dynamic Property. In this section, we introduce two functionals of Perelman [103], $\mathcal{F}$ and $\mathcal{W}$, and discuss their relations with the Ricci flow. It was not known whether the Ricci flow is a gradient flow until Perelman [103] showed that the Ricci flow is, in a certain sense, the gradient flow of the functional $\mathcal{F}$. If we consider the Ricci flow as a dynamical system on the space of Riemannian metrics, then these two functionals are of Lyapunov type for this dynamical system. Obviously, Ricci flat metrics are fixed points of the dynamical system. When we consider the space of Riemannian metrics modulo diffeomorphism and scaling, fixed points of the Ricci flow dynamical system correspond to steady, or shrinking, or expanding Ricci solitons. The following concept corresponds to a periodic orbit.

Definition 1.5.1. A metric $g_{i j}(t)$ evolving by the Ricci flow is called a breather if for some $t_{1}<t_{2}$ and $\alpha>0$ the metrics $\alpha g_{i j}\left(t_{1}\right)$ and $g_{i j}\left(t_{2}\right)$ differ only by a diffeomorphism; the case $\alpha=1, \alpha<1, \alpha>1$ correspond to steady, shrinking and expanding breathers, respectively.

Clearly, (steady, shrinking or expanding) Ricci solitons are trivial breathers for which the metrics $g_{i j}\left(t_{1}\right)$ and $g_{i j}\left(t_{2}\right)$ differ only by diffeomorphism and scaling for every pair $t_{1}$ and $t_{2}$.

We always assume $M$ is a compact $n$-dimensional manifold in this section. Let us first consider the functional

$$
\mathcal{F}\left(g_{i j}, f\right)=\int_{M}\left(R+|\nabla f|^{2}\right) e^{-f} d V
$$

of Perelman [103] defined on the space of Riemannian metrics, and smooth functions on $M$. Here $R$ is the scalar curvature of $g_{i j}$.

Lemma 1.5.2 (Perelman [103]). If $\delta g_{i j}=v_{i j}$ and $\delta f=h$ are variations of $g_{i j}$ and $f$ respectively, then the first variation of $\mathcal{F}$ is given by

$$
\delta \mathcal{F}\left(v_{i j}, h\right)=\int_{M}\left[-v_{i j}\left(R_{i j}+\nabla_{i} \nabla_{j} f\right)+\left(\frac{v}{2}-h\right)\left(2 \Delta f-|\nabla f|^{2}+R\right)\right] e^{-f} d V
$$

where $v=g^{i j} v_{i j}$. 
Proof. In any normal coordinates at a fixed point, we have

$$
\begin{aligned}
\delta R_{i j l}^{h}= & \frac{\partial}{\partial x^{i}}\left(\delta \Gamma_{j l}^{h}\right)-\frac{\partial}{\partial x^{j}}\left(\delta \Gamma_{i l}^{h}\right) \\
= & \frac{\partial}{\partial x^{i}}\left[\frac{1}{2} g^{h m}\left(\nabla_{j} v_{l m}+\nabla_{l} v_{j m}-\nabla_{m} v_{j l}\right)\right] \\
& -\frac{\partial}{\partial x^{j}}\left[\frac{1}{2} g^{h m}\left(\nabla_{i} v_{l m}+\nabla_{l} v_{i m}-\nabla_{m} v_{i l}\right)\right], \\
\delta R_{j l}= & \frac{\partial}{\partial x^{i}}\left[\frac{1}{2} g^{i m}\left(\nabla_{j} v_{l m}+\nabla_{l} v_{j m}-\nabla_{m} v_{j l}\right)\right] \\
& -\frac{\partial}{\partial x^{j}}\left[\frac{1}{2} g^{i m}\left(\nabla_{i} v_{l m}+\nabla_{l} v_{i m}-\nabla_{m} v_{i l}\right)\right] \\
= & \frac{1}{2} \frac{\partial}{\partial x^{i}}\left[\nabla_{j} v_{l}^{i}+\nabla_{l} v_{j}^{i}-\nabla^{i} v_{j l}\right]-\frac{1}{2} \frac{\partial}{\partial x^{j}}\left[\nabla_{l} v\right], \\
\delta R= & \delta\left(g^{j l} R_{j l}\right) \\
= & -v_{j l} R_{j l}+g^{j l} \delta R_{j l} \\
= & -v_{j l} R_{j l}+\frac{1}{2} \frac{\partial}{\partial x^{i}}\left[\nabla^{l} v_{l}^{i}+\nabla_{l} v^{i l}-\nabla^{i} v\right]-\frac{1}{2} \frac{\partial}{\partial x^{j}}\left[\nabla^{j} v\right] \\
= & -v_{j l} R_{j l}+\nabla_{i} \nabla_{l} v_{i l}-\Delta v .
\end{aligned}
$$

Thus

$$
\delta R\left(v_{i j}\right)=-\Delta v+\nabla_{i} \nabla_{j} v_{i j}-v_{i j} R_{i j} .
$$

The first variation of the functional $\mathcal{F}\left(g_{i j}, f\right)$ is

$$
\begin{aligned}
& \delta\left(\int_{M}\left(R+|\nabla f|^{2}\right) e^{-f} d V\right) \\
& =\int_{M}\left(\left[\delta R\left(v_{i j}\right)+\delta\left(g^{i j} \nabla_{i} f \nabla_{j} f\right)\right] e^{-f} d V\right. \\
& \left.\quad+\left(R+|\nabla f|^{2}\right)\left[-h e^{-f} d V+e^{-f} \frac{v}{2} d V\right]\right) \\
& =\int_{M}\left[-\Delta v+\nabla_{i} \nabla_{j} v_{i j}-R_{i j} v_{i j}-v_{i j} \nabla_{i} f \nabla_{j} f\right. \\
& \left.\quad+2\langle\nabla f, \nabla h\rangle+\left(R+|\nabla f|^{2}\right)\left(\frac{v}{2}-h\right)\right] e^{-f} d V .
\end{aligned}
$$

On the other hand,

$$
\begin{aligned}
\int_{M}\left(\nabla_{i} \nabla_{j} v_{i j}-v_{i j} \nabla_{i} f \nabla_{j} f\right) e^{-f} d V & =\int_{M}\left(\nabla_{i} f \nabla_{j} v_{i j}-v_{i j} \nabla_{i} f \nabla_{j} f\right) e^{-f} d V \\
& =-\int_{M}\left(\nabla_{i} \nabla_{j} f\right) v_{i j} e^{-f} d V \\
\int_{M} 2\langle\nabla f, \nabla h\rangle e^{-f} d V & =-2 \int_{M} h \Delta f e^{-f} d V+2 \int_{M}|\nabla f|^{2} h e^{-f} d V
\end{aligned}
$$


and

$$
\begin{aligned}
\int_{M}(-\Delta v) e^{-f} d V & =-\int_{M}\langle\nabla f, \nabla v\rangle e^{-f} d V \\
& =\int_{M} v \Delta f e^{-f} d V-\int_{M}|\nabla f|^{2} v e^{-f} d V .
\end{aligned}
$$

Plugging these identities into (1.5.3) the first variation formula follows.

Now let us study the functional $\mathcal{F}$ when the metric evolves under the Ricci flow and the function evolves by a backward heat equation.

Proposition 1.5.3 (Perelman [103]). Let $g_{i j}(t)$ and $f(t)$ evolve according to the coupled flow

$$
\left\{\begin{aligned}
\frac{\partial g_{i j}}{\partial t} & =-2 R_{i j} \\
\frac{\partial f}{\partial t} & =-\Delta f+|\nabla f|^{2}-R .
\end{aligned}\right.
$$

Then

$$
\frac{d}{d t} \mathcal{F}\left(g_{i j}(t), f(t)\right)=2 \int_{M}\left|R_{i j}+\nabla_{i} \nabla_{j} f\right|^{2} e^{-f} d V
$$

and $\int_{M} e^{-f} d V$ is constant. In particular $\mathcal{F}\left(g_{i j}(t), f(t)\right)$ is nondecreasing in time and the monotonicity is strict unless we are on a steady gradient soliton.

Proof. Under the coupled flow and using the first variation formula in Lemma 1.5.2, we have

$$
\begin{aligned}
& \frac{d}{d t} \mathcal{F}\left(g_{i j}(t), f(t)\right) \\
& =\int_{M}\left[-\left(-2 R_{i j}\right)\left(R_{i j}+\nabla_{i} \nabla_{j} f\right)\right. \\
& \left.\quad+\left(\frac{1}{2}(-2 R)-\frac{\partial f}{\partial t}\right)\left(2 \Delta f-|\nabla f|^{2}+R\right)\right] e^{-f} d V \\
& =\int_{M}\left[2 R_{i j}\left(R_{i j}+\nabla_{i} \nabla_{j} f\right)+\left(\Delta f-|\nabla f|^{2}\right)\left(2 \Delta f-|\nabla f|^{2}+R\right)\right] e^{-f} d V .
\end{aligned}
$$

Now

$$
\begin{aligned}
& \int_{M}\left(\Delta f-|\nabla f|^{2}\right)\left(2 \Delta f-|\nabla f|^{2}\right) e^{-f} d V \\
& =\int_{M}-\nabla_{i} f \nabla_{i}\left(2 \Delta f-|\nabla f|^{2}\right) e^{-f} d V \\
& =\int_{M}-\nabla_{i} f\left(2 \nabla_{j}\left(\nabla_{i} \nabla_{j} f\right)-2 R_{i j} \nabla_{j} f-2\left\langle\nabla f, \nabla_{i} \nabla f\right\rangle\right) e^{-f} d V \\
& =-2 \int_{M}\left[\left(\nabla_{i} f \nabla_{j} f-\nabla_{i} \nabla_{j} f\right) \nabla_{i} \nabla_{j} f-R_{i j} \nabla_{i} f \nabla_{j} f-\left\langle\nabla f, \nabla_{i} \nabla f\right\rangle \nabla_{i} f\right] e^{-f} d V \\
& =2 \int_{M}\left[\left|\nabla_{i} \nabla_{j} f\right|^{2}+R_{i j} \nabla_{i} f \nabla_{j} f\right] e^{-f} d V,
\end{aligned}
$$


and

$$
\begin{aligned}
& \int_{M}\left(\Delta f-|\nabla f|^{2}\right) R e^{-f} d V \\
& =\int_{M}-\nabla_{i} f \nabla_{i} R e^{-f} d V \\
& =2 \int_{M} \nabla_{i} \nabla_{j} f R_{i j} e^{-f} d V-2 \int_{M} \nabla_{i} f \nabla_{j} f R_{i j} e^{-f} d V .
\end{aligned}
$$

Here we have used the contracted second Bianchi identity. Therefore we obtain

$$
\begin{aligned}
& \frac{d}{d t} \mathcal{F}\left(g_{i j}(t), f(t)\right) \\
& =\int_{M}\left[2 R_{i j}\left(R_{i j}+\nabla_{i} \nabla_{j} f\right)+2\left(\nabla_{i} \nabla_{j} f\right)\left(\nabla_{i} \nabla_{j} f+R_{i j}\right)\right] e^{-f} d V \\
& =2 \int_{M}\left|R_{i j}+\nabla_{i} \nabla_{j} f\right|^{2} e^{-f} d V .
\end{aligned}
$$

It remains to show $\int_{M} e^{-f} d V$ is a constant. Note that the volume element $d V=$ $\sqrt{\operatorname{det} g_{i j}} d x$ evolves under the Ricci flow by

$$
\begin{aligned}
\frac{\partial}{\partial t} d V & =\frac{\partial}{\partial t}\left(\sqrt{\operatorname{det} g_{i j}}\right) d x \\
& =\frac{1}{2}\left(\frac{\partial}{\partial t} \log \left(\operatorname{det} g_{i j}\right)\right) d V \\
& =\frac{1}{2}\left(g^{i j} \frac{\partial}{\partial t} g_{i j}\right) d V \\
& =-R d V .
\end{aligned}
$$

Hence

$$
\begin{aligned}
\frac{\partial}{\partial t}\left(e^{-f} d V\right) & =e^{-f}\left(-\frac{\partial f}{\partial t}-R\right) d V \\
& =\left(\Delta f-|\nabla f|^{2}\right) e^{-f} d V \\
& =-\Delta\left(e^{-f}\right) d V
\end{aligned}
$$

It then follows that

$$
\frac{d}{d t} \int_{M} e^{-f} d V=-\int_{M} \Delta\left(e^{-f}\right) d V=0 .
$$

This finishes the proof of the proposition. $\square$

Next we define the associated energy

$$
\lambda\left(g_{i j}\right)=\inf \left\{\mathcal{F}\left(g_{i j}, f\right) \mid f \in C^{\infty}(M), \int_{M} e^{-f} d V=1\right\} .
$$

If we set $u=e^{-f / 2}$, then the functional $\mathcal{F}$ can be expressed in terms of $u$ as

$$
\mathcal{F}=\int_{M}\left(R u^{2}+4|\nabla u|^{2}\right) d V,
$$


and the constraint $\int_{M} e^{-f} d V=1$ becomes $\int_{M} u^{2} d V=1$. Therefore $\lambda\left(g_{i j}\right)$ is just the first eigenvalue of the operator $-4 \Delta+R$. Let $u_{0}>0$ be a first eigenfunction of the operator $-4 \Delta+R$ satisfying

$$
-4 \Delta u_{0}+R u_{0}=\lambda\left(g_{i j}\right) u_{0}
$$

The $f_{0}=-2 \log u_{0}$ is a minimizer:

$$
\lambda\left(g_{i j}\right)=\mathcal{F}\left(g_{i j}, f_{0}\right)
$$

Note that $f_{0}$ satisfies the equation

$$
-2 \Delta f_{0}+\left|\nabla f_{0}\right|^{2}-R=-\lambda\left(g_{i j}\right) .
$$

Observe that the evolution equation

$$
\frac{\partial f}{\partial t}=-\Delta f+|\nabla f|^{2}-R
$$

can be rewritten as the following linear equation

$$
\frac{\partial}{\partial t}\left(e^{-f}\right)=-\Delta\left(e^{-f}\right)+R\left(e^{-f}\right) .
$$

Thus we can always solve the evolution equation for $f$ backwards in time. Suppose at $t=t_{0}$, the infimum $\lambda\left(g_{i j}\right)$ is achieved by some function $f_{0}$ with $\int_{M} e^{-f_{0}} d V=1$. We solve the backward heat equation

$$
\left\{\begin{array}{l}
\frac{\partial f}{\partial t}=-\Delta f+|\nabla f|^{2}-R \\
\left.f\right|_{t=t_{0}}=f_{0}
\end{array}\right.
$$

to obtain a solution $f(t)$ for $t \leq t_{0}$ which satisfies $\int_{M} e^{-f} d V=1$. It then follows from Proposition 1.5.3 that

$$
\lambda\left(g_{i j}(t)\right) \leq \mathcal{F}\left(g_{i j}(t), f(t)\right) \leq \mathcal{F}\left(g_{i j}\left(t_{0}\right), f\left(t_{0}\right)\right)=\lambda\left(g_{i j}\left(t_{0}\right)\right) .
$$

Also note $\lambda\left(g_{i j}\right)$ is invariant under diffeomorphism. Thus we have proved

\section{COROLlary 1.5.4.}

(i) $\lambda\left(g_{i j}(t)\right)$ is nondecreasing along the Ricci flow and the monotonicity is strict unless we are on a steady gradient soliton;

(ii) A steady breather is necessarily a steady gradient soliton.

To deal with the expanding case we consider a scale invariant version

$$
\bar{\lambda}\left(g_{i j}\right)=\lambda\left(g_{i j}\right) V^{\frac{2}{n}}\left(g_{i j}\right) .
$$

Here $V=\operatorname{Vol}\left(g_{i j}\right)$ denotes the volume of $M$ with respect to the metric $g_{i j}$. 
COROllary 1.5.5.

(i) $\bar{\lambda}\left(g_{i j}\right)$ is nondecreasing along the Ricci flow whenever it is nonpositive; moreover, the monotonicity is strict unless we are on a gradient expanding soliton;

(ii) An expanding breather is necessarily an expanding gradient soliton.

Proof. Let $f_{0}$ be a minimizer of $\lambda\left(g_{i j}(t)\right)$ at $t=t_{0}$ and solve the backward heat equation

$$
\frac{\partial f}{\partial t}=-\Delta f+|\nabla f|^{2}-R
$$

to obtain $f(t), t \leq t_{0}$, with $\int_{M} e^{-f(t)} d V=1$. We compute the derivative (understood in the barrier sense) at $t=t_{0}$,

$$
\begin{aligned}
& \frac{d}{d t} \bar{\lambda}\left(g_{i j}(t)\right) \\
& \geq \frac{d}{d t}\left(\mathcal{F}\left(g_{i j}(t), f(t)\right) \cdot V^{\frac{2}{n}}\left(g_{i j}(t)\right)\right) \\
& =V^{\frac{2}{n}} \int_{M} 2\left|R_{i j}+\nabla_{i} \nabla_{j} f\right|^{2} e^{-f} d V \\
& \quad+\frac{2}{n} V^{\frac{2-n}{n}} \int_{M}(-R) d V \cdot \int_{M}\left(R+|\nabla f|^{2}\right) e^{-f} d V \\
& =2 V^{\frac{2}{n}}\left[\int_{M}\left|R_{i j}+\nabla_{i} \nabla_{j} f-\frac{1}{n}(R+\Delta f) g_{i j}\right|^{2} e^{-f} d V\right. \\
& \left.\quad+\frac{1}{n} \int_{M}(R+\Delta f)^{2} e^{-f} d V+\frac{1}{n}\left(-\int_{M}\left(R+|\nabla f|^{2}\right) e^{-f} d V\right)\left(\frac{1}{V} \int_{M} R d V\right)\right],
\end{aligned}
$$

where we have used the formula (1.5.4) in the computation of $d V / d t$.

Suppose $\lambda\left(g_{i j}\left(t_{0}\right)\right) \leq 0$, then the last term on the RHS is given by,

$$
\begin{aligned}
& \frac{1}{n}\left(-\int_{M}\left(R+|\nabla f|^{2}\right) e^{-f} d V\right)\left(\frac{1}{V} \int_{M} R d V\right) \\
& \geq \frac{1}{n}\left(-\int_{M}\left(R+|\nabla f|^{2}\right) e^{-f} d V\right)\left(\int_{M}\left(R+|\nabla f|^{2}\right) e^{-f} d V\right) \\
& =-\frac{1}{n}\left(\int_{M}(R+\Delta f) e^{-f} d V\right)^{2} .
\end{aligned}
$$

Thus at $t=t_{0}$,

$$
\begin{aligned}
& \frac{d}{d t} \bar{\lambda}\left(g_{i j}(t)\right) \\
& \geq 2 V^{\frac{2}{n}}\left[\int_{M}\left|R_{i j}+\nabla_{i} \nabla_{j} f-\frac{1}{n}(R+\Delta f) g_{i j}\right|^{2} e^{-f} d V\right. \\
& \left.\quad+\frac{1}{n}\left(\int_{M}(R+\Delta f)^{2} e^{-f} d V-\left(\int_{M}(R+\Delta f) e^{-f} d V\right)^{2}\right)\right] \geq 0
\end{aligned}
$$

by the Cauchy-Schwarz inequality. Thus we have proved statement (i).

We note that on an expanding breather on $\left[t_{1}, t_{2}\right]$ with $\alpha g_{i j}\left(t_{1}\right)$ and $g_{i j}\left(t_{2}\right)$ differ only by a diffeomorphism for some $\alpha>1$, it would necessary have

$$
\frac{d V}{d t}>0, \text { for some } t \in\left[t_{1}, t_{2}\right]
$$


On the other hand, for every $t$,

$$
-\frac{d}{d t} \log V=\frac{1}{V} \int_{M} R d V \geq \lambda\left(g_{i j}(t)\right)
$$

by the definition of $\lambda\left(g_{i j}(t)\right)$. It follows that on an expanding breather on $\left[t_{1}, t_{2}\right]$,

$$
\bar{\lambda}\left(g_{i j}(t)\right)=\lambda\left(g_{i j}(t)\right) V^{\frac{2}{n}}\left(g_{i j}(t)\right)<0
$$

for some $t \in\left[t_{1}, t_{2}\right]$. Then by using statement (i), it implies

$$
\bar{\lambda}\left(g_{i j}\left(t_{1}\right)\right)<\bar{\lambda}\left(g_{i j}\left(t_{2}\right)\right)
$$

unless we are on an expanding gradient soliton. We also note that $\bar{\lambda}\left(g_{i j}(t)\right)$ is invariant under diffeomorphism and scaling which implies

$$
\bar{\lambda}\left(g_{i j}\left(t_{1}\right)\right)=\bar{\lambda}\left(g_{i j}\left(t_{2}\right)\right) .
$$

Therefore the breather must be an expanding gradient soliton.

In particular part (ii) of Corollaries 1.5.4 and 1.5.5 imply that all compact steady or expanding Ricci solitons are gradient ones. Combining this fact with Proposition (1.1.1), we immediately get

Proposition 1.5.6. On a compact manifold, a steady or expanding breather is necessarily an Einstein metric.

In order to handle the shrinking case, we introduce the following important functional, also due to Perelman [103],

$$
\mathcal{W}\left(g_{i j}, f, \tau\right)=\int_{M}\left[\tau\left(R+|\nabla f|^{2}\right)+f-n\right](4 \pi \tau)^{-\frac{n}{2}} e^{-f} d V
$$

where $g_{i j}$ is a Riemannian metric, $f$ is a smooth function on $M$, and $\tau$ is a positive scale parameter. Clearly the functional $\mathcal{W}$ is invariant under simultaneous scaling of $\tau$ and $g_{i j}$ (or equivalently the parabolic scaling), and invariant under diffeomorphism. Namely, for any positive number $a$ and any diffeomorphism $\varphi$

$$
\mathcal{W}\left(a \varphi^{*} g_{i j}, \varphi^{*} f, a \tau\right)=\mathcal{W}\left(g_{i j}, f, \tau\right) .
$$

Similar to Lemma 1.5.2, we have the following first variation formula for $\mathcal{W}$.

Lemma 1.5.7 (Perelman [103]). If $v_{i j}=\delta g_{i j}, h=\delta f$, and $\eta=\delta \tau$, then

$$
\begin{aligned}
& \delta \mathcal{W}\left(v_{i j}, h, \eta\right) \\
& =\int_{M}-\tau v_{i j}\left(R_{i j}+\nabla_{i} \nabla_{j} f-\frac{1}{2 \tau} g_{i j}\right)(4 \pi \tau)^{-\frac{n}{2}} e^{-f} d V \\
& \quad+\int_{M}\left(\frac{v}{2}-h-\frac{n}{2 \tau} \eta\right)\left[\tau\left(R+2 \Delta f-|\nabla f|^{2}\right)+f-n-1\right](4 \pi \tau)^{-\frac{n}{2}} e^{-f} d V \\
& \quad+\int_{M} \eta\left(R+|\nabla f|^{2}-\frac{n}{2 \tau}\right)(4 \pi \tau)^{-\frac{n}{2}} e^{-f} d V .
\end{aligned}
$$

Here $v=g^{i j} v_{i j}$ as before. 
Proof. Arguing as in the proof of Lemma 1.5.2, the first variation of the functional $\mathcal{W}$ can be computed as follows,

$$
\begin{aligned}
& \delta W\left(v_{i j}, h, \eta\right) \\
& =\int_{M}\left[\eta\left(R+|\nabla f|^{2}\right)+\tau\left(-\Delta v+\nabla_{i} \nabla_{j} v_{i j}-R_{i j} v_{i j}-v_{i j} \nabla_{i} f \nabla_{j} f\right.\right. \\
& +2\langle\nabla f, \nabla h\rangle)+h](4 \pi \tau)^{-\frac{n}{2}} e^{-f} d V \\
& +\int_{M}\left[\left(\tau\left(R+|\nabla f|^{2}\right)+f-n\right)\left(-\frac{n}{2} \frac{\eta}{\tau}+\frac{v}{2}-h\right)\right](4 \pi \tau)^{-\frac{n}{2}} e^{-f} d V \\
& =\int_{M}\left[\eta\left(R+|\nabla f|^{2}\right)+h\right](4 \pi \tau)^{-\frac{n}{2}} e^{-f} d V \\
& +\int_{M}\left[-\tau v_{i j}\left(R_{i j}+\nabla_{i} \nabla_{j} f\right)+\tau(v-2 h)\left(\Delta f-|\nabla f|^{2}\right)\right](4 \pi \tau)^{-\frac{n}{2}} e^{-f} d V \\
& +\int_{M}\left[\left(\tau\left(R+|\nabla f|^{2}\right)+f-n\right)\left(-\frac{n}{2} \frac{\eta}{\tau}+\frac{v}{2}-h\right)\right](4 \pi \tau)^{-\frac{n}{2}} e^{-f} d V \\
& =-\int_{M} \tau v_{i j}\left(R_{i j}+\nabla_{i} \nabla_{j} f-\frac{1}{2 \tau} g_{i j}\right)(4 \pi \tau)^{-\frac{n}{2}} e^{-f} d V \\
& +\int_{M}\left(\frac{v}{2}-h-\frac{n}{2 \tau} \eta\right)\left[\tau\left(R+|\nabla f|^{2}\right)\right. \\
& \left.+f-n+2 \tau\left(\Delta f-|\nabla f|^{2}\right)\right](4 \pi \tau)^{-\frac{n}{2}} e^{-f} d V \\
& +\int_{M}\left[\eta\left(R+|\nabla f|^{2}-\frac{n}{2 \tau}\right)+\left(h-\frac{v}{2}+\frac{n}{2 \tau} \eta\right)\right](4 \pi \tau)^{-\frac{n}{2}} e^{-f} d V \\
& =\int_{M}-\tau v_{i j}\left(R_{i j}+\nabla_{i} \nabla_{j} f-\frac{1}{2 \tau} g_{i j}\right)(4 \pi \tau)^{-\frac{n}{2}} e^{-f} d V \\
& +\int_{M}\left(\frac{v}{2}-h-\frac{n}{2 \tau} \eta\right)\left[\tau\left(R+2 \Delta f-|\nabla f|^{2}\right)+f-n-1\right](4 \pi \tau)^{-\frac{n}{2}} e^{-f} d V \\
& +\int_{M} \eta\left(R+|\nabla f|^{2}-\frac{n}{2 \tau}\right)(4 \pi \tau)^{-\frac{n}{2}} e^{-f} d V
\end{aligned}
$$

The following result is analogous to Proposition 1.5.3.

Proposition 1.5.8. If $g_{i j}(t), f(t)$ and $\tau(t)$ evolve according to the system

$$
\left\{\begin{array}{l}
\frac{\partial g_{i j}}{\partial t}=-2 R_{i j} \\
\frac{\partial f}{\partial t}=-\Delta f+|\nabla f|^{2}-R+\frac{n}{2 \tau} \\
\frac{\partial \tau}{\partial t}=-1
\end{array}\right.
$$

then we have the identity

$$
\frac{d}{d t} \mathcal{W}\left(g_{i j}(t), f(t), \tau(t)\right)=\int_{M} 2 \tau\left|R_{i j}+\nabla_{i} \nabla_{j} f-\frac{1}{2 \tau} g_{i j}\right|^{2}(4 \pi \tau)^{-\frac{n}{2}} e^{-f} d V
$$

and $\int_{M}(4 \pi \tau)^{-\frac{n}{2}} e^{-f} d V$ is constant. In particular $\mathcal{W}\left(g_{i j}(t), f(t), \tau(t)\right)$ is nondecreasing in time and the monotonicity is strict unless we are on a shrinking gradient soliton. 
Proof. Using Lemma 1.5.7, we have

$$
\begin{aligned}
& \frac{d}{d t} \mathcal{W}\left(g_{i j}(t), f(t), \tau(t)\right) \\
& =\int_{M} 2 \tau R_{i j}\left(R_{i j}+\nabla_{i} \nabla_{j} f-\frac{1}{2 \tau} g_{i j}\right)(4 \pi \tau)^{-\frac{n}{2}} e^{-f} d V \\
& \quad+\int_{M}\left(\Delta f-|\nabla f|^{2}\right)\left[\tau\left(R+2 \Delta f-|\nabla f|^{2}\right)+f\right](4 \pi \tau)^{-\frac{n}{2}} e^{-f} d V \\
& \quad-\int_{M}\left(R+|\nabla f|^{2}-\frac{n}{2 \tau}\right)(4 \pi \tau)^{-\frac{n}{2}} e^{-f} d V .
\end{aligned}
$$

Here we have used the fact that $\int_{M}\left(\Delta f-|\nabla f|^{2}\right) e^{-f} d V=0$.

The second term on the RHS of (1.5.11) is

$$
\begin{aligned}
& \int_{M}\left(\Delta f-|\nabla f|^{2}\right)\left[\tau\left(R+2 \Delta f-|\nabla f|^{2}\right)+f\right](4 \pi \tau)^{-\frac{n}{2}} e^{-f} d V \\
& =\int_{M}\left(\Delta f-|\nabla f|^{2}\right)\left(2 \tau \Delta f-\tau|\nabla f|^{2}\right)(4 \pi \tau)^{-\frac{n}{2}} e^{-f} d V \\
& -\int_{M}|\nabla f|^{2}(4 \pi \tau)^{-\frac{n}{2}} e^{-f} d V+\tau \int_{M}\left(-\nabla_{i} f\right)\left(\nabla_{i} R\right)(4 \pi \tau)^{-\frac{n}{2}} e^{-f} d V \\
& =\tau \int_{M}\left(-\nabla_{i} f\right)\left(\nabla_{i}\left(2 \Delta f-|\nabla f|^{2}\right)\right)(4 \pi \tau)^{-\frac{n}{2}} e^{-f} d V \\
& -\int_{M} \Delta f(4 \pi \tau)^{-\frac{n}{2}} e^{-f} d V-2 \tau \int_{M} \nabla_{i} f \nabla_{j} R_{i j}(4 \pi \tau)^{-\frac{n}{2}} e^{-f} d V \\
& =-2 \tau \int_{M}\left(\nabla_{i} f\right)\left(\nabla_{i} \Delta f-\left\langle\nabla f, \nabla_{i} \nabla f\right\rangle\right)(4 \pi \tau)^{-\frac{n}{2}} e^{-f} d V \\
& +2 \tau \int_{M}\left[\left(\nabla_{i} \nabla_{j} f\right) R_{i j}-\nabla_{i} f \nabla_{j} f R_{i j}\right](4 \pi \tau)^{-\frac{n}{2}} e^{-f} d V \\
& +2 \tau \int_{M}\left(-\frac{1}{2 \tau} g_{i j}\right)\left(\nabla_{i} \nabla_{j} f\right)(4 \pi \tau)^{-\frac{n}{2}} e^{-f} d V \\
& =-2 \tau \int_{M}\left[\left(\nabla_{i} f \nabla_{j} f-\nabla_{i} \nabla_{j} f\right) \nabla_{i} \nabla_{j} f-R_{i j} \nabla_{i} f \nabla_{j} f\right. \\
& \left.-\nabla_{i} \nabla_{j} f \nabla_{i} f \nabla_{j} f\right](4 \pi \tau)^{-\frac{n}{2}} e^{-f} d V \\
& +2 \tau \int_{M}\left[\left(\nabla_{i} \nabla_{j} f\right) R_{i j}-\nabla_{i} f \nabla_{j} f R_{i j}\right](4 \pi \tau)^{-\frac{n}{2}} e^{-f} d V \\
& +2 \tau \int_{M}\left(-\frac{1}{2 \tau} g_{i j}\right)\left(\nabla_{i} \nabla_{j} f\right)(4 \pi \tau)^{-\frac{n}{2}} e^{-f} d V \\
& =2 \tau \int_{M}\left(\nabla_{i} \nabla_{j} f\right)\left(\nabla_{i} \nabla_{j} f+R_{i j}-\frac{1}{2 \tau} g_{i j}\right)(4 \pi \tau)^{-\frac{n}{2}} e^{-f} d V .
\end{aligned}
$$

Also the third term on the RHS of (1.5.11) is

$$
\begin{aligned}
& \int_{M}-\left(R+|\nabla f|^{2}-\frac{n}{2 \tau}\right)(4 \pi \tau)^{-\frac{n}{2}} e^{-f} d V \\
& =\int_{M}-\left(R+\Delta f-\frac{n}{2 \tau}\right)(4 \pi \tau)^{-\frac{n}{2}} e^{-f} d V \\
& =2 \tau \int_{M}\left(\frac{-1}{2 \tau} g_{i j}\right)\left(R_{i j}+\nabla_{i} \nabla_{j} f-\frac{1}{2 \tau} g_{i j}\right)(4 \pi \tau)^{-\frac{n}{2}} e^{-f} d V .
\end{aligned}
$$


Therefore, by combining the above identities, we obtain

$$
\frac{d}{d t} \mathcal{W}\left(g_{i j}(t), f(t), \tau(t)\right)=2 \tau \int_{M}\left|R_{i j}+\nabla_{i} \nabla_{j} f-\frac{1}{2 \tau} g_{i j}\right|^{2}(4 \pi \tau)^{-\frac{n}{2}} e^{-f} d V .
$$

Finally, by using the computations in (1.5.5) and the evolution equations of $f$ and $\tau$, we have

$$
\begin{aligned}
\frac{\partial}{\partial t}\left((4 \pi \tau)^{-\frac{n}{2}} e^{-f} d V\right) & =(4 \pi \tau)^{-\frac{n}{2}}\left[\frac{\partial}{\partial t}\left(e^{-f} d V\right)+\frac{n}{2 \tau} e^{-f} d V\right] \\
& =-(4 \pi \tau)^{-\frac{n}{2}} \Delta\left(e^{-f}\right) d V
\end{aligned}
$$

Hence

$$
\frac{d}{d t} \int_{M}(4 \pi \tau)^{-\frac{n}{2}} e^{-f} d V=-(4 \pi \tau)^{-\frac{n}{2}} \int_{M} \Delta\left(e^{-f}\right) d V=0
$$

Now we set

$$
\mu\left(g_{i j}, \tau\right)=\inf \left\{\mathcal{W}\left(g_{i j}, f, \tau\right) \mid f \in C^{\infty}(M), \frac{1}{(4 \pi \tau)^{n / 2}} \int_{M} e^{-f} d V=1\right\}
$$

and

$$
\nu\left(g_{i j}\right)=\inf \left\{\mathcal{W}(g, f, \tau) \mid f \in C^{\infty}(M), \tau>0, \frac{1}{(4 \pi \tau)^{n / 2}} \int e^{-f} d V=1\right\} .
$$

Note that if we let $u=e^{-f / 2}$, then the functional $\mathcal{W}$ can be expressed as

$$
\mathcal{W}\left(g_{i j}, f, \tau\right)=\int_{M}\left[\tau\left(R u^{2}+4|\nabla u|^{2}\right)-u^{2} \log u^{2}-n u^{2}\right](4 \pi \tau)^{-\frac{n}{2}} d V
$$

and the constraint $\int_{M}(4 \pi \tau)^{-\frac{n}{2}} e^{-f} d V=1$ becomes $\int_{M} u^{2}(4 \pi \tau)^{-\frac{n}{2}} d V=1$. Thus $\mu\left(g_{i j}, \tau\right)$ corresponds to the best constant of a logarithmic Sobolev inequality. Since the nonquadratic term is subcritical (in view of Sobolev exponent), it is rather straightforward to show that

$$
\inf \left\{\int_{M}\left[\tau\left(4|\nabla u|^{2}+R u^{2}\right)-u^{2} \log u^{2}-n u^{2}\right](4 \pi \tau)^{-\frac{n}{2}} d V \mid \int_{M} u^{2}(4 \pi \tau)^{-\frac{n}{2}} d V=1\right\}
$$

is achieved by some nonnegative function $u \in H^{1}(M)$ which satisfies the EulerLagrange equation

$$
\tau(-4 \Delta u+R u)-2 u \log u-n u=\mu\left(g_{i j}, \tau\right) u .
$$

One can further show that $u$ is positive (see [108]). Then the standard regularity theory of elliptic PDEs shows that $u$ is smooth. We refer the reader to Rothaus [108] for more details. It follows that $\mu\left(g_{i j}, \tau\right)$ is achieved by a minimizer $f$ satisfying the nonlinear equation

$$
\tau\left(2 \Delta f-|\nabla f|^{2}+R\right)+f-n=\mu\left(g_{i j}, \tau\right) .
$$


Corollary 1.5.9.

(i) $\mu\left(g_{i j}(t), \tau-t\right)$ is nondecreasing along the Ricci flow; moveover, the monotonicity is strict unless we are on a shrinking gradient soliton;

(ii) A shrinking breather is necessarily a shrinking gradient soliton.

Proof. Fix any time $t_{0}$, let $f_{0}$ be a minimizer of $\mu\left(g_{i j}\left(t_{0}\right), \tau-t_{0}\right)$. Note that the backward heat equation

$$
\frac{\partial f}{\partial t}=-\Delta f+|\nabla f|^{2}-R+\frac{n}{2 \tau}
$$

is equivalent to the linear equation

$$
\frac{\partial}{\partial t}\left((4 \pi \tau)^{-\frac{n}{2}} e^{-f}\right)=-\Delta\left((4 \pi \tau)^{-\frac{n}{2}} e^{-f}\right)+R\left((4 \pi \tau)^{-\frac{n}{2}} e^{-f}\right) .
$$

Thus we can solve the backward heat equation of $f$ with $\left.f\right|_{t=t_{0}}=f_{0}$ to obtain $f(t), t \leq t_{0}$, with $\int_{M}(4 \pi \tau)^{-\frac{n}{2}} e^{-f(t)} d V=1$. It then follows from Proposition 1.5.8 that

$$
\begin{aligned}
\mu\left(g_{i j}(t), \tau-t\right) & \leq \mathcal{W}\left(g_{i j}(t), f(t), \tau-t\right) \\
& \leq \mathcal{W}\left(g_{i j}\left(t_{0}\right), f\left(t_{0}\right), \tau-t_{0}\right) \\
& =\mu\left(g_{i j}\left(t_{0}\right), \tau-t_{0}\right)
\end{aligned}
$$

for $t \leq t_{0}$ and the second inequality is strict unless we are on a shrinking gradient soliton. This proves statement (i).

Consider a shrinking breather on $\left[t_{1}, t_{2}\right]$ with $\alpha g_{i j}\left(t_{1}\right)$ and $g_{i j}\left(t_{2}\right)$ differ only by a diffeomorphism for some $\alpha<1$. Recall that the functional $\mathcal{W}$ is invariant under simultaneous scaling of $\tau$ and $g_{i j}$ and invariant under diffeomorphism. Then for $\tau>0$ to be determined,

$$
\mu\left(g_{i j}\left(t_{1}\right), \tau-t_{1}\right)=\mu\left(\alpha g_{i j}\left(t_{1}\right), \alpha\left(\tau-t_{1}\right)\right)=\mu\left(g_{i j}\left(t_{2}\right), \alpha\left(\tau-t_{1}\right)\right)
$$

and by the monotonicity of $\mu\left(g_{i j}(t), \tau-t\right)$,

$$
\mu\left(g_{i j}\left(t_{1}\right), \tau-t_{1}\right) \leq \mu\left(g_{i j}\left(t_{2}\right), \tau-t_{2}\right) .
$$

Now take $\tau>0$ such that

$$
\alpha\left(\tau-t_{1}\right)=\tau-t_{2},
$$

i.e.,

$$
\tau=\frac{t_{2}-\alpha t_{1}}{1-\alpha} .
$$

This shows the equality holds in the monotonicity of $\mu\left(g_{i j}(t), \tau-t\right)$. So the shrinking breather must be a shrinking gradient soliton. $\square$

Finally, we remark that Hamilton, Ilmanen and the first author [18] have obtained the second variation formulas for both $\lambda$-energy and $\nu$-energy. We refer the reader to their paper [18] for more details and related stability questions. 
2. Maximum Principle and Li-Yau-Hamilton Inequalities. The maximum principle is a fundamental tool in the study of parabolic equations in general. In this chapter, we present various maximum principles for tensors developed by Hamilton in the Ricci flow. As an immediate consequence, the Ricci flow preserves the nonnegativity of the curvature operator. We also present the two crucial estimates in the Ricci flow: the Hamilton-Ivey curvature pinching estimate (when dimension $n=3$ ), and the Li-Yau-Hamilton estimate from which one obtains the Harnack inequality for the evolved scalar curvature via a Li-Yau path integral. Finally, we describe Perelman's Li-Yau type estimate for solutions to the conjugate heat equation and show how Li-Yau type path integral leads to a space-time distance function (i.e., what Perelman called the reduced distance).

2.1. Preserving Positive Curvature. Let $M$ be an $n$-dimensional complete manifold. Consider a family of smooth metrics $g_{i j}(t)$ evolving by the Ricci flow with uniformly bounded curvature for $t \in[0, T]$ with $T<+\infty$. Denote by $d_{t}(x, y)$ the distance between two points $x, y \in M$ with respect to the metric $g_{i j}(t)$.

LEMma 2.1.1. There exists a smooth function $f$ on $M$ such that $f \geq 1$ everywhere, $f(x) \rightarrow+\infty$ as $d_{0}\left(x, x_{0}\right) \rightarrow+\infty\left(\right.$ for some fixed $\left.x_{0} \in M\right)$,

$$
|\nabla f|_{g_{i j}(t)} \leq C \quad \text { and } \quad\left|\nabla^{2} f\right|_{g_{i j}(t)} \leq C
$$

on $M \times[0, T]$ for some positive constant $C$.

Proof. Let $\varphi(v)$ be a smooth function on $\mathbb{R}^{n}$ which is nonnegative, rotationally symmetric and has compact support in a small ball centered at the origin with $\int_{\mathbb{R}^{n}} \varphi(v) d v=1$.

For each $x \in M$, set

$$
f(x)=\int_{\mathbb{R}^{n}} \varphi(v)\left(d_{0}\left(x_{0}, \exp _{x}(v)\right)+1\right) d v,
$$

where the integral is taken over the tangent space $T_{x} M$ at $x$ which we have identified with $\mathbb{R}^{n}$. If the size of the support of $\varphi(v)$ is small compared to the maximum curvature, then it is well known that this defines a smooth function $f$ on $M$ with $f(x) \rightarrow+\infty$ as $d_{0}\left(x, x_{0}\right) \rightarrow+\infty$, while the bounds on the first and second covariant derivatives of $f$ with respect to the metric $g_{i j}(\cdot, 0)$ follow from the Hessian comparison theorem. Thus it remains to show these bounds hold with respect to the evolving metric $g_{i j}(t)$.

We compute, using the frame $\left\{F_{a}^{i} \nabla_{i} f\right\}$ introduced in Section 1.3,

$$
\frac{\partial}{\partial t} \nabla_{a} f=\frac{\partial}{\partial t}\left(F_{a}^{i} \nabla_{i} f\right)=R_{a b} \nabla_{b} f
$$

Hence

$$
|\nabla f| \leq C_{1} \cdot e^{C_{2} t},
$$

where $C_{1}, C_{2}$ are some positive constants depending only on the dimension. Also

$$
\begin{aligned}
\frac{\partial}{\partial t}\left(\nabla_{a} \nabla_{b} f\right) & =\frac{\partial}{\partial t}\left(F_{a}^{i} F_{b}^{j}\left(\frac{\partial^{2} f}{\partial x^{i} \partial x^{j}}-\Gamma_{i j}^{k} \frac{\partial f}{\partial x^{k}}\right)\right) \\
& =R_{a c} \nabla_{b} \nabla_{c} f+R_{b c} \nabla_{a} \nabla_{c} f+\left(\nabla_{c} R_{a b}-\nabla_{a} R_{b c}-\nabla_{b} R_{a c}\right) \nabla_{c} f .
\end{aligned}
$$


Then by Shi's derivative estimate (Theorem 1.4.1), we have

$$
\frac{\partial}{\partial t}\left|\nabla^{2} f\right| \leq C_{3}\left|\nabla^{2} f\right|+\frac{C_{3}}{\sqrt{t}},
$$

which implies

$$
\left|\nabla^{2} f\right|_{g_{i j}(t)} \leq e^{C_{3} t}\left(\left|\nabla^{2} f\right|_{g_{i j}(0)}+\int_{0}^{t} \frac{C_{3}}{\sqrt{\tau}} e^{-C_{3} \tau} d \tau\right)
$$

for some positive constants $C_{3}$ depending only on the dimension and the curvature bound.

We now use the weak maximum principle to derive the following

Proposition 2.1.2. If the scalar curvature $R$ of the solution $g_{i j}(t), 0 \leq t \leq T$, to the Ricci flow is nonnegative at $t=0$, then it remains so on $0 \leq t \leq T$.

Proof. Let $f$ be the function constructed in Lemma 2.1.1 and recall

$$
\frac{\partial R}{\partial t}=\Delta R+2|\operatorname{Ric}|^{2}
$$

For any small constant $\varepsilon>0$ and large constant $A>0$, we have

$$
\begin{aligned}
\frac{\partial}{\partial t}\left(R+\varepsilon e^{A t} f\right) & =\frac{\partial R}{\partial t}+\varepsilon A e^{A t} f \\
& =\Delta\left(R+\varepsilon e^{A t} f\right)+2|R i c|^{2}+\varepsilon e^{A t}(A f-\Delta f) \\
& >\Delta\left(R+\varepsilon e^{A t} f\right)
\end{aligned}
$$

by choosing $A$ large enough.

We claim that

$$
R+\varepsilon e^{A t} f>0 \quad \text { on } \quad M \times[0, T] .
$$

Suppose not, then there exist a first time $t_{0}>0$ and a point $x_{0} \in M$ such that

$$
\begin{aligned}
\left(R+\varepsilon e^{A t} f\right)\left(x_{0}, t_{0}\right) & =0, \\
\nabla\left(R+\varepsilon e^{A t} f\right)\left(x_{0}, t_{0}\right) & =0, \\
\Delta\left(R+\varepsilon e^{A t} f\right)\left(x_{0}, t_{0}\right) & \geq 0, \\
\text { and } \quad \frac{\partial}{\partial t}\left(R+\varepsilon e^{A t} f\right)\left(x_{0}, t_{0}\right) & \leq 0 .
\end{aligned}
$$

Then

$$
0 \geq \frac{\partial}{\partial t}\left(R+\varepsilon e^{A t} f\right)\left(x_{0}, t_{0}\right)>\Delta\left(R+\varepsilon e^{A t} f\right)\left(x_{0}, t_{0}\right) \geq 0,
$$

which is a contradiction. So we have proved that

$$
R+\varepsilon e^{A t} f>0 \quad \text { on } \quad M \times[0, T] .
$$

Letting $\varepsilon \rightarrow 0$, we get

$$
R \geq 0 \quad \text { on } \quad M \times[0, T] .
$$


This finishes the proof of the proposition. $\square$

Next we derive a maximum principle of Hamilton for tensors. Let $M$ be a complete manifold with a metric $g=\left\{g_{i j}\right\}, V$ a vector bundle over $M$ with a metric $h=\left\{h_{\alpha \beta}\right\}$ and a connection $\nabla=\left\{\Gamma_{i \beta}^{\alpha}\right\}$ compatible with $h$, and suppose $h$ is fixed but $g$ and $\nabla$ may vary smoothly with time $t$. Let $\Gamma(V)$ be the vector space of $C^{\infty}$ sections of $V$. The Laplacian $\Delta$ acting on a section $\sigma \in \Gamma(V)$ is defined by

$$
\Delta \sigma=g^{i j} \nabla_{i} \nabla_{j} \sigma
$$

Let $M_{\alpha \beta}$ be a symmetric bilinear form on $V$. We say $M_{\alpha \beta} \geq 0$ if $M_{\alpha \beta} v^{\alpha} v^{\beta} \geq 0$ for all vectors $v=\left\{v^{\alpha}\right\}$. Assume $N_{\alpha \beta}=\mathcal{P}\left(M_{\alpha \beta}, h_{\alpha \beta}\right)$ is a polynomial in $M_{\alpha \beta}$ formed by contracting products of $M_{\alpha \beta}$ with itself using the metric $h=\left\{h_{\alpha \beta}\right\}$. Assume that the tensor $M_{\alpha \beta}$ is uniformly bounded in space-time and let $g_{i j}$ evolve by the Ricci flow with bounded curvature.

LEMMA 2.1.3. Suppose that on $0 \leq t \leq T$,

$$
\frac{\partial}{\partial t} M_{\alpha \beta}=\Delta M_{\alpha \beta}+u^{i} \nabla_{i} M_{\alpha \beta}+N_{\alpha \beta}
$$

where $u^{i}(t)$ is a time-dependent vector field on $M$ with uniform bound and $N_{\alpha \beta}=$ $\mathcal{P}\left(M_{\alpha \beta}, h_{\alpha \beta}\right)$ satisfies

$$
N_{\alpha \beta} v^{\alpha} v^{\beta} \geq 0 \quad \text { whenever } M_{\alpha \beta} v^{\beta}=0
$$

If $M_{\alpha \beta} \geq 0$ at $t=0$, then it remains so on $0 \leq t \leq T$.

Proof. Set

$$
\tilde{M}_{\alpha \beta}=M_{\alpha \beta}+\varepsilon e^{A t} f h_{\alpha \beta},
$$

where $A>0$ is a suitably large constant (to be chosen later) and $f$ is the function constructed in Lemma 2.1.1.

We claim that $\tilde{M}_{\alpha \beta}>0$ on $M \times[0, T]$ for every $\varepsilon>0$. If not, then for some $\varepsilon>0$, there will be a first time $t_{0}>0$ where $\tilde{M}_{\alpha \beta}$ acquires a null vector $v^{\alpha}$ of unit length at some point $x_{0} \in M$. At $\left(x_{0}, t_{0}\right)$,

$$
\begin{aligned}
N_{\alpha \beta} v^{\alpha} v^{\beta} & \geq N_{\alpha \beta} v^{\alpha} v^{\beta}-\tilde{N}_{\alpha \beta} v^{\alpha} v^{\beta} \\
& \geq-C \varepsilon e^{A t_{0}} f\left(x_{0}\right)
\end{aligned}
$$

where $\tilde{N}_{\alpha \beta}=\mathcal{P}\left(\tilde{M}_{\alpha \beta}, h_{\alpha \beta}\right)$, and $C$ is a positive constant (depending on the bound of $M_{\alpha \beta}$, but independent of $A$ ).

Let us extend $v^{\alpha}$ to a local vector field in a neighborhood of $x_{0}$ by parallel translating $v^{\alpha}$ along geodesics (with respect to the metric $g_{i j}\left(t_{0}\right)$ ) emanating radially out of $x_{0}$, with $v^{\alpha}$ independent of $t$. Then, at $\left(x_{0}, t_{0}\right)$, we have

$$
\begin{aligned}
\frac{\partial}{\partial t}\left(\tilde{M}_{\alpha \beta} v^{\alpha} v^{\beta}\right) & \leq 0, \\
\nabla\left(\tilde{M}_{\alpha \beta} v^{\alpha} v^{\beta}\right) & =0, \\
\text { and } \quad \Delta\left(\tilde{M}_{\alpha \beta} v^{\alpha} v^{\beta}\right) & \geq 0 .
\end{aligned}
$$


But

$$
\begin{aligned}
0 \geq \frac{\partial}{\partial t}\left(\tilde{M}_{\alpha \beta} v^{\alpha} v^{\beta}\right)= & \frac{\partial}{\partial t}\left(M_{\alpha \beta} v^{\alpha} v^{\beta}+\varepsilon e^{A t} f\right), \\
= & \Delta\left(\tilde{M}_{\alpha \beta} v^{\alpha} v^{\beta}\right)-\Delta\left(\varepsilon e^{A t} f\right)+u^{i} \nabla_{i}\left(\tilde{M}_{\alpha \beta} v^{\alpha} v^{\beta}\right) \\
& -u^{i} \nabla_{i}\left(\varepsilon e^{A t} f\right)+N_{\alpha \beta} v^{\alpha} v^{\beta}+\varepsilon A e^{A t_{0}} f\left(x_{0}\right) \\
\geq & -C \varepsilon e^{A t_{0}} f\left(x_{0}\right)+\varepsilon A e^{A t_{0}} f\left(x_{0}\right)>0
\end{aligned}
$$

when $A$ is chosen sufficiently large. This is a contradiction.

By applying Lemma 2.1.3 to the evolution equation

$$
\frac{\partial}{\partial t} M_{\alpha \beta}=\Delta M_{\alpha \beta}+M_{\alpha \beta}^{2}+M_{\alpha \beta}^{\#}
$$

of the curvature operator $M_{\alpha \beta}$, we immediately obtain the following important result.

Proposition 2.1.4 (Hamilton [59]). Nonnegativity of the curvature operator $M_{\alpha \beta}$ is preserved by the Ricci flow.

In the Kähler case, the nonnegativity of the holomorpic bisectional curvature is preserved under the Kähler-Ricci flow. This result is proved by Bando [5] for complex dimension $n=3$ and by Mok [92] for general dimension $n$ when the manifold is compact, and by Shi [115] when the manifold is noncompact.

Proposition 2.1.5. Under the Kähler-Ricci flow if the initial metric has positive (nonnegative) holomorphic bisectional curvature then the evolved metric also has positive (nonnegative) holomorphic bisectional curvature.

2.2. Strong Maximum Principle. Let $\Omega$ be a bounded, connected open set of a complete $n$-dimensional manifold $M$, and let $g_{i j}(x, t)$ be a smooth solution to the Ricci flow on $\Omega \times[0, T]$. Consider a vector bundle $V$ over $\Omega$ with a fixed metric $h_{\alpha \beta}$ (independent of time), and a connection $\nabla=\left\{\Gamma_{i \beta}^{\alpha}\right\}$ which is compatible with $h_{\alpha \beta}$ and may vary with time $t$. Let $\Gamma(V)$ be the vector space of $C^{\infty}$ sections of $V$ over $\Omega$. The Laplacian $\Delta$ acting on a section $\sigma \in \Gamma(V)$ is defined by

$$
\Delta \sigma=g^{i j}(x, t) \nabla_{i} \nabla_{j} \sigma .
$$

Consider a family of smooth symmetric bilinear forms $M_{\alpha \beta}$ evolving by

$$
\frac{\partial}{\partial t} M_{\alpha \beta}=\Delta M_{\alpha \beta}+N_{\alpha \beta}, \quad \text { on } \Omega \times[0, T],
$$

where $N_{\alpha \beta}=P\left(M_{\alpha \beta}, h_{\alpha \beta}\right)$ is a polynomial in $M_{\alpha \beta}$ formed by contracting products of $M_{\alpha \beta}$ with itself using the metric $h_{\alpha \beta}$ and satisfies

$$
N_{\alpha \beta} \geq 0 \text {, whenever } M_{\alpha \beta} \geq 0 .
$$

The following result, due to Hamilton [59], shows that the solution of (2.2.1) satisfies a strong maximum principle.

Theorem 2.2.1 (Hamilton's strong maximum principle). Let $M_{\alpha \beta}$ be a smooth solution of the equation (2.2.1). Suppose $M_{\alpha \beta} \geq 0$ on $\Omega \times[0, T]$. Then there exists a positive constant $0<\delta \leq T$ such that on $\Omega \times(0, \delta)$, the rank of $M_{\alpha \beta}$ is constant, and 
the null space of $M_{\alpha \beta}$ is invariant under parallel translation and invariant in time and also lies in the null space of $N_{\alpha \beta}$.

Proof. Set

$$
l=\max _{x \in \Omega}\left\{\operatorname{rank} \text { of } M_{\alpha \beta}(x, 0)\right\} .
$$

Then we can find a nonnegative smooth function $\rho(x)$, which is positive somewhere and has compact support in $\Omega$, so that at every point $x \in \Omega$,

$$
\sum_{i=1}^{n-l+1} M_{\alpha \beta}(x, 0) v_{i}^{\alpha} v_{i}^{\beta} \geq \rho(x)
$$

for any $(n-l+1)$ orthogonal unit vectors $\left\{v_{1}, \ldots, v_{n-l+1}\right\}$ at $x$.

Let us evolve $\rho(x)$ by the heat equation

$$
\frac{\partial}{\partial t} \rho=\Delta \rho
$$

with the Dirichlet condition $\left.\rho\right|_{\partial \Omega}=0$ to get a smooth function $\rho(x, t)$ defined on $\Omega \times[0, T]$. By the standard strong maximum principle, we know that $\rho(x, t)$ is positive everywhere in $\Omega$ for all $t \in(0, T]$.

For every $\varepsilon>0$, we claim that at every point $(x, t) \in \Omega \times[0, T]$, there holds

$$
\sum_{i=1}^{n-l+1} M_{\alpha \beta}(x, t) v_{i}^{\alpha} v_{i}^{\beta}+\varepsilon e^{t}>\rho(x, t)
$$

for any $(n-l+1)$ orthogonal unit vectors $\left\{v_{1}, \ldots, v_{n-l+1}\right\}$ at $x$.

We argue by contradiction. Suppose not, then for some $\varepsilon>0$, there will be a first time $t_{0}>0$ and some $(n-l+1)$ orthogonal unit vectors $\left\{v_{1}, \ldots, v_{n-l+1}\right\}$ at some point $x_{0} \in \Omega$ so that

$$
\sum_{i=1}^{n-l+1} M_{\alpha \beta}\left(x_{0}, t_{0}\right) v_{i}^{\alpha} v_{i}^{\beta}+\varepsilon e^{t_{0}}=\rho\left(x_{0}, t_{0}\right)
$$

Let us extend each $v_{i}(i=1, \ldots, n-l+1)$ to a local vector field, independent of $t$, in a neighborhood of $x_{0}$ by parallel translation along geodesics (with respect to the metric $\left.g_{i j}\left(t_{0}\right)\right)$ emanating radially out of $x_{0}$. Clearly $\left\{v_{1}, \ldots, v_{n-l+1}\right\}$ remain orthogonal unit vectors in the neighborhood. Then, at $\left(x_{0}, t_{0}\right)$, we have

$$
\begin{aligned}
\frac{\partial}{\partial t}\left(\sum_{i=1}^{n-l+1} M_{\alpha \beta} v_{i}^{\alpha} v_{i}^{\beta}+\varepsilon e^{t}-\rho\right) & \leq 0, \\
\text { and } \Delta\left(\sum_{i=1}^{n-l+1} M_{\alpha \beta} v_{i}^{\alpha} v_{i}^{\beta}+\varepsilon e^{t}-\rho\right) & \geq 0 .
\end{aligned}
$$


But, since $N_{\alpha \beta} \geq 0$ by our assumption, we have

$$
\begin{aligned}
0 & \geq \frac{\partial}{\partial t}\left(\sum_{i=1}^{n-l+1} M_{\alpha \beta} v_{i}^{\alpha} v_{i}^{\beta}+\varepsilon e^{t}-\rho\right) \\
& =\sum_{i=1}^{n-l+1}\left(\Delta M_{\alpha \beta}+N_{\alpha \beta}\right) v_{i}^{\alpha} v_{i}^{\beta}+\varepsilon e^{t}-\Delta \rho \\
& \geq \sum_{i=1}^{n-l+1} \Delta\left(M_{\alpha \beta} v_{i}^{\alpha} v_{i}^{\beta}\right)+\varepsilon e^{t}-\Delta \rho \\
& =\sum_{i=1}^{n-l+1} \Delta\left(M_{\alpha \beta} v_{i}^{\alpha} v_{i}^{\beta}+\varepsilon e^{t}-\rho\right)+\varepsilon e^{t} \\
& \geq \varepsilon e^{t}>0 .
\end{aligned}
$$

This is a contradiction. Thus by letting $\varepsilon \rightarrow 0$, we prove that

$$
\sum_{i=1}^{n-l+1} M_{\alpha \beta}(x, t) v_{i}^{\alpha} v_{i}^{\beta} \geq \rho(x, t)
$$

for any $(n-l+1)$ orthogonal unit vectors $\left\{v_{1}, \ldots, v_{n-l+1}\right\}$ at $x \in \Omega$ and $t \in[0, T]$. Hence $M_{\alpha \beta}$ has at least rank $l$ everywhere in the open set $\Omega$ for all $t \in(0, T]$. Therefore we can find a positive constant $\delta(\leq T)$ such that the rank $M_{\alpha \beta}$ is constant over $\Omega \times(0, \delta)$.

Next we proceed to analyze the null space of $M_{\alpha \beta}$. Let $v$ be any smooth section of $V$ in the null of $M_{\alpha \beta}$ on $0<t<\delta$. Then

$$
\begin{aligned}
0 & =\frac{\partial}{\partial t}\left(M_{\alpha \beta} v^{\alpha} v^{\beta}\right) \\
& =\left(\frac{\partial}{\partial t} M_{\alpha \beta}\right) v^{\alpha} v^{\beta}+2 M_{\alpha \beta} v^{\alpha} \frac{\partial v^{\beta}}{\partial t} \\
& =\left(\frac{\partial}{\partial t} M_{\alpha \beta}\right) v^{\alpha} v^{\beta}
\end{aligned}
$$

and

$$
\begin{aligned}
0= & \Delta\left(M_{\alpha \beta} v^{\alpha} v^{\beta}\right) \\
= & \left(\Delta M_{\alpha \beta}\right) v^{\alpha} v^{\beta}+4 g^{k l} \nabla_{k} M_{\alpha \beta} \cdot v^{\alpha} \nabla_{l} v^{\beta} \\
& +2 M_{\alpha \beta} g^{k l} \nabla_{k} v^{\alpha} \cdot \nabla_{l} v^{\beta}+2 M_{\alpha \beta} v^{\alpha} \Delta v^{\beta} \\
= & \left(\Delta M_{\alpha \beta}\right) v^{\alpha} v^{\beta}+4 g^{k l} \nabla_{k} M_{\alpha \beta} \cdot v^{\alpha} \nabla_{l} v^{\beta}+2 M_{\alpha \beta} g^{k l} \nabla_{k} v^{\alpha} \cdot \nabla_{l} v^{\beta} .
\end{aligned}
$$

By noting that

$$
0=\nabla_{k}\left(M_{\alpha \beta} v^{\beta}\right)=\left(\nabla_{k} M_{\alpha \beta}\right) v^{\alpha}+M_{\alpha \beta} \nabla_{k} v^{\alpha}
$$

and using the evolution equation (2.2.1), we get

$$
N_{\alpha \beta} v^{\alpha} v^{\beta}+2 M_{\alpha \beta} g^{k l} \nabla_{k} v^{\alpha} \cdot \nabla_{l} v^{\beta}=0 .
$$


Since $M_{\alpha \beta} \geq 0$ and $N_{\alpha \beta} \geq 0$, we must have

$$
v \in \operatorname{null}\left(N_{\alpha \beta}\right) \quad \text { and } \quad \nabla_{i} v \in \operatorname{null}\left(M_{\alpha \beta}\right), \quad \text { for all } i .
$$

The first inclusion shows that null $\left(M_{\alpha \beta}\right) \subset$ null $\left(N_{\alpha \beta}\right)$, and the second inclusion shows that null $\left(M_{\alpha \beta}\right)$ is invariant under parallel translation.

To see null $\left(M_{\alpha \beta}\right)$ is also invariant in time, we first note that

$$
\Delta v=\nabla^{i}\left(\nabla_{i} v\right) \in \operatorname{null}\left(M_{\alpha \beta}\right)
$$

and then

$$
g^{k l} \nabla_{k} M_{\alpha \beta} \cdot \nabla_{l} v^{\alpha}=g^{k l} \nabla_{k}\left(M_{\alpha \beta} \nabla_{l} v^{\alpha}\right)-M_{\alpha \beta} \Delta v^{\alpha}=0 .
$$

Thus we have

$$
\begin{aligned}
0 & =\Delta\left(M_{\alpha \beta} v^{\alpha}\right) \\
& =\left(\Delta M_{\alpha \beta}\right) v^{\alpha}+2 g^{k l} \nabla_{k} M_{\alpha \beta} \cdot \nabla_{l} v^{\alpha}+M_{\alpha \beta} \Delta v^{\alpha} \\
& =\left(\Delta M_{\alpha \beta}\right) v^{\alpha},
\end{aligned}
$$

and hence

$$
\begin{aligned}
0 & =\frac{\partial}{\partial t}\left(M_{\alpha \beta} v^{\alpha}\right) \\
& =\left(\Delta M_{\alpha \beta}+N_{\alpha \beta}\right) v^{\alpha}+M_{\alpha \beta} \frac{\partial v^{\alpha}}{\partial t} \\
& =M_{\alpha \beta} \frac{\partial v^{\alpha}}{\partial t} .
\end{aligned}
$$

This shows that

$$
\frac{\partial v}{\partial t} \in \operatorname{null}\left(M_{\alpha \beta}\right)
$$

so the null space of $M_{\alpha \beta}$ is invariant in time.

We now apply Hamilton's strong maximum principle to the evolution equation of the curvature operator $M_{\alpha \beta}$. Recall

$$
\frac{\partial M_{\alpha \beta}}{\partial t}=\Delta M_{\alpha \beta}+M_{\alpha \beta}^{2}+M_{\alpha \beta}^{\#}
$$

where $M_{\alpha \beta}^{\#}=C_{\alpha}^{\xi \gamma} C_{\beta}^{\eta \theta} M_{\xi \eta} M_{\gamma \theta}$. Suppose we have a solution to the Ricci flow with nonnegative curvature operator. Then by Theorem 2.2.1, the null space of the curvature operator $M_{\alpha \beta}$ of the solution has constant rank and is invariant in time and under parallel translation over some time interval $0<t<\delta$. Moreover the null space of $M_{\alpha \beta}$ must also lie in the null space of $M_{\alpha \beta}^{\#}$.

Denote by $(n-k)$ the rank of $M_{\alpha \beta}$ on $0<t<\delta$. Let us diagonalize $M_{\alpha \beta}$ so that $M_{\alpha \alpha}=0$ if $\alpha \leq k$ and $M_{\alpha \alpha}>0$ if $\alpha>k$. Then we have $M_{\alpha \alpha}^{\#}=0$ also for $\alpha \leq k$ from the evolution equation of $M_{\alpha \alpha}$. Since

$$
0=M_{\alpha \alpha}^{\#}=C_{\alpha}^{\xi \gamma} C_{\alpha}^{\eta \theta} M_{\xi \eta} M_{\gamma \theta},
$$


it follows that

$$
\begin{aligned}
C_{\alpha}^{\xi \gamma} & =\left\langle v^{\alpha},\left[v^{\xi}, v^{\gamma}\right]\right\rangle \\
& =0, \quad \text { if } \quad \alpha \leq k \text { and } \xi, \gamma>k
\end{aligned}
$$

This says that the image of $M_{\alpha \beta}$ is a Lie subalgebra (in fact it is the subalgebra of the restricted holonomy group by using the Ambrose-Singer holonomy theorem [3]). This proves the following result.

THEOREM 2.2.2 (Hamilton [59]). Suppose the curvature operator $M_{\alpha \beta}$ of the initial metric is nonnegative. Then, under the Ricci flow, for some interval $0<t<\delta$ the image of $M_{\alpha \beta}$ is a Lie subalgebra of $s o(n)$ which has constant rank and is invariant under parallel translation and invariant in time.

2.3. Advanced Maximum Principle for Tensors. In this section we present Hamilton's advanced maximum principle for tensors which generalizes Lemma 2.1.3 and shows how a tensor evolving by a nonlinear heat equation may be controlled by a system of ODEs. An important application of the advanced maximum principle is the Hamilton-Ivey curvature pinching estimate for the Ricci flow on three-manifolds given in the next section. More applications will be given in Chapter 5 .

Let $M$ be a complete manifold equipped with a one-parameter family of Riemannian metrics $g_{i j}(t), 0 \leq t \leq T$, with $T<+\infty$. Let $V \rightarrow M$ be a vector bundle with a time-independent bundle metric $h_{a b}$ and $\Gamma(V)$ be the vector space of $C^{\infty}$ sections of $V$. Let

$$
\nabla_{t}: \quad \Gamma(V) \rightarrow \Gamma\left(V \otimes T^{*} M\right), \quad t \in[0, T]
$$

be a smooth family of time-dependent connections compatible with $h_{a b}$, i.e.

$$
\left(\nabla_{t}\right)_{i} h_{a b} \triangleq\left(\nabla_{t}\right)_{\frac{\partial}{\partial x^{i}}} h_{a b}=0
$$

for any local coordinate $\left\{\frac{\partial}{\partial x^{1}}, \ldots, \frac{\partial}{\partial x^{n}}\right\}$. The Laplacian $\Delta_{t}$ acting on a section $\sigma \in$ $\Gamma(V)$ is defined by

$$
\Delta_{t} \sigma=g^{i j}(x, t)\left(\nabla_{t}\right)_{i}\left(\nabla_{t}\right)_{j} \sigma
$$

For the application to the Ricci flow, we will always assume that the metrics $g_{i j}(\cdot, t)$ evolve by the Ricci flow. Since $M$ may be noncompact, we assume that, for the sake of simplicity, the curvature of $g_{i j}(t)$ is uniformly bounded on $M \times[0, T]$.

Let $N: V \times[0, T] \rightarrow V$ be a fiber preserving map, i.e., $N(x, \sigma, t)$ is a timedependent vector field defined on the bundle $V$ and tangent to the fibers. We assume that $N(x, \sigma, t)$ is continuous in $x, t$ and satisfies

$$
\left|N\left(x, \sigma_{1}, t\right)-N\left(x, \sigma_{2}, t\right)\right| \leq C_{B}\left|\sigma_{1}-\sigma_{2}\right|
$$

for all $x \in M, t \in[0, T]$ and $\left|\sigma_{1}\right| \leq B,\left|\sigma_{2}\right| \leq B$, where $C_{B}$ is a positive constant depending only on $B$. Then we can form the nonlinear heat equation

$$
\frac{\partial}{\partial t} \sigma(x, t)=\Delta_{t} \sigma(x, t)+u^{i}\left(\nabla_{t}\right)_{i} \sigma(x, t)+N(x, \sigma(x, t), t)
$$

where $u^{i}=u^{i}(t)$ is a time-dependent vector field on $M$ which is uniformly bounded on $M \times[0, T]$. Let $K$ be a closed subset of $V$. One important question is under what conditions will solutions of the PDE which start in $K$ remain in $K$. To answer this question, Hamilton [59] imposed the following two conditions on $K$ : 
(H1) $K$ is invariant under parallel translation defined by the connection $\nabla_{t}$ for each $t \in[0, T]$

(H2) in each fiber $V_{x}$, the set $K_{x} \triangleq V_{x} \cap K$ is closed and convex.

Then one can judge the behavior of the PDE by comparing to that of the following ODE

(ODE)

$$
\frac{d \sigma_{x}}{d t}=N\left(x, \sigma_{x}, t\right)
$$

for $\sigma_{x}=\sigma_{x}(t)$ in each fiber $V_{x}$.

Theorem 2.3.1 (Hamilton's advanced maximum principle [59]). Let $K$ be a closed subset of $V$ satisfying the hypothesis (H1) and (H2). Suppose that for any $x \in M$ and any initial time $t_{0} \in[0, T)$, any solution $\sigma_{x}(t)$ of the (ODE) which starts in $K_{x}$ at $t_{0}$ will remain in $K_{x}$ for all later times. Then for any initial time $t_{0} \in[0, T)$ the solution $\sigma(x, t)$ of the (PDE) will remain in $K$ for all later times provided $\sigma(x, t)$ starts in $K$ at time $t_{0}$ and $\sigma(x, t)$ is uniformly bounded with respect to the bundle metric $h_{a b}$ on $M \times\left[t_{0}, T\right]$.

We remark that Lemma 2.1.3 is a special case of the above theorem where $V$ is given by a symmetric tensor product of a vector bundle and $K$ corresponds to the convex set consisting of all nonnegative symmetric bilinear forms. We also remark that Hamilton [59] established the above theorem for a general evolving metric $g_{i j}(x, t)$ which does not necessarily satisfy the Ricci flow.

Before proving Theorem 2.3.1, we need to establish three lemmas. Let $\varphi:[a, b] \rightarrow$ $\mathbb{R}$ be a Lipschitz function. We consider $\frac{d \varphi}{d t}(t)$ at $t \in[a, b)$ in the sense of limsup of the forward difference quotients, i.e.,

$$
\frac{d \varphi}{d t}(t)=\limsup _{h \rightarrow 0^{+}} \frac{\varphi(t+h)-\varphi(t)}{h}
$$

Lemma 2.3.2. Suppose $\varphi:[a, b] \rightarrow \mathbb{R}$ is Lipschitz continuous and suppose for some constant $C<+\infty$,

$$
\frac{d}{d t} \varphi(t) \leq C \varphi(t), \quad \text { whenever } \varphi(t) \geq 0 \text { on }[a, b),
$$

$$
\text { and } \varphi(a) \leq 0 .
$$

Then $\varphi(t) \leq 0$ on $[a, b]$.

Proof. By replacing $\varphi$ by $e^{-C t} \varphi$, we may assume

$$
\frac{d}{d t} \varphi(t) \leq 0, \quad \text { whenever } \varphi(t) \geq 0 \text { on }[a, b)
$$

and

$$
\varphi(a) \leq 0 \text {. }
$$

For arbitrary $\varepsilon>0$, we shall show $\varphi(t) \leq \varepsilon(t-a)$ on $[a, b]$. Clearly we may assume $\varphi(a)=0$. Since

$$
\limsup _{h \rightarrow 0^{+}} \frac{\varphi(a+h)-\varphi(a)}{h} \leq 0,
$$

there must be some interval $a \leq t<\delta$ on which $\varphi(t) \leq \varepsilon(t-a)$. 
Let $a \leq t<c$ be the largest interval with $c \leq b$ such that $\varphi(t) \leq \varepsilon(t-a)$ on $[a, c)$. Then by continuity $\varphi(t) \leq \varepsilon(t-a)$ on the closed interval $[a, c]$. We claim that $c=b$. Suppose not, then we can find $\delta>0$ such that $\varphi(t) \leq \varepsilon(t-a)$ on $[a, c+\delta]$ since

$$
\limsup _{h \rightarrow 0^{+}} \frac{\varphi(c+h)-\varphi(c)}{h} \leq 0 .
$$

This contradicts the choice of the largest interval $[a, c)$. Therefore, since $\varepsilon>0$ can be arbitrary small, we have proved $\varphi(t) \leq 0$ on $[a, b]$.

The second lemma below is a general principle on the derivative of a sup-function which will bridge solutions between ODEs and PDEs. Let $X$ be a complete smooth manifold and $Y$ be a compact subset of $X$. Let $\psi(x, t)$ be a smooth function on $X \times[a, b]$ and let $\varphi(t)=\sup \{\psi(y, t) \mid y \in Y\}$. Then it is clear that $\varphi(t)$ is Lipschitz continuous. We have the following useful estimate on its derivative.

LEMMA 2.3.3.

$$
\frac{d}{d t} \varphi(t) \leq \sup \left\{\frac{\partial \psi}{\partial t}(y, t) \mid y \in Y \text { satisfies } \psi(y, t)=\varphi(t)\right\} .
$$

Proof. Choose a sequence of times $\left\{t_{j}\right\}$ decreasing to $t$ for which

$$
\lim _{t_{j} \rightarrow t} \frac{\varphi\left(t_{j}\right)-\varphi(t)}{t_{j}-t}=\frac{d \varphi(t)}{d t} .
$$

Since $Y$ is compact, we can choose $y_{j} \in Y$ with $\varphi\left(t_{j}\right)=\psi\left(y_{j}, t_{j}\right)$. By passing to a subsequence, we can assume $y_{j} \rightarrow y$ for some $y \in Y$. By continuity, we have $\varphi(t)=\psi(y, t)$. It follows that $\psi\left(y_{j}, t\right) \leq \psi(y, t)$, and then

$$
\begin{aligned}
\varphi\left(t_{j}\right)-\varphi(t) & \leq \psi\left(y_{j}, t_{j}\right)-\psi\left(y_{j}, t\right) \\
& =\frac{\partial}{\partial t} \psi\left(y_{j}, \tilde{t}_{j}\right) \cdot\left(t_{j}-t\right)
\end{aligned}
$$

for some $\tilde{t}_{j} \in\left[t, t_{j}\right]$ by the mean value theorem. Thus we have

$$
\lim _{t_{j} \rightarrow t} \frac{\varphi\left(t_{j}\right)-\varphi(t)}{t_{j}-t} \leq \frac{\partial}{\partial t} \psi(y, t)
$$

This proves the result.

We remark that the above two lemmas are somewhat standard facts in the theory of PDEs and we have implicitly used them in the previous sections when we apply the maximum principle. The third lemma gives a characterization of when a system of ODEs preserve closed convex sets in Euclidean space. Let $Z \subset \mathbb{R}^{n}$ be a closed convex subset. We define the tangent cone $T_{\varphi} Z$ to the closed convex set $Z$ at a point $\varphi \in \partial Z$ as the smallest closed convex cone with vertex at $\varphi$ which contains $Z$.

Lemma 2.3.4. Let $U \subset R^{n}$ be an open set and $Z \subset U$ be a closed convex subset. Consider the ODE

$$
\frac{d \varphi}{d t}=N(\varphi, t)
$$

where $N: U \times[0, T] \rightarrow R^{n}$ is continuous and Lipschitz in $\varphi$. Then the following two statements are equivalent. 
(i) For any initial time $t_{0} \in[0, T]$, any solution of the ODE (2.3.1) which starts in $Z$ at $t_{0}$ will remain in $Z$ for all later times;

(ii) $\varphi+N(\varphi, t) \in T_{\varphi} Z$ for all $\varphi \in \partial Z$ and $t \in[0, T)$.

Proof. We say that a linear function $l$ on $\mathbb{R}^{n}$ is a support function for $Z$ at $\varphi \in \partial Z$ and write $l \in S_{\varphi} Z$ if $|l|=1$ and $l(\varphi) \geq l(\eta)$ for all $\eta \in Z$. Then $\varphi+N(\varphi, t) \in T_{\varphi} Z$ if and only if $l(N(\varphi, t)) \leq 0$ for all $l \in S_{\varphi} Z$. Suppose $l(N(\varphi, t))>0$ for some $\varphi \in \partial Z$ and some $l \in S_{\varphi} Z$. Then

$$
\frac{d}{d t} l(\varphi)=l\left(\frac{d \varphi}{d t}\right)=l(N(\varphi, t))>0,
$$

so $l(\varphi)$ is strictly increasing and the solution $\varphi(t)$ of the ODE (2.3.1) cannot remain in $Z$.

To see the converse, first note that we may assume $Z$ is compact. This is because we can modify the vector field $N(\varphi, t)$ by multiplying a cutoff function which is everywhere nonnegative, equals one on a large ball and equals zero on the complement of a larger ball. The paths of solutions of the ODE are unchanged inside the first large ball, so we can intersect $Z$ with the second ball to make $Z$ convex and compact. If there were a counterexample before the modification there would still be one after as we chose the first ball large enough.

Let $s(\varphi)$ be the distance from $\varphi$ to $Z$ in $\mathbb{R}^{n}$. Clearly $s(\varphi)=0$ if $\varphi \in Z$. Then

$$
s(\varphi)=\sup \left\{l(\varphi-\eta) \mid \eta \in \partial Z \text { and } l \in S_{\eta} Z\right\} .
$$

The sup is taken over a compact subset of $\mathbb{R}^{n} \times \mathbb{R}^{n}$. Hence by Lemma 2.3 .3

$$
\frac{d}{d t} s(\varphi) \leq \sup \left\{l(N(\varphi, t)) \mid \eta \in \partial Z, l \in S_{\eta} Z \text { and } s(\varphi)=l(\varphi-\eta)\right\} .
$$

It is clear that the sup on the RHS of the above inequality can be takeen only when $\eta$ is the unique closest point in $Z$ to $\varphi$ and $l$ is the linear function of length one with gradient in the direction of $\varphi-\eta$. Since $N(\varphi, t)$ is Lipschitz in $\varphi$ and continuous in $t$, we have

$$
|N(\varphi, t)-N(\eta, t)| \leq C|\varphi-\eta|
$$

for some constant $C$ and all $\varphi$ and $\eta$ in the compact set $Z$.

By hypothesis (ii),

$$
l(N(\eta, t)) \leq 0,
$$

and for the unique $\eta$, the closest point in $Z$ to $\varphi$,

$$
|\varphi-\eta|=s(\varphi)
$$

Thus

$$
\begin{aligned}
\frac{d}{d t} s(\varphi) & \leq \sup \left\{\begin{array}{c}
l(N(\eta, t))+|l(N(\varphi, t))-l(N(\eta, t))| \quad \mid \eta \in \partial Z, \\
l \in S_{\eta} Z, \quad \text { and } \quad s(\varphi)=l(\varphi-\eta)
\end{array}\right\} \\
& \leq C s(\varphi) .
\end{aligned}
$$


Since $s(\varphi)=0$ to start at $t_{0}$, it follows from Lemma 2.3.2 that $s(\varphi)=0$ for $t \in\left[t_{0}, T\right]$. This proves the lemma.

We are now ready to prove Theorem 2.3.1.

Proof of Theorem 2.3.1. Since the solution $\sigma(x, t)$ of the (PDE) is uniformly bounded with respect to the bundle metric $h_{a b}$ on $M \times\left[t_{0}, T\right]$ by hypothesis, we may assume that $K$ is contained in a tubular neighborhood $V(r)$ of the zero section in $V$ whose intersection with each fiber $V_{x}$ is a ball of radius $r$ around the origin measured by the bundle metric $h_{a b}$ for some large $r>0$.

Recall that $g_{i j}(\cdot, t), t \in[0, T]$, is a smooth solution to the Ricci flow with uniformly bounded curvature on $M \times[0, T]$. From Lemma 2.1.1, we have a smooth function $f$ such that $f \geq 1$ everywhere, $f(x) \rightarrow+\infty$ as $d_{0}\left(x, x_{0}\right) \rightarrow+\infty$ for some fixed point $x_{0} \in M$, and the first and second covariant derivatives with respect to the metrics $g_{i j}(\cdot, t)$ are uniformly bounded on $M \times[0, T]$. Using the metric $h_{a b}$ in each fiber $V_{x}$ and writing $|\varphi-\eta|$ for the distance between $\varphi \in V_{x}$ and $\eta \in V_{x}$, we set

$$
s(t)=\sup _{x \in M}\left\{\inf \left\{|\sigma(x, t)-\eta| \mid \eta \in K_{x} \triangleq K \cap V_{x}\right\}-\epsilon e^{A t} f(x)\right\}
$$

where $\epsilon$ is an arbitrarily small positive number and $A$ is a positive constant to be determined. We rewrite the function $s(t)$ as

$$
s(t)=\sup \left\{l(\sigma(x, t)-\eta)-\epsilon e^{A t} f(x) \mid x \in M, \eta \in \partial K_{x} \text { and } l \in S_{\eta} K_{x}\right\} .
$$

By the construction of the function $f$, we see that the sup is taken in a compact subset of $M \times V \times V^{*}$ for all $t$. Then by Lemma 2.3.3,

$$
\frac{d s(t)}{d t} \leq \sup \left\{\frac{\partial}{\partial t} l(\sigma(x, t)-\eta)-\epsilon A e^{A t} f(x)\right\}
$$

where the sup is over all $x \in M, \eta \in \partial K_{x}$ and $l \in S_{\eta} K_{x}$ such that

$$
l(\sigma(x, t)-\eta)-\epsilon e^{A t} f(x)=s(t)
$$

in particular we have $|\sigma(x, t)-\eta|=l(\sigma(x, t)-\eta)$, where $\eta$ is the unique closest point in $K_{x}$ to $\sigma(x, t)$, and $l$ is the linear function of length one on the fiber $V_{x}$ with gradient in the direction of $\eta$ to $\sigma(x, t)$. We compute at these $(x, \eta, l)$,

$$
\begin{aligned}
& \frac{\partial}{\partial t} l(\sigma(x, t)-\eta)-\epsilon A e^{A t} f(x) \\
& =l\left(\frac{\partial \sigma(x, t)}{\partial t}\right)-\epsilon A e^{A t} f(x) \\
& =l\left(\Delta_{t} \sigma(x, t)\right)+l\left(u^{i}(x, t)\left(\nabla_{t}\right)_{i} \sigma(x, t)\right)+l(N(x, \sigma(x, t), t))-\epsilon A e^{A t} f(x) .
\end{aligned}
$$

By the assumption and Lemma 2.3.4 we have $\eta+N(x, \eta, t) \in T_{\eta} K_{x}$. Hence, for those $(x, \eta, l), l(N(x, \eta, t)) \leq 0$ and then

$$
\begin{aligned}
& l(N(x, \sigma(x, t), t)) \\
& \leq l(N(x, \eta, t))+|N(x, \sigma(x, t), t)-N(x, \eta, t)| \\
& \leq C|\sigma(x, t)-\eta|=C\left(s(t)+\epsilon e^{A t} f(x)\right)
\end{aligned}
$$


for some positive constant $C$ by the assumption that $N(x, \sigma, t)$ is Lipschitz in $\sigma$ and the fact that the sup is taken on a compact set. Thus the combination of $(2.3 .2)-(2.3 .4)$ gives

$$
\frac{d s(t)}{d t} \leq l\left(\Delta_{t} \sigma(x, t)\right)+l\left(u^{i}(x, t)\left(\nabla_{t}\right)_{i} \sigma(x, t)\right)+C s(t)+\epsilon(C-A) e^{A t} f(x)
$$

for those $x \in M, \eta \in \partial K_{x}$ and $l \in S_{\eta} K_{x}$ such that $l(\sigma(x, t)-\eta)-\epsilon e^{A t} f(x)=s(t)$.

Next we estimate the first two terms of (2.3.5). As we extend a vector in a bundle from a point $x$ by parallel translation along geodesics emanating radially out of $x$, we will get a smooth section of the bundle in some small neighborhood of $x$ such that all the symmetrized covariant derivatives at $x$ are zero. Now let us extend $\eta \in V_{x}$ and $l \in V_{x}^{*}$ in this manner. Clearly, we continue to have $|l|(\cdot)=1$. Since $K$ is invariant under parallel translations, we continue to have $\eta(\cdot) \in \partial K$ and $l(\cdot)$ as a support function for $K$ at $\eta(\cdot)$. Therefore

$$
l(\sigma(\cdot, t)-\eta(\cdot))-\epsilon e^{A t} f(\cdot) \leq s(t)
$$

in the neighborhood. It follows that the function $l(\sigma(\cdot, t)-\eta(\cdot))-\epsilon e^{A t} f(\cdot)$ has a local maximum at $x$, so at $x$

$$
\begin{array}{r}
\left(\nabla_{t}\right)_{i}\left(l(\sigma(x, t)-\eta)-\epsilon e^{A t} f(x)\right)=0, \\
\text { and } \Delta_{t}\left(l(\sigma(x, t)-\eta)-\epsilon e^{A t} f(x)\right) \leq 0 .
\end{array}
$$

Hence at $x$

$$
\begin{aligned}
& l\left(\left(\nabla_{t}\right)_{i} \sigma(x, t)\right)-\epsilon e^{A t}\left(\nabla_{t}\right)_{i} f(x)=0, \\
& \text { and } l\left(\Delta_{t} \sigma(x, t)\right)-\epsilon e^{A t} \Delta_{t} f(x) \leq 0
\end{aligned}
$$

Therefore by combining with (2.3.5), we have

$$
\begin{aligned}
\frac{d}{d t} s(t) & \leq C s(t)+\epsilon\left(\Delta_{t} f(x)+u^{i}\left(\nabla_{t}\right)_{i} f(x)+(C-A) f(x)\right) e^{A t} \\
& \leq C s(t)
\end{aligned}
$$

for $A>0$ large enough, since $f(x) \geq 1$ and the first and second covariant derivatives of $f$ are uniformly bounded on $M \times[0, T]$. So by applying Lemma 2.3 .2 and the arbitrariness of $\epsilon$, we have completed the proof of Theorem 2.3.1.

Finally, we would like to state a useful generalization of Theorem 2.3.1 by Chow and $\mathrm{Lu}$ in [40] which allows the set $K$ to depend on time. One can consult the paper [40] for the proof.

TheOrem 2.3.5 (Chow and Lu [40]). Let $K(t) \subset V, t \in[0, T]$ be closed subsets which satisfy the following hypotheses

(H3) $K(t)$ is invariant under parallel translation defined by the connection $\nabla_{t}$ for each $t \in[0, T]$

(H4) in each fiber $V_{x}$, the set $K_{x}(t) \triangleq K(t) \cap V_{x}$ is nonempty, closed and convex for each $t \in[0, T]$;

(H5) the space-time track $\bigcup_{t \in[0, T]}(\partial K(t) \times\{t\})$ is a closed subset of $V \times[0, T]$. 
Suppose that, for any $x \in M$ and any initial time $t_{0} \in[0, T)$, and for any solution $\sigma_{x}(t)$ of the $(\mathrm{ODE})$ which starts in $K_{x}\left(t_{0}\right)$, the solution $\sigma_{x}(t)$ will remain in $K_{x}(t)$ for all later times. Then for any initial time $t_{0} \in[0, T)$ the solution $\sigma(x, t)$ of the (PDE) will remain in $K(t)$ for all later times if $\sigma(x, t)$ starts in $K\left(t_{0}\right)$ at time $t_{0}$ and the solution $\sigma(x, t)$ is uniformly bounded with respect to the bundle metric $h_{a b}$ on $M \times\left[t_{0}, T\right]$.

2.4. Hamilton-Ivey Curvature Pinching Estimate. The Hamilton-Ivey curvature pinching estimate roughly says that if a solution to the Ricci flow on a three-manifold becomes singular (i.e., the curvature goes to infinity) as time $t$ approaches the maximal time $T$, then the most negative sectional curvature will be small compared to the most positive sectional curvature. This pinching estimate plays a crucial role in analyzing the formation of singularities in the Ricci flow on three-manifolds.

Consider a complete solution to the Ricci flow

$$
\frac{\partial}{\partial t} g_{i j}=-2 R_{i j}
$$

on a complete three-manifold with bounded curvature in space for each time $t \geq 0$. Recall from Section 1.3 that the evolution equation of the curvature operator $M_{\alpha \beta}$ is given by

$$
\frac{\partial}{\partial t} M_{\alpha \beta}=\Delta M_{\alpha \beta}+M_{\alpha \beta}^{2}+M_{\alpha \beta}^{\#}
$$

where $M_{\alpha \beta}^{2}$ is the operator square

$$
M_{\alpha \beta}^{2}=M_{\alpha \gamma} M_{\beta \gamma}
$$

and $M_{\alpha \beta}^{\#}$ is the Lie algebra $s o(n)$ square

$$
M_{\alpha \beta}^{\#}=C_{\alpha}^{\gamma \zeta} C_{\beta}^{\eta \theta} M_{\gamma \eta} M_{\zeta \theta} .
$$

In dimension $n=3$, we know that $M_{\alpha \beta}^{\#}$ is the adjoint matrix of $M_{\alpha \beta}$. If we diagonalize $M_{\alpha \beta}$ with eigenvalues $\lambda \geq \mu \geq \nu$ so that

$$
\left(M_{\alpha \beta}\right)=\left(\begin{array}{ccc}
\lambda & & \\
& \mu & \\
& & \nu
\end{array}\right),
$$

then $M_{\alpha \beta}^{2}$ and $M_{\alpha \beta}^{\#}$ are also diagonal, with

$$
\left(M_{\alpha \beta}^{2}\right)=\left(\begin{array}{ccc}
\lambda^{2} & & \\
& \mu^{2} & \\
& & \nu^{2}
\end{array}\right) \text { and }\left(M_{\alpha \beta}^{\#}\right)=\left(\begin{array}{ccc}
\mu \nu & & \\
& \lambda \nu & \\
& & \lambda \mu
\end{array}\right) .
$$

Thus the ODE corresponding to PDE (2.4.1) for $M_{\alpha \beta}$ (in the space of $3 \times 3$ matrices) is given by the following system

$$
\left\{\begin{array}{l}
\frac{d}{d t} \lambda=\lambda^{2}+\mu \nu, \\
\frac{d}{d t} \mu=\mu^{2}+\lambda \nu, \\
\frac{d}{d t} \nu=\nu^{2}+\lambda \mu .
\end{array}\right.
$$


Let $P$ be the principal bundle of the manifold and form the associated bundle $V=P \times_{G} E$, where $G=O(3)$ and $E$ is the vector space of symmetric bilinear forms on $s o(3)$. The curvature operator $M_{\alpha \beta}$ is a smooth section of $V=P \times_{G} E$. According to Theorem 2.3.1, any closed convex set of curvature operator matrices $M_{\alpha \beta}$ which is $O(3)$-invariant (and hence invariant under parallel translation) and preserved by ODE (2.4.2) is also preserved by the Ricci flow.

We are now ready to state and prove the Hamilton-Ivey pinching estimate .

Theorem 2.4.1 (Hamilton [63], Ivey [73]). Suppose we have a solution to the Ricci flow on a three-manifold which is complete with bounded curvature for each $t \geq 0$. Assume at $t=0$ the eigenvalues $\lambda \geq \mu \geq \nu$ of the curvature operator at each point are bounded below by $\nu \geq-1$. The scalar curvature $R=\lambda+\mu+\nu$ is their sum. Then at all points and all times $t \geq 0$ we have the pinching estimate

$$
R \geq(-\nu)[\log (-\nu)-3],
$$

whenever $\nu<0$.

Proof. Consider the function

$$
y=f(x)=x(\log x-3)
$$

defined on $e^{2} \leq x<+\infty$. It is easy to check that $f$ is increasing and convex with range $-e^{2} \leq y<+\infty$. Let $f^{-1}(y)=x$ be the inverse function, which is also increasing but concave and satisfies

$$
\lim _{y \rightarrow \infty} \frac{f^{-1}(y)}{y}=0
$$

Consider also the set $K$ of matrices $M_{\alpha \beta}$ defined by the inequalities

$$
K:\left\{\begin{array}{l}
\lambda+\mu+\nu \geq-3, \\
\nu+f^{-1}(\lambda+\mu+\nu) \geq 0 .
\end{array}\right.
$$

By Theorem 2.3.1 and the assumptions in Theorem 2.4.1 at $t=0$, we only need to check that the set $K$ defined above is closed, convex and preserved by the ODE (2.4.2).

Clearly $K$ is closed because $f^{-1}$ is continuous. $\lambda+\mu+\nu$ is just the trace function of $3 \times 3$ matrices which is a linear function. Hence the first inequality in (2.4.4) defines a linear half-space, which is convex. The function $\nu$ is the least eigenvalue function, which is concave. Also note that $f^{-1}$ is concave. Thus the second inequality in (2.4.4) defines a convex set as well. Therefore we proved $K$ is closed and convex.

Under the ODE (2.4.2)

$$
\begin{aligned}
\frac{d}{d t}(\lambda+\mu+\nu) & =\lambda^{2}+\mu^{2}+\nu^{2}+\lambda \mu+\lambda \nu+\mu \nu \\
& =\frac{1}{2}\left[(\lambda+\mu)^{2}+(\lambda+\nu)^{2}+(\mu+\nu)^{2}\right] \\
& \geq 0
\end{aligned}
$$

Thus the first inequality in (2.4.4) is preserved by the ODE. 
The second inequality in (2.4.4) can be written as

$$
\lambda+\mu+\nu \geq f(-\nu), \quad \text { whenever } \nu \leq-e^{2},
$$

which becomes

$$
\lambda+\mu \geq(-\nu)[\log (-\nu)-2], \quad \text { whenever } \nu \leq-e^{2} .
$$

To show the inequality is preserved we only need to look at points on the boundary of the set. If $\nu+f^{-1}(\lambda+\mu+\nu)=0$ then $\nu=-f^{-1}(\lambda+\mu+\nu) \leq-e^{2}$ since $f^{-1}(y) \geq e^{2}$. Hence the RHS of (2.4.5) is nonnegative. We thus have $\lambda \geq 0$ because $\lambda \geq \mu$. But $\mu$ may have either sign. We split our consideration into two cases:

Case (i): $\mu \geq 0$.

We need to verify

$$
\frac{d \lambda}{d t}+\frac{d \mu}{d t} \geq(\log (-\nu)-1) \frac{d(-\nu)}{d t}
$$

when $\lambda+\mu=(-\nu)[\log (-\nu)-2]$. Solving for

$$
\log (-\nu)-2=\frac{\lambda+\mu}{(-\nu)}
$$

and substituting above, we must show

$$
\lambda^{2}+\mu \nu+\mu^{2}+\lambda \nu \geq\left(\frac{\lambda+\mu}{(-\nu)}+1\right)\left(-\nu^{2}-\lambda \mu\right)
$$

which is equivalent to

$$
\left(\lambda^{2}+\mu^{2}\right)(-\nu)+\lambda \mu(\lambda+\mu+(-\nu))+(-\nu)^{3} \geq 0 .
$$

Since $\lambda, \mu$ and $(-\nu)$ are all nonnegative we are done in the first case.

Case (ii): $\mu<0$.

We need to verify

$$
\frac{d \lambda}{d t} \geq \frac{d(-\mu)}{d t}+(\log (-\nu)-1) \frac{d(-\nu)}{d t}
$$

when $\lambda=(-\mu)+(-\nu)[\log (-\nu)-2]$. Solving for

$$
\log (-\nu)-2=\frac{\lambda-(-\mu)}{(-\nu)}
$$

and substituting above, we need to show

$$
\lambda^{2}+\mu \nu \geq-\mu^{2}-\lambda \nu+\left(\frac{\lambda-(-\mu)}{(-\nu)}+1\right)\left(-\nu^{2}-\lambda \mu\right)
$$

or

$$
\lambda^{2}+(-\mu)(-\nu) \geq \lambda(-\nu)-(-\mu)^{2}+\left(\frac{\lambda-(-\mu)}{(-\nu)}+1\right)\left(\lambda(-\mu)-(-\nu)^{2}\right)
$$


which reduces to

$$
\lambda^{2}(-\nu)+\lambda(-\mu)^{2}+(-\mu)^{2}(-\nu)+(-\nu)^{3} \geq \lambda^{2}(-\mu)+\lambda(-\mu)(-\nu)
$$

or equivalently

$$
\left(\lambda^{2}-\lambda(-\mu)+(-\mu)^{2}\right)((-\nu)-(-\mu))+(-\mu)^{3}+(-\nu)^{3} \geq 0
$$

Since $\lambda^{2}-\lambda(-\mu)+(-\mu)^{2} \geq 0$ and $(-\nu)-(-\mu) \geq 0$ we are also done in the second case.

Therefore the proof is completed.

2.5. Li-Yau-Hamilton Estimates. In [82], Li-Yau developed a fundamental gradient estimate, now called Li-Yau estimate, for positive solutions to the heat equation on a complete Riemannian manifold with nonnegative Ricci curvature. They used it to derive the Harnack inequality for such solutions by path integration. Then based on the suggestion of Yau, Hamilton [60] developed a similar estimate for the scalar curvature of solutions to the Ricci flow on a Riemann surface with positive curvature, and later obtained a matrix version of the Li-Yau estimate for solutions to the Ricci flow with positive curvature operator in all dimensions. This matrix version of the Li-Yau estimate is the Li-Yau-Hamilton estimate, which we will present in this section. The Li-Yau-Hamilton estimate plays a central role in the analysis of formation of singularities and the application of the Ricci flow to three-manifold topology.

We have seen that in the Ricci flow the curvature tensor satisfies a nonlinear heat equation, and the nonnegativity of the curvature operator is preserved by the Ricci flow. Roughly speaking the Li-Yau-Hamilton estimate says the nonnegativity of a certain combination of the derivatives of the curvature up to second order is also preserved by the Ricci flow.

Let us begin by describing the Li-Yau estimate for positive solutions to the heat equation on a complete Riemannian manifold with nonnegative Ricci curvature.

TheOrem 2.5.1 (Li-Yau [82]). Let $\left(M, g_{i j}\right)$ be an $n$-dimensional complete Riemannian manifold with nonnegative Ricci curvature. Let $u(x, t)$ be any positive solution to the heat equation

$$
\frac{\partial u}{\partial t}=\Delta u \quad \text { on } \quad M \times[0, \infty)
$$

Then we have

$$
\frac{\partial u}{\partial t}-\frac{|\nabla u|^{2}}{u}+\frac{n}{2 t} u \geq 0 \quad \text { on } \quad M \times(0, \infty) .
$$

We remark that one can in fact prove the following quadratic version that for any vector field $V^{i}$ on $M$,

$$
\frac{\partial u}{\partial t}+2 \nabla u \cdot V+u|V|^{2}+\frac{n}{2 t} u \geq 0
$$

If we take the optimal vector field $V=-\nabla u / u$, we recover the inequality (2.5.1).

Now we consider the Ricci flow on a Riemann surface. Since in dimension two the Ricci curvature is given by

$$
R_{i j}=\frac{1}{2} R g_{i j}
$$


the Ricci flow (1.1.5) becomes

$$
\frac{\partial g_{i j}}{\partial t}=-R g_{i j}
$$

Now let $g_{i j}(x, t)$ be a complete solution of the Ricci flow (2.5.3) on a Riemann surface $M$ and $0 \leq t<T$. Then the scalar curvature $R(x, t)$ evolves by the semilinear equation

$$
\frac{\partial R}{\partial t}=\triangle R+R^{2}
$$

on $M \times[0, T)$. Suppose the scalar curvature of the initial metric is bounded, nonnegative everywhere and positive somewhere. Then it follows from Proposition 2.1.2 that the scalar curvature $R(x, t)$ of the evolving metric remains nonnegative. Moreover, from the standard strong maximum principle (which works in each local coordinate neighborhood), the scalar curvature is positive everywhere for $t>0$. In [60], Hamilton obtained the following Li-Yau estimate for the scalar curvature $R(x, t)$.

TheOREm 2.5.2 (Hamilton [60]). Let $g_{i j}(x, t)$ be a complete solution of the Ricci flow on a surface $M$. Assume the scalar curvature of the initial metric is bounded, nonnegative everywhere and positive somewhere. Then the scalar curvature $R(x, t)$ satisfies the Li-Yau estimate

$$
\frac{\partial R}{\partial t}-\frac{|\nabla R|^{2}}{R}+\frac{R}{t} \geq 0 .
$$

Proof. By the above discussion, we know $R(x, t)>0$ for $t>0$. If we set

$$
L=\log R(x, t) \quad \text { for } \quad t>0,
$$

then

$$
\begin{aligned}
\frac{\partial}{\partial t} L & =\frac{1}{R}\left(\triangle R+R^{2}\right) \\
& =\triangle L+|\nabla L|^{2}+R
\end{aligned}
$$

and (2.5.4) is equivalent to

$$
\frac{\partial L}{\partial t}-|\nabla L|^{2}+\frac{1}{t}=\triangle L+R+\frac{1}{t} \geq 0 .
$$

Following Li-Yau [82] in the linear heat equation case, we consider the quantity

$$
Q=\frac{\partial L}{\partial t}-|\nabla L|^{2}=\triangle L+R .
$$

Then by a direct computation,

$$
\begin{aligned}
\frac{\partial Q}{\partial t} & =\frac{\partial}{\partial t}(\triangle L+R) \\
& =\triangle\left(\frac{\partial L}{\partial t}\right)+R \triangle L+\frac{\partial R}{\partial t} \\
& =\triangle Q+2 \nabla L \cdot \nabla Q+2\left|\nabla^{2} L\right|^{2}+2 R(\triangle L)+R^{2} \\
& \geq \triangle Q+2 \nabla L \cdot \nabla Q+Q^{2} .
\end{aligned}
$$


So we get

$$
\frac{\partial}{\partial t}\left(Q+\frac{1}{t}\right) \geq \triangle\left(Q+\frac{1}{t}\right)+2 \nabla L \cdot \nabla\left(Q+\frac{1}{t}\right)+\left(Q-\frac{1}{t}\right)\left(Q+\frac{1}{t}\right) .
$$

Hence by a similar maximum principle argument as in the proof of Lemma 2.1.3, we obtain

$$
Q+\frac{1}{t} \geq 0
$$

This proves the theorem.

As an immediate consequence, we obtain the following Harnack inequality for the scalar curvature $R$ by taking the Li-Yau type path integral as in [82].

Corollary 2.5.3 (Hamilton [60]). Let $g_{i j}(x, t)$ be a complete solution of the Ricci flow on a surface with bounded and nonnegative scalar curvature. Then for any points $x_{1}, x_{2} \in M$, and $0<t_{1}<t_{2}$, we have

$$
R\left(x_{2}, t_{2}\right) \geq \frac{t_{1}}{t_{2}} e^{-d_{t_{1}}\left(x_{1}, x_{2}\right)^{2} / 4\left(t_{2}-t_{1}\right)} R\left(x_{1}, t_{1}\right) .
$$

Proof. Take the geodesic path $\gamma(\tau), \tau \in\left[t_{1}, t_{2}\right]$, from $x_{1}$ to $x_{2}$ at time $t_{1}$ with constant velocity $d_{t_{1}}\left(x_{1}, x_{2}\right) /\left(t_{2}-t_{1}\right)$. Consider the space-time path $\eta(\tau)=(\gamma(\tau), \tau)$, $\tau \in\left[t_{1}, t_{2}\right]$. We compute

$$
\begin{aligned}
\log \frac{R\left(x_{2}, t_{2}\right)}{R\left(x_{1}, t_{1}\right)} & =\int_{t_{1}}^{t_{2}} \frac{d}{d \tau} L(\gamma(\tau), \tau) d \tau \\
& =\int_{t_{1}}^{t_{2}} \frac{1}{R}\left(\frac{\partial R}{\partial \tau}+\nabla R \cdot \frac{d \gamma}{d \tau}\right) d \tau \\
& \geq \int_{t_{1}}^{t_{2}}\left(\frac{\partial L}{\partial \tau}-|\nabla L|_{g_{i j}(\tau)}^{2}-\frac{1}{4}\left|\frac{d \gamma}{d \tau}\right|_{g_{i j}(\tau)}^{2}\right) d \tau .
\end{aligned}
$$

Then by Theorem 2.5.2 and the fact that the metric is shrinking (since the scalar curvature is nonnegative), we have

$$
\begin{aligned}
\log \frac{R\left(x_{2}, t_{2}\right)}{R\left(x_{1}, t_{1}\right)} & \geq \int_{t_{1}}^{t_{2}}\left(-\frac{1}{\tau}-\frac{1}{4}\left|\frac{d \gamma}{d \tau}\right|_{g_{i j}(\tau)}^{2}\right) d \tau \\
& =\log \frac{t_{1}}{t_{2}}-\frac{d_{t_{1}}\left(x_{1}, x_{2}\right)^{2}}{4\left(t_{2}-t_{1}\right)}
\end{aligned}
$$

After exponentiating above, we obtain the desired Harnack inequality.

To prove a similar inequality as (2.5.4) for the scalar curvature of solutions to the Ricci flow in higher dimensions is not so simple. First of all, we will need to require nonnegativity of the curvature operator (which we know is preserved under the Ricci flow). Secondly, one does not get inequality (2.5.4) directly, but rather indirectly as the trace of certain matrix estimate. The key ingredient in formulating this matrix version is to derive some identities from the soliton solutions and prove an elliptic 
inequality based on these quantities. Hamilton found such a general principle which was based on the idea of Li-Yau [82] when an identity is checked on the heat kernel before an inequality was found. To illustrate this point, let us first examine the heat equation case. Consider the heat kernel

$$
u(x, t)=(4 \pi t)^{-n / 2} e^{-|x|^{2} / 4 t}
$$

for the standard heat equation on $\mathbb{R}^{n}$ which can be considered as an expanding soliton solution.

Differentiating the function $u$, we get

$$
\nabla_{j} u=-u \frac{x_{j}}{2 t} \text { or } \nabla_{j} u+u V_{j}=0
$$

where

$$
V_{j}=\frac{x_{j}}{2 t}=-\frac{\nabla_{j} u}{u}
$$

Differentiating (2.5.6), we have

$$
\nabla_{i} \nabla_{j} u+\nabla_{i} u V_{j}+\frac{u}{2 t} \delta_{i j}=0 .
$$

To make the expression in (2.5.7) symmetric in $i, j$, we multiply $V_{i}$ to (2.5.6) and add to $(2.5 .7)$ and obtain

$$
\nabla_{i} \nabla_{j} u+\nabla_{i} u V_{j}+\nabla_{j} u V_{i}+u V_{i} V_{j}+\frac{u}{2 t} \delta_{i j}=0 .
$$

Taking the trace in (2.5.8) and using the equation $\partial u / \partial t=\Delta u$, we arrive at

$$
\frac{\partial u}{\partial t}+2 \nabla u \cdot V+u|V|^{2}+\frac{n}{2 t} u=0
$$

which shows that the Li-Yau inequality (2.5.1) becomes an equality on our expanding soliton solution $u$ ! Moreover, we even have the matrix identity (2.5.8).

Based on the above observation and using a similar process, Hamilton found a matrix quantity, which vanishes on expanding gradient Ricci solitons and is nonnegative for any solution to the Ricci flow with nonnegative curvature operator. Now we describe the process of finding the Li-Yau-Hamilton quadratic for the Ricci flow in arbitrary dimension.

Consider a homothetically expanding gradient soliton $g$, we have

$$
R_{a b}+\frac{1}{2 t} g_{a b}=\nabla_{a} V_{b}
$$

in the orthonormal frame coordinate chosen as in Section 1.3. Here $V_{b}=\nabla_{b} f$ for some function $f$. Differentiating (2.5.9) and commuting give the first order relations

$$
\begin{aligned}
\nabla_{a} R_{b c}-\nabla_{b} R_{a c} & =\nabla_{a} \nabla_{b} V_{c}-\nabla_{b} \nabla_{a} V_{c} \\
& =R_{a b c d} V_{d},
\end{aligned}
$$

and differentiating again, we get

$$
\begin{aligned}
\nabla_{a} \nabla_{b} R_{c d}-\nabla_{a} \nabla_{c} R_{b d} & =\nabla_{a}\left(R_{b c d e} V_{e}\right) \\
& =\nabla_{a} R_{b c d e} V_{e}+R_{b c d e} \nabla_{a} V_{e} \\
& =\nabla_{a} R_{b c d e} V_{e}+R_{a e} R_{b c d e}+\frac{1}{2 t} R_{b c d a}
\end{aligned}
$$


We further take the trace of this on $a$ and $b$ to get

$$
\Delta R_{c d}-\nabla_{a} \nabla_{c} R_{a d}-R_{a e} R_{a c d e}+\frac{1}{2 t} R_{c d}-\nabla_{a} R_{a c d e} V_{e}=0,
$$

and then by commuting the derivatives and second Bianchi identity,

$$
\Delta R_{c d}-\frac{1}{2} \nabla_{c} \nabla_{d} R+2 R_{c a d e} R_{a e}-R_{c e} R_{d e}+\frac{1}{2 t} R_{c d}+\left(\nabla_{e} R_{c d}-\nabla_{d} R_{c e}\right) V_{e}=0 .
$$

Let us define

$$
\begin{aligned}
M_{a b} & =\Delta R_{a b}-\frac{1}{2} \nabla_{a} \nabla_{b} R+2 R_{a c b d} R_{c d}-R_{a c} R_{b c}+\frac{1}{2 t} R_{a b}, \\
P_{a b c} & =\nabla_{a} R_{b c}-\nabla_{b} R_{a c} .
\end{aligned}
$$

Then

$$
M_{a b}+P_{c b a} V_{c}=0
$$

We rewrite (2.5.10) as

$$
P_{a b c}=R_{a b c d} V_{d}
$$

and then

$$
P_{c a b} V_{c}+R_{a c b d} V_{c} V_{d}=0 .
$$

Adding (2.5.11) and (2.5.12) we have

$$
M_{a b}+\left(P_{c a b}+P_{c b a}\right) V_{c}+R_{a c b d} V_{c} V_{d}=0
$$

and then

$$
M_{a b} W_{a} W_{b}+\left(P_{c a b}+P_{c b a}\right) W_{a} W_{b} V_{c}+R_{a c b d} W_{a} V_{c} W_{b} V_{d}=0 .
$$

If we write

$$
U_{a b}=\frac{1}{2}\left(V_{a} W_{b}-V_{b} W_{a}\right)=V \wedge W,
$$

then the above identity can be rearranged as

$$
Q \triangleq M_{a b} W_{a} W_{b}+2 P_{a b c} U_{a b} W_{c}+R_{a b c d} U_{a b} U_{c d}=0 .
$$

This is the Li-Yau-Hamilton quadratic we look for. Note that the proof of the LiYau-Hamilton estimate below does not depend on the existence of such an expanding gradient Ricci soliton. It is only used as inspiration.

Now we are ready to state the remarkable Li-Yau-Hamilton estimate for the Ricci flow.

TheOREM 2.5.4 (Hamilton [61]). Let $g_{i j}(x, t)$ be a complete solution with bounded curvature to the Ricci flow on a manifold $M$ for $t$ in some time interval $(0, T)$ and suppose the curvature operator of $g_{i j}(x, t)$ is nonnegative. Then for any one-form $W_{a}$ and any two-form $U_{a b}$ we have

$$
M_{a b} W_{a} W_{b}+2 P_{a b c} U_{a b} W_{c}+R_{a b c d} U_{a b} U_{c d} \geq 0
$$


on $M \times(0, T)$.

The proof of this theorem requires some rather intense calculations. Here we only give a sketch of the proof. For more details, we refer the reader to Hamilton's original paper [61].

Sketch of the Proof. Let $g_{i j}(x, t)$ be the complete solution with bounded and nonnegative curvature operator. Recall that in the orthonormal frame coordinate system, the curvatures evolve by

$$
\left\{\begin{array}{l}
\frac{\partial}{\partial t} R_{a b c d}=\Delta R_{a b c d}+2\left(B_{a b c d}-B_{a b d c}-B_{a d b c}+B_{a c b d}\right) \\
\frac{\partial}{\partial t} R_{a b}=\Delta R_{a b}+2 R_{a c b d} R_{c d} \\
\frac{\partial}{\partial t} R=\Delta R+2|R i c|^{2}
\end{array}\right.
$$

where $B_{a b c d}=R_{a e b f} R_{c e d f}$.

By a long but straightforward computation from these evolution equations, one can get

$$
\left(\frac{\partial}{\partial t}-\Delta\right) P_{a b c}=2 R_{a d b e} P_{d e c}+2 R_{a d c e} P_{d b e}+2 R_{b d c e} P_{a d e}-2 R_{d e} \nabla_{d} R_{a b c e}
$$

and

$$
\begin{aligned}
\left(\frac{\partial}{\partial t}-\Delta\right) M_{a b}= & 2 R_{a c b d} M_{c d}+2 R_{c d}\left(\nabla_{c} P_{d a b}+\nabla_{c} P_{d b a}\right) \\
& +2 P_{a c d} P_{b c d}-4 P_{a c d} P_{b d c}+2 R_{c d} R_{c e} R_{a d b e}-\frac{1}{2 t^{2}} R_{a b}
\end{aligned}
$$

Now consider

$$
Q \triangleq M_{a b} W_{a} W_{b}+2 P_{a b c} U_{a b} W_{c}+R_{a b c d} U_{a b} U_{c d}
$$

At a point where

$$
\left(\frac{\partial}{\partial t}-\Delta\right) W_{a}=\frac{1}{t} W_{a}, \quad\left(\frac{\partial}{\partial t}-\Delta\right) U_{a b}=0
$$

and

$$
\nabla_{a} W_{b}=0, \quad \nabla_{a} U_{b c}=\frac{1}{2}\left(R_{a b} W_{c}-R_{a c} W_{b}\right)+\frac{1}{4 t}\left(g_{a b} W_{c}-g_{a c} W_{b}\right),
$$

we have

$$
\begin{aligned}
\left(\frac{\partial}{\partial t}-\Delta\right) Q= & 2 R_{a c b d} M_{c d} W_{a} W_{b}-2 P_{a c d} P_{b d c} W_{a} W_{b} \\
& +8 R_{a d c e} P_{d b e} U_{a b} W_{c}+4 R_{a e c f} R_{b e d f} U_{a b} U_{c d} \\
& +\left(P_{a b c} W_{c}+R_{a b c d} U_{c d}\right)\left(P_{a b e} W_{e}+R_{a b e f} U_{e f}\right)
\end{aligned}
$$

For simplicity we assume the manifold is compact and the curvature operator is strictly positive. (For the general case we shall mess the formula up a bit to sneak in the term $\epsilon e^{A t} f$, as done in Lemma 2.1.3). Suppose not; then there will be a first time 
when the quantity $Q$ is zero, and a point where this happens, and a choice of $U$ and $W$ giving the null eigenvectors. We can extend $U$ and $W$ any way we like in space and time and still have $Q \geq 0$, up to the critical time. In particular we can make the first derivatives in space and time to be anything we like, so we can extend first in space to make (2.5.15) hold at that point. And then, knowing $\Delta W_{a}$ and $\Delta U_{a b}$, we can extend in time to make (2.5.14) hold at that point and that moment. Thus we have (2.5.16) at the point.

In the RHS of (2.5.16) the quadratic term

$$
\left(P_{a b c} W_{c}+R_{a b c d} U_{c d}\right)\left(P_{a b e} W_{e}+R_{a b e f} U_{e f}\right)
$$

is clearly nonnegative. By similar argument as in the proof of Lemma 2.1.3, to get a contradiction we only need to show the remaining part in the RHS of (2.5.16) is also nonnegative.

A nonnegative quadratic form can always be written as a sum of squares of linear forms. This is equivalent to diagonalizing a symmetric matrix and writing each nonnegative eigenvalue as a square. Write

$$
Q=\sum_{k}\left(X_{a}^{k} W_{a}+Y_{a b}^{k} U_{a b}\right)^{2}, \quad\left(1 \leq k \leq n+\frac{n(n-1)}{2}\right) .
$$

This makes

$$
M_{a b}=\sum_{k} X_{a}^{k} X_{b}^{k}, \quad P_{a b c}=\sum_{k} Y_{a b}^{k} X_{c}^{k}
$$

and

$$
R_{a b c d}=\sum_{k} Y_{a b}^{k} Y_{c d}^{k}
$$

It is then easy to compute

$$
\begin{aligned}
2 & R_{a c b d} M_{c d} W_{a} W_{b}-2 P_{a c d} P_{b d c} W_{a} W_{b}+8 R_{a d c e} P_{d b e} U_{a b} W_{e} \\
& +4 R_{a e c f} R_{b e d f} U_{a b} U_{c d} \\
= & 2\left(\sum_{k} Y_{a c}^{k} Y_{b d}^{k}\right)\left(\sum_{l} X_{a}^{l} Y_{c}^{l}\right) W_{a} W_{b} \\
& -2\left(\sum_{k} Y_{a c}^{k} X_{d}^{k}\right)\left(\sum_{l} Y_{b d}^{l} X_{c}^{l}\right) W_{a} W_{b} \\
& +8\left(\sum_{k} Y_{a d}^{k} Y_{c e}^{k}\right)\left(\sum_{l} Y_{d b}^{l} X_{e}^{l}\right) U_{a b} W_{c} \\
& +4\left(\sum_{k} Y_{a e}^{k} Y_{c f}^{k}\right)\left(\sum_{l} Y_{b e}^{l} Y_{d f}^{l}\right) U_{a b} U_{c d} \\
= & \sum_{k, l}\left(Y_{a c}^{k} X_{c}^{l} W_{a}-Y_{a c}^{l} X_{c}^{k} W_{a}-2 Y_{a c}^{k} Y_{b c}^{l} U_{a b}\right)^{2} \\
\geq & 0 .
\end{aligned}
$$

This says that the remaining part in the RHS of (2.5.16) is also nonnegative. Therefore we have completed the sketch of the proof. $\square$ 
By taking $U_{a b}=\frac{1}{2}\left(V_{a} W_{b}-V_{b} W_{a}\right)$ and tracing over $W_{a}$, we immediately get

Corollary 2.5.5 (Hamilton [61]). For any one-form $V_{a}$ we have

$$
\frac{\partial R}{\partial t}+\frac{R}{t}+2 \nabla_{a} R \cdot V_{a}+2 R_{a b} V_{a} V_{b} \geq 0 .
$$

In particular by taking $V \equiv 0$, we see that the function $t R(x, t)$ is pointwise nondecreasing in time. By combining this property with the local derivative estimate of curvature, we have the following elliptic type estimate.

Corollary 2.5.6. Suppose we have a solution to the Ricci flow for $t>0$ which is complete with bounded curvature, and has nonnegative curvature operator. Suppose also that at some time $t>0$ we have the scalar curvature $R \leq M$ for some constant $M$ in the ball of radius $r$ around some point $p$. Then for $k=1,2, \ldots$, the $k^{\text {th }}$ order derivatives of the curvature at $p$ at the time $t$ satisfy a bound

$$
\left|\nabla^{k} R m(p, t)\right|^{2} \leq C_{k} M^{2}\left(\frac{1}{r^{2 k}}+\frac{1}{t^{k}}+M^{k}\right)
$$

for some constant $C_{k}$ depending only on the dimension and $k$.

Proof. Since $t R$ is nondecreasing in time, we get a bound $R \leq 2 M$ in the given region for times between $t / 2$ and $t$. The nonnegative curvature hypothesis tells us the metric is shrinking. So we can apply the local derivative estimate in Theorem 1.4.2 to deduce the result.

By a similar argument as in Corollary 2.5.3, one readily has the following Harnack inequality.

COROLlary 2.5.7. Let $g_{i j}(x, t)$ be a complete solution of the Ricci flow on a manifold with bounded and nonnegative curvature operator, and let $x_{1}, x_{2} \in M, 0<$ $t_{1}<t_{2}$. Then the following inequality holds

$$
R\left(x_{2}, t_{2}\right) \geq \frac{t_{1}}{t_{2}} e^{-d_{t_{1}}\left(x_{1}, x_{2}\right)^{2} / 2\left(t_{2}-t_{1}\right)} \cdot R\left(x_{1}, t_{1}\right) .
$$

In the above discussion, we assumed that the solution to the Ricci flow exists on $0 \leq t<T$, and we derived the Li-Yau-Hamilton estimate with terms $1 / t$ in it. When the solution happens to be ancient, i.e., defined on $-\infty<t<T$, Hamilton [61] found an interesting and simple procedure for getting rid of them. Suppose we have a solution on $\alpha<t<T$ we can replace $t$ by $t-\alpha$ in the Li-Yau-Hamilton estimate. If we let $\alpha \rightarrow-\infty$, then the expression $1 /(t-\alpha) \rightarrow 0$ and disappears! In particular the trace Li-Yau-Hamilton estimate in Corollary 2.5.5 becomes

$$
\frac{\partial R}{\partial t}+2 \nabla_{a} R \cdot V_{a}+2 R_{a b} V_{a} V_{b} \geq 0
$$

By taking $V=0$, we see that $\frac{\partial R}{\partial t} \geq 0$. Thus, we have the following

COROllary 2.5.8 (Hamilton [61]). Let $g_{i j}(x, t)$ be a complete ancient solution of the Ricci flow on $M \times(-\infty, T)$ with bounded and nonnegative curvature operator, then the scalar curvature $R(x, t)$ is pointwise nondecreasing in time $t$. 
Corollary 2.5 .8 will be very useful later on when we study ancient $\kappa$-solutions in Chapter 6, especially combined with Shi's derivative estimate.

We end this section by stating the Li-Yau-Hamilton estimate for the Kähler-Ricci flow, due to the first author [12], under the weaker curvature assumption of nonnegative holomorphic bisectional curvature. Note that the following Li-Yau-Hamilton estimate in the Kähler case is really a Li-Yau-Hamilton estimate for the Ricci tensor of the evolving metric, so not only can we derive an estimate on the scalar curvature, which is the trace of the Ricci curvature, similar to Corollary 2.5.5 but also an estimate on the determinant of the Ricci curvature as well.

TheOREM 2.5.9 (Cao [12]). Let $g_{\alpha \bar{\beta}}(x, t)$ be a complete solution to the KählerRicci flow on a complex manifold $M$ with bounded curvature and nonnegtive bisectional curvature and $0 \leq t<T$. For any point $x \in M$ and any vector $V$ in the holomorphic tangent space $T_{x}^{1,0} M$, let

$$
Q_{\alpha \bar{\beta}}=\frac{\partial}{\partial t} R_{\alpha \bar{\beta}}+R_{\alpha \bar{\gamma}} R_{\gamma \bar{\beta}}+\nabla_{\gamma} R_{\alpha \bar{\beta}} V^{\gamma}+\nabla_{\bar{\gamma}} R_{\alpha \bar{\beta}} V^{\bar{\gamma}}+R_{\alpha \bar{\beta} \gamma \bar{\delta}} V^{\gamma} V^{\bar{\delta}}+\frac{1}{t} R_{\alpha \bar{\beta}} .
$$

Then we have

$$
Q_{\alpha \bar{\beta}} W^{\alpha} W^{\bar{\beta}} \geq 0
$$

for all $x \in M, V, W \in T_{x}^{1,0} M$, and $t>0$.

Corollary 2.5.10 (Cao [12]). Under the assumptions of Theorem 2.5.9, we have

(i) the scalar curvature $R$ satisfies the estimate

$$
\frac{\partial R}{\partial t}-\frac{|\nabla R|^{2}}{R}+\frac{R}{t} \geq 0
$$

and

(ii) assuming $R_{\alpha \bar{\beta}}>0$, the determinant $\phi=\operatorname{det}\left(R_{\alpha \bar{\beta}}\right) / \operatorname{det}\left(g_{\alpha \bar{\beta}}\right)$ of the Ricci curvature satisfies the estimate

$$
\frac{\partial \phi}{\partial t}-\frac{|\nabla \phi|^{2}}{n \phi}+\frac{n \phi}{t} \geq 0
$$

for all $x \in M$ and $t>0$.

2.6. Perelman's Estimate for Conjugate Heat Equations. In [103] Perelman obtained a Li-Yau type estimate for fundamental solutions of the conjugate heat equation, which is a backward heat equation, when the metric evolves by the Ricci flow. In this section we shall describe how to get this estimate along the same line as in the previous section. More importantly, we shall show how the Li-Yau path integral, when applied to Perelman's Li-Yau type estimate, leads to an important space-time distance function introduced by Perelman [103]. We learned from Hamilton [67] this idea of looking at Perelman's Li-Yau estimate.

We saw in the previous section that the Li-Yau quantity and the Li-Yau-Hamilton quantity vanish on expanding solutions. Note that when we consider a backward heat equation, shrinking solitons can be viewed as expanding backward in time. So we start by looking at shrinking gradient Ricci solitons. 
Suppose we have a shrinking gradient Ricci soliton $g_{i j}$ with potential function $f$ on manifold $M$ and $-\infty<t<0$ so that, for $\tau=-t$,

$$
R_{i j}+\nabla_{j} \nabla_{i} f-\frac{1}{2 \tau} g_{i j}=0 .
$$

Then, by taking the trace, we have

$$
R+\Delta f-\frac{n}{2 \tau}=0
$$

Also, by similar calculations as in deriving (1.1.15), we get

$$
R+|\nabla f|^{2}-\frac{f}{\tau}=C
$$

where $C$ is a constant which we can set to be zero.

Moreover, observe

$$
\frac{\partial f}{\partial t}=|\nabla f|^{2}
$$

because $f$ evolves in time with the rate of change given by the Lie derivative in the direction of $\nabla f$ generating the one-parameter family of diffeomorphisms.

Combining (2.6.2) with (2.6.4), we see $f$ satisfies the backward heat equation

$$
\frac{\partial f}{\partial t}=-\Delta f+|\nabla f|^{2}-R+\frac{n}{2 \tau}
$$

or equivalently

$$
\frac{\partial f}{\partial \tau}=\Delta f-|\nabla f|^{2}+R-\frac{n}{2 \tau} .
$$

Recall the Li-Yau-Hamilton quadratic is a certain combination of the second order space derivative (or first order time derivative), first order space derivatives and zero orders. Multiplying (2.6.2) by a factor of 2 and subtracting (2.6.3) yields

$$
2 \Delta f-|\nabla f|^{2}+R+\frac{1}{\tau} f-\frac{n}{\tau}=0
$$

valid for our potential function $f$ of the shrinking gradient Ricci soliton. The quantity on the LHS of the above identity is precisely the Li-Yau-Hamilton type quadratic found by Perelman [103].

Note that a function $f$ satisfies the backward heat equation (2.6.6) if and only if the function

$$
u=(4 \pi \tau)^{-\frac{n}{2}} e^{-f}
$$

satisfies the so called conjugate heat equation

$$
\square^{*} u \triangleq \frac{\partial u}{\partial \tau}-\Delta u+R u=0
$$

Lemma 2.6.1 (Perelman [103]). Let $g_{i j}(x, t), 0 \leq t<T$, be a complete solution to the Ricci flow on an n-dimensional manifold $M$ and let $u=(4 \pi \tau)^{-\frac{n}{2}} e^{-f}$ be a solution to the conjugate equation (2.6.7) with $\tau=T-t$. Set

$$
H=2 \Delta f-|\nabla f|^{2}+R+\frac{f-n}{\tau}
$$


and

$$
v=\tau H u=\left(\tau\left(R+2 \Delta f-|\nabla f|^{2}\right)+f-n\right) u .
$$

Then we have

$$
\frac{\partial H}{\partial \tau}=\Delta H-2 \nabla f \cdot \nabla H-\frac{1}{\tau} H-2\left|R_{i j}+\nabla_{i} \nabla_{j} f-\frac{1}{2 \tau} g_{i j}\right|^{2},
$$

and

$$
\frac{\partial v}{\partial \tau}=\Delta v-R v-2 \tau u\left|R_{i j}+\nabla_{i} \nabla_{j} f-\frac{1}{2 \tau} g_{i j}\right|^{2}
$$

Proof. By direct computations, we have

$$
\begin{aligned}
\frac{\partial}{\partial \tau} H= & \frac{\partial}{\partial \tau}\left(2 \triangle f-|\nabla f|^{2}+R+\frac{f-n}{\tau}\right) \\
= & 2 \triangle\left(\frac{\partial f}{\partial \tau}\right)-2\left\langle 2 R_{i j}, f_{i j}\right\rangle-2\left\langle\nabla f, \nabla\left(\frac{\partial f}{\partial \tau}\right)\right\rangle+2 \operatorname{Ric}(\nabla f, \nabla f) \\
& +\frac{\partial}{\partial \tau} R+\frac{1}{\tau} \frac{\partial}{\partial \tau} f-\frac{f-n}{\tau^{2}} \\
= & 2 \triangle\left(\triangle f-|\nabla f|^{2}+R-\frac{n}{2 \tau}\right)-4\left\langle R_{i j}, f_{i j}\right\rangle+2 \operatorname{Ric}(\nabla f, \nabla f) \\
& -2\left\langle\nabla f, \nabla\left(\triangle f-|\nabla f|^{2}+R-\frac{n}{2 \tau}\right)\right\rangle-\triangle R-2\left|R_{i j}\right|^{2} \\
& +\frac{1}{\tau}\left(\triangle f-|\nabla f|^{2}+R-\frac{n}{2 \tau}\right)-\frac{f-n}{\tau^{2}}, \\
\nabla H= & \nabla\left(2 \triangle f-|\nabla f|^{2}+R+\frac{f-n}{\tau}\right) \\
= & 2 \nabla(\triangle f)-2\left\langle\nabla \nabla_{i} f, \nabla_{i} f\right\rangle+\nabla R+\frac{1}{\tau} \nabla f \\
\triangle H= & \triangle\left(2 \triangle f-|\nabla f|^{2}+R+\frac{f-n}{\tau}\right) \\
= & 2 \triangle(\triangle f)-\triangle\left(|\nabla f|^{2}\right)+\triangle R+\frac{1}{\tau} \triangle f
\end{aligned}
$$

and

$$
\begin{aligned}
2 \nabla H \cdot \nabla f & =2\left\langle 2 \nabla(\triangle f)-2\left\langle\nabla \nabla_{i} f, \nabla_{i} f\right\rangle+\nabla R+\frac{1}{\tau} \nabla f, \nabla f\right\rangle \\
& =2\left[\langle 2 \nabla(\triangle f), \nabla f\rangle-2\left\langle f_{i j}, f_{i} f_{j}\right\rangle+\langle\nabla R, \nabla f\rangle\right]+\frac{2}{\tau}|\nabla f|^{2}
\end{aligned}
$$


Thus we get

$$
\begin{aligned}
& \frac{\partial}{\partial \tau} H-\triangle H+2 \nabla f \cdot \nabla H+\frac{1}{\tau} H \\
& =-4\left\langle R_{i j}, f_{i j}\right\rangle+2 \operatorname{Ric}(\nabla f, \nabla f)-2\left|R_{i j}\right|^{2}-\triangle\left(|\nabla f|^{2}\right)+2\langle\nabla(\triangle f), \nabla f\rangle \\
& \quad+\frac{2}{\tau} \triangle f+\frac{2}{\tau} R-\frac{n}{2 \tau^{2}} \\
& =-2\left[\left|R_{i j}\right|^{2}+\left|f_{i j}\right|^{2}+\frac{n}{4 \tau^{2}}+2\left\langle R_{i j}, f_{i j}\right\rangle-\frac{R}{\tau}-\frac{1}{\tau} \triangle f\right] \\
& =-2\left|R_{i j}+\nabla_{i} \nabla_{j} f-\frac{1}{2 \tau} g_{i j}\right|^{2},
\end{aligned}
$$

and

$$
\begin{aligned}
\left(\frac{\partial}{\partial \tau}-\Delta+R\right) v & =\left(\frac{\partial}{\partial \tau}-\triangle+R\right)(\tau H u) \\
& =\left(\frac{\partial}{\partial \tau}-\triangle\right)(\tau H) \cdot u-2\langle\nabla(\tau H), \nabla u\rangle \\
& =\left(\left(\frac{\partial}{\partial \tau}-\triangle\right)(\tau H)-2\langle\nabla(\tau H), \nabla f\rangle\right) u \\
& =\tau\left(\frac{\partial H}{\partial \tau}-\triangle H+2 \nabla f \cdot \nabla H+\frac{1}{\tau} H\right) u \\
& =-2 \tau u\left|R_{i j}+\nabla_{i} \nabla_{j} f-\frac{1}{2 \tau} g_{i j}\right|^{2} .
\end{aligned}
$$

Note that, since $f$ satisfies the equation (2.6.6), we can rewrite $H$ as

$$
H=2 \frac{\partial f}{\partial \tau}+|\nabla f|^{2}-R+\frac{1}{\tau} f .
$$

Then, by Lemma 2.6.1, we have

$$
\frac{\partial}{\partial \tau}(\tau H)=\Delta(\tau H)-2 \nabla f \cdot \nabla(\tau H)-2 \tau\left|\operatorname{Ric}+\nabla^{2} f-\frac{1}{2 \tau} g\right|^{2}
$$

So by the maximum principle, we find $\max (\tau H)$ is nonincreasing as $\tau$ increasing. When $u$ is chosen to be a fundamental solution to (2.6.7), one can show that $\lim _{\tau \rightarrow 0} \tau H \leq 0$ and hence $H \leq 0$ on $M$ for all $\tau \in(0, T]$ (see, for example, [99]). Since this fact is not used in later chapters and will be only used in the rest of the section to introduce a space-time distance via Li-Yau path integral, we omit the details of the proof.

Once we have Perelman's Li-Yau type estimate $H \leq 0$, we can apply the Li-Yau path integral as in [82] to estimate the above solution $u$ (i.e., a heat kernel estimate for the conjugate heat equation, see also the earlier work of Cheeger-Yau [28]). Let $p, q \in M$ be two points and $\gamma(\tau), \tau \in[0, \bar{\tau}]$, be a curve joining $p$ and $q$, with $\gamma(0)=p$ 
and $\gamma(\bar{\tau})=q$. Then along the space-time path $(\gamma(\tau), \tau), \tau \in[0, \bar{\tau}]$, we have

$$
\begin{aligned}
\frac{d}{d \tau}(2 \sqrt{\tau} f(\gamma(\tau), \tau)) & =2 \sqrt{\tau}\left(\frac{\partial f}{\partial \tau}+\nabla f \cdot \dot{\gamma}(\tau)\right)+\frac{1}{\sqrt{\tau}} f \\
& \leq \sqrt{\tau}\left(-|\nabla f|_{g_{i j}(\tau)}^{2}+2 \nabla f \cdot \dot{\gamma}(\tau)\right)+\sqrt{\tau} R \\
& =-\sqrt{\tau}|\nabla f-\dot{\gamma}(\tau)|_{g_{i j}(\tau)}^{2}+\sqrt{\tau}\left(R+|\dot{\gamma}(\tau)|_{g_{i j}(\tau)}^{2}\right) \\
& \leq \sqrt{\tau}\left(R+|\dot{\gamma}(\tau)|_{g_{i j}(\tau)}^{2}\right)
\end{aligned}
$$

where we have used the fact that $H \leq 0$ and the expression for $H$ in (2.6.8).

Integrating the above inequality from $\tau=0$ to $\tau=\bar{\tau}$, we obtain

$$
2 \sqrt{\bar{\tau}} f(q, \bar{\tau}) \leq \int_{0}^{\bar{\tau}} \sqrt{\tau}\left(R+|\dot{\gamma}(\tau)|_{g_{i j}(\tau)}^{2}\right) d \tau
$$

or

$$
f(q, \bar{\tau}) \leq \frac{1}{2 \sqrt{\bar{\tau}}} \mathcal{L}(\gamma)
$$

where

$$
\mathcal{L}(\gamma) \triangleq \int_{0}^{\bar{\tau}} \sqrt{\tau}\left(R+|\dot{\gamma}(\tau)|_{g_{i j}(\tau)}^{2}\right) d \tau
$$

Denote by

$$
l(q, \bar{\tau}) \triangleq \inf _{\gamma} \frac{1}{2 \sqrt{\bar{\tau}}} \mathcal{L}(\gamma),
$$

where the inf is taken over all space curves $\gamma(\tau), 0 \leq \tau \leq \bar{\tau}$, joining $p$ and $q$. The space-time distance function $l(q, \bar{\tau})$ obtained by the above Li-Yau path integral argument is first introduced by Perelman in [103] and is what Perelman calls reduced distance. Since Perelman pointed out in page 19 of [103] that "an even closer reference is [82], where they use 'length', associated to a linear parabolic equation, which is pretty much the same as in our case", it is natural to call $l(q, \bar{\tau})$ the Li-YauPerelman distance. See Chapter 3 for much more detailed discussions.

Finally, we conclude this section by relating the quantity $H$ (or $v$ ) and the $\mathcal{W}$ functional of Perelman defined in (1.5.9). Observe that $v$ happens to be the integrand of the $\mathcal{W}$-functional,

$$
\mathcal{W}\left(g_{i j}(t), f, \tau\right)=\int_{M} v d V
$$

Hence, when $M$ is compact,

$$
\begin{aligned}
\frac{d}{d \tau} \mathcal{W} & =\int_{M}\left(\frac{\partial}{\partial \tau} v+R v\right) d V \\
& =-2 \tau \int_{M}\left|\operatorname{Ric}+\nabla^{2} f-\frac{1}{2 \tau} g\right|^{2} u d V \\
& \leq 0
\end{aligned}
$$

or equivalently,

$$
\frac{d}{d t} \mathcal{W}\left(g_{i j}(t), f(t), \tau(t)\right)=\int_{M} 2 \tau\left|R_{i j}+\nabla_{i} \nabla_{j} f-\frac{1}{2 \tau} g_{i j}\right|^{2}(4 \pi \tau)^{-\frac{n}{2}} e^{-f} d V,
$$

which is the same as stated in Proposition 1.5.8. 
3. Perelman's Reduced Volume. In Section 1.5 we introduced the $\mathcal{F}$ functional and the $\mathcal{W}$-functional of Perelman and proved their monotonicity properties under the Ricci flow. In the last section of the previous chapter we have defined the Li-Yau-Perelman distance. The main purpose of this chapter is to use the Li-YauPerelman distance to define the Perelman's reduced volume, which was introduced by Perelman in [103], and prove the monotonicity property of the reduced volume under the Ricci flow. This new monotonicity formula is more useful for local considerations, especially when we consider the formation of singularities in Chapter 6 and work on the Ricci flow with surgery in Chapter 7. As first applications we will present two no local collapsing theorems of Perelman [103] in this chapter. More applications can be found in Chapter 6 and 7 .

3.1. Riemannian Formalism in Potentially Infinite Dimensions. In Section 2.6, from an analytic view point, we saw how the Li-Yau path integral of Perelman's estimate for fundamental solutions to the conjugate heat equation leads to the Li-Yau-Perelman distance. In this section we present, from a geometric view point, another motivation why one is lead to the consideration of the Li-Yau-Perelman distance function, as well as a reduced volume concept. Interestingly enough, the LiYau-Hamilton quadratic introduced in Section 2.5 appears again in this geometric consideration.

We consider the Ricci flow

$$
\frac{\partial}{\partial t} g_{i j}=-2 R_{i j}
$$

on a manifold $M$ where we assume that $g_{i j}(\cdot, t)$ are complete and have uniformly bounded curvatures.

Recall from Section 2.5 that the Li-Yau-Hamilton quadratic introduced in [61] is

$$
Q=M_{i j} W_{i} W_{j}+2 P_{i j k} U_{i j} W_{k}+R_{i j k l} U_{i j} U_{k l}
$$

where

$$
\begin{aligned}
M_{i j} & =\Delta R_{i j}-\frac{1}{2} \nabla_{i} \nabla_{j} R+2 R_{i k j l} R_{k l}-R_{i k} R_{j k}+\frac{1}{2 t} R_{i j}, \\
P_{i j k} & =\nabla_{i} R_{j k}-\nabla_{j} R_{i k}
\end{aligned}
$$

and $U_{i j}$ is any two-form and $W_{i}$ is any 1-form. Here and throughout this chapter we do not always bother to raise indices; repeated indices is short hand for contraction with respect to the metric.

In [63], Hamilton predicted that the Li-Yau-Hamilton quadratic is some sort of jet extension of positive curvature operator on some larger space. Such an interpretation of the Li-Yau-Hamilton quadratic as a curvature operator on the space $M \times \mathbb{R}^{+}$was found by Chow and Chu [38] where a potentially degenerate Riemannian metric on $M \times \mathbb{R}^{+}$was constructed. The degenerate Riemannian metric on $M \times \mathbb{R}^{+}$is the limit of the following two-parameter family of Riemannian metrics

$$
g_{N, \delta}(x, t)=g(x, t)+\left(R(x, t)+\frac{N}{2(t+\delta)}\right) d t^{2}
$$

as $N$ tends to infinity and $\delta$ tends to zero, where $g(x, t)$ is the solution of the Ricci flow on $M$ and $t \in \mathbb{R}^{+}$. 
To avoid the degeneracy, Perelman [103] considers the manifold $\tilde{M}=M \times \mathbb{S}^{N} \times \mathbb{R}^{+}$ with the following metric:

$$
\begin{aligned}
\tilde{g}_{i j} & =g_{i j}, \\
\tilde{g}_{\alpha \beta} & =\tau g_{\alpha \beta}, \\
\tilde{g}_{o o} & =\frac{N}{2 \tau}+R, \\
\tilde{g}_{i \alpha} & =\tilde{g}_{i o}=\tilde{g}_{\alpha o}=0,
\end{aligned}
$$

where $i, j$ are coordinate indices on $M ; \alpha, \beta$ are coordinate indices on $\mathbb{S}^{N}$; and the coordinate $\tau$ on $R^{+}$has index $o$. Let $\tau=T-t$ for some fixed constant $T$. Then $g_{i j}$ will evolve with $\tau$ by the backward Ricci flow $\frac{\partial}{\partial \tau} g_{i j}=2 R_{i j}$. The metric $g_{\alpha \beta}$ on $\mathbb{S}^{N}$ is a metric with constant sectional curvature $\frac{1}{2 N}$.

We remark that the metric $\tilde{g}_{\alpha \beta}$ on $\mathbb{S}^{N}$ is chosen so that the product metric $\left(\tilde{g}_{i j}, \tilde{g}_{\alpha \beta}\right)$ on $M \times \mathbb{S}^{N}$ evolves by the Ricci flow, while the component $\tilde{g}_{o o}$ is just the scalar curvature of $\left(\tilde{g}_{i j}, \tilde{g}_{\alpha \beta}\right)$. Thus the metric $\tilde{g}$ defined on $\tilde{M}=M \times \mathbb{S}^{N} \times \mathbb{R}^{+}$is exactly a "regularization" of Chow-Chu's degenerate metric on $M \times \mathbb{R}^{+}$.

Proposition 3.1.1. The components of the curvature tensor of the metric $\tilde{g}$ coincide (modulo $\mathrm{N}^{-1}$ ) with the components of the Li-Yau-Hamilton quadratic.

Proof. By definition, the Christoffel symbols of the metric $\tilde{g}$ are given by the following list:

$$
\begin{aligned}
\tilde{\Gamma}_{i j}^{k} & =\Gamma_{i j}^{k}, \\
\tilde{\Gamma}_{i \beta}^{k} & =0 \text { and } \tilde{\Gamma}_{i j}^{\gamma}=0 \\
\tilde{\Gamma}_{\alpha \beta}^{k} & =0 \text { and } \tilde{\Gamma}_{i \beta}^{\gamma}=0 \\
\tilde{\Gamma}_{i o}^{k} & =g^{k l} R_{l i} \text { and } \tilde{\Gamma}_{i j}^{o}=-\tilde{g}^{o o} R_{i j}, \\
\tilde{\Gamma}_{o o}^{k} & =-\frac{1}{2} g^{k l} \frac{\partial}{\partial x^{l}} R \quad \text { and } \quad \tilde{\Gamma}_{i o}^{o}=\frac{1}{2} \tilde{g}^{o o} \frac{\partial}{\partial x^{i}} R \\
\tilde{\Gamma}_{i \beta}^{o} & =0, \tilde{\Gamma}_{o \beta}^{k}=0 \quad \text { and } \tilde{\Gamma}_{o j}^{\gamma}=0 \\
\tilde{\Gamma}_{\alpha \beta}^{\gamma} & =\Gamma_{\alpha \beta}^{\gamma}, \\
\tilde{\Gamma}_{\alpha o}^{\gamma} & =\frac{1}{2 \tau} \delta_{\alpha}^{\gamma} \text { and } \tilde{\Gamma}_{\alpha \beta}^{o}=-\frac{1}{2} \tilde{g}^{o o} g_{\alpha \beta}, \\
\tilde{\Gamma}_{o o}^{\gamma} & =0 \text { and } \tilde{\Gamma}_{o \beta}^{o}=0, \\
\tilde{\Gamma}_{o o}^{o} & =\frac{1}{2} \tilde{g}^{o o}\left(-\frac{N}{2 \tau^{2}}+\frac{\partial}{\partial \tau} R\right) .
\end{aligned}
$$

Fix a point $(p, s, \tau) \in M \times \mathbb{S}^{N} \times \mathbb{R}^{+}$and choose normal coordinates around $p \in M$ and normal coordinates around $s \in \mathbb{S}^{N}$ such that $\Gamma_{i j}^{k}(p)=0$ and $\Gamma_{\alpha \beta}^{\gamma}(s)=0$ for all $i, j, k$ and $\alpha, \beta, \gamma$. We compute the curvature tensor $\tilde{R} m$ of the metric $\tilde{g}$ at the point 
as follows:

$$
\begin{aligned}
\tilde{R}_{i j k l} & =R_{i j k l}+\tilde{\Gamma}_{i o}^{k} \tilde{\Gamma}_{j l}^{o}-\tilde{\Gamma}_{j o}^{k} \tilde{\Gamma}_{i l}^{o}=R_{i j k l}+O\left(\frac{1}{N}\right) \\
\tilde{R}_{i j k \delta} & =0 \\
\tilde{R}_{i j \gamma \delta} & =0 \text { and } \tilde{R}_{i \beta k \delta}=\tilde{\Gamma}_{i o}^{k} \tilde{\Gamma}_{\beta \delta}^{o}-\tilde{\Gamma}_{\beta o}^{k} \tilde{\Gamma}_{i \delta}^{o}=-\frac{1}{2} \tilde{g}^{o o} g_{\beta \delta} g^{k l} R_{l i}=O\left(\frac{1}{N}\right), \\
\tilde{R}_{i \beta \gamma \delta} & =0 \\
\tilde{R}_{i j k o} & =\frac{\partial}{\partial x^{i}} R_{j k}-\frac{\partial}{\partial x^{j}} R_{i k}+\tilde{\Gamma}_{i o}^{k} \tilde{\Gamma}_{j o}^{o}-\tilde{\Gamma}_{j o}^{k} \tilde{\Gamma}_{i o}^{o}=P_{i j k}+O\left(\frac{1}{N}\right), \\
\tilde{R}_{i o k o} & =-\frac{1}{2} \frac{\partial^{2}}{\partial x^{i} \partial x^{k}} R-\frac{\partial}{\partial \tau}\left(R_{i l} g^{l k}\right)+\tilde{\Gamma}_{i o}^{k} \tilde{\Gamma}_{o o}^{o}-\tilde{\Gamma}_{o j}^{k} \tilde{\Gamma}_{i o}^{j}-\tilde{\Gamma}_{o o}^{k} \tilde{\Gamma}_{i o}^{o} \\
& =-\frac{1}{2} \nabla_{i} \nabla_{k} R-\frac{\partial}{\partial \tau} R_{i k}+2 R_{i k} R_{l k}-\frac{1}{2 \tau} R_{i k}-R_{i j} R_{j k}+O\left(\frac{1}{N}\right) \\
& =M_{i k}+O\left(\frac{1}{N}\right), \\
\tilde{R}_{i j \gamma o} & =0 \quad \text { and } \quad \tilde{R}_{i \gamma j o}=0, \\
\tilde{R}_{i \beta \gamma o} & =-\tau \tilde{\Gamma}_{\beta o}^{\gamma} \tilde{\Gamma}_{i o}^{o}=O\left(\frac{1}{N}\right) \quad \text { and } \quad \tilde{R}_{i o \gamma \delta}=0 \\
\tilde{R}_{i o \gamma o} & =0, \\
\tilde{R}_{\alpha \beta \gamma o} & =0, \\
\tilde{R}_{\alpha o \gamma o} & =\left(\frac{1}{2 \tau^{2}} \delta_{\alpha}^{\gamma}+\tilde{\Gamma}_{\alpha o}^{\gamma} \tilde{\Gamma}_{o o}^{o}-\tilde{\Gamma}_{o \beta}^{\gamma} \tilde{\Gamma}_{\alpha o}^{\beta}\right) \tau=O\left(\frac{1}{N}\right) \\
\tilde{R}_{\alpha \beta \gamma \delta} & =O\left(\frac{1}{N}\right) .
\end{aligned}
$$

Thus the components of the curvature tensor of the metric $\tilde{g}$ coincide (modulo $N^{-1}$ ) with the components of the Li-Yau-Hamilton quadratic. $\square$

The following observation due to Perelman [103] gives an important motivation to define Perelman's reduced volume.

Corollary 3.1.2. All components of the Ricci tensor of $\tilde{g}$ are zero (modulo $\left.N^{-1}\right)$.

Proof. From the list of the components of the curvature tensor of $\tilde{g}$ given above, 
we have

$$
\begin{aligned}
\tilde{R}_{i j} & =\tilde{g}^{k l} \tilde{R}_{i j k l}+\tilde{g}^{\alpha \beta} \tilde{R}_{i \alpha j \beta}+\tilde{g}^{o o} \tilde{R}_{i o j o} \\
& =R_{i j}-\frac{1}{2 \tau} g^{\alpha \beta} \tilde{g}^{o o} g^{\alpha \beta} R_{i j}+\tilde{g}^{o o}\left(M_{i j}-\frac{1}{2 \tau} R_{i j}+O\left(\frac{1}{N}\right)\right) \\
& =R_{i j}-\frac{N}{2 \tau} \tilde{g}^{o o} R_{i j}+O\left(\frac{1}{N}\right) \\
& =O\left(\frac{1}{N}\right) \\
\tilde{R}_{i \gamma} & =\tilde{g}^{k l} \tilde{R}_{i k \gamma l}+\tilde{g}^{\alpha \beta} \tilde{R}_{i \alpha \gamma \beta}+\tilde{g}^{o o} \tilde{R}_{i o \gamma o}=0, \\
\tilde{R}_{i o} & =\tilde{g}^{k l} \tilde{R}_{i k o l}+\tilde{g}^{\alpha \beta} \tilde{R}_{i \alpha o \beta}+\tilde{g}^{o o} \tilde{R}_{i o o o} \\
& =-g^{k l} P_{i k l}+O\left(\frac{1}{N}\right), \\
\tilde{R}_{\alpha \beta} & =\tilde{g}^{k l} \tilde{R}_{\alpha k \beta l}+\tilde{g}^{\gamma \delta} \tilde{R}_{\alpha \gamma \beta \delta}+\tilde{g}^{o o} \tilde{R}_{\alpha o \beta o} \\
& =O\left(\frac{1}{N}\right), \\
\tilde{R}_{\alpha o} & =\tilde{g}^{k l} \tilde{R}_{\alpha k o l}+\tilde{g}^{\beta \gamma} \tilde{R}_{\alpha \beta o \gamma}+\tilde{g}^{o o} \tilde{R}_{\alpha o o o}=0, \\
\tilde{R}_{o o} & =\tilde{g}^{k l} \tilde{R}_{o k o l}+\tilde{g}^{\alpha \beta} \tilde{R}_{o \alpha o \beta}+\tilde{g}^{o o} \tilde{R}_{o o o o} \\
& =g^{k l}\left(M_{k l}+O\left(\frac{1}{N}\right)\right)+O\left(\frac{1}{N}\right) .
\end{aligned}
$$

Since $\tilde{g}^{o o}$ is of order $N^{-1}$, we see that the norm of the Ricci tensor is given by

$$
|\tilde{R i c}|_{\tilde{g}}=O\left(\frac{1}{N}\right)
$$

This proves the result.

We now use the Ricci-flatness of the metric $\tilde{g}$ to interpret the Bishop-Gromov relative volume comparison theorem which will motivate another monotonicity formula for the Ricci flow. The argument in the following will not be rigorous. However it gives an intuitive picture of what one may expect. Consider a metric ball in $(\tilde{M}, \tilde{g})$ centered at some point $(p, s, 0) \in \tilde{M}$. Note that the metric of the sphere $\mathbb{S}^{N}$ at $\tau=0$ degenerates and it shrinks to a point. Then the shortest geodesic $\gamma(\tau)$ between $(p, s, 0)$ and an arbitrary point $(q, \bar{s}, \bar{\tau}) \in \tilde{M}$ is always orthogonal to the $\mathbb{S}^{N}$ fibre. The length of $\gamma(\tau)$ can be computed as

$$
\begin{aligned}
& \int_{0}^{\bar{\tau}} \sqrt{\left(\frac{N}{2 \tau}+R\right)+|\dot{\gamma}(\tau)|_{g_{i j}(\tau)}^{2}} d \tau \\
& =\sqrt{2 N \bar{\tau}}+\frac{1}{\sqrt{2 N}} \int_{0}^{\bar{\tau}} \sqrt{\tau}\left(R+|\dot{\gamma}(\tau)|_{g_{i j}}^{2}\right) d \tau+O\left(N^{-\frac{3}{2}}\right) .
\end{aligned}
$$

Thus a shortest geodesic should minimize

$$
\mathcal{L}(\gamma)=\int_{0}^{\bar{\tau}} \sqrt{\tau}\left(R+|\dot{\gamma}(\tau)|_{g_{i j}}^{2}\right) d \tau
$$


Let $L(q, \bar{\tau})$ denote the corresponding minimum. We claim that a metric sphere $S_{\tilde{M}}(\sqrt{2 N \bar{\tau}})$ in $\tilde{M}$ of radius $\sqrt{2 N \bar{\tau}}$ centered at $(p, s, 0)$ is $O\left(N^{-1}\right)$-close to the hypersurface $\{\tau=\bar{\tau}\}$. Indeed, if $\left(x, s^{\prime}, \tau(x)\right)$ lies on the metric sphere $S_{\tilde{M}}(\sqrt{2 N \bar{\tau}})$, then the distance between $\left(x, s^{\prime}, \tau(x)\right)$ and $(p, s, 0)$ is

$$
\sqrt{2 N \bar{\tau}}=\sqrt{2 N \tau(x)}+\frac{1}{\sqrt{2 N}} L(x, \tau(x))+O\left(N^{-\frac{3}{2}}\right)
$$

which can be written as

$$
\sqrt{\tau(x)}-\sqrt{\bar{\tau}}=-\frac{1}{2 N} L(x, \tau(x))+O\left(N^{-2}\right)=O\left(N^{-1}\right) .
$$

This shows that the metric sphere $S_{\tilde{M}}(\sqrt{2 N \bar{\tau}})$ is $O\left(N^{-1}\right)$-close to the hypersurface $\{\tau=\bar{\tau}\}$. Note that the metric $g_{\alpha \beta}$ on $\mathbb{S}^{N}$ has constant sectional curvature $\frac{1}{2 N}$. Thus

$$
\begin{aligned}
& \mathrm{Vol}\left(S_{\tilde{M}}(\sqrt{2 N \bar{\tau}})\right) \\
& \approx \int_{M}\left(\int_{S^{N}} d V_{\tau(x) g_{\alpha \beta}}\right) d V_{g_{i j}}(x) \\
& =\int_{M}(\tau(x))^{\frac{N}{2}} \operatorname{Vol}\left(S^{N}\right) d V_{M} \\
& \approx(2 N)^{\frac{N}{2}} \omega_{N} \int_{M}\left(\sqrt{\bar{\tau}}-\frac{1}{2 N} L(x, \tau(x))+O\left(N^{-2}\right)\right)^{N} d V_{M} \\
& \approx(2 N)^{\frac{N}{2}} \omega_{N} \int_{M}\left(\sqrt{\bar{\tau}}-\frac{1}{2 N} L(x, \bar{\tau})+o\left(N^{-1}\right)\right)^{N} d V_{M},
\end{aligned}
$$

where $\omega_{N}$ is the volume of the standard $N$-dimensional sphere. Now the volume of Euclidean sphere of radius $\sqrt{2 N \bar{\tau}}$ in $\mathbb{R}^{n+N+1}$ is

$$
\operatorname{Vol}\left(S_{\mathbb{R}^{n+N+1}}(\sqrt{2 N \bar{\tau}})\right)=(2 N \bar{\tau})^{\frac{N+n}{2}} \omega_{n+N} .
$$

Thus we have

$$
\frac{\operatorname{Vol}\left(S_{\tilde{M}}(\sqrt{2 N \bar{\tau}})\right)}{\operatorname{Vol}\left(S_{\mathbb{R}^{n+N+1}}(\sqrt{2 N \bar{\tau}})\right)} \approx \text { const } \cdot N^{-\frac{n}{2}} \cdot \int_{M}(\bar{\tau})^{-\frac{n}{2}} \exp \left\{-\frac{1}{2 \sqrt{\bar{\tau}}} L(x, \bar{\tau})\right\} d V_{M}
$$

Since the Ricci curvature of $\tilde{M}$ is zero (modulo $N^{-1}$ ), the Bishop-Gromov volume comparison theorem then suggests that the integral

$$
\tilde{V}(\bar{\tau}) \triangleq \int_{M}(4 \pi \bar{\tau})^{-\frac{n}{2}} \exp \left\{-\frac{1}{2 \sqrt{\bar{\tau}}} L(x, \bar{\tau})\right\} d V_{M}
$$

which we will call Perelman's reduced volume, should be nonincreasing in $\bar{\tau}$. A rigorous proof of this monotonicity property will be given in the next section. One should note the analog of reduced volume with the heat kernel and there is a parallel calculation for the heat kernel of the Shrödinger equation in the paper of Li-Yau [82].

3.2. Comparison Theorems for Perelman's Reduced Volume. In this section we will write the Ricci flow in the backward version

$$
\frac{\partial}{\partial \tau} g_{i j}=2 R_{i j}
$$


on a manifold $M$ with $\tau=\tau(t)$ satisfying $d \tau / d t=-1$ (in practice we often take $\tau=t_{0}-t$ for some fixed time $t_{0}$ ). We always assume that either $M$ is compact or $g_{i j}(\tau)$ are complete and have uniformly bounded curvature. To each (smooth) space curve $\gamma(\tau), 0<\tau_{1} \leq \tau \leq \tau_{2}$, in $M$, we define its $\mathcal{L}$-length as

$$
\mathcal{L}(\gamma)=\int_{\tau_{1}}^{\tau_{2}} \sqrt{\tau}\left(R(\gamma(\tau), \tau)+|\dot{\gamma}(\tau)|_{g_{i j}(\tau)}^{2}\right) d \tau
$$

Let $X(\tau)=\dot{\gamma}(\tau)$, and let $Y(\tau)$ be any (smooth) vector field along $\gamma(\tau)$. First of all, we compute the first variation formula for $\mathcal{L}$-length.

LEMMA 3.2.1 (First variation formula).

$$
\delta_{Y}(\mathcal{L})=\left.2 \sqrt{\tau}\langle X, Y\rangle\right|_{\tau_{1}} ^{\tau_{2}}+\int_{\tau_{1}}^{\tau_{2}} \sqrt{\tau}\left\langle Y, \nabla R-2 \nabla_{X} X-4 \operatorname{Ric}(\cdot, X)-\frac{1}{\tau} X\right\rangle d \tau
$$

where $\langle\cdot, \cdot\rangle$ denotes the inner product with respect to the metric $g_{i j}(\tau)$.

Proof. By direct computations,

$$
\begin{aligned}
\delta_{Y}(\mathcal{L}) & =\int_{\tau_{1}}^{\tau_{2}} \sqrt{\tau}\left(\langle\nabla R, Y\rangle+2\left\langle X, \nabla_{Y} X\right\rangle\right) d \tau \\
& =\int_{\tau_{1}}^{\tau_{2}} \sqrt{\tau}\left(\langle\nabla R, Y\rangle+2\left\langle X, \nabla_{X} Y\right\rangle\right) d \tau \\
& =\int_{\tau_{1}}^{\tau_{2}} \sqrt{\tau}\left(\langle\nabla R, Y\rangle+2 \frac{d}{d \tau}\langle X, Y\rangle-2\left\langle\nabla_{X} X, Y\right\rangle-4 \operatorname{Ric}(X, Y)\right) d \tau \\
& =\left.2 \sqrt{\tau}\langle X, Y\rangle\right|_{\tau_{1}} ^{\tau_{2}}+\int_{\tau_{1}}^{\tau_{2}} \sqrt{\tau}\left\langle Y, \nabla R-2 \nabla_{X} X-4 \operatorname{Ric}(\cdot, X)-\frac{1}{\tau} X\right\rangle d \tau
\end{aligned}
$$

A smooth curve $\gamma(\tau)$ in $M$ is called an $\mathcal{L}$-geodesic if it satisfies the following $\mathcal{L}$-geodesic equation

$$
\nabla_{X} X-\frac{1}{2} \nabla R+\frac{1}{2 \tau} X+2 \operatorname{Ric}(X, \cdot)=0 .
$$

Given any two points $p, q \in M$ and $\tau_{2}>\tau_{1}>0$, there always exists an $\mathcal{L}$-shortest curve (or shortest $\mathcal{L}$-geodesic) $\gamma(\tau):\left[\tau_{1}, \tau_{2}\right] \rightarrow M$ connecting $p$ to $q$ which satisfies the above $\mathcal{L}$-geodesic equation. Multiplying the $\mathcal{L}$-geodesic equation $(3.2 .1)$ by $\sqrt{\tau}$, we get

$$
\nabla_{X}(\sqrt{\tau} X)=\frac{\sqrt{\tau}}{2} \nabla R-2 \sqrt{\tau} \operatorname{Ric}(X, \cdot) \quad \text { on } \quad\left[\tau_{1}, \tau_{2}\right],
$$

or equivalently

$$
\frac{d}{d \tau}(\sqrt{\tau} X)=\frac{\sqrt{\tau}}{2} \nabla R-2 \operatorname{Ric}(\sqrt{\tau} X, \cdot) \quad \text { on } \quad\left[\tau_{1}, \tau_{2}\right] .
$$

Thus if a continuous curve, defined on $\left[0, \tau_{2}\right]$, satisfies the $\mathcal{L}$-geodesic equation on every subinterval $0<\tau_{1} \leq \tau \leq \tau_{2}$, then $\sqrt{\tau_{1}} X\left(\tau_{1}\right)$ has a limit as $\tau_{1} \rightarrow 0^{+}$. This allows us to extend the definition of the $\mathcal{L}$-length to include the case $\tau_{1}=0$ for 
all those (continuous) curves $\gamma:\left[0, \tau_{2}\right] \rightarrow M$ which are smooth on $\left(0, \tau_{2}\right]$ and have limits $\lim _{\tau \rightarrow 0^{+}} \sqrt{\tau} \dot{\gamma}(\tau)$. Clearly, there still exists an $\mathcal{L}$-shortest curve $\gamma(\tau):\left[0, \tau_{2}\right] \rightarrow M$ connecting arbitrary two points $p, q \in M$ and satisfying the $\mathcal{L}$-geodesic equation $(3.2 .1)$ on $\left(0, \tau_{2}\right]$. Moreover, for any vector $v \in T_{p} M$, we can find an $\mathcal{L}$-geodesic $\gamma(\tau)$ starting at $p$ with $\lim _{\tau \rightarrow 0^{+}} \sqrt{\tau} \dot{\gamma}(\tau)=v$.

From now on, we fix a point $p \in M$ and set $\tau_{1}=0$. The $\mathcal{L}$-distance function on the space-time $M \times \mathbb{R}^{+}$is denoted by $L(q, \bar{\tau})$ and defined to be the $\mathcal{L}$-length of the $\mathcal{L}$-shortest curve $\gamma(\tau)$ connecting $p$ and $q$ with $0 \leq \tau \leq \bar{\tau}$.

Consider a shortest $\mathcal{L}$-geodesic $\gamma:[0, \bar{\tau}] \rightarrow M$ connecting $p$ to $q$. In the computations below we pretend that $\mathcal{L}$-shortest geodesics between $p$ and $q$ are unique for all pairs $(q, \bar{\tau})$; if this is not the case, the inequalities that we obtain are still valid, by a standard barrier argument, when understood in the sense of distributions (see, for example, [112]).

The first variation formula in Lemma 3.2.1 implies that

$$
\nabla_{Y} L(q, \bar{\tau})=\langle 2 \sqrt{\bar{\tau}} X(\bar{\tau}), Y(\bar{\tau})\rangle
$$

Thus

$$
\nabla L(q, \bar{\tau})=2 \sqrt{\bar{\tau}} X(\bar{\tau})
$$

and

$$
|\nabla L|^{2}=4 \bar{\tau}|X|^{2}=-4 \bar{\tau} R+4 \bar{\tau}\left(R+|X|^{2}\right)
$$

We also compute

$$
\begin{aligned}
L_{\bar{\tau}}(\gamma(\bar{\tau}), \bar{\tau}) & =\left.\frac{d}{d \tau} L(\gamma(\tau), \tau)\right|_{\tau=\bar{\tau}}-\langle\nabla L, X\rangle \\
& =\sqrt{\bar{\tau}}\left(R+|X|^{2}\right)-2 \sqrt{\bar{\tau}}|X|^{2} \\
& =2 \sqrt{\bar{\tau}} R-\sqrt{\bar{\tau}}\left(R+|X|^{2}\right) .
\end{aligned}
$$

To evaluate $R+|X|^{2}$, we compute by using (3.2.1),

$$
\begin{aligned}
& \frac{d}{d \tau}\left(R(\gamma(\tau), \tau)+|X(\tau)|_{g_{i j}(\tau)}^{2}\right) \\
& =R_{\tau}+\langle\nabla R, X\rangle+2\left\langle\nabla_{X} X, X\right\rangle+2 \operatorname{Ric}(X, X) \\
& =R_{\tau}+\frac{1}{\tau} R+2\langle\nabla R, X\rangle-2 \operatorname{Ric}(X, X)-\frac{1}{\tau}\left(R+|X|^{2}\right) \\
& =-Q(X)-\frac{1}{\tau}\left(R+|X|^{2}\right),
\end{aligned}
$$

where

$$
Q(X)=-R_{\tau}-\frac{R}{\tau}-2\langle\nabla R, X\rangle+2 \operatorname{Ric}(X, X)
$$

is the trace Li-Yau-Hamilton quadratic in Corollary 2.5.5. Hence

$$
\begin{aligned}
\left.\frac{d}{d \tau}\left(\tau^{\frac{3}{2}}\left(R+|X|^{2}\right)\right)\right|_{\tau=\bar{\tau}} & =\frac{1}{2} \sqrt{\bar{\tau}}\left(R+|X|^{2}\right)-\bar{\tau}^{\frac{3}{2}} Q(X) \\
& =\left.\frac{1}{2} \frac{d}{d \tau} L(\gamma(\tau), \tau)\right|_{\tau=\bar{\tau}}-\bar{\tau}^{\frac{3}{2}} Q(X) .
\end{aligned}
$$


Therefore,

$$
\bar{\tau}^{\frac{3}{2}}\left(R+|X|^{2}\right)=\frac{1}{2} L(q, \bar{\tau})-K,
$$

where

$$
K=\int_{0}^{\bar{\tau}} \tau^{\frac{3}{2}} Q(X) d \tau
$$

Combining (3.2.2) with (3.2.3), we obtain

$$
|\nabla L|^{2}=-4 \bar{\tau} R+\frac{2}{\sqrt{\bar{\tau}}} L-\frac{4}{\sqrt{\bar{\tau}}} K
$$

and

$$
L_{\bar{\tau}}=2 \sqrt{\bar{\tau}} R-\frac{1}{2 \bar{\tau}} L+\frac{1}{\bar{\tau}} K .
$$

Next we compute the second variation of an $\mathcal{L}$-geodesic.

Lemma 3.2.2 (Second variation formula). For any $\mathcal{L}$-geodesic $\gamma$, we have

$$
\begin{aligned}
\delta_{Y}^{2}(\mathcal{L})= & \left.2 \sqrt{\tau}\left\langle\nabla_{Y} Y, X\right\rangle\right|_{0} ^{\bar{\tau}}+\int_{0}^{\bar{\tau}} \sqrt{\tau}\left[2\left|\nabla_{X} Y\right|^{2}+2\langle R(Y, X) Y, X\rangle\right. \\
& \left.+\nabla_{Y} \nabla_{Y} R+2 \nabla_{X} \operatorname{Ric}(Y, Y)-4 \nabla_{Y} \operatorname{Ric}(Y, X)\right] d \tau .
\end{aligned}
$$

Proof. We compute

$$
\begin{aligned}
\delta_{Y}^{2}(\mathcal{L}) & =Y\left(\int_{0}^{\bar{\tau}} \sqrt{\tau}\left(Y(R)+2\left\langle\nabla_{Y} X, X\right\rangle\right) d \tau\right) \\
& =\int_{0}^{\bar{\tau}} \sqrt{\tau}\left(Y(Y(R))+2\left\langle\nabla_{Y} \nabla_{Y} X, X\right\rangle+2\left|\nabla_{Y} X\right|^{2}\right) d \tau \\
& =\int_{0}^{\bar{\tau}} \sqrt{\tau}\left(Y(Y(R))+2\left\langle\nabla_{Y} \nabla_{X} Y, X\right\rangle+2\left|\nabla_{X} Y\right|^{2}\right) d \tau
\end{aligned}
$$

and

$$
\begin{aligned}
2\left\langle\nabla_{Y} \nabla_{X} Y, X\right\rangle & =2\left\langle\nabla_{X} \nabla_{Y} Y, X\right\rangle+2\langle R(Y, X) Y, X\rangle \\
= & 2 \frac{d}{d \tau}\left\langle\nabla_{Y} Y, X\right\rangle-4 \operatorname{Ric}\left(\nabla_{Y} Y, X\right)-2\left\langle\nabla_{Y} Y, \nabla_{X} X\right\rangle \\
& -\left(2\left\langle\frac{d}{d \tau} \nabla_{Y} Y, X\right\rangle-2\left\langle\nabla_{X} \nabla_{Y} Y, X\right\rangle\right)+2\langle R(Y, X) Y, X\rangle \\
= & 2 \frac{d}{d \tau}\left\langle\nabla_{Y} Y, X\right\rangle-4 \operatorname{Ric}\left(\nabla_{Y} Y, X\right)-2\left\langle\nabla_{Y} Y, \nabla_{X} X\right\rangle \\
& -2\left\langle Y^{i} Y^{j}\left(g^{k l}\left(\nabla_{i} R_{l j}+\nabla_{j} R_{l i}-\nabla_{l} R_{i j}\right)\right) \frac{\partial}{\partial x^{k}}, X\right\rangle+2\langle R(Y, X) Y, X\rangle \\
= & 2 \frac{d}{d \tau}\left\langle\nabla_{Y} Y, X\right\rangle-4 \operatorname{Ric}\left(\nabla_{Y} Y, X\right)-2\left\langle\nabla_{Y} Y, \nabla_{X} X\right\rangle-4 \nabla_{Y} \operatorname{Ric}(X, Y) \\
& +2 \nabla_{X} \operatorname{Ric}(Y, Y)+2\langle R(Y, X) Y, X\rangle,
\end{aligned}
$$


where we have used the computation

$$
\frac{\partial}{\partial \tau} \Gamma_{i j}^{k}=g^{k l}\left(\nabla_{i} R_{l j}+\nabla_{j} R_{l i}-\nabla_{l} R_{i j}\right) .
$$

Thus by using the $\mathcal{L}$-geodesic equation (3.2.1), we get

$$
\begin{aligned}
\delta_{Y}^{2}(\mathcal{L})= & \int_{0}^{\bar{\tau}} \sqrt{\tau}\left[Y(Y(R))+2 \frac{d}{d \tau}\left\langle\nabla_{Y} Y, X\right\rangle-4 \operatorname{Ric}\left(\nabla_{Y} Y, X\right)\right. \\
& -2\left\langle\nabla_{Y} Y, \nabla_{X} X\right\rangle-4 \nabla_{Y} \operatorname{Ric}(X, Y)+2 \nabla_{X} \operatorname{Ric}(Y, Y) \\
& \left.+2\langle R(Y, X) Y, X\rangle+2\left|\nabla_{X} Y\right|^{2}\right] d \tau \\
= & \int_{0}^{\bar{\tau}}\left[2 \sqrt{\tau} \frac{d}{d \tau}\left\langle\nabla_{Y} Y, X\right\rangle+\frac{1}{\sqrt{\tau}}\left\langle\nabla_{Y} Y, X\right\rangle\right] d \tau \\
& +\int_{0}^{\bar{\tau}} \sqrt{\tau}\left[Y(Y(R))-\left\langle\nabla_{Y} Y, \nabla R\right\rangle-4 \nabla_{Y} \operatorname{Ric}(X, Y)\right. \\
& \left.+2 \nabla_{X} \operatorname{Ric}(Y, Y)+2\langle R(Y, X) Y, X\rangle+2\left|\nabla_{X} Y\right|^{2}\right] d \tau \\
= & \left.2 \sqrt{\tau}\left\langle\nabla_{Y} Y, X\right\rangle\right|_{0} ^{\bar{\tau}}+\int_{0}^{\bar{\tau}} \sqrt{\tau}\left[2\left|\nabla_{X} Y\right|^{2}+2\langle R(Y, X) Y, X\rangle\right. \\
& \left.+\nabla_{Y} \nabla_{Y} R-4 \nabla_{Y} \operatorname{Ric}(X, Y)+2 \nabla_{X} \operatorname{Ric}(Y, Y)\right] d \tau .
\end{aligned}
$$

We now use the above second variation formula to estimate the Hessian of the $\mathcal{L}$-distance function.

Let $\gamma(\tau):[0, \bar{\tau}] \rightarrow M$ be an $\mathcal{L}$-shortest curve connecting $p$ and $q$ so that the $\mathcal{L}$-distance function $L=L(q, \bar{\tau})$ is given by the $\mathcal{L}$-length of $\gamma$. We fix a vector $Y$ at $\tau=\bar{\tau}$ with $|Y|_{g_{i j}(\bar{\tau})}=1$, and extend $Y$ along the $\mathcal{L}$-shortest geodesic $\gamma$ on $[0, \bar{\tau}]$ by solving the following ODE

$$
\nabla_{X} Y=-\operatorname{Ric}(Y, \cdot)+\frac{1}{2 \tau} Y
$$

This is similar to the usual parallel translation and multiplication with proportional parameter. Indeed, suppose $\left\{Y_{1}, \ldots, Y_{n}\right\}$ is an orthonormal basis at $\tau=\bar{\tau}$ (with respect to the metric $g_{i j}(\bar{\tau})$ ) and extend this basis along the $\mathcal{L}$-shortest geodesic $\gamma$ by solving the above ODE (3.2.8). Then

$$
\begin{aligned}
\frac{d}{d \tau}\left\langle Y_{i}, Y_{j}\right\rangle & =2 \operatorname{Ric}\left(Y_{i}, Y_{j}\right)+\left\langle\nabla_{X} Y_{i}, Y_{j}\right\rangle+\left\langle Y_{i}, \nabla_{X} Y_{j}\right\rangle \\
& =\frac{1}{\tau}\left\langle Y_{i}, Y_{j}\right\rangle
\end{aligned}
$$

for all $i, j$. Hence,

$$
\left\langle Y_{i}(\tau), Y_{j}(\tau)\right\rangle=\frac{\tau}{\bar{\tau}} \delta_{i j}
$$

and $\left\{Y_{1}(\tau), \ldots, Y_{n}(\tau)\right\}$ remains orthogonal on $[0, \bar{\tau}]$ with $Y_{i}(0)=0, i=1, \ldots, n$.

Proposition 3.2.3. Given any unit vector $Y$ at any point $q \in M$ with $\tau=\bar{\tau}$, consider an $\mathcal{L}$-shortest geodesic $\gamma$ connecting $p$ to $q$ and extend $Y$ along $\gamma$ by solving 
the ODE (3.2.8). Then the Hessian of the $\mathcal{L}$-distance function $L$ on $M$ with $\tau=\bar{\tau}$ satisfies

$$
\operatorname{Hess}_{L}(Y, Y) \leq \frac{1}{\sqrt{\bar{\tau}}}-2 \sqrt{\bar{\tau}} \operatorname{Ric}(Y, Y)-\int_{0}^{\bar{\tau}} \sqrt{\tau} Q(X, Y) d \tau
$$

in the sense of distributions, where

$$
\begin{aligned}
Q(X, Y)= & -\nabla_{Y} \nabla_{Y} R-2\langle R(Y, X) Y, X\rangle-4 \nabla_{X} \operatorname{Ric}(Y, Y)+4 \nabla_{Y} \operatorname{Ric}(Y, X) \\
& -2 \operatorname{Ric}_{\tau}(Y, Y)+2|\operatorname{Ric}(Y, \cdot)|^{2}-\frac{1}{\tau} \operatorname{Ric}(Y, Y)
\end{aligned}
$$

is the Li-Yau-Hamilton quadratic. Moreover the equality holds if and only if the vector field $Y(\tau), \tau \in[0, \bar{\tau}]$, is an $\mathcal{L}$-Jacobian field (i.e., $Y$ is the derivative of a variation of $\gamma$ by $\mathcal{L}$-geodesics).

Proof. As said before, we pretend that the shortest $\mathcal{L}$-geodesics between $p$ and $q$ are unique so that $L(q, \bar{\tau})$ is smooth. Otherwise, the inequality is still valid, by a standard barrier argument, when understood in the sense of distributions (see, for example, [112]).

Recall that $\nabla L(q, \bar{\tau})=2 \sqrt{\bar{\tau}} X$. Then $\left\langle\nabla_{Y} Y, \nabla L\right\rangle=2 \sqrt{\bar{\tau}}\left\langle\nabla_{Y} Y, X\right\rangle$. We compute by using Lemma $3.2 .2,(3.2 .8)$ and (3.2.9),

$$
\begin{aligned}
\operatorname{Hess}_{L}(Y, Y)= & Y(Y(L))(\bar{\tau})-\left\langle\nabla_{Y} Y, \nabla L\right\rangle(\bar{\tau}) \\
\leq & \delta_{Y}^{2}(\mathcal{L})-2 \sqrt{\bar{\tau}}\left\langle\nabla_{Y} Y, X\right\rangle(\bar{\tau}) \\
= & \int_{0}^{\bar{\tau}} \sqrt{\tau}\left[2\left|\nabla_{X} Y\right|^{2}+2\langle R(Y, X) Y, X\rangle+\nabla_{Y} \nabla_{Y} R\right. \\
& \left.+2 \nabla_{X} \operatorname{Ric}(Y, Y)-4 \nabla_{Y} \operatorname{Ric}(Y, X)\right] d \tau \\
= & \int_{0}^{\bar{\tau}} \sqrt{\tau}\left[2\left|-\operatorname{Ric}(Y, \cdot)+\frac{1}{2 \tau} Y\right|^{2}+2\langle R(Y, X) Y, X\rangle+\nabla_{Y} \nabla_{Y} R\right. \\
& \left.+2 \nabla_{X} \operatorname{Ric}(Y, Y)-4 \nabla_{Y} \operatorname{Ric}(Y, X)\right] d \tau \\
= & \int_{0}^{\bar{\tau}} \sqrt{\tau}\left[2|\operatorname{Ric}(Y, \cdot)|^{2}-\frac{2}{\tau} \operatorname{Ric}(Y, Y)+\frac{1}{2 \tau \bar{\tau}}+2\langle R(Y, X) Y, X\rangle\right. \\
& \left.+\nabla_{Y} \nabla_{Y} R+2 \nabla_{X} \operatorname{Ric}(Y, Y)-4 \nabla_{Y} \operatorname{Ric}(Y, X)\right] d \tau .
\end{aligned}
$$

Since

$$
\begin{aligned}
\frac{d}{d \tau} \operatorname{Ric}(Y, Y) & =\operatorname{Ric}_{\tau}(Y, Y)+\nabla_{X} \operatorname{Ric}(Y, Y)+2 \operatorname{Ric}\left(\nabla_{X} Y, Y\right) \\
& =\operatorname{Ric}_{\tau}(Y, Y)+\nabla_{X} \operatorname{Ric}(Y, Y)-2|\operatorname{Ric}(Y, \cdot)|^{2}+\frac{1}{\tau} \operatorname{Ric}(Y, Y),
\end{aligned}
$$


we have

$$
\begin{aligned}
\operatorname{Hess}_{L}(Y, Y) \\
\leq \int_{0}^{\bar{\tau}} \sqrt{\tau}\left[2|\operatorname{Ric}(Y, \cdot)|^{2}-\frac{2}{\tau} \operatorname{Ric}(Y, Y)+\frac{1}{2 \tau \bar{\tau}}+2\langle R(Y, X) Y, X\rangle\right. \\
\quad+\nabla_{Y} \nabla_{Y} R-4\left(\nabla_{Y} \operatorname{Ric}\right)(X, Y)-\left(2 \frac{d}{d \tau} \operatorname{Ric}(Y, Y)-2 \operatorname{Ric}_{\tau}(Y, Y)\right. \\
\left.\left.\quad+4|\operatorname{Ric}(Y, \cdot)|^{2}-\frac{2}{\tau} \operatorname{Ric}(Y, Y)\right)+4 \nabla_{X} \operatorname{Ric}(Y, Y)\right] d \tau \\
=-\int_{0}^{\bar{\tau}}\left[2 \sqrt{\tau} \frac{d}{d \tau} \operatorname{Ric}(Y, Y)+\frac{1}{\sqrt{\tau}} \operatorname{Ric}(Y, Y)\right] d \tau+\frac{1}{2 \bar{\tau}} \int_{0}^{\bar{\tau}} \frac{1}{\sqrt{\tau}} d \tau \\
\quad+\int_{0}^{\bar{\tau}} \sqrt{\tau}\left[2\langle R(Y, X) Y, X\rangle+\nabla_{Y} \nabla_{Y} R+\frac{1}{\tau} \operatorname{Ric}(Y, Y)\right. \\
\left.\quad+4\left(\nabla_{X} \operatorname{Ric}(Y, Y)-\nabla_{Y} \operatorname{Ric}(X, Y)\right)+2 \operatorname{Ric}_{\tau}(Y, Y)-2|\operatorname{Ric}(Y, \cdot)|^{2}\right] d \tau \\
=\frac{1}{\sqrt{\bar{\tau}}}-2 \sqrt{\bar{\tau}} \operatorname{Ric}(Y, Y)-\int_{0}^{\bar{\tau}} \sqrt{\tau} Q(X, Y) d \tau .
\end{aligned}
$$

This proves the inequality.

As usual, the quadratic form

$$
\begin{aligned}
I(V, V)= & \int_{0}^{\bar{\tau}} \sqrt{\tau}\left[2\left|\nabla_{X} V\right|^{2}+2\langle R(V, X) V, X\rangle+\nabla_{V} \nabla_{V} R\right. \\
& \left.+2 \nabla_{X} \operatorname{Ric}(V, V)-4 \nabla_{V} \operatorname{Ric}(V, X)\right] d \tau,
\end{aligned}
$$

for any vector field $V$ along $\gamma$, is called the index form. Since $\gamma$ is shortest, the standard Dirichlet principle for $I(V, V)$ implies that the equality holds if and only if the vector field $Y$ is the derivative of a variation of $\gamma$ by $\mathcal{L}$-geodesics. $\square$

Corollary 3.2.4. We have

$$
\Delta L \leq \frac{n}{\sqrt{\bar{\tau}}}-2 \sqrt{\bar{\tau}} R-\frac{1}{\bar{\tau}} K
$$

in the sense of distribution. Moreover, the equality holds if and only if we are on a gradient shrinking soliton with

$$
R_{i j}+\frac{1}{2 \sqrt{\bar{\tau}}} \nabla_{i} \nabla_{j} L=\frac{1}{2 \bar{\tau}} g_{i j}
$$

Proof. Choose an orthonormal basis $\left\{Y_{1}, \ldots, Y_{n}\right\}$ at $\tau=\bar{\tau}$ and extend them along the shortest $\mathcal{L}$-geodesic $\gamma$ to get vector fields $Y_{i}(\tau), i=1, \ldots, n$, by solving the ODE (3.2.8), with $\left\langle Y_{i}(\tau), Y_{j}(\tau)\right\rangle=\frac{\tau}{\bar{\tau}} \delta_{i j}$ on $[0, \bar{\tau}]$. Taking $Y=Y_{i}$ in Proposition 3.2.3 and 
summing over $i$, we get

$$
\begin{aligned}
\Delta L & \leq \frac{n}{\sqrt{\bar{\tau}}}-2 \sqrt{\bar{\tau}} R-\sum_{i=1}^{n} \int_{0}^{\bar{\tau}} \sqrt{\tau} Q\left(X, Y_{i}\right) d \tau \\
& =\frac{n}{\sqrt{\bar{\tau}}}-2 \sqrt{\bar{\tau}} R-\int_{0}^{\bar{\tau}} \sqrt{\tau}\left(\frac{\tau}{\bar{\tau}}\right) Q(X) d \tau \\
& =\frac{n}{\sqrt{\bar{\tau}}}-2 \sqrt{\bar{\tau}} R-\frac{1}{\bar{\tau}} K .
\end{aligned}
$$

Moreover, by Proposition 3.2.3, the equality in (3.2.10) holds everywhere if and only if for each $(q, \bar{\tau})$ and any shortest $\mathcal{L}$-geodesic $\gamma$ on $[0, \bar{\tau}]$ connecting $p$ and $q$, and for any unit vector $Y$ at $\tau=\bar{\tau}$, the extended vector field $Y(\tau)$ along $\gamma$ by the ODE (3.2.8) must be an $\mathcal{L}$-Jacobian field. When $Y_{i}(\tau), i=1, \ldots, n$ are $\mathcal{L}$-Jacobian fields along $\gamma$, we have

$$
\begin{aligned}
& \frac{d}{d \tau}\left\langle Y_{i}(\tau), Y_{j}(\tau)\right\rangle \\
& =2 \operatorname{Ric}\left(Y_{i}, Y_{j}\right)+\left\langle\nabla_{X} Y_{i}, Y_{j}\right\rangle+\left\langle Y_{i}, \nabla_{X} Y_{j}\right\rangle \\
& =2 \operatorname{Ric}\left(Y_{i}, Y_{j}\right)+\left\langle\nabla_{Y_{i}}\left(\frac{1}{2 \sqrt{\bar{\tau}}} \nabla L\right), Y_{j}\right\rangle+\left\langle Y_{i}, \nabla_{Y_{j}}\left(\frac{1}{2 \sqrt{\bar{\tau}}} \nabla L\right)\right\rangle \\
& =2 \operatorname{Ric}\left(Y_{i}, Y_{j}\right)+\frac{1}{\sqrt{\bar{\tau}}} \operatorname{Hess}_{L}\left(Y_{i}, Y_{j}\right)
\end{aligned}
$$

and then by (3.2.9),

$$
2 \operatorname{Ric}\left(Y_{i}, Y_{j}\right)+\frac{1}{\sqrt{\bar{\tau}}} \operatorname{Hess}_{L}\left(Y_{i}, Y_{j}\right)=\frac{1}{\bar{\tau}} \delta_{i j}, \quad \text { at } \tau=\bar{\tau} .
$$

Therefore the equality in (3.2.10) holds everywhere if and only if we are on a gradient shrinking soliton with

$$
R_{i j}+\frac{1}{2 \sqrt{\bar{\tau}}} \nabla_{i} \nabla_{j} L=\frac{1}{2 \bar{\tau}} g_{i j}
$$

In summary, from (3.2.6), (3.2.7) and Corollary 3.2.4, we have

$$
\left\{\begin{array}{l}
\frac{\partial L}{\partial \bar{\tau}}=2 \sqrt{\bar{\tau}} R-\frac{L}{2 \bar{\tau}}+\frac{K}{\bar{\tau}}, \\
|\nabla L|^{2}=-4 \bar{\tau} R+\frac{2}{\sqrt{\bar{\tau}}} L-\frac{4}{\sqrt{\bar{\tau}}} K, \\
\Delta L \leq-2 \sqrt{\bar{\tau}} R+\frac{n}{\sqrt{\bar{\tau}}}-\frac{K}{\bar{\tau}}
\end{array}\right.
$$

in the sense of distributions.

Now the Li-Yau-Perelman distance $l=l(q, \bar{\tau})$ is defined by

$$
l(q, \bar{\tau})=L(q, \bar{\tau}) / 2 \sqrt{\bar{\tau}} .
$$

We thus have the following 
Lemma 3.2.5. For the Li-Yau-Perelman distance $l(q, \bar{\tau})$ defined above, we have

$$
\begin{aligned}
\frac{\partial l}{\partial \bar{\tau}} & =-\frac{l}{\bar{\tau}}+R+\frac{1}{2 \bar{\tau}^{3 / 2}} K, \\
|\nabla l|^{2} & =-R+\frac{l}{\bar{\tau}}-\frac{1}{\bar{\tau}^{3 / 2}} K, \\
\Delta l & \leq-R+\frac{n}{2 \bar{\tau}}-\frac{1}{2 \bar{\tau}^{3 / 2}} K,
\end{aligned}
$$

in the sense of distributions. Moreover, the equality in (3.2.13) holds if and only if we are on a gradient shrinking soliton.

As the first consequence, we derive the following upper bound on the minimum of $l(\cdot, \tau)$ for every $\tau$ which will be useful in proving the no local collapsing theorem in the next section.

Corollary 3.2.6. Let $_{i j}(\tau), \tau \geq 0$, be a family of metrics evolving by the Ricci flow $\frac{\partial}{\partial \tau} g_{i j}=2 R_{i j}$ on a compact $n$-dimensional manifold $M$. Fix a point $p$ in $M$ and let $l(q, \tau)$ be the Li-Yau-Perelman distance from $(p, 0)$. Then for all $\tau$,

$$
\min \{l(q, \tau) \mid q \in M\} \leq \frac{n}{2}
$$

Proof. Let

$$
\bar{L}(q, \tau)=4 \tau l(q, \tau)
$$

Then, it follows from (3.2.11) and (3.2.13) that

$$
\frac{\partial \bar{L}}{\partial \tau}=4 \tau R+\frac{2 K}{\sqrt{\tau}}
$$

and

$$
\Delta \bar{L} \leq-4 \tau R+2 n-\frac{2 K}{\sqrt{\tau}}
$$

Hence

$$
\frac{\partial \bar{L}}{\partial \tau}+\Delta \bar{L} \leq 2 n
$$

Thus, by a standard maximum principle argument, $\min \{\bar{L}(q, \tau)-2 n \tau \mid q \in M\}$ is nonincreasing and therefore $\min \{\bar{L}(q, \tau) \mid q \in M\} \leq 2 n \tau$. $\mathbf{Q}$

As another consequence of Lemma 3.2.5, we obtain

$$
\frac{\partial l}{\partial \bar{\tau}}-\Delta l+|\nabla l|^{2}-R+\frac{n}{2 \bar{\tau}} \geq 0 .
$$

or equivalently

$$
\left(\frac{\partial}{\partial \bar{\tau}}-\Delta+R\right)\left((4 \pi \bar{\tau})^{-\frac{n}{2}} \exp (-l)\right) \leq 0 .
$$


If $M$ is compact, we define Perelman's reduced volume by

$$
\tilde{V}(\tau)=\int_{M}(4 \pi \tau)^{-\frac{n}{2}} \exp (-l(q, \tau)) d V_{\tau}(q),
$$

where $d V_{\tau}$ denotes the volume element with respect to the metric $g_{i j}(\tau)$. Note that Perelman's reduced volume resembles the expression in Huisken's monotonicity formula for the mean curvature flow [72]. It follows, from the above computation, that

$$
\begin{aligned}
& \frac{d}{d \bar{\tau}} \int_{M}(4 \pi \bar{\tau})^{-\frac{n}{2}} \exp (-l(q, \bar{\tau})) d V_{\bar{\tau}}(q) \\
& =\int_{M}\left[\frac{\partial}{\partial \bar{\tau}}\left((4 \pi \bar{\tau})^{-\frac{n}{2}} \exp (-l(q, \bar{\tau}))\right)+R(4 \pi \bar{\tau})^{-\frac{n}{2}} \exp (-l(q, \bar{\tau}))\right] d V_{\bar{\tau}}(q) \\
& \leq \int_{M} \Delta\left((4 \pi \bar{\tau})^{-\frac{n}{2}} \exp (-l(q, \bar{\tau}))\right) d V_{\bar{\tau}}(q) \\
& =0 .
\end{aligned}
$$

This says that if $M$ is compact, then Perelman's reduced volume $\tilde{V}(\tau)$ is nonincreasing in $\tau$; moreover, the monotonicity is strict unless we are on a gradient shrinking soliton.

In order to define and to obtain the monotonicity of Perelman's reduced volume for a complete noncompact manifold, we need to formulate the monotonicity of Perelman's reduced volume in a local version. This local version is very important and will play a crucial role in the analysis of the Ricci flow with surgery in Chapter 7 .

We define the $\mathcal{L}$-exponential map (with parameter $\bar{\tau}$ ) $\mathcal{L} \exp (\bar{\tau}): T_{p} M \rightarrow M$ as follows: for any $X \in T_{p} M$, we set

$$
\mathcal{L} \exp _{X}(\bar{\tau})=\gamma(\bar{\tau})
$$

where $\gamma(\tau)$ is the $\mathcal{L}$-geodesic, starting at $p$ and having $X$ as the limit of $\sqrt{\tau} \dot{\gamma}(\tau)$ as $\tau \rightarrow 0^{+}$. The associated Jacobian of the $\mathcal{L}$-exponential map is called $\mathcal{L}$-Jacobian. We denote by $\mathcal{J}(\tau)$ the $\mathcal{L}$-Jacobian of $\mathcal{L} \exp (\tau): T_{p} M \rightarrow M$. We can now deduce an estimate for the $\mathcal{L}$-Jacobian as follows.

Let $q=\mathcal{L} \exp _{X}(\bar{\tau})$ and $\gamma(\tau), \tau \in[0, \bar{\tau}]$, be the shortest $\mathcal{L}$-geodesic connecting $p$ and $q$ with $\sqrt{\tau} \dot{\gamma}(\tau) \rightarrow X$ as $\tau \rightarrow 0^{+}$. For any vector $v \in T_{p} M$, we consider the family of $\mathcal{L}$-geodesics:

$$
\gamma_{s}(\tau)=\mathcal{L} \exp _{(X+s v)}(\tau), \quad 0 \leq \tau \leq \bar{\tau}, \quad s \in(-\epsilon, \epsilon) .
$$

The associated variation vector field $V(\tau), 0 \leq \tau \leq \bar{\tau}$, is an $\mathcal{L}$-Jacobian field with $V(0)=0$ and $V(\tau)=\left(\mathcal{L} \exp _{X}(\tau)\right)_{*}(v)$.

Let $v_{1}, \ldots, v_{n}$ be $n$ linearly independent vectors in $T_{p} M$. Then

$$
V_{i}(\tau)=\left(\mathcal{L} \exp _{X}(\tau)\right)_{*}\left(v_{i}\right), \quad i=1,2, \ldots, n,
$$

are $n \mathcal{L}$-Jacobian fields along $\gamma(\tau), \tau \in[0, \bar{\tau}]$. The $\mathcal{L}$-Jacobian $\mathcal{J}(\tau)$ is given by

$$
\mathcal{J}(\tau)=\left|V_{1}(\tau) \wedge \cdots \wedge V_{n}(\tau)\right|_{g_{i j}(\tau)} /\left|v_{1} \wedge \cdots \wedge v_{n}\right| .
$$


Now for fixed $b \in(0, \bar{\tau})$, we can choose linearly independent vectors $v_{1}, \ldots, v_{n} \in$ $T_{p} M$ such that $\left\langle V_{i}(b), V_{j}(b)\right\rangle_{g_{i j}(b)}=\delta_{i j}$. We compute

$$
\begin{aligned}
& \frac{d}{d \tau} \mathcal{J}^{2} \\
& =\frac{2}{\left|v_{1} \wedge \cdots \wedge v_{n}\right|^{2}} \sum_{j=1}^{n}\left\langle V_{1} \wedge \cdots \wedge \nabla_{X} V_{j} \wedge \cdots \wedge V_{n}, V_{1} \wedge \cdots \wedge V_{n}\right\rangle_{g_{i j}(\tau)} \\
& +\frac{2}{\left|v_{1} \wedge \cdots \wedge v_{n}\right|^{2}} \sum_{j=1}^{n}\left\langle V_{1} \wedge \cdots \wedge \operatorname{Ric}\left(V_{j}, \cdot\right) \wedge \cdots \wedge V_{n}, V_{1} \wedge \cdots \wedge V_{n}\right\rangle_{g_{i j}(\tau)}
\end{aligned}
$$

At $\tau=b$,

$$
\frac{d}{d \tau} \mathcal{J}^{2}(b)=\frac{2}{\left|v_{1} \wedge \cdots \wedge v_{n}\right|^{2}} \sum_{j=1}^{n}\left(\left\langle\nabla_{X} V_{j}, V_{j}\right\rangle_{g_{i j}(b)}+\operatorname{Ric}\left(V_{j}, V_{j}\right)\right) .
$$

Thus,

$$
\begin{aligned}
\frac{d}{d \tau} \log \mathcal{J}(b) & =\sum_{j=1}^{n}\left(\left\langle\nabla_{X} V_{j}, V_{j}\right\rangle_{g_{i j}(b)}+\operatorname{Ric}\left(V_{j}, V_{j}\right)\right) \\
& =\sum_{j=1}^{n}\left(\left\langle\nabla_{V_{j}}\left(\frac{1}{2 \sqrt{b}} \nabla L\right), V_{j}\right\rangle_{g_{i j}(b)}+\operatorname{Ric}\left(V_{j}, V_{j}\right)\right) \\
& =\frac{1}{2 \sqrt{b}}\left(\sum_{j=1}^{n} \operatorname{Hess}_{L}\left(V_{j}, V_{j}\right)\right)+R \\
& =\frac{1}{2 \sqrt{b}} \Delta L+R .
\end{aligned}
$$

Therefore, in view of Corollary 3.2.4, we obtain the following estimate for $\mathcal{L}$-Jacobian:

$$
\frac{d}{d \tau} \log \mathcal{J}(\tau) \leq \frac{n}{2 \tau}-\frac{1}{2 \tau^{3 / 2}} K \quad \text { on } \quad[0, \bar{\tau}] .
$$

On the other hand, by the definition of the Li-Yau-Perelman distance and (3.2.4), we have

$$
\begin{aligned}
\frac{d}{d \tau} l(\tau) & =-\frac{1}{2 \tau} l+\frac{1}{2 \sqrt{\tau}} \frac{d}{d \tau} L \\
& =-\frac{1}{2 \tau} l+\frac{1}{2 \sqrt{\tau}}\left(\sqrt{\tau}\left(R+|X|^{2}\right)\right) \\
& =-\frac{1}{2 \tau^{3 / 2}} K .
\end{aligned}
$$

Here and in the following we denote by $l(\tau)=l(\gamma(\tau), \tau)$. Now the combination of (3.2.14) and (3.2.15) implies the following important Jacobian comparison theorem of Perelman [103].

Theorem 3.2.7 (Perelman's Jacobian comparison theorem). Let $g_{i j}(\tau)$ be a family of complete solutions to the Ricci flow $\frac{\partial}{\partial \tau} g_{i j}=2 R_{i j}$ on a manifold $M$ with 
bounded curvature. Let $\gamma:[0, \bar{\tau}] \rightarrow M$ be a shortest $\mathcal{L}$-geodesic starting from a fixed point $p$. Then Perelman's reduced volume element

$$
(4 \pi \tau)^{-\frac{n}{2}} \exp (-l(\tau)) \mathcal{J}(\tau)
$$

is nonincreasing in $\tau$ along $\gamma$.

We now show how to integrate Perelman's reduced volume element over $T_{p} M$ to deduce the following monotonicity result of Perelman [103].

TheOREM 3.2.8 (Monotonicity of Perelman's reduced volume). Let $g_{i j}$ be a family of complete metrics evolving by the Ricci flow $\frac{\partial}{\partial \tau} g_{i j}=2 R_{i j}$ on a manifold $M$ with bounded curvature. Fix a point $p$ in $M$ and let $l(q, \tau)$ be the reduced distance from $(p, 0)$. Then

(i) Perelman's reduced volume

$$
\tilde{V}(\tau)=\int_{M}(4 \pi \tau)^{-\frac{n}{2}} \exp (-l(q, \tau)) d V_{\tau}(q)
$$

is finite and nonincreasing in $\tau$;

(ii) the monotonicity is strict unless we are on a gradient shrinking soliton.

Proof. For any $v \in T_{p} M$ we can find an $\mathcal{L}$-geodesic $\gamma(\tau)$, starting at $p$, with $\lim _{\tau \rightarrow 0^{+}} \sqrt{\tau} \dot{\gamma}(\tau)=v$. Recall that $\gamma(\tau)$ satisfies the $\mathcal{L}$-geodesic equation

$$
\nabla_{\dot{\gamma}(\tau)} \dot{\gamma}(\tau)-\frac{1}{2} \nabla R+\frac{1}{2 \tau} \dot{\gamma}(\tau)+2 \operatorname{Ric}(\dot{\gamma}(\tau), \cdot)=0 .
$$

Multiplying this equation by $\sqrt{\tau}$, we get

$$
\frac{d}{d \tau}(\sqrt{\tau} \dot{\gamma})-\frac{1}{2} \sqrt{\tau} \nabla R+2 \operatorname{Ric}(\sqrt{\tau} \dot{\gamma}(\tau), \cdot)=0
$$

Since the curvature of the metric $g_{i j}(\tau)$ is bounded, it follows from Shi's derivative estimate (Theorem 1.4.1) that $|\nabla R|$ is also bounded for small $\tau>0$. Thus by integrating (3.2.16), we have

$$
|\sqrt{\tau} \dot{\gamma}(\tau)-v| \leq C \tau(|v|+1)
$$

for $\tau$ small enough and for some positive constant $C$ depending only the curvature bound.

Let $v_{1}, \ldots, v_{n}$ be $n$ linearly independent vectors in $T_{p} M$ and let

$$
V_{i}(\tau)=\left(\mathcal{L} \exp _{v}(\tau)\right)_{*}\left(v_{i}\right)=\left.\frac{d}{d s}\right|_{s=0} \mathcal{L} \exp _{\left(v+s v_{i}\right)}(\tau), \quad i=1, \ldots, n .
$$

The $\mathcal{L}$-Jacobian $\mathcal{J}(\tau)$ is given by

$$
\mathcal{J}(\tau)=\left|V_{1}(\tau) \wedge \cdots \wedge V_{n}(\tau)\right|_{g_{i j}(\tau)} /\left|v_{1} \wedge \cdots \wedge v_{n}\right|
$$

By (3.2.17), we see that

$$
\left|\sqrt{\tau} \frac{d}{d \tau} \mathcal{L} \exp _{\left(v+s v_{i}\right)}(\tau)-\left(v+s v_{i}\right)\right| \leq C \tau\left(|v|+\left|v_{i}\right|+1\right)
$$


for $\tau$ small enough and all $s \in(-\epsilon, \epsilon)$ (for some $\epsilon>0$ small) and $i=1, \ldots, n$. This implies that

$$
\lim _{\tau \rightarrow 0^{+}} \sqrt{\tau} \dot{V}_{i}(\tau)=v_{i}, \quad i=1, \ldots, n,
$$

so we deduce that

$$
\lim _{\tau \rightarrow 0^{+}} \tau^{-\frac{n}{2}} \mathcal{J}(\tau)=1
$$

Meanwhile, by using (3.2.17), we have

$$
\begin{aligned}
l(\tau) & =\frac{1}{2 \sqrt{\tau}} \int_{0}^{\tau} \sqrt{\tau}\left(R+|\dot{\gamma}(\tau)|^{2}\right) d \tau \\
& \rightarrow|v|^{2} \text { as } \tau \rightarrow 0^{+} .
\end{aligned}
$$

Thus

$$
l(0)=|v|^{2} .
$$

Combining (3.2.18) and (3.2.19) with Theorem 3.2.7, we get

$$
\begin{aligned}
\tilde{V}(\tau) & =\int_{M}(4 \pi \tau)^{-\frac{n}{2}} \exp (-l(q, \tau)) d V_{\tau}(q) \\
& \leq\left.\int_{T_{p} M}(4 \pi \tau)^{-\frac{n}{2}} \exp (-l(\tau)) \mathcal{J}(\tau)\right|_{\tau=0} d v \\
& =(4 \pi)^{-\frac{n}{2}} \int_{\mathbb{R}^{n}} \exp \left(-|v|^{2}\right) d v \\
& <+\infty
\end{aligned}
$$

This proves that Perelman's reduced volume is always finite and hence well defined. Now the monotonicity assertion in (i) follows directly from Theorem 3.2.7.

For the assertion (ii), we note that the equality in (3.2.13) holds everywhere when the monotonicity of Perelman's reduced volume is not strict. Therefore we have completed the proof of the theorem.

3.3. No Local Collapsing Theorem I. In this section we apply the monotonicity of Perelman's reduced volume in Theorem 3.2.8 to prove Perelman's no local collapsing theorem $\mathbf{I}$, which is extremely important not only because it gives a local injectivity radius estimate in terms of local curvature bound but also it will survive the surgeries in Chapter 7.

Definition 3.3.1. Let $\kappa, r$ be two positive constants and let $g_{i j}(t), 0 \leq t<T$, be a solution to the Ricci flow on an $n$-dimensional manifold $M$. We call the solution $g_{i j}(t) \kappa$-noncollapsed at $\left(x_{0}, t_{0}\right) \in M \times[0, T)$ on the scale $r$ if it satisfies the following property: whenever

$$
|R m|(x, t) \leq r^{-2}
$$

for all $x \in B_{t_{0}}\left(x_{0}, r\right)$ and $t \in\left[t_{0}-r^{2}, t_{0}\right]$, we have

$$
\operatorname{Vol}_{t_{0}}\left(B_{t_{0}}\left(x_{0}, r\right)\right) \geq \kappa r^{n} .
$$


Here $B_{t_{0}}\left(x_{0}, r\right)$ is the geodesic ball centered at $x_{0} \in M$ and of radius $r$ with respect to the metric $g_{i j}\left(t_{0}\right)$.

Now we are ready to state the no local collapsing theorem I of Perelman [103].

TheOREm 3.3.2 (No local collapsing theorem I). Given any metric $g_{i j}$ on an $n$-dimensional compact manifold $M$. Let $g_{i j}(t)$ be the solution to the Ricci flow on $[0, T)$, with $T<+\infty$, starting at $g_{i j}$. Then there exist positive constants $\kappa$ and $\rho_{0}$ such that for any $t_{0} \in[0, T)$ and any point $x_{0} \in M$, the solution $g_{i j}(t)$ is $\kappa$-noncollapsed at $\left(x_{0}, t_{0}\right)$ on all scales less than $\rho_{0}$.

Proof. We argue by contradiction. Suppose that there are sequences $p_{k} \in M$, $t_{k} \in[0, T)$ and $r_{k} \rightarrow 0$ such that

$$
|R m|(x, t) \leq r_{k}^{-2}
$$

for $x \in B_{k}=B_{t_{k}}\left(p_{k}, r_{k}\right)$ and $t_{k}-r_{k}^{2} \leq t \leq t_{k}$, but

$$
\epsilon_{k}=r_{k}^{-1} \operatorname{Vol}_{t_{k}}\left(B_{k}\right)^{\frac{1}{n}} \rightarrow 0 \text { as } k \rightarrow \infty .
$$

Without loss of generality, we may assume that $t_{k} \rightarrow T$ as $k \rightarrow+\infty$.

Let $\bar{\tau}(t)=t_{k}-t, p=p_{k}$ and

$$
\tilde{V}_{k}(\bar{\tau})=\int_{M}(4 \pi \bar{\tau})^{-\frac{n}{2}} \exp (-l(q, \bar{\tau})) d V_{t_{k}-\bar{\tau}}(q)
$$

where $l(q, \bar{\tau})$ is the Li-Yau-Perelman distance with respect to $p=p_{k}$.

Step 1 . We first want to show that for $k$ large enough,

$$
\tilde{V}_{k}\left(\epsilon_{k} r_{k}^{2}\right) \leq 2 \epsilon_{k}^{\frac{n}{2}}
$$

For any $v \in T_{p} M$ we can find an $\mathcal{L}$-geodesic $\gamma(\tau)$ starting at $p$ with $\lim _{\tau \rightarrow 0} \sqrt{\tau} \dot{\gamma}(\tau)$ $=v$. Recall that $\gamma(\tau)$ satisfies the equation (3.2.16). It follows from assumption (3.3.1) and Shi's local derivative estimate (Theorem 1.4.2) that $|\nabla R|$ has a bound in the order of $1 / r_{k}^{3}$ for $t \in\left[t_{k}-\epsilon_{k} r_{k}^{2}, t_{k}\right]$. Thus by integrating (3.2.16) we see that for $\tau \leq \epsilon_{k} r_{k}^{2}$ satisfying the property that $\gamma(\sigma) \in B_{k}$ as long as $\sigma<\tau$, there holds

$$
|\sqrt{\tau} \dot{\gamma}(\tau)-v| \leq C \epsilon_{k}(|v|+1)
$$

where $C$ is some positive constant depending only on the dimension. Here we have implicitly used the fact that the metric $g_{i j}(t)$ is equivalent for $x \in B_{k}$ and $t \in\left[t_{k}-\right.$ $\left.\epsilon_{k} r_{k}^{2}, t_{k}\right]$. In fact since $\frac{\partial g_{i j}}{\partial t}=-2 R_{i j}$ and $|R m| \leq r_{k}^{-2}$ on $B_{k} \times\left[t_{k}-r_{k}^{2}, t_{k}\right]$, we have

$$
e^{-2 \epsilon_{k}} g_{i j}\left(x, t_{k}\right) \leq g_{i j}(x, t) \leq e^{2 \epsilon_{k}} g_{i j}\left(x, t_{k}\right),
$$

for $x \in B_{k}$ and $t \in\left[t_{k}-\epsilon_{k} r_{k}^{2}, t_{k}\right]$.

Suppose $v \in T_{p} M$ with $|v| \leq \frac{1}{4} \epsilon_{k}^{-\frac{1}{2}}$. Let $\tau \leq \epsilon_{k} r_{k}^{2}$ such that $\gamma(\sigma) \in B_{k}$ as long as $\sigma<\tau$, where $\gamma$ is the $\mathcal{L}$-geodesic starting at $p$ with $\lim _{\tau \rightarrow 0} \sqrt{\tau} \dot{\gamma}(\tau)=v$. Then, by (3.3.3) 
and (3.3.4), for $k$ large enough,

$$
\begin{aligned}
d_{t_{k}}\left(p_{k}, \gamma(\tau)\right) & \leq \int_{0}^{\tau}|\dot{\gamma}(\sigma)|_{g_{i j}\left(t_{k}\right)} d \sigma \\
& <\frac{1}{2} \epsilon_{k}^{-\frac{1}{2}} \int_{0}^{\tau} \frac{d \sigma}{\sqrt{\sigma}} \\
& =\epsilon_{k}^{-\frac{1}{2}} \sqrt{\tau} \\
& \leq r_{k} .
\end{aligned}
$$

This shows that for $k$ large enough,

$$
\mathcal{L} \exp _{\left\{|v| \leq \frac{1}{4} \epsilon_{k}^{-1 / 2}\right\}}\left(\epsilon_{k} r_{k}^{2}\right) \subset B_{k}=B_{t_{k}}\left(p_{k}, r_{k}\right) .
$$

We now estimate the integral of $\tilde{V}_{k}\left(\epsilon_{k} r_{k}^{2}\right)$ as follows,

$$
\begin{aligned}
\tilde{V}_{k}\left(\epsilon_{k} r_{k}^{2}\right)= & \int_{M}\left(4 \pi \epsilon_{k} r_{k}^{2}\right)^{-\frac{n}{2}} \exp \left(-l\left(q, \epsilon_{k} r_{k}^{2}\right)\right) d V_{t_{k}-\epsilon_{k} r_{k}^{2}}(q) \\
= & \int_{\mathcal{L} \exp _{\left\{|v| \leq \frac{1}{4} \epsilon_{k}^{-1 / 2}\right\}}\left(\epsilon_{k} r_{k}^{2}\right)}\left(4 \pi \epsilon_{k} r_{k}^{2}\right)^{-\frac{n}{2}} \exp \left(-l\left(q, \epsilon_{k} r_{k}^{2}\right)\right) d V_{t_{k}-\epsilon_{k} r_{k}^{2}}(q) \\
& +\int_{M \backslash \mathcal{L} \exp _{\left\{|v| \leq \frac{1}{4} \epsilon_{k}^{-1 / 2}\right\}}\left(\epsilon_{k} r_{k}^{2}\right)}\left(4 \pi \epsilon_{k} r_{k}^{2}\right)^{-\frac{n}{2}} \exp \left(-l\left(q, \epsilon_{k} r_{k}^{2}\right)\right) d V_{t_{k}-\epsilon_{k} r_{k}^{2}}(q) .
\end{aligned}
$$

We observe that for each $q \in B_{k}$,

$$
L\left(q, \epsilon_{k} r_{k}^{2}\right)=\int_{0}^{\epsilon_{k} r_{k}^{2}} \sqrt{\tau}\left(R+|\dot{\gamma}|^{2}\right) d \tau \geq-C(n) r_{k}^{-2}\left(\epsilon_{k} r_{k}^{2}\right)^{\frac{3}{2}}=-C(n) \epsilon_{k}^{\frac{3}{2}} r_{k}
$$

hence $l\left(q, \epsilon_{k} r_{k}^{2}\right) \geq-C(n) \epsilon_{k}$. Thus, the first term on the RHS of (3.3.6) can be estimated by

$$
\begin{aligned}
& \quad \int_{\mathcal{L} \exp _{\left\{|v| \leq \frac{1}{4} \epsilon_{k}^{-1 / 2}\right\}}\left(\epsilon_{k} r_{k}^{2}\right)}\left(4 \pi \epsilon_{k} r_{k}^{2}\right)^{-\frac{n}{2}} \exp \left(-l\left(q, \epsilon_{k} r_{k}^{2}\right)\right) d V_{t_{k}-\epsilon_{k} r_{k}^{2}}(q) \\
& \leq e^{n \epsilon_{k}} \int_{B_{k}}\left(4 \pi \epsilon_{k} r_{k}^{2}\right)^{-\frac{n}{2}} \exp \left(-l\left(q, \epsilon_{k} r_{k}^{2}\right)\right) d V_{t_{k}}(q) \\
& \leq e^{n \epsilon_{k}}(4 \pi)^{-\frac{n}{2}} \cdot e^{C(n) \epsilon_{k}} \cdot \epsilon_{k}^{-\frac{n}{2}} \cdot\left(r_{k}^{-n} \operatorname{Vol}_{t_{k}}\left(B_{k}\right)\right) \\
& =e^{(n+C(n)) \epsilon_{k}}(4 \pi)^{-\frac{n}{2}} \cdot \epsilon_{k}^{\frac{n}{2}}
\end{aligned}
$$

where we have also used (3.3.5) and (3.3.4).

Meanwhile, by using (3.2.18), (3.2.19) and the Jacobian Comparison Theorem 
3.2.7, the second term on the RHS of (3.3.6) can be estimated as follows

$$
\begin{aligned}
& \int_{M \backslash \mathcal{L} \exp }\left(4 \pi \epsilon_{k} r_{k}^{2}\right)^{-\frac{n}{2}} \exp \left(-l\left(q, \epsilon_{k} r_{k}^{2}\right)\right) d V_{t_{k}-\epsilon_{k} r_{k}^{2}}(q) \\
\leq & \int_{\left\{|v|>\frac{1}{4} \epsilon_{k}^{-\frac{1}{2}}\right\}}\left(\epsilon_{k} r_{k}^{2}\right) \\
\left.=(4 \pi)^{-\frac{1}{2}}\right\} & \left.(4 \pi \tau)^{-\frac{n}{2}} \exp (-l(\tau)) \mathcal{J}(\tau)\right|_{\tau=0} d v \\
\leq & \exp \left(-|v|^{2}\right) d v \\
\leq \epsilon_{k}^{\frac{n}{2}} &
\end{aligned}
$$

for $k$ sufficiently large. Combining (3.3.6)-(3.3.8), we finish the proof of Step 1.

Step 2. We next want to show

$$
\tilde{V}_{k}\left(t_{k}\right)=\left(4 \pi t_{k}\right)^{-\frac{n}{2}} \int_{M} \exp \left(-l\left(q, t_{k}\right)\right) d V_{0}(q)>C^{\prime}
$$

for all $k$, where $C^{\prime}$ is some positive constant independent of $k$.

It suffices to show the Li-Yau-Perelman distance $l\left(\cdot, t_{k}\right)$ is uniformly bounded from above on $M$. By Corollary 3.2.6 we know that the minimum of $l(\cdot, \tau)$ does not exceed $\frac{n}{2}$ for each $\tau>0$. Choose $q_{k} \in M$ such that the minimum of $l\left(\cdot, t_{k}-\frac{T}{2}\right)$ is attained at $q_{k}$. We now construct a path $\gamma:\left[0, t_{k}\right] \rightarrow M$ connecting $p_{k}$ to any given point $q \in M$ as follows: the first half path $\left.\gamma\right|_{\left[0, t_{k}-\frac{T}{2}\right]}$ connects $p_{k}$ to $q_{k}$ so that

$$
l\left(q_{k}, t_{k}-\frac{T}{2}\right)=\frac{1}{2 \sqrt{t_{k}-\frac{T}{2}}} \int_{0}^{t_{k}-\frac{T}{2}} \sqrt{\tau}\left(R+|\dot{\gamma}(\tau)|^{2}\right) d \tau \leq \frac{n}{2}
$$

and the second half path $\left.\gamma\right|_{\left[t_{k}-\frac{T}{2}, t_{k}\right]}$ is a shortest geodesic connecting $q_{k}$ to $q$ with respect to the initial metric $g_{i j}(0)$. Then, for any $q \in M^{n}$,

$$
\begin{aligned}
l\left(q, t_{k}\right) & =\frac{1}{2 \sqrt{t_{k}}} L\left(q, t_{k}\right) \\
& \leq \frac{1}{2 \sqrt{t_{k}}}\left(\int_{0}^{t_{k}-\frac{T}{2}}+\int_{t_{k}-\frac{T}{2}}^{t_{k}}\right) \sqrt{\tau}\left(R+|\dot{\gamma}(\tau)|^{2}\right) d \tau \\
& \leq \frac{1}{2 \sqrt{t_{k}}}\left(n \sqrt{t_{k}-\frac{T}{2}}+\int_{t_{k}-\frac{T}{2}}^{t_{k}} \sqrt{\tau}\left(R+|\dot{\gamma}(\tau)|^{2}\right) d \tau\right) \\
& \leq C
\end{aligned}
$$

for some constant $C>0$, since all geometric quantities in $g_{i j}$ are uniformly bounded when $t \in\left[0, \frac{T}{2}\right]$ (or equivalently, $\tau \in\left[t_{k}-\frac{T}{2}, t_{k}\right]$ ).

Combining Step 1 with Step 2, and using the monotonicity of $\tilde{V}_{k}(\tau)$, we get

$$
C^{\prime}<\tilde{V}_{k}\left(t_{k}\right) \leq \tilde{V}_{k}\left(\epsilon_{k} r_{k}^{2}\right) \leq 2 \epsilon_{k}^{\frac{n}{2}} \rightarrow 0
$$


as $k \rightarrow \infty$. This gives the desired contradiction. Therefore we have proved the theorem. $\square$

The above no local collapsing theorem I says that if $|R m| \leq r^{-2}$ on the parabolic ball $\left\{(x, t) \mid d_{t_{0}}\left(x, x_{0}\right) \leq r, t_{0}-r^{2} \leq t \leq t_{0}\right\}$, then the volume of the geodesic ball $B_{t_{0}}\left(x_{0}, r\right)$ (with respect to the metric $g_{i j}\left(t_{0}\right)$ ) is bounded from below by $\kappa r^{n}$. In [103], Perelman used the monotonicity of the $\mathcal{W}$-functional (defined by (1.5.9)) to obtain a stronger version of the no local collapsing theorem, where the curvature bound assumption on the parabolic ball is replaced by that on the geodesic ball $B_{t_{0}}\left(x_{0}, r\right)$. The following result, called no local collapsing theorem $\mathbf{I}^{\prime}$, gives a further extension where the bound on the curvature tensor is replaced by the bound on the scalar curvature only. We now follow a clever argument by Bing-Long Chen.

Theorem 3.3.3 (No local collapsing theorem I'). Suppose $M$ is a compact Riemannian manifold, and $g_{i j}(t), 0 \leq t<T<+\infty$, is a solution to the Ricci flow. Then there exists a positive constant $\kappa$ depending only the initial metric and $T$ such that for any $\left(x_{0}, t_{0}\right) \in M \times(0, T)$ if

$$
R\left(x, t_{0}\right) \leq r^{-2}, \quad \forall x \in B_{t_{0}}\left(x_{0}, r\right)
$$

with $0<r \leq \sqrt{T}$, then we have

$$
\operatorname{Vol}_{t_{0}}\left(B_{t_{0}}\left(x_{0}, r\right)\right) \geq \kappa r^{n} .
$$

Proof. We will prove the assertion

$$
\operatorname{Vol}_{t_{0}}\left(B_{t_{0}}\left(x_{0}, a\right)\right) \geq \kappa a^{n}
$$

for all $0<a \leq r$. Recall that

$$
\mu\left(g_{i j}, \tau\right)=\inf \left\{\mathcal{W}\left(g_{i j}, f, \tau\right) \mid \int_{M}(4 \pi \tau)^{-\frac{n}{2}} e^{-f} d V=1\right\}
$$

Set

$$
\mu_{0}=\inf _{0 \leq \tau \leq 2 T} \mu\left(g_{i j}(0), \tau\right)>-\infty .
$$

By Corollary 1.5.9, we have

$$
\begin{aligned}
\mu\left(g_{i j}\left(t_{0}\right), b\right) & \geq \mu\left(g_{i j}(0), t_{0}+b\right) \\
& \geq \mu_{0}
\end{aligned}
$$

for $0<b \leq r^{2}$. Let $0<\zeta \leq 1$ be a positive smooth function on $\mathbb{R}$ where $\zeta(s)=1$ for $|s| \leq \frac{1}{2},\left|\zeta^{\prime}\right|^{2} / \zeta \leq 20$ everywhere, and $\zeta(s)$ is very close to zero for $|s| \geq 1$. Define a function $f$ on $M$ by

$$
\left(4 \pi r^{2}\right)^{-\frac{n}{2}} e^{-f(x)}=e^{-c}\left(4 \pi r^{2}\right)^{-\frac{n}{2}} \zeta\left(\frac{d_{t_{0}}\left(x, x_{0}\right)}{r}\right),
$$

where the constant $c$ is chosen so that $\int_{M}\left(4 \pi r^{2}\right)^{-\frac{n}{2}} e^{-f} d V_{t_{0}}=1$. Then it follows from (3.3.9) that

$$
\begin{aligned}
\mathcal{W}\left(g_{i j}\left(t_{0}\right), f, r^{2}\right) & =\int_{M}\left[r^{2}\left(|\nabla f|^{2}+R\right)+f-n\right]\left(4 \pi r^{2}\right)^{-\frac{n}{2}} e^{-f} d V_{t_{0}} \\
& \geq \mu_{0}
\end{aligned}
$$


Note that

$$
\begin{aligned}
1 & =\int_{M}\left(4 \pi r^{2}\right)^{-\frac{n}{2}} e^{-c} \zeta\left(\frac{d_{t_{0}}\left(x, x_{0}\right)}{r}\right) d V_{t_{0}} \\
& \geq \int_{B_{t_{0}}\left(x_{0}, \frac{r}{2}\right)}\left(4 \pi r^{2}\right)^{-\frac{n}{2}} e^{-c} d V_{t_{0}} \\
& =\left(4 \pi r^{2}\right)^{-\frac{n}{2}} e^{-c} \operatorname{Vol}_{t_{0}}\left(B_{t_{0}}\left(x_{0}, \frac{r}{2}\right)\right) .
\end{aligned}
$$

By combining with (3.3.10) and the scalar curvature bound, we have

$$
\begin{aligned}
c & \geq-\int_{M}\left(\frac{\left(\zeta^{\prime}\right)^{2}}{\zeta}-\log \zeta \cdot \zeta\right) e^{-c}\left(4 \pi r^{2}\right)^{-\frac{n}{2}} d V_{t_{0}}+(n-1)+\mu_{0} \\
& \geq-2\left(20+e^{-1}\right) e^{-c}\left(4 \pi r^{2}\right)^{-\frac{n}{2}} \operatorname{Vol}_{t_{0}}\left(B_{t_{0}}\left(x_{0}, r\right)\right)+(n-1)+\mu_{0} \\
& \geq-2\left(20+e^{-1}\right) \frac{\operatorname{Vol}_{t_{0}}\left(B_{t_{0}}\left(x_{0}, r\right)\right)}{\operatorname{Vol}_{t_{0}}\left(B_{t_{0}}\left(x_{0}, \frac{r}{2}\right)\right)}+(n-1)+\mu_{0},
\end{aligned}
$$

where we used the fact that $\zeta(s)$ is very close to zero for $|s| \geq 1$. Note also that

$$
2 \int_{B_{t_{0}}\left(x_{0}, r\right)} e^{-c}\left(4 \pi r^{2}\right)^{-\frac{n}{2}} d V_{t_{0}} \geq \int_{M}\left(4 \pi r^{2}\right)^{-\frac{n}{2}} e^{-f} d V_{t_{0}}=1
$$

Let us set

$$
\kappa=\min \left\{\frac{1}{2} \exp \left(-2\left(20+e^{-1}\right) 3^{-n}+(n-1)+\mu_{0}\right), \frac{1}{2} \alpha_{n}\right\}
$$

where $\alpha_{n}$ is the volume of the unit ball in $\mathbb{R}^{n}$. Then we obtain

$$
\begin{aligned}
\operatorname{Vol}_{t_{0}}\left(B_{t_{0}}\left(x_{0}, r\right)\right) & \geq \frac{1}{2} e^{c}\left(4 \pi r^{2}\right)^{\frac{n}{2}} \\
& \geq \frac{1}{2}(4 \pi)^{\frac{n}{2}} \exp \left(-2\left(20+e^{-1}\right) 3^{-n}+(n-1)+\mu_{0}\right) \cdot r^{n} \\
& \geq \kappa r^{n}
\end{aligned}
$$

provided $\operatorname{Vol}_{t_{0}}\left(B_{t_{0}}\left(x_{0}, \frac{r}{2}\right)\right) \geq 3^{-n} \operatorname{Vol}_{t_{0}}\left(B_{t_{0}}\left(x_{0}, r\right)\right)$.

Note that the above argument also works for any smaller radius $a \leq r$. Thus we have proved the following assertion:

$$
\operatorname{Vol}_{t_{0}}\left(B_{t_{0}}\left(x_{0}, a\right)\right) \geq \kappa a^{n}
$$

whenever $a \in(0, r]$ and $\operatorname{Vol}_{t_{0}}\left(B_{t_{0}}\left(x_{0}, \frac{a}{2}\right)\right) \geq 3^{-n} \operatorname{Vol}_{t_{0}}\left(B_{t_{0}}\left(x_{0}, a\right)\right)$.

Now we argue by contradiction to prove the assertion $(*)_{a}$ for any $a \in(0, r]$. Suppose $(*)_{a}$ fails for some $a \in(0, r]$. Then by (3.3.11) we have

$$
\begin{aligned}
\operatorname{Vol}_{t_{0}}\left(B_{t_{0}}\left(x_{0}, \frac{a}{2}\right)\right) & <3^{-n} \operatorname{Vol}_{t_{0}}\left(B_{t_{0}}\left(x_{0}, a\right)\right) \\
& <3^{-n} \kappa a^{n} \\
& <\kappa\left(\frac{a}{2}\right)^{n}
\end{aligned}
$$

This says that $(*) \frac{a}{2}$ would also fail. By induction, we deduce that

$$
\operatorname{Vol}_{t_{0}}\left(B_{t_{0}}\left(x_{0}, \frac{a}{2^{k}}\right)\right)<\kappa\left(\frac{a}{2^{k}}\right)^{n} \quad \text { for all } k \geq 1 .
$$

This is a contradiction since $\lim _{k \rightarrow \infty} \operatorname{Vol}_{t_{0}}\left(B_{t_{0}}\left(x_{0}, \frac{a}{2^{k}}\right)\right) /\left(\frac{a}{2^{k}}\right)^{n}=\alpha_{n}$. $\mathbf{\square}$ 
3.4. No Local Collapsing Theorem II. By inspecting the arguments in the previous section, one can see that if the injectivity radius of the initial metric is uniformly bounded from below, then the no local collapsing theorem I also holds for complete solutions with bounded curvature on a complete noncompact manifold. In this section we will use a cut-off argument to extend the no local collapsing theorem to any complete solution with bounded curvature. In some sense, the second no local collapsing theorem gives a good relative estimate of the volume element for the Ricci flow.

We first need the following useful lemma which contains two assertions. The first one is a parabolic version of the Laplacian comparison theorem (where the curvature sign restriction in the ordinary Laplacian comparison is essentially removed in the Ricci flow). The second one is a generalization of a result of Hamilton (Theorem 17.2 in [63]), where it was derived by an integral version of Bonnet-Myers' theorem.

Lemma 3.4.1 (Perelman [103]). Let $g_{i j}(x, t)$ be a solution to the Ricci flow on an $n$-dimensional manifold $M$ and denote by $d_{t}\left(x, x_{0}\right)$ the distance between $x$ and $x_{0}$ with respect to the metric $g_{i j}(t)$.

(i) Suppose $\operatorname{Ric}\left(\cdot, t_{0}\right) \leq(n-1) K$ on $B_{t_{0}}\left(x_{0}, r_{0}\right)$ for some $x_{0} \in M$ and some positive constants $K$ and $r_{0}$. Then the distance function $d(x, t)=d_{t}\left(x, x_{0}\right)$ satisfies, at $t=t_{0}$ and outside $B_{t_{0}}\left(x_{0}, r_{0}\right)$, the differential inequality:

$$
\frac{\partial}{\partial t} d-\Delta d \geq-(n-1)\left(\frac{2}{3} K r_{0}+r_{0}^{-1}\right) .
$$

(ii) Suppose Ric $\left(\cdot, t_{0}\right) \leq(n-1) K$ on $B_{t_{0}}\left(x_{0}, r_{0}\right) \cup B_{t_{0}}\left(x_{1}, r_{0}\right)$ for some $x_{0}, x_{1} \in$ $M$ and some positive constants $K$ and $r_{0}$. Then, at $t=t_{0}$,

$$
\frac{d}{d t} d_{t}\left(x_{0}, x_{1}\right) \geq-2(n-1)\left(\frac{2}{3} K r_{0}+r_{0}^{-1}\right) \text {. }
$$

Proof. Let $\gamma:\left[0, d\left(x, t_{0}\right)\right] \rightarrow M$ be a shortest normal geodesic from $x_{0}$ to $x$ with respect to the metric $g_{i j}\left(t_{0}\right)$. As usual, we may assume that $x$ and $x_{0}$ are not conjugate to each other in the metric $g_{i j}\left(t_{0}\right)$, otherwise we can understand the differential inequality in the barrier sense. Let $X=\dot{\gamma}(0)$ and let $\left\{X, e_{1}, \ldots, e_{n-1}\right\}$ be an orthonormal basis of $T_{x_{0}} M$. Extend this basis parallel along $\gamma$ to form a parallel orthonormal basis $\left\{X(s), e_{1}(s), \ldots, e_{n-1}(s)\right\}$ along $\gamma$.

(i) Let $X_{i}(s), i=1, \ldots, n-1$, be the Jacobian fields along $\gamma$ such that $X_{i}(0)=0$ and $X_{i}\left(d\left(x, t_{0}\right)\right)=e_{i}\left(d\left(x, t_{0}\right)\right)$ for $i=1, \ldots, n-1$. Then it is well-known that (see for example [112])

$$
\Delta d_{t_{0}}\left(x, x_{0}\right)=\sum_{i=1}^{n-1} \int_{0}^{d\left(x, t_{0}\right)}\left(\left|\dot{X}_{i}\right|^{2}-R\left(X, X_{i}, X, X_{i}\right)\right) d s
$$

(in Proposition 3.2.3 we actually did this for the more complicated $\mathcal{L}$-distance function).

Define vector fields $Y_{i}, i=1, \ldots, n-1$, along $\gamma$ as follows:

$$
Y_{i}(s)= \begin{cases}\frac{s}{r_{0}} e_{i}(s), & \text { if } s \in\left[0, r_{0}\right], \\ e_{i}(s), & \text { if } s \in\left[r_{0}, d\left(x, t_{0}\right)\right] .\end{cases}
$$


which have the same value as the corresponding Jacobian fields $X_{i}(s)$ at the two end points of $\gamma$. Then by using the standard index comparison theorem (see for example [22]) we have

$$
\begin{aligned}
\Delta d_{t_{0}}\left(x, x_{0}\right) & =\sum_{i=1}^{n-1} \int_{0}^{d\left(x, t_{0}\right)}\left(\left|\dot{X}_{i}\right|^{2}-R\left(X, X_{i}, X, X_{i}\right)\right) d s \\
& \leq \sum_{i=1}^{n-1} \int_{0}^{d\left(x, t_{0}\right)}\left(\left|\dot{Y}_{i}\right|^{2}-R\left(X, Y_{i}, X, Y_{i}\right)\right) d s \\
& =\int_{0}^{r_{0}} \frac{1}{r_{0}^{2}}\left(n-1-s^{2} \operatorname{Ric}(X, X)\right) d s+\int_{r_{0}}^{d\left(x, t_{0}\right)}(-\operatorname{Ric}(X, X)) d s \\
& =-\int_{\gamma} \operatorname{Ric}(X, X)+\int_{0}^{r_{0}}\left(\frac{(n-1)}{r_{0}^{2}}+\left(1-\frac{s^{2}}{r_{0}^{2}}\right) \operatorname{Ric}(X, X)\right) d s \\
& \leq-\int_{\gamma} \operatorname{Ric}(X, X)+(n-1)\left(\frac{2}{3} K r_{0}+r_{0}^{-1}\right) .
\end{aligned}
$$

On the other hand,

$$
\begin{aligned}
\frac{\partial}{\partial t} d_{t}\left(x, x_{0}\right) & =\frac{\partial}{\partial t} \int_{0}^{d\left(x, t_{0}\right)} \sqrt{g_{i j} X^{i} X^{j}} d s \\
& =-\int_{\gamma} \operatorname{Ric}(X, X) d s .
\end{aligned}
$$

Hence we obtain the desired differential inequality.

(ii) The proof is divided into three cases.

Case (1): $d_{t_{0}}\left(x_{0}, x_{1}\right) \geq 2 r_{0}$.

Define vector fields $Y_{i}, i=1, \ldots, n-1$, along $\gamma$ as follows:

$$
Y_{i}(s)= \begin{cases}\frac{s}{r_{0}} e_{i}(s), & \text { if } s \in\left[0, r_{0}\right], \\ e_{i}(s), & \text { if } s \in\left[r_{0}, d\left(x_{1}, t_{0}\right)\right], \\ \frac{d\left(x_{1}, t_{0}\right)-s}{r_{0}} e_{i}(s), & \text { if } s \in\left[d\left(x_{1}, t_{0}\right)-r_{0}, d\left(x_{1}, t_{0}\right)\right] .\end{cases}
$$

Then by the second variation formula, we have

$$
\sum_{i=1}^{n-1} \int_{0}^{d\left(x_{1}, t_{0}\right)} R\left(X, Y_{i}, X, Y_{i}\right) d s \leq \sum_{i=1}^{n-1} \int_{0}^{d\left(x_{1}, t_{0}\right)}\left|\dot{Y}_{i}\right|^{2} d s
$$

which implies

$$
\begin{aligned}
& \int_{0}^{r_{0}} \frac{s^{2}}{r_{0}^{2}} \operatorname{Ric}(X, X) d s+\int_{r_{0}}^{d\left(x, t_{0}\right)-r_{0}} \operatorname{Ric}(X, X) d s \\
& \quad+\int_{d\left(x_{1}, t_{0}\right)-r_{0}}^{d\left(x_{1}, t_{0}\right)}\left(\frac{d\left(x_{1}, t_{0}\right)-s}{r_{0}}\right)^{2} \operatorname{Ric}(X, X) d s \leq \frac{2(n-1)}{r_{0}} .
\end{aligned}
$$


Thus

$$
\begin{aligned}
\frac{d}{d t} & \left(d_{t}\left(x_{0}, x_{1}\right)\right) \\
\geq & -\int_{0}^{r_{0}}\left(1-\frac{s^{2}}{r_{0}^{2}}\right) \operatorname{Ric}(X, X) d s \\
& -\int_{d\left(x_{1}, t_{0}\right)-r_{0}}^{d\left(x_{1}, t_{0}\right)}\left(1-\left(\frac{d\left(x_{1}, t_{0}\right)-s}{r_{0}}\right)^{2}\right) \operatorname{Ric}(X, X) d s-\frac{2(n-1)}{r_{0}} \\
\geq & -2(n-1)\left(\frac{2}{3} K r_{0}+r_{0}^{-1}\right) .
\end{aligned}
$$

Case (2): $\frac{2}{\sqrt{\frac{2 K}{3}}} \leq d_{t_{0}}\left(x_{0}, x_{1}\right) \leq 2 r_{0}$. get

In this case, letting $r_{1}=\frac{1}{\sqrt{\frac{2 K}{3}}}$ and applying case (1) with $r_{0}$ replaced by $r_{1}$, we

$$
\begin{aligned}
\frac{d}{d t}\left(d_{t}\left(x_{0}, x_{1}\right)\right) & \geq-2(n-1)\left(\frac{2}{3} K r_{1}+r_{1}^{-1}\right) \\
& \geq-2(n-1)\left(\frac{2}{3} K r_{0}+r_{0}^{-1}\right)
\end{aligned}
$$

Case (3): $d_{t_{0}}\left(x_{0}, x_{1}\right) \leq \min \left\{\frac{2}{\sqrt{\frac{2 K}{3}}}, 2 r_{0}\right\}$.

In this case,

$$
\int_{0}^{d\left(x_{1}, t_{0}\right)} \operatorname{Ric}(X, X) d s \leq(n-1) K \frac{2}{\sqrt{\frac{2 K}{3}}}=(n-1) \sqrt{6 K},
$$

and

$$
2(n-1)\left(\frac{2}{3} K r_{0}+r_{0}^{-1}\right) \geq(n-1) \sqrt{\frac{32}{3} K} .
$$

This proves the lemma.

The following result, called the no local collapsing theorem II, was obtained by Perelman in [103].

Theorem 3.4.2 (No local collapsing theorem II). For any $A>0$ there exists $\kappa=\kappa(A)>0$ with the following property: if $g_{i j}(t)$ is a complete solution to the Ricci flow on $0 \leq t \leq r_{0}^{2}$ with bounded curvature and satifying

$$
|R m|(x, t) \leq r_{0}^{-2} \quad \text { on } B_{0}\left(x_{0}, r_{0}\right) \times\left[0, r_{0}^{2}\right]
$$

and

$$
\operatorname{Vol}_{0}\left(B_{0}\left(x_{0}, r_{0}\right)\right) \geq A^{-1} r_{0}^{n},
$$

then $g_{i j}(t)$ is $\kappa$-noncollapsed on all scales less than $r_{0}$ at every point $\left(x, r_{0}^{2}\right)$ with $d_{r_{0}^{2}}\left(x, x_{0}\right) \leq A r_{0}$. 
Proof. From the evolution equation of the Ricci flow, we know that the metrics $g_{i j}(\cdot, t)$ are equivalent to each other on $B_{0}\left(x_{0}, r_{0}\right) \times\left[0, r_{0}^{2}\right]$. Thus, without loss of generality, we may assume that the curvature of the solution is uniformly bounded for all $t \in\left[0, r_{0}^{2}\right]$ and all points in $B_{t}\left(x_{0}, r_{0}\right)$. Fix a point $\left(x, r_{0}^{2}\right) \in M \times\left\{r_{0}^{2}\right\}$. By scaling we may assume $r_{0}=1$. We may also assume $d_{1}\left(x, x_{0}\right)=A$. Let $p=x$, $\bar{\tau}=1-t$, and consider Perelman's reduced volume

$$
\tilde{V}(\bar{\tau})=\int_{M}(4 \pi \bar{\tau})^{-\frac{n}{2}} \exp (-l(q, \bar{\tau})) d V_{1-\bar{\tau}}(q),
$$

where

$$
\begin{aligned}
l(q, \bar{\tau})=\inf \left\{\frac{1}{2 \sqrt{\bar{\tau}}} \int_{0}^{\bar{\tau}} \sqrt{\tau}\left(R+|\dot{\gamma}|^{2}\right) d \tau \mid \gamma:[0, \bar{\tau}] \rightarrow\right. & \\
& \text { with } \gamma(0)=p, \gamma(\bar{\tau})=q\}
\end{aligned}
$$

is the Li-Yau-Perelman distance. We argue by contradiction. Suppose for some $0<$ $r<1$ we have

$$
|R m|(y, t) \leq r^{-2}
$$

whenever $y \in B_{1}(x, r)$ and $1-r^{2} \leq t \leq 1$, but $\epsilon=r^{-1} \operatorname{Vol}_{1}\left(B_{1}(x, r)\right)^{\frac{1}{n}}$ is very small. Then arguing as in the proof of the no local collapsing theorem I (Theorem 3.3.2), we see that Perelman's reduced volume

$$
\tilde{V}\left(\epsilon r^{2}\right) \leq 2 \epsilon^{\frac{n}{2}} .
$$

On the other hand, from the monotonicity of Perelman's reduced volume we have

$$
(4 \pi)^{-\frac{n}{2}} \int_{M} \exp (-l(q, 1)) d V_{0}(q)=\tilde{V}(1) \leq \tilde{V}\left(\epsilon r^{2}\right) .
$$

Thus once we bound the function $l(q, 1)$ over $B_{0}\left(x_{0}, 1\right)$ from above, we will get the desired contradiction and will prove the theorem.

For any $q \in B_{0}\left(x_{0}, 1\right)$, exactly as in the proof of the no local collapsing theorem I, we choose a path $\gamma:[0,1] \rightarrow M$ with $\gamma(0)=x, \gamma(1)=q, \gamma\left(\frac{1}{2}\right)=y \in B_{\frac{1}{2}}\left(x_{0}, \frac{1}{10}\right)$ and $\gamma(\tau) \in B_{1-\tau}\left(x_{0}, 1\right)$ for $\tau \in\left[\frac{1}{2}, 1\right]$ such that

$$
\mathcal{L}\left(\left.\gamma\right|_{\left[0, \frac{1}{2}\right]}\right)=2 \sqrt{\frac{1}{2}} l\left(y, \frac{1}{2}\right) \quad\left(=L\left(y, \frac{1}{2}\right)\right) .
$$

Now $\mathcal{L}\left(\left.\gamma\right|_{\left[\frac{1}{2}, 1\right]}\right)=\int_{\frac{1}{2}}^{1} \sqrt{\tau}\left(R(\gamma(\tau), 1-\tau)+|\dot{\gamma}(\tau)|_{g_{i j}(1-\tau)}^{2}\right) d \tau$ is bounded from above by a uniform constant since all geometric quantities in $g_{i j}$ are uniformly bounded on $\left\{(y, t) \mid t \in[0,1 / 2], y \in B_{t}\left(x_{0}, 1\right)\right\}$ (where $t \in[0,1 / 2]$ is equivalent to $\tau \in[1 / 2,1]$ ). Thus all we need is to estimate the minimum of $l\left(\cdot, \frac{1}{2}\right)$, or equivalently $\bar{L}\left(\cdot, \frac{1}{2}\right)=$ $4 \frac{1}{2} l\left(\cdot, \frac{1}{2}\right)$, in the ball $B_{\frac{1}{2}}\left(x_{0}, \frac{1}{10}\right)$.

Recall that $\bar{L}$ satisfies the differential inequality

$$
\frac{\partial \bar{L}}{\partial \tau}+\Delta \bar{L} \leq 2 n .
$$


We will use this in a maximum principle argument. Let us define

$$
h(y, t)=\phi(d(y, t)-A(2 t-1)) \cdot(\bar{L}(y, 1-t)+2 n+1)
$$

where $d(y, t)=d_{t}\left(y, x_{0}\right)$, and $\phi$ is a function of one variable, equal to 1 on $\left(-\infty, \frac{1}{20}\right)$, and rapidly increasing to infinity on $\left(\frac{1}{20}, \frac{1}{10}\right)$ in such a way that:

$$
2 \frac{\left(\phi^{\prime}\right)^{2}}{\phi}-\phi^{\prime \prime} \geq(2 A+100 n) \phi^{\prime}-C(A) \phi
$$

for some constant $C(A)<+\infty$. The existence of such a function $\phi$ can be justified as follows: put $v=\frac{\phi^{\prime}}{\phi}$, then the condition (3.4.2) for $\phi$ can be written as

$$
3 v^{2}-v^{\prime} \geq(2 A+100 n) v-C(A)
$$

which can be solved for $v$.

Since the scalar curvature $R$ evolves by

$$
\frac{\partial R}{\partial t}=\Delta R+2|R c|^{2} \geq \Delta R+\frac{2}{n} R^{2}
$$

we can apply the maximum principle as in Chapter 2 to deduce

$$
R(x, t) \geq-\frac{n}{2 t} \text { for } t \in(0,1] \text { and } x \in M .
$$

Thus for $\bar{\tau}=1-t \in\left[0, \frac{1}{2}\right]$,

$$
\begin{aligned}
\bar{L}(\cdot, \bar{\tau}) & =2 \sqrt{\bar{\tau}} \int_{0}^{\bar{\tau}} \sqrt{\tau}\left(R+|\dot{\gamma}|^{2}\right) d \tau \\
& \geq 2 \sqrt{\bar{\tau}} \int_{0}^{\bar{\tau}} \sqrt{\tau}\left(-\frac{n}{2(1-\tau)}\right) d \tau \\
& \geq 2 \sqrt{\bar{\tau}} \int_{0}^{\bar{\tau}} \sqrt{\tau}(-n) d \tau \\
& >-2 n
\end{aligned}
$$

That is

$$
\bar{L}(\cdot, 1-t)+2 n+1 \geq 1, \text { for } t \in\left[\frac{1}{2}, 1\right]
$$

Clearly $\min _{y \in M} h\left(y, \frac{1}{2}\right)$ is achieved by some $y \in B_{\frac{1}{2}}\left(x_{0}, \frac{1}{10}\right)$ and

$$
\min _{y \in M} h(y, 1) \leq h(x, 1)=2 n+1 .
$$


We compute

$$
\begin{aligned}
\left(\frac{\partial}{\partial t}-\Delta\right) h= & \left(\frac{\partial}{\partial t}-\Delta\right) \phi \cdot(\bar{L}(y, 1-t)+2 n+1) \\
& +\phi \cdot\left(\frac{\partial}{\partial t}-\Delta\right) \bar{L}(y, 1-t)-2\langle\nabla \phi, \nabla \bar{L}(y, 1-t)\rangle \\
= & \left(\phi^{\prime}\left[\left(\frac{\partial}{\partial t}-\Delta\right) d-2 A\right]-\phi^{\prime \prime}|\nabla d|^{2}\right) \cdot(\bar{L}+2 n+1) \\
& +\phi \cdot\left(-\frac{\partial}{\partial \tau}-\Delta\right) \bar{L}-2\langle\nabla \phi, \nabla \bar{L}\rangle \\
\geq & \left(\phi^{\prime}\left[\left(\frac{\partial}{\partial t}-\Delta\right) d-2 A\right]-\phi^{\prime \prime}\right) \cdot(\bar{L}+2 n+1) \\
& -2 n \phi-2\langle\nabla \phi, \nabla \bar{L}\rangle
\end{aligned}
$$

by using (3.4.1). At a minimizing point of $h$ we have

$$
\frac{\nabla \phi}{\phi}=-\frac{\nabla \bar{L}}{(\bar{L}+2 n+1)} \text {. }
$$

Hence

$$
-2\langle\nabla \phi, \nabla \bar{L}\rangle=2 \frac{|\nabla \phi|^{2}}{\phi}(\bar{L}+2 n+1)=2 \frac{\left(\phi^{\prime}\right)^{2}}{\phi}(\bar{L}+2 n+1) .
$$

Then at the minimizing point of $h$, we compute

$$
\begin{aligned}
\left(\frac{\partial}{\partial t}-\Delta\right) h \geq & \left(\phi^{\prime}\left[\left(\frac{\partial}{\partial t}-\Delta\right) d-2 A\right]-\phi^{\prime \prime}\right) \cdot(\bar{L}+2 n+1) \\
& -2 n \phi+2 \frac{\left(\phi^{\prime}\right)^{2}}{\phi}(\bar{L}+2 n+1) \\
\geq & \left(\phi^{\prime}\left[\left(\frac{\partial}{\partial t}-\Delta\right) d-2 A\right]-\phi^{\prime \prime}\right) \cdot(\bar{L}+2 n+1) \\
& -2 n h+2 \frac{\left(\phi^{\prime}\right)^{2}}{\phi}(\bar{L}+2 n+1)
\end{aligned}
$$

for $t \in\left[\frac{1}{2}, 1\right]$ and

$$
\Delta h \geq 0 .
$$

Let us denote by $h_{\min }(t)=\min _{y \in M} h(y, t)$. By applying Lemma 3.4.1(i) to the set where $\phi^{\prime} \neq 0$, we further obtain

$$
\begin{aligned}
\frac{d}{d t} h_{\min } & \geq(\bar{L}+2 n+1) \cdot\left[\phi^{\prime}(-100 n-2 A)-\phi^{\prime \prime}+2 \frac{\left(\phi^{\prime}\right)^{2}}{\phi}\right]-2 n h_{\min } \\
& \geq-(2 n+C(A)) h_{\min }, \quad \text { for } t \in\left[\frac{1}{2}, 1\right] .
\end{aligned}
$$

This implies that $h_{\min }(t)$ cannot decrease too fast. By combining (3.4.3) and (3.4.4) we get the required estimate for the minimum $\bar{L}\left(\cdot, \frac{1}{2}\right)$ in the ball $B_{\frac{1}{2}}\left(x_{0}, \frac{1}{10}\right)$.

Therefore we have completed the proof of the theorem. $\square$ 
4. Formation of Singularities. Let $g_{i j}(x, t)$ be a solution to the Ricci flow on $M \times[0, T)$ and suppose $[0, T), T \leq \infty$, is the maximal time interval. If $T<+\infty$, then the short time existence theorem tells us the curvature of the solution becomes unbounded as $t \rightarrow T$. We then say the solution develops a singularity as $t \rightarrow T$. As in the minimal surface theory and harmonic map theory, one usually tries to understand the structure of a singularity of the Ricci flow by rescaling the solution (or blow up) to obtain a sequence of solutions to the Ricci flow with uniformly bounded curvature on compact subsets and looking at its limit.

The main purpose of this chapter is to establish a convergence theorem for a sequence of solutions to the Ricci flow with uniform bounded curvature on compact subsets and to use the convergence theorem to give a rough classification for singularities of solutions to the Ricci flow. Further studies on the structures of singularities of the Ricci flow will be given in Chapter 6 and 7 .

4.1. Cheeger Type Compactness. We begin with the concept of $C_{l o c}^{\infty}$ convergence of tensors on a given manifold $M$. Let $T_{i}$ be a sequence of tensors on $M$. We say that $\boldsymbol{T}_{\boldsymbol{i}}$ converges to a tensor $\boldsymbol{T}$ in the $C_{\text {loc }}^{\infty}$ topology if we can find a covering $\left\{\left(U_{s}, \varphi_{s}\right)\right\}, \varphi_{s}: U_{s} \rightarrow \mathbb{R}^{n}$, of $C^{\infty}$ coordinate charts so that for every compact set $K \subset M$, the components of $T_{i}$ converge in the $C^{\infty}$ topology to the components of $T$ in the intersections of $K$ with these coordinate charts, considered as functions on $\varphi_{s}\left(U_{s}\right) \subset \mathbb{R}^{n}$. Consider a Riemannian manifold $(M, g)$. A marking on $M$ is a choice of a point $p \in M$ which we call the origin. We will refer to such a triple $(M, g, p)$ as a marked Riemannian manifold.

Definition 4.1.1. Let $\left(M_{k}, g_{k}, p_{k}\right)$ be a sequence of marked complete Riemannian manifolds, with metrics $g_{k}$ and marked points $p_{k} \in M_{k}$. Let $B\left(p_{k}, s_{k}\right) \subset M_{k}$ denote the geodesic ball centered at $p_{k} \in M_{k}$ and of radius $s_{k}\left(0<s_{k} \leq+\infty\right)$. We say a sequence of marked geodesic balls $\left(B\left(p_{k}, s_{k}\right), g_{k}, p_{k}\right)$ with $s_{k} \rightarrow s_{\infty}(\leq+\infty)$ converges in the $C_{\text {loc }}^{\infty}$ topology to a marked (maybe noncomplete) manifold $\left(B_{\infty}, g_{\infty}, p_{\infty}\right)$, which is an open geodesic ball centered at $p_{\infty} \in B_{\infty}$ and of radius $s_{\infty}$ with respect to the metric $g_{\infty}$, if we can find a sequence of exhausting open sets $U_{k}$ in $B_{\infty}$ containing $p_{\infty}$ and a sequence of diffeomorphisms $f_{k}$ of the sets $U_{k}$ in $B_{\infty}$ to open sets $V_{k}$ in $B\left(p_{k}, s_{k}\right) \subset M_{k}$ mapping $p_{\infty}$ to $p_{k}$ such that the pull-back metrics $\tilde{g}_{k}=\left(f_{k}\right)^{*} g_{k}$ converge in $C^{\infty}$ topology to $g_{\infty}$ on every compact subset of $B_{\infty}$.

We remark that this concept of $C_{\mathrm{loc}}^{\infty}$-convergence of a sequence of marked manifolds $\left(M_{k}, g_{k}, p_{k}\right)$ is not the same as that of $C_{\mathrm{loc}}^{\infty}$-convergence of metric tensors on a given manifold, even when we are considering the sequence of Riemannian metric $g_{k}$ on the same space $M$. This is because one can have a sequence of diffeomorphisms $f_{k}: M \rightarrow M$ such that $\left(f_{k}\right)^{*} g_{k}$ converges in $C_{\text {loc }}^{\infty}$ topology while $g_{k}$ itself does not converge.

There have been a lot of work in Riemannian geometry on the convergence of a sequence of compact manifolds with bounded curvature, diameter and injectivity radius (see for example Gromov [53], Peters [106], and Greene and $\mathrm{Wu}$ [51]). The following theorem, which is a slight generalization of Hamilton's convergence theorem [62], modifies these results in three aspects: the first one is to allow noncompact limits and then to avoid any diameter bound; the second one is to avoid having to assume a uniform lower bound for the injectivity radius over the whole manifold, a hypothesis which is much harder to satisfy in applications; the last one is to avoid a uniform curvature bound over the whole manifold so that we can take a local limit.

TheOrem 4.1.2 (Hamilton [62]). Let $\left(M_{k}, g_{k}, p_{k}\right)$ be a sequence of marked 
complete Riemannian manifolds of dimension $n$. Consider a sequence of geodesic balls $B\left(p_{k}, s_{k}\right) \subset M_{k}$ of radius $s_{k}\left(0<s_{k} \leq \infty\right)$, with $s_{k} \rightarrow s_{\infty}(\leq \infty)$, around the base point $p_{k}$ of $M_{k}$ in the metric $g_{k}$. Suppose

(a) for every radius $r<s_{\infty}$ and every integer $l \geq 0$ there exists a constant $B_{l, r}$, independent of $k$, and positive integer $k(r, l)<+\infty$ such that as $k \geq$ $k(r, l)$, the curvature tensors $\operatorname{Rm}\left(g_{k}\right)$ of the metrics $g_{k}$ and their $l^{\text {th }}$-covariant derivatives satisfy the bounds

$$
\left|\nabla^{l} R m\left(g_{k}\right)\right| \leq B_{l, r}
$$

on the balls $B\left(p_{k}, r\right)$ of radius $r$ around $p_{k}$ in the metrics $g_{k}$; and

(b) there exists a constant $\delta>0$ independent of $k$ such that the injectivity radii $\operatorname{inj}\left(M_{k}, p_{k}\right)$ of $M_{k}$ at $p_{k}$ in the metric $g_{k}$ satisfy the bound

$$
\operatorname{inj}\left(M_{k}, p_{k}\right) \geq \delta .
$$

Then there exists a subsequence of the marked geodesic balls $\left(B\left(p_{k}, s_{k}\right), g_{k}, p_{k}\right) \quad$ which converges to a marked geodesic ball $\left(B\left(p_{\infty}, s_{\infty}\right), g_{\infty}, p_{\infty}\right)$ in $C_{\mathrm{loc}}^{\infty}$ topology. Moreover the limit is complete if $s_{\infty}=+\infty$.

Proof. In [62] and Theorem 16.1 of [63], Hamilton proved this convergence theorem for the case $s_{\infty}=+\infty$. In the following we only need to modify Hamilton's argument to prove the remaining case of $s_{\infty}<+\infty$. Suppose we are given a sequence of geodesic balls $\left(B\left(p_{k}, s_{k}\right), g_{k}, p_{k}\right) \subset\left(M_{k}, g_{k}, p_{k}\right)$, with $s_{k} \rightarrow s_{\infty}(<+\infty)$, satisfying the assumptions of Theorem 4.1.2. We will split the proof into three steps.

Step 1: Picking the subsequence.

By the local injectivity radius estimate (4.2.2) in Corollary 4.2 .3 of the next section, we can find a positive decreasing $C^{1}$ function $\rho(r), 0 \leq r<s_{\infty}$, independent of $k$ such that

$$
\begin{aligned}
\rho(r) & <\frac{1}{100}\left(s_{\infty}-r\right), \\
0 & \geq \rho^{\prime}(r) \geq-\frac{1}{1000},
\end{aligned}
$$

and a sequence of positive constants $\varepsilon_{k} \rightarrow 0$ so that the injectivity radius at any point $x \in B\left(p_{k}, s_{k}\right)$ with $r_{k}=d_{k}\left(x, p_{k}\right)<s_{\infty}-\varepsilon_{k}$ is bounded from below by

$$
\operatorname{inj}\left(M_{k}, x\right) \geq 500 \rho\left(r_{k}(x)\right),
$$

where $r_{k}(x)=d_{k}\left(x, p_{k}\right)$ is the distance from $x$ to $p_{k}$ in the metric $g_{k}$ of $M_{k}$. We define

$$
\tilde{\rho}(r)=\rho(r+20 \rho(r)), \quad \tilde{\tilde{\rho}}(r)=\tilde{\rho}(r+20 \tilde{\rho}(r)) .
$$

By (4.1.2) we know that both $\tilde{\rho}(r)$ and $\tilde{\tilde{\rho}}(r)$ are nonincreasing positive functions on $\left[0, s_{\infty}\right)$.

In each $B\left(p_{k}, s_{\infty}\right)$ we choose inductively a sequence of points $x_{k}^{\alpha}$ for $\alpha=0,1,2, \ldots$ in the following way. First we let $x_{k}^{0}=p_{k}$. Once $x_{k}^{\alpha}$ are chosen for $\alpha=0,1,2, \ldots, \sigma$, we pick $x_{k}^{\sigma+1}$ closest to $p_{k}$ so that $r_{k}^{\sigma+1}=r_{k}\left(x_{k}^{\sigma+1}\right)$ is as small as possible, subject to the requirement that the open ball $B\left(x_{k}^{\sigma+1}, \tilde{\tilde{\rho}}_{k}^{\sigma+1}\right)$ around $x_{k}^{\sigma+1}$ of radius $\tilde{\tilde{\rho}}_{k}^{\sigma+1}$ is disjoint 
from the balls $B\left(x_{k}^{\alpha}, \tilde{\tilde{\rho}}_{k}^{\alpha}\right)$ for $\alpha=0,1,2, \ldots, \sigma$, where $\tilde{\tilde{\rho}}_{k}^{\alpha}=\tilde{\tilde{\rho}}\left(r_{k}^{\alpha}\right)$ and $r_{k}^{\alpha}=r_{k}\left(x_{k}^{\alpha}\right)$. In particular, the open balls $B\left(x_{k}^{\alpha}, \tilde{\tilde{\rho}}_{k}^{\alpha}\right), \alpha=0,1,2, \ldots$, are all disjoint. We claim the balls $B\left(x_{k}^{\alpha}, 2 \tilde{\tilde{\rho}}_{k}^{\alpha}\right)$ cover $B\left(p_{k}, s_{\infty}-\varepsilon_{k}\right)$ and moreover for any $r, 0<r<s_{\infty}-\varepsilon_{k}$, we can find $\lambda(r)$ independent of $k$ such that for $k$ large enough, the geodesic balls $B\left(x_{k}^{\alpha}, 2 \tilde{\tilde{\rho}}_{k}^{\alpha}\right)$ for $\alpha \leq \lambda(r)$ cover the ball $B\left(p_{k}, r\right)$.

To see this, let $x \in B\left(p_{k}, s_{\infty}-\varepsilon_{k}\right)$ and let $r(x)$ be the distance from $x$ to $p_{k}$ and let $\tilde{\tilde{\rho}}=\tilde{\tilde{\rho}}(r(x))$. Consider those $\alpha$ with $r_{k}^{\alpha} \leq r(x)<s_{\infty}-\varepsilon_{k}$. Then

$$
\tilde{\tilde{\rho}} \leq \tilde{\tilde{\rho}}_{k}^{\alpha} \text {. }
$$

Now the given point $x$ must lie in one of the balls $B\left(x_{k}^{\alpha}, 2 \tilde{\tilde{\rho}}_{k}^{\alpha}\right)$. If not, we could choose the next point in the sequence of $x_{k}^{\beta}$ to be $x$ instead, for since $\tilde{\tilde{\rho}}_{k}^{\alpha}+\tilde{\tilde{\rho}} \leq 2 \tilde{\tilde{\rho}}_{k}^{\alpha}$ the ball $B(x, \tilde{\tilde{\rho}})$ would miss $B\left(x_{k}^{\alpha}, \tilde{\tilde{\rho}}_{k}^{\alpha}\right)$ with $r_{k}^{\alpha} \leq r(x)$. But this is a contradiction. Moreover for any $r, 0<r<s_{\infty}-\varepsilon_{k}$, using the curvature bound and the injectivity radius bound, each ball $B\left(x_{k}^{\alpha}, \tilde{\tilde{\rho}}_{k}^{\alpha}\right)$ with $r_{k}^{\alpha} \leq r$ has volume at least $\epsilon(r) \tilde{\tilde{\rho}}^{n}$ where $\epsilon(r)>0$ is some constant depending on $r$ but independent of $k$. Now these balls are all disjoint and contained in the ball $B\left(p_{k},\left(r+s_{\infty}\right) / 2\right)$. On the other hand, for large enough $k$, we can estimate the volume of this ball from above, again using the curvature bound, by a positive function of $r$ that is independent of $k$. Thus there is a $k^{\prime}(r)>0$ such that for each $k \geq k^{\prime}(r)$, there holds

$$
\#\left\{\alpha \mid r_{k}^{\alpha} \leq r\right\} \leq \lambda(r)
$$

for some positive constant $\lambda(r)$ depending only on $r$, and the geodesic balls $B\left(x_{k}^{\alpha}, 2 \tilde{\tilde{\rho}}_{k}^{\alpha}\right)$ for $\alpha \leq \lambda(r)$ cover the ball $B\left(p_{k}, r\right)$.

By the way, since

$$
\begin{aligned}
r_{k}^{\alpha} & \leq r_{k}^{\alpha-1}+\tilde{\tilde{\rho}}_{k}^{\alpha-1}+\tilde{\tilde{\rho}}_{k}^{\alpha} \\
& \leq r_{k}^{\alpha-1}+2 \tilde{\tilde{\rho}}_{k}^{\alpha-1}
\end{aligned}
$$

and by (4.1.1)

$$
\tilde{\tilde{\rho}}_{k}^{\alpha-1} \leq \frac{1}{100}\left(s_{\infty}-r_{k}^{\alpha-1}\right)
$$

we get by induction

$$
\begin{aligned}
r_{k}^{\alpha} & \leq \frac{49}{50} r_{k}^{\alpha-1}+\frac{1}{50} s_{\infty} \\
& \leq\left(\frac{49}{50}\right)^{\alpha} r_{k}^{0}+\frac{1}{50}\left(1+\frac{49}{50}+\cdots+\left(\frac{49}{50}\right)^{\alpha-1}\right) s_{\infty} \\
& =\left(1-\left(\frac{49}{50}\right)^{\alpha}\right) s_{\infty} .
\end{aligned}
$$

So for each $\alpha$, with $\alpha \leq \lambda(r)\left(r<s_{\infty}\right)$, there holds

$$
r_{k}^{\alpha} \leq\left(1-\left(\frac{49}{50}\right)^{\lambda(r)}\right) s_{\infty}
$$

for all $k$. And by passing to a subsequence (using a diagonalization argument) we may assume that $r_{k}^{\alpha}$ converges to some $r^{\alpha}$ for each $\alpha$. Then $\tilde{\tilde{\rho}}_{k}^{\alpha}$ (respectively $\tilde{\rho}_{k}^{\alpha}, \rho_{k}^{\alpha}$ ) converges to $\tilde{\tilde{\rho}}^{\alpha}=\tilde{\rho}\left(r^{\alpha}\right)$ (respectively $\tilde{\rho}^{\alpha}=\tilde{\rho}\left(r^{\alpha}\right), \rho^{\alpha}=\rho\left(r^{\alpha}\right)$ ). 
Hence for all $\alpha$ we can find $k(\alpha)$ such that

$$
\begin{gathered}
\frac{1}{2} \tilde{\tilde{\rho}}^{\alpha} \leq \tilde{\tilde{\rho}}_{k}^{\alpha} \leq 2 \tilde{\tilde{\rho}}^{\alpha} \\
\frac{1}{2} \tilde{\rho}^{\alpha} \leq \tilde{\rho}_{k}^{\alpha} \leq 2 \tilde{\rho}^{\alpha} \text { and } \frac{1}{2} \rho^{\alpha} \leq \rho_{k}^{\alpha} \leq 2 \rho^{\alpha}
\end{gathered}
$$

whenever $k \geq k(\alpha)$. Thus for all $\alpha, \tilde{\tilde{\rho}}_{k}^{\alpha}$ and $\tilde{\tilde{\rho}}^{\alpha}$ are comparable when $k$ is large enough so we can work with balls of a uniform size, and the same is true for $\tilde{\rho}_{k}^{\alpha}$ and $\tilde{\rho}^{\alpha}$, and $\rho_{k}^{\alpha}$ and $\rho^{\alpha}$. Let $\hat{B}_{k}^{\alpha}=B\left(x_{k}^{\alpha}, 4 \tilde{\tilde{\rho}}^{\alpha}\right)$, then $\tilde{\tilde{\rho}}_{k}^{\alpha} \leq 2 \tilde{\tilde{\rho}}^{\alpha}$ and $B\left(x_{k}^{\alpha}, 2 \tilde{\tilde{\rho}}_{k}^{\alpha}\right) \subset B\left(x_{k}^{\alpha}, 4 \tilde{\tilde{\rho}}^{\alpha}\right)=\hat{B}_{k}^{\alpha}$. So for every $r$ if we let $k(r)=\max \{k(\alpha) \mid \alpha \leq \lambda(r)\}$ then when $k \geq k(r)$, the balls $\hat{B}_{k}^{\alpha}$ for $\alpha \leq \lambda(r)$ cover the ball $B\left(p_{k}, r\right)$ as well. Suppose that $\hat{B}_{k}^{\alpha}$ and $\hat{B}_{k}^{\beta}$ meet for $k \geq k(\alpha)$ and $k \geq k(\beta)$, and suppose $r_{k}^{\beta} \leq r_{k}^{\alpha}$. Then, by the triangle inequality, we must have

$$
r_{k}^{\alpha} \leq r_{k}^{\beta}+4 \tilde{\tilde{\rho}}^{\alpha}+4 \tilde{\tilde{\rho}}^{\beta} \leq r_{k}^{\beta}+8 \tilde{\tilde{\rho}}^{\beta}<r_{k}^{\beta}+16 \tilde{\rho}_{k}^{\beta} .
$$

This then implies

$$
\tilde{\tilde{\rho}}_{k}^{\beta}=\tilde{\tilde{\rho}}\left(r_{k}^{\beta}\right)=\tilde{\rho}\left(r_{k}^{\beta}+20 \tilde{\rho}\left(r_{k}^{\beta}\right)\right)<\tilde{\rho}\left(r_{k}^{\alpha}\right)=\tilde{\rho}_{k}^{\alpha}
$$

and hence

$$
\tilde{\tilde{\rho}}^{\beta} \leq 4 \tilde{\rho}^{\alpha}
$$

Therefore $\hat{B}_{k}^{\beta} \subset B\left(x_{k}^{\alpha}, 36 \tilde{\rho}^{\alpha}\right)$ whenever $\hat{B}_{k}^{\alpha}$ and $\hat{B}_{k}^{\beta}$ meet and $k \geq \max \{k(\alpha), k(\beta)\}$.

Next we define the balls $B_{k}^{\alpha}=B\left(x_{k}^{\alpha}, 5 \tilde{\tilde{\rho}}^{\alpha}\right)$ and $\tilde{B}_{k}^{\alpha}=B\left(x_{k}^{\alpha}, \tilde{\tilde{\rho}}^{\alpha} / 2\right)$. Note that $\tilde{B}_{k}^{\alpha}$ are disjoint since $\tilde{B}_{k}^{\alpha} \subset B\left(x_{k}^{\alpha}, \tilde{\tilde{\rho}}_{k}^{\alpha}\right)$. Since $\hat{B}_{k}^{\alpha} \subset B_{k}^{\alpha}$, the balls $B_{k}^{\alpha}$ cover $B\left(p_{k}, r\right)$ for $\alpha \leq \lambda(r)$ as before. If $B_{k}^{\alpha}$ and $B_{k}^{\beta}$ meet for $k \geq k(\alpha)$ and $k \geq k(\beta)$ and $r_{k}^{\beta} \leq r_{k}^{\alpha}$, then by the triangle inequality we get

$$
r_{k}^{\alpha} \leq r_{k}^{\beta}+10 \tilde{\tilde{\rho}}^{\beta}<r_{k}^{\beta}+20 \tilde{\rho}_{k}^{\beta},
$$

and hence

$$
\tilde{\tilde{\rho}}^{\beta} \leq 4 \tilde{\rho}^{\alpha}
$$

again. Similarly,

$$
\tilde{\rho}_{k}^{\beta}=\tilde{\rho}\left(r_{k}^{\beta}\right)=\rho\left(r_{k}^{\beta}+20 \rho\left(r_{k}^{\beta}\right)\right)<\rho\left(r_{k}^{\alpha}\right)=\rho_{k}^{\alpha} .
$$

This makes

$$
\tilde{\rho}^{\beta} \leq 4 \rho^{\alpha} .
$$

Now any point in $B_{k}^{\beta}$ has distance at most

$$
5 \tilde{\tilde{\rho}}^{\alpha}+5 \tilde{\tilde{\rho}}^{\beta}+5 \tilde{\tilde{\rho}}^{\beta} \leq 45 \tilde{\rho}^{\alpha}
$$

from $x_{k}^{\alpha}$, so $B_{k}^{\beta} \subset B\left(x_{k}^{\alpha}, 45 \tilde{\rho}^{\alpha}\right)$. Likewise, whenever $B_{k}^{\alpha}$ and $B_{k}^{\beta}$ meet for $k \geq k(\alpha)$ and $k \geq k(\beta)$, any point in the larger ball $B\left(x_{k}^{\beta}, 45 \tilde{\rho}^{\beta}\right)$ has distance at most

$$
5 \tilde{\tilde{\rho}}^{\alpha}+5 \tilde{\tilde{\rho}}^{\beta}+45 \tilde{\rho}^{\beta} \leq 205 \rho^{\alpha}
$$


from $x_{k}^{\alpha}$ and hence $B\left(x_{k}^{\beta}, 45 \tilde{\rho}^{\beta}\right) \subset B\left(x_{k}^{\alpha}, 205 \rho^{\alpha}\right)$. Now we define $\bar{B}_{k}^{\alpha}=B\left(x_{k}^{\alpha}, 45 \tilde{\rho}^{\alpha}\right)$ and $\overline{\bar{B}}_{k}^{\alpha}=B\left(x_{k}^{\alpha}, 205 \rho^{\alpha}\right)$. Then the above discussion says that whenever $B_{k}^{\alpha}$ and $B_{k}^{\beta}$ meet for $k \geq k(\alpha)$ and $k \geq k(\beta)$, we have

$$
B_{k}^{\beta} \subset \bar{B}_{k}^{\alpha} \quad \text { and } \quad \bar{B}_{k}^{\beta} \subset \overline{\bar{B}}_{k}^{\alpha} .
$$

Note that $\overline{\bar{B}}_{k}^{\alpha}$ is still a nice embedded ball since, by (4.1.3), $205 \rho^{\alpha} \leq 410 \rho_{k}^{\alpha}<$ $\operatorname{inj}\left(M_{k}, x_{k}^{\alpha}\right)$.

We claim there exist positive numbers $N(r)$ and $k^{\prime \prime}(r)$ such that for any given $\alpha$ with $r^{\alpha}<r$, as $k \geq k^{\prime \prime}(r)$, there holds

$$
\#\left\{\beta \mid B_{k}^{\alpha} \cap B_{k}^{\beta} \neq \phi\right\} \leq N(r) .
$$

Indeed, if $B_{k}^{\alpha}$ meets $B_{k}^{\beta}$ then there is a positive $k^{\prime \prime}(\alpha)$ such that as $k \geq k^{\prime \prime}(\alpha)$,

$$
\begin{aligned}
r_{k}^{\beta} & \leq r_{k}^{\alpha}+10 \tilde{\tilde{\rho}}_{k}^{\alpha} \\
& \leq r+20 \rho(r) \\
& \leq r+\frac{1}{5}\left(s_{\infty}-r\right),
\end{aligned}
$$

where we used (4.1.2) in the third inequality. Set

$$
k^{\prime \prime}(r)=\max \left\{k^{\prime \prime}(\alpha), k^{\prime}(r) \mid \alpha \leq \lambda(r)\right\}
$$

and

$$
N(r)=\lambda\left(r+\frac{1}{5}\left(s_{\infty}-r\right)\right) .
$$

Then by combining with (4.1.4), these give the desired estimate (4.1.7)

Next we observe that by passing to another subsequence we can guarantee that for any pair $\alpha$ and $\beta$ we can find a number $k(\alpha, \beta)$ such that if $k \geq k(\alpha, \beta)$ then either $B_{k}^{\alpha}$ always meets $B_{k}^{\beta}$ or it never does.

Hence by setting

$$
\begin{aligned}
& \bar{k}(r)=\max \left\{k(\alpha, \beta), k(\alpha), k(\beta), k^{\prime \prime}(r) \mid \alpha \leq \lambda(r)\right. \text { and } \\
&\left.\qquad \quad \beta \leq \lambda\left(r+\frac{1}{5}\left(s_{\infty}-r\right)\right)\right\},
\end{aligned}
$$

we have shown the following results: for every $r<s_{\infty}$, if $k \geq \bar{k}(r)$, we have

(i) the ball $B\left(p_{k}, r\right)$ in $M_{k}$ is covered by the balls $B_{k}^{\alpha}$ for $\alpha \leq \lambda(r)$,

(ii) whenever $B_{k}^{\alpha}$ and $B_{k}^{\beta}$ meet for $\alpha \leq \lambda(r)$, we have

$$
B_{k}^{\beta} \subset \bar{B}_{k}^{\alpha} \quad \text { and } \quad \bar{B}_{k}^{\beta} \subset \overline{\bar{B}}_{k}^{\alpha}
$$

(iii) for each $\alpha \leq \lambda(r)$, there no more than $N(r)$ balls ever meet $B_{k}^{\alpha}$, and

(iv) for any $\alpha \leq \lambda(r)$ and any $\beta$, either $B_{k}^{\alpha}$ meets $B_{k}^{\beta}$ for all $k \geq \bar{k}(r)$ or none for all $k \geq \bar{k}(r)$. 
Now we let $\hat{E}^{\alpha}, E^{\alpha}, \bar{E}^{\alpha}$, and $\overline{\bar{E}}^{\alpha}$ be the balls of radii $4 \tilde{\tilde{\rho}}^{\alpha}, 5 \tilde{\tilde{\rho}}^{\alpha}, 45 \tilde{\rho}^{\alpha}$, and $205 \rho^{\alpha}$ around the origin in Euclidean space $\mathbb{R}^{n}$. At each point $x_{k}^{\alpha} \in B\left(p_{k}, s_{k}\right)$ we define coordinate charts $H_{k}^{\alpha}: E^{\alpha} \rightarrow B_{k}^{\alpha}$ as the composition of a linear isometry of $\mathbb{R}^{n}$ to the tangent space $T_{x_{k}^{\alpha}} M_{k}$ with the exponential map $\exp _{x_{k}^{\alpha}}$ at $x_{k}^{\alpha}$. We also get maps $\bar{H}_{k}^{\alpha}: \bar{E}^{\alpha} \rightarrow \bar{B}_{k}^{\alpha}$ and $\overline{\bar{H}}_{k}^{\alpha}: \overline{\bar{E}}^{\alpha} \rightarrow \overline{\bar{B}}_{k}^{\alpha}$ in the same way. Note that (4.1.3) implies that these maps are all well defined. We denote by $g_{k}^{\alpha}$ (and $\bar{g}_{k}^{\alpha}$ and $\overline{\bar{g}}_{k}^{\alpha}$ ) the pull-backs of the metric $g_{k}$ by $H_{k}^{\alpha}$ (and $\bar{H}_{k}^{\alpha}$ and $\overline{\bar{H}}_{k}^{\alpha}$ ). We also consider the coordinate transition functions $J_{k}^{\alpha \beta}: E^{\beta} \rightarrow \bar{E}^{\alpha}$ and $\bar{J}_{k}^{\alpha \beta}: \bar{E}^{\beta} \rightarrow \bar{E}^{\alpha}$ defined by

$$
J_{k}^{\alpha \beta}=\left(\bar{H}_{k}^{\alpha}\right)^{-1} H_{k}^{\beta} \quad \text { and } \quad \bar{J}_{k}^{\alpha \beta}=\left(\overline{\bar{H}}_{k}^{\alpha}\right)^{-1} \bar{H}_{k}^{\beta}
$$

Clearly $\bar{J}_{k}^{\alpha \beta} J_{k}^{\beta \alpha}=I$. Moreover $J_{k}^{\alpha \beta}$ is an isometry from $g_{k}^{\beta}$ to $\bar{g}_{k}^{\alpha}$ and $\bar{J}_{k}^{\alpha \beta}$ from $\bar{g}_{k}^{\beta}$ to $\overline{\bar{g}}_{k}^{\alpha}$.

Now for each fixed $\alpha$, the metrics $g_{k}^{\alpha}$ are in geodesic coordinates and have their curvatures and their covariant derivatives uniformly bounded.

Claim 1. By passing to another subsequence we can guarantee that for each $\alpha$ (and indeed all $\alpha$ by diagonalization) the metrics $g_{k}^{\alpha}$ (or $\bar{g}_{k}^{\alpha}$ or $\overline{\bar{g}}_{k}^{\alpha}$ ) converge uniformly with their derivatives to a smooth metric $g^{\alpha}$ (or $\bar{g}^{\alpha}$ or $\left.\overline{\bar{g}}^{\alpha}\right)$ on $E^{\alpha}$ (or $\bar{E}^{\alpha}$ or $\overline{\bar{E}}^{\alpha}$ ) which is also in geodesic coordinates.

Look now at any pair $\alpha, \beta$ for which the balls $B_{k}^{\alpha}$ and $B_{k}^{\beta}$ always meet for large $k$, and thus the maps $J_{k}^{\alpha \beta}$ (and $\bar{J}_{k}^{\alpha \beta}$ and $J_{k}^{\beta \alpha}$ and $\bar{J}_{k}^{\beta \alpha}$ ) are always defined for large $k$.

Claim 2. The isometries $J_{k}^{\alpha \beta}$ (and $\bar{J}_{k}^{\alpha \beta}$ and $J_{k}^{\beta \alpha}$ and $\bar{J}_{k}^{\beta \alpha}$ ) always have a convergent subsequence.

So by passing to another subsequence we may assume $J_{k}^{\alpha \beta} \rightarrow J^{\alpha \beta}$ (and $\bar{J}_{k}^{\alpha \beta} \rightarrow$ $\bar{J}^{\alpha \beta}$ and $J_{k}^{\beta \alpha} \rightarrow J^{\beta \alpha}$ and $\left.\bar{J}_{k}^{\beta \alpha} \rightarrow \bar{J}^{\beta \alpha}\right)$. The limit maps $J^{\alpha \beta}: E^{\beta} \rightarrow \bar{E}^{\alpha}$ and $\bar{J}^{\alpha \beta}: \bar{E}^{\beta} \rightarrow \bar{E}^{\alpha}$ are isometries in the limit metrics $g^{\beta}$ and $g^{\alpha}$. Moreover

$$
J^{\alpha \beta} \bar{J}^{\beta \alpha}=I
$$

We are now done picking subsequences, except we still owe the reader the proofs of Claim 1 and Claim 2.

Step 2: Finding local diffeomorphisms which are approximate isometries.

Take the subsequence $\left(B\left(p_{k}, s_{k}\right), g_{k}, p_{k}\right)$ chosen in Step 1 above. We claim that for every $r<s_{\infty}$ and every $\left(\epsilon_{1}, \epsilon_{2}, \ldots, \epsilon_{p}\right)$, and for all $k$ and $l$ sufficiently large in comparison, we can find a diffeomorphism $F_{k l}$ of a neighborhood of the ball $B\left(p_{k}, r\right) \subset$ $B\left(p_{k}, s_{k}\right)$ into an open set in $B\left(p_{l}, s_{l}\right)$ which is an $\left(\epsilon_{1}, \epsilon_{2}, \ldots, \epsilon_{p}\right)$ approximate isometry in the sense that

$$
{ }^{t} \nabla F_{k l} \nabla F_{k l}-I \mid<\epsilon_{1}
$$

and

$$
\left|\nabla^{2} F_{k l}\right|<\epsilon_{2}, \ldots,\left|\nabla^{p} F_{k l}\right|<\epsilon_{p}
$$

where $\nabla^{p} F_{k l}$ is the $p^{t h}$ covariant derivative of $F_{k l}$.

The idea (following Peters [106] or Greene and $\mathrm{Wu}$ [51]) of proving the claim is to define the $\operatorname{map} F_{k \ell}^{\alpha}=H_{l}^{\alpha} \circ\left(H_{k}^{\alpha}\right)^{-1}$ (or $\bar{F}_{k \ell}^{\alpha}=\bar{H}_{l}^{\alpha} \circ\left(\bar{H}_{k}^{\alpha}\right)^{-1}$, resp.) from $B_{k}^{\alpha}$ to $B_{\ell}^{\alpha}$ 
(or $\bar{B}_{k}^{\alpha}$ to $\bar{B}_{\ell}^{\alpha}$, resp.) for $k$ and $\ell$ large compared to $\alpha$ so as to be the identity map on $E^{\alpha}$ (or $\bar{E}^{\alpha}$, resp.) in the coordinate charts $H_{k}^{\alpha}$ and $H_{\ell}^{\alpha}$ (or $\bar{H}_{k}^{\alpha}$ and $\bar{H}_{\ell}^{\alpha}$, resp.), and then to define $F_{k \ell}$ on a neighborhood of $B\left(p_{k}, r\right)$ for $k, \ell \geq \bar{k}(r)$ be averaging the maps $\bar{F}_{k \ell}^{\beta}$ for $\beta \leq \lambda\left(r+\frac{1}{5}\left(s_{\infty}-r\right)\right)$. To describe the averaging process on $B_{k}^{\alpha}$ with $\alpha \leq \lambda(r)$ we only need to consider those $B_{k}^{\beta}$ which meet $B_{k}^{\alpha}$; there are never more than $N(r)$ of them and each $\beta \leq \lambda\left(r+\frac{1}{5}\left(s_{\infty}-r\right)\right)$, and they are the same for $k$ and $\ell$ when $k, \ell \geq \bar{k}(r)$. The averaging process is defined by taking $F_{k \ell}(x)$ to be the center of mass of the $\bar{F}_{k \ell}^{\beta}(x)$ for $x \in B_{k}^{\alpha}$ averaging over those $\beta$ where $B_{k}^{\beta}$ meets $B_{k}^{\alpha}$ using weights $\mu_{k}^{\beta}(x)$ defined by a partition of unity. The center of mass of the points $y^{\beta}=F_{k \ell}^{\beta}(x)$ with weights $\mu^{\beta}$ is defined to be the point $y$ such that

$$
\exp _{y} V^{\beta}=y^{\beta} \quad \text { and } \quad \sum \mu^{\beta} V^{\beta}=0 .
$$

When the points $y^{\beta}$ are all close and the weights $\mu^{\beta}$ satisfy $0 \leq \mu^{\beta} \leq 1$ then there will be a unique solution $y$ close to $y^{\beta}$ which depends smoothly on the $y^{\beta}$ and the $\mu^{\beta}$ (see [51] for the details). The point $y$ is found by the inverse function theorem, which also provides bounds on all the derivatives of $y$ as a function of the $y^{\beta}$ and the $\mu^{\beta}$.

Since $B_{k}^{\alpha} \subseteq \bar{B}_{k}^{\beta}$ and $\bar{B}_{\ell}^{\beta} \subseteq \bar{B}_{\ell}^{\alpha}$, the map $\bar{F}_{k \ell}^{\beta}=\bar{H}_{l}^{\beta} \circ\left(\bar{H}_{k}^{\beta}\right)^{-1}$ can be represented in local coordinates by the map

$$
P_{k \ell}^{\alpha \beta}: E^{\alpha} \rightarrow \overline{\bar{E}}^{\alpha}
$$

defined by

$$
P_{k \ell}^{\alpha \beta}=\bar{J}_{\ell}^{\alpha \beta} \circ J_{k}^{\beta \alpha} .
$$

Since $J_{k}^{\beta \alpha} \rightarrow J^{\beta \alpha}$ as $k \rightarrow \infty$ and $\bar{J}_{\ell}^{\alpha \beta} \rightarrow \bar{J}^{\alpha \beta}$ as $\ell \rightarrow \infty$ and $\bar{J}^{\alpha \beta} \circ J^{\beta \alpha}=I$, we see that the maps $P_{k \ell}^{\alpha \beta} \rightarrow I$ as $k, \ell \rightarrow \infty$ for each choice of $\alpha$ and $\beta$. The weights $\mu_{k}^{\beta}$ are defined in the following way. We pick for each $\beta$ a smooth function $\psi^{\beta}$ which equals 1 on $\hat{E}^{\beta}$ and equals 0 outside $E^{\beta}$. We then transfer $\psi^{\beta}$ to a function $\psi_{k}^{\beta}$ on $M_{k}$ by the coordinate map $\overline{\bar{H}}_{k}^{\beta}$ (i.e. $\left.\psi_{k}^{\beta}=\psi^{\beta} \circ\left(\overline{\bar{H}}_{k}^{\beta}\right)^{-1}\right)$. Then let

$$
\mu_{k}^{\beta}=\psi_{k}^{\beta} / \sum_{\gamma} \psi_{k}^{\gamma}
$$

as usual. In the coordinate chart $E^{\alpha}$ the function $\psi_{k}^{\beta}$ looks like the composition of $J_{k}^{\beta \alpha}$ with $\psi^{\beta}$. Call this function

$$
\psi_{k}^{\alpha \beta}=\psi^{\beta} \circ J_{k}^{\beta \alpha} .
$$

Then as $k \rightarrow \infty, \psi_{k}^{\alpha \beta} \rightarrow \psi^{\alpha \beta}$ where

$$
\psi^{\alpha \beta}=\psi^{\beta} \circ J^{\beta \alpha} .
$$

In the coordinate chart $E^{\alpha}$ the function $\mu_{k}^{\beta}$ looks like

$$
\mu_{k}^{\alpha \beta}=\psi_{k}^{\alpha \beta} / \sum_{\gamma} \psi_{k}^{\alpha \gamma}
$$

and $\mu_{k}^{\alpha \beta} \rightarrow \mu^{\alpha \beta}$ as $k \rightarrow \infty$ where

$$
\mu^{\alpha \beta}=\psi^{\alpha \beta} / \sum_{\gamma} \psi^{\alpha \gamma} .
$$


Since the sets $\hat{B}_{k}^{\alpha}$ cover $B\left(p_{k}, r\right)$, it follows that $\sum_{\gamma} \psi_{k}^{\gamma} \geq 1$ on this set and by combining with (4.1.5) and (4.1.7) there is no problem bounding all these functions and their derivatives. There is a small problem in that we want to guarantee that the averaged map still takes $p_{k}$ to $p_{\ell}$. This is true at least for the map $F_{k \ell}^{0}$. Therefore it will suffice to guarantee that $\mu_{k}^{\alpha}=0$ in a neighborhood of $p_{k}$ if $\alpha \neq 0$. This happens if the same is true for $\psi_{k}^{\alpha}$. If not, we can always replace $\psi_{k}^{\alpha}$ by $\tilde{\psi}_{k}^{\alpha}=\left(1-\psi_{k}^{0}\right) \psi_{k}^{\alpha}$ which still leaves $\tilde{\psi}_{k}^{\alpha} \geq \frac{1}{2} \psi_{k}^{\alpha}$ or $\psi_{k}^{0} \geq \frac{1}{2}$ everywhere, and this is sufficient to make $\sum_{\gamma} \tilde{\psi}_{k}^{\gamma} \geq \frac{1}{2}$ everywhere.

Now in the local coordinate $E^{\alpha}$ we are averaging maps $P_{k \ell}^{\alpha \beta}$ which converge to the identity with respect to weights $\mu_{k}^{\alpha \beta}$ which converge. It follows that the averaged map converges to the identity in these coordinates. Thus $F_{k \ell}$ can be made to be an $\left(\epsilon_{1}, \epsilon_{2}, \ldots, \epsilon_{p}\right)$ approximate isometry on $B\left(p_{k}, r\right)$ when $k$ and $\ell$ are suitably large. At least the estimates

$$
{ }^{t} \nabla F_{k \ell} \cdot \nabla F_{k \ell}-I \mid<\epsilon_{1}
$$

and $\left|\nabla^{2} F_{k \ell}\right|<\epsilon_{2}, \ldots,\left|\nabla^{p} F_{k \ell}\right|<\epsilon_{p}$ on $B\left(p_{k}, r\right)$ follow from the local coordinates. We still need to check that $F_{k \ell}$ is a diffeomorphism on a neighborhood of $B\left(p_{k}, r\right)$.

This, however, follows quickly enough from the fact that we also get a map $F_{\ell k}$ on a slightly larger ball $B\left(p_{\ell}, r^{\prime}\right)$ which contains the image of $F_{k \ell}$ on $B\left(p_{k}, r\right)$ if we take $r^{\prime}=\left(1+\epsilon_{1}\right) r$, and $F_{\ell k}$ also satisfies the above estimates. Also $F_{k \ell}$ and $F_{\ell k}$ fix the markings, so the composition $F_{\ell k} \circ F_{k \ell}$ satisfies the same sort of estimates and fixes the origin $p_{k}$.

Since the maps $P_{k \ell}^{\alpha \beta}$ and $P_{\ell k}^{\alpha \beta}$ converge to the identity as $k, \ell$ tend to infinity, $F_{\ell k} \circ F_{k \ell}$ must be very close to the identity on $B\left(p_{k}, r\right)$. It follows that $F_{k \ell}$ is invertible. This finishes the proof of the claim and the Step 2.

Step 3: Constructing the limit geodesic ball $\left(B_{\infty}, g_{\infty}, p_{\infty}\right)$.

We now know the geodesic balls $\left(B\left(p_{k}, s_{k}\right), g_{k}, p_{k}\right)$ are nearly isometric for large $k$. We are now going to construct the limit $B_{\infty}$. For a sequence of positive numbers $r_{j} \nearrow s_{\infty}$ with each $r_{j}<s_{j}$, we choose the numbers $\left(\epsilon_{1}\left(r_{j}\right), \ldots, \epsilon_{j}\left(r_{j}\right)\right)$ so small that when we choose $k\left(r_{j}\right)$ large in comparison and find the maps $F_{k\left(r_{j}\right), k\left(r_{j+1}\right)}$ constructed above on neighborhoods of $B\left(p_{k\left(r_{j}\right)}, r_{j}\right)$, in $M_{k\left(r_{j}\right)}$ into $M_{k\left(r_{j+1}\right)}$ the image always lies in $B\left(p_{k\left(r_{j+1}\right)}, r_{j+1}\right)$ and the composition of $F_{k\left(r_{j}\right), k\left(r_{j+1}\right)}$ with $F_{k\left(r_{j+1}\right), k\left(r_{j+2}\right)}$ and $\cdots$ and $F_{k\left(r_{s-1}\right), k\left(r_{s}\right)}$ for any $s>j$ is still an $\left(\eta_{1}\left(r_{j}\right), \ldots, \eta_{j}\left(r_{j}\right)\right)$ isometry for any choice of $\eta_{i}\left(r_{j}\right)$, say $\eta_{i}\left(r_{j}\right)=1 / j$ for $1 \leq i \leq j$. Now we simplify the notation by writing $M_{j}$ in place of $M_{k\left(r_{j}\right)}$ and $F_{j}$ in place of $F_{k\left(r_{j}\right), k\left(r_{j+1}\right)}$. Then

$$
F_{j}: B\left(p_{j}, r_{j}\right) \rightarrow B\left(p_{j+1}, r_{j+1}\right)
$$

is a diffeomorphism map from $B\left(p_{j}, r_{j}\right)$ into $B\left(p_{j+1}, r_{j+1}\right)$, and the composition

$$
F_{s-1} \circ \cdots \circ F_{j}: B\left(p_{j}, j\right) \rightarrow B\left(p_{s}, s\right)
$$

is always an $\left(\eta_{1}\left(r_{j}\right), \ldots, \eta_{j}\left(r_{j}\right)\right)$ approximate isometry.

We now construct the limit $B_{\infty}$ as a topological space by identifying the balls $B\left(p_{j}, r_{j}\right)$ with each other using the homeomorphisms $F_{j}$. Given any two points $x$ and $y$ in $B_{\infty}$, we have $x \in B\left(p_{j}, r_{j}\right)$ and $y \in B\left(p_{s}, r_{s}\right)$ for some $j$ and $s$. If $j \leq s$ then $x \in B\left(p_{s}, r_{s}\right)$ also, by identification. A set in $B_{\infty}$ is open if and only if it intersects each $B\left(p_{j}, r_{j}\right)$ in an open set. Then choosing disjoint neighborhoods of $x$ and $y$ in 
$B\left(p_{s}, r_{s}\right)$ gives disjoint neighborhoods of $x$ and $y$ in $B_{\infty}$. Thus $B_{\infty}$ is a Hausdorff space.

Any smooth chart on $B\left(p_{j}, r_{j}\right)$ also gives a smooth chart on $B\left(p_{s}, r_{s}\right)$ for all $s>j$. The union of all such charts gives a smooth atlas on $B_{\infty}$. It is fairly easy to see the metrics $g_{j}$ on $B\left(p_{j}, r_{j}\right)$, converge to a smooth metric $g_{\infty}$ on $B_{\infty}$ uniformly together with all derivatives on compact sets. For since the $F_{s-1} \circ \cdots \circ F_{j}$ are very good approximate isometries, the $g_{j}$ are very close to each other, and hence form a Cauchy sequence (together with their derivatives, in the sense that the covariant derivatives of $g_{j}$ with respect to $g_{s}$ are very small when $j$ and $s$ are both large). One checks in the usual way that such a Cauchy sequence converges.

The origins $p_{j}$ are identified with each other, and hence with an origin $p_{\infty}$ in $B_{\infty}$. Now it is the inverses of the maps identifying $B\left(p_{j}, r_{j}\right)$ with open subsets of $B_{\infty}$ that provide the diffeomorphisms of (relatively compact) open sets in $B_{\infty}$ into the geodesic balls $B\left(p_{j}, s_{j}\right) \subset M_{j}$ such that the pull-backs of the metrics $g_{j}$ converge to $g_{\infty}$. This completes the proof of Step 3.

Now it remains to prove both Claim 1 and Claim 2 in Step 1.

Proof of Claim 1. It suffices to show the following general result:

There exists a constant $c>0$ depending only on the dimension, and constants $C_{q}$ depending only on the dimension and $q$ and bounds $B_{j}$ on the curvature and its derivatives for $j \leq q$ where $\left|D^{j} R m\right| \leq B_{j}$, so that for any metric $g_{k \ell}$ in geodesic coordinates in the ball $|x| \leq r \leq c / \sqrt{B_{0}}$, we have

$$
\frac{1}{2} I_{k \ell} \leq g_{k \ell} \leq 2 I_{k \ell}
$$

and

$$
\left|\frac{\partial}{\partial x^{j_{1}}} \cdots \frac{\partial}{\partial x^{j_{q}}} g_{k \ell}\right| \leq C_{q}
$$

where $I_{k \ell}$ is the Euclidean metric.

Suppose we are given a metric $g_{i j}(x) d x^{i} d x^{j}$ in geodesic coordinates in the ball $|x| \leq r \leq c / \sqrt{B_{0}}$ as in Claim 1 . Then by definition every line through the origin is a geodesic (parametrized proportional to arc length) and $g_{i j}=I_{i j}$ at the origin. Also, the Gauss Lemma says that the metric $g_{i j}$ is in geodesic coordinates if and only if $g_{i j} x^{i}=I_{i j} x^{i}$. Note in particular that in geodesic coordinates

$$
|x|^{2}=g_{i j} x^{i} x^{j}=I_{i j} x^{i} x^{j}
$$

is unambiguously defined. Also, in geodesic coordinates we have $\Gamma_{i j}^{k}(0)=0$, and all the first derivatives for $g_{j k}$ vanish at the origin.

Introduce the symmetric tensor

$$
A_{i j}=\frac{1}{2} x^{k} \frac{\partial}{\partial x^{k}} g_{i j}
$$

Since we have $g_{j k} x^{k}=I_{j k} x^{k}$, we get

$$
x^{k} \frac{\partial}{\partial x^{i}} g_{j k}=I_{i j}-g_{i j}=x^{k} \frac{\partial}{\partial x^{j}} g_{i k}
$$


and hence from the formula for $\Gamma_{j k}^{i}$

$$
x^{j} \Gamma_{j k}^{i}=g^{i \ell} A_{k \ell} .
$$

Hence $A_{k \ell} x^{k}=0$. Let $D_{i}$ be the covariant derivative with respect to the metric $g_{i j}$. Then

$$
D_{i} x^{k}=I_{i}^{k}+\Gamma_{i j}^{k} x^{j}=I_{i}^{k}+g^{k \ell} A_{i \ell} .
$$

Introduce the potential function

$$
P=|x|^{2} / 2=\frac{1}{2} g_{i j} x^{i} x^{j} .
$$

We can use the formulas above to compute

$$
D_{i} P=g_{i j} x^{j}
$$

Also we get

$$
D_{i} D_{j} P=g_{i j}+A_{i j}
$$

The defining equation for $P$ gives

$$
g^{i j} D_{i} P D_{j} P=2 P
$$

If we take the covariant derivative of this equation we get

$$
g^{k \ell} D_{j} D_{k} P D_{\ell} P=D_{j} P
$$

which is equivalent to $A_{j k} x^{k}=0$. But if we take the covariant derivative again we get

$$
g^{k \ell} D_{i} D_{j} D_{k} P D_{\ell} P+g^{k \ell} D_{j} D_{k} P D_{i} D_{\ell} P=D_{i} D_{j} P .
$$

Now switching derivatives

$$
D_{i} D_{j} D_{k} P=D_{i} D_{k} D_{j} P=D_{k} D_{i} D_{j} P+R_{i k j \ell} g^{\ell m} D_{m} P
$$

and if we use this and $D_{i} D_{j} P=g_{i j}+A_{i j}$ and $g^{k \ell} D_{\ell} P=x^{k}$ we find that

$$
x^{k} D_{k} A_{i j}+A_{i j}+g^{k \ell} A_{i k} A_{j \ell}+R_{i k j \ell} x^{k} x^{\ell}=0 .
$$

From our assumed curvature bounds we can take $\left|R_{i j k \ell}\right| \leq B_{0}$. Then we get the following estimate:

$$
\left|x^{k} D_{k} A_{i j}+A_{i j}\right| \leq C\left|A_{i j}\right|^{2}+C B_{0} r^{2}
$$

on the ball $|x| \leq r$ for some constant $C$ depending only on the dimension.

We now show how to use the maximum principle on such equations. First of all, by a maximum principle argument, it is easy to show that if $f$ is a function on a ball $|x| \leq r$ and $\lambda>0$ is a constant, then

$$
\lambda \sup |f| \leq \sup \left|x^{k} \frac{\partial f}{\partial x^{k}}+\lambda f\right|
$$


For any tensor $T=\left\{T_{i \cdots j}\right\}$ and any constant $\lambda>0$, setting $f=|T|^{2}$ in the above inequality, we have

$$
\lambda \sup |T| \leq \sup \left|x^{k} D_{k} T+\lambda T\right|
$$

Applying this to the tensor $A_{i j}$ we get

$$
\sup _{|x| \leq r}\left|A_{i j}\right| \leq C \sup _{|x| \leq r}\left|A_{i j}\right|^{2}+C B_{0} r^{2}
$$

for some constant depending only on the dimension.

It is fairly elementary to see that there exist constants $c>0$ and $C_{0}<\infty$ such that if the metric $g_{i j}$ is in geodesic coordinates with $\left|R_{i j k \ell}\right| \leq B_{0}$ in the ball of radius $r \leq c / \sqrt{B_{0}}$ then

$$
\left|A_{i j}\right| \leq C_{0} B_{0} r^{2}
$$

Indeed, since the derivatives of $g_{i j}$ vanish at the origin, so does $A_{i j}$. Hence the estimate holds near the origin. But the inequality

$$
\sup _{|x| \leq r}\left|A_{i j}\right| \leq C \sup _{|x| \leq r}\left|A_{i j}\right|^{2}+C B_{0} r^{2}
$$

says that $\left|A_{i j}\right|$ avoids an interval when $c$ is chosen small. In fact the inequality

$$
X \leq C X^{2}+D
$$

is equivalent to

$$
|2 C X-1| \geq \sqrt{1-4 C D}
$$

which makes $X$ avoid an interval if $4 C D<1$. (Hence in our case we need to choose $c$ with $4 C^{2} c^{2}<1$.) Then if $X$ is on the side containing 0 we get

$$
X \leq \frac{1-\sqrt{1-4 C D}}{2 C} \leq 2 D .
$$

This gives $\left|A_{i j}\right| \leq C_{0} B_{0} r^{2}$ with $C_{0}=2 C$.

We can also derive bounds on all the covariant derivatives of $P$ in terms of bounds on the covariant derivatives of the curvature. To simplify the notation, we let

$$
D^{q} P=\left\{D_{j_{1}} D_{j_{2}} \cdots D_{j_{q}} P\right\}
$$

denote the $q^{\text {th }}$ covariant derivative, and in estimating $D^{q} P$ we will lump all the lower order terms into a general slush term $\Phi^{q}$ which will be a polynomial in $D^{1} P, D^{2} P, \ldots, D^{q-1} P$ and $R m, D^{1} R m, \ldots, D^{q-2} R m$. We already have estimates on a ball of radius $r$

$$
\begin{gathered}
P \leq r^{2} / 2 \\
\left|D^{1} P\right| \leq r \\
\left|A_{i j}\right| \leq C_{0} B_{0} r^{2}
\end{gathered}
$$


and since $D_{i} D_{j} P=g_{i j}+A_{i j}$ and $r \leq c / \sqrt{B_{0}}$ if we choose $c$ small we can make

$$
\left|A_{i j}\right| \leq 1 / 2
$$

and we get

$$
\left|D^{2} P\right| \leq C_{2}
$$

for some constant $C_{2}$ depending only on the dimension.

Start with the equation $g^{i j} D_{i} P D_{j} P=2 P$ and apply repeated covariant derivatives. Observe that we get an equation which starts out

$$
g^{i j} D_{i} P D^{q} D_{j} P+\cdots=0
$$

where the omitted terms only contain derivatives $D^{q} P$ and lower. If we switch two derivatives in a term $D^{q+1} P$ or lower, we get a term which is a product of a covariant derivative of $R m$ of order at most $q-2$ (since the two closest to $P$ commute) and a covariant derivative of $P$ of order at most $q-1$; such a term can be lumped in with the slush term $\Phi^{q}$. Therefore up to terms in $\Phi^{q}$ we can regard the derivatives as commuting. Then paying attention to the derivatives in $D^{1} P$ we get an equation

$g^{i j} D_{i} P D_{j} D_{k_{1}} \cdots D_{k_{q}} P+g^{i j} D_{i} D_{k_{1}} P D_{j} D_{k_{2}} \cdots D_{k_{q}} P$

$+g^{i j} D_{i} D_{k_{2}} P D_{j} D_{k_{1}} D_{k_{3}} \cdots D_{k_{q}} P+\cdots+g^{i j} D_{i} D_{k_{q}} P D_{j} D_{k_{1}} \cdots D_{k_{q-1}} P$

$=D_{k_{1}} \cdots D_{k_{q}} P+\Phi^{q}$.

Recalling that $D_{i} D_{j} P=g_{i j}+A_{i j}$ we can rewrite this as

$$
\begin{aligned}
\Phi^{q}= & g^{i j} D_{i} P D_{j} D_{k_{1}} \cdots D_{k_{q}}+(q-1) D_{k_{1}} \cdots D_{k_{q}} P \\
& +g^{i j} A_{i k_{1}} D_{j} D_{k_{2}} \cdots D_{k_{q}} P+\cdots+g^{i j} A_{i k_{q}} D_{j} D_{k_{1}} \cdots D_{k_{q-1}} P .
\end{aligned}
$$

Estimating the product of tensors in the usual way gives

$$
\left|x^{i} D_{i} D^{q} P+(q-1) D^{q} P\right| \leq q|A|\left|D^{q} P\right|+\left|\Phi^{q}\right| .
$$

Applying the inequality $\lambda \sup |T| \leq \sup \left|x^{k} D_{k} T+\lambda T\right|$ with $T=D^{q} P$ gives

$$
(q-1) \sup \left|D^{q} P\right| \leq \sup \left(q|A|\left|D^{q} P\right|+\left|\Phi^{q}\right|\right) .
$$

Now we can make $|A| \leq 1 / 2$ by making $r \leq c / \sqrt{B_{0}}$ with $c$ small; it is important here that $c$ is independent of $q$ ! Then we get

$$
(q-2) \sup \left|D^{q} P\right| \leq 2 \sup \left|\Phi^{q}\right|
$$

which is a good estimate for $q \geq 3$. The term $\Phi^{q}$ is estimated inductively from the terms $D^{q-1} P$ and $D^{q-2} R m$ and lower. This proves that there exist constants $C_{q}$ for $q \geq 3$ depending only on $q$ and the dimension and on $\left|D^{j} R m\right|$ for $j \leq q-2$ such that

$$
\left|D^{q} P\right| \leq C_{q}
$$

on the ball $r \leq c / \sqrt{B_{0}}$.

Now we turn our attention to estimating the Euclidean metric $I_{j k}$ and its covariant derivatives with respect to $g_{j k}$. We will need the following elementary fact: suppose that $f$ is a function on a ball $|x| \leq r$ with $f(0)=0$ and

$$
\left|x^{i} \frac{\partial f}{\partial x^{i}}\right| \leq C|x|^{2}
$$


for some constant $C$. Then

$$
|f| \leq C|x|^{2}
$$

for the same constant $C$. As a consequence, if $T=\left\{T_{j \ldots k}\right\}$ is a tensor which vanishes at the origin and if

$$
\left|x^{i} D_{i} T\right| \leq C|x|^{2}
$$

on a ball $|x| \leq r$ then $|T| \leq C|x|^{2}$ with the same constant $C$. (Simply apply the inequality (4.1.9) to the function $f=|T|$. In case this is not smooth, we can use $f=\sqrt{|T|^{2}+\epsilon^{2}}-\epsilon$ and then let $\epsilon \rightarrow 0$.)

Our application will be to the tensor $I_{j k}$ which gives the Euclidean metric as a tensor in geodesic coordinates. We have

$$
D_{i} I_{j k}=-\Gamma_{i j}^{p} I_{p k}-\Gamma_{i k}^{p} I_{p j}
$$

and since

$$
x^{i} \Gamma_{i j}^{p}=g^{p q} A_{j q}
$$

we get the equation

$$
x^{i} D_{i} I_{j k}=-g^{p q} A_{j p} I_{k q}-g^{p q} A_{k p} I_{j q} .
$$

We already have $\left|A_{j k}\right| \leq C_{0} B_{0}|x|^{2}$ for $|x| \leq r \leq c / \sqrt{B_{0}}$. The tensor $I_{j k}$ doesn't vanish at the origin, but the tensor

$$
h_{j k}=I_{j k}-g_{j k}
$$

does. We can then use

$$
x^{i} D_{i} h_{j k}=-g^{p q} A_{j p} h_{k q}-g^{p q} A_{k q} h_{j q}-2 A_{j k} .
$$

Suppose $M(s)=\sup _{|x| \leq s}\left|h_{j k}\right|$. Then

$$
\left|x^{i} D_{i} h_{j k}\right| \leq 2[1+M(s)] C_{0} B_{0}|x|^{2}
$$

and we get

$$
\left|h_{j k}\right| \leq 2[1+M(s)] C_{0} B_{0}|x|^{2}
$$

on $|x| \leq s$. This makes

$$
M(s) \leq 2[1+M(s)] C_{0} B_{0} s^{2} .
$$

Then for $s \leq r \leq c / \sqrt{B_{0}}$ with $c$ small compared to $C_{0}$ we get $2 C_{0} B_{0} s^{2} \leq 1 / 2$ and $M(s) \leq 4 C_{0} B_{0} s^{2}$. Thus

$$
\left|I_{j k}-g_{j k}\right|=\left|h_{j k}\right| \leq 4 C_{0} B_{0}|x|^{2}
$$

for $|x| \leq r \leq c / \sqrt{B_{0}}$, and hence for $c$ small enough

$$
\frac{1}{2} g_{j k} \leq I_{j k} \leq 2 g_{j k} .
$$


Thus the metrics are comparable. Note that this estimate only needs $r$ small compared to $B_{0}$ and does not need any bounds on the derivatives of the curvature.

Now to obtain bounds on the covariant derivative of the Eucliden metric $I_{k \ell}$ with respect to the Riemannian metric $g_{k \ell}$ we want to start with the equation

$$
x^{i} D_{i} I_{k \ell}+g^{m n} A_{k m} I_{\ell n}+g^{m n} A_{\ell m} I_{k n}=0
$$

and apply $q$ covariant derivatives $D_{j_{1}} \cdots D_{j_{q}}$. Each time we do this we must interchange $D_{j}$ and $x^{i} D_{i}$, and since this produces a term which helps we should look at it closely. If we write $R_{j i}=\left[D_{j}, D_{i}\right]$ for the commutator, this operator on tensors involves the curvature but no derivatives. Since

$$
D_{j} x^{i}=I_{j}^{i}+g^{i m} A_{j m}
$$

we can compute

$$
\left[D_{j}, x^{i} D_{i}\right]=D_{j}+g^{i m} A_{j m} D_{i}+x^{i} R_{j i}
$$

and the term $D_{j}$ in the commutator helps, while $A_{j m}$ can be kept small and $R_{j i}$ is zero order. It follows that we get an equation of the form

$$
\begin{aligned}
0= & x^{i} D_{i} D_{j_{1}} \cdots D_{j_{q}} I_{k \ell}+q D_{j_{1}} \cdots D_{j_{q}} I_{k \ell} \\
& +\sum_{h=1}^{q} g^{i m} A_{j_{h} m} D_{j_{1}} \cdots D_{j_{h-1}} D_{i} D_{j_{h+1}} \cdots D_{j_{q}} I_{k \ell} \\
& +g^{m n} A_{k m} D_{j_{1}} \cdots D_{j_{q}} I_{\ell n}+g^{m n} A_{\ell m} D_{j_{1}} \cdots D_{j_{q}} I_{k n}+\Psi^{q}
\end{aligned}
$$

where the slush term $\Psi^{q}$ is a polynomial in derivatives of $I_{k \ell}$ of degree no more than $q-1$ and derivatives of $P$ of degree no more than $q+2$ (remember $x^{i}=g^{i j} D_{j} P$ and $\left.A_{i j}=D_{i} D_{j} P-g_{i j}\right)$ and derivatives of the curvature $R m$ of degree no more than $q-1$. We now estimate

$$
D^{q} I_{k \ell}=\left\{D_{j_{1}} \cdots D_{j_{q}} I_{k \ell}\right\}
$$

by induction on $q$ using (4.1.8) with $\lambda=q$. Noticing a total of $q+2$ terms contracting $A_{i j}$ with a derivative of $I_{k \ell}$ of degree $q$, we get the estimate

$$
q \sup \left|D^{q} I_{k \ell}\right| \leq(q+2) \sup |A| \sup \left|D^{q} I_{k \ell}\right|+\sup \left|\Psi^{q}\right| .
$$

and everything works. This proves that there exists a constant $c>0$ depending only on the dimension, and constants $C_{q}$ depending only on the dimension and $q$ and bounds $B_{j}$ on the curvature and its derivatives for $j \leq q$ where $\left|D^{j} R m\right| \leq B_{j}$, so that for any metric $g_{k \ell}$ in geodesic coordinates in the ball $|x| \leq r \leq c / \sqrt{B_{0}}$ the Euclidean metric $I_{k \ell}$ satisfies

$$
\frac{1}{2} g_{k \ell} \leq I_{k \ell} \leq 2 g_{k \ell}
$$

and the covariant derivatives of $I_{k \ell}$ with respect to $g_{k \ell}$ satisfy

$$
\left|D_{j_{1}} \cdots D_{j_{q}} I_{k \ell}\right| \leq C_{q}
$$

The difference between a covariant derivative and an ordinary derivative is given by the connection

$$
-\Gamma_{i j}^{p} I_{p k}-\Gamma_{i k}^{p} I_{p j}
$$


to get

$$
\Gamma_{i j}^{k}=\frac{1}{2} I^{k \ell}\left(D_{\ell} I_{i j}-D_{i} I_{j \ell}-D_{j} I_{i \ell}\right)
$$

This gives us bounds on $\Gamma_{i j}^{k}$. We then obtain bounds on the first derivatives of $g_{i j}$ from

$$
\frac{\partial}{\partial x^{i}} g_{j k}=g_{k \ell} \Gamma_{i j}^{\ell}+g_{j \ell} \Gamma_{i k}^{\ell} .
$$

Always proceeding inductively on the order of the derivative, we now get bounds on covariant derivatives of $\Gamma_{i j}^{k}$ from the covariant derivatives of $I_{p k}$ and bounds of the ordinary derivatives of $\Gamma_{i j}^{k}$ by relating the to the covariant derivatives using the $\Gamma_{i j}^{k}$, and bounds on the ordinary derivatives of the $g_{j k}$ from bounds on the ordinary derivatives of the $\Gamma_{i j}^{\ell}$. Consequently, we have estimates

$$
\frac{1}{2} I_{k \ell} \leq g_{k \ell} \leq 2 I_{k \ell}
$$

and

$$
\left|\frac{\partial}{\partial x^{j_{1}}} \cdots \frac{\partial}{\partial x^{j_{q}}} g_{k \ell}\right| \leq \tilde{C}_{q}
$$

for similar constants $\tilde{C}_{q}$.

Therefore we have finished the proof of Claim 1.

Proof of Claim 2. We need to show how to estimate the derivatives of an isometry. We will prove that if $y=F(x)$ is an isometry from a ball in Euclidean space with a metric $g_{i j} d x^{i} d x^{j}$ to a ball in Euclidean space with a metric $h_{k l} d y^{k} d y^{l}$. Then we can bound all of the derivatives of $y$ with respect to $x$ in terms of bounds on $g_{i j}$ and its derivatives with respect to $x$ and bound on $h_{k l}$ and its derivatives with respect to $y$. This would imply Claim 2 .

Since $y=F(x)$ is an isometry we have the equation

$$
h_{p q} \frac{\partial y^{p}}{\partial x^{j}} \frac{\partial y^{q}}{\partial x^{k}}=g_{j k}
$$

Using bounds $g_{j k} \leq C I_{j k}$ and $h_{p q} \geq c I_{p q}$ comparing to the Euclidean metric, we easily get estimates

$$
\left|\frac{\partial y^{p}}{\partial x^{j}}\right| \leq C
$$

Now if we differentiate the equation with respect to $x^{i}$ we get

$$
h_{p q} \frac{\partial^{2} y^{p}}{\partial x^{i} \partial x^{j}} \frac{\partial y^{q}}{\partial x^{k}}+h_{p q} \frac{\partial y^{p}}{\partial x^{j}} \frac{\partial^{2} y^{q}}{\partial x^{i} \partial x^{k}}=\frac{\partial g_{j k}}{\partial x^{i}}-\frac{\partial h_{p q}}{\partial y^{r}} \frac{\partial y^{r}}{\partial x^{i}} \frac{\partial y^{p}}{\partial x^{j}} \frac{\partial y^{q}}{\partial x^{k}} .
$$

Now let

$$
T_{i j k}=h_{p q} \frac{\partial y^{p}}{\partial x^{i}} \frac{\partial^{2} y^{q}}{\partial x^{j} \partial x^{k}}
$$


and let

$$
U_{i j k}=\frac{\partial g_{j k}}{\partial x^{i}}-\frac{\partial h_{p q}}{\partial y^{r}} \frac{\partial y^{r}}{\partial x^{i}} \frac{\partial y^{p}}{\partial x^{j}} \frac{\partial y^{q}}{\partial x^{k}} .
$$

Then the above equation says

$$
T_{k i j}+T_{j i k}=U_{i j k}
$$

Using the obvious symmetries $T_{i j k}=T_{i k j}$ and $U_{i j k}=U_{i k j}$ we can solve this in the usual way to obtain

$$
T_{i j k}=\frac{1}{2}\left(U_{j i k}+U_{k i j}-U_{i j k}\right) .
$$

We can recover the second derivatives of $y$ with respect to $x$ from the formula

$$
\frac{\partial^{2} y^{p}}{\partial x^{i} \partial x^{j}}=g^{k \ell} T_{k i j} \frac{\partial y^{p}}{\partial x^{\ell}} .
$$

Combining these gives an explicit formula giving $\partial^{2} y^{p} / \partial x^{i} \partial x^{j}$ as a function of $g^{i j}, h_{p q}, \partial g_{j k} / \partial x^{i}, \partial h_{p q} / \partial y^{r}$, and $\partial y^{p} / \partial y^{i}$. This gives bounds

$$
\left|\frac{\partial^{2} y^{p}}{\partial y^{i} \partial y^{j}}\right| \leq C
$$

and bounds on all higher derivatives follow by differentiating the formula and using induction. This completes the proof of Claim 2 and hence the proof of Theorem 4.1.2. $\mathrm{\square}$

We now want to show how to use this convergence result on solutions to the Ricci flow. Let us first state the definition for the convergence of evolving manifolds.

DeFinition 4.1.3. Let $\left(M_{k}, g_{k}(t), p_{k}\right)$ be a sequence of evolving marked complete Riemannian manifolds, with the evolving metrics $g_{k}(t)$ over a fixed time interval $t \in(A, \Omega], A<0 \leq \Omega$, and with the marked points $p_{k} \in M_{k}$. We say a sequence of evolving marked $\left(B_{0}\left(p_{k}, s_{k}\right), g_{k}(t), p_{k}\right)$ over $t \in(A, \Omega]$, where $B_{0}\left(p_{k}, s_{k}\right)$ are geodesic balls of $\left(M_{k}, g_{k}(0)\right)$ centered at $p_{k}$ with the radii $s_{k} \rightarrow s_{\infty}(\leq+\infty)$, converges in the $C_{\mathrm{loc}}^{\infty}$ topology to an evolving marked (maybe noncomplete) manifold $\left(B_{\infty}, g_{\infty}(t), p_{\infty}\right)$ over $t \in(A, \Omega]$, where, at the time $t=0, B_{\infty}$ is a geodesic open ball centered at $p_{\infty} \in B_{\infty}$ with the radius $s_{\infty}$, if we can find a sequence of exhausting open sets $U_{k}$ in $B_{\infty}$ containing $p_{\infty}$ and a sequence of diffeomorphisms $f_{k}$ of the sets $U_{k}$ in $B_{\infty}$ to open sets $V_{k}$ in $B\left(p_{k}, s_{k}\right) \subset M_{k}$ mapping $p_{\infty}$ to $p_{k}$ such that the pull-back metrics $\tilde{g}_{k}(t)=\left(f_{k}\right)^{*} g_{k}(t)$ converge in $C^{\infty}$ topology to $g_{\infty}(t)$ on every compact subset of $B_{\infty} \times(A, \Omega]$.

Now we fix a time interval $A<t \leq \Omega$ with $-\infty<A<0$ and $0 \leq \Omega<+\infty$. Consider a sequence of marked evolving complete manifolds $\left(M_{k}, g_{k}(t), p_{k}\right), t \in(A, \Omega]$, with each $g_{k}(t), k=1,2, \ldots$, being a solution of the Ricci flow

$$
\frac{\partial}{\partial t} g_{k}(t)=-2 \operatorname{Ric}_{k}(t)
$$

on $B_{0}\left(p_{k}, s_{k}\right) \times(A, \Omega]$, where $R i c_{k}$ is the Ricci curvature tensor of $g_{k}$, and $B_{0}\left(p_{k}, s_{k}\right)$ is the geodesic ball of $\left(M_{k}, g_{k}(0)\right)$ centered at $p_{k}$ with the radii $s_{k} \rightarrow s_{\infty}(\leq+\infty)$. 
Assume that for each $r<s_{\infty}$ there are positive constants $C(r)$ and $k(r)$ such that the curvatures of $g_{k}(t)$ satisfy the bound

$$
\left|R m\left(g_{k}\right)\right| \leq C(r)
$$

on $B_{0}\left(p_{k}, r\right) \times(A, \Omega]$ for all $k \geq k(r)$. We also assume that $\left(M_{k}, g_{k}(t), p_{k}\right), k=1,2, \ldots$, have a uniform injectivity radius bound at the origins $p_{k}$ at $t=0$. By Shi's derivatives estimate (Theorem 1.4.1), the above assumption of uniform bound of the curvatures on the geodesic balls $B_{0}\left(p_{k}, r\right)\left(r<s_{\infty}\right)$ implies the uniform bounds on all the derivatives of the curvatures at $t=0$ on the geodesic balls $B_{0}\left(p_{k}, r\right)\left(r<s_{\infty}\right)$. Then by Theorem 4.1.2 we can find a subsequence of marked evolving manifolds, still denoted by $\left(M_{k}, g_{k}(t), p_{k}\right)$ with $t \in(A, \Omega]$, so that the geodesic balls $\left(B_{0}\left(p_{k}, s_{k}\right), g_{k}(0), p_{k}\right)$ converge in the $C_{\text {loc }}^{\infty}$ topology to a geodesic ball $\left(B_{\infty}\left(p_{\infty}, s_{\infty}\right), g_{\infty}(0), p_{\infty}\right)$. From now on, we consider this subsequence of marked evolving manifolds. By Definition 4.1.1, we have a sequence of (relatively compact) exhausting covering $\left\{U_{k}\right\}$ of $B_{\infty}\left(p_{\infty}, s_{\infty}\right)$ containing $p_{\infty}$ and a sequence of diffeomorphisms $f_{k}$ of the sets $U_{k}$ in $B_{\infty}\left(p_{\infty}, s_{\infty}\right)$ to open sets $V_{k}$ in $B_{0}\left(p_{k}, s_{k}\right)$ mapping $p_{\infty}$ to $p_{k}$ such that the pull-back metrics at $t=0$

$$
\tilde{g}_{k}(0)=\left(f_{k}\right)^{*} g_{k}(0) \stackrel{C_{\mathrm{loc}}^{\infty}}{\longrightarrow} g_{\infty}(0), \quad \text { as } k \rightarrow+\infty, \quad \text { on } B_{\infty}\left(p_{\infty}, s_{\infty}\right) .
$$

However, the pull-back metrics $\tilde{g}_{k}(t)=\left(f_{k}\right)^{*} g_{k}(t)$ are also defined at all times $A<$ $t \leq \Omega$ (although $g_{\infty}(t)$ is not yet). We also have uniform bounds on the curvature of the pull-back metrics $\tilde{g}_{k}(t)$ and all their derivatives, by Shi's derivative estimates (Theorem 1.4.1), on every compact subset of $B_{\infty}\left(p_{\infty}, s_{\infty}\right) \times(A, \Omega]$. What we claim next is that we can find uniform bounds on all the covariant derivatives of the $\tilde{g}_{k}$ taken with respect to the fixed metric $g_{\infty}(0)$.

Lemma 4.1.4. Let $(M, g)$ be a Riemannian manifold, $K$ a compact subset of $M$, and $\tilde{g}_{k}(t)$ a collection of solutions to Ricci flow defined on neighborhoods of $K \times[\alpha, \beta]$ with $[\alpha, \beta]$ containing 0 . Suppose that for each $l \geq 0$,

(a) $C_{0}^{-1} g \leq \tilde{g}_{k}(0) \leq C_{0} g$, on $K$, for all $k$,

(b) $\left|\nabla^{l} \tilde{g}_{k}(0)\right| \leq C_{l}$, on $K$, for all $k$,

(c) $\left|\tilde{\nabla}_{k}^{l} R m\left(\tilde{g}_{k}\right)\right|_{k} \leq C_{l}^{\prime}$, on $K \times[\alpha, \beta]$, for all $k$, for some positive constants $C_{l}, C_{l}^{\prime}, l=0,1, \ldots$, independent of $k$, where $R m\left(\tilde{g}_{k}\right)$ are the curvature tensors of the metrics $\tilde{g}_{k}(t), \tilde{\nabla}_{k}$ denote covariant derivative with respect to $\tilde{g}_{k}(t),|\cdot|_{k}$ are the length of a tensor with respect to $\tilde{g}_{k}(t)$, and $|\cdot|$ is the length with respect to $\mathrm{g}$. Then the metrics $\tilde{g}_{k}(t)$ satisfy

$$
\tilde{C}_{0}^{-1} g \leq \tilde{g}_{k}(t) \leq \tilde{C}_{0} g, \text { on } K \times[\alpha, \beta]
$$

and

$$
\left|\nabla^{l} \tilde{g}_{k}\right| \leq \tilde{C}_{l}, \text { on } K \times[\alpha, \beta], \quad l=1,2, \ldots,
$$

for all $k$, where $\tilde{C}_{l}, l=0,1, \ldots$, are positive constants independent of $k$.

Proof. First by using the equation

$$
\frac{\partial}{\partial t} \tilde{g}_{k}=-2 \tilde{R i c}_{k}
$$

and the assumption (c) we immediately get

$$
\tilde{C}_{0}^{-1} g \leq \tilde{g}_{k}(t) \leq \tilde{C}_{0} g, \text { on } K \times[\alpha, \beta]
$$


for some positive constant $\tilde{C}_{0}$ independent of $k$.

Next we want to bound $\nabla \tilde{g}_{k}$. The difference of the connection $\tilde{\Gamma}_{k}$ of $\tilde{g}_{k}$ and the connection $\Gamma$ of $g$ is a tensor. Taking $\Gamma$ to be fixed in time, we get

$$
\begin{aligned}
\frac{\partial}{\partial t}\left(\tilde{\Gamma}_{k}-\Gamma\right)= & \frac{\partial}{\partial t}\left(\frac{1}{2}\left(\tilde{g}_{k}\right)^{\gamma \delta}\left[\frac{\partial}{\partial x^{\alpha}}\left(\tilde{g}_{k}\right)_{\delta \beta}+\frac{\partial}{\partial x^{\beta}}\left(\tilde{g}_{k}\right)_{\delta \alpha}-\frac{\partial}{\partial x^{\delta}}\left(\tilde{g}_{k}\right)_{\alpha \beta}\right]\right) \\
= & \frac{1}{2}\left(\tilde{g}_{k}\right)^{\gamma \delta}\left[\left(\tilde{\nabla}_{k}\right)_{\alpha}\left(-2\left(\tilde{R i c}_{k}\right)_{\beta \delta}\right)+\left(\tilde{\nabla}_{k}\right)_{\beta}\left(-2\left(\tilde{R i c}_{k}\right)_{\alpha \delta}\right)\right. \\
& \left.-\left(\tilde{\nabla}_{k}\right)_{\delta}\left(-2\left(\tilde{R i c}_{k}\right)_{\alpha \beta}\right)\right]
\end{aligned}
$$

and then by the assumption (c) and (4.1.10),

$$
\left|\frac{\partial}{\partial t}\left(\tilde{\Gamma}_{k}-\Gamma\right)\right| \leq C, \text { for all } k \text {. }
$$

Note also that at a normal coordinate of the metric $g$ at a fixed point and at the time $t=0$

$$
\begin{aligned}
\left(\tilde{\Gamma}_{k}\right)_{\alpha \beta}^{\gamma}-\Gamma_{\alpha \beta}^{\gamma} & =\frac{1}{2}\left(\tilde{g}_{k}\right)^{\gamma \delta}\left(\frac{\partial}{\partial x^{\alpha}}\left(\tilde{g}_{k}\right)_{\delta \beta}+\frac{\partial}{\partial x^{\beta}}\left(\tilde{g}_{k}\right)_{\delta \alpha}-\frac{\partial}{\partial x^{\delta}}\left(\tilde{g}_{k}\right)_{\alpha \beta}\right) \\
& =\frac{1}{2}\left(\tilde{g}_{k}\right)^{\gamma \delta}\left(\nabla_{\alpha}\left(\tilde{g}_{k}\right)_{\delta \beta}+\nabla_{\beta}\left(\tilde{g}_{k}\right)_{\delta \alpha}-\nabla_{\delta}\left(\tilde{g}_{k}\right)_{\alpha \beta}\right),
\end{aligned}
$$

thus by the assumption (b) and (4.1.10),

$$
\left|\tilde{\Gamma}_{k}(0)-\Gamma\right| \leq C, \text { for all } k .
$$

Integrating over time we deduce that

$$
\left|\tilde{\Gamma}_{k}-\Gamma\right| \leq C, \text { on } K \times[\alpha, \beta], \text { for all } k \text {. }
$$

By using the assumption (c) and (4.1.10) again, we have

$$
\begin{aligned}
\left|\frac{\partial}{\partial t}\left(\nabla \tilde{g}_{k}\right)\right| & =\left|-2 \nabla \tilde{R}_{c}\right| \\
& =\left|-2 \tilde{\nabla}_{k} \tilde{\operatorname{Ric}}_{k}+\left(\tilde{\Gamma}_{k}-\Gamma\right) * \tilde{\operatorname{Ric}}_{k}\right| \\
& \leq C, \text { for all } k .
\end{aligned}
$$

Hence by combining with the assumption (b) we get bounds

$$
\left|\nabla \tilde{g}_{k}\right| \leq \tilde{C}_{1}, \quad \text { on } K \times[\alpha, \beta],
$$

for some positive constant $\tilde{C}_{1}$ independent of $k$.

Further we want to bound $\nabla^{2} \tilde{g}_{k}$. Again regarding $\nabla$ as fixed in time, we see

$$
\frac{\partial}{\partial t}\left(\nabla^{2} \tilde{g}_{k}\right)=-2 \nabla^{2}\left(\tilde{R} i c_{k}\right) .
$$

Write

$$
\begin{aligned}
& \nabla^{2} \tilde{R}_{i} c_{k}=\left(\nabla-\tilde{\nabla}_{k}\right)\left(\nabla \tilde{R}_{i}\right)+\tilde{\nabla}_{k}\left(\nabla-\tilde{\nabla}_{k}\right) \tilde{R}_{i}{ }_{k}+\tilde{\nabla}_{k}^{2} \tilde{R i c}_{k} \\
& =\left(\Gamma-\tilde{\Gamma}_{k}\right) * \nabla \operatorname{Ric} c_{k}+\tilde{\nabla}_{k}\left(\left(\Gamma-\tilde{\Gamma}_{k}\right) * \tilde{R}_{i c}\right)+\tilde{\nabla}_{k}^{2} \tilde{R i c}_{k} \\
& =\left(\Gamma-\tilde{\Gamma}_{k}\right) *\left[\left(\nabla-\tilde{\nabla}_{k}\right) \tilde{R}_{i}{ }_{k}+\tilde{\nabla}_{k} \tilde{R i c}_{k}\right] \\
& +\tilde{\nabla}_{k}\left(\tilde{g}_{k}^{-1} * \nabla \tilde{g}_{k} * \tilde{R i c}_{k}\right)+\tilde{\nabla}_{k}^{2} \tilde{R i c}_{k} \\
& =\left(\Gamma-\tilde{\Gamma}_{k}\right) *\left[\left(\Gamma-\tilde{\Gamma}_{k}\right) * \tilde{R}_{i c}+\tilde{\nabla}_{k} \operatorname{Ric}_{k}\right] \\
& +\tilde{\nabla}_{k}\left(\tilde{g}_{k}^{-1} * \nabla \tilde{g}_{k} * \tilde{R i c}_{k}\right)+\tilde{\nabla}_{k}^{2} \operatorname{Ric}{ }_{k}
\end{aligned}
$$


where we have used (4.1.11). Then by the assumption (c), (4.1.10), (4.1.12) and (4.1.13) we have

$$
\begin{aligned}
\left|\frac{\partial}{\partial t} \nabla^{2} \tilde{g}_{k}\right| & \leq C+C \cdot\left|\tilde{\nabla}_{k} \nabla \tilde{g}_{k}\right| \\
& =C+C \cdot\left|\nabla^{2} \tilde{g}_{k}+\left(\tilde{\Gamma}_{k}-\Gamma\right) * \nabla \tilde{g}_{k}\right| \\
& \leq C+C\left|\nabla^{2} \tilde{g}_{k}\right| .
\end{aligned}
$$

Hence by combining with the assumption (b) we get

$$
\left|\nabla^{2} \tilde{g}_{k}\right| \leq \tilde{C}_{2}, \quad \text { on } K \times[\alpha, \beta],
$$

for some positive constant $\tilde{C}_{2}$ independent of $k$.

The bounds on the higher derivatives can be derived by the same argument. Therefore we have completed the proof of the lemma.

We now apply the lemma to the pull-back metrics $\tilde{g}_{k}(t)=\left(f_{k}\right)^{*} g_{k}(t)$ on $B_{\infty}\left(p_{\infty}, s_{\infty}\right) \times(A, \Omega]$. Since the metrics $\tilde{g}_{k}(0)$ have uniform bounds on their curvature and all derivatives of their curvature on every compact set of $B_{\infty}\left(p_{\infty}, s_{\infty}\right)$ and converge to the metric $g_{\infty}(0)$ in $C_{l o c}^{\infty}$ topology, the assumptions (a) and (b) are certainly held for every compact subset $K \subset B_{\infty}\left(p_{\infty}, s_{\infty}\right)$ with $g=g_{\infty}(0)$. For every compact subinterval $[\alpha, \beta] \subset(A, \Omega]$, we have already seen from Shi's derivative estimates (Theorem 1.4.1) that the assumption (c) is also held on $K \times[\alpha, \beta]$. Then all of the $\nabla^{l} \tilde{g}_{k}$ are uniformly bounded with respect to the fixed metric $g=g_{\infty}(0)$ on every compact set of $B_{\infty}\left(p_{\infty}, s_{\infty}\right) \times(A, \Omega]$. By using the classical Arzela-Ascoli theorem, we can find a subsequence which converges uniformly together with all its derivatives on every compact subset of $B_{\infty}\left(p_{\infty}, s_{\infty}\right) \times(A, \Omega]$. The limit metric will agree with that obtained previously at $t=0$, where we know its convergence already. The limit $g_{\infty}(t), t \in(A, \Omega]$, is now clearly itself a solution of the Ricci flow. Thus we obtain the following Cheeger type compactness theorem to the Ricci flow, which is essentially obtained by Hamilton in [62] and is called Hamilton's compactness theorem.

Theorem 4.1.5 (Hamilton's compactness theorem). Let $\left(M_{k}, g_{k}(t), p_{k}\right), t \in$ $(A, \Omega]$ with $A<0 \leq \Omega$, be a sequence of evolving marked complete Riemannian manifolds. Consider a sequence of geodesic balls $B_{0}\left(p_{k}, s_{k}\right) \subset M_{k}$ of radii $s_{k}(0<$ $\left.s_{k} \leq+\infty\right)$, with $s_{k} \rightarrow s_{\infty}(\leq+\infty)$, around the base points $p_{k}$ in the metrics $g_{k}(0)$. Suppose each $g_{k}(t)$ is a solution to the Ricci flow on $B_{0}\left(p_{k}, s_{k}\right) \times(A, \Omega]$. Suppose also

(i) for every radius $r<s_{\infty}$ there exist positive constants $C(r)$ and $k(r)$ independent of $k$ such that the curvature tensors $\operatorname{Rm}\left(g_{k}\right)$ of the evolving metrics $g_{k}(t)$ satisfy the bound

$$
\left|R m\left(g_{k}\right)\right| \leq C(r),
$$

on $B_{0}\left(p_{k}, r\right) \times(A, \Omega]$ for all $k \geq k(r)$, and

(ii) there exists a constant $\delta>0$ such that the injectivity radii of $M_{k}$ at $p_{k}$ in the metric $g_{k}(0)$ satisfy the bound

$$
\operatorname{inj}\left(M_{k}, p_{k}, g_{k}(0)\right) \geq \delta>0,
$$

for all $k=1,2, \ldots$.

Then there exists a subsequence of evolving marked $\left(B_{0}\left(p_{k}, s_{k}\right), g_{k}(t), p_{k}\right)$ over $t \in$ $(A, \Omega]$ which converge in $C_{\text {loc }}^{\infty}$ topology to a solution $\left(B_{\infty}, g_{\infty}(t), p_{\infty}\right)$ over $t \in(A, \Omega]$ to the Ricci flow, where, at the time $t=0, B_{\infty}$ is a geodesic open ball centered at $p_{\infty} \in B_{\infty}$ with the radius $s_{\infty}$. Moreover the limiting solution is complete if $s_{\infty}=+\infty$. 
4.2. Injectivity Radius Estimates. We will use rescaling arguments to understand the formation of singularities and long-time behaviors of the Ricci flow. In view of the compactness property obtained in the previous section, on one hand one needs to control the bounds on the curvature, and on the other hand one needs to control the lower bounds of the injectivity radius. In applications we usually rescale the solution so that the (rescaled) curvatures become uniformly bounded on compact subsets and leave the injectivity radii of the (rescaled) solutions to be estimated in terms of curvatures. In this section we will review a number of such injectivity radius estimates in Riemannian geometry. In the end we will combine these injectivity estimates with Perelman's no local collapsing theorem I' to give the well-known little loop lemma to the Ricci flow which was conjectured by Hamilton in [63].

Let $M$ be a Riemannian manifold. Recall that the injectivity radius at a point $p \in M$ is defined by

$$
\operatorname{inj}(M, p)=\sup \left\{r>0 \mid \exp _{p}: B(O, r)\left(\subset T_{p} M\right) \rightarrow M \text { is injective }\right\},
$$

and the injectivity radius of $M$ is

$$
\operatorname{inj}(M)=\inf \{\operatorname{inj}(M, p) \mid p \in M\} .
$$

We begin with a basic lemma due to Klingenberg (see for example, Corollary 5.7 in Cheeger \& Ebin [22]).

Klingenberg's Lemma. Let $M$ be a complete Riemannian manifold and let $p \in M$. Let $l_{M}(p)$ denote the minimal length of a nontrivial geodesic loop starting and ending at $p$ (maybe not smooth at $p$ ). Then the injectivity radius of $M$ at $p$ satisfies the inequality

$$
\operatorname{inj}(M, p) \geq \min \left\{\frac{\pi}{\sqrt{K_{\max }}}, \frac{1}{2} l_{M}(p)\right\}
$$

where $K_{\max }$ denotes the supermum of the sectional curvature on $M$ and we understand $\pi / \sqrt{K_{\max }}$ to be positive infinity if $K_{\max } \leq 0$.

Based on this lemma and a second variation argument, Klingenberg proved that the injectivity radius of an even-dimensional, compact, simply connected Riemannian manifold of positive sectional curvature is bounded from below by $\pi / \sqrt{K_{\max }}$. For odddimensional, compact, simply connected Riemannian manifold of positive sectional curvature, the same injectivity radius estimates was also proved by Klingenberg under an additional assumption that the sectional curvature is strictly $\frac{1}{4}$-pinched (see for example Theorem 5.9 and 5.10 in Cheeger \& Ebin [22]). We also remark that in dimension 7, there exists a sequence of simply connected, homogeneous Einstein spaces whose sectional curvatures are positive and uniformly bounded from above but their injectivity radii converge to zero. (See [2].)

The next result due to Gromoll and Meyer [52] shows that for complete, noncompact Riemannian manifold with positive sectional curvature, the above injectivity radius estimate actually holds without any restriction on dimension. Since the result and proof were not explicitly given in [52], we include a proof here.

Theorem 4.2.1 (The Gromoll-Meyer injectivity radius estimate). Let $M$ be a complete, noncompact Riemannian manifold with positive sectional curvature. Then the injectivity radius of $M$ satisfies the following estimate

$$
\operatorname{inj}(M) \geq \frac{\pi}{\sqrt{K_{\max }}} .
$$


Proof. Let $O$ be an arbitrary fixed point in $M$. We need to show that the injectivity radius at $O$ is not less than $\pi / \sqrt{K_{\max }}$. We argue by contradiction. Suppose not, then by Klingenberg's lemma there exists a closed geodesic loop $\gamma$ on $M$ starting and ending at $O$ (may be not smooth at $O$ ).

Since $M$ has positive sectional curvature, we know from the work of GromollMeyer [52] (see also Proposition 8.5 in Cheeger \& Ebin [22]) that there exists a compact totally convex subset $C$ of $M$ containing the geodesic loop $\gamma$. Among all geodesic loops starting and ending at the same point and lying entirely in the compact totally convex set $C$ there will be a shortest one. Call it $\gamma_{0}$, and suppose $\gamma_{0}$ starts and ends at a point we call $p_{0}$.

First we claim that $\gamma_{0}$ must be also smooth at the point $p_{0}$. Indeed by the curvature bound and implicit function theorem, there will be a geodesic loop $\tilde{\gamma}$ close to $\gamma_{0}$ starting and ending at any point $\tilde{p}$ close to $p_{0}$. Let $\tilde{p}$ be along $\gamma_{0}$. Then by total convexity of the set $C, \tilde{\gamma}$ also lies entirely in $C$. If $\gamma_{0}$ makes an angle different from $\pi$ at $p_{0}$, the first variation formula will imply that $\tilde{\gamma}$ is shorter than $\gamma_{0}$. This contradicts with the choice of the geodesic loop $\gamma_{0}$ being the shortest.

Now let $L:[0,+\infty) \rightarrow M$ be a ray emanating from $p_{0}$. Choose $r>0$ large enough and set $q=L(r)$. Consider the distance between $q$ and the geodesic loop $\gamma_{0}$. It is clear that the distance can be realized by a geodesic $\beta$ connecting the point $q$ to a point $p$ on $\gamma_{0}$.

Let $X$ be the unit tangent vector of the geodesic loop $\gamma_{0}$ at $p$. Clearly $X$ is orthogonal to the tangent vector of $\beta$ at $p$. We then translate the vector $X$ along the geodesic $\beta$ to get a parallel vector field $X(t), 0 \leq t \leq r$. By using this vector field we can form a variation fixing one endpoint $q$ and the other on $\gamma_{0}$ such that the variational vector field is $\left(1-\frac{t}{r}\right) X(t)$. The second variation of the arclength of this family of curves is given by

$$
\begin{aligned}
& I\left(\left(1-\frac{t}{r}\right) X(t),\left(1-\frac{t}{r}\right) X(t)\right) \\
& =\int_{0}^{r}\left[\left|\nabla_{\frac{\partial}{\partial t}}\left(\left(1-\frac{t}{r}\right) X(t)\right)\right|^{2}\right. \\
& \left.\quad-R\left(\frac{\partial}{\partial t},\left(1-\frac{t}{r}\right) X(t), \frac{\partial}{\partial t},\left(1-\frac{t}{r}\right) X(t)\right)\right] d t \\
& =\frac{1}{r}-\int_{0}^{r}\left(1-\frac{t}{r}\right)^{2} R\left(\frac{\partial}{\partial t}, X(t), \frac{\partial}{\partial t}, X(t)\right) d t \\
& <0
\end{aligned}
$$

when $r$ is sufficiently large, since the sectional curvature of $M$ is strictly positive everywhere. This contradicts with the fact that $\beta$ is the shortest geodesic connecting the point $q$ to the shortest geodesic loop $\gamma_{0}$. Thus we have proved the injectivity radius estimate. $\mathrm{Q}$

In contrast to the above injectivity radius estimates, the following well-known injectivity radius estimate of Cheeger (see for example, Theorem 5.8 in Cheeger \& Ebin [22]) does not impose the restriction on the sign of the sectional curvature.

Cheeger's Lemma. Let $M$ be an n-dimensional compact Riemannian manifold with the sectional curvature $\left|K_{M}\right| \leq \lambda$, the diameter $d(M) \leq D$, and the volume $\operatorname{Vol}(M) \geq v>0$. Then, we have

$$
\operatorname{inj}(M) \geq C_{n}(\lambda, D, v)
$$


for some positive constant $C_{n}(\lambda, D, v)$ depending only on $\lambda, D, v$ and the dimension $n$.

For general complete manifolds, it is possible to relate a lower injectivity radius bound to some lower volume bound provided one localizes the relevant geometric quantities appropriately. The following injectivity radius estimate, which was first obtained by Cheng-Li-Yau [35] for heat kernel estimates and later by Cheeger-Gromov-Taylor [27] with a wave equation argument, is a localized version of the above Cheeger's Lemma. We now present an argument adapted from Abresch and Meyer [1].

Theorem 4.2.2 (Cheng-Li-Yau [35]). Let $B\left(x_{0}, 4 r_{0}\right), 0<r_{0}<\infty$, be a geodesic ball in an $n$-dimensional complete Riemannian manifold $(M, g)$ such that the sectional curvature $K$ of the metric $g$ on $B\left(x_{0}, 4 r_{0}\right)$ satisfies the bounds

$$
\lambda \leq K \leq \Lambda
$$

for some constants $\lambda$ and $\Lambda$. Then for any positive constant $r \leq r_{0}$ (we will also require $r \leq \pi /(4 \sqrt{\Lambda})$ if $\Lambda>0)$ the injectivity radius of $M$ at $x_{0}$ can be bounded from below by

$$
\operatorname{inj}\left(M, x_{0}\right) \geq r \cdot \frac{\operatorname{Vol}\left(B\left(x_{0}, r\right)\right)}{\operatorname{Vol}\left(B\left(x_{0}, r\right)\right)+V_{\lambda}^{n}(2 r)},
$$

where $V_{\lambda}^{n}(2 r)$ denotes the volume of a geodesic ball of radius $2 r$ in the $n$-dimensional simply connected space form $M_{\lambda}$ with constant sectional curvature $\lambda$.

Proof. It is well known (cf. Lemma 5.6 in Cheeger and Ebin [22]) that

$$
\operatorname{inj}\left(M, x_{0}\right)=\min \left\{\text { conjugate radius of } x_{0}, \frac{1}{2} l_{M}\left(x_{0}\right)\right\}
$$

where $l_{M}\left(x_{0}\right)$ denotes the length of the shortest (nontrivial) closed geodesic starting and ending at $x_{0}$. Since by assumption $r \leq \pi /(4 \sqrt{\Lambda})$ if $\Lambda>0$, the conjugate radius of $x_{0}$ is at least $4 r$. Thus it suffices to show

$$
l_{M}\left(x_{0}\right) \geq 2 r \cdot \frac{\operatorname{Vol}\left(B\left(x_{0}, r\right)\right)}{\operatorname{Vol}\left(B\left(x_{0}, r\right)\right)+V_{\lambda}^{n}(2 r)} .
$$

Now we follow the argument presented in [1]. The idea for proving this inequality, as indicated in [1], is to compare the geometry of the ball $B\left(x_{0}, 4 r\right) \subseteq B\left(x_{0}, 4 r_{0}\right) \subset$ $M$ with the geometry of its lifting $\tilde{B}_{4 r} \subset T_{x_{0}}(M)$, via the exponential map $\exp _{x_{0}}$, equipped with the pull-back metric $\tilde{g}=\exp _{x_{0}}^{*} g$. Thus $\exp _{x_{0}}: \tilde{B}_{4 r} \rightarrow B\left(x_{0}, 4 r\right)$ is a length-preserving local diffeomorphism.

Let $\tilde{x}_{0}, \tilde{x}_{1}, \ldots, \tilde{x}_{N}$ be the preimages of $x_{0}$ in $\tilde{B}_{r} \subset \tilde{B}_{4 r}$ with $\tilde{x}_{0}=0$. Clearly they one-to-one correspond to the geodesic loops $\gamma_{0}, \gamma_{1}, \ldots, \gamma_{N}$ at $x_{0}$ of length less than $r$, where $\gamma_{0}$ is the trivial loop. Now for each point $\tilde{x}_{i}$ there exists exactly one isometric immersion $\varphi_{i}: \tilde{B}_{r} \rightarrow \tilde{B}_{4 r}$ mapping 0 to $\tilde{x}_{i}$ and such that $\exp _{x_{0}} \varphi_{i}=\exp _{x_{0}}$.

Without loss of generality, we may assume $\gamma_{1}$ is the shortest nontrivial geodesic loop at $x_{0}$. By analyzing short homotopies, one finds that $\varphi_{i}(\tilde{x}) \neq \varphi_{j}(\tilde{x})$ for all $\tilde{x} \in \tilde{B}_{r}$ and $0 \leq i<j \leq N$. This fact has two consequences:

(a) $N \geq 2 m$, where $m=\left[r / l_{M}\left(x_{0}\right)\right]$. To see this, we first observe that the points $\varphi_{1}^{k}(0),-m \leq k \leq m$, are preimages of $x_{0}$ in $\tilde{B}_{r}$ because $\varphi_{1}$ is an isometric immersion 
satisfying $\exp _{x_{0}} \varphi_{1}=\exp _{x_{0}}$. Moreover we claim they are distinct. For otherwise $\varphi_{1}$ would act as a permutation on the set $\left\{\varphi_{1}^{k}(0) \mid-m \leq k \leq m\right\}$. Since the induced metric $\tilde{g}$ at each point in $\tilde{B}_{r}$ has the injectivity radius at least $2 r$, it follows from the Whitehead theorem (see for example $[22]$ ) that $\tilde{B}_{r}$ is geodesically convex. Then there would exist the unique center of mass $\tilde{y} \in \tilde{B}_{r}$. But then $\tilde{y}=\varphi_{0}(\tilde{y})=\varphi_{1}(\tilde{y})$, a contradiction.

(b) Each point in $B\left(x_{0}, r\right)$ has at least $N+1$ preimages in $\Omega=\cup_{i=0}^{N} B\left(\tilde{x}_{i}, r\right) \subset \tilde{B}_{2 r}$. Hence by the Bishop volume comparison,

$$
(N+1) \operatorname{Vol}\left(B\left(x_{0}, r\right)\right) \leq \operatorname{Vol}_{\tilde{g}}(\Omega) \leq \operatorname{Vol}_{\tilde{g}}\left(\tilde{B}_{2 r}\right) \leq V_{\lambda}^{n}(2 r) .
$$

Now the inequality (4.2.1) follows by combining the fact $N \geq 2\left[r / l_{M}\left(x_{0}\right)\right]$ with the above volume estimate. $\square$

For our purpose of application, we now consider in a complete Riemannian manifold $M$ a geodesic ball $B\left(p_{0}, s_{0}\right)\left(0<s_{0} \leq \infty\right)$ with the property that there exists a positive increasing function $\Lambda:\left[0, s_{0}\right) \rightarrow[0, \infty)$ such that for any $0<s<s_{0}$ the sectional curvature $K$ on the ball $B\left(p_{0}, s\right)$ of radius $s$ around $p_{0}$ satisfies the bound

$$
|K| \leq \Lambda(s) .
$$

Using Theorem 4.2.2, we can control the injectivity radius at any point $p \in B\left(p_{0}, s_{0}\right)$ in terms a positive constant that depends only on the dimension $n$, the injectivity radius at the base point $p_{0}$, the function $\Lambda$ and the distance $d\left(p_{0}, p\right)$ from $p$ to $p_{0}$. We now proceed to derive such an estimate. The geometric insight of the following argument belongs to Yau [128] where he obtained a lower bound estimate for volume by comparing various geodesic balls. Indeed, it is a finite version of Yau's Busemann function argument which gives the information on comparing geodesic balls with centers far apart.

For any point $p \in B\left(p_{0}, s_{0}\right)$ with $d\left(p_{0}, p\right)=s$, set $r_{0}=\left(s_{0}-s\right) / 4$ (we define $r_{0}=1$ if $s_{0}=\infty$ ). Define the set $S$ to be the union of minimal geodesic segments that connect $p$ to each point in $B\left(p_{0}, r_{0}\right)$. Now any point $q \in S$ has distance at most

$$
r_{0}+r_{0}+s=s+2 r_{0}
$$

from $p_{0}$ and hence $S \subseteq B\left(p_{0}, s+2 r_{0}\right)$. For any $0<r \leq \min \left\{\pi / 4 \sqrt{\Lambda\left(s+2 r_{0}\right)}, r_{0}\right\}$, we denote by $\alpha(p, r)$ the sector $S \cap B(p, r)$ of radius $r$ and by $\alpha\left(p, s+r_{0}\right)=S \cap B\left(p, s+r_{0}\right)$. Let $\alpha_{-\Lambda\left(s+2 r_{0}\right)}\left(r_{0}\right)$ (resp. $\left.\alpha_{-\Lambda\left(s+2 r_{0}\right)}\left(s+r_{0}\right)\right)$ be a corresponding sector of the same "angles" with radius $r_{0}$ (resp. $s+r_{0}$ ) in the $n$-dimensional simply connected space form with constant sectional curvature $-\Lambda\left(s+2 r_{0}\right)$. Since $B\left(p_{0}, r_{0}\right) \subset S \subset \alpha\left(p, s+r_{0}\right)$ and $\alpha(p, r) \subset B(p, r)$, the Bishop-Gromov volume comparison theorem implies that

$$
\begin{aligned}
\frac{\operatorname{Vol}\left(B\left(p_{0}, r_{0}\right)\right)}{\operatorname{Vol}(B(p, r))} & \leq \frac{\operatorname{Vol}\left(\alpha\left(p, s+r_{0}\right)\right)}{\operatorname{Vol}(\alpha(p, r))} \\
& \leq \frac{\operatorname{Vol}\left(\alpha_{-\Lambda\left(s+2 r_{0}\right)}\left(s+r_{0}\right)\right)}{\operatorname{Vol}\left(\alpha_{-\Lambda\left(s+2 r_{0}\right)}(r)\right)}=\frac{V_{-\Lambda\left(s+2 r_{0}\right)}^{n}\left(s+r_{0}\right)}{V_{-\Lambda\left(s+2 r_{0}\right)}^{n}(r)} .
\end{aligned}
$$

Combining this inequality with the local injectivity radius estimate in Theorem 4.2.2, we get

$$
\begin{aligned}
& \operatorname{inj}(M, p) \\
& \geq r \frac{V_{-\Lambda\left(s+2 r_{0}\right)}^{n}(r) \cdot \operatorname{Vol}\left(B\left(p_{0}, r_{0}\right)\right)}{V_{-\Lambda\left(s+2 r_{0}\right)}^{n}(r) \operatorname{Vol}\left(B\left(p_{0}, r_{0}\right)\right)+V_{-\Lambda\left(s+2 r_{0}\right)}^{n}(2 r) V_{-\Lambda\left(s+2 r_{0}\right)}^{n}\left(s+2 r_{0}\right)} .
\end{aligned}
$$


Thus, we have proved the following

Corollary 4.2.3. Suppose $B\left(p_{0}, s_{0}\right)\left(0<s_{0} \leq \infty\right)$ is a geodesic ball in an $n$-dimensional complete Riemannian manifold $M$ having the property that for any $0<s<s_{0}$ the sectional curvature $K$ on $B\left(p_{0}, s\right)$ satisfies the bound

$$
|K| \leq \Lambda(s)
$$

for some positive increasing function $\Lambda$ defined on $\left[0, s_{0}\right)$. Then for any point $p \in B\left(p_{0}, s_{0}\right)$ with $d\left(p_{0}, p\right)=s$ and any positive number $r \leq$ $\min \left\{\pi / 4 \sqrt{\Lambda\left(s+2 r_{0}\right)}, r_{0}\right\}$ with $r_{0}=\left(s_{0}-s\right) / 4$, the injectivity radius of $M$ at $p$ is bounded below by

$$
\begin{aligned}
& \operatorname{inj}(M, p) \\
& \geq r \frac{V_{-\Lambda\left(s+2 r_{0}\right)}^{n}(r) \cdot \operatorname{Vol}\left(B\left(p_{0}, r_{0}\right)\right)}{V_{-\Lambda\left(s+2 r_{0}\right)}^{n}(r) \operatorname{Vol}\left(B\left(p_{0}, r_{0}\right)\right)+V_{-\Lambda\left(s+2 r_{0}\right)}^{n}(2 r) V_{-\Lambda\left(s+2 r_{0}\right)}^{n}\left(s+2 r_{0}\right)} .
\end{aligned}
$$

In particular, we have

$$
\operatorname{inj}(M, p) \geq \rho_{n, \delta, \Lambda}(s)
$$

where $\delta>0$ is a lower bound of the injectivity radius $\operatorname{inj}\left(M, p_{0}\right)$ at the origin $p_{0}$ and $\rho_{n, \delta, \Lambda}:\left[0, s_{0}\right) \rightarrow \mathbb{R}^{+}$is a positive decreasing function that depends only on the dimension $n$, the lower bound $\delta$ of the injectivity radius $\operatorname{inj}\left(M, p_{0}\right)$, and the function $\Lambda$.

We remark that in the above discussion if $s_{0}=\infty$ then we can apply the standard Bishop relative volume comparison theorem to geodesic balls directly. Indeed, for any $p \in M$ and any positive constants $r$ and $r_{0}$, we have $B\left(p_{0}, r_{0}\right) \subseteq B(p, \hat{r})$ with $\hat{r} \triangleq$ $\max \left\{r, r_{0}+d\left(p_{0}, p\right)\right\}$. Suppose in addition the curvature $K$ on $M$ is uniformly bounded by $\lambda \leq K \leq \Lambda$ for some constants $\lambda$ and $\Lambda$, then the Bishop volume comparison theorem implies that

$$
\frac{\operatorname{Vol}\left(B\left(p_{0}, r_{0}\right)\right)}{\operatorname{Vol}(B(p, r))} \leq \frac{\operatorname{Vol}(B(p, \hat{r}))}{\operatorname{Vol}(B(p, r))} \leq \frac{V_{\lambda}(\hat{r})}{V_{\lambda}(r)} .
$$

Hence

$$
\operatorname{inj}(M, p) \geq r \frac{V_{\lambda}^{n}(r) \cdot \operatorname{Vol}\left(B\left(p_{0}, r_{0}\right)\right)}{V_{\lambda}^{n}(r) \operatorname{Vol}\left(B\left(p_{0}, r_{0}\right)\right)+V_{\lambda}^{n}(2 r) V_{\lambda}^{n}(\hat{r})} .
$$

So we see that the injectivity radius $\operatorname{inj}(M, p)$ at $p$ falls off at worst exponentially as the distance $d\left(p_{0}, p\right)$ goes to infinity. In other words,

$$
\operatorname{inj}(M, p) \geq \frac{c}{\sqrt{B}}(\delta \sqrt{B})^{n} e^{-C \sqrt{B} d\left(p, p_{0}\right)}
$$

where $B$ is an upper bound on the absolute value of the sectional curvature, $\delta$ is a lower bound on the injectivity radius at $p_{0}$ with $\delta<c / \sqrt{B}$, and $c>0$ and $C<+\infty$ are positive constants depending only on the dimension $n$.

Finally, by combining Theorem 4.2.2 with Perelman's no local collapsing Theorem I' (Theorem 3.3.3) we immediately obtain the following important Little Loop Lemma conjectured by Hamilton [63]. 
Theorem 4.2.4 (Little Loop Lemma). Let $g_{i j}(t), 0 \leq t<T<+\infty$, be a solution of the Ricci flow on a compact manifold $M$. Then there exists a constant $\rho>0$ having the following property: if at a point $x_{0} \in M$ and a time $t_{0} \in[0, T)$,

$$
|R m|\left(\cdot, t_{0}\right) \leq r^{-2} \quad \text { on } B_{t_{0}}\left(x_{0}, r\right)
$$

for some $r \leq \sqrt{T}$, then the injectivity radius of $M$ with respect to the metric $g_{i j}\left(t_{0}\right)$ at $x_{0}$ is bounded from below by

$$
\operatorname{inj}\left(M, x_{0}, g_{i j}\left(t_{0}\right)\right) \geq \rho r .
$$

4.3. Limiting Singularity Models. Consider a solution $g_{i j}(x, t)$ of the Ricci flow on $M \times[0, T), T \leq+\infty$, where either $M$ is compact or at each time $t$ the metric $g_{i j}(\cdot, t)$ is complete and has bounded curvature. We say that $g_{i j}(x, t)$ is a maximal solution if either $T=+\infty$ or $T<+\infty$ and $|R m|$ is unbounded as $t \rightarrow T$.

Denote by

$$
K_{\max }(t)=\sup _{x \in M}|R m(x, t)|_{g_{i j}(t)}
$$

Definition 4.3.1. We say that $\left\{\left(x_{k}, t_{k}\right) \in M \times[0, T)\right\}, k=1,2, \ldots$, is a sequence of (almost) maximum points if there exist positive constants $c_{1}$ and $\alpha \in(0,1]$ such that

$$
\left|R m\left(x_{k}, t_{k}\right)\right| \geq c_{1} K_{\max }(t), \quad t \in\left[t_{k}-\frac{\alpha}{K_{\max }\left(t_{k}\right)}, t_{k}\right]
$$

for all $k$.

DEFINITION 4.3.2. We say that the solution satisfies injectivity radius condition if for any sequence of (almost) maximum points $\left\{\left(x_{k}, t_{k}\right)\right\}$, there exists a constant $c_{2}>0$ independent of $k$ such that

$$
\operatorname{inj}\left(M, x_{k}, g_{i j}\left(t_{k}\right)\right) \geq \frac{c_{2}}{\sqrt{K_{\max }\left(t_{k}\right)}} \text { for all } k \text {. }
$$

Clearly, by the Little Loop Lemma, a maximal solution on a compact manifold with the maximal time $T<+\infty$ always satisfies the injectivity radius condition. Also by the Gromoll-Meyer injectivity radius estimate, a solution on a complete noncompact manifold with positive sectional curvature also satisfies the injectivity radius condition.

According to Hamilton [63], we classify maximal solutions into three types; every maximal solution is clearly of one and only one of the following three types:

Type I: $\quad T<+\infty$ and $\sup _{t \in[0, T)}(T-t) K_{\max }(t)<+\infty$;

Type II: (a) $T<+\infty$ but $\sup _{t \in[0, T)}(T-t) K_{\max }(t)=+\infty$;

(b) $T=+\infty$ but $\sup _{t \in[0, T)} t K_{\max }(t)=+\infty$; 
Type III: (a) $T=+\infty, \quad \sup _{t \in[0, T)} t K_{\max }(t)<+\infty$, and

$$
\begin{aligned}
& \limsup _{t \rightarrow+\infty} t K_{\max }(t)>0 \\
& \text { (b) } T=+\infty, \quad \sup _{t \in[0, T)} t K_{\max }(t)<+\infty, \text { and } \\
& \limsup _{t \rightarrow+\infty} t K_{\max }(t)=0
\end{aligned}
$$

It seems that Type III (b) is not compatible with the injectivity radius condition unless it is a trivial flat solution. Indeed under the Ricci flow the length of a curve $\gamma$ connecting two points $x_{0}, x_{1} \in M$ evolves by

$$
\begin{aligned}
\frac{d}{d t} L_{t}(\gamma) & =\int_{\gamma}-\operatorname{Ric}(\dot{\gamma}, \dot{\gamma}) d s \\
& \leq C(n) K_{\max }(t) \cdot L_{t}(\gamma) \\
& \leq \frac{\epsilon}{t} L_{t}(\gamma), \text { as } t \text { large enough, }
\end{aligned}
$$

for arbitrarily fixed $\epsilon>0$. Thus when we are considering the Ricci flow on a compact manifold, the diameter of the evolving manifold grows at most as $t^{\epsilon}$. But the curvature of the evolving manifold decays faster than $t^{-1}$. This says, as choosing $\epsilon>0$ small enough,

$$
\operatorname{diam}_{t}(M)^{2} \cdot|R m(\cdot, t)| \rightarrow 0, \text { as } t \rightarrow+\infty .
$$

Then it is well-known from Cheeger-Gromov [54] that the manifold is a nilmanifold and the injectivity radius condition can not be satisfied as $t$ large enough. When we are considering the Ricci flow on a complete noncompact manifold with nonnegative curvature operator or on a complete noncompact Kähler manifold with nonnegative holomorphic bisectional curvature, Li-Yau-Hamilton inequalities imply that $t R(x, t)$ is increasing in time $t$. Then Type III(b) occurs only when the solution is a trivial flat metric.

For each type of solution we define a corresponding type of limiting singularity model.

Definition 4.3.3. A solution $g_{i j}(x, t)$ to the Ricci flow on the manifold $M$, where either $M$ is compact or at each time $t$ the metric $g_{i j}(\cdot, t)$ is complete and has bounded curvature, is called a singularity model if it is not flat and of one of the following three types:

Type I: The solution exists for $t \in(-\infty, \Omega)$ for some constant $\Omega$ with $0<\Omega<+\infty$ and

$$
|R m| \leq \Omega /(\Omega-t)
$$

everywhere with equality somewhere at $t=0$; 
Type II: The solution exists for $t \in(-\infty,+\infty)$ and

$$
|R m| \leq 1
$$

everywhere with equality somewhere at $t=0$;

Type III: The solution exists for $t \in(-A,+\infty)$ for some constant $A$ with $0<A<$ $+\infty$ and

$$
|R m| \leq A /(A+t)
$$

everywhere with equality somewhere at $t=0$.

THEOREM 4.3.4. For any maximal solution to the Ricci flow which satisfies the injectivity radius condition and is of Type I, II(a), (b), or III(a), there exists a sequence of dilations of the solution along (almost) maximum points which converges in the $C_{\mathrm{loc}}^{\infty}$ topology to a singularity model of the corresponding type.

Proof.

Type I: We consider a maximal solution $g_{i j}(x, t)$ on $M \times[0, T)$ with $T<+\infty$ and

$$
\Omega \triangleq \limsup _{t \rightarrow T}(T-t) K_{\max }(t)<+\infty .
$$

First we note that $\Omega>0$. Indeed by the evolution equation of curvature,

$$
\frac{d}{d t} K_{\max }(t) \leq \text { Const } \cdot K_{\max }^{2}(t)
$$

This implies that

$$
K_{\max }(t) \cdot(T-t) \geq \text { Const }>0,
$$

because

$$
\limsup _{t \rightarrow T} K_{\max }(t)=+\infty
$$

Thus $\Omega$ must be positive.

Choose a sequence of points $x_{k}$ and times $t_{k}$ such that $t_{k} \rightarrow T$ and

$$
\lim _{k \rightarrow \infty}\left(T-t_{k}\right)\left|R m\left(x_{k}, t_{k}\right)\right|=\Omega .
$$

Denote by

$$
\epsilon_{k}=\frac{1}{\sqrt{\left|R m\left(x_{k}, t_{k}\right)\right|}}
$$

We translate in time so that $t_{k}$ becomes 0 , dilate in space by the factor $\epsilon_{k}$ and dilate in time by $\epsilon_{k}^{2}$ to get

$$
\tilde{g}_{i j}^{(k)}(\cdot, \tilde{t})=\epsilon_{k}^{-2} g_{i j}\left(\cdot, t_{k}+\epsilon_{k}^{2} \tilde{t}\right), \quad \tilde{t} \in\left[-t_{k} / \epsilon_{k}^{2},\left(T-t_{k}\right) / \epsilon_{k}^{2}\right) .
$$


Then

$$
\begin{aligned}
\frac{\partial}{\partial \tilde{t}} \tilde{g}_{i j}^{(k)}(\cdot, \tilde{t}) & =\epsilon_{k}^{-2} \frac{\partial}{\partial t} g_{i j}(\cdot, t) \cdot \epsilon_{k}^{2} \\
& =-2 R_{i j}\left(\cdot, t_{k}+\epsilon_{k}^{2} \tilde{t}\right) \\
& =-2 \tilde{R}_{i j}^{(k)}(\cdot, \tilde{t}),
\end{aligned}
$$

where $\tilde{R}_{i j}^{(k)}$ is the Ricci curvature of the metric $\tilde{g}_{i j}^{(k)}$. So $\tilde{g}_{i j}^{(k)}(\cdot, \tilde{t})$ is still a solution to the Ricci flow which exists on the time interval $\left[-t_{k} / \epsilon_{k}^{2},\left(T-t_{k}\right) / \epsilon_{k}^{2}\right)$, where

$$
t_{k} / \epsilon_{k}^{2}=t_{k}\left|R m\left(x_{k}, t_{k}\right)\right| \rightarrow+\infty
$$

and

$$
\left(T-t_{k}\right) / \epsilon_{k}^{2}=\left(T-t_{k}\right)\left|R m\left(x_{k}, t_{k}\right)\right| \rightarrow \Omega .
$$

For any $\epsilon>0$ we can find a time $\tau<T$ such that for $t \in[\tau, T)$,

$$
|R m| \leq(\Omega+\epsilon) /(T-t)
$$

by the assumption. Then for $\tilde{t} \in\left[\left(\tau-t_{k}\right) / \epsilon_{k}^{2},\left(T-t_{k}\right) / \epsilon_{k}^{2}\right)$, the curvature of $\tilde{g}_{i j}^{(k)}(\cdot, \tilde{t})$ is bounded by

$$
\begin{aligned}
\left|\tilde{R} m^{(k)}\right| & =\epsilon_{k}^{2}|R m| \\
& \leq(\Omega+\epsilon) /\left((T-t)\left|R m\left(x_{k}, t_{k}\right)\right|\right) \\
& =(\Omega+\epsilon) /\left(\left(T-t_{k}\right)\left|R m\left(x_{k}, t_{k}\right)\right|+\left(t_{k}-t\right)\left|R m\left(x_{k}, t_{k}\right)\right|\right) \\
& \rightarrow(\Omega+\epsilon) /(\Omega-\tilde{t}), \quad \text { as } k \rightarrow+\infty .
\end{aligned}
$$

This implies that $\left\{\left(x_{k}, t_{k}\right)\right\}$ is a sequence of (almost) maximum points. And then by the injectivity radius condition and Hamilton's compactness theorem 4.1.5, there exists a subsequence of the metrics $\tilde{g}_{i j}^{(k)}(\tilde{t})$ which converges in the $C_{l o c}^{\infty}$ topology to a limit metric $\tilde{g}_{i j}^{(\infty)}(\tilde{t})$ on a limiting manifold $\tilde{M}$ with $\tilde{t} \in(-\infty, \Omega)$ such that $\tilde{g}_{i j}^{(\infty)}(\tilde{t})$ is a complete solution of the Ricci flow and its curvature satisfies the bound

$$
\left|\tilde{R} m^{(\infty)}\right| \leq \Omega /(\Omega-\tilde{t})
$$

everywhere on $\tilde{M} \times(-\infty, \Omega)$ with the equality somewhere at $\tilde{t}=0$.

Type II(a): We consider a maximal solution $g_{i j}(x, t)$ on $M \times[0, T)$ with

$$
T<+\infty \quad \text { and } \quad \limsup _{t \rightarrow T}(T-t) K_{\max }(t)=+\infty .
$$

Let $T_{k}<T<+\infty$ with $T_{k} \rightarrow T$, and $\gamma_{k} \nearrow 1$, as $k \rightarrow+\infty$. Pick points $x_{k}$ and times $t_{k}$ such that, as $k \rightarrow+\infty$,

$$
\left(T_{k}-t_{k}\right)\left|R m\left(x_{k}, t_{k}\right)\right| \geq \gamma_{k} \sup _{x \in M, t \leq T_{k}}\left(T_{k}-t\right)|R m(x, t)| \rightarrow+\infty .
$$

Again denote by

$$
\epsilon_{k}=\frac{1}{\sqrt{\left|R m\left(x_{k}, t_{k}\right)\right|}}
$$


and dilate the solution as before to get

$$
\tilde{g}_{i j}^{(k)}(\cdot, \tilde{t})=\epsilon_{k}^{-2} g_{i j}\left(\cdot, t_{k}+\epsilon_{k}^{2} \tilde{t}\right), \quad \tilde{t} \in\left[-t_{k} / \epsilon_{k}^{2},\left(T_{k}-t_{k}\right) / \epsilon_{k}^{2}\right),
$$

which is still a solution to the Ricci flow and satisfies the curvature bound

$$
\begin{aligned}
\left|\tilde{R} m^{(k)}\right| & =\epsilon_{k}^{2}|R m| \\
& \leq \frac{1}{\gamma_{k}} \cdot \frac{\left(T_{k}-t_{k}\right)}{\left(T_{k}-t\right)} \\
& =\frac{1}{\gamma_{k}} \frac{\left(T_{k}-t_{k}\right)\left|R m\left(x_{k}, t_{k}\right)\right|}{\left[\left(T_{k}-t_{k}\right)\left|R m\left(x_{k}, t_{k}\right)\right|-\tilde{t}\right]} \quad \text { for } \tilde{t} \in\left[-\frac{t_{k}}{\epsilon_{k}^{2}}, \frac{\left(T_{k}-t_{k}\right)}{\epsilon_{k}^{2}}\right),
\end{aligned}
$$

since $t=t_{k}+\epsilon_{k}^{2} \tilde{t}$ and $\epsilon_{k}=1 / \sqrt{\left|R m\left(x_{k}, t_{k}\right)\right|}$. Hence $\left\{\left(x_{k}, t_{k}\right)\right\}$ is a sequence of (almost) maximum points. And then as before, by applying Hamilton's compactness theorem 4.1.5, there exists a subsequence of the metrics $\tilde{g}_{i j}^{(k)}(\tilde{t})$ which converges in the $C_{l o c}^{\infty}$ topology to a limit $\tilde{g}_{i j}^{(\infty)}(\tilde{t})$ on a limiting manifold $\tilde{M}$ and $\tilde{t} \in(-\infty,+\infty)$ such that $\tilde{g}_{i j}^{(\infty)}(\tilde{t})$ is a complete solution of the Ricci flow and its curvature satisfies

$$
\left|\tilde{R} m^{(\infty)}\right| \leq 1
$$

everywhere on $\tilde{M} \times(-\infty,+\infty)$ and the equality holds somewhere at $\tilde{t}=0$.

Type II(b): We consider a maximal solution $g_{i j}(x, t)$ on $M \times[0, T)$ with

$$
T=+\infty \quad \text { and } \quad \limsup _{t \rightarrow T} t K_{\max }(t)=+\infty .
$$

Again let $T_{k} \rightarrow T=+\infty$, and $\gamma_{k} \nearrow 1$, as $k \rightarrow+\infty$. Pick $x_{k}$ and $t_{k}$ such that

$$
t_{k}\left(T_{k}-t_{k}\right)\left|R m\left(x_{k}, t_{k}\right)\right| \geq \gamma_{k} \sup _{x \in M, t \leq T_{k}} t\left(T_{k}-t\right)|R m(x, t)| .
$$

Define

$$
\tilde{g}_{i j}^{(k)}(\cdot, \tilde{t})=\epsilon_{k}^{-2} g_{i j}\left(\cdot, t_{k}+\epsilon_{k}^{2} \tilde{t}\right), \quad \tilde{t} \in\left[-t_{k} / \epsilon_{k}^{2},\left(T_{k}-t_{k}\right) / \epsilon_{k}^{2}\right),
$$

where $\epsilon_{k}=1 / \sqrt{\left|R m\left(x_{k}, t_{k}\right)\right|}$.

Since

$$
\begin{aligned}
t_{k}\left(T_{k}-t_{k}\right)\left|R m\left(x_{k}, t_{k}\right)\right| & \geq \gamma_{k} \sup _{x \in M, t \leq T_{k}} t\left(T_{k}-t\right)|\operatorname{Rm}(x, t)| \\
& \geq \gamma_{k} \sup _{x \in M, t \leq T_{k} / 2} t\left(T_{k}-t\right)|\operatorname{Rm}(x, t)| \\
& \geq \frac{T_{k}}{2} \gamma_{k} \sup _{x \in M, t \leq T_{k} / 2} t|\operatorname{Rm}(x, t)|,
\end{aligned}
$$

we have

$$
\frac{t_{k}}{\epsilon_{k}^{2}}=t_{k}\left|R m\left(x_{k}, t_{k}\right)\right| \geq \frac{\gamma_{k}}{2}\left(\frac{T_{k}}{T_{k}-t_{k}}\right) \sup _{x \in M, t \leq T_{k} / 2} t|R m(x, t)| \rightarrow+\infty,
$$

and

$$
\frac{\left(T_{k}-t_{k}\right)}{\epsilon_{k}^{2}}=\left(T_{k}-t_{k}\right)\left|R m\left(x_{k}, t_{k}\right)\right| \geq \frac{\gamma_{k}}{2}\left(\frac{T_{k}}{t_{k}}\right) \sup _{x \in M, t \leq T_{k} / 2} t|R m(x, t)| \rightarrow+\infty,
$$


as $k \rightarrow+\infty$. As before, we also have

$$
\frac{\partial}{\partial \tilde{t}^{(k)}}(\cdot, \tilde{t})=-2 \tilde{R}_{i j}^{(k)}(\cdot, \tilde{t})
$$

and

$$
\begin{aligned}
& \left|\tilde{R} m^{(k)}\right| \\
& =\epsilon_{k}^{2}|R m| \\
& \leq \frac{1}{\gamma_{k}} \cdot \frac{t_{k}\left(T_{k}-t_{k}\right)}{t\left(T_{k}-t\right)} \\
& =\frac{1}{\gamma_{k}} \cdot \frac{t_{k}\left(T_{k}-t_{k}\right)\left|R m\left(x_{k}, t_{k}\right)\right|}{\left(t_{k}+\epsilon_{k}^{2} \tilde{t}\right)\left[\left(T_{k}-t_{k}\right)-\epsilon_{k}^{2} \tilde{t}\right] \cdot\left|R m\left(x_{k}, t_{k}\right)\right|} \\
& =\frac{1}{\gamma_{k}} \cdot \frac{t_{k}\left(T_{k}-t_{k}\right)\left|R m\left(x_{k}, t_{k}\right)\right|}{\left(t_{k}+\epsilon_{k}^{2} \tilde{t}\right)\left[\left(T_{k}-t_{k}\right)\left|R m\left(x_{k}, t_{k}\right)\right|-\tilde{t}\right]} \\
& =\frac{t_{k}\left(T_{k}-t_{k}\right)\left|R m\left(x_{k}, t_{k}\right)\right|}{\gamma_{k}\left(1+\tilde{t} /\left(t_{k}\left|R m\left(x_{k}, t_{k}\right)\right|\right)\right)\left[t_{k}\left(T_{k}-t_{k}\right)\left|\operatorname{Rm}\left(x_{k}, t_{k}\right)\right|\right]\left(1-\tilde{t} /\left(\left(T_{k}-t_{k}\right)\left|\operatorname{Rm}\left(x_{k}, t_{k}\right)\right|\right)\right)} \\
& \rightarrow 1, \quad \text { as } k \rightarrow+\infty .
\end{aligned}
$$

Hence $\left\{\left(x_{k}, t_{k}\right)\right\}$ is again a sequence of (almost) maximum points. As before, there exists a subsequence of the metrics $\tilde{g}_{i j}^{(k)}(\tilde{t})$ which converges in the $C_{l o c}^{\infty}$ topology to a limit $\tilde{g}_{i j}^{(\infty)}(\tilde{t})$ on a limiting manifold $\tilde{M}$ and $\tilde{t} \in(-\infty,+\infty)$ such that $\tilde{g}_{i j}^{(\infty)}(\tilde{t})$ is a complete solution of the Ricci flow and its curvature satisfies

$$
\left|\tilde{R} m^{(\infty)}\right| \leq 1
$$

everywhere on $\tilde{M} \times(-\infty,+\infty)$ with the equality somewhere at $\tilde{t}=0$.

Type III(a): We consider a maximal solution $g_{i j}(x, t)$ on $M \times[0, T)$ with $T=+\infty$ and

$$
\limsup _{t \rightarrow T} t K_{\max }(t)=A \in(0,+\infty) .
$$

Choose a sequence of $x_{k}$ and $t_{k}$ such that $t_{k} \rightarrow+\infty$ and

$$
\lim _{k \rightarrow \infty} t_{k}\left|R m\left(x_{k}, t_{k}\right)\right|=A .
$$

Set $\epsilon_{k}=1 / \sqrt{\left|R m\left(x_{k}, t_{k}\right)\right|}$ and dilate the solution as before to get

$$
\tilde{g}_{i j}^{(k)}(\cdot, \tilde{t})=\epsilon_{k}^{-2} g_{i j}\left(\cdot, t_{k}+\epsilon_{k}^{2} \tilde{t}\right), \quad \tilde{t} \in\left[-t_{k} / \epsilon_{k}^{2},+\infty\right)
$$

which is still a solution to the Ricci flow. Also, for arbitrarily fixed $\epsilon>0$, there exists a sufficiently large positive constant $\tau$ such that for $t \in[\tau,+\infty)$,

$$
\begin{aligned}
\left|\tilde{R} m^{(k)}\right| & =\epsilon_{k}^{2}|R m| \\
& \leq \epsilon_{k}^{2}\left(\frac{A+\epsilon}{t}\right) \\
& =\epsilon_{k}^{2}\left(\frac{A+\epsilon}{t_{k}+\epsilon_{k}^{2} \tilde{t}}\right) \\
& =(A+\epsilon) /\left(t_{k}\left|R m\left(x_{k}, t_{k}\right)\right|+\tilde{t}\right), \quad \text { for } \tilde{t} \in\left[\left(\tau-t_{k}\right) / \epsilon_{k}^{2},+\infty\right) .
\end{aligned}
$$


Note that

$$
(A+\epsilon) /\left(t_{k}\left|\operatorname{Rm}\left(x_{k}, t_{k}\right)\right|+\tilde{t}\right) \rightarrow(A+\epsilon) /(A+\tilde{t}), \quad \text { as } k \rightarrow+\infty
$$

and

$$
\left(\tau-t_{k}\right) / \epsilon_{k}^{2} \rightarrow-A, \quad \text { as } k \rightarrow+\infty .
$$

Hence $\left\{\left(x_{k}, t_{k}\right)\right\}$ is a sequence of (almost) maximum points. And then as before, there exists a subsequence of the metrics $\tilde{g}_{i j}^{(k)}(\tilde{t})$ which converges in the $C_{l o c}^{\infty}$ topology to a limit $\tilde{g}_{i j}^{(\infty)}(\tilde{t})$ on a limiting manifold $\tilde{M}$ and $\tilde{t} \in(-A,+\infty)$ such that $\tilde{g}_{i j}^{(\infty)}(\tilde{t})$ is a complete solution of the Ricci flow and its curvature satisfies

$$
\left|\tilde{R} m^{(\infty)}\right| \leq A /(A+\tilde{t})
$$

everywhere on $\tilde{M} \times(-A,+\infty)$ with the equality somewhere at $\tilde{t}=0$.

In the case of manifolds with nonnegative curvature operator, or Kähler metrics with nonnegative holomorphic bisectional curvature, we can bound the Riemannian curvature by the scalar curvature $R$ upto a constant factor depending only on the dimension. Then we can slightly modify the statements in the previous theorem as follows

Corollary 4.3.5. For any complete maximal solution to the Ricci flow with bounded and nonnegative curvature operator on a Riemannian manifold, or on a Kähler manifold with bounded and nonnegative holomorphic bisectional curvature, there exists a sequence of dilations of the solution along (almost) maximum points which converges to a singular model.

For Type I solutions: the limit model exists for $t \in(-\infty, \Omega)$ with $0<\Omega<+\infty$ and has

$$
R \leq \Omega /(\Omega-t)
$$

everywhere with equality somewhere at $t=0$.

For Type II solutions: the limit model exists for $t \in(-\infty,+\infty)$ and has

$$
R \leq 1
$$

everywhere with equality somewhere at $t=0$.

For Type III solutions: the limit model exists for $t \in(-A,+\infty)$ with $0<A<+\infty$ and has

$$
R \leq A /(A+t)
$$

everywhere with equality somewhere at $t=0$.

A natural and important question is to understand each of the three types of singularity models. The following results obtained by Hamilton [66] and Chen-Zhu [30] characterize the Type II and Type III singularity models with nonnegative curvature operator and positive Ricci curvature respectively. The corresponding results in the Kähler case with nonnegative holomorphic bisectional curvature were obtained by the first author [14]. 
THEOREM 4.3.6.

(i) (Hamilton [66]) Any Type II singularity model with nonnegative curvature operator and positive Ricci curvature to the Ricci flow on a manifold M must be a (steady) Ricci soliton.

(ii) (Chen-Zhu [30]) Any Type III singularity model with nonnegative curvature operator and positive Ricci curvature on a manifold $M$ must be a homothetically expanding Ricci soliton.

Proof. We only give the proof of (ii), since the proof of (i) is similar and easier.

After a shift of the time variable, we may assume the Type III singularity model is defined on $0<t<+\infty$ and $t R$ assumes its maximum in space-time.

Recall from the Li-Yau-Hamilton inequality (Theorem 2.5.4) that for any vectors $V^{i}$ and $W^{i}$,

$$
M_{i j} W^{i} W^{j}+\left(P_{k i j}+P_{k j i}\right) V^{k} W^{i} W^{j}+R_{i k j l} W^{i} W^{j} V^{k} V^{l} \geq 0
$$

where

$$
M_{i j}=\Delta R_{i j}-\frac{1}{2} \nabla_{i} \nabla_{j} R+2 R_{i p j q} R^{p q}-g^{p q} R_{i p} R_{j q}+\frac{1}{2 t} R_{i j}
$$

and

$$
P_{i j k}=\nabla_{i} R_{j k}-\nabla_{j} R_{i k}
$$

Take the trace on $W$ to get

$$
Q \triangleq \frac{\partial R}{\partial t}+\frac{R}{t}+2 \nabla_{i} R \cdot V^{i}+2 R_{i j} V^{i} V^{j} \geq 0
$$

for any vector $V^{i}$. Let us choose $V$ to be the vector field minimizing $Q$, i.e.,

$$
V^{i}=-\frac{1}{2}\left(\operatorname{Ric}^{-1}\right)^{i k} \nabla_{k} R
$$

where $\left(R i c^{-1}\right)^{i k}$ is the inverse of the Ricci tensor $R_{i j}$. Substitute this vector field $V$ into $Q$ to get a smooth function $\tilde{Q}$. By a direct computation from the evolution equations of curvatures (see [61] for details),

$$
\frac{\partial}{\partial t} \tilde{Q} \geq \Delta \tilde{Q}-\frac{2}{t} \tilde{Q}
$$

Suppose $t R$ assumes its maximum at $\left(x_{0}, t_{0}\right)$ with $t_{0}>0$, then

$$
\frac{\partial R}{\partial t}+\frac{R}{t}=0, \quad \text { at } \quad\left(x_{0}, t_{0}\right)
$$

This implies that the quantity

$$
Q=\frac{\partial R}{\partial t}+\frac{R}{t}+2 \nabla_{i} R \cdot V^{i}+2 R_{i j} V^{i} V^{j}
$$

vanishes in the direction $V=0$ at $\left(x_{0}, t_{0}\right)$. We claim that for any earlier time $t<t_{0}$ and any point $x \in M$, there is a vector $V \in T_{x} M$ such that $Q=0$. 
We argue by contradiction. Suppose not, then there is $\bar{x} \in M$ and $0<\bar{t}<t_{0}$ such that $\tilde{Q}$ is positive at $x=\bar{x}$ and $t=\bar{t}$. We can find a nonnegative smooth function $\rho$ on $M$ with support in a neighborhood of $\bar{x}$ so that $\rho(\bar{x})>0$ and

$$
\tilde{Q} \geq \frac{\rho}{\overline{t^{2}}}
$$

at $t=\bar{t}$. Let $\rho$ evolve by the heat equation

$$
\frac{\partial \rho}{\partial t}=\Delta \rho
$$

It then follows from the standard strong maximum principle that $\rho>0$ everywhere for any $t>\bar{t}$. From (4.3.4) we see that

$$
\frac{\partial}{\partial t}\left(\tilde{Q}-\frac{\rho}{t^{2}}\right) \geq \Delta\left(\tilde{Q}-\frac{\rho}{t^{2}}\right)-\frac{2}{t}\left(\tilde{Q}-\frac{\rho}{t^{2}}\right)
$$

Then by the maximum principle as in Chapter 2, we get

$$
\tilde{Q} \geq \frac{\rho}{t^{2}}>0, \quad \text { for all } t \geq \bar{t}
$$

This gives a contradiction with the fact $Q=0$ for $V=0$ at $\left(x_{0}, t_{0}\right)$. We thus prove the claim.

Consider each time $t<t_{0}$. The null vector field of $Q$ satisfies the equation

$$
\nabla_{i} R+2 R_{i j} V^{j}=0
$$

by the first variation of $Q$ in $V$. Since $R_{i j}$ is positive, we see that such a null vector field is unique and varies smoothly in space-time.

Substituting (4.3.5) into the expression of $Q$, we have

$$
\frac{\partial R}{\partial t}+\frac{R}{t}+\nabla_{i} R \cdot V^{i}=0
$$

Denote by

$$
Q_{i j}=M_{i j}+\left(P_{k i j}+P_{k j i}\right) V^{k}+R_{i k j l} V^{k} V^{l} .
$$

From (4.3.1) we see that $Q_{i j}$ is nonnegative definite with its trace $Q=0$ for such a null vector $V$. It follows that

$$
Q_{i j}=M_{i j}+\left(P_{k i j}+P_{k j i}\right) V^{k}+R_{i k j l} V^{k} V^{l}=0 .
$$

Again from the first variation of $Q_{i j}$ in $V$, we see that

$$
\left(P_{k i j}+P_{k j i}\right)+\left(R_{i k j l}+R_{j k i l}\right) V^{l}=0,
$$

and hence

$$
M_{i j}-R_{i k j l} V^{k} V^{l}=0 .
$$

Applying the heat operator to (4.3.5) and (4.3.6) we get

$$
\begin{aligned}
0= & \left(\frac{\partial}{\partial t}-\Delta\right)\left(\nabla_{i} R+2 R_{i j} V^{j}\right) \\
= & 2 R_{i j}\left(\frac{\partial}{\partial t}-\Delta\right) V^{j}+\left(\frac{\partial}{\partial t}-\Delta\right)\left(\nabla_{i} R\right) \\
& +2 V^{j}\left(\frac{\partial}{\partial t}-\Delta\right) R_{i j}-4 \nabla_{k} R_{i j} \nabla^{k} V^{j}
\end{aligned}
$$


and

$$
\begin{aligned}
0= & \left(\frac{\partial}{\partial t}-\Delta\right)\left(\frac{\partial R}{\partial t}+\frac{R}{t}+\nabla_{i} R \cdot V^{i}\right) \\
= & \nabla_{i} R\left(\frac{\partial}{\partial t}-\Delta\right) V^{i}+V^{i}\left(\frac{\partial}{\partial t}-\Delta\right)\left(\nabla_{i} R\right)-2 \nabla_{k} \nabla_{i} R \cdot \nabla^{k} V^{i} \\
& +\left(\frac{\partial}{\partial t}-\Delta\right)\left(\frac{\partial R}{\partial t}+\frac{R}{t}\right) .
\end{aligned}
$$

Multiplying (4.3.9) by $V^{i}$, summing over $i$ and adding (4.3.10), as well as using the evolution equations on curvature, we get

$$
\begin{aligned}
0= & 2 V^{i}\left(2 \nabla_{i}\left(|R c|^{2}\right)-R_{i l} \nabla^{l} R\right)+2 V^{i} V^{j}\left(2 R_{p i q j} R^{p q}-2 g^{p q} R_{p i} R_{q j}\right) \\
& -4 \nabla_{k} R_{i j} \cdot \nabla^{k} V^{j} \cdot V^{i}-2 \nabla_{k} \nabla_{i} R \cdot \nabla^{k} V^{i}+4 R^{i j} \nabla_{i} \nabla_{j} R \\
& +4 g^{k l} g^{m n} g^{p q} R_{k m} R_{n p} R_{q l}+4 R_{i j k l} R^{i k} V^{j} V^{l}-\frac{R}{t^{2}}
\end{aligned}
$$

From (4.3.5), we have the following equalities

$$
\left\{\begin{array}{l}
-2 V^{i} R_{i l} \nabla^{l} R-4 V^{i} V^{j} g^{p q} R_{p i} R_{q j}=0 \\
-4 \nabla_{k} R_{i j} \cdot \nabla^{k} V^{j} \cdot V^{i}-2 \nabla_{k} \nabla_{i} R \cdot \nabla^{k} V^{i}=4 R_{i j} \nabla_{k} V^{i} \cdot \nabla^{k} V^{j}, \\
\nabla_{i} \nabla_{j} R=-2 \nabla_{i} R_{j l} \cdot V^{l}-2 R_{j l} \nabla_{i} V^{l} .
\end{array}\right.
$$

Substituting (4.3.12) into (4.3.11), we obtain

$$
\begin{aligned}
& 8 R^{i j}\left(\nabla_{k} R_{i j} \cdot V^{k}+R_{i k j l} V^{k} V^{l}-\nabla_{i} R_{j l} \cdot V^{l}-R_{j l} \nabla_{i} V^{l}\right) \\
& \quad+4 R_{i j} \nabla_{k} V^{i} \cdot \nabla^{k} V^{j}+4 g^{k l} g^{m n} g^{p q} R_{k m} R_{n p} R_{q l}-\frac{R}{t^{2}}=0 .
\end{aligned}
$$

By using (4.3.7), we know

$$
R^{i j}\left(\nabla_{k} R_{i j} \cdot V^{k}+R_{i k j l} V^{k} V^{l}-\nabla_{i} R_{j l} \cdot V^{l}\right)=0 .
$$

Then we have

$$
-8 R^{i j} R_{j l} \nabla_{i} V^{l}+4 R_{i j} \nabla_{k} V^{i} \cdot \nabla^{k} V^{j}+4 g^{k l} g^{m n} g^{p q} R_{k m} R_{n p} R_{q l}-\frac{R}{t^{2}}=0 .
$$

By taking the trace in the last equality in (4.3.12) and using (4.3.6) and the evolution equation of the scalar curvature, we can get

$$
R^{i j}\left(R_{i j}+\frac{g_{i j}}{2 t}-\nabla_{i} V_{j}\right)=0
$$

Finally by combining (4.3.13) and (4.3.14), we deduce

$$
4 R^{i j} g^{k l}\left(R_{i k}+\frac{g_{i k}}{2 t}-\nabla_{k} V_{i}\right)\left(R_{j k}+\frac{g_{j k}}{2 t}-\nabla_{k} V_{j}\right)=0 .
$$

Since $R_{i j}$ is positive definite, we get

$$
\nabla_{i} V_{j}=R_{i j}+\frac{g_{i j}}{2 t}, \quad \text { for all } i, j .
$$


This means that $g_{i j}(t)$ is a homothetically expanding Ricci soliton.

REMARK 4.3.7. Recall from Section 1.5 that any compact steady Ricci soliton or expanding Ricci soliton must be Einstein. If the manifold $M$ in Theorem 4.3.6 is noncompact and simply connected, then the steady (or expanding) Ricci soliton must be a steady (or expanding) gradient Ricci soliton. For example, we know that $\nabla_{i} V_{j}$ is symmetric from (4.3.15). Also, by the simply connectedness of $M$ there exists a function $F$ such that

$$
\nabla_{i} \nabla_{j} F=\nabla_{i} V_{j}, \quad \text { on } M
$$

So

$$
R_{i j}=\nabla_{i} \nabla_{j} F-\frac{g_{i j}}{2 t}, \quad \text { on } M
$$

This means that $g_{i j}$ is an expanding gradient Ricci soliton.

In the Kähler case, we have the following results for Type II and Type III singularity models with nonnegative holomorphic bisectional curvature obtained by the first author in [14].

THEOREM 4.3.8 (Cao [14]).

(i) Any Type II singularity model on a Kähler manifold with nonnegative holomorphic bisectional curvature and positive Ricci curvature must be a steady Kähler-Ricci soliton.

(ii) Any Type III singularity model on a Kähler manifold with nonnegative holomorphic bisectional curvature and positive Ricci curvature must be an expanding Kähler-Ricci soliton.

To conclude this section, we state a result of Sesum [113] on compact Type I singularity models. Recall that Perelman's functional $\mathcal{W}$, introduced in Section 1.5, is given by

$$
\mathcal{W}(g, f, \tau)=\int_{M}(4 \pi \tau)^{-\frac{n}{2}}\left[\tau\left(|\nabla f|^{2}+R\right)+f-n\right] e^{-f} d V_{g}
$$

with the function $f$ satisfying the constraint

$$
\int_{M}(4 \pi \tau)^{-\frac{n}{2}} e^{-f} d V_{g}=1
$$

And recall from Corollary 1.5.9 that

$$
\mu(g(t))=\inf \left\{\mathcal{W}(g(t), f, T-t) \mid \int_{M}(4 \pi(T-t))^{-\frac{n}{2}} e^{-f} d V_{g(t)}=1\right\}
$$

is strictly increasing along the Ricci flow unless we are on a gradient shrinking soliton. If one can show that $\mu(g(t))$ is uniformly bounded from above and the minimizing functions $f=f(\cdot, t)$ have a limit as $t \rightarrow T$, then the rescaling limit model will be a shrinking gradient soliton. As shown by Natasa Sesum in [113], Type I assumption guarantees the boundedness of $\mu(g(t))$, while the compactness assumption of the rescaling limit guarantees the existence of the limit for the minimizing functions $f(\cdot, t)$. Therefore we have 
TheOREM 4.3.9 (Sesum [113]). Let $\left(M, g_{i j}(t)\right)$ be a Type I singularity model obtained as a rescaling limit of a Type I maximal solution. Suppose $M$ is compact. Then $\left(M, g_{i j}(t)\right)$ must be a gradient shrinking Ricci soliton.

It seems that the assumption on the compactness of the rescaling limit is superfluous. We conjecture that any noncompact Type I limit is also a gradient shrinking soliton.

4.4. Ricci Solitons. We will now examine the structure of a steady Ricci soliton of the sort we get as a Type II limit.

Lemma 4.4.1. Suppose we have a complete gradient steady Ricci soliton $g_{i j}$ with bounded curvature so that

$$
R_{i j}=\nabla_{i} \nabla_{j} F
$$

for some function $F$ on $M$. Assume the Ricci curvature is positive and the scalar curvature $R$ attains its maximum $R_{\max }$ at a point $x_{0} \in M$. Then

$$
|\nabla F|^{2}+R=R_{\max }
$$

everywhere on $M$, and furthermore $F$ is convex and attains its minimum at $x_{0}$.

Proof. Recall that, from (1.1.15) and noting our $F$ here is $-f$ there, the steady gradient Ricci soliton has the property

$$
|\nabla F|^{2}+R=C_{0}
$$

for some constant $C_{0}$. Clearly, $C_{0} \geq R_{\max }$.

If $C_{0}=R_{\max }$, then $\nabla F=0$ at the point $x_{0}$. Since $\nabla_{i} \nabla_{j} F=R_{i j}>0$, we see that $F$ is convex and $F$ attains its minimum at $x_{0}$.

If $C_{0}>R_{\max }$, consider a gradient path of $F$ in a local coordinate neighborhood through $x_{0}=\left(x_{0}^{1}, \ldots, x_{0}^{n}\right)$ :

$$
\left\{\begin{array}{l}
x^{i}=x^{i}(u), \quad u \in(-\varepsilon, \varepsilon), i=1, \ldots, n \\
x_{0}^{i}=x^{i}(0),
\end{array}\right.
$$

and

$$
\frac{d x^{i}}{d u}=g^{i j} \nabla_{j} F, \quad u \in(-\varepsilon, \varepsilon) .
$$

Now $|\nabla F|^{2}=C_{0}-R \geq C_{0}-R_{\max }>0$ everywhere, while $|\nabla F|^{2}$ is smallest at $x=x_{0}$ since $R$ is largest there. But we compute

$$
\begin{aligned}
\frac{d}{d u}|\nabla F|^{2} & =2 g^{j l}\left(\frac{d}{d u} \nabla_{j} F\right) \nabla_{l} F \\
& =2 g^{i k} g^{j l} \nabla_{i} \nabla_{j} F \cdot \nabla_{k} F \nabla_{l} F \\
& =2 g^{i k} g^{j l} R_{i j} \nabla_{k} F \nabla_{l} F \\
& >0
\end{aligned}
$$

since $R_{i j}>0$ and $|\nabla F|^{2}>0$. Then $|\nabla F|^{2}$ is not smallest at $x_{0}$, and we have a contradiction. $\square$ 
We remark that when we are considering a complete expanding gradient Ricci soliton on $M$ with positive Ricci curvature and

$$
R_{i j}+\rho g_{i j}=\nabla_{i} \nabla_{j} F
$$

for some constant $\rho>0$ and some function $F$, the above argument gives

$$
|\nabla F|^{2}+R-2 \rho F=C
$$

for some positive constant $C$. Moreover the function $F$ is an exhausting and convex function. In particular, such an expanding gradient Ricci soliton is diffeomorphic to the Euclidean space $\mathbb{R}^{n}$.

Let us introduce a geometric invariant as follows. Let $O$ be a fixed point in a Riemannian manifold $M, s$ the distance to the fixed point $O$, and $R$ the scalar curvature. We define the asymptotic scalar curvature ratio

$$
A=\limsup _{s \rightarrow+\infty} R s^{2} .
$$

Clearly the definition is independent of the choice of the fixed point $O$ and invariant under dilation. This concept is particular useful on manifolds with positive sectional curvature. The first type of gap theorem was obtained by Mok-Siu-Yau [93] in understanding the hypothesis of the paper of Siu-Yau [120]. Yau (see [49]) suggested that this should be a general phenomenon. This was later conformed by Greene-Wu [49, 50], Eschenberg-Shrader-Strake [45] and Drees [44] where they show that any complete noncompact $n$-dimensional (except $n=4$ or 8 ) Riemannian manifold of positive sectional curvature must have $A>0$. Similar results on complete noncompact Kähler manifolds of positive holomorphic bisectional curvature were obtained by Chen-Zhu [31] and Ni-Tam [100].

THEOREM 4.4.2 (Hamilton [63]). For a complete noncompact steady gradient Ricci soliton with bounded curvature and positive sectional curvature of dimension $n \geq$ 3 where the scalar curvature assume its maximum at a point $O \in M$, the asymptotic scalar curvature ratio is infinite, i.e.,

$$
A=\limsup _{s \rightarrow+\infty} R s^{2}=+\infty
$$

where $s$ is the distance to the point $O$.

Proof. The solution to the Ricci flow corresponding to the soliton exists for $-\infty<t<+\infty$ and is obtained by flowing along the gradient of a potential function $F$ of the soliton. We argue by contradiction. Suppose $R s^{2} \leq C$. We will show that the limit

$$
\bar{g}_{i j}(x)=\lim _{t \rightarrow-\infty} g_{i j}(x, t)
$$

exists for $x \neq O$ on the manifold $M$ and is a complete flat metric on $M \backslash\{O\}$. Since the sectional curvature of $M$ is positive everywhere, it follows from Cheeger-Gromoll [23] that $M$ is diffeomorphic to $\mathbb{R}^{n}$. Thus $M \backslash\{O\}$ is diffeomorphic to $\mathbb{S}^{n-1} \times \mathbb{R}$. But for $n \geq 3$ there is no flat metric on $\mathbb{S}^{n-1} \times \mathbb{R}$, and this will finish the proof. 
To see the limit metric exists, we note that $R \rightarrow 0$ as $s \rightarrow+\infty$, so $|\nabla F|^{2} \rightarrow R_{\max }$ as $s \rightarrow+\infty$ by (4.4.1). The function $F$ itself can be taken to evolve with time, using the definition

$$
\frac{\partial F}{\partial t}=\nabla_{i} F \cdot \frac{\partial x^{i}}{\partial t}=-|\nabla F|^{2}=\Delta F-R_{\max }
$$

which pulls $F$ back by the flow along the gradient of $F$. Then we continue to have $\nabla_{i} \nabla_{j} F=R_{i j}$ for all time, and $|\nabla F|^{2} \rightarrow R_{\max }$ as $s \rightarrow+\infty$ for each time.

When we go backward in time, this is equivalent to flowing outwards along the gradient of $F$, and our speed approaches $\sqrt{R_{\max }}$. So, starting outside of any neighborhood of $O$ we have

$$
\frac{s}{|t|}=\frac{d_{t}(\cdot, O)}{|t|} \rightarrow \sqrt{R_{\max }}, \quad \text { as } t \rightarrow-\infty
$$

and

$$
R(\cdot, t) \leq \frac{C}{R_{\max } \cdot|t|^{2}}, \quad \text { as }|t| \text { large enough. }
$$

Hence for $|t|$ sufficiently large,

$$
\begin{aligned}
0 & \geq-2 R_{i j} \\
& =\frac{\partial}{\partial t} g_{i j} \\
& \geq-2 R g_{i j} \\
& \geq-\frac{2 C}{R_{\max } \cdot|t|^{2}} g_{i j}
\end{aligned}
$$

which implies that for any tangent vector $V$,

$$
0 \leq \frac{d}{d|t|}\left(\log \left(g_{i j}(t) V^{i} V^{j}\right)\right) \leq \frac{2 C}{R_{\max } \cdot|t|^{2}} .
$$

These two inequalities show that $g_{i j}(t) V^{i} V^{j}$ has a limit $\bar{g}_{i j} V^{i} V^{j}$ as $t \rightarrow-\infty$.

Since the metrics are all essentially the same, it always takes an infinite length to get out to the infinity. This shows the limit $\bar{g}_{i j}$ is complete at the infinity. One the other hand, any point $P$ other than $O$ will eventually be arbitrarily far from $O$, so the limit metric $\bar{g}_{i j}$ is also complete away from $O$ in $M \backslash\{O\}$. Using Shi's derivative estimates in Chapter 1 , it follows that $g_{i j}(\cdot, t)$ converges in the $C_{\text {loc }}^{\infty}$ topology to a complete smooth limit metric $\bar{g}_{i j}$ as $t \rightarrow-\infty$, and the limit metric is flat by (4.4.2). $\mathrm{\square}$

The above argument actually shows that

$$
\limsup _{s \rightarrow+\infty} R s^{1+\varepsilon}=+\infty
$$

for arbitrarily small $\varepsilon>0$ and for any complete gradient Ricci soliton with bounded and positive sectional curvature of dimension $n \geq 3$ where the scalar curvature assumes its maximum at a fixed point $O$.

Finally we conclude this section with the important uniqueness of complete Ricci soliton on two-dimensional Riemannian manifolds. 
THEOREM 4.4.3 (Hamilton [60]). The only complete steady Ricci soliton on a two-dimensional manifold with bounded curvature which assumes its maximum 1 at an origin is the "cigar" soliton on the plane $\mathbb{R}^{2}$ with the metric

$$
d s^{2}=\frac{d x^{2}+d y^{2}}{1+x^{2}+y^{2}}
$$

Proof. Recall that the scalar curvature evolves by

$$
\frac{\partial R}{\partial t}=\Delta R+R^{2}
$$

on a two-dimensional manifold $M$. Denote by $R_{\min }(t)=\inf \{R(x, t) \mid x \in M\}$. We see from the maximum principle (see for example Chapter 2) that $R_{\min }(t)$ is strictly increasing whenever $R_{\min }(t) \neq 0$, for $-\infty<t<+\infty$. This shows that the curvature of a steady Ricci soliton on a two-dimensional manifold $M$ must be nonnegative and $R_{\min }(t)=0$ for all $t \in(-\infty,+\infty)$. Further by the strong maximum principle we see that the curvature is actually positive everywhere. In particular, the manifold must be noncompact. So the manifold $M$ is diffiomorphic to $\mathbb{R}^{2}$ and the Ricci soliton must be a gradient soliton. Let $F$ be a potential function of the gradient Ricci soliton. Then, by definition, we have

$$
\nabla_{i} V_{j}+\nabla_{j} V_{i}=R g_{i j}
$$

with $V_{i}=\nabla_{i} F$. This says that the vector field $V$ must be conformal. In complex coordinate a conformal vector field is holomorphic. Hence $V$ is locally given by $V(z) \frac{\partial}{\partial z}$ for a holomorphic function $V(z)$. At a zero of $V$ there will be a power series expansion

$$
V(z)=a z^{p}+\cdots,(a \neq 0)
$$

and if $p>1$ the vector field will have closed orbits in any neighborhood of the zero. Now the vector field is gradient and a gradient flow cannot have a closed orbit. Hence $V(z)$ has only simple zeros. By Lemma 4.4.1, we know that $F$ is strictly convex with the only critical point being the minima, chosen to be the origin of $\mathbb{R}^{2}$. So the holomorphic vector field $V$ must be

$$
V(z) \frac{\partial}{\partial z}=c z \frac{\partial}{\partial z}, \quad \text { for } z \in C,
$$

for some complex number $c$.

We now claim that $c$ is real. Let us write the metric as

$$
d s^{2}=g(x, y)\left(d x^{2}+d y^{2}\right)
$$

with $z=x+\sqrt{-1} y$. Then $\nabla F=c z \frac{\partial}{\partial z}$ means that if $c=a+\sqrt{-1} b$, then

$$
\frac{\partial F}{\partial x}=(a x-b y) g, \quad \frac{\partial F}{\partial y}=(b x+a y) g
$$

Taking the mixed partial derivatives $\frac{\partial^{2} F}{\partial x \partial y}$ and equating them at the origin $x=y=0$ gives $b=0$, so $c$ is real. 
Let

$$
\begin{cases}x=e^{u} \cos v, & -\infty<u<+\infty, \\ y=e^{u} \sin v, & 0 \leq v \leq 2 \pi\end{cases}
$$

Write

$$
\begin{aligned}
d s^{2} & =g(x, y)\left(d x^{2}+d y^{2}\right) \\
& =g\left(e^{u} \cos v, e^{u} \sin v\right) e^{2 u}\left(d u^{2}+d v^{2}\right) \\
& \triangleq g(u, v)\left(d u^{2}+d v^{2}\right) .
\end{aligned}
$$

Then we get the equations

$$
\frac{\partial F}{\partial u}=a g, \quad \frac{\partial F}{\partial v}=0
$$

since the gradient of $F$ is just $a \frac{\partial}{\partial u}$ for a real constant $a$. The second equation shows that $F=F(u)$ is a function of $u$ only, then the first equation shows that $g=g(u)$ is also a function of $u$ only. Then we can write the metric as

$$
\begin{aligned}
d s^{2} & =g(u)\left(d u^{2}+d v^{2}\right) \\
& =g(u) e^{-2 u}\left(d x^{2}+d y^{2}\right) .
\end{aligned}
$$

This implies that $e^{-2 u} g(u)$ must be a smooth function of $x^{2}+y^{2}=e^{2 u}$. So as $u \rightarrow-\infty$,

$$
g(u)=b_{1} e^{2 u}+b_{2}\left(e^{2 u}\right)^{2}+\cdots
$$

with $b_{1}>0$.

The curvature of the metric is given by

$$
R=-\frac{1}{g}\left(\frac{g^{\prime}}{g}\right)^{\prime}
$$

where $(\cdot)^{\prime}$ is the derivative with respect to $u$. Note that the soliton is by translation in $u$ with velocity $c$. Hence $g=g(u+c t)$ satisfies

$$
\frac{\partial g}{\partial t}=-R g
$$

which becomes

$$
c g^{\prime}=\left(\frac{g^{\prime}}{g}\right)^{\prime} .
$$

Thus by (4.4.5),

$$
\frac{g^{\prime}}{g}=c g+2
$$

and then by integrating

$$
e^{2 u}\left(\frac{1}{g}\right)=-\frac{c}{2} e^{2 u}+b_{1}
$$


i.e.,

$$
g(u)=\frac{e^{2 u}}{b_{1}-\frac{c}{2} e^{2 u}}
$$

In particular, we have $c<0$ since the Ricci soliton is not flat. Therefore

$$
d s^{2}=g(u) e^{-2 u}\left(d x^{2}+d y^{2}\right)=\frac{d x^{2}+d y^{2}}{\alpha_{1}+\alpha_{2}\left(x^{2}+y^{2}\right)}
$$

for some constants $\alpha_{1}, \alpha_{2}>0$. By the normalization condition that the curvature attains its maximum 1 at the origin, we conclude that

$$
d s^{2}=\frac{d x^{2}+d y^{2}}{1+\left(x^{2}+y^{2}\right)}
$$

5. Long Time Behaviors. Let $M$ be a complete manifold of dimension $n$. Consider a solution of the Ricci flow $g_{i j}(x, t)$ on $M$ and on a maximal time interval $[0, T)$. When $M$ is compact, we usually consider the normalized Ricci flow

$$
\frac{\partial g_{i j}}{\partial t}=\frac{2}{n} r g_{i j}-2 R_{i j}
$$

where $r=\int_{M} R d V / \int_{M} d V$ is the average scalar curvature. The factor $r$ serves to normalize the Ricci flow so that the volume is constant. To see this we observe that $d V=\sqrt{\operatorname{det} g_{i j}} d x$ and then

$$
\begin{gathered}
\frac{\partial}{\partial t} \log \sqrt{\operatorname{det} g_{i j}}=\frac{1}{2} g^{i j} \frac{\partial}{\partial t} g_{i j}=r-R, \\
\frac{d}{d t} \int_{M} d V=\int_{M}(r-R) d V=0 .
\end{gathered}
$$

The Ricci flow and the normalized Ricci flow differ only by a change of scale in space and a change of parametrization in time. Indeed, we first assume that $g_{i j}(t)$ evolves by the (unnormalized) Ricci flow and choose the normalization factor $\psi=\psi(t)$ so that $\tilde{g}_{i j}=\psi g_{i j}$, and $\int_{M} d \tilde{\mu}=1$. Next we choose a new time scale $\tilde{t}=\int \psi(t) d t$. Then for the normalized metric $\tilde{g}_{i j}$ we have

$$
\tilde{R}_{i j}=R_{i j}, \tilde{R}=\frac{1}{\psi} R, \tilde{r}=\frac{1}{\psi} r .
$$

Because $\int_{M} d \tilde{V}=1$, we see that $\int_{M} d V=\psi^{-\frac{n}{2}}$. Then

$$
\begin{aligned}
\frac{d}{d t} \log \psi & =\left(-\frac{2}{n}\right) \frac{d}{d t} \log \int_{M} d V \\
& =\left(-\frac{2}{n}\right) \frac{\int_{M} \frac{\partial}{\partial t} \sqrt{\operatorname{det} g_{i j}} d x}{\int_{M} d V} \\
& =\frac{2}{n} r,
\end{aligned}
$$


since $\frac{\partial}{\partial t} g_{i j}=-2 R_{i j}$ for the Ricci flow. Hence it follows that

$$
\begin{aligned}
\frac{\partial}{\partial \tilde{t}} \tilde{g}_{i j} & =\frac{\partial}{\partial t} g_{i j}+\left(\frac{d}{d t} \log \psi\right) g_{i j} \\
& =\frac{2}{n} \tilde{r} \tilde{g}_{i j}-2 \tilde{R}_{i j} .
\end{aligned}
$$

Thus studying the behavior of the Ricci flow near the maximal time is equivalent to studying the long-time behavior of the normalized Ricci flow.

In this chapter we will obtain long-time behavior of the normalized Ricci flow for the following special cases: (1) compact two-manifolds; (2) compact three-manifolds with nonnegative Ricci curvature; (3) compact four-manifolds with nonnegative curvature operator; and (4) compact three-manifolds with uniformly bounded normalized curvature.

5.1. The Ricci Flow on Two-manifolds. Let $M$ be a compact surface, we will discuss in this section the evolution of a Riemannian metric $g_{i j}$ under the normalized Ricci flow. On a surface, the Ricci curvature is given by

$$
R_{i j}=\frac{1}{2} R g_{i j}
$$

so the normalized Ricci flow equation becomes

$$
\frac{\partial}{\partial t} g_{i j}=(r-R) g_{i j}
$$

Recall the Gauss-Bonnet formula says

$$
\int_{M} R d V=4 \pi \chi(M)
$$

where $\chi(M)$ is the Euler characteristic number of $M$. Thus the average scalar curvature $r=4 \pi \chi(M) / \int_{M} d V$ is constant in time.

To obtain the evolution equation of the normalized curvature, we recall a simple principle in [58] for converting from the unnormalized to the normalized evolution equation on an $n$-dimensional manifold. Let $P$ and $Q$ be two expressions formed from the metric and curvature tensors, and let $\tilde{P}$ and $\tilde{Q}$ be the corresponding expressions for the normalized Ricci flow. Since they differ by dilations, they differ by a power of the normalized factor $\psi=\psi(t)$. We say $P$ has degree $k$ if $\tilde{P}=\psi^{k} P$. Thus $g_{i j}$ has degree $1, R_{i j}$ has degree $0, R$ has degree -1 .

Lemma 5.1.1. Suppose $P$ satisfies

$$
\frac{\partial P}{\partial t}=\Delta P+Q
$$

for the unnormalized Ricci flow, and $P$ has degree $k$. Then $Q$ has degree $k-1$, and for the normalized Ricci flow,

$$
\frac{\partial \tilde{P}}{\partial \tilde{t}}=\tilde{\Delta} \tilde{P}+\tilde{Q}+\frac{2}{n} k \tilde{r} \tilde{P}
$$

Proof. We first see $Q$ has degree $k-1$ since $\partial \tilde{t} / \partial t=\psi$ and $\Delta=\psi \tilde{\Delta}$. Then

$$
\psi \frac{\partial}{\partial \tilde{t}}\left(\psi^{-k} \tilde{P}\right)=\psi \tilde{\Delta}\left(\psi^{-k} \tilde{P}\right)+\psi^{-k+1} \tilde{Q}
$$


which implies

$$
\begin{aligned}
\frac{\partial \tilde{P}}{\partial \tilde{t}} & =\tilde{\Delta} \tilde{P}+\tilde{Q}+\frac{k}{\psi} \frac{\partial \psi}{\partial \tilde{t}} \tilde{P} \\
& =\tilde{\Delta} \tilde{P}+\tilde{Q}+\frac{2}{n} k \tilde{r} \tilde{P}
\end{aligned}
$$

since $\frac{\partial}{\partial t} \log \psi=\left(\frac{\partial}{\partial t} \log \psi\right) \psi^{-1}=\frac{2}{n} \tilde{r}$.

We now come back to the normalized Ricci flow (5.1.1) on a compact surface. By applying the above lemma to the evolution equation of unnormalized scalar curvature, we have

$$
\frac{\partial R}{\partial t}=\Delta R+R^{2}-r R
$$

for the normalized scalar curvature $R$. As a direct consequence, by using the maximum principle, both nonnegative scalar curvature and nonpositive scalar curvature are preserved for the normalized Ricci flow on surfaces.

Let us introduce a potential function $\varphi$ as in the Kähler-Ricci flow (see for example [11]). Since $R-r$ has mean value zero on a compact surface, there exists a unique function $\varphi$, with mean value zero, such that

$$
\Delta \varphi=R-r
$$

Differentiating (5.1.3) in time, we have

$$
\begin{aligned}
\frac{\partial}{\partial t} R & =\frac{\partial}{\partial t}(\Delta \varphi) \\
& =(R-r) \Delta \varphi+g^{i j} \frac{\partial}{\partial t}\left(\frac{\partial^{2} \varphi}{\partial x^{i} \partial x^{j}}-\Gamma_{i j}^{k} \frac{\partial \varphi}{\partial x^{k}}\right) \\
& =(R-r) \Delta \varphi+\Delta\left(\frac{\partial \varphi}{\partial t}\right) .
\end{aligned}
$$

Combining with the equation (5.1.2), we get

$$
\Delta\left(\frac{\partial \varphi}{\partial t}\right)=\Delta(\Delta \varphi)+r \Delta \varphi
$$

which implies that

$$
\frac{\partial \varphi}{\partial t}=\Delta \varphi+r \varphi-b(t)
$$

for some function $b(t)$ of time only. Since $\int_{M} \varphi d V=0$ for all $t$, we have

$$
\begin{aligned}
0=\frac{d}{d t} \int_{M} \varphi d \mu & =\int_{M}(\Delta \varphi+r \varphi-b(t)) d \mu+\int_{M} \varphi(r-R) d \mu \\
& =-b(t) \int_{M} d \mu+\int_{M}|\nabla \varphi|^{2} d \mu .
\end{aligned}
$$

Thus the function $b(t)$ is given by

$$
b(t)=\frac{\int_{M}|\nabla \varphi|^{2} d \mu}{\int_{M} d \mu} .
$$


Define a function $h$ by

$$
h=\Delta \varphi+|\nabla \varphi|^{2}=(R-r)+|\nabla \varphi|^{2},
$$

and set

$$
M_{i j}=\nabla_{i} \nabla_{j} \varphi-\frac{1}{2} \Delta \varphi g_{i j}
$$

to be the traceless part of $\nabla_{i} \nabla_{j} \varphi$.

LEMma 5.1.2. The function $h$ satisfies the evolution equation

$$
\frac{\partial h}{\partial t}=\Delta h-2\left|M_{i j}\right|^{2}+r h .
$$

Proof. Under the normalized Ricci flow,

$$
\begin{aligned}
\frac{\partial}{\partial t}|\nabla \varphi|^{2} & =\left(\frac{\partial}{\partial t} g^{i j}\right) \nabla_{i} \varphi \nabla_{j} \varphi+2 g^{i j}\left(\frac{\partial}{\partial t} \nabla_{i} \varphi\right)\left(\nabla_{j} \varphi\right) \\
& =(R-r)|\nabla \varphi|^{2}+2 g^{i j} \nabla_{i}(\Delta \varphi+r \varphi-b(t)) \nabla_{j} \varphi \\
& =(R+r)|\nabla \varphi|^{2}+2 g^{i j}\left(\Delta \nabla_{i} \varphi-R_{i k} \nabla_{k} \varphi\right) \nabla_{j} \varphi \\
& =(R+r)|\nabla \varphi|^{2}+\Delta|\nabla \varphi|^{2}-2\left|\nabla^{2} \varphi\right|^{2}-2 g^{i j} R_{i k} \nabla_{k} \varphi \nabla_{j} \varphi \\
& =\Delta|\nabla \varphi|^{2}-2\left|\nabla^{2} \varphi\right|^{2}+r|\nabla \varphi|^{2},
\end{aligned}
$$

where $R_{i k}=\frac{1}{2} R g_{i k}$ on a surface.

On the other hand we may rewrite the evolution equation (5.1.2) as

$$
\frac{\partial}{\partial t}(R-r)=\Delta(R-r)+(\Delta \varphi)^{2}+r(R-r) .
$$

Then the combination of above two equations yields

$$
\begin{aligned}
\frac{\partial}{\partial t} h & =\Delta h-2\left(\left|\nabla^{2} \varphi\right|^{2}-\frac{1}{2}(\Delta \varphi)^{2}\right)+r h \\
& =\Delta h-2\left|M_{i j}\right|^{2}+r h
\end{aligned}
$$

as desired.

As a direct consequence of the evolution equation (5.1.5) and the maximum principle, we have

$$
R \leq C_{1} e^{r t}+r
$$

for some positive constant $C_{1}$ depending only on the initial metric.

On the other hand, it follows from (5.1.2) that $R_{\min }(t)=\min _{x \in M} R(x, t)$ satisfies

$$
\frac{d}{d t} R_{\min } \geq R_{\min }\left(R_{\min }-r\right) \geq 0
$$

whenever $R_{\min } \leq 0$. This says that

$$
R_{\min }(t) \geq-C_{2}, \quad \text { for all } t>0
$$


for some positive constant $C_{2}$ depending only on the initial metric.

Thus the combination of (5.1.6) and (5.1.7) implies the following long time existence result.

Proposition 5.1.3. For any initial metric on a compact surface, the normalized Ricci flow (5.1.1) has a solution for all time.

To investigate the long-time behavior of the solution, let us now divide the discussion into three cases: $\chi(M)<0 ; \chi(M)=0$; and $\chi(M)>0$.

Case (1): $\chi(M)<0$ (i.e., $r<0$ ).

From the evolution equation (5.1.2), we have

$$
\begin{aligned}
\frac{d}{d t} R_{\min } & \geq R_{\min }\left(R_{\min }-r\right) \\
& \geq r\left(R_{\min }-r\right), \text { on } M \times[0,+\infty)
\end{aligned}
$$

which implies that

$$
R-r \geq-\tilde{C}_{1} e^{r t}, \text { on } M \times[0,+\infty)
$$

for some positive constant $\tilde{C}_{1}$ depending only on the initial metric. Thus by combining with (5.1.6) we have

$$
-\tilde{C}_{1} e^{r t} \leq R-r \leq C_{1} e^{r t}, \text { on } M \times[0,+\infty) .
$$

THEOREM 5.1.4 (Hamilton [60]). On a compact surface with $\chi(M)<0$, for any initial metric the solution of the normalized Ricci flow (5.1.1) exists for all time and converges in the $C^{\infty}$ topology to a metric with negative constant curvature.

Proof. The estimate (5.1.8) shows that the scalar curvature $R(x, t)$ converges exponentially to the negative constant $r$ as $t \rightarrow+\infty$.

Fix a tangent vector $v \in T_{x} M$ at a point $x \in M$ and let $|v|_{t}^{2}=g_{i j}(x, t) v^{i} v^{j}$. Then we have

$$
\begin{aligned}
\frac{d}{d t}|v|_{t}^{2} & =\left(\frac{\partial}{\partial t} g_{i j}(x, t)\right) v^{i} v^{j} \\
& =(r-R)|v|_{t}^{2}
\end{aligned}
$$

which implies

$$
\left.\left|\frac{d}{d t} \log \right| v\right|_{t} ^{2} \mid \leq C e^{r t}, \text { for all } t>0
$$

for some positive constant $C$ depending only on the initial metric (by using (5.1.8)). Thus $|v|_{t}^{2}$ converges uniformly to a continuous function $|v|_{\infty}^{2}$ as $t \rightarrow+\infty$ and $|v|_{\infty}^{2} \neq 0$ if $v \neq 0$. Since the parallelogram law continues to hold to the limit, the limiting norm $|v|_{\infty}^{2}$ comes from an inner product $g_{i j}(\infty)$. This says, the metrics $g_{i j}(t)$ are all equivalent and as $t \rightarrow+\infty$, the metric $g_{i j}(t)$ converges uniformly to a positive-definite metric tensor $g_{i j}(\infty)$ which is continuous and equivalent to the initial metric.

By the virtue of Shi's derivative estimates of the unnormalized Ricci flow in Section 1.4, we see that all derivatives and higher order derivatives of the curvature of the solution $g_{i j}$ of the normalized flow are uniformly bounded on $M \times[0,+\infty)$. 
This shows that the limiting metric $g_{i j}(\infty)$ is a smooth metric with negative constant curvature and the solution $g_{i j}(t)$ converges to the limiting metric $g_{i j}(\infty)$ in the $C^{\infty}$ topology as $t \rightarrow+\infty$.

Case (2): $\chi(M)=0$, i.e., $r=0$.

From (5.1.6) and (5.1.7) we know that the curvature remains bounded above and below. To get the convergence, we consider the potential function $\varphi$ of (5.1.3) again. The evolution of $\varphi$ is given by (5.1.4). We renormalize the function $\varphi$ by

$$
\tilde{\varphi}(x, t)=\varphi(x, t)+\int b(t) d t, \quad \text { on } M \times[0,+\infty) .
$$

Then, since $r=0, \tilde{\varphi}$ evolves by

$$
\frac{\partial \tilde{\varphi}}{\partial t}=\Delta \tilde{\varphi}, \quad \text { on } M \times[0,+\infty) .
$$

From the proof of Lemma 5.1.2, we get

$$
\frac{\partial}{\partial t}|\nabla \tilde{\varphi}|^{2}=\Delta|\nabla \tilde{\varphi}|^{2}-2\left|\nabla^{2} \tilde{\varphi}\right|^{2}
$$

Clearly, we have

$$
\frac{\partial}{\partial t} \tilde{\varphi}^{2}=\Delta \tilde{\varphi}^{2}-2|\nabla \tilde{\varphi}|^{2}
$$

Thus it follows that

$$
\frac{\partial}{\partial t}\left(t|\nabla \tilde{\varphi}|^{2}+\tilde{\varphi}^{2}\right) \leq \Delta\left(t|\nabla \tilde{\varphi}|^{2}+\tilde{\varphi}^{2}\right)
$$

Hence by applying the maximum principle, there exists a positive constant $C_{3}$ depending only on the initial metric such that

$$
|\nabla \tilde{\varphi}|^{2}(x, t) \leq \frac{C_{3}}{1+t}, \quad \text { on } M \times[0,+\infty) .
$$

In the following we will use this decay estimate to obtain a decay estimate for the scalar curvature.

By the evolution equations (5.1.2) and (5.1.10), we have

$$
\begin{aligned}
\frac{\partial}{\partial t}\left(R+2|\nabla \tilde{\varphi}|^{2}\right) & =\Delta\left(R+2|\nabla \tilde{\varphi}|^{2}\right)+R^{2}-4\left|\nabla^{2} \tilde{\varphi}\right|^{2} \\
& \leq \Delta\left(R+2|\nabla \tilde{\varphi}|^{2}\right)-R^{2}
\end{aligned}
$$

since $R^{2}=(\Delta \tilde{\varphi})^{2} \leq 2\left|\nabla^{2} \tilde{\varphi}\right|^{2}$. Thus by using (5.1.12), we have

$$
\begin{aligned}
& \frac{\partial}{\partial t}\left[t\left(R+2|\nabla \tilde{\varphi}|^{2}\right)\right] \\
& \leq \Delta\left[t\left(R+2|\nabla \tilde{\varphi}|^{2}\right)\right]-t R^{2}+R+2|\nabla \tilde{\varphi}|^{2} \\
& \leq \Delta\left[t\left(R+2|\nabla \tilde{\varphi}|^{2}\right)\right]-t\left(R+2|\nabla \tilde{\varphi}|^{2}\right)^{2}+\left(1+4 t|\nabla \tilde{\varphi}|^{2}\right)\left(R+2|\nabla \tilde{\varphi}|^{2}\right) \\
& \leq \Delta\left[t\left(R+2|\nabla \tilde{\varphi}|^{2}\right)\right]-\left[t\left(R+2|\nabla \tilde{\varphi}|^{2}\right)-\left(1+4 C_{3}\right)\right]\left(R+2|\nabla \tilde{\varphi}|^{2}\right) \\
& \leq \Delta\left[t\left(R+2|\nabla \tilde{\varphi}|^{2}\right)\right]
\end{aligned}
$$


wherever $t\left(R+2|\nabla \tilde{\varphi}|^{2}\right) \geq\left(1+4 C_{3}\right)$. Hence by the maximum principle, there holds

$$
R+2|\nabla \tilde{\varphi}|^{2} \leq \frac{C_{4}}{1+t}, \quad \text { on } M \times[0,+\infty)
$$

for some positive constant $C_{4}$ depending only on the initial metric.

On the other hand, the scalar curvature satisfies

$$
\frac{\partial R}{\partial t}=\Delta R+R^{2}, \quad \text { on } M \times[0,+\infty) .
$$

It is not hard to see that

$$
R \geq \frac{R_{\min }(0)}{1-R_{\min }(0) t}, \quad \text { on } \quad M \times[0,+\infty),
$$

by using the maximum principle. So we obtain the decay estimate for the scalar curvature

$$
|R(x, t)| \leq \frac{C_{5}}{1+t}, \quad \text { on } M \times[0,+\infty)
$$

for some positive constant $C_{5}$ depending only on the initial metric.

THEOREM 5.1 .5 (Hamilton [60]). On a compact surface with $\chi(M)=0$, for any initial metric the solution of the normalized Ricci flow (5.1.1) exists for all time and converges in $C^{\infty}$ topology to a flat metric.

Proof. Since $\frac{\partial \tilde{\varphi}}{\partial t}=\Delta \tilde{\varphi}$, it follows from the maximum principle that

$$
|\tilde{\varphi}(x, t)| \leq C_{6}, \quad \text { on } M \times[0,+\infty)
$$

for some positive constant $C_{6}$ depending only on the initial metric. Recall that $\Delta \tilde{\varphi}=$ $R$. We thus obtain for any tangent vector $v \in T_{x} M$ at a point $x \in M$,

$$
\begin{aligned}
\frac{d}{d t}|v|_{t}^{2} & =\left(\frac{\partial}{\partial t} g_{i j}(x, t)\right) v^{i} v^{j} \\
& =-R(x, t)|v|_{t}^{2}
\end{aligned}
$$

and then

$$
\begin{aligned}
\left|\log \frac{|v|_{t}^{2}}{|v|_{0}^{2}}\right| & =\left.\left|\int_{0}^{t} \frac{d}{d t} \log \right| v_{t}\right|^{2} d t \mid \\
& =\left|\int_{0}^{t} R(x, t) d t\right| \\
& =|\tilde{\varphi}(x, t)-\tilde{\varphi}(x, 0)| \\
& \leq 2 C_{6},
\end{aligned}
$$

for all $x \in M$ and $t \in[0,+\infty)$. This shows that the solution $g_{i j}(t)$ of the normalized Ricci flow are all equivalent. This gives us control of the diameter and injectivity radius.

As before, by Shi's derivative estimates of the unnormalized Ricci flow, all derivatives and higher order derivatives of the curvature of the solution $g_{i j}$ of the normalized Ricci flow (5.1.1) are uniformly bounded on $M \times[0,+\infty)$. By the virtue of Hamilton's 
compactness theorem (Theorem 4.1.5) we see that the solution $g_{i j}(t)$ subsequentially converges in $C^{\infty}$ topology. The decay estimate (5.1.15) implies that each limit must be a flat metric on $M$. Clearly, we will finish the proof if we can show that limit is unique.

Note that the solution $g_{i j}(t)$ is changing conformally under the Ricci flow (5.1.1) on surfaces. Thus each limit must be conformal to the initial metric, denoted by $\bar{g}_{i j}$. Let us denote $g_{i j}(\infty)=e^{u} \bar{g}_{i j}$ to be a limiting metric. Since $g_{i j}(\infty)$ is flat, it is easy to compute

$$
0=e^{-u}(\bar{R}-\bar{\Delta} u), \quad \text { on } M,
$$

where $\bar{R}$ is the curvature of $\bar{g}_{i j}$ and $\bar{\Delta}$ is the Laplacian in the metric $\bar{g}_{i j}$. The solution of Poission equation

$$
\bar{\Delta} u=\bar{R}, \quad \text { on } M
$$

is unique up to constant. Moreover the constant must be also uniquely determined since the area of the solution of the normalized Ricci flow (5.1.1) is constant in time. So the limit is unique and we complete the proof of Theorem 5.1.5.

Case (3): $\chi(M)>0$, i.e., $r>0$.

This is the most difficult case. There exist several proofs by now but, in contrast to the previous two cases, none of them depend only on the maximum principle type of argument. In fact, all the proofs rely on some combination of the maximum principle argument and certain integral estimate of the curvature. In the pioneer work [60], Hamilton introduced an integral quantity

$$
E=\int_{M} R \log R d V
$$

which he calls entropy, for the (normalized) Ricci flow on a surface $M$ with positive curvature, and showed that the entropy is monotone decreasing under the flow. By combining this entropy estimate with the Harnack inequality for the curvature (Corollary 2.5.3), Hamilton obtained the uniform bound on the curvature of the normalized Ricci flow on $M$ with positive curvature. Furthermore, he showed that the evolving metric converges to a shrinking Ricci soliton on $M$ and that the shrinking Ricci soliton must be a round metric on the 2 -sphere $\mathbb{S}^{2}$. Subsequently, Chow [36] extended Hamilton's work to the general case when the curvature may change signs. More precisely, he proved that given any initial metric on a compact surface $M$ with $\chi(M)>0$, the evolving metric under the (normalized) Ricci flow will have positive curvature after a finite time. Hence, when combined with Hamilton's result, we know the evolving metric on $M$ converges to the round metric on $\mathbb{S}^{2}$.

In the following we present a new argument by combining the Li-Yau-Hamilton inequality of the curvature with Perelman's no local collapsing theorem I', as was done in the recent joint work of Bing-Long Chen and the authors [15] where they considered the Kähler-Ricci flow on higher dimensional Kähler manifolds of nonnegative holomorphic bisectional curvature (see [15] for more details). (There are also other proofs for Case (3) by Bartz-Struwe-Ye [6] and Struwe [121].)

Given any initial metric on $M$ with $\chi(M)>0$, we consider the solution $g_{i j}(t)$ of the normalized Ricci flow (5.1.1). Recall that the (scalar) curvature $R$ satisfies the evolution equation

$$
\frac{\partial}{\partial t} R=\Delta R+R^{2}-r R
$$


The corresponding ODE is

$$
\frac{d s}{d t}=s^{2}-r s
$$

Let us choose $c>1$ and close to 1 so that $r /(1-c)<\min _{x \in M} R(x, 0)$. It is clear that the function $s(t)=r /\left(1-c e^{r t}\right)<0$ is a solution of the ODE (5.1.16) with $s(0)<\min _{x \in M} R(x, 0)$. Then the difference of $R$ and $s$ evolves by

$$
\frac{\partial}{\partial t}(R-s)=\Delta(R-s)+(R-r+s)(R-s)
$$

Since $\min _{x \in M} R(x, 0)-s(0)>0$, the maximum principle implies that $R-s>0$ for all times.

We first extend the Li-Yau-Hamilton inequality (Theorem 2.5.2) to the normalized Ricci flow whose curvature may change signs. As in the proof of Theorem 2.5.2, we consider the quantity

$$
L=\log (R-s)
$$

It is easy to compute

$$
\frac{\partial L}{\partial t}=\Delta L+|\nabla L|^{2}+R-r+s
$$

Then we set

$$
Q=\frac{\partial L}{\partial t}-|\nabla L|^{2}-s=\Delta L+R-r
$$

By a direct computation and using the estimate (5.1.8), we have

$$
\begin{aligned}
\frac{\partial}{\partial t} Q= & \Delta\left(\frac{\partial L}{\partial t}\right)+(R-r) \Delta L+\frac{\partial R}{\partial t} \\
= & \Delta Q+2\left|\nabla^{2} L\right|^{2}+2\langle\nabla L, \nabla(\Delta L)\rangle+R|\nabla L|^{2} \\
& +(R-r) \Delta L+\Delta R+R(R-r) \\
= & \Delta Q+2\left|\nabla^{2} L\right|^{2}+2\langle\nabla L, \nabla Q\rangle+2(R-r) \Delta L+(R-r)^{2} \\
& +(r-s) \Delta L+s|\nabla L|^{2}+r(R-r) \\
= & \Delta Q+2\langle\nabla L, \nabla Q\rangle+2\left|\nabla^{2} L\right|^{2}+2(R-r) \Delta L+(R-r)^{2} \\
& +(r-s) Q+s|\nabla L|^{2}+s(R-r) \\
\geq & \Delta Q+2\langle\nabla L, \nabla Q\rangle+Q^{2}+(r-s) Q+s|\nabla L|^{2}-C .
\end{aligned}
$$

Here and below $C$ is denoted by various positive constants depending only on the initial metric.

In order to control the bad term $s|\nabla L|^{2}$, we consider

$$
\begin{aligned}
\frac{\partial}{\partial t}(s L) & =\Delta(s L)+s|\nabla L|^{2}+s(R-r+s)+s(s-r) L \\
& \geq \Delta(s L)+2\langle\nabla L, \nabla(s L)\rangle-s|\nabla L|^{2}-C
\end{aligned}
$$


by using the estimate (5.1.8) again. Thus

$$
\begin{aligned}
\frac{\partial}{\partial t}(Q+s L) & \geq \Delta(Q+s L)+2\langle\nabla L, \nabla(Q+s L)\rangle+Q^{2}+(r-s) Q-C \\
& \geq \Delta(Q+s L)+2\langle\nabla L, \nabla(Q+s L)\rangle+\frac{1}{2}\left[(Q+s L)^{2}-C^{2}\right],
\end{aligned}
$$

since $s L$ is bounded by (5.1.8). This, by the maximum principle, implies that

$$
Q \geq-C, \quad \text { for all } t \in[0,+\infty) .
$$

Then for any two points $x_{1}, x_{2} \in M$ and two times $t_{2}>t_{1} \geq 0$, and a path $\gamma$ : $\left[t_{1}, t_{2}\right] \rightarrow M$ connecting $x_{1}$ to $x_{2}$, we have

$$
\begin{aligned}
L\left(x_{2}, t_{2}\right)-L\left(x_{1}, t_{1}\right) & =\int_{t_{1}}^{t_{2}} \frac{d}{d t} L(\gamma(t), t) d t \\
& =\int_{t_{1}}^{t_{2}}\left(\frac{\partial L}{\partial t}+\langle\nabla L, \dot{\gamma}\rangle\right) d t \\
& \geq-\frac{1}{4} \Delta-C\left(t_{2}-t_{1}\right)
\end{aligned}
$$

where

$$
\begin{aligned}
\Delta & =\Delta\left(x_{1}, t_{1} ; x_{2}, t_{2}\right) \\
& =\inf \left\{\int_{t_{1}}^{t_{2}}|\dot{\gamma}(t)|_{g_{i j}(t)}^{2} d t \mid \gamma:\left[t_{1}, t_{2}\right] \rightarrow M \text { with } \gamma\left(t_{1}\right)=x_{1}, \gamma\left(t_{2}\right)=x_{2}\right\}
\end{aligned}
$$

Thus we have proved the following Harnack inequality.

Lemma 5.1.6 (Chow [36]). There exists a positive constant $C$ depending only on the initial metric such that for any $x_{1}, x_{2} \in M$ and $t_{2}>t_{1} \geq 0$,

$$
R\left(x_{1}, t_{1}\right)-s\left(t_{1}\right) \leq e^{\frac{\Delta}{4}+C\left(t_{2}-t_{1}\right)}\left(R\left(x_{2}, t_{2}\right)-s\left(t_{2}\right)\right)
$$

where

$$
\Delta=\inf \left\{\int_{t_{1}}^{t_{2}}|\dot{\gamma}(t)|_{t}^{2} d t \mid \gamma:\left[t_{1}, t_{2}\right] \rightarrow M \text { with } \gamma\left(t_{1}\right)=x_{1}, \gamma\left(t_{2}\right)=x_{2}\right\} .
$$

We now state and prove the uniform bound estimate for the curvature.

Proposition 5.1.7. Let $\left(M, g_{i j}(t)\right)$ be a solution of the normalized Ricci flow on a compact surface with $\chi(M)>0$. Then there exist a time $t_{0}>0$ and a positive constant $C$ such that the estimate

$$
C^{-1} \leq R(x, t) \leq C
$$

holds for all $x \in M$ and $t \in\left[t_{0},+\infty\right)$.

Proof. Recall that

$$
R(x, t) \geq s(t)=\frac{r}{1-c e^{r t}}, \quad \text { on } M \times[0,+\infty) .
$$


For any $\varepsilon \in(0, r)$, there exists a large enough $t_{0}>0$ such that

$$
R(x, t) \geq-\varepsilon^{2}, \quad \text { on } M \times\left[t_{0},+\infty\right) .
$$

Let $t$ be any fixed time with $t \geq t_{0}+1$. Obviously there is some point $x_{0} \in M$ such that $R\left(x_{0}, t+1\right)=r$.

Consider the geodesic ball $B_{t}\left(x_{0}, 1\right)$, centered at $x_{0}$ and radius 1 with respect to the metric at the fixed time $t$. For any point $x \in B_{t}\left(x_{0}, 1\right)$, we choose a geodesic $\gamma$ : $[t, t+1] \rightarrow M$ connecting $x$ and $x_{0}$ with respect to the metric at the fixed time $t$. Since

$$
\frac{\partial}{\partial t} g_{i j}=(r-R) g_{i j} \leq 2 r g_{i j} \quad \text { on } M \times\left[t_{0},+\infty\right),
$$

we have

$$
\int_{t}^{t+1}|\dot{\gamma}(\tau)|_{\tau}^{2} d \tau \leq e^{2 r} \int_{t}^{t+1}|\dot{\gamma}(\tau)|_{t}^{2} d \tau \leq e^{2 r}
$$

Then by Lemma 5.1.6, we have

$$
\begin{aligned}
R(x, t) & \leq s(t)+\exp \left\{\frac{1}{4} e^{2 r}+C\right\} \cdot\left(R\left(x_{0}, t+1\right)-s(t+1)\right) \\
& \leq C_{1}, \quad \text { as } x \in B_{t}\left(x_{0}, 1\right),
\end{aligned}
$$

for some positive constant $C_{1}$ depending only on the initial metric. Note the the corresponding unnormalized Ricci flow in this case has finite maximal time since its volume decreases at a fixed rate $-4 \pi \chi(M)<0$. Hence the no local collapsing theorem $\mathrm{I}^{\prime}$ (Theorem 3.3.3) implies that the volume of $B_{t}\left(x_{0}, 1\right)$ with respect to the metric at the fixed time $t$ is bounded from below by

$$
\operatorname{Vol}_{t}\left(B_{t}\left(x_{0}, 1\right)\right) \geq C_{2}
$$

for some positive constant $C_{2}$ depending only on the initial metric.

We now want to bound the diameter of $\left(M, g_{i j}(t)\right)$ from above. The following argument is analogous to Yau in [128] where he got a lower bound for the volume of geodesic balls of a complete Riemannian manifold with nonnegative Ricci curvature. Without loss of generality, we may assume that the diameter of $\left(M, g_{i j}(t)\right)$ is at least 3 . Choose a point $x_{1} \in M$ such that the distance $d_{t}\left(x_{0}, x_{1}\right)$ between $x_{1}$ and $x_{0}$ with respect to the metric at the fixed time $t$ is at least a half of the diameter of $\left(M, g_{i j}(t)\right)$. By (5.1.18), the standard Laplacian comparison theorem (c.f. [112]) implies

$$
\Delta \rho^{2}=2 \rho \Delta \rho+2 \leq 2(1+\varepsilon \rho)+2
$$

in the sense of distribution, where $\rho$ is the distance function from $x_{1}$ (with respect to the metric $\left.g_{i j}(t)\right)$. That is, for any $\varphi \in C_{0}^{\infty}(M), \varphi \geq 0$, we have

$$
-\int_{M} \nabla \rho^{2} \cdot \nabla \varphi \leq \int_{M}[2(1+\varepsilon \rho)+2] \varphi .
$$

Since $C_{0}^{\infty}(M)$ functions can be approximated by Lipschitz functions in the above inequality, we can set $\varphi(x)=\psi(\rho(x)), x \in M$, where $\psi(s)$ is given by

$$
\psi(s)= \begin{cases}1, & 0 \leq s \leq d_{t}\left(x_{0}, x_{1}\right)-1, \\ \psi^{\prime}(s)=-\frac{1}{2}, & d_{t}\left(x_{0}, x_{1}\right)-1 \leq s \leq d_{t}\left(x_{0}, x_{1}\right)+1, \\ 0, & s \geq d_{t}\left(x_{0}, x_{1}\right)+1\end{cases}
$$


Thus, by using (5.1.20), the left hand side of (5.1.21) is

$$
\begin{aligned}
& -\int_{M} \nabla \rho^{2} \cdot \nabla \varphi \\
& =\int_{B_{t}\left(x_{1}, d_{t}\left(x_{0}, x_{1}\right)+1\right) \backslash B_{t}\left(x_{1}, d_{t}\left(x_{0}, x_{1}\right)-1\right)} \rho \\
& \geq\left(d_{t}\left(x_{0}, x_{1}\right)-1\right) \operatorname{Vol}_{t}\left(B_{t}\left(x_{1}, d_{t}\left(x_{0}, x_{1}\right)+1\right) \backslash B_{t}\left(x_{1}, d_{t}\left(x_{0}, x_{1}\right)-1\right)\right) \\
& \geq\left(d_{t}\left(x_{0}, x_{1}\right)-1\right) \operatorname{Vol}_{t}\left(B_{t}\left(x_{0}, 1\right)\right) \\
& \geq\left(d_{t}\left(x_{0}, x_{1}\right)-1\right) C_{2},
\end{aligned}
$$

and the right hand side of (5.1.21) is

$$
\begin{aligned}
\int_{M}[2(1+\varepsilon \rho)+2] \varphi & \leq \int_{B_{t}\left(x_{1}, d_{t}\left(x_{0}, x_{1}\right)+1\right)}[2(1+\varepsilon \rho)+2] \\
& \leq\left[2\left(1+\varepsilon d_{t}\left(x_{0}, x_{1}\right)\right)+4\right] \operatorname{Vol}_{t}\left(B_{t}\left(x_{1}, d_{t}\left(x_{0}, x_{1}\right)+1\right)\right) \\
& \leq\left[2\left(1+\varepsilon d_{t}\left(x_{0}, x_{1}\right)\right)+4\right] A
\end{aligned}
$$

where $A$ is the area of $M$ with respect to the initial metric. Here we have used the fact that the area of solution of the normalized Ricci flow is constant in time. Hence

$$
C_{2}\left(d_{t}\left(x_{0}, x_{1}\right)-1\right) \leq\left[2\left(1+\varepsilon d_{t}\left(x_{0}, x_{1}\right)\right)+4\right] A,
$$

which implies, by choosing $\varepsilon>0$ small enough,

$$
d_{t}\left(x_{0}, x_{1}\right) \leq C_{3}
$$

for some positive constant $C_{3}$ depending only on the initial metric. Therefore, the diameter of $\left(M, g_{i j}(t)\right)$ is uniformly bounded above by

$$
\operatorname{diam}\left(M, g_{i j}(t)\right) \leq 2 C_{3}
$$

for all $t \in\left[t_{0},+\infty\right)$.

We then argue, as in deriving (5.1.19), by applying Lemma 5.1.6 again to obtain

$$
R(x, t) \leq C_{4}, \quad \text { on } M \times\left[t_{0},+\infty\right)
$$

for some positive constant $C_{4}$ depending only on the initial metric.

It remains to prove a positive lower bound estimate of the curvature. First, we note that the function $s(t) \rightarrow 0$ as $t \rightarrow+\infty$, and the average scalar curvature of the solution equals to $r$, a positive constant. Thus the Harnack inequality in Lemma 5.1.6 and the diameter estimate (5.1.22) imply a positive lower bound for the curvature. Therefore we have completed the proof of Proposition 5.1.7.

Next we consider long-time convergence of the normalized flow.

Recall that the trace-free part of the Hessian of the potential $\varphi$ of the curvature is the tensor $M_{i j}$ defined by

$$
M_{i j}=\nabla_{i} \nabla_{j} \varphi-\frac{1}{2} \Delta \varphi \cdot g_{i j},
$$

where by (5.1.3),

$$
\Delta \varphi=R-r
$$


Lemma 5.1.8. We have

$$
\frac{\partial}{\partial t}\left|M_{i j}\right|^{2}=\Delta\left|M_{i j}\right|^{2}-2\left|\nabla_{k} M_{i j}\right|^{2}-2 R\left|M_{i j}\right|^{2}, \quad \text { on } M \times[0,+\infty) .
$$

Proof. First we note the time-derivative of the Levi-Civita connection is

$$
\begin{aligned}
\frac{\partial}{\partial t} \Gamma_{i j}^{k} & =\frac{1}{2} g^{k l}\left(\nabla_{i} \frac{\partial}{\partial t} g_{j l}+\nabla_{j} \frac{\partial}{\partial t} g_{i l}-\nabla_{l} \frac{\partial}{\partial t} g_{i j}\right) \\
& =\frac{1}{2}\left(-\nabla_{i} R \cdot \delta_{j}^{k}-\nabla_{j} R \cdot \delta_{i}^{k}+\nabla^{k} R \cdot g_{i j}\right) .
\end{aligned}
$$

By using this and (5.1.4), we have

$$
\begin{aligned}
\frac{\partial}{\partial t} M_{i j}= & \nabla_{i} \nabla_{j}\left(\frac{\partial \varphi}{\partial t}\right)-\left(\frac{\partial}{\partial t} \Gamma_{i j}^{k}\right) \nabla_{k} \varphi-\frac{1}{2} \frac{\partial}{\partial t}\left[(R-r) g_{i j}\right] \\
= & \nabla_{i} \nabla_{j} \Delta \varphi+\frac{1}{2}\left(\nabla_{i} R \cdot \nabla_{j} \varphi+\nabla_{j} R \cdot \nabla_{i} \varphi-\langle\nabla R, \nabla \varphi\rangle g_{i j}\right) \\
& -\frac{1}{2} \Delta R \cdot g_{i j}+r M_{i j} .
\end{aligned}
$$

Since on a surface,

$$
R_{i j k l}=\frac{1}{2} R\left(g_{i l} g_{j k}-g_{i k} g_{j l}\right)
$$

we have

$$
\begin{aligned}
\nabla_{i} \nabla_{j} \Delta \varphi \\
=\nabla_{i} \nabla_{k} \nabla_{j} \nabla^{k} \varphi-\nabla_{i}\left(R_{j l} \nabla^{l} \varphi\right) \\
=\nabla_{k} \nabla_{i} \nabla_{j} \nabla^{k} \varphi-R_{i k j}^{l} \nabla_{l} \nabla^{k} \varphi-R_{i l} \nabla_{j} \nabla^{l} \varphi-R_{j l} \nabla_{i} \nabla^{l} \varphi-\nabla_{i} R_{j l} \nabla^{l} \varphi \\
=\Delta \nabla_{i} \nabla_{j} \varphi-\nabla^{k}\left(R_{i k j}^{l} \nabla_{l} \varphi\right)-R_{i k j}^{l} \nabla_{l} \nabla^{k} \varphi \\
\quad-R_{i l} \nabla_{j} \nabla^{l} \varphi-R_{j l} \nabla_{i} \nabla^{l} \varphi-\nabla_{i} R_{j l} \nabla^{l} \varphi \\
=\Delta \nabla_{i} \nabla_{j} \varphi-\frac{1}{2}\left(\nabla_{i} R \cdot \nabla_{j} \varphi+\nabla_{j} R \cdot \nabla_{i} \varphi-\langle\nabla R, \nabla \varphi\rangle g_{i j}\right) \\
\\
-2 R\left(\nabla_{i} \nabla_{j} \varphi-\frac{1}{2} \Delta \varphi \cdot g_{i j}\right) .
\end{aligned}
$$

Combining these identities, we get

$$
\begin{aligned}
\frac{\partial}{\partial t} M_{i j} & =\Delta \nabla_{i} \nabla_{j} \varphi-\frac{1}{2} \Delta R \cdot g_{i j}+(r-2 R) M_{i j} \\
& =\Delta\left(\nabla_{i} \nabla_{j} \varphi-\frac{1}{2}(R-r) g_{i j}\right)+(r-2 R) M_{i j}
\end{aligned}
$$

Thus the evolution $M_{i j}$ is given by

$$
\frac{\partial M_{i j}}{\partial t}=\Delta M_{i j}+(r-2 R) M_{i j}
$$

Now the lemma follows from (5.1.24) and a straightforward computation. $\mathrm{Q}$ 
Proposition 5.1.7 tells us that the curvature $R$ of the solution to the normalized Ricci flow is uniformly bounded from below by a positive constant for $t$ large. Thus we can apply the maximum principle to the equation (5.1.23) in Lemma 5.1.8 to obtain the following estimate.

Proposition 5.1.9. Let $\left(M, g_{i j}(t)\right)$ be a solution of the normalized Ricci flow on a compact surface with $\chi(M)>0$. Then there exist positive constants $c$ and $C$ depending only on the initial metric such that

$$
\left|M_{i j}\right|^{2} \leq C e^{-c t}, \quad \text { on } M \times[0,+\infty) .
$$

Now we consider a modification of the normalized Ricci flow. Consider the equation

$$
\frac{\partial}{\partial t} g_{i j}=2 M_{i j}=(r-R) g_{i j}+2 \nabla_{i} \nabla_{j} \varphi .
$$

As we saw in Section 1.3, the solution of this modified flow differs from that of the normalized Ricci flow only by a one parameter family of diffeomorphisms generated by the gradient vector field of the potential function $\varphi$. Since the quantity $\left|M_{i j}\right|^{2}$ is invariant under diffeomorphisms, the estimate $\left|M_{i j}\right|^{2} \leq C e^{-c t}$ also holds for the solution of the modified flow (5.1.25). This exponential decay estimate then implies the solution $g_{i j}(x, t)$ of the modified flow (5.1.25) converges exponentially to a continuous metric $g_{i j}(\infty)$ as $t \rightarrow+\infty$. Furthermore, by the virtue of Hamilton's compactness theorem (Theorem 4.1.5) we see that the solution $g_{i j}(x, t)$ of the modified flow actually converges exponentially in $C^{\infty}$ topology to $g_{i j}(\infty)$. Moreover the limiting metric $g_{i j}(\infty)$ satisfies

$$
M_{i j}=(r-R) g_{i j}+2 \nabla_{i} \nabla_{j} \varphi=0, \quad \text { on } M .
$$

That is, the limiting metric is a shrinking gradient Ricci soliton on the surface $M$.

The next result was first obtained by Hamilton in [60]. The following simplified proof by using the Kazdan-Warner identity was widely known to experts in the field.

Proposition 5.1.10. On a compact surface there are no shrinking Ricci solitons other than constant curvature. by

Proof. By definition, a shrinking Ricci soliton on a compact surface $M$ is given

$$
\nabla_{i} X_{j}+\nabla_{j} X_{i}=(R-r) g_{i j}
$$

for some vector field $X=X_{j}$. By contracting the above equation by $R g^{-1}$, we have

$$
2 R(R-r)=2 R \operatorname{div} X,
$$

and hence

$$
\int_{M}(R-r)^{2} d V=\int_{M} R(R-r) d V=\int_{M} R \operatorname{div} X d V .
$$

Since $X$ is a conformal vector field (by the Ricci soliton equation (5.1.26)), by integrating by parts and applying the Kazdan-Warner identity [77], we obtain

$$
\int_{M}(R-r)^{2} d V=-\int_{M}\langle\nabla R, X\rangle d V=0 .
$$


Hence $R \equiv r$, and the lemma is proved.

Now back to the solution of the modified flow (5.1.25). We have seen the curvature converges exponentially to its limiting value in the $C^{\infty}$ topology. But since there are no nontrivial soliton on $M$, we must have $R$ converging exponentially to the constant value $r$ in the $C^{\infty}$ topology. This then implies that the unmodified flow (5.1.1) will converge to a metric of positive constant curvature in the $C^{\infty}$ topology.

In conclusion, we have proved the following main theorem of this section.

TheOREM 5.1.11 (Hamilton [60], Chow [36]). On a compact surface with $\chi(M)>$ 0 , for any initial metric, the solution of the normalized Ricci flow (5.1.1) exists for all time, and converges in the $C^{\infty}$ topology to a metric with positive constant curvature.

5.2. Differentiable Sphere Theorems in $3-D$ and $4-D$. An important problem in Riemannian geometry is to understand the influence of curvatures, in particular the sign of curvatures, on the topology of underlying manifolds. Classical results of this type include sphere theorem and its refinements stated below (see, for example, Theorem 6.1 and Theorem 7.16 in Cheeger-Ebin [22], and Theorem 6.6 in CheegerEbin [22]). In this section we shall use the long-time behavior of the Ricci flow on positively curved manifolds to establish Hamilton's differentiable sphere theorems in dimensions three and four.

Let us first recall the classical sphere theorems. Given a Remannian manifold $M$, we denote by $K_{M}$ the sectional curvature of $M$.

Classical Sphere Theorems. Let $M$ be a complete, simply connected $n$ dimensional manifold.

(i) If $\frac{1}{4}<K_{M} \leq 1$, then $M$ is homeomorphic to the $n$-sphere $\mathbb{S}^{n}$.

(ii) There exists a positive constant $\delta \in\left(\frac{1}{4}, 1\right)$ such that if $\delta<K_{M} \leq 1$, then $M$ is diffeomorphic to the $n$-sphere $\mathbb{S}^{n}$.

Result (ii) is called the differentiable sphere theorem. If we relax the assumptions on the strict lower bound in (i), then we have the following rigidity result.

Berger's Rigidity Theorem. Let $M$ be a complete, simply connected $n$ dimensional manifold with $\frac{1}{4} \leq K_{M} \leq 1$. Then either $M$ is homeomorphic to $\mathbb{S}^{n}$ or $M$ is isometric to a symmetric space.

We remark that it follows from the classification of symmetric spaces (see for example [68]) that the only simply connected symmetric spaces with positive curvature are $\mathbb{S}^{n}, \mathbb{C P}^{\frac{n}{2}}, \mathbb{Q P}^{\frac{n}{4}}$, and the Cayley plane.

In early and mid 80's respectively, Hamilton [58], [59] used the Ricci flow to prove the following differential sphere theorems.

TheOREM 5.2.1 (Hamilton [58]). A compact three-manifold with positive Ricci curvature must be diffeomorphic to the three-sphere $\mathbb{S}^{3}$ or a quotient of it by a finite group of fixed point free isometries in the standard metric.

THEOREM 5.2.2 (Hamilton [59]). A compact four-manifold with positive curvature operator is diffeomorphic to the four-sphere $\mathbb{S}^{4}$ or the real projective space $\mathbb{R} \mathbb{P}^{4}$.

Note that in above two theorems, we only assume curvatures to be strictly positive, but not any strong pinching conditions as in the classical sphere theorems. In fact, one of the important special features discovered by Hamilton is that if the initial metric has positive curvature, then the metric will get rounder and rounder as it 
evolves under the Ricci flow, at least in dimension three and four, so any small initial pinching will get improved. Indeed, the pinching estimate is a key step in proving both Theorem 5.2.1 and 5.2.2.

The following results are concerned with compact three-manifolds or fourmanifolds with weakly positive curvatures.

Theorem 5.2.3 (Hamilton [59]).

(i) A compact three-manifold with nonnegative Ricci curvature is diffeomorphic to $\mathbb{S}^{3}$, or a quotient of one of the spaces $\mathbb{S}^{3}$ or $\mathbb{S}^{2} \times \mathbb{R}^{1}$ or $\mathbb{R}^{3}$ by a group of fixed point free isometries in the standard metrics.

(ii) A compact four-manifold with nonnegative curvature operator is diffeomorphic to $\mathbb{S}^{4}$ or $\mathbb{C} \mathbb{P}^{2}$ or $\mathbb{S}^{2} \times \mathbb{S}^{2}$, or a quotient of one of the spaces $\mathbb{S}^{4}$ or $\mathbb{C P}^{2}$ or $\mathbb{S}^{3} \times \mathbb{R}^{1}$ or $\mathbb{S}^{2} \times \mathbb{S}^{2}$ or $\mathbb{S}^{2} \times \mathbb{R}^{2}$ or $\mathbb{R}^{4}$ by a group of fixed point free isometries in the standard metrics.

The rest of the section will be devoted to prove Theorems 5.2.1-5.2.3.

Recall that the curvature operator $M_{\alpha \beta}$ evolves by

$$
\frac{\partial}{\partial t} M_{\alpha \beta}=\Delta M_{\alpha \beta}+M_{\alpha \beta}^{2}+M_{\alpha \beta}^{\#}
$$

where (see Section 1.3 and Section 2.4) $M_{\alpha \beta}^{2}$ is the operator square

$$
M_{\alpha \beta}^{2}=M_{\alpha \gamma} M_{\beta \gamma}
$$

and $M_{\alpha \beta}^{\#}$ is the Lie algebra $s o(n)$ square

$$
M_{\alpha \beta}^{\#}=C_{\alpha}^{\gamma \zeta} C_{\beta}^{\eta \theta} M_{\gamma \eta} M_{\zeta \theta} .
$$

We begin with the curvature pinching estimates of the Ricci flow in three dimensions. In dimension $n=3$, we know that $M_{\alpha \beta}^{\#}$ is the adjoint matrix of $M_{\alpha \beta}$. If we diagonalize $M_{\alpha \beta}$ with eigenvalues $\lambda \geq \mu \geq \nu$ so that

$$
\left(M_{\alpha \beta}\right)=\left(\begin{array}{ccc}
\lambda & & \\
& \mu & \\
& & \nu
\end{array}\right),
$$

then $M_{\alpha \beta}^{2}$ and $M_{\alpha \beta}^{\#}$ are also diagonal, with

$$
\left(M_{\alpha \beta}^{2}\right)=\left(\begin{array}{ccc}
\lambda^{2} & & \\
& \mu^{2} & \\
& & \nu^{2}
\end{array}\right) \text { and }\left(M_{\alpha \beta}^{\#}\right)=\left(\begin{array}{ccc}
\mu \nu & & \\
& \lambda \nu & \\
& & \lambda \mu
\end{array}\right),
$$

and the ODE corresponding to PDE (5.2.1) is then given by the system

$$
\left\{\begin{array}{l}
\frac{d}{d t} \lambda=\lambda^{2}+\mu \nu \\
\frac{d}{d t} \mu=\mu^{2}+\lambda \nu \\
\frac{d}{d t} \nu=\nu^{2}+\lambda \mu .
\end{array}\right.
$$

Lemma 5.2.4. For any $\varepsilon \in\left[0, \frac{1}{3}\right]$, the pinching condition

$$
R_{i j} \geq 0 \quad \text { and } \quad R_{i j} \geq \varepsilon R g_{i j}
$$


is preserved by the Ricci flow.

Proof. If we diagonalize the $3 \times 3$ curvature operator matrix $M_{\alpha \beta}$ with eigenvalues $\lambda \geq \mu \geq \nu$, then nonnegative sectional curvature corresponds to $\nu \geq 0$ and nonnegative Ricci curvature corresponds to the inequality $\mu+\nu \geq 0$. Also, the scalar curvature $R=\lambda+\mu+\nu$. So we need to show

$$
\mu+\nu \geq 0 \text { and } \mu+\nu \geq \delta \lambda, \text { with } \delta=2 \varepsilon /(1-2 \varepsilon),
$$

are preserved by the Ricci flow. By Hamilton's advanced maximum principle (Theorem 2.3.1), it suffices to show that the closed convex set

$$
K=\left\{M_{\alpha \beta} \mid \mu+\nu \geq 0 \text { and } \mu+\nu \geq \delta \lambda\right\}
$$

is preserved by the ODE system (5.2.2).

Now suppose we have diagonalized $M_{\alpha \beta}$ with eigenvalues $\lambda \geq \mu \geq \nu$ at $t=0$, then both $M_{\alpha \beta}^{2}$ and $M_{\alpha \beta}^{\#}$ are diagonal, so the matrix $M_{\alpha \beta}$ remains diagonal for $t>0$. Moreover, since

$$
\frac{d}{d t}(\mu-\nu)=(\mu-\nu)(\mu+\nu-\lambda),
$$

it is clear that $\mu \geq \nu$ for $t>0$ also. Similarly, we have $\lambda \geq \mu$ for $t>0$. Hence the inequalities $\lambda \geq \mu \geq \nu$ persist. This says that the solutions of the ODE system (5.2.2) agree with the original choice for the eigenvalues of the curvature operator.

The condition $\mu+\nu \geq 0$ is clearly preserved by the ODE, because

$$
\frac{d}{d t}(\mu+\nu)=\mu^{2}+\nu^{2}+\lambda(\mu+\nu) \geq 0 .
$$

It remains to check

$$
\frac{d}{d t}(\mu+\nu) \geq \delta \frac{d}{d t} \lambda
$$

or

$$
\mu^{2}+\lambda \nu+\nu^{2}+\lambda \mu \geq \delta\left(\lambda^{2}+\mu \nu\right)
$$

on the boundary where

$$
\mu+\nu=\delta \lambda \geq 0 .
$$

In fact, since

$$
(\lambda-\nu) \mu^{2}+(\lambda-\mu) \nu^{2} \geq 0,
$$

we have

$$
\lambda\left(\mu^{2}+\nu^{2}\right) \geq(\mu+\nu) \mu \nu .
$$

Hence

$$
\begin{aligned}
\mu^{2}+\mu \lambda+\nu^{2}+\nu \lambda & \geq\left(\frac{\mu+\nu}{\lambda}\right)\left(\lambda^{2}+\mu \nu\right) \\
& =\delta\left(\lambda^{2}+\mu \nu\right) .
\end{aligned}
$$


So we get the desired pinching estimate. $\mathrm{Q}$

Proposition 5.2.5. Suppose that the initial metric of the solution to the Ricci flow on $M^{3} \times[0, T)$ has positive Ricci curvature. Then for any $\varepsilon>0$ we can find $C_{\varepsilon}<+\infty$ such that

$$
\left|R_{i j}-\frac{1}{3} R g_{i j}\right| \leq \varepsilon R+C_{\varepsilon}
$$

for all subsequent $t \in[0, T)$.

Proof. Again we consider the ODE system (5.2.2). Let $M_{\alpha \beta}$ be diagonalized with eigenvalues $\lambda \geq \mu \geq \nu$ at $t=0$. We saw in the proof of Lemma 5.2.4 the inequalities $\lambda \geq \mu \geq \nu$ persist for $t>0$. We only need to show that there are positive constants $\delta$ and $C$ such that the closed convex set

$$
K=\left\{M_{\alpha \beta} \mid \lambda-\nu \leq C(\lambda+\mu+\nu)^{1-\delta}\right\}
$$

is preserved by the ODE.

We compute

$$
\frac{d}{d t}(\lambda-\nu)=(\lambda-\nu)(\lambda+\nu-\mu)
$$

and

$$
\begin{aligned}
\frac{d}{d t}(\lambda+\mu+\nu)= & (\lambda+\mu+\nu)(\lambda+\nu-\mu)+\mu^{2} \\
& +\mu(\mu+\nu)+\lambda(\mu-\nu) \\
\geq & (\lambda+\mu+\nu)(\lambda+\nu-\mu)+\mu^{2} .
\end{aligned}
$$

Thus, without loss of generality, we may assume $\lambda-\nu>0$ and get

$$
\frac{d}{d t} \log (\lambda-\nu)=\lambda+\nu-\mu
$$

and

$$
\frac{d}{d t} \log (\lambda+\mu+\nu) \geq \lambda+\nu-\mu+\frac{\mu^{2}}{\lambda+\mu+\nu} .
$$

By Lemma 5.2.4, there exists a positive constant $C$ depending only on the initial metric such that

$$
\begin{aligned}
& \lambda \leq \lambda+\mu \leq C(\mu+\nu) \leq 2 C \mu, \\
& \lambda+\nu-\mu \leq \lambda+\mu+\nu \leq 6 C \mu,
\end{aligned}
$$

and hence with $\epsilon=1 / 36 C^{2}$,

$$
\frac{d}{d t} \log (\lambda+\mu+\nu) \geq(1+\epsilon)(\lambda+\nu-\mu) .
$$


Therefore with $(1-\delta)=1 /(1+\epsilon)$,

$$
\frac{d}{d t} \log \left((\lambda-\nu) /(\lambda+\mu+\nu)^{1-\delta}\right) \leq 0
$$

This proves the proposition.

We now are ready to prove Theorem 5.2.1.

Proof of Theorem 5.2.1. Let $M$ be a compact three-manifold with positive Ricci curvature and let the metric evolve by the Ricci flow. By Lemma 5.2.4 we know that there exists a positive constant $\beta>0$ such that

$$
R_{i j} \geq \beta R g_{i j}
$$

for all $t \geq 0$ as long as the solution exists. The scalar curvature evolves by

$$
\begin{aligned}
\frac{\partial R}{\partial t} & =\Delta R+2\left|R_{i j}\right|^{2} \\
& \geq \Delta R+\frac{2}{3} R^{2},
\end{aligned}
$$

which implies, by the maximum principle, that the scalar curvature remains positive and tends to $+\infty$ in finite time.

We now use a blow up argument as in Section 4.3 to get the following gradient estimate.

Claim. For any $\varepsilon>0$, there exists a positive constant $C_{\varepsilon}<+\infty$ such that for any time $\tau \geq 0$, we have

$$
\max _{t \leq \tau} \max _{x \in M}|\nabla R m(x, t)| \leq \varepsilon \max _{t \leq \tau} \max _{x \in M}|R m(x, t)|^{\frac{3}{2}}+C_{\varepsilon} .
$$

We argue by contradiction. Suppose the above gradient estimate fails for some fixed $\varepsilon_{0}>0$. Pick a sequence $C_{j} \rightarrow+\infty$, and pick points $x_{j} \in M$ and times $\tau_{j}$ such that

$$
\left|\nabla R m\left(x_{j}, \tau_{j}\right)\right| \geq \varepsilon_{0} \max _{t \leq \tau_{j}} \max _{x \in M}|R m(x, t)|^{\frac{3}{2}}+C_{j}, \quad j=1,2, \ldots
$$

Choose $x_{j}$ to be the origin, and pull the metric back to a small ball on the tangent space $T_{x_{j}} M$ of radius $r_{j}$ proportional to the reciprocal of the square root of the maximum curvature up to time $\tau_{j}$ (i.e., $\left.\max _{t \leq \tau_{j}} \max _{x \in M}|R m(x, t)|\right)$. Clearly the maximum curvatures go to infinity by Shi's derivative estimate of curvature (Theorem 1.4.1). Dilate the metrics so that the maximum curvature

$$
\max _{t \leq \tau_{j}} \max _{x \in M}|R m(x, t)|
$$

becomes 1 and translate time so that $\tau_{j}$ becomes the time 0. By Theorem 4.1.5, we can take a (local) limit. The limit metric satisfies

$$
|\nabla R m(0,0)| \geq \varepsilon_{0}>0 .
$$

However the pinching estimate in Proposition 5.2.5 tells us the limit metric has

$$
R_{i j}-\frac{1}{3} R g_{i j} \equiv 0 .
$$


By using the contracted second Bianchi identity

$$
\frac{1}{2} \nabla_{i} R=\nabla^{j} R_{i j}=\nabla^{j}\left(R_{i j}-\frac{1}{3} R g_{i j}\right)+\frac{1}{3} \nabla_{i} R
$$

we get

$$
\nabla_{i} R \equiv 0 \quad \text { and then } \quad \nabla_{i} R_{j k} \equiv 0 .
$$

For a three-manifold, this in turn implies

$$
\nabla R m=0
$$

which is a contradiction. Hence we have proved the gradient estimate claimed.

We can now show that the solution to the Ricci flow becomes round as the time $t$ tends to the maximal time $T$. We have seen that the scalar curvature goes to infinity in finite time. Pick a sequence of points $x_{j} \in M$ and times $\tau_{j}$ where the curvature at $x_{j}$ is as large as it has been anywhere for $0 \leq t \leq \tau_{j}$ and $\tau_{j}$ tends to the maximal time. Since $|\nabla R m|$ is very small compared to $\left|R m\left(x_{j}, \tau_{j}\right)\right|$ by the above gradient estimate and $\left|R_{i j}-\frac{1}{3} R g_{i j}\right|$ is also very small compared to $\left|R m\left(x_{j}, \tau_{j}\right)\right|$ by Proposition 5.2.5, the curvature is nearly constant and positive in a large ball around $x_{j}$ at the time $\tau_{j}$. But then the Bonnet-Myers' theorem tells us this is the whole manifold. For $j$ large enough, the sectional curvature of the solution at the time $\tau_{j}$ is sufficiently pinched. Then it follows from the Klingenberg injectivity radius estimate (see Section 4.2) that the injectivity radius of the metric at time $\tau_{j}$ is bounded from below by $c / \sqrt{\left|R m\left(x_{j}, \tau_{j}\right)\right|}$ for some positive constant $c$ independent of $j$. Dilate the metrics so that the maximum curvature $\left|R m\left(x_{j}, \tau_{j}\right)\right|=\max _{t \leq \tau_{j}} \max _{x \in M}|R m(x, t)|$ becomes 1 and shift the time $\tau_{j}$ to the new time 0 . Then we can apply Hamilton's compactness theorem (Theorem 4.1.5) to take a limit. By the pinching estimate in Proposition 5.2.5, we know that the limit has positive constant curvature which is either the round $\mathbb{S}^{3}$ or a metric quotient of the round $\mathbb{S}^{3}$. Consequently, the compact three-manifold $M$ is diffeomorphic to the round $\mathbb{S}^{3}$ or a metric quotient of the round $\mathbb{S}^{3} \cdot \mathrm{q}$

Next we consider the pinching estimates of the Ricci flow on a compact fourmanifold $M$ with positive curvature operator.

In dimension 4, we saw in Section 1.3 when we decompose orthogonally $\Lambda^{2}=$ $\Lambda_{+}^{2} \oplus \Lambda_{-}^{2}$ into the eigenspaces of Hodge star with eigenvalue \pm 1 , we have a block decomposition of $M_{\alpha \beta}$ as

$$
M_{\alpha \beta}=\left(\begin{array}{cc}
A & B \\
{ }^{t} B & C
\end{array}\right)
$$

and then

$$
M_{\alpha \beta}^{\#}=2\left(\begin{array}{cc}
A^{\#} & B^{\#} \\
{ }^{t} B^{\#} & C^{\#}
\end{array}\right)
$$

where $A^{\#}, B^{\#}, C^{\#}$ are the adjoints of $3 \times 3$ submatrices as before.

Thus the ODE

$$
\frac{d}{d t} M_{\alpha \beta}=M_{\alpha \beta}^{2}+M_{\alpha \beta}^{\#}
$$


corresponding to the PDE (5.2.1) breaks up into the system of three equations

$$
\left\{\begin{array}{l}
\frac{d}{d t} A=A^{2}+B^{t} B+2 A^{\#} \\
\frac{d}{d t} B=A B+B C+2 B^{\#} \\
\frac{d}{d t} C=C^{2}+{ }^{t} B B+2 C^{\#}
\end{array}\right.
$$

As shown in Section 1.3, by the Bianchi identity, we know that $\operatorname{tr} A=\operatorname{tr} C$. For the symmetric matrices $A$ and $C$, we can choose an orthonormal basis $x_{1}, x_{2}, x_{3}$ of $\Lambda_{+}^{2}$ such that

$$
A=\left(\begin{array}{ccc}
a_{1} & 0 & 0 \\
0 & a_{2} & 0 \\
0 & 0 & a_{3}
\end{array}\right),
$$

and an orthonormal basis $z_{1}, z_{2}, z_{3}$ of $\Lambda_{-}^{2}$ such that

$$
C=\left(\begin{array}{ccc}
c_{1} & 0 & 0 \\
0 & c_{2} & 0 \\
0 & 0 & c_{3}
\end{array}\right) .
$$

For matrix $B$, we can choose orthonormal basis $y_{1}^{+}, y_{2}^{+}, y_{3}^{+}$of $\Lambda_{+}^{2}$ and $y_{1}^{-}, y_{2}^{-}, y_{3}^{-}$of $\Lambda_{-}^{2}$ such that

$$
B=\left(\begin{array}{ccc}
b_{1} & 0 & 0 \\
0 & b_{2} & 0 \\
0 & 0 & b_{3}
\end{array}\right) .
$$

with $0 \leq b_{1} \leq b_{2} \leq b_{3}$. We may also arrange the eigenvalues of $A$ and $C$ as $a_{1} \leq a_{2} \leq$ $a_{3}$ and $c_{1} \leq c_{2} \leq c_{3}$. In view of the advanced maximum principle Theorem 2.3.1, we only need to establish the pinching estimates for the ODE (5.2.3).

Note that

$$
\begin{aligned}
& a_{1}=\inf \left\{A(x, x) \mid x \in \Lambda_{+}^{2} \text { and }|x|=1\right\}, \\
& a_{3}=\sup \left\{A(x, x) \mid x \in \Lambda_{+}^{2} \text { and }|x|=1\right\}, \\
& c_{1}=\inf \left\{C(z, z) \mid z \in \Lambda_{-}^{2} \text { and }|z|=1\right\}, \\
& c_{3}=\sup \left\{C(z, z) \mid z \in \Lambda_{-}^{2} \text { and }|z|=1\right\} .
\end{aligned}
$$

We can compute their derivatives by Lemma 2.3 .3 as follows:

$$
\left\{\begin{aligned}
\frac{d}{d t} a_{1} & \geq a_{1}^{2}+b_{1}^{2}+2 a_{2} a_{3}, \\
\frac{d}{d t} a_{3} & \leq a_{3}^{2}+b_{3}^{2}+2 a_{1} a_{2}, \\
\frac{d}{d t} c_{1} & \geq c_{1}^{2}+b_{1}^{2}+2 c_{2} c_{3}, \\
\frac{d}{d t} c_{3} & \leq c_{3}^{2}+b_{3}^{2}+2 c_{1} c_{2} .
\end{aligned}\right.
$$


We shall make the pinching estimates by using the functions $b_{2}+b_{3}$ and $a-2 b+c$, where $a=a_{1}+a_{2}+a_{3}=c=c_{1}+c_{2}+c_{3}$ and $b=b_{1}+b_{2}+b_{3}$. Since

$$
\begin{aligned}
b_{2}+b_{3}= & B\left(y_{2}^{+}, y_{2}^{-}\right)+B\left(y_{3}^{+}, y_{3}^{-}\right) \\
= & \sup \left\{B\left(y^{+}, y^{-}\right)+B\left(\tilde{y}^{+}, \tilde{y}^{-}\right) \mid y^{+}, \tilde{y}^{+} \in \Lambda_{+}^{2} \text { with }\left|y^{+}\right|=\left|\tilde{y}^{+}\right|=1,\right. \\
& \left.y^{+} \perp \tilde{y}^{+}, \text {and } y^{-}, \tilde{y}^{-} \in \Lambda_{-}^{2} \text { with }\left|y^{-}\right|=\left|\tilde{y}^{-}\right|=1, y^{-} \perp \tilde{y}^{-}\right\},
\end{aligned}
$$

We compute by Lemma 2.3.3,

$$
\begin{aligned}
\frac{d}{d t}\left(b_{2}+b_{3}\right) \leq & \frac{d}{d t} B\left(y_{2}^{+}, y_{2}^{-}\right)+\frac{d}{d t} B\left(y_{3}^{+}, y_{3}^{-}\right) \\
= & A B\left(y_{2}^{+}, y_{2}^{-}\right)+B C\left(y_{2}^{+}, y_{2}^{-}\right)+2 B^{\#}\left(y_{2}^{+}, y_{2}^{-}\right) \\
& +A B\left(y_{3}^{+}, y_{3}^{-}\right)+B C\left(y_{3}^{+}, y_{3}^{-}\right)+2 B^{\#}\left(y_{3}^{+}, y_{3}^{-}\right) \\
= & b_{2} A\left(y_{2}^{+}, y_{2}^{+}\right)+b_{2} C\left(y_{2}^{-}, y_{2}^{-}\right)+2 b_{1} b_{3} \\
& +b_{3} A\left(y_{3}^{+}, y_{3}^{+}\right)+b_{3} C\left(y_{3}^{-}, y_{3}^{-}\right)+2 b_{1} b_{2} \\
\leq & a_{2} b_{2}+a_{3} b_{3}+b_{2} c_{2}+b_{3} c_{3}+2 b_{1} b_{2}+2 b_{1} b_{3}
\end{aligned}
$$

where we used the facts that $A\left(y_{2}^{+}, y_{2}^{+}\right)+A\left(y_{3}^{+}, y_{3}^{+}\right) \leq a_{2}+a_{3}$ and $C\left(y_{2}^{-}, y_{2}^{-}\right)+$ $C\left(y_{3}^{-}, y_{3}^{-}\right) \leq c_{2}+c_{3}$.

Note also that the function $a=\operatorname{tr} A=c=\operatorname{tr} C$ is linear, and the function $b$ is given by

$$
\begin{aligned}
b & =B\left(y_{1}^{+}, y_{1}^{-}\right)+B\left(y_{2}^{+}, y_{2}^{-}\right)+B\left(y_{3}^{+}, y_{3}^{-}\right) \\
& =\sup \left\{B\left(T y_{1}^{+}, \tilde{T} y_{1}^{-}\right)+B\left(T y_{2}^{+}, \tilde{T} y_{2}^{-}\right)+B\left(T y_{3}^{+}, \tilde{T} y_{3}^{-}\right) \mid T, \tilde{T}\right. \text { are }
\end{aligned}
$$

othogonal transformations of $\Lambda_{+}^{2}$ and $\Lambda_{-}^{2}$ respectively $\}$.

Indeed,

$$
\begin{aligned}
& B\left(T y_{1}^{+}, \tilde{T} y_{1}^{-}\right)+B\left(T y_{2}^{+}, \tilde{T} y_{2}^{-}\right)+B\left(T y_{3}^{+}, \tilde{T} y_{3}^{-}\right) \\
& =B\left(y_{1}^{+}, T^{-1} \tilde{T}\left(y_{1}^{-}\right)\right)+B\left(y_{2}^{+}, T^{-1} \tilde{T}\left(y_{2}^{-}\right)\right)+B\left(y_{3}^{+}, T^{-1} \tilde{T}\left(y_{3}^{-}\right)\right) \\
& =b_{1} t_{11}+b_{2} t_{22}+b_{3} t_{33}
\end{aligned}
$$

where $t_{11}, t_{22}, t_{33}$ are diagonal elements of the orthogonal matrix $T^{-1} \tilde{T}$ with $t_{11}, t_{22}, t_{33} \leq 1$. Thus by using Lemma 2.3 .3 again, we compute

$$
\begin{aligned}
\frac{d}{d t}(a-2 b+c) & \geq \operatorname{tr}\left(\frac{d}{d t} A-2 \frac{d}{d t} B+\frac{d}{d t} C\right) \\
& =\operatorname{tr}\left((A-B)^{2}+(C-B)^{2}+2\left(A^{\#}-2 B^{\#}+C^{\#}\right)\right)
\end{aligned}
$$

evaluated in those coordinates where $B$ is diagonal as above. Recalling the definition of Lie algebra product

$$
P \# Q=\frac{1}{2} \varepsilon_{\alpha \beta \gamma} \varepsilon_{\zeta \eta \theta} P_{\beta \eta} Q_{\gamma \theta}
$$

with $\varepsilon_{\alpha \beta \gamma}$ being the permutation tensor, we see that the Lie algebra product \# gives 
a symmetric bilinear operation on matrices, and then

$$
\begin{aligned}
\operatorname{tr} & \left(2\left(A^{\#}-2 B^{\#}+C^{\#}\right)\right) \\
= & \operatorname{tr}\left((A-C)^{\#}+(A+2 B+C) \#(A-2 B+C)\right) \\
= & -\frac{1}{2} \operatorname{tr}(A-C)^{2}+\frac{1}{2}(\operatorname{tr}(A-C))^{2} \\
& +\operatorname{tr}((A+2 B+C) \#(A-2 B+C)) \\
= & -\frac{1}{2} \operatorname{tr}(A-C)^{2}+\operatorname{tr}((A+2 B+C) \#(A-2 B+C))
\end{aligned}
$$

by the Bianchi identity. It is easy to check that

$$
\operatorname{tr}(A-B)^{2}+\operatorname{tr}(C-B)^{2}-\frac{1}{2} \operatorname{tr}(A-C)^{2}=\frac{1}{2} \operatorname{tr}(A-2 B+C)^{2} \geq 0 .
$$

Thus we obtain

$$
\frac{d}{d t}(a-2 b+c) \geq \operatorname{tr}((A+2 B+C) \#(A-2 B+C))
$$

Since $M_{\alpha \beta} \geq 0$ and

$$
M_{\alpha \beta}=\left(\begin{array}{cc}
A & B \\
{ }^{t} B & C
\end{array}\right),
$$

we see that $A+2 B+C \geq 0$ and $A-2 B+C \geq 0$, by applying $M_{\alpha \beta}$ to the vectors $(x, x)$ and $(x,-x)$. It is then not hard to see

$$
\operatorname{tr}((A+2 B+C) \#(A-2 B+C)) \geq\left(a_{1}+2 b_{1}+c_{1}\right)(a-2 b+c) .
$$

Hence we obtain

$$
\frac{d}{d t}(a-2 b+c) \geq\left(a_{1}+2 b_{1}+c_{1}\right)(a-2 b+c) .
$$

We now state and prove the following pinching estimates of Hamilton for the associated ODE (5.2.3).

Proposition 5.2.6 (Hamilton [59]). If we choose successively positive constants $G$ large enough, $H$ large enough, $\delta$ small enough, $J$ large enough, $\varepsilon$ small enough, $K$ large enough, $\theta$ small enough, and $L$ large enough, with each depending on those chosen before, then the closed convex subset $X$ of $\left\{M_{\alpha \beta} \geq 0\right\}$ defined by the inequalities

(1) $\left(b_{2}+b_{3}\right)^{2} \leq G a_{1} c_{1}$,

(2) $a_{3} \leq H a_{1}$ and $c_{3} \leq H c_{1}$,

(3) $\left(b_{2}+b_{3}\right)^{2+\delta} \leq J a_{1} c_{1}(a-2 b+c)^{\delta}$,

(4) $\left(b_{2}+b_{3}\right)^{2+\varepsilon} \leq K a_{1} c_{1}$,

(5) $a_{3} \leq a_{1}+L a_{1}^{1-\theta}$ and $c_{3} \leq c_{1}+L c_{1}^{1-\theta}$,

is preserved by ODE (5.2.3). Moreover every compact subset of $\left\{M_{\alpha \beta}>0\right\}$ lies in some such set $X$.

Proof. Clearly the subset $X$ is closed and convex. We first note that we may assume $b_{2}+b_{3}>0$ because if $b_{2}+b_{3}=0$, then from (5.2.5), $b_{2}+b_{3}$ will remain 
zero and then the inequalities (1), (3) and (4) concerning $b_{2}+b_{3}$ are automatically satisfied. Likewise we may assume $a_{3}>0$ and $c_{3}>0$ from (5.2.4). check

Let $G$ be a fixed positive constant. To prove the inequality (1) we only need to

$$
\frac{d}{d t} \log \frac{a_{1} c_{1}}{\left(b_{2}+b_{3}\right)^{2}} \geq 0
$$

whenever $\left(b_{2}+b_{3}\right)^{2}=G a_{1} c_{1}$ and $b_{2}+b_{3}>0$. Indeed, it follows from (5.2.4) and (5.2.5) that

$$
\begin{aligned}
& \frac{d}{d t} \log a_{1} \geq 2 b_{1}+2 a_{3}+\frac{\left(a_{1}-b_{1}\right)^{2}}{a_{1}}+2 \frac{a_{3}}{a_{1}}\left(a_{2}-a_{1}\right), \\
& \frac{d}{d t} \log c_{1} \geq 2 b_{1}+2 c_{3}+\frac{\left(c_{1}-b_{1}\right)^{2}}{c_{1}}+2 \frac{c_{3}}{c_{1}}\left(c_{2}-c_{1}\right),
\end{aligned}
$$

and

$$
\frac{d}{d t} \log \left(b_{2}+b_{3}\right) \leq 2 b_{1}+a_{3}+c_{3}-\frac{b_{2}}{b_{2}+b_{3}}\left[\left(a_{3}-a_{2}\right)+\left(c_{3}-c_{2}\right)\right],
$$

which immediately give the desired inequality (5.2.7).

By (5.2.4), we have

$$
\frac{d}{d t} \log a_{3} \leq a_{3}+2 a_{1}+\frac{b_{3}^{2}}{a_{3}}-\frac{2 a_{1}}{a_{3}}\left(a_{3}-a_{2}\right) .
$$

From the inequality (1) there holds $b_{3}^{2} \leq G a_{1} c_{1}$. Since $\operatorname{tr} A=\operatorname{tr} C, c_{1} \leq c_{1}+c_{2}+c_{3}=$ $a_{1}+a_{2}+a_{3} \leq 3 a_{3}$ which shows

$$
\frac{b_{3}^{2}}{a_{3}} \leq 3 G a_{1}
$$

Thus by (5.2.8) and (5.2.11),

$$
\frac{d}{d t} \log \frac{a_{3}}{a_{1}} \leq(3 G+2) a_{1}-a_{3} .
$$

So if $H \geq(3 G+2)$, then the inequalities $a_{3} \leq H a_{1}$ and likewise $c_{3} \leq H c_{1}$ are preserved.

For the inequality (3), we compute from (5.2.8)-(5.2.10)

$$
\begin{aligned}
\frac{d}{d t} \log \frac{a_{1} c_{1}}{\left(b_{2}+b_{3}\right)^{2}} \geq & \frac{\left(a_{1}-b_{1}\right)^{2}}{a_{1}}+\frac{\left(c_{1}-b_{1}\right)^{2}}{c_{1}}+2 \frac{a_{3}}{a_{1}}\left(a_{2}-a_{1}\right)+2 \frac{c_{3}}{c_{1}}\left(c_{2}-c_{1}\right) \\
& +\frac{2 b_{2}}{b_{2}+b_{3}}\left[\left(a_{3}-a_{2}\right)+\left(c_{3}-c_{2}\right)\right] .
\end{aligned}
$$

If $b_{1} \leq a_{1} / 2$, then

$$
\frac{\left(a_{1}-b_{1}\right)^{2}}{a_{1}} \geq \frac{a_{1}}{4} \geq \frac{1}{4 H} a_{3},
$$

and if $b_{1} \geq a_{1} / 2$, then

$$
\frac{2 b_{2}}{b_{2}+b_{3}} \geq \frac{2 b_{2}}{\sqrt{G a_{1} c_{1}}} \geq \frac{2 b_{2}}{\sqrt{3 G a_{1} a_{3}}} \geq \frac{2 b_{2}}{\sqrt{3 G H} \cdot a_{1}} \geq \frac{1}{\sqrt{3 G H}} .
$$


Thus by combining with $3 a_{3} \geq c_{3}$, we have

$$
\frac{d}{d t} \log \frac{a_{1} c_{1}}{\left(b_{2}+b_{3}\right)^{2}} \geq \delta\left(a_{3}-a_{1}\right)+\delta\left(c_{3}-c_{1}\right)
$$

provided $\delta \leq \min \left(\frac{1}{24 H}, \frac{1}{\sqrt{3 G H}}\right)$. On the other hand, it follows from (5.2.6) and (5.2.10) that

$$
\frac{d}{d t} \log \frac{b_{2}+b_{3}}{a-2 b+c} \leq\left(a_{3}-a_{1}\right)+\left(c_{3}-c_{1}\right) .
$$

Therefore the inequality (3)

$$
\left(b_{2}+b_{3}\right)^{2+\delta} \leq J a_{1} c_{1}(a-2 b+c)^{\delta}
$$

will be preserved by any positive constant $J$.

To verify the inequality (4), we first note that there is a small $\eta>0$ such that

$$
b \leq(1-\eta) a,
$$

on the set defined by the inequality (3). Indeed, if $b \leq \frac{a}{2}$, this is trivial and if $b \geq \frac{a}{2}$, then

$$
\left(\frac{a}{3}\right)^{2+\delta} \leq\left(b_{2}+b_{3}\right)^{2+\delta} \leq 2^{\delta} J a^{2}(a-b)^{\delta}
$$

which makes $b \leq(1-\eta) a$ for some $\eta>0$ small enough. Consequently,

$$
\eta a \leq a-b \leq 3\left(a_{3}-b_{1}\right)
$$

which implies either

$$
a_{3}-a_{1} \geq \frac{1}{6} \eta a
$$

or

$$
a_{1}-b_{1} \geq \frac{1}{6} \eta a
$$

Thus as in the proof of the inequality (3), we have

$$
\frac{d}{d t} \log \frac{a_{1} c_{1}}{\left(b_{2}+b_{3}\right)^{2}} \geq \delta\left(a_{3}-a_{1}\right)
$$

and

$$
\frac{d}{d t} \log \frac{a_{1} c_{1}}{\left(b_{2}+b_{3}\right)^{2}} \geq \frac{\left(a_{1}-b_{1}\right)^{2}}{a_{1}}
$$

which in turn implies

$$
\frac{d}{d t} \log \frac{a_{1} c_{1}}{\left(b_{2}+b_{3}\right)^{2}} \geq\left(\max \left\{\frac{1}{6} \eta \delta, \frac{1}{36} \eta^{2}\right\}\right) \cdot a .
$$

On the other hand, it follows from (5.2.10) that

$$
\frac{d}{d t} \log \left(b_{2}+b_{3}\right) \leq 2 b_{1}+a_{3}+c_{3} \leq 4 a
$$


since $M_{\alpha \beta} \geq 0$. Then if $\varepsilon>0$ is small enough

$$
\frac{d}{d t} \log \frac{a_{1} c_{1}}{\left(b_{2}+b_{3}\right)^{2+\varepsilon}} \geq 0
$$

and it follows that the inequality (4) is preserved by any positive $K$.

Finally we consider the inequality (5). From (5.2.8) we have

$$
\frac{d}{d t} \log a_{1} \geq a_{1}+2 a_{3}
$$

and then for $\theta \in(0,1)$,

$$
\frac{d}{d t} \log \left(a_{1}+L a_{1}^{1-\theta}\right) \geq \frac{a_{1}+(1-\theta) L a_{1}^{1-\theta}}{a_{1}+L a_{1}^{1-\theta}}\left(a_{1}+2 a_{3}\right) .
$$

On the other hand, the inequality (4) tells us

$$
b_{3}^{2} \leq \tilde{K} a_{1}^{1-\theta} a_{3}
$$

for some positive constant $\tilde{K}$ large enough with $\theta$ to be fixed small enough. And then

$$
\frac{d}{d t} \log a_{3} \leq a_{3}+2 a_{1}+\tilde{K} a_{1}^{1-\theta}
$$

by combining with (5.2.11). Thus by choosing $\theta \leq \frac{1}{6 H}$ and $L \geq 2 \tilde{K}$,

$$
\begin{aligned}
\frac{d}{d t} \log \frac{a_{1}+L a_{1}^{1-\theta}}{a_{3}} & \geq\left(a_{3}-a_{1}\right)-\theta \frac{L a_{1}^{1-\theta}}{a_{1}+L a_{1}^{1-\theta}}\left(a_{1}+2 a_{3}\right)-\tilde{K} a_{1}^{1-\theta} \\
& \geq\left(a_{3}-a_{1}\right)-\theta \frac{L a_{1}^{1-\theta}}{a_{1}+L a_{1}^{1-\theta}} \cdot 3 H a_{1}-\tilde{K} a_{1}^{1-\theta} \\
& \geq\left(a_{3}-a_{1}\right)-(3 \theta H L+\tilde{K}) a_{1}^{1-\theta} \\
& =[L-(3 \theta H L+\tilde{K})] a_{1}^{1-\theta} \\
& \geq 0
\end{aligned}
$$

whenever $a_{1}+L a_{1}^{1-\theta}=a_{3}$. Consequently the set $\left\{a_{1}+L a_{1}^{1-\theta} \geq a_{3}\right\}$ is preserved. A similar argument works for the inequality in $C$. This completes the proof of Proposition 5.2.6.

The combination of the advanced maximum principle Theorem 2.3.1 and the pinching estimates of the ODE (5.2.3) in Proposition 5.2.6 immediately gives the following pinching estimate for the Ricci flow on a compact four-manifold.

Corollary 5.2.7. Suppose that the initial metric of the solution to the Ricci flow on a compact four-manifold has positive curvature operator. Then for any $\varepsilon>0$ we can find positive constant $C_{\varepsilon}<+\infty$ such that

$$
|\stackrel{\circ}{R m}| \leq \varepsilon R+C_{\varepsilon}
$$

for all $t \geq 0$ as long as the solution exists, where $\stackrel{\circ}{\mathrm{R} m}$ is the traceless part of the curvature operator. 
Proof of Theorem 5.2.2. Let $M$ be a compact four-manifold with positive curvature operator and let us evolve the metric by the Ricci flow. Again the evolution equation of the scalar curvature tells us that the scalar curvature remains positive and becomes unbounded in finite time. Pick a sequence of points $x_{j} \in M$ and times $\tau_{j}$ where the curvature at $x_{j}$ is as large as it has been anywhere for $0 \leq t \leq \tau_{j}$. Dilate the metrics so that the maximum curvature $\left|R m\left(x_{j}, \tau_{j}\right)\right|=\max _{t \leq \tau_{j}} \max _{x \in M}|R m(x, t)|$ becomes 1 and shift the time so that the time $\tau_{j}$ becomes the new time 0 . The Klingenberg injectivity radius estimate in Section 4.2 tells us that the injectivity radii of the rescaled metrics at the origins $x_{j}$ and at the new time 0 are uniformly bounded from below. Then we can apply the Hamilton's compactness theorem (Theorem 4.1.5) to take a limit. By the pinching estimate in Corollary 5.2.7, we know that the limit metric has positive constant curvature which is either $\mathbb{S}^{4}$ or $\mathbb{R} \mathbb{P}^{4}$. Therefore the compact four-manifold $M$ is diffeomorphic to the sphere $\mathbb{S}^{4}$ or the real projective space $\mathbb{R} \mathbb{P}^{4}$.

REMARK 5.2.8. The proofs of Theorem 5.2.1 and Theorem 5.2.2 also show that the Ricci flow on a compact three-manifold with positive Ricci curvature or a compact four-manifold with positive curvature operator is subsequentially converging (up to scalings) in the $C^{\infty}$ topology to the same underlying compact manifold with a metric of positive constant curvature. Of course, this subsequential convergence is in the sense of Hamilton's compactness theorem (Theorem 4.1.5) which is also up to the pullbacks of diffeomorphisms. Actually in [58] and [59], Hamilton obtained the convergence in the stronger sense that the (rescaled) metrics converge (in the $C^{\infty}$ topology) to a constant (positive) curvature metric.

In the following we use the Hamilton's strong maximum principle in Section 2.4 to prove Theorem 5.2.3.

Proof of Theorem 5.2.3. In views of Theorem 5.2.1 and Theorem 5.2.2, we may assume the Ricci curvature (in dimension 3 ) and the curvature operator (in dimension 4) always have nontrivial kernels somewhere along the Ricci flow.

(i) In the case of dimension 3, we consider the evolution equation (1.3.5) of the Ricci curvature

$$
\frac{\partial R_{a b}}{\partial t}=\triangle R_{a b}+2 R_{a c b d} R_{c d}
$$

in an orthonormal frame coordinate. At each point, we diagonalize $R_{a b}$ with eigenvectors $e_{1}, e_{2}, e_{3}$ and eigenvalues $\lambda_{1} \leq \lambda_{2} \leq \lambda_{3}$. Since

$$
\begin{aligned}
R_{1 c 1 d} R_{c d} & =R_{1212} R_{22}+R_{1313} R_{33} \\
& =\frac{1}{2}\left(\left(\lambda_{3}-\lambda_{2}\right)^{2}+\lambda_{1}\left(\lambda_{2}+\lambda_{3}\right)\right)
\end{aligned}
$$

we know that if $R_{a b} \geq 0$, then $R_{a c b d} R_{c d} \geq 0$. By Hamilton's strong maximum principle (Theorem 2.2.1), there exists an interval $0<t<\delta$ on which the rank of $R_{a b}$ is constant and the null space of $R_{a b}$ is invariant under parallel translation and invariant in time and also lies in the null space of $R_{a c b d} R_{c d}$. If the null space of $R_{a b}$ has rank one, then $\lambda_{1}=0$ and $\lambda_{2}=\lambda_{3}>0$. In this case, by De Rham decomposition theorem, the universal cover $\tilde{M}$ of the compact $M$ splits isometrically as $\mathbb{R} \times \Sigma^{2}$ and the curvature of $\Sigma^{2}$ has a positive lower bound. Hence $\Sigma^{2}$ is diffeomorphic to $\mathbb{S}^{2}$. Assume $M=\mathbb{R} \times \Sigma^{2} / \Gamma$, for some isometric subgroup $\Gamma$ of $\mathbb{R} \times \Sigma^{2}$. Note that $\Gamma$ 
remains to be an isometric subgroup of $\mathbb{R} \times \Sigma^{2}$ during the Ricci flow by the uniqueness (Theorem 1.2.4). Since the Ricci flow on $\mathbb{R} \times \Sigma^{2} / \Gamma$ converges to the standard metric by Theorem 5.1.11, $\Gamma$ must be an isometric subgroup of $\mathbb{R} \times \mathbb{S}^{2}$ in the standard metric. If the null space of $R_{a b}$ has rank greater than one, then $R_{a b}=0$ and the manifold is flat. This proves Theorem 5.2.3 part (i).

(ii) In the case of dimension 4, we classify the manifolds according to the (restricted) holonomy algebra $\mathcal{G}$. Note that the curvature operator has nontrivial kernel and $\mathcal{G}$ is the image of the the curvature operator, we see that $\mathcal{G}$ is a proper subalgebra of $s o(4)$. We divide the argument into two cases.

Case 1. $\mathcal{G}$ is reducible.

In this case the universal cover $\tilde{M}$ splits isometrically as $\tilde{M}_{1} \times \tilde{M}_{2}$. By the above results on two and three dimensional Ricci flow, we see that $M$ is diffeomorphic to a quotient of one of the spaces $\mathbb{R}^{4}, \mathbb{R} \times \mathbb{S}^{3}, \mathbb{R}^{2} \times \mathbb{S}^{2}, \mathbb{S}^{2} \times \mathbb{S}^{2}$ by a group of fixed point free isometries. As before by running the Ricci flow until it converges and using the uniqueness (Theorem 1.2.4), we see that this group is actually a subgroup of the isometries in the standard metrics.

Case 2. $\mathcal{G}$ is not reducible (i.e., irreducible).

If the manifold is not Einstein, then by Berger's classification theorem for holonomy groups [7], $\mathcal{G}=s o(4)$ or $u(2)$. Since the curvature operator is not strictly positive, $\mathcal{G}=u(2)$, and the universal cover $\tilde{M}$ of $M$ is Kähler and has positive bisectional curvature. In this case $\tilde{M}$ is biholomorphic to $\mathbb{C P}^{2}$ by the result of AndreottiFrankel [47] (also the resolution of the Frankel conjecture by Mori [96] and Siu-Yau $[120])$.

If the manifold is Einstein, then by the block decomposition of the curvature operator matrix in four-manifolds (see the third section of Chapter 1),

$$
\operatorname{Rm}\left(\Lambda_{+}^{2}, \Lambda_{-}^{2}\right)=0 .
$$

Let $\varphi \neq 0$, and

$$
\varphi=\varphi_{+}+\varphi_{-} \in \Lambda_{+}^{2} \oplus \Lambda_{-}^{2},
$$

lies in the kernel of the curvature operator, then

$$
0=R m\left(\varphi_{+}, \varphi_{+}\right)+R m\left(\varphi_{-}, \varphi_{-}\right) .
$$

It follows that

$$
\operatorname{Rm}\left(\varphi_{+}, \varphi_{+}\right)=0 \text {, and } \operatorname{Rm}\left(\varphi_{-}, \varphi_{-}\right)=0 .
$$

We may assume $\varphi_{+} \neq 0$ (the argument for the other case is similar). We consider the restriction of $R m$ to $\Lambda_{+}^{2}$, since $\Lambda_{+}^{2}$ is an invariant subspace of $R m$ and the intersection of $\Lambda_{+}^{2}$ with the null space of $R m$ is nontrivial. By considering the null space of $R m$ and its orthogonal complement in $\Lambda_{+}^{2}$, we obtain a parallel distribution of rank one in $\Lambda_{+}^{2}$. This parallel distribution gives a parallel translation invariant two-form $\omega \in \Lambda_{+}^{2}$ on the universal cover $\tilde{M}$ of $M$. This two-form is nondegenerate, so it induces a Kähler structure of $\tilde{M}$. Since the Kähler metric is parallel with respect to the original metric and the manifold is irreducible, the Kähler metric is proportional to the original metric. Hence the manifold $\tilde{M}$ is Kähler-Einstein with nonnegative 
curvature operator. Taking into account the irreducibility of $\mathcal{G}$, it follows that $\tilde{M}$ is biholomorphic to $\mathbb{C P}^{2}$. Therefore the proof of Theorem 5.2.3 is completed.

To end this section, we mention the generalizations of Hamilton's differential sphere theorem (Theorem 5.2.1 and Theorem 5.2.2) to higher dimensions.

Using minimal surface theory, Micallef and Moore [88] proved that any compact simply connected $n$-dimensional manifold with positive curvature operator is homeomorphic to the sphere $\mathbb{S}^{n}$. But it is still an open question whether a compact simply connected $n$-dimensional manifold with positive curvature operator is diffeomorphic to the sphere $\mathbb{S}^{n}$.

It is well-known that the curvature tensor $R m=\left\{R_{i j k l}\right\}$ of a Riemannian manifold can be decomposed into three orthogonal components which have the same symmetries as $R m$ :

$$
R m=W+V+U
$$

Here $W=\left\{W_{i j k l}\right\}$ is the Weyl conformal curvature tensor, whereas $V=\left\{V_{i j k l}\right\}$ and $U=\left\{U_{i j k l}\right\}$ denote the traceless Ricci part and the scalar curvature part respectively. The following pointwisely pinching sphere theorem under the additional assumption that the manifold is compact was first obtained by Huisken [71], Margerin [83], [84] and Nishikawa [101] by using the Ricci flow. The compactness assumption was later removed by Chen and the second author in [30].

THEOREM 5.2.9. Let $n \geq 4$. Suppose $M$ is a complete $n$-dimensional manifold with positive and bounded scalar curvature and satisfies the pointwisely pinching condition

$$
|W|^{2}+|V|^{2} \leq \delta_{n}(1-\varepsilon)^{2}|U|^{2}
$$

where $\varepsilon>0, \delta_{4}=\frac{1}{5}, \delta_{5}=\frac{1}{10}$, and

$$
\delta_{n}=\frac{2}{(n-2)(n+1)}, n \geq 6
$$

Then $M$ is diffeomorphic to the sphere $\mathbb{S}^{n}$ or a quotient of it by a finite group of fixed point free isometries in the standard metric.

In [30], Chen and the second author also used the Ricci flow to obtain the following flatness theorem for noncompact three-manifolds.

TheOREM 5.2.10. Let $M$ be a three-dimensional complete noncompact Riemannian manifold with bounded and nonnegative sectional curvature. Suppose $M$ satisfies the following Ricci pinching condition

$$
R_{i j} \geq \varepsilon R g_{i j}, \quad \text { on } M,
$$

for some $\varepsilon>0$. Then $M$ is flat.

The basic idea of proofs of these two theorems is to analyze the asymptotic behavior of the solution to the Ricci flow. For the details, one can consult the above cited literatures. 
5.3. Nonsingular Solutions on Three-manifolds. We have seen in the previous section that a good understanding of the long time behaviors for solutions to the Ricci flow could lead to remarkable topological or geometric consequences for the underlying manifolds. Since one of the central themes of the Ricci flow is to study the geometry and topology of three-manifolds, we will start to analyze the long time behavior of the Ricci flow on a compact three-manifold by first considering a special class of solutions (i.e., the nonsingular solutions defined below) in this section. Our presentation follows closely the paper of Hamilton [65].

Let $M$ be a compact three-manifold. We will consider the (unnormalized) Ricci flow

$$
\frac{\partial}{\partial t} g_{i j}=-2 R_{i j}
$$

and the normalized Ricci flow

$$
\frac{\partial}{\partial t} g_{i j}=\frac{2}{3} r g_{i j}-2 R_{i j}
$$

where $r=r(t)$ is the function of the average of the scalar curvature. Recall that the normalized flow differs from the unnormalized flow only by rescaling in space and time so that the total volume $V=\int_{M} d \mu$ remains constant.

In this section we only consider a special class of solutions that we now define.

Definition 5.3.1. A nonsingular solution of the Ricci flow is one where the solution of the normalized flow exists for all time $0 \leq t<\infty$, and the curvature remains bounded $|R m| \leq C<+\infty$ for all time with some constant $C$ independent of $t$.

Clearly any solution to the Ricci flow on a compact three-manifold with nonnegative Ricci curvature is nonsingular. Currently there are few conditions which guarantee a solution will remain nonsingular. Nevertheless, the ideas and arguments of this section is extremely important. One will see in Chapter 7 that these arguments will be modified to analyze the long-time behavior of arbitrary solutions, or even the solutions with surgery, to the Ricci flow on three-manifolds.

We begin with an improvement of Hamilton-Ivey pinching result (Theorem 2.4.1).

TheOREM 5.3.2 (Hamilton [65]). Suppose we have a solution to the (unnormalized) Ricci flow on a three-manifold which is complete with bounded curvature for each $t \geq 0$. Assume at $t=0$ the eigenvalues $\lambda \geq \mu \geq \nu$ of the curvature operator at each point are bounded below by $\nu \geq-1$. Then at all points and all times $t \geq 0$ we have the pinching estimate

$$
R \geq(-\nu)[\log (-\nu)+\log (1+t)-3]
$$

whenever $\nu<0$.

Proof. As before, we study the ODE system

$$
\left\{\begin{array}{l}
\frac{d \lambda}{d t}=\lambda^{2}+\mu \nu, \\
\frac{d \mu}{d t}=\mu^{2}+\lambda \nu, \\
\frac{d \nu}{d t}=\nu^{2}+\lambda \mu .
\end{array}\right.
$$


Consider again the function

$$
y=f(x)=x(\log x-3)
$$

for $e^{2} \leq x<+\infty$, which is increasing and convex with range $-e^{2} \leq y<+\infty$. Its inverse function $x=f^{-1}(y)$ is increasing and concave on $-e^{2} \leq y<+\infty$. For each $t \geq 0$, we consider the set $K(t)$ of $3 \times 3$ symmetric matrices defined by the inequalities:

$$
\lambda+\mu+\nu \geq-\frac{3}{1+t}
$$

and

$$
\nu(1+t)+f^{-1}((\lambda+\mu+\nu)(1+t)) \geq 0,
$$

which is closed and convex (as we saw in the proof of Theorem 2.4.1). By the assumptions at $t=0$ and the advanced maximum principle Theorem 2.3.5, we only need to check that the set $K(t)$ is preserved by the ODE system.

Since $R=\lambda+\mu+\nu$, we get from the ODE that

$$
\frac{d R}{d t} \geq \frac{2}{3} R^{2} \geq \frac{1}{3} R^{2}
$$

which implies that

$$
R \geq-\frac{3}{1+t}, \quad \text { for all } t \geq 0 .
$$

Thus the first inequality (5.3.1) is preserved. Note that the second inequality (5.3.2) is automatically satisfied when $(-\nu) \leq 3 /(1+t)$. Now we compute from the ODE system,

$$
\begin{aligned}
\frac{d}{d t}\left(\frac{R}{(-\nu)}-\log (-\nu)\right) & =\frac{1}{(-\nu)^{2}}\left[(-\nu) \cdot \frac{d R}{d t}-(R+(-\nu)) \frac{d(-\nu)}{d t}\right] \\
& =\frac{1}{(-\nu)^{2}}\left[(-\nu)^{3}+(-\nu) \mu^{2}+\lambda^{2}((-\nu)+\mu)-\lambda \mu(\nu-\mu)\right] \\
& \geq(-\nu) \\
& \geq \frac{3}{(1+t)} \\
& \geq \frac{d}{d t}[\log (1+t)-3]
\end{aligned}
$$

whenever $R=(-\nu)[\log (-\nu)+\log (1+t)-3]$ and $(-\nu) \geq 3 /(1+t)$. Thus the second inequality (5.3.2) is also preserved under the system of ODE.

Therefore we have proved the theorem.

Denote by

$$
\hat{\rho}(t)=\max \left\{\operatorname{inj}\left(x, g_{i j}(t)\right) \mid x \in M\right\}
$$

where $\operatorname{inj}\left(x, g_{i j}(t)\right)$ is the injectivity radius of the manifold $M$ at $x$ with respect to the metric $g_{i j}(t)$. 
DEFINITION 5.3.3. We say a solution to the normalized Ricci flow is collapsed if there is a sequence of times $t_{k} \rightarrow+\infty$ such that $\hat{\rho}\left(t_{k}\right) \rightarrow 0$ as $k \rightarrow+\infty$.

When a nonsingular solution of the Ricci flow on $M$ is collapsed, it follows from the work of Cheeger-Gromov [24] [25] or Cheeger-Gromov-Fukaya [26] that the manifold $M$ has an $\mathcal{F}$-structure and then its topology is completely understood. In the following we always assume nonsingular solutions are not collapsed.

Now suppose that we have a nonsingular solution which does not collapse. Then for arbitrary sequence of times $t_{j} \rightarrow \infty$, we can find a sequence of points $x_{j}$ and some $\delta>0$ so that the injectivity radius of $M$ at $x_{j}$ in the metric at time $t_{j}$ is at least $\delta$. Clearly the Hamilton's compactness theorem (Theorem 4.1.5) also holds for the normalized Ricci flow. Then by taking the $x_{j}$ as origins and the $t_{j}$ as initial times, we can extract a convergent subsequence. We call such a limit a noncollapsing limit. Of course the limit has also finite volume. However the volume of the limit may be smaller than the original one if the diameter goes to infinity.

The main result of this section is the following theorem of Hamilton [65].

Theorem 5.3.4 (Hamilton [65]). Let $g_{i j}(t), 0 \leq t<+\infty$, be a noncollapsing nonsingular solution of the normalized Ricci flow on a compact three-manifold $M$. Then either

(i) there exist a sequence of times $t_{k} \rightarrow+\infty$ and a sequence of diffeomorphisms $\varphi_{k}: M \rightarrow M$ so that the pull-back of the metric $g_{i j}\left(t_{k}\right)$ by $\varphi_{k}$ converges in the $C^{\infty}$ topology to a metric on $M$ with constant sectional curvature; or

(ii) we can find a finite collection of complete noncompact hyperbolic threemanifolds $\mathcal{H}_{1}, \ldots, \mathcal{H}_{m}$ with finite volume, and for all $t$ beyond some time $T<+\infty$ we can find compact subsets $K_{1}, \ldots, K_{m}$ of $\mathcal{H}_{1}, \ldots, \mathcal{H}_{m}$ respectively obtained by truncating each cusp of the hyperbolic manifolds along constant mean curvature torus of small area, and diffeomorphisms $\varphi_{l}(t), 1 \leq l \leq m$, of $K_{l}$ into $M$ so that as long as $t$ sufficiently large, the pull-back of the solution metric $g_{i j}(t)$ by $\varphi_{l}(t)$ is as close as to the hyperbolic metric as we like on the compact sets $K_{1}, \ldots, K_{m}$; and moreover if we call the exceptional part of $M$ those points where they are not in the image of any $\varphi_{l}$, we can take the injectivity radii of the exceptional part at everywhere as small as we like and the boundary tori of each $K_{l}$ are incompressible in the sense that each $\varphi_{l}$ injects $\pi_{1}\left(\partial K_{l}\right)$ into $\pi_{1}(M)$.

REMARK 5.3.5. The exceptional part has bounded curvature and arbitrarily small injectivity radii everywhere as $t$ large enough. Moreover the boundary of the exceptional part consists of a finite disjoint union of tori with sufficiently small area and is convex. Then by the work of Cheeger-Gromov [24], [25] or Cheeger-GromovFukaya [26], there exists an $\mathcal{F}$-structure on the exceptional part. In particular, the exceptional part is a graph manifold, which have been topologically classified. Hence any nonsingular solution to the normalized Ricci flow is geometrizable in the sense of Thurston (see the last section of Chapter 7 for details).

The rest of this section is devoted to the proof of Theorem 5.3.4. We will divide the proof into three parts.

\section{Part I: Subsequence Convergence}

According to Lemma 5.1.1, the scalar curvature of the normalized flow evolves by 
the equation

$$
\begin{aligned}
\frac{\partial}{\partial t} R & =\Delta R+2|R i c|^{2}-\frac{2}{3} r R \\
& =\Delta R+2|R i c|+\frac{2}{3} R(R-r)
\end{aligned}
$$

where $\stackrel{\circ}{R} i c$ is the traceless part of the Ricci tensor. As before, we denote by $R_{\min }(t)=$ $\min _{x \in M} R(x, t)$. It then follows from the maximum principle that

$$
\frac{d}{d t} R_{\min } \geq \frac{2}{3} R_{\min }\left(R_{\min }-r\right)
$$

which implies that if $R_{\min } \leq 0$ it must be nondecreasing, and if $R_{\min } \geq 0$ it cannot go negative again. We can then divide the noncollapsing solutions of the normalized Ricci flow into three cases.

Case (1): $R_{\min }(t)>0$ for some $t>0$;

Case (2): $R_{\min }(t) \leq 0$ for all $t \in[0,+\infty)$ and $\lim _{t \rightarrow+\infty} R_{\min }(t)=0$;

Case (3): $R_{\min }(t) \leq 0$ for all $t \in[0,+\infty)$ and $\lim _{t \rightarrow+\infty} R_{\min }(t)<0$.

Let us first consider Case (1). In this case the maximal time interval $[0, T)$ of the corresponding solution of the unnormalized flow is finite, since the unnormalized scalar curvature $\tilde{R}$ satisfies

$$
\begin{aligned}
\frac{\partial}{\partial t} \tilde{R} & =\Delta \tilde{R}+2|\tilde{R} i c|^{2} \\
& \geq \Delta \tilde{R}+\frac{2}{3} \tilde{R}^{2}
\end{aligned}
$$

which implies that the curvature of the unnormalized solution blows up in finite time. Without loss of generality, we may assume that for the initial metric at $t=0$, the eigenvalues $\tilde{\lambda} \geq \tilde{\mu} \geq \tilde{\nu}$ of the curvature operator are bounded below by $\tilde{\nu} \geq-1$. It follows from Theorem 5.3.2 that the pinching estimate

$$
\tilde{R} \geq(-\tilde{\nu})[\log (-\tilde{\nu})+\log (1+t)-3]
$$

holds whenever $\tilde{\nu}<0$. This shows that when the unnormalized curvature big, the negative ones are not nearly as large as the positive ones. Note that the unnormalized curvature becomes unbounded in finite time. Thus when we rescale the unnormalized flow to the normalized flow, the scaling factor must go to infinity. In the nonsingular case the rescaled positive curvature stay finite, so the rescaled negative curvature (if any) go to zero. Hence we can take a noncollapsing limit for the nonsingular solution of the normalized flow so that it has nonnegative sectional curvature.

Since the volume of the limit is finite, it follows from a result of Calabi and Yau [112] that the limit must be compact and the limiting manifold is the original one. Then by the strong maximum principle as in the proof of Theorem 5.2.3 (i), either the limit is flat, or it is a compact metric quotient of the product of a positively curved surface $\Sigma^{2}$ with $\mathbb{R}$, or it has strictly positive curvature. By the work of Schoen-Yau [110], a flat three-manifold cannot have a metric of positive scalar curvature, but our manifold does in Case (1). This rules out the possibility of a flat limit. Clearly the limit is also a nonsingular solution to the normalized Ricci flow. Note that the 
curvature of the surface $\Sigma^{2}$ has a positive lower bound and is compact since it comes from the lifting of the compact limiting manifold. From Theorem 5.1.11, we see the metric of the two-dimensional factor $\Sigma^{2}$ converges to the round two-sphere $\mathbb{S}^{2}$ in the normalized Ricci flow. Note also that the normalized factors in two-dimension and three-dimension are different. This implies that the compact quotient of the product $\Sigma^{2} \times \mathbb{R}$ cannot be nonsingular, which is also ruled out for the limit. Thus the limit must have strictly positive sectional curvature. Since the convergence takes place everywhere for the compact limit, it follows that as $t$ large enough the original nonsingular solution has strictly positive sectional curvature. This in turn shows that the corresponding unnormalized flow has strictly positive sectional curvature after some finite time. Then in views of the proof of Theorem 5.2.1, in particular the pinching estimate in Proposition 5.2.5, the limit has constant Ricci curvature and then constant sectional curvature for three-manifolds. This finishes the proof in Case (1).

We next consider Case (2). In this case we only need to show that we can take a noncollapsing limit which has nonnegative sectional curvature. Indeed, if this is true, then as in the previous case, the limit is compact and either it is flat, or it splits as a product (or a quotient of a product) of a positively curved $S^{2}$ with a circle $S^{1}$, or it has strictly positive curvature. But the assumption $R_{\min }(t) \leq 0$ for all times $t \geq 0$ in this case implies the limit must be flat.

Let us consider the corresponding unnormalized flow $\tilde{g}_{i j}(t)$ associated to the noncollapsing nonsingular solution. The pinching estimate in Theorem 5.3.2 tells us that we may assume the unnormalized flow $\tilde{g}_{i j}(t)$ exists for all times $0 \leq t<+\infty$, for otherwise, the scaling factor approaches infinity as in the previous case which implies the limit has nonnegative sectional curvature. The volume $\tilde{V}(t)$ of the unnormalized solution $\tilde{g}_{i j}(t)$ now changes. We divide the discussion into three subcases.

Subcase (2.1): there is a sequence of times $\tilde{t}_{k} \rightarrow+\infty$ such that $\tilde{V}\left(\tilde{t}_{k}\right) \rightarrow+\infty$;

Subcase (2.2): there is a sequence of times $\tilde{t}_{k} \rightarrow+\infty$ such that $\tilde{V}\left(\tilde{t}_{k}\right) \rightarrow 0$;

Subcase (2.3): there exist two positive constants $C_{1}, C_{2}$ such that $C_{1} \leq \tilde{V}(t) \leq C_{2}$ for all $0 \leq t<+\infty$.

For Subcase (2.1), because

$$
\frac{d \tilde{V}}{d t}=-r \tilde{V}
$$

we have

$$
\log \frac{\tilde{V}\left(\tilde{t}_{k}\right)}{\tilde{V}(0)}=-\int_{0}^{\tilde{t}_{k}} r(t) d t \rightarrow+\infty, \quad \text { as } k \rightarrow+\infty,
$$

which implies that there exists another sequence of times, still denoted by $\tilde{t}_{k}$, such that $\tilde{t}_{k} \rightarrow+\infty$ and $r\left(\tilde{t}_{k}\right) \leq 0$. Let $t_{k}$ be the corresponding times for the normalized flow. Thus there holds for the normalized flow

$$
r\left(t_{k}\right) \rightarrow 0, \quad \text { as } k \rightarrow \infty
$$

since $0 \geq r\left(t_{k}\right) \geq R_{\min }\left(t_{k}\right) \rightarrow 0$ as $k \rightarrow+\infty$. Then

$$
\int_{M}\left(R-R_{\min }\right) d \mu\left(t_{k}\right)=\left(r\left(t_{k}\right)-R_{\min }\left(t_{k}\right)\right) V \rightarrow 0, \quad \text { as } k \rightarrow \infty .
$$


As we take a noncollapsing limit along the time sequence $t_{k}$, we get

$$
\int_{M^{\infty}} R d \mu^{\infty}=0
$$

for the limit of the normalized solutions at the new time $t=0$. But $R \geq 0$ for the limit because $\lim _{t \rightarrow+\infty} R_{\min }(t)=0$ for the nonsingular solution. So $R=0$ at $t=0$ for the limit. Since the limit flow exists for $-\infty<t<+\infty$ and the scalar curvature of the limit flow evolves by

$$
\frac{\partial}{\partial t} R=\Delta R+2|\mathrm{Ric}|^{2}-\frac{2}{3} r^{\infty} R, \quad t \in(-\infty,+\infty)
$$

where $r^{\infty}$ is the limit of the function $r(t)$ by translating the times $t_{k}$ as the new time $t=0$. It follows from the strong maximum principle that

$$
R \equiv 0, \quad \text { on } M^{\infty} \times(-\infty,+\infty) .
$$

This in turn implies, in view of the above evolution equation, that

$$
\operatorname{Ric} \equiv 0, \quad \text { on } M^{\infty} \times(-\infty,+\infty)
$$

Hence this limit must be flat. Since the limit $M^{\infty}$ is complete and has finite volume, the flat manifold $M^{\infty}$ must be compact. Thus the underlying manifold $M^{\infty}$ must agree with the original $M$ (as a topological manifold). This says that the limit was taken on $M$.

For Subcase (2.2), we may assume as before that for the initial metric at $t=0$ of the unnormalized flow $\tilde{g}_{i j}(t)$, the eigenvalues $\tilde{\lambda} \geq \tilde{\mu} \geq \tilde{\nu}$ of the curvature operator satisfy $\tilde{\nu} \geq-1$. It then follows from Theorem 5.3.2 that

$$
\tilde{R} \geq(-\tilde{\nu})[\log (-\tilde{\nu})+\log (1+t)-3], \quad \text { for all } t \geq 0
$$

whenever $\tilde{\nu}<0$.

Let $t_{k}$ be the sequence of times in the normalized flow which corresponds to the sequence of times $\tilde{t}_{k}$. Take a noncollapsing limit for the normalized flow along the times $t_{k}$. Since $\tilde{V}\left(\tilde{t}_{k}\right) \rightarrow 0$, the normalized curvatures at the times $t_{k}$ are reduced by multiplying the factor $\left(\tilde{V}\left(\tilde{t}_{k}\right)\right)^{\frac{2}{3}}$. We claim the noncollapsing limit has nonnegative sectional curvature. Indeed if the maximum value of $(-\tilde{\nu})$ at the time $\tilde{t}_{k}$ does not go to infinity, the normalized eigenvalue $-\nu$ at the corresponding time $t_{k}$ must get rescaled to tend to zero; while if the maximum value of $(-\tilde{\nu})$ at the time $\tilde{t}_{k}$ does go to infinity, the maximum value of $\tilde{R}$ at $\tilde{t}_{k}$ will go to infinity even faster from the pinching estimate, and when we normalize to keep the normalized scalar curvature $R$ bounded at the time $t_{k}$ so the normalized $(-\nu)$ at the time $t_{k}$ will go to zero. Thus in either case the noncollapsing limit has nonnegative sectional curvature at the initial time $t=0$ and then has nonnegative sectional curvature for all times $t \geq 0$.

For Subcase (2.3), normalizing the flow only changes quantities in a bounded way. As before we have the pinching estimate

$$
R \geq(-\nu)[\log (-\nu)+\log (1+t)-C]
$$

for the normalized Ricci flow, where $C$ is a positive constant depending only on the constants $C_{1}, C_{2}$ in the assumption of Subcase (2.3). If

$$
(-\nu) \leq \frac{A}{1+t}
$$


for any fixed positive constant $A$, then $(-\nu) \rightarrow 0$ as $t \rightarrow+\infty$ and we can take a noncollapsing limit which has nonnegative sectional curvature. On the other hand if we can pick a sequence of times $t_{k} \rightarrow \infty$ and points $x_{k}$ where $(-\nu)\left(x_{k}, t_{k}\right)=$ $\max _{x \in M}(-\nu)\left(x, t_{k}\right)$ satisfies

$$
(-\nu)\left(x_{k}, t_{k}\right)\left(1+t_{k}\right) \rightarrow+\infty, \quad \text { as } k \rightarrow+\infty,
$$

then from the pinching estimate, we have

$$
\frac{R\left(x_{k}, t_{k}\right)}{(-\nu)\left(x_{k}, t_{k}\right)} \rightarrow+\infty, \quad \text { as } \quad k \rightarrow+\infty .
$$

But $R\left(x_{k}, t_{k}\right)$ are uniformly bounded since normalizing the flow only changes quantities in bounded way. This shows $\sup (-\nu)\left(\cdot, t_{k}\right) \rightarrow 0$ as $k \rightarrow+\infty$. Thus we can take a noncollapsing limit along $t_{k}$ which has nonnegative sectional curvature. Hence we have completed the proof of Case (2).

We now come to the most interesting Case (3) where $R_{\text {min }}$ increases monotonically to a limit strictly less than zero. By scaling we can assume $R_{\min }(t) \rightarrow-6$ as $t \rightarrow+\infty$.

LEMMA 5.3.6. In Case (3) where $R_{\min } \rightarrow-6$ as $t \rightarrow+\infty$, all noncollapsing limit are hyperbolic with constant sectional curvature -1 .

Proof. By (5.3.4) and the fact $R_{\min }(t) \leq-6$, we have

$$
\frac{d}{d t} R_{\min }(t) \geq 4\left(r(t)-R_{\min }(t)\right)
$$

and

$$
\int_{0}^{\infty}\left(r(t)-R_{\min }(t)\right) d t<+\infty
$$

Since $r(t)-R_{\min }(t) \geq 0$ and $R_{\min }(t) \rightarrow-6$ as $t \rightarrow+\infty$, it follows that the function $r(t)$ has the limit

$$
r=-6
$$

for any convergent subsequence. And since

$$
\int_{M}\left(R-R_{\min }(t)\right) d \mu=\left(r(t)-R_{\min }(t)\right) \cdot V,
$$

it then follows that

$$
R \equiv-6 \text { for the limit. }
$$

The limit still has the following evolution equation for the limiting scalar curvature

$$
\frac{\partial}{\partial t} R=\Delta R+2|\stackrel{\circ}{\mathrm{Ric}}|^{2}+\frac{2}{3} R(R-r)
$$

Since $R \equiv r \equiv-6$ in space and time for the limit, it follows directly that $\mid \mathrm{Ric}^{\circ} \equiv 0$ for the limit. Thus the limit metric has $\lambda=\mu=\nu=-2$, so it has constant sectional curvature -1 as desired. 
If in the discussion above there exists a compact noncollapsing limit, then we know that the underlying manifold $M$ is compact and we fall into the conclusion of Theorem 5.3.4(i) for the constant negative sectional curvature limit. Thus it remains to show when every noncollapsing limit is a complete noncompact hyperbolic manifold with finite volume, we have conclusion (ii) in Theorem 5.3.4.

Now we first want to find a finite collection of persistent complete noncompact hyperbolic manifolds as stated in Theorem 5.3.4 (ii).

\section{Part II: Persistence of Hyperbolic Pieces}

We begin with the definition of the topology of $C^{\infty}$ convergence on compact sets for maps $F: M \rightarrow N$ of one Riemannian manifold to another. For any compact set $K \subset \subset M$ and any two maps $F, G: M \rightarrow N$, we define

$$
d_{K}(F, G)=\sup _{x \in K} d(F(x), G(x))
$$

where $d(y, z)$ is the geodesic distance from $y$ to $z$ on $N$. This gives the $C_{l o c}^{0}$ topology for maps between $M$ and $N$. To define $C_{l o c}^{k}$ topology for any positive integer $k \geq 1$, we consider the $\boldsymbol{k}$-jet space $J^{k} M$ of a manifold $M$ which is the collection of all

$$
\left(x, J^{1}, J^{2}, \ldots, J^{k}\right)
$$

where $x$ is a point on $M$ and $J^{i}$ is a tangent vector for $1 \leq i \leq k$ defined by the $i^{t h}$ covariant derivative $J^{i}=\nabla_{\frac{\partial}{\partial t}}^{i} \gamma(0)$ for a path $\gamma$ passing through the point $x$ with $\gamma(0)=x$. A smooth map $F: M \rightarrow N$ induces a map

$$
J^{k} F: \quad J^{k} M \rightarrow J^{k} N
$$

defined by

$$
J^{k} F\left(x, J^{1}, \ldots, J^{k}\right)=\left(F(x), \nabla_{\frac{\partial}{\partial t}}(F(\gamma))(0), \ldots, \nabla_{\frac{\partial}{\partial t}}^{k}(F(\gamma))(0)\right)
$$

where $\gamma$ is a path passing through the point $x$ with $J^{i}=\nabla_{\frac{\partial}{\partial t}}^{i} \gamma(0), 1 \leq i \leq k$.

Define the k-jet distance between $F$ and $G$ on a compact set $K \subset \subset M$ by

$$
d_{C^{k}(K)}(F, G)=d_{B J^{k} K}\left(J^{k} F, J^{k} G\right)
$$

where $B J^{k} K$ consists of all $k$-jets $\left(x, J^{1}, \ldots, J^{k}\right)$ with $x \in K$ and

$$
\left|J^{1}\right|^{2}+\left|J^{2}\right|^{2}+\cdots+\left|J^{k}\right|^{2} \leq 1
$$

Then the convergence in the metric $d_{C^{k}(K)}$ for all positive integers $k$ and all compact sets $K$ defines the topology of $C^{\infty}$ convergence on compact sets for the space of maps.

We will need the following Mostow type rigidity result.

LEMma 5.3.7. For any complete noncompact hyperbolic three-manifold $\mathcal{H}$ with finite volume with metric $h$, we can find a compact set $\mathcal{K}$ of $\mathcal{H}$ such that for every integer $k$ and every $\varepsilon>0$, there exist an integer $q$ and $a \delta>0$ with the following property: if $F$ is a diffeomorphism of $\mathcal{K}$ into another complete noncompact hyperbolic three-manifold $\tilde{\mathcal{H}}$ with no fewer cusps $($ than $\mathcal{H})$, finite volume with metric $\tilde{h}$ such that

$$
\left\|F^{*} \tilde{h}-h\right\|_{C^{q}(\mathcal{K})}<\delta
$$


then there exists an isometry I of $\mathcal{H}$ to $\tilde{\mathcal{H}}$ such that

$$
d_{C^{k}(\mathcal{K})}(F, I)<\varepsilon .
$$

Proof. First we claim that $\mathcal{H}$ is isometric to $\tilde{\mathcal{H}}$ for an appropriate choice of compact set $\mathcal{K}$, positive integer $q$ and positive number $\delta$. Let $l: \mathcal{H} \rightarrow \mathbb{R}$ be a function defined at each point by the length of the shortest non-contractible loop starting and ending at this point. Denote the Margulis constant by $\mu$. Then by Margulis lemma (see for example [55] or [76]), for any $0<\varepsilon_{0}<\frac{1}{2} \mu$, the set $l^{-1}\left(\left[0, \varepsilon_{0}\right]\right) \subset \mathcal{H}$ consists of finitely many components and each of these components is isometric to a cusp or to a tube. Topologically, a tube is just a solid torus. Let $\varepsilon_{0}$ be even smaller than one half of the minimum of the lengths of the all closed geodesics on the tubes. Then $l^{-1}\left(\left[0, \varepsilon_{0}\right]\right)$ consists of finite number of cusps. Set $\mathcal{K}_{0}=l^{-1}\left(\left[\varepsilon_{0}, \infty\right)\right)$. The boundary of $\mathcal{K}_{0}$ consists of flat tori with constant mean curvatures. Note that each embedded torus in a complete hyperbolic three-manifold with finite volume either bounds a solid torus or is isotopic to a standard torus in a cusp. The diffeomorphism $F$ implies the boundary $F\left(\partial \mathcal{K}_{0}\right)$ are embedded tori. If one of components bounds a solid torus, then as $\delta$ sufficiently small and $q$ sufficiently large, $\tilde{\mathcal{H}}$ would have fewer cusps than $\mathcal{H}$, which contradicts with our assumption. Consequently, $\tilde{\mathcal{H}}$ is diffeomorphic to $F\left(\mathcal{K}_{0}\right)$. Here $\mathcal{K}_{0}$ is the interior of the set $\mathcal{K}_{0}$. Since $\mathcal{H}$ is diffeomorphic to $\mathcal{K}_{0}, \mathcal{H}$ is diffeomorphic to $\tilde{\mathcal{H}}$. Hence by Mostow's rigidity theorem (see [97] and [107]), $\mathcal{H}$ is isometric to $\tilde{\mathcal{H}}$.

So we can assume $\tilde{\mathcal{H}}=\mathcal{H}$. For $\mathcal{K}=\mathcal{K}_{0}$, we argue by contradiction. Suppose there is some $k>0$ and $\varepsilon>0$ so that there exist sequences of integers $q_{j} \rightarrow \infty, \delta_{j} \rightarrow 0^{+}$ and diffeomorphisms $F_{j}$ mapping $\mathcal{K}$ into $\mathcal{H}$ with

$$
\left\|F_{j}^{*} h-h\right\|_{C^{q_{j}}(\mathcal{K})}<\delta_{j}
$$

and

$$
d_{C^{k}(\mathcal{K})}\left(F_{j}, I\right) \geq \varepsilon
$$

for all isometries $I$ of $\mathcal{H}$ to itself. We can extract a subsequence of $F_{j}$ convergent to a map $F_{\infty}$ with $F_{\infty}^{*} h=h$ on $\mathcal{K}$.

We need to check that $F_{\infty}$ is still a diffeomorphism on $\mathcal{K}$. Since $F_{\infty}$ is a local diffeomorphism and is the limit of diffeomorphisms, we can find an inverse of $F_{\infty}$ on $F_{\infty}(\mathcal{K})$. So $F_{\infty}$ is a diffeomorphism on $\mathcal{K}$. We claim the image of the boundary can not touch the image of the interior. Indeed, if $F_{\infty}\left(x_{1}\right)=F_{\infty}\left(x_{2}\right)$ with $x_{1} \in \partial \mathcal{K}$ and

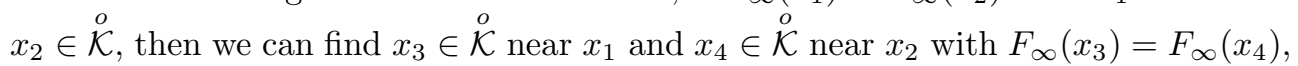
since $F_{\infty}$ is a local diffeomorphism. This contradicts with the fact that $F_{\infty}$ is a diffeomorphism on $\mathcal{K}$. This proves our claim. Hence, the only possible overlap is at the boundary. But the image $F_{\infty}(\partial \mathcal{K})$ is strictly concave, this prevents the boundary from touching itself. We conclude that the mapping $F_{\infty}$ is a diffeomorphism on $\mathcal{K}$, hence an isometry.

To extend $F_{\infty}$ to a global isometry, we argue as follows. For each truncated cusp end of $\mathcal{K}$, the area of constant mean curvature flat torus is strictly decreasing. Since $F_{\infty}$ takes each such torus to another of the same area, we see that $F_{\infty}$ takes the foliation of an end by constant mean curvature flat tori to another such foliation. So $F_{\infty}$ takes cusps to cusps and preserves their foliations. Note that the isometric type 
of a cusp is just the isometric type of the torus, more precisely, let $\left(N, d r^{2}+e^{-2 r} g_{\mathcal{V}}\right)$ be a cusp (where $g_{\mathcal{V}}$ is the flat metric on the torus $\mathcal{V}$ ), $0<a<b$ are two constants, any isometry of $N \cap l^{-1}[a, b]$ to itself is just an isometry of $\mathcal{V}$. Hence the isometry $F_{\infty}$ can be extended to the whole cusps. This gives a global isometry $I$ contradicting our assumption when $j$ large enough.

The proof of the Lemma 5.3.7 is completed.

In order to obtain the persistent hyperbolic pieces stated in Theorem 5.3.4 (ii), we will need to use a special parametrization given by harmonic maps.

Lemma 5.3.8. Let $(X, g)$ be a compact Riemannian manifold with strictly negative Ricci curvature and with strictly concave boundary. Then there are positive integer $l_{0}$ and small number $\varepsilon_{0}>0$ such that for each positive integer $l \geq l_{0}$ and positive number $\varepsilon \leq \varepsilon_{0}$ we can find positive integer $q$ and positive number $\delta>0$ such that for every metric $\tilde{g}$ on $X$ with $\|\tilde{g}-g\|_{C^{q}(X)} \leq \delta$ we can find a unique diffeomorphism $F$ of $X$ to itself so that

(a) $F:(X, g) \rightarrow(X, \tilde{g})$ is harmonic,

(b) F takes the boundary $\partial X$ to itself and satisfies the free boundary condition that the normal derivative $\nabla_{N} F$ of $F$ at the boundary is normal to the boundary,

(c) $d_{C^{l}(X)}(F, I d)<\varepsilon$, where Id is the identity map.

Proof. Let $\Phi(X, \partial X)$ be the space of maps of $X$ to itself which take $\partial X$ to itself. Then $\Phi(X, \partial X)$ is a Banach manifold and the tangent space to $\Phi(X, \partial X)$ at the identity is the space of vector fields $V=V^{i} \frac{\partial}{\partial x^{i}}$ tangent to the boundary. Consider the map sending $F \in \Phi(X, \partial X)$ to the pair $\left\{\Delta F,\left(\nabla_{N} F\right)_{/ /}\right\}$consisting of the harmonic map Laplacian and the tangential component (in the target) of the normal derivative of $F$ at the boundary. By using the inverse function theorem, we only need to check that the derivative of this map is an isomorphism at the identity with $\tilde{g}=g$.

Let $\left\{x^{i}\right\}_{i=1, \ldots, n}$ be a local coordinates of $(X, g)$ and $\left\{y^{\alpha}\right\}_{\alpha=1, \ldots, n}$ be a local coordinates of $(X, \tilde{g})$. The harmonic map Laplacian of $F:(X, g) \rightarrow(X, \tilde{g})$ is given in local coordinates by

$$
(\Delta F)^{\alpha}=\Delta\left(F^{\alpha}\right)+g^{i j}\left(\tilde{\Gamma}_{\beta \gamma}^{\alpha} \circ F\right) \frac{\partial F^{\beta}}{\partial x^{i}} \frac{\partial F^{\gamma}}{\partial x^{j}}
$$

where $\Delta\left(F^{\alpha}\right)$ is the Laplacian of the function $F^{\alpha}$ on $X$ and $\tilde{\Gamma}_{\beta \gamma}^{\alpha}$ is the connection of $\tilde{g}$. Let $F$ be a one-parameter family with $\left.F\right|_{s=0}=I d$ and $\left.\frac{d F}{d s}\right|_{s=0}=V$, a smooth vector field on $X$ tangent to the boundary (with respect to $g$ ). At an arbitrary given point $x \in X$, we choose the coordinates $\left\{x^{i}\right\}_{i=1, \ldots, n}$ so that $\Gamma_{j k}^{i}(x)=0$. We compute at the point $x$ with $\tilde{g}=g$,

$$
\left.\frac{d}{d s}\right|_{s=0}(\Delta F)^{\alpha}=\Delta\left(V^{\alpha}\right)+g^{i j}\left(\frac{\partial}{\partial x^{k}} \Gamma_{i j}^{\alpha}\right) V^{k}
$$

Since

$$
\left(\nabla_{i} V\right)^{\alpha}=\nabla_{i} V^{\alpha}+\left(\Gamma_{i \beta}^{\alpha} \circ F\right) V^{\beta},
$$

we have, at $s=0$ and the point $x$,

$$
(\Delta V)^{\alpha}=\Delta\left(V^{\alpha}\right)+g^{i j} \frac{\partial}{\partial x^{i}} \Gamma_{j k}^{\alpha} V^{k} .
$$


Thus we obtain

$$
\begin{aligned}
\left.\frac{d}{d s}\right|_{s=0}(\Delta F)^{\alpha} & =(\Delta V)^{\alpha}+g^{i j}\left(\frac{\partial}{\partial x^{k}} \Gamma_{i j}^{\alpha}-\frac{\partial}{\partial x^{i}} \Gamma_{j k}^{\alpha}\right) V^{k} \\
& =(\Delta V)^{\alpha}+g^{\alpha i} R_{i k} V^{k}
\end{aligned}
$$

Since

$$
\left(\nabla_{N} F\right)\left(F^{-1}(x)\right)=N^{i}\left(F^{-1}(x)\right) \frac{\partial F^{j}}{\partial x^{i}}\left(F^{-1}(x)\right) \frac{\partial}{\partial x^{j}}(x) \quad \text { on } \partial X,
$$

we have

$$
\begin{aligned}
\left.\frac{d}{d s}\right|_{s=0}\left\{\left(\nabla_{N} F\right)_{/ /}\right\}= & \left.\frac{d}{d s}\right|_{s=0}\left(\nabla_{N} F-\left\langle\nabla_{N} F, N\right\rangle N\right) \\
= & \left.\frac{d}{d s}\right|_{s=0}\left(\nabla_{N} F\right)-\left\langle\left.\frac{d}{d s}\right|_{s=0} \nabla_{N} F, N\right\rangle N \\
& -\left.\left\langle\nabla_{N} F, \nabla_{V} N\right\rangle N\right|_{s=0}-\left.\left\langle\nabla_{N} F, N\right\rangle \nabla_{V} N\right|_{s=0} \\
= & \left(\left.\frac{d}{d s}\right|_{s=0} \nabla_{N} F\right)_{/ /}-\nabla_{V} N \\
= & \left(-V\left(N^{i}\right) \frac{\partial}{\partial x^{i}}+N\left(V^{j}\right) \frac{\partial}{\partial x^{j}}\right)_{/ /}-I I(V) \\
= & {[N, V]_{/ /}-I I(V) } \\
= & \left(\nabla_{N} V\right)_{/ /}-2 I I(V)
\end{aligned}
$$

where $I I$ is the second fundamental form of the boundary (as an automorphism of $T(\partial X)$ ). Thus by (5.3.5) and (5.3.6), the kernel of the map sending $F \in \Phi(X, \partial X)$ to the pair $\left\{\Delta F,\left(\nabla_{N} F\right)_{/ /}\right\}$is the space of solutions of elliptic boundary value problem

$$
\begin{cases}\Delta V+\operatorname{Ric}(V)=0 & \text { on } X \\ V_{\perp}=0, & \text { at } \partial X \\ \left(\nabla_{N} V\right)_{/ /}-2 I I(V)=0, & \text { at } \partial X\end{cases}
$$

where $V_{\perp}$ is the normal component of $V$.

Now using these equations and integrating by parts gives

$$
\iint_{X}|\nabla V|^{2}=\iint_{X} \operatorname{Ric}(V, V)+2 \int_{\partial X} I I(V, V) .
$$

Since $R c<0$ and $I I<0$ we conclude that the kernel is trivial. Clearly this elliptic boundary value is self-adjoint because of the free boundary condition. Thus the cokernel is trivial also. This proves the lemma.

Now we can prove the persistence of hyperbolic pieces. Let $g_{i j}(t), 0 \leq t<+\infty$, be a noncollapsing nonsingular solution of the normalized Ricci flow on a compact three-manifold $M$. Assume that any noncollapsing limit of the nonsingular solution is a complete noncompact hyperbolic three-manifold with finite volume. Consider all the possible hyperbolic limits of the given nonsingular solution, and among them choose one such complete noncompact hyperbolic three-manifold $\mathcal{H}$ with the least possible number of cusps. In particular, we can find a sequence of times $t_{k} \rightarrow+\infty$ and a 
sequence of points $P_{k}$ on $M$ such that the marked three-manifolds $\left(M, g_{i j}\left(t_{k}\right), P_{k}\right)$ converge in the $C_{l o c}^{\infty}$ topology to $\mathcal{H}$ with hyperbolic metric $h_{i j}$ and marked point $P \in \mathcal{H}$.

For any small enough $a>0$ we can truncate each cusp of $\mathcal{H}$ along a constant mean curvature torus of area $a$ which is uniquely determined; the remainder we denote by $\mathcal{H}_{a}$. Clearly as $a \rightarrow 0$ the $\mathcal{H}_{a}$ exhaust $\mathcal{H}$. Pick a sufficiently small number $a>0$ to truncate cusps so that Lemma 5.3.7 is applicable for the compact set $\mathcal{K}=\mathcal{H}_{a}$. Choose an integer $l_{0}$ large enough and an $\varepsilon_{0}$ sufficiently small to guarantee from Lemma 5.3.8 the uniqueness of the identity map $I d$ among maps close to $I d$ as a harmonic map $F$ from $\mathcal{H}_{a}$ to itself with taking $\partial \mathcal{H}_{a}$ to itself, with the normal derivative of $F$ at the boundary of the domain normal to the boundary of the target, and with $d_{C^{l_{0}\left(\mathcal{H}_{a}\right)}}(F, I d)<\varepsilon_{0}$. Then choose positive integer $q_{0}$ and small number $\delta_{0}>0$ from Lemma 5.3.7 such that if $\tilde{F}$ is a diffeomorphism of $\mathcal{H}_{a}$ into another complete noncompact hyperbolic three-manifold $\tilde{\mathcal{H}}$ with no fewer cusps (than $\mathcal{H}$ ), finite volume with metric $\tilde{h}_{i j}$ satisfying

$$
\left\|\tilde{F}^{*} \tilde{h}_{i j}-h_{i j}\right\|_{C^{q_{0}\left(\mathcal{H}_{a}\right)}} \leq \delta_{0}
$$

then there exists an isometry $I$ of $\mathcal{H}$ to $\tilde{\mathcal{H}}$ such that

$$
d_{C^{l_{0}\left(\mathcal{H}_{a}\right)}}(\tilde{F}, I)<\varepsilon_{0} .
$$

And we further require $q_{0}$ and $\delta_{0}$ from Lemma 5.3.8 to guarantee the existence of harmonic diffeomorphism from $\left(\mathcal{H}_{a}, \tilde{g}_{i j}\right)$ to $\left(\mathcal{H}_{a}, h_{i j}\right)$ for any metric $\tilde{g}_{i j}$ on $\mathcal{H}_{a}$ with $\left\|\tilde{g}_{i j}-h_{i j}\right\|_{C^{q_{0}}\left(\mathcal{H}_{a}\right)} \leq \delta_{0}$.

By definition, there exist a sequence of exhausting compact sets $U_{k}$ of $\mathcal{H}$ (each $U_{k} \supset \mathcal{H}_{a}$ ) and a sequence of diffeomorphisms $F_{k}$ from $U_{k}$ into $M$ such that $F_{k}(P)=P_{k}$ and $\left\|F_{k}^{*} g_{i j}\left(t_{k}\right)-h_{i j}\right\|_{C^{m}\left(U_{k}\right)} \rightarrow 0$ as $k \rightarrow+\infty$ for all positive integers $m$. Note that $\partial \mathcal{H}_{a}$ is strictly concave and we can foliate a neighborhood of $\partial \mathcal{H}_{a}$ with constant mean curvature hypersurfaces where the area $a$ has a nonzero gradient. As the approximating maps $F_{k}:\left(U_{k}, h_{i j}\right) \rightarrow\left(M, g_{i j}\left(t_{k}\right)\right)$ are close enough to isometries on this collar of $\partial \mathcal{H}_{a}$, the metrics $g_{i j}\left(t_{k}\right)$ on $M$ will also admit a unique constant mean curvature hypersurface with the same area $a$ near $F_{k}\left(\partial \mathcal{H}_{a}\right)(\subset M)$ by the inverse function theorem. Thus we can change the map $F_{k}$ by an amount which goes to zero as $k \rightarrow \infty$ so that now $F_{k}\left(\partial \mathcal{H}_{a}\right)$ has constant mean curvature with the area $a$. Furthermore, by applying Lemma 5.3 .8 we can again change $F_{k}$ by an amount which goes to zero as $k \rightarrow \infty$ so as to make $F_{k}$ a harmonic diffeomorphism and take $\partial \mathcal{H}_{a}$ to the constant mean curvature hypersurface $F_{k}\left(\partial \mathcal{H}_{a}\right)$ and also satisfy the free boundary condition that the normal derivative of $F_{k}$ at the boundary of the domain is normal to the boundary of the target. Hence for arbitrarily given positive integer $q \geq q_{0}$ and positive number $\delta<\delta_{0}$, there exists a positive integer $k_{0}$ such that for the modified harmonic diffeomorphism $F_{k}$, when $k \geq k_{0}$,

$$
\left\|F_{k}^{*} g_{i j}\left(t_{k}\right)-h_{i j}\right\|_{C^{q}\left(\mathcal{H}_{a}\right)}<\delta .
$$

For each fixed $k \geq k_{0}$, by the implicit function theorem we can first find a constant mean curvature hypersurface near $F_{k}\left(\partial \mathcal{H}_{a}\right)$ in $M$ with the metric $g_{i j}(t)$ for $t$ close to $t_{k}$ and with the same area for each component since $\partial \mathcal{H}_{a}$ is strictly concave and a neighborhood of $\partial \mathcal{H}_{a}$ is foliated by constant mean curvature hypersurfaces where the area $a$ has a nonzero gradient and $F_{k}:\left(\mathcal{H}_{a}, h_{i j}\right) \rightarrow\left(M, g_{i j}\left(t_{k}\right)\right)$ is close enough to an isometry and $g_{i j}(t)$ varies smoothly. Then by applying Lemma 5.3 .8 we can smoothly 
continue the harmonic diffeomorphism $F_{k}$ forward in time a little to a family of harmonic diffeomorphisms $F_{k}(t)$ from $\mathcal{H}_{a}$ into $M$ with the metric $g_{i j}(t)$, with $F_{k}\left(t_{k}\right)=$ $F_{k}$, where each $F_{k}(t)$ takes $\partial \mathcal{H}_{a}$ into the constant mean curvature hypersurface we just found in $\left(M, g_{i j}(t)\right)$ and satisfies the free boundary condition, and also satisfies

$$
\left\|F_{k}^{*}(t) g_{i j}(t)-h_{i j}\right\|_{C^{q}\left(\mathcal{H}_{a}\right)}<\delta .
$$

We claim that for all sufficiently large $k$, we can smoothly extend the harmonic diffeomorphism $F_{k}$ to the family harmonic diffeomorphisms $F_{k}(t)$ with $\| F_{k}^{*}(t) g_{i j}(t)-$ $h_{i j} \|_{C^{q}\left(\mathcal{H}_{a}\right)} \leq \delta$ on a maximal time interval $t_{k} \leq t \leq \omega_{k}$ (or $t_{k} \leq t<\omega_{k}$ when $\left.\omega_{k}=+\infty\right)$; and if $\omega_{k}<+\infty$, then

$$
\left\|F_{k}^{*}\left(\omega_{k}\right) g_{i j}\left(\omega_{k}\right)-h_{i j}\right\|_{C^{q}\left(\mathcal{H}_{a}\right)}=\delta .
$$

Clearly the above argument shows that the set of $t$ where we can extend the harmonic diffeomorphisms as desired is open. To verify claim (5.3.9), we thus only need to show that if we have a family of harmonic diffeomorphisms $F_{k}(t)$ such as we desire for $t_{k} \leq t<\omega(<+\infty)$, we can take the limit of $F_{k}(t)$ as $t \rightarrow \omega$ to get a harmonic diffeomorphism $F_{k}(\omega)$ satisfying

$$
\left\|F_{k}^{*}(\omega) g_{i j}(\omega)-h_{i j}\right\|_{C^{q}\left(\mathcal{H}_{a}\right)} \leq \delta,
$$

and if

$$
\left\|F_{k}^{*}(\omega) g_{i j}(\omega)-h_{i j}\right\|_{C^{q}\left(\mathcal{H}_{a}\right)}<\delta,
$$

then we can extend $F_{k}(\omega)$ forward in time a little (i.e., we can find a constant mean curvature hypersurface near $F_{k}(\omega)\left(\partial \mathcal{H}_{a}\right)$ in $M$ with the metric $g_{i j}(t)$ for each $t$ close to $\omega$ and with the same area $a$ for each component). Note that

$$
\left\|F_{k}^{*}(t) g_{i j}(t)-h_{i j}\right\|_{C^{q}\left(\mathcal{H}_{a}\right)}<\delta .
$$

for $t_{k} \leq t<\omega$ and the metrics $g_{i j}(t)$ for $t_{k} \leq t \leq \omega$ are uniformly equivalent. We can find a subsequence $t_{n} \rightarrow \omega$ for which $F_{k}\left(t_{n}\right)$ converge to $F_{k}(\omega)$ in $C^{q-1}\left(\mathcal{H}_{a}\right)$ and the limit map has

$$
\left\|F_{k}^{*}(\omega) g_{i j}(\omega)-h_{i j}\right\|_{C^{q-1}\left(\mathcal{H}_{a}\right)} \leq \delta .
$$

We need to check that $F_{k}(\omega)$ is still a diffeomorphism. We at least know $F_{k}(\omega)$ is a local diffeomorphism, and $F_{k}(\omega)$ is the limit of diffeomorphisms, so the only possibility of overlap is at the boundary. Hence we use the fact that $F_{k}(\omega)\left(\partial \mathcal{H}_{a}\right)$ is still strictly concave since $q$ is large and $\delta$ is small to prevent the boundary from touching itself. Thus $F_{k}(\omega)$ is a diffeomorphism. A limit of harmonic maps is harmonic, so $F_{k}(\omega)$ is a harmonic diffeomorphism from $\mathcal{H}_{a}$ into $M$ with the metric $g_{i j}(\omega)$. Moreover $F_{k}(\omega)$ takes $\partial \mathcal{H}_{a}$ to the constant mean curvature hypersurface $\partial\left(F_{k}(\omega)\left(\mathcal{H}_{a}\right)\right)$ of the area $a$ in $\left(M, g_{i j}(\omega)\right)$ and continue to satisfy the free boundary condition. As a consequence of the standard regularity result of elliptic partial differential equations (see for example [48]), the map $F_{k}(\omega) \in C^{\infty}\left(\mathcal{H}_{a}\right)$ and then from (5.3.10) we have

$$
\left\|F_{k}^{*}(\omega) g_{i j}(\omega)-h_{i j}\right\|_{C^{q}\left(\mathcal{H}_{a}\right)} \leq \delta .
$$

If $\left\|F_{k}^{*}(\omega) g_{i j}(\omega)-h_{i j}\right\|_{C^{q}\left(\mathcal{H}_{a}\right)}=\delta$, we then finish the proof of the claim. So we may assume that $\left\|F_{k}^{*}(\omega) g_{i j}(\omega)-h_{i j}\right\|_{C^{q}\left(\mathcal{H}_{a}\right)}<\delta$. We want to show that $F_{k}(\omega)$ can be extended forward in time a little. 
We argue by contradiction. Suppose not, then we consider the new sequence of the manifolds $M$ with metric $g_{i j}(\omega)$ and the origins $F_{k}(\omega)(P)$. Since $F_{k}(\omega)$ are close to isometries, the injectivity radii of the metrics $g_{i j}(\omega)$ at $F_{k}(\omega)(P)$ do not go to zero, and we can extract a subsequence which converges to a hyperbolic limit $\widetilde{\mathcal{H}}$ with the metric $\widetilde{h}_{i j}$ and the origin $\widetilde{P}$ and with finite volume. The new limit $\widetilde{\mathcal{H}}$ has at least as many cusps as the old limit $\mathcal{H}$, since we choose $\mathcal{H}$ with cusps as few as possible. By the definition of convergence, we can find a sequence of compact sets $\widetilde{B}_{k}$ exhausting $\widetilde{\mathcal{H}}$ and containing $\widetilde{P}$, and a sequence of diffeomorphisms $\widetilde{F}_{k}$ of neighborhoods of $\widetilde{B}_{k}$ into $M$ with $\widetilde{F}_{k}(\widetilde{P})=F_{k}(\omega)(P)$ such that for each compact set $\widetilde{B}$ in $\widetilde{\mathcal{H}}$ and each integer $m$

$$
\left\|\widetilde{F}_{k}^{*}\left(g_{i j}(\omega)\right)-\widetilde{h}_{i j}\right\|_{C^{m}(\widetilde{B})} \rightarrow 0
$$

as $k \rightarrow+\infty$. For large enough $k$ the set $\widetilde{F}_{k}\left(\widetilde{B}_{k}\right)$ will contain all points out to any fixed distance we need from the point $F_{k}(\omega)(P)$, and then

$$
\widetilde{F}_{k}\left(\widetilde{B}_{k}\right) \supset F_{k}(\omega)\left(\mathcal{H}_{a}\right)
$$

since the points of $\mathcal{H}_{a}$ have a bounded distance from $P$ and $F_{k}(\omega)$ are reasonably close to preserving the metrics. Hence we can form the composition

$$
G_{k}=\widetilde{F}_{k}^{-1} \circ F_{k}(\omega): \mathcal{H}_{a} \rightarrow \widetilde{\mathcal{H}}
$$

Arbitrarily fix $\delta^{\prime} \in\left(\delta, \delta_{0}\right)$. Since the $\widetilde{F}_{k}$ are as close to preserving the metric as we like, we have

$$
\left\|G_{k}^{*} \widetilde{h}_{i j}-h_{i j}\right\|_{C^{q}\left(\mathcal{H}_{a}\right)}<\delta^{\prime}
$$

for all sufficiently large $k$. By Lemma 5.3.7, we deduce that there exists an isometry $I$ of $\mathcal{H}$ to $\widetilde{\mathcal{H}}$, and then $\left(M, g_{i j}(\omega), F_{k}(\omega)(P)\right)$ (on compact subsets) is very close to $\left(\mathcal{H}, h_{i j}, P\right)$ as long as $\delta$ small enough and $k$ large enough. Since $F_{k}(\omega)\left(\partial \mathcal{H}_{a}\right)$ is strictly concave and the foliation of a neighborhood of $F_{k}(\omega)\left(\partial \mathcal{H}_{a}\right)$ by constant mean curvature hypersurfaces has the area as a function with nonzero gradient, by the implicit function theorem, there exists a unique constant mean curvature hypersurface with the same area $a$ near $F_{k}(\omega)\left(\partial \mathcal{H}_{a}\right)$ in $M$ with the metric $g_{i j}(t)$ for $t$ close to $\omega$. Hence, when $k$ sufficiently large, $F_{k}(\omega)$ can be extended forward in time a little. This is a contradiction and we have proved claim (5.3.9).

We further claim that there must be some $k$ such that $\omega_{k}=+\infty$ (i.e., we can smoothly continue the family of harmonic diffeomorphisms $F_{k}(t)$ for all $t_{k} \leq t<+\infty$, in other words, there must be at least one hyperbolic piece persisting). We argue by contradiction. Suppose for each $k$ large enough, we can continue the family $F_{k}(t)$ for $t_{k} \leq t \leq \omega_{k}<+\infty$ with

$$
\left\|F_{k}^{*}\left(\omega_{k}\right) g_{i j}\left(\omega_{k}\right)-h_{i j}\right\|_{C^{q}\left(\mathcal{H}_{a}\right)}=\delta .
$$

Then as before, we consider the new sequence of the manifolds $M$ with metrics $g_{i j}\left(\omega_{k}\right)$ and origins $F_{k}\left(\omega_{k}\right)(P)$. For sufficiently large $k$, we can obtain diffeomorphisms $\widetilde{F}_{k}$ of neighborhoods of $\widetilde{B}_{k}$ into $M$ with $\widetilde{F}_{k}(\widetilde{P})=F_{k}\left(\omega_{k}\right)(P)$ which are as close to preserving the metric as we like, where $\widetilde{B}_{k}$ is a sequence of compact sets, exhausting some hyperbolic three-manifold $\tilde{\mathcal{H}}$, of finite volume and with no fewer cusps (than $\mathcal{H}$ ), and 
containing $\tilde{P}$; moreover, the set $\widetilde{F}_{k}\left(\widetilde{B}_{k}\right)$ will contain all the points out to any fixed distance we need from the point $F_{k}\left(\omega_{k}\right)(P)$; and hence

$$
\widetilde{F}_{k}\left(\widetilde{B}_{k}\right) \supseteq F_{k}\left(\omega_{k}\right)\left(\mathcal{H}_{a}\right)
$$

since $\mathcal{H}_{a}$ is at bounded distance from $P$ and $F_{k}\left(\omega_{k}\right)$ is reasonably close to preserving the metrics. Then we can form the composition

$$
G_{k}=\widetilde{F}_{k}^{-1} \circ F_{k}\left(\omega_{k}\right): \quad \mathcal{H}_{a} \rightarrow \tilde{\mathcal{H}}
$$

Since the $\widetilde{F}_{k}$ are as close to preserving the metric as we like, for any $\widetilde{\delta}>\delta$ we have

$$
\left\|G_{k}^{*} \widetilde{h}_{i j}-h_{i j}\right\|_{C^{q}\left(\mathcal{H}_{a}\right)}<\widetilde{\delta}
$$

for large enough $k$. Then a subsequence of $G_{k}$ converges at least in $C^{q-1}\left(\mathcal{H}_{a}\right)$ topology to a map $G_{\infty}$ of $\mathcal{H}_{a}$ into $\widetilde{\mathcal{H}}$. By the same reason as in the argument of previous two paragraphs, the limit map $G_{\infty}$ is a smooth harmonic diffeomorphism from $\mathcal{H}_{a}$ into $\widetilde{\mathcal{H}}$ with the metric $\tilde{h}_{i j}$, and takes $\partial \mathcal{H}_{a}$ to a constant mean curvature hypersurface $G_{\infty}\left(\partial \mathcal{H}_{a}\right)$ of $\left(\tilde{\mathcal{H}}, \widetilde{h}_{i j}\right)$ with the area $a$, and also satisfies the free boundary condition. Moreover we still have

$$
\left\|G_{\infty}^{*} \widetilde{h}_{i j}-h_{i j}\right\|_{C^{q}\left(\mathcal{H}_{a}\right)}=\delta .
$$

Now by Lemma 5.3 .7 we deduce that there exists an isometry $I$ of $\mathcal{H}$ to $\widetilde{\mathcal{H}}$ with

$$
d_{C^{l_{0}\left(\mathcal{H}_{a}\right)}}\left(G_{\infty}, I\right)<\varepsilon_{0}
$$

By using $I$ to identify $\widetilde{\mathcal{H}}_{a}$ and $\mathcal{H}_{a}$, we see that the map $I^{-1} \circ G_{\infty}$ is a harmonic diffeomorphism of $\mathcal{H}_{a}$ to itself which satisfies the free boundary condition and

$$
d_{C^{l_{0}\left(\mathcal{H}_{a}\right)}}\left(I^{-1} \circ G_{\infty}, I d\right)<\varepsilon_{0} .
$$

From the uniqueness in Lemma 5.3.8 we conclude that $I^{-1} \circ G_{\infty}=I d$ which contradicts with (5.3.11). This shows at least one hyperbolic piece persists. Moreover the pull-back of the solution metric $g_{i j}(t)$ by $F_{k}(t)$, for $t_{k} \leq t<+\infty$, is as close to the hyperbolic metric $h_{i j}$ as we like.

We can continue to form other persistent hyperbolic pieces in the same way as long as there are any points $P_{k}$ outside of the chosen pieces where the injectivity radius at times $t_{k} \rightarrow \infty$ are all at least some fixed positive number $\rho>0$. The only modification in the proof is to take the new limit $\mathcal{H}$ to have the least possible number of cusps out of all remaining possible limits.

Note that the volume of the normalized Ricci flow is constant in time. Therefore by combining with Margulis lemma (see for example [55] [76]), we have proved that there exists a finite collection of complete noncompact hyperbolic three-manifolds $\mathcal{H}_{1}, \ldots, \mathcal{H}_{m}$ with finite volume, a small number $a>0$ and a time $T<+\infty$ such that for all $t$ beyond $T$ we can find diffeomorphisms $\varphi_{l}(t)$ of $\left(\mathcal{H}_{l}\right)_{a}$ into $M, 1 \leq l \leq m$, so that the pull-back of the solution metric $g_{i j}(t)$ by $\varphi_{l}(t)$ is as close to the hyperbolic metrics as we like and the exceptional part of $M$ where the points are not in the image of any $\varphi_{l}$ has the injectivity radii everywhere as small as we like. 


\section{Part III: Incompressibility}

We remain to show that the boundary tori of any persistent hyperbolic piece are incompressible, in the sense that the fundamental group of the torus injects into that of the whole manifold. The argument of this part is a parabolic version of Schoen and Yau's minimal surface argument in $[109,110,111]$.

Let $B$ be a small positive number and assume the above positive number $a$ is much smaller than $B$. Denote by $M_{a}$ a persistent hyperbolic piece of the manifold $M$ truncated by boundary tori of area $a$ with constant mean curvature and denote by $M_{a}^{c}=M \backslash \stackrel{\circ}{M}_{a}$ the part of $M$ exterior to $M_{a}$. Thus there is a persistent hyperbolic piece $M_{B} \subset M_{a}$ of the manifold $M$ truncated by boundary tori of area $B$ with constant mean curvature. We also denote by $M_{B}^{c}=M \backslash \stackrel{\circ}{M}_{B}$. By Van Kampen's Theorem, if $\pi_{1}\left(\partial M_{B}\right)$ injects into $\pi_{1}\left(M_{B}^{c}\right)$ then it injects into $\pi_{1}(M)$ also. Thus we only need to show $\pi_{1}\left(\partial M_{B}\right)$ injects into $\pi_{1}\left(M_{B}^{c}\right)$.

We will argue by contradiction. Let $T$ be a torus in $\partial M_{B}$. Suppose $\pi_{1}(T)$ does not inject into $\pi_{1}\left(M_{B}^{c}\right)$, then by Dehn's Lemma the kernel is a cyclic subgroup of $\pi_{1}(T)$ generated by a primitive element. The work of Meeks-Yau [86] or Meeks-Simon-Yau [87] shows that among all disks in $M_{B}^{c}$ whose boundary curve lies in $T$ and generates the kernel, there is a smooth embedded disk normal to the boundary which has the least possible area. Let $A=A(t)$ be the area of this disk. This is defined for all $t$ sufficiently large. We will show that $A(t)$ decreases at a rate bounded away from zero which will be a contradiction.

Let us compute the rate at which $A(t)$ changes under the Ricci flow. We need to show $A(t)$ decrease at least at a certain rate, and since $A(t)$ is the minimum area to bound any disk in the given homotopy class, it suffices to find some such disk whose area decreases at least that fast. We choose this disk as follows. Pick the minimal disk at time $t_{0}$, and extend it smoothly a little past the boundary torus since the minimal disk is normal to the boundary. For times $t$ a little bigger than $t_{0}$, the boundary torus may need to move a little to stay constant mean curvature with area $B$ as the metrics change, but we leave the surface alone and take the bounding disk to be the one cut off from it by the new torus. The change of the area $\tilde{A}(t)$ of such disk comes from the change in the metric and the change in the boundary.

For the change in the metric, we choose an orthonormal frame $X, Y, Z$ at a point $x$ in the disk so that $X$ and $Y$ are tangent to the disk while $Z$ is normal and compute the rate of change of the area element $d \sigma$ on the disk as

$$
\begin{aligned}
\frac{\partial}{\partial t} d \sigma & =\frac{1}{2}\left(g^{i j}\right)^{T}\left(\frac{2}{3} r\left(g_{i j}\right)^{T}-2\left(R_{i j}\right)^{T}\right) d \sigma \\
& =\left[\frac{2}{3} r-\operatorname{Ric}(X, X)-\operatorname{Ric}(Y, Y)\right] d \sigma,
\end{aligned}
$$

since the metric evolves by the normalized Ricci flow. Here $(\cdot)^{T}$ denotes the tangential projections on the disk. Notice the torus $T$ may move in time to preserve constant mean curvature and constant area $B$. Suppose the boundary of the disk evolves with a normal velocity $N$. The change of the area at boundary along a piece of length $d s$ is given by $N d s$. Thus the total change of the area $\tilde{A}(t)$ is given by

$$
\frac{d \tilde{A}}{d t}=\iint\left(\frac{2}{3} r-\operatorname{Ric}(X, X)-\operatorname{Ric}(Y, Y)\right) d \sigma+\int_{\partial} N d s .
$$


Note that

$$
\begin{aligned}
\operatorname{Ric}(X, X)+\operatorname{Ric}(Y, Y)= & R(X, Y, X, Y)+R(X, Z, X, Z) \\
& +R(Y, X, Y, X)+R(Y, Z, Y, Z) \\
= & \frac{1}{2} R+R(X, Y, X, Y) .
\end{aligned}
$$

By the Gauss equation, the Gauss curvature $K$ of the disk is given by

$$
K=R(X, Y, X, Y)+\operatorname{det} I I
$$

where $I I$ is the second fundamental form of the disk in $M_{B}^{c}$. This gives at $t=t_{0}$,

$$
\frac{d A}{d t} \leq \iint\left(\frac{2}{3} r-\frac{1}{2} R\right) d \sigma-\iint(K-\operatorname{det} I I) d \sigma+\int_{\partial} N d s
$$

Since the bounding disk is a minimal surface, we have

$$
\operatorname{det} I I \leq 0
$$

The Gauss-Bonnet Theorem tells us that for a disk

$$
\iint K d \sigma+\int_{\partial} k d s=2 \pi
$$

where $k$ is the geodesic curvature of the boundary. Thus we obtain

$$
\frac{d A}{d t} \leq \iint\left(\frac{2}{3} r-\frac{1}{2} R\right) d \sigma+\int_{\partial} k d s+\int_{\partial} N d s-2 \pi
$$

Recall that we are assuming $R_{\min }(t)$ increases monotonically to -6 as $t \rightarrow+\infty$. By the evolution equation of the scalar curvature,

$$
\frac{d}{d t} R_{\min }(t) \geq 4\left(r(t)-R_{\min }(t)\right)
$$

and then

$$
\int_{0}^{\infty}\left(r(t)-R_{\min }(t)\right) d t<+\infty
$$

This implies that $r(t) \rightarrow-6$ as $t \rightarrow+\infty$ by using the derivatives estimate for the curvatures. Thus for every $\varepsilon>0$ we have

$$
\frac{2}{3} r-\frac{1}{2} R \leq-(1-\varepsilon)
$$

for $t$ sufficiently large. And then the first term on RHS of (5.3.12) is bounded above by

$$
\iint\left(\frac{2}{3} r-\frac{1}{2} R\right) d \sigma \leq-(1-\varepsilon) A .
$$

The geodesic curvature $k$ of the boundary of the minimal disk is the acceleration of a curve moving with unit speed along the intersection of the disk with the torus; 
since the disk and torus are normal, this is the same as the second fundamental form of the torus in the direction of the curve of intersection. Now if the metric were actually hyperbolic, the second fundamental form of the torus would be exactly 1 in all directions. Note that the persistent hyperbolic pieces are as close to the standard hyperbolic as we like. This makes that the second term of RHS of (5.3.12) is bounded above by

$$
\int_{\partial} k d s \leq\left(1+\varepsilon_{0}\right) L
$$

for some sufficiently small positive number $\varepsilon_{0}>0$, where $L$ is the length of the boundary curve. Also since the metric on the persistent hyperbolic pieces are close to the standard hyperbolic as we like, its change under the normalized Ricci flow is as small as we like; So the motion of the constant mean curvature torus of fixed area $B$ will have a normal velocity $N$ as small as we like. This again makes the third term of RHS of (5.3.12) bounded above by

$$
\int_{\partial} N d s \leq \varepsilon_{0} L
$$

Combining these estimates, we obtain

$$
\frac{d A}{d t} \leq\left(1+2 \varepsilon_{0}\right) L-\left(1-\varepsilon_{0}\right) A-2 \pi
$$

on the persistent hyperbolic piece, where $\varepsilon_{0}$ is some sufficiently small positive number.

We next need to bound the length $L$ in terms of the area $A$. Since $a$ is much smaller than $B$, for large $t$ the metric is as close as we like to the standard hyperbolic one; not just on the persistent hyperbolic piece $M_{B}$ but as far beyond as we like. Thus for a long distance into $M_{B}^{c}$ the metric will look nearly like a standard hyperbolic cusplike collar.

Let us first recall a special coordinate system on the standard hyperbolic cusp projecting beyond torus $T_{1}$ in $\partial \mathcal{H}_{1}$ as follows. The universal cover of the flat torus $T_{1}$ can be mapped conformally to the $x-y$ plane so that the deck transformation of $T_{1}$ become translations in $x$ and $y$, and so that the Euclidean area of the quotient is 1 ; then these coordinates are unique up to a translation. The hyperbolic cusp projecting beyond the torus $T_{1}$ in $\partial \mathcal{H}_{1}$ can be parametrized by $\left\{(x, y, z) \in \mathbb{R}^{3} \mid z>0\right\}$ with the hyperbolic metric

$$
d s^{2}=\frac{d x^{2}+d y^{2}+d z^{2}}{z^{2}} .
$$

Note that we can make the solution metric, in an arbitrarily large neighborhood of the torus $T$ (of $\partial M_{B}$ ), as close to hyperbolic as we wish (in the sense that there exists a diffeomorphism from a large neighborhood of the torus $T_{B}$ (of $\partial \mathcal{H}_{B}$ ) on the standard hyperbolic cusp to the above neighborhood of the torus $T$ (of $\partial M_{B}$ ) such that the pull-back of the solution metric by the diffeomorphism is as close to the hyperbolic metric as we wish). Then by using this diffeomorphism (up to a slight modification) we can parametrize the cusplike tube of $M_{B}^{c}$ projecting beyond the torus $T$ in $\partial M_{B}$ by $\{(x, y, z) \mid z \geq \zeta\}$ where the height $\zeta$ is chosen so that the torus in the hyperbolic cusp at height $\zeta$ has the area $B$.

Now consider our minimal disk, and let $L(z)$ be the length of the curve of the intersection of the disk with the torus at height $z$ in the above coordinate system, 
and also let $A(z)$ be the area of the part of the disk between $\zeta$ and $z$. We now want to derive a monotonicity formula on the area $A(z)$ for the minimal surface.

For almost every $z$ the intersection of the disk with the torus at height $z$ is a smooth embedded curve or a finite union of them by the standard transversality theorem. If there is more than one curve, at least one of them is not homotopic to a point in $T$ and represents the primitive generator in the kernel of $\pi_{1}(T)$ such that a part of the original disk beyond height $z$ continues to a disk that bounds it. We extend this disk back to the initial height $\zeta$ by dropping the curve straight down. Let $\tilde{L}(z)$ be the length of the curve we picked at height $z$; of course $\tilde{L}(z) \leq L(z)$ with equality if it is the only piece. Let $\tilde{L}(w)$ denote the length of the same curve in the $x-y$ plane dropped down to height $w$ for $\zeta \leq w \leq z$. In the hyperbolic space we would have

$$
\tilde{L}(w)=\frac{z}{w} \tilde{L}(z)
$$

exactly. In our case there is a small error proportional to $\tilde{L}(z)$ and we can also take it proportional to the distance $z-w$ by which it drops since $\left.\tilde{L}(w)\right|_{w=z}=\tilde{L}(z)$ and the solution metric is close to the hyperbolic in the $C_{l o c}^{\infty}$ topology. Thus, for arbitrarily given $\delta>0$ and $\zeta^{*}>\zeta$, as the solution metric is sufficiently close to hyperbolic, we have

$$
\left|\tilde{L}(w)-\frac{z}{w} \tilde{L}(z)\right| \leq \delta(z-w) \tilde{L}(z)
$$

for all $z$ and $w$ in $\zeta \leq w \leq z \leq \zeta^{*}$. Now given $\varepsilon$ and $\zeta^{*}$ pick $\delta=2 \varepsilon / \zeta^{*}$. Then

$$
\tilde{L}(w) \leq \frac{z}{w} \tilde{L}(z)\left[1+\frac{2 \varepsilon(z-w)}{w}\right]
$$

When we drop the curve vertically for the construction of the new disk we get an area $\tilde{A}(z)$ between $\zeta$ and $z$ given by

$$
\tilde{A}(z)=(1+o(1)) \int_{\zeta}^{z} \frac{\tilde{L}(w)}{w} d w .
$$

Here and in the following $o(1)$ denotes various small error quantities as the solution metric close to hyperbolic. On the other hand if we do not drop vertically we pick up even more area, so the area $A(z)$ of the original disk between $\zeta$ and $z$ has

$$
A(z) \geq(1-o(1)) \int_{\zeta}^{z} \frac{L(w)}{w} d w .
$$

Since the original disk minimized among all disks bounded a curve in the primitive generator of the kernel of $\pi_{1}(T)$, and the new disk beyond the height $z$ is part of the original disk, we have

$$
A(z) \leq \tilde{A}(z)
$$

and then by combining with (5.3.15),

$$
\begin{aligned}
\int_{\zeta}^{z} \frac{L(w)}{w} d w & \leq(1+o(1)) z \tilde{L}(z) \int_{\zeta}^{z}\left[\frac{1-2 \varepsilon}{w^{2}}+\frac{2 \varepsilon z}{w^{3}}\right] d w \\
& \leq(1+o(1)) L(z) \frac{(z-\zeta)}{\zeta}\left[1+\varepsilon\left(\frac{z-\zeta}{\zeta}\right)\right]
\end{aligned}
$$


Here we used the fact that $\tilde{L}(z) \leq L(z)$. Since the solution metric is sufficiently close to hyperbolic, we have

$$
\begin{aligned}
\frac{d}{d z}\left(\int_{\zeta}^{z} \frac{L(w)}{w} d w\right) & =\frac{L(z)}{z} \\
& \geq(1-o(1)) \frac{\zeta}{z(z-\zeta)}\left[1-\varepsilon\left(\frac{z-\zeta}{\zeta}\right)\right] \int_{\zeta}^{z} \frac{L(w)}{w} d w \\
& \geq\left[\frac{1}{z-\zeta}-\frac{1+2 \varepsilon}{z}\right] \int_{\zeta}^{z} \frac{L(w)}{w} d w
\end{aligned}
$$

or equivalently

$$
\frac{d}{d z} \log \left\{\frac{z^{1+2 \varepsilon}}{(z-\zeta)} \int_{\zeta}^{z} \frac{L(w)}{w} d w\right\} \geq 0 .
$$

This is the desired monotonicity formula for the area $A(z)$.

It follows directly from (5.3.16) and (5.3.17) that

$$
\frac{z^{1+2 \varepsilon}}{(z-\zeta)} A(z) \geq(1-o(1)) \zeta^{2 \varepsilon} L(\zeta)
$$

or equivalently

$$
L(\zeta) \leq(1+o(1))\left(\frac{z}{\zeta}\right)^{2 \varepsilon} \frac{z}{z-\zeta} A(z)
$$

for all $z \in\left[\zeta, \zeta^{*}\right]$. Since the solution metric, in an arbitrarily large neighborhood of the torus $T$ ( of $\partial M_{B}$ ), as close to hyperbolic as we wish, we may assume that $\zeta^{*}$ is so large that $\sqrt{\zeta^{*}}>\zeta$ and $\frac{\sqrt{\zeta^{*}}}{\sqrt{\zeta^{*}}-\zeta}$ is close to 1 , and also $\varepsilon>0$ is so small that $\left(\frac{\sqrt{\zeta^{*}}}{\zeta}\right)^{2 \varepsilon}$ is close to 1 . Thus for arbitrarily small $\delta_{0}>0$, we have

$$
L(\zeta) \leq\left(1+\delta_{0}\right) A\left(\sqrt{\zeta^{*}}\right)
$$

Now recall that (5.3.13) states

$$
\frac{d A}{d t} \leq\left(1+2 \varepsilon_{0}\right) L-\left(1-\varepsilon_{0}\right) A-2 \pi
$$

We now claim that if

$$
\left(1+2 \varepsilon_{0}\right) L-\left(1-\varepsilon_{0}\right) A \geq 0
$$

then $L=L(\zeta)$ is uniformly bounded from above.

Indeed by the assumption we have

$$
A\left(\zeta^{*}\right) \leq \frac{\left(1+2 \varepsilon_{0}\right)}{\left(1-\varepsilon_{0}\right)} L(\zeta)
$$

since $A\left(\zeta^{*}\right) \leq A$. By combining with $(5.3 .16)$ we have some $z_{0} \in\left(\sqrt{\zeta^{*}}, \zeta^{*}\right)$ satisfying

$$
\begin{aligned}
\frac{L\left(z_{0}\right)}{z_{0}}\left(\zeta^{*}-\sqrt{\zeta^{*}}\right) & \leq \int_{\sqrt{\zeta^{*}}}^{\zeta^{*}} \frac{L(w)}{w} d w \\
& \leq(1+o(1)) A\left(\zeta^{*}\right) \\
& \leq(1+o(1))\left(\frac{1+2 \varepsilon_{0}}{1-\varepsilon_{0}}\right) L(\zeta) .
\end{aligned}
$$


Thus for $\zeta^{*}$ suitably large, by noting that the solution metric on a large neighborhood of $T$ (of $\partial M_{B}$ ) is sufficiently close to hyperbolic, we have

$$
L\left(z_{0}\right) \leq\left(1+4 \varepsilon_{0}\right) \frac{z_{0}}{\zeta^{*}} L(\zeta)
$$

for some $z_{0} \in\left(\sqrt{\zeta^{*}}, \zeta^{*}\right)$. It is clear that we may assume the intersection curve between the minimal disk with the torus at this height $z_{0}$ is smooth and embedded. If the intersection curve at the height $z_{0}$ has more than one piece, as before one of them will represent the primitive generator in the kernel of $\pi_{1}(T)$, and we can ignore the others. Let us move (the piece of) the intersection curve on the torus at height $z_{0}$ through as small as possible area in the same homotopy class of $\pi_{1}(T)$ to a curve which is a geodesic circle in the flat torus coming from our special coordinates, and then drop this geodesic circle vertically in the special coordinates to obtain another new disk. We will compare the area of this new disk with the original minimal disk as follows.

Denote by $G$ the length of the geodesic circle in the standard hyperbolic cusp at height 1 . Then the length of the geodesic circle at height $z_{0}$ will be $G / z_{0}$. Observe that given an embedded curve of length $l$ circling the cylinder $S^{1} \times \mathbb{R}$ of circumference $w$ once, it is possible to deform the curve through an area not bigger than $l w$ into a meridian circle. Note that (the piece of) the intersection curve represents the primitive generator in the kernel of $\pi_{1}(T)$. Note also that the solution metric is sufficiently close to the hyperbolic metric. Then the area of the deformation from (the piece of) the intersection curve on the torus at height $z_{0}$ to the geodesic circle at height $z_{0}$ is bounded by

$$
(1+o(1))\left(\frac{G}{z_{0}}\right) \cdot L\left(z_{0}\right) .
$$

The area to drop the geodesic circle from height $z_{0}$ to height $\zeta$ is bounded by

$$
(1+o(1)) \int_{\zeta}^{z_{0}} \frac{G}{w^{2}} d w .
$$

Hence comparing the area of the original minimal disk to that of this new disk gives

$$
A\left(z_{0}\right) \leq(1+o(1)) G\left[\frac{L\left(z_{0}\right)}{z_{0}}+\left(\frac{1}{\zeta}-\frac{1}{z_{0}}\right)\right] .
$$

By (5.3.18), (5.3.19) and the fact that $z_{0} \in\left(\sqrt{\zeta^{*}}, \zeta^{*}\right)$, this in turn gives

$$
\begin{aligned}
L(\zeta) & \leq\left(1+\delta_{0}\right) A\left(z_{0}\right) \\
& \leq\left(1+\delta_{0}\right) G\left[\left(1+4 \varepsilon_{0}\right) \frac{L(\zeta)}{\zeta^{*}}+\frac{1}{\zeta}\right] .
\end{aligned}
$$

Since $\zeta^{*}$ is suitably large, we obtain

$$
L(\zeta) \leq 2 G / \zeta
$$

This gives the desired assertion since $G$ is fixed from the geometry of the limit hyperbolic manifold $\mathcal{H}$ and $\zeta$ is very large as long as the area $B$ of $\partial M_{B}$ small enough.

Thus the combination of (5.3.13), (5.3.18) and the assertion implies that either

$$
\frac{d}{d t} A \leq-2 \pi
$$


or

$$
\begin{aligned}
\frac{d}{d t} A & \leq\left(1+2 \varepsilon_{0}\right) L-\left(1-\varepsilon_{0}\right) A-2 \pi \\
& \leq\left(1+2 \varepsilon_{0}\right) \frac{2 G}{\zeta}-2 \pi \\
& \leq-\pi
\end{aligned}
$$

since the solution metric on a very large neighborhood of the torus $T$ (of $\partial M_{B}$ ) is sufficiently close to hyperbolic and $\zeta$ is very large as the area $B$ of $\partial M_{B}$ small enough. This is impossible because $A \geq 0$ and the persistent hyperbolic pieces go on forever. The contradiction shows that $\pi_{1}(T)$ in fact injects into $\pi_{1}\left(M_{B}^{c}\right)$. This proves that $\pi_{1}\left(\partial M_{B}\right)$ injects into $\pi_{1}(M)$.

Therefore we have completed the proof of Theorem 5.3.4. $\mathrm{C}$

6. Ancient $\kappa$-solutions. Let us consider a solution of the Ricci flow on a compact manifold. If the solution blows up in finite time (i.e., the maximal solution exists only on a finite time interval), then as we saw in Chapter 4 a sequence of rescalings of the solution around the singularities converge to a solution which exists at least on the time interval $(-\infty, T)$ for some finite number $T$. Furthermore, by Perelman's no local collapsing theorem I (Theorem 3.3.2), we see that the limit is $\kappa$-noncollapsed on all scales for some positive constant $\kappa$. In addition, if the dimension $n=3$ then the Hamilton-Ivey pinching estimate implies that the limiting solution must have nonnegative curvature operator.

We call a solution to the Ricci flow an ancient $\kappa$-solution if it is complete (either compact or noncompact) and defined on an ancient time interval $(-\infty, T)$ with $T>0$, has nonnegative curvature operator and bounded curvature, and is $\kappa$-noncollapsed on all scales for some positive constant $\kappa$.

In this chapter we study ancient $\kappa$-solutions of the Ricci flow. We will obtain crucial curvature estimates of such solutions and determine their structures in lower dimensional cases.

6.1. Preliminaries. We first present a useful geometric property, given by Chen and the second author in [34], for complete noncompact Riemannian manifolds with nonnegative sectional curvature.

Let $\left(M, g_{i j}\right)$ be an $n$-dimensional complete Riemannian manifold and let $\varepsilon$ be a positive constant. We call an open subset $N \subset M$ an $\varepsilon$-neck of radius $r$ if $\left(N, r^{-2} g_{i j}\right)$ is $\varepsilon$-close, in the $C^{\left[\varepsilon^{-1}\right]}$ topology, to a standard neck $\mathbb{S}^{n-1} \times \mathbb{I}$, where $\mathbb{S}^{n-1}$ is the round $(n-1)$-sphere with scalar curvature 1 and $\mathbb{I}$ is an interval of length $2 \varepsilon^{-1}$. The following result is, to some extent, in similar spirit of Yau's volume lower bound estimate [128].

Proposition 6.1.1 (Chen-Zhu [34]). There exists a positive constant $\varepsilon_{0}=\varepsilon_{0}(n)$ such that every complete noncompact $n$-dimensional Riemannian manifold $\left(M, g_{i j}\right)$ of nonnegative sectional curvature has a positive constant $r_{0}$ such that any $\varepsilon$-neck of radius $r$ on $\left(M, g_{i j}\right)$ with $\varepsilon \leq \varepsilon_{0}$ must have $r \geq r_{0}$.

Proof. We argue by contradiction. Suppose there exist a sequence of positive constants $\varepsilon^{\alpha} \rightarrow 0$ and a sequence of $n$-dimensional complete noncompact Riemannian manifolds $\left(M^{\alpha}, g_{i j}^{\alpha}\right)$ such that for each fixed $\alpha$, there exists a sequence of $\varepsilon^{\alpha}$-necks $N_{k}$ of radius at most $1 / k$ in $M^{\alpha}$ with centers $P_{k}$ divergent to infinity. 
Fix a point $P$ on the manifold $M^{\alpha}$ and connect each $P_{k}$ to $P$ by a minimizing geodesic $\gamma_{k}$. By passing to a subsequence we may assume the angle $\theta_{k l}$ between geodesic $\gamma_{k}$ and $\gamma_{l}$ at $P$ is very small and tends to zero as $k, l \rightarrow+\infty$, and the length of $\gamma_{k+1}$ is much bigger than the length of $\gamma_{k}$. Let us connect $P_{k}$ to $P_{l}$ by a minimizing geodesic $\eta_{k l}$. For each fixed $l>k$, let $\tilde{P}_{k}$ be a point on the geodesic $\gamma_{l}$ such that the geodesic segment from $P$ to $\tilde{P}_{k}$ has the same length as $\gamma_{k}$ and consider the triangle $\triangle P P_{k} \tilde{P}_{k}$ in $M^{\alpha}$ with vertices $P, P_{k}$ and $\tilde{P}_{k}$. By comparing with the corresponding triangle in the Euclidean plane $\mathbb{R}^{2}$ whose sides have the same corresponding lengths, Toponogov's comparison theorem implies

$$
d\left(P_{k}, \tilde{P}_{k}\right) \leq 2 \sin \left(\frac{1}{2} \theta_{k l}\right) \cdot d\left(P_{k}, P\right)
$$

Since $\theta_{k l}$ is very small, the distance from $P_{k}$ to the geodesic $\gamma_{l}$ can be realized by a geodesic $\zeta_{k l}$ which connects $P_{k}$ to a point $P_{k}^{\prime}$ on the interior of the geodesic $\gamma_{l}$ and has length at most $2 \sin \left(\frac{1}{2} \theta_{k l}\right) \cdot d\left(P_{k}, P\right)$. Clearly the angle between $\zeta_{k l}$ and $\gamma_{l}$ at the intersection point $P_{k}^{\prime}$ is $\frac{\pi}{2}$. Consider $\alpha$ to be fixed and sufficiently large. We claim that for large enough $k$, each minimizing geodesic $\gamma_{l}$ with $l>k$, connecting $P$ to $P_{l}$, goes through the neck $N_{k}$.

Suppose not; then the angle between $\gamma_{k}$ and $\zeta_{k l}$ at $P_{k}$ is close to either zero or $\pi$ since $P_{k}$ is in the center of an $\varepsilon^{\alpha}$-neck and $\alpha$ is sufficiently large. If the angle between $\gamma_{k}$ and $\zeta_{k l}$ at $P_{k}$ is close to zero, we consider the triangle $\Delta P P_{k} P_{k}^{\prime}$ in $M^{\alpha}$ with vertices $P, P_{k}$, and $P_{k}^{\prime}$. Note that the length between $P_{k}$ and $P_{k}^{\prime}$ is much smaller than the lengths from $P_{k}$ or $P_{k}^{\prime}$ to $P$. By comparing the angles of this triangle with those of the corresponding triangle in the Euclidean plane with the same corresponding lengths and using Toponogov's comparison theorem, we find that it is impossible. Thus the angle between $\gamma_{k}$ and $\zeta_{k l}$ at $P_{k}$ is close to $\pi$. We now consider the triangle $\Delta P_{k} P_{k}^{\prime} P_{l}$ in $M^{\alpha}$ with the three sides $\zeta_{k l}, \eta_{k l}$ and the geodesic segment from $P_{k}^{\prime}$ to $P_{l}$ on $\gamma_{l}$. We have seen that the angle of $\Delta P_{k} P_{k}^{\prime} P_{l}$ at $P_{k}$ is close to zero and the angle at $P_{k}^{\prime}$ is $\frac{\pi}{2}$. By comparing with corresponding triangle $\bar{\Delta} \bar{P}_{k} \bar{P}_{k}^{\prime} \bar{P}_{l}$ in the Euclidean plane $\mathbb{R}^{2}$ whose sides have the same corresponding lengths, Toponogov's comparison theorem implies

$$
\angle \bar{P}_{l} \bar{P}_{k} \bar{P}_{k}^{\prime}+\angle \bar{P}_{l} \bar{P}_{k}^{\prime} \bar{P}_{k} \leq \angle P_{l} P_{k} P_{k}^{\prime}+\angle P_{l} P_{k}^{\prime} P_{k}<\frac{3}{4} \pi .
$$

This is impossible since the length between $\bar{P}_{k}$ and $\bar{P}_{k}^{\prime}$ is much smaller than the length from $\bar{P}_{l}$ to either $\bar{P}_{k}$ or $\bar{P}_{k}^{\prime}$. So we have proved each $\gamma_{l}$ with $l>k$ passes through the neck $N_{k}$.

Hence by taking a limit, we get a geodesic ray $\gamma$ emanating from $P$ which passes through all the necks $N_{k}, k=1,2, \ldots$, except a finite number of them. Throwing these finite number of necks away, we may assume $\gamma$ passes through all necks $N_{k}$, $k=1,2, \ldots$ Denote the center sphere of $N_{k}$ by $S_{k}$, and their intersection points with $\gamma$ by $p_{k} \in S_{k} \cap \gamma, k=1,2, \ldots$

Take a sequence of points $\gamma(m)$ with $m=1,2, \ldots$. For each fixed neck $N_{k}$, arbitrarily choose a point $q_{k} \in N_{k}$ near the center sphere $S_{k}$ and draw a geodesic segment $\gamma^{k m}$ from $q_{k}$ to $\gamma(m)$. Now we claim that for any neck $N_{l}$ with $l>k, \gamma^{k m}$ will pass through $N_{l}$ for all sufficiently large $m$.

We argue by contradiction. Let us place all the necks $N_{i}$ horizontally so that the geodesic $\gamma$ passes through each $N_{i}$ from the left to the right. We observe that the geodesic segment $\gamma^{k m}$ must pass through the right half of $N_{k}$; otherwise $\gamma^{k m}$ cannot be minimal. Then for large enough $m$, the distance from $p_{l}$ to the geodesic segment 
$\gamma^{k m}$ must be achieved by the distance from $p_{l}$ to some interior point $p_{k}{ }^{\prime}$ of $\gamma^{k m}$. Let us draw a minimal geodesic $\eta$ from $p_{l}$ to the interior point $p_{k}{ }^{\prime}$ with the angle at the intersection point $p_{k}{ }^{\prime} \in \eta \cap \gamma^{k m}$ to be $\frac{\pi}{2}$. Suppose the claim is false. Then the angle between $\eta$ and $\gamma$ at $p_{l}$ is close to 0 or $\pi$ since $\varepsilon^{\alpha}$ is small.

If the angle between $\eta$ and $\gamma$ at $p_{l}$ is close to 0 , we consider the triangle $\Delta p_{l} p_{k}{ }^{\prime} \gamma(m)$ and construct a comparison triangle $\bar{\Delta} \bar{p}_{l} \bar{p}_{k}{ }^{\prime} \bar{\gamma}(m)$ in the plane with the same corresponding length. Then by Toponogov's comparison theorem, we see the sum of the inner angles of the comparison triangle $\bar{\Delta} \bar{p}_{l} \bar{p}_{k}{ }^{\prime} \bar{\gamma}(m)$ is less than $3 \pi / 4$, which is impossible.

If the angle between $\eta$ and $\gamma$ at $p_{l}$ is close to $\pi$, by drawing a minimal geodesic $\xi$ from $q_{k}$ to $p_{l}$, we see that $\xi$ must pass through the right half of $N_{k}$ and the left half of $N_{l}$; otherwise $\xi$ cannot be minimal. Thus the three inner angles of the triangle $\Delta p_{l} p_{k}{ }^{\prime} q_{k}$ are almost $0, \pi / 2$, and 0 respectively. This is also impossible by the Toponogov comparison theorem.

Hence we have proved that the geodesic segment $\gamma^{k m}$ passes through $N_{l}$ for $m$ large enough.

Consider the triangle $\Delta p_{k} q_{k} \gamma(m)$ with two long sides $\overline{p_{k} \gamma(m)}(\subset \gamma)$ and $\overline{q_{k} \gamma(m)}(=$ $\left.\gamma^{k m}\right)$. For any $s>0$, choose points $\tilde{p}_{k}$ on $\overline{p_{k} \gamma(m)}$ and $\tilde{q}_{k}$ on $\overline{q_{k} \gamma(m)}$ with $d\left(p_{k}, \tilde{p}_{k}\right)=$ $d\left(q_{k}, \tilde{q}_{k}\right)=s$. By Toponogov's comparison theorem, we have

$$
\begin{aligned}
& \left(\frac{d\left(\tilde{p}_{k}, \tilde{q}_{k}\right)}{d\left(p_{k}, q_{k}\right)}\right)^{2} \\
& =\frac{d\left(\tilde{p}_{k}, \gamma(m)\right)^{2}+d\left(\tilde{q}_{k}, \gamma(m)\right)^{2}-2 d\left(\tilde{p}_{k}, \gamma(m)\right) d\left(\tilde{q}_{k}, \gamma(m)\right) \cos \bar{\measuredangle}\left(\tilde{p}_{k} \gamma(m) \tilde{q}_{k}\right)}{d\left(p_{k}, \gamma(m)\right)^{2}+d\left(q_{k}, \gamma(m)\right)^{2}-2 d\left(p_{k}, \gamma(m)\right) d\left(q q_{k}, \gamma(m)\right) \cos \bar{\measuredangle}\left(p_{k} \gamma(m) q_{k}\right)} \\
& \geq \frac{d\left(\tilde{p}_{k}, \gamma(m)\right)^{2}+d\left(\tilde{q}_{k}, \gamma(m)\right)^{2}-2 d\left(\tilde{p}_{k}, \gamma(m)\right) d\left(\tilde{q}_{k}, \gamma(m)\right) \cos \bar{\measuredangle}\left(\tilde{p}_{k} \gamma(m) \tilde{q}_{k}\right)}{d\left(p_{k}, \gamma(m)\right)^{2}+d\left(q_{k}, \gamma(m)\right)^{2}-2 d\left(p_{k}, \gamma(m)\right) d\left(q_{k}, \gamma(m)\right) \cos \bar{\measuredangle}\left(\tilde{p}_{k} \gamma(m) \tilde{q}_{k}\right)} \\
& =\frac{\left(d\left(\tilde{p}_{k}, \gamma(m)\right)-d\left(\tilde{q}_{k}, \gamma(m)\right)\right)^{2}+2 d\left(\tilde{p}_{k}, \gamma(m)\right) d\left(\tilde{q}_{k}, \gamma(m)\right)\left(1-\cos \bar{\measuredangle}\left(\tilde{p}_{k} \gamma(m) \tilde{q}_{k}\right)\right)}{\left(d\left(\tilde{p}_{k}, \gamma(m)\right)-d\left(\tilde{q}_{k}, \gamma(m)\right)\right)^{2}+2 d\left(p_{k}, \gamma(m)\right) d\left(q_{k}, \gamma(m)\right)\left(1-\cos \bar{\measuredangle}\left(\tilde{p}_{k} \gamma(m) \tilde{q}_{k}\right)\right)} \\
& \geq \frac{d\left(\tilde{p}_{k}, \gamma(m)\right) d\left(\tilde{q}_{k}, \gamma(m)\right)}{d\left(p_{k}, \gamma(m)\right) d\left(q_{k}, \gamma(m)\right)} \\
& \rightarrow 1
\end{aligned}
$$

as $m \rightarrow \infty$, where $\bar{\measuredangle}\left(p_{k} \gamma(m) q_{k}\right)$ and $\bar{\measuredangle}\left(\tilde{p}_{k} \gamma(m) \tilde{q}_{k}\right)$ are the corresponding angles of the comparison triangles.

Letting $m \rightarrow \infty$, we see that $\gamma^{k m}$ has a convergent subsequence whose limit $\gamma^{k}$ is a geodesic ray passing through all $N_{l}$ with $l>k$. Let us denote by $p_{j}=\gamma\left(t_{j}\right), j=$ $1,2, \ldots$. From the above computation, we deduce that

$$
d\left(p_{k}, q_{k}\right) \leq d\left(\gamma\left(t_{k}+s\right), \gamma^{k}(s)\right)
$$

for all $s>0$.

Let $\varphi(x)=\lim _{t \rightarrow+\infty}(t-d(x, \gamma(t)))$ be the Busemann function constructed from the ray $\gamma$. Note that the level set $\varphi^{-1}\left(\varphi\left(p_{j}\right)\right) \cap N_{j}$ is close to the center sphere $S_{j}$ for any $j=1,2, \ldots$. Now let $q_{k}$ be any fixed point in $\varphi^{-1}\left(\varphi\left(p_{k}\right)\right) \cap N_{k}$. By the definition of Busemann function $\varphi$ associated to the ray $\gamma$, we see that $\varphi\left(\gamma^{k}\left(s_{1}\right)\right)-\varphi\left(\gamma^{k}\left(s_{2}\right)\right)=$ $s_{1}-s_{2}$ for any $s_{1}, s_{2} \geq 0$. Consequently, for each $l>k$, by choosing $s=t_{l}-t_{k}$, we see $\gamma^{k}\left(t_{l}-t_{k}\right) \in \varphi^{-1}\left(\varphi\left(p_{l}\right)\right) \cap N_{l}$. Since $\gamma\left(t_{k}+t_{l}-t_{k}\right)=p_{l}$, it follows that

$$
d\left(p_{k}, q_{k}\right) \leq d\left(p_{l}, \gamma^{k}(s)\right) .
$$


with $s=t_{l}-t_{k}>0$. This implies that the diameter of $\varphi^{-1}\left(\varphi\left(p_{k}\right)\right) \cap N_{k}$ is not greater than the diameter of $\varphi^{-1}\left(\varphi\left(p_{l}\right)\right) \cap N_{l}$ for any $l>k$, which is a contradiction for $l$ much larger than $k$.

Therefore we have proved the proposition.

In [63], Hamilton discovered an important repulsion principle (cf. Theorem 21.4 of [63]) about the influence of a bump of strictly positive curvature in a complete noncompact manifold of nonnegative sectional curvature. Namely minimal geodesic paths that go past the bump have to avoid it. As a consequence he obtained a finite bump theorem (cf. Theorem 21.5 of [63]) that gives a bound on the number of bumps of curvature.

Let $M$ be a complete noncompact Riemannian manifold with nonnegative sectional curvature $K \geq 0$. A geodesic ball $B(p, r)$ of radius $r$ centered at a point $p \in M$ is called a curvature $\beta$-bump if sectional curvature $K \geq \beta / r^{2}$ at all points in the ball. The ball $B(p, r)$ is called $\lambda$-remote from an origin $O$ if $d(p, O) \geq \lambda r$.

Finite Bump Theorem (Hamilton [63]). For every $\beta>0$ there exists $\lambda<\infty$ such that in any complete manifold of nonnegative sectional curvature there are at most a finite number of disjoint balls which are $\lambda$-remote curvature $\beta$-bumps.

This finite bump theorem played an important role in Hamilton's study of the behavior of singularity models at infinity and in the dimension reduction argument he developed for the Ricci flow (cf. Section 22 of [63], see also [29] for application to the Kähler-Ricci flow and uniformization problem in complex dimension two). A special consequence of the finite bump theorem is that if we have a complete noncompact solution to the Ricci flow on an ancient time interval $-\infty<t<T$ with $T>0$ satisfying certain local injectivity radius bound, with curvature bounded at each time and with asymptotic scalar curvature ratio $A=\lim \sup R s^{2}=\infty$, then we can find a sequence of points $p_{j}$ going to $\infty$ (as in the following Lemma 6.1.3) such that a cover of the limit of dilations around these points at time $t=0$ splits as a product with a flat factor. The following result, obtained by Chen and the second author in [34], is in similar spirit as Hamilton's finite bumps theorem and its consequence. The advantage is that we will get in the limit of dilations a product of the line with a lower dimensional manifold, instead of a quotient of such a product.

Proposition 6.1.2 (Chen-Zhu [34]). Suppose $\left(M, g_{i j}\right)$ is a complete $n$ dimensional Riemannian manifold with nonnegative sectional curvature. Let $P \in M$ be fixed, and $P_{k} \in M$ a sequence of points and $\lambda_{k}$ a sequence of positive numbers with $d\left(P, P_{k}\right) \rightarrow+\infty$ and $\lambda_{k} d\left(P, P_{k}\right) \rightarrow+\infty$. Suppose also that the marked manifolds $\left(M, \lambda_{k}^{2} g_{i j}, P_{k}\right)$ converge in the $C_{\text {loc }}^{\infty}$ topology to a Riemannian manifold $\widetilde{M}$. Then the limit $\widetilde{M}$ splits isometrically as the metric product of the form $\mathbb{R} \times N$, where $N$ is a Riemannian manifold with nonnegative sectional curvature.

Proof. Let us denote by $|O Q|=d(O, Q)$ the distance between two points $O, Q \in$ $M$. Without loss of generality, we may assume that for each $k$,

$$
1+2\left|P P_{k}\right| \leq\left|P P_{k+1}\right| .
$$

Draw a minimal geodesic $\gamma_{k}$ from $P$ to $P_{k}$ and a minimal geodesic $\sigma_{k}$ from $P_{k}$ to $P_{k+1}$, both parametrized by arclength. We may further assume

$$
\theta_{k}=\left|\measuredangle\left(\dot{\gamma}_{k}(0), \dot{\gamma}_{k+1}(0)\right)\right|<\frac{1}{k} .
$$


By assumption, the sequence $\left(M, \lambda_{k}^{2} g_{i j}, P_{k}\right)$ converges (in the $C_{l o c}^{\infty}$ topology) to a Riemannian manifold $\left(\widetilde{M}, \widetilde{g}_{i j}, \widetilde{P}\right)$ with nonnegative sectional curvature. By a further choice of subsequences, we may also assume $\gamma_{k}$ and $\sigma_{k}$ converge to geodesic rays $\widetilde{\gamma}$ and $\widetilde{\sigma}$ starting at $\widetilde{P}$ respectively. We will prove that $\tilde{\gamma} \cup \tilde{\sigma}$ forms a line in $\widetilde{M}$, and then by the Toponogov splitting theorem [89] the limit $\widetilde{M}$ must be splitted as $\mathbb{R} \times N$.

We argue by contradiction. Suppose $\widetilde{\gamma} \cup \widetilde{\sigma}$ is not a line; then for each $k$, there exist two points $A_{k} \in \gamma_{k}$ and $B_{k} \in \sigma_{k}$ such that as $k \rightarrow+\infty$,

$$
\left\{\begin{array}{l}
\lambda_{k} d\left(P_{k}, A_{k}\right) \rightarrow A>0, \\
\lambda_{k} d\left(P_{k}, B_{k}\right) \rightarrow B>0, \\
\lambda_{k} d\left(A_{k}, B_{k}\right) \rightarrow C>0, \\
\text { but } A+B>C .
\end{array}\right.
$$

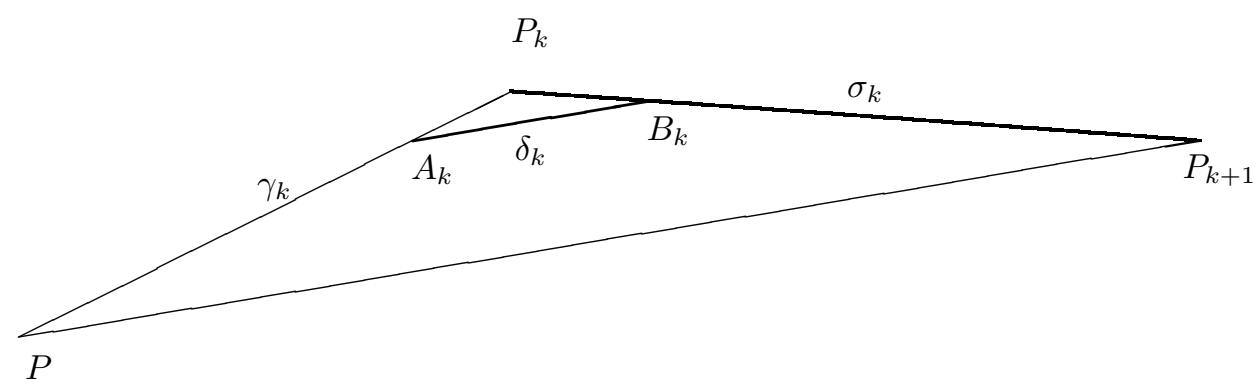

Now draw a minimal geodesic $\delta_{k}$ from $A_{k}$ to $B_{k}$. Consider comparison triangles $\bar{\triangle} \bar{P}_{k} \bar{P} \bar{P}_{k+1}$ and $\bar{\triangle} \bar{P}_{k} \bar{A}_{k} \bar{B}_{k}$ in $\mathbb{R}^{2}$ with

$$
\begin{aligned}
& \left|\bar{P}_{k} \bar{P}\right|=\left|P_{k} P\right|,\left|\bar{P}_{k} \bar{P}_{k+1}\right|=\left|P_{k} P_{k+1}\right|,\left|\bar{P} \bar{P}_{k+1}\right|=\left|P P_{k+1}\right|, \\
& \text { and }\left|\bar{P}_{k} \bar{A}_{k}\right|=\left|P_{k} A_{k}\right|,\left|\bar{P}_{k} \bar{B}_{k}\right|=\left|P_{k} B_{k}\right|,\left|\bar{A}_{k} \bar{B}_{k}\right|=\left|A_{k} B_{k}\right|
\end{aligned}
$$

By Toponogov's comparison theorem [8], we have

$$
\measuredangle \bar{A}_{k} \bar{P}_{k} \bar{B}_{k} \geq \measuredangle \bar{P} \bar{P}_{k} \bar{P}_{k+1} .
$$

On the other hand, by (6.1.2) and using Toponogov's comparison theorem again, we have

$$
\measuredangle \bar{P}_{k} \bar{P} \bar{P}_{k+1} \leq \measuredangle P_{k} P P_{k+1}<\frac{1}{k},
$$

and since $\left|\bar{P}_{k} \bar{P}_{k+1}\right|>\left|\bar{P} \bar{P}_{k}\right|$ by (6.1.1), we further have

$$
\measuredangle \bar{P}_{k} \bar{P}_{k+1} \bar{P} \leq \measuredangle \bar{P}_{k} \bar{P} \bar{P}_{k+1}<\frac{1}{k} .
$$

Thus the above inequalities (6.1.4)-(6.1.6) imply that

$$
\measuredangle \bar{A}_{k} \bar{P}_{k} \bar{B}_{k}>\pi-\frac{2}{k} .
$$


Hence

$$
\left|\bar{A}_{k} \bar{B}_{k}\right|^{2} \geq\left|\bar{A}_{k} \bar{P}_{k}\right|^{2}+\left|\bar{P}_{k} \bar{B}_{k}\right|^{2}-2\left|\bar{A}_{k} \bar{P}_{k}\right| \cdot\left|\bar{P}_{k} \bar{B}_{k}\right| \cos \left(\pi-\frac{2}{k}\right) .
$$

Multiplying the above inequality by $\lambda_{k}^{2}$ and letting $k \rightarrow+\infty$, we get

$$
C \geq A+B
$$

which contradicts (6.1.3).

Therefore we have proved the proposition.

Let $M$ be an $n$-dimensional complete noncompact Riemannian manifold. Pick an origin $O \in M$. Let $s$ be the geodesic distance to the origin $O$ of $M$, and $R$ the scalar curvature. Recall that in Chapter 4 we have defined the asymptotic scalar curvature ratio

$$
A=\limsup _{s \rightarrow+\infty} R s^{2} .
$$

We now state a useful lemma of Hamilton (Lemma 22.2 in [63]) about picking local (almost) maximum curvature points at infinity.

Lemma 6.1.3. Given a complete noncompact Riemannian manifold with bounded curvature and with asymptotic scalar curvature ratio

$$
A=\limsup _{s \rightarrow+\infty} R s^{2}=+\infty
$$

we can find a sequence of points $x_{j}$ divergent to infinity, a sequence of radii $r_{j}$ and a sequence of positive numbers $\delta_{j} \rightarrow 0$ such that

(i) $R(x) \leq\left(1+\delta_{j}\right) R\left(x_{j}\right)$ for all $x$ in the ball $B\left(x_{j}, r_{j}\right)$ of radius $r_{j}$ around $x_{j}$,

(ii) $r_{j}^{2} R\left(x_{j}\right) \rightarrow+\infty$,

(iii) $\lambda_{j}=d\left(x_{j}, O\right) / r_{j} \rightarrow+\infty$,

(iv) the balls $B\left(x_{j}, r_{j}\right)$ are disjoint,

where $d\left(x_{j}, O\right)$ is the distance of $x_{j}$ from the origin $O$.

Proof. Pick a sequence of positive numbers $\epsilon_{j} \rightarrow 0$, then choose $A_{j} \rightarrow+\infty$ so that $A_{j} \epsilon_{j}^{2} \rightarrow+\infty$. Let $\sigma_{j}$ be the largest number such that

$$
\sup \left\{R(x) d(x, O)^{2} \mid d(x, O) \leq \sigma_{j}\right\} \leq A_{j} .
$$

Then there exists some $y_{j} \in M$ such that

$$
R\left(y_{j}\right) d\left(y_{j}, O\right)^{2}=A_{j} \quad \text { and } \quad d\left(y_{j}, O\right)=\sigma_{j} .
$$

Now pick $x_{j} \in M$ so that $d\left(x_{j}, O\right) \geq \sigma_{j}$ and

$$
R\left(x_{j}\right) \geq \frac{1}{1+\epsilon_{j}} \sup \left\{R(x) \mid d(x, O) \geq \sigma_{j}\right\}
$$

Finally pick $r_{j}=\epsilon_{j} \sigma_{j}$. We check the properties (i)-(iv) as follows.

(i) If $x \in B\left(x_{j}, r_{j}\right) \cap\left\{d(\cdot, O) \geq \sigma_{j}\right\}$, we have

$$
R(x) \leq\left(1+\epsilon_{j}\right) R\left(x_{j}\right)
$$


by the choice of the point $x_{j}$; while if $x \in B\left(x_{j}, r_{j}\right) \cap\left\{d(\cdot, O) \leq \sigma_{j}\right\}$, we have

$$
\begin{aligned}
R(x) & \leq A_{j} / d(x, O)^{2} \\
& \leq \frac{1}{\left(1-\epsilon_{j}\right)^{2}}\left(A_{j} / \sigma_{j}^{2}\right) \\
& =\frac{1}{\left(1-\epsilon_{j}\right)^{2}} R\left(y_{j}\right) \\
& \leq \frac{\left(1+\epsilon_{j}\right)}{\left(1-\epsilon_{j}\right)^{2}} R\left(x_{j}\right),
\end{aligned}
$$

since $d(x, O) \geq d\left(x_{j}, O\right)-d\left(x, x_{j}\right) \geq \sigma_{j}-r_{j}=\left(1-\epsilon_{j}\right) \sigma_{j}$. Thus we have obtained

$$
R(x) \leq\left(1+\delta_{j}\right) R\left(x_{j}\right), \quad \forall x \in B\left(x_{j}, r_{j}\right),
$$

where $\delta_{j}=\frac{\left(1+\epsilon_{j}\right)}{\left(1-\epsilon_{j}\right)^{2}}-1 \rightarrow 0$ as $j \rightarrow+\infty$.

(ii) By the choices of $r_{j}, x_{j}$ and $y_{j}$, we have

$$
\begin{aligned}
r_{j}^{2} R\left(x_{j}\right) & =\epsilon_{j}^{2} \sigma_{j}^{2} R\left(x_{j}\right) \\
& \geq \epsilon_{j}^{2} \sigma_{j}^{2}\left[\frac{1}{1+\epsilon_{j}} R\left(y_{j}\right)\right] \\
& =\frac{\epsilon_{j}^{2}}{1+\epsilon_{j}} A_{j} \rightarrow+\infty, \quad \text { as } j \rightarrow+\infty .
\end{aligned}
$$

(iii) Since $d\left(x_{j}, O\right) \geq \sigma_{j}=r_{j} / \epsilon_{j}$, it follows that $\lambda_{j}=d\left(x_{j}, O\right) / r_{j} \rightarrow+\infty$ as $j \rightarrow+\infty$.

(iv) For any $x \in B\left(x_{j}, r_{j}\right)$, the distance from the origin

$$
\begin{aligned}
d(x, O) & \geq d\left(x_{j}, O\right)-d\left(x, x_{j}\right) \\
& \geq \sigma_{j}-r_{j} \\
& =\left(1-\epsilon_{j}\right) \sigma_{j} \rightarrow+\infty, \text { as } j \rightarrow+\infty .
\end{aligned}
$$

Thus any fixed compact set does not meet the balls $B\left(x_{j}, r_{j}\right)$ for large enough $j$. If we pass to a subsequence, the balls will all avoid each other. $\square$

The above point picking lemma of Hamilton, as written down in Lemma 22.2 of [63], requires the curvature of the manifold to be bounded. When the manifold has unbounded curvature, we will appeal to the following simple lemma.

Lemma 6.1.4. Given a complete noncompact Riemannian manifold with unbounded curvature, we can find a sequence of points $x_{j}$ divergent to infinity such that for each positive integer $j$, we have $\left|R m\left(x_{j}\right)\right| \geq j$, and

$$
|R m(x)| \leq 4\left|R m\left(x_{j}\right)\right|
$$

for $x \in B\left(x_{j}, \frac{j}{\sqrt{\left|R m\left(x_{j}\right)\right|}}\right)$.

Proof. Each $x_{j}$ can be constructed as a limit of a finite sequence $\left\{y_{i}\right\}$, defined as follows. Let $y_{0}$ be any fixed point with $\left|R m\left(y_{0}\right)\right| \geq j$. Inductively, if $y_{i}$ cannot be 
taken as $x_{j}$, then there is a $y_{i+1}$ such that

$$
\left\{\begin{aligned}
\left|R m\left(y_{i+1}\right)\right| & >4\left|R m\left(y_{i}\right)\right|, \\
d\left(y_{i}, y_{i+1}\right) & \leqslant \frac{j}{\sqrt{\left|R m\left(y_{i}\right)\right|}} .
\end{aligned}\right.
$$

Thus we have

$$
\begin{gathered}
\left|R m\left(y_{i}\right)\right|>4^{i}\left|\operatorname{Rm}\left(y_{0}\right)\right| \geq 4^{i} j, \\
d\left(y_{i}, y_{0}\right) \leq j \sum_{k=1}^{i} \frac{1}{\sqrt{4^{k-1} j}}<2 \sqrt{j} .
\end{gathered}
$$

Since the manifold is smooth, the sequence $\left\{y_{i}\right\}$ must be finite. The last element fits. $\mathrm{c}$

6.2. Asymptotic Shrinking Solitons. We begin with the study of the asymptotic behavior of an ancient $\kappa$-solution $g_{i j}(x, t)$, on $M \times(-\infty, T)$ with $T>0$, to the Ricci flow as $t \rightarrow-\infty$.

Pick an arbitrary point $\left(p, t_{0}\right) \in M \times(-\infty, 0]$ and recall from Chapter 3 that

$$
\tau=t_{0}-t, \quad \text { for } t<t_{0},
$$

$$
\begin{aligned}
& l(q, \tau)=\frac{1}{2 \sqrt{\tau}} \inf \left\{\begin{array}{l|l}
\int_{0}^{\tau} \sqrt{s}\left(R\left(\gamma(s), t_{0}-s\right)\right. \\
\left.+|\dot{\gamma}(s)|_{g_{i j}\left(t_{0}-s\right)}^{2}\right) d s & \begin{array}{l}
\gamma:[0, \tau] \rightarrow M \text { with } \\
\gamma(0)=p, \gamma(\tau)=q
\end{array}
\end{array}\right\}
\end{aligned}
$$

and

$$
\tilde{V}(\tau)=\int_{M}(4 \pi \tau)^{-\frac{n}{2}} \exp (-l(q, \tau)) d V_{t_{0}-\tau}(q) .
$$

We first observe that Corollary 3.2.6 also holds for the general complete manifold $M$. Indeed, since the scalar curvature is nonnegative, the function $\bar{L}(\cdot, \tau)=4 \tau l(\cdot, \tau)$ achieves its minimum on $M$ for each fixed $\tau>0$. Thus the same argument in the proof of Corollary 3.2.6 shows there exists $q=q(\tau)$ such that

$$
l(q(\tau), \tau) \leq \frac{n}{2}
$$

for each $\tau>0$.

Recall from (3.2.11)-(3.2.13), the Li-Yau-Perelman distance $l$ satisfies the following

$$
\begin{aligned}
\frac{\partial}{\partial \tau} l & =-\frac{l}{\tau}+R+\frac{1}{2 \tau^{3 / 2}} K, \\
|\nabla l|^{2} & =-R+\frac{l}{\tau}-\frac{1}{\tau^{3 / 2}} K, \\
\Delta l & \leq-R+\frac{n}{2 \tau}-\frac{1}{2 \tau^{3 / 2}} K,
\end{aligned}
$$


and the equality in (6.2.4) holds everywhere if and only if we are on a gradient shrinking soliton. Here $K=\int_{0}^{\tau} s^{3 / 2} Q(X) d s, Q(X)$ is the trace Li-Yau-Hamilton quadratic given by

$$
Q(X)=-R_{\tau}-\frac{R}{\tau}-2\langle\nabla R, X\rangle+2 \operatorname{Ric}(X, X)
$$

and $X$ is the tangential (velocity) vector field of an $\mathcal{L}$-shortest curve $\gamma:[0, \tau] \rightarrow M$ connecting $p$ to $q$.

By applying the trace Li-Yau-Hamilton inequality (Corollary 2.5.5) to the ancient $\kappa$-solution, we have

$$
\begin{aligned}
Q(X) & =-R_{\tau}-\frac{R}{\tau}-2\langle\nabla R, X\rangle+2 \operatorname{Ric}(X, X) \\
& \geq-\frac{R}{\tau}
\end{aligned}
$$

and hence

$$
\begin{aligned}
K & =\int_{0}^{\tau} s^{3 / 2} Q(X) d s \\
& \geq-\int_{0}^{\tau} \sqrt{s} R d s \\
& \geq-L(q, \tau)
\end{aligned}
$$

Thus by (6.2.3) we get

$$
|\nabla l|^{2}+R \leq \frac{3 l}{\tau}
$$

We now state and prove a result of Perelman [103] about the asymptotic shapes of ancient $\kappa$-solutions as the time $t \rightarrow-\infty$.

Theorem 6.2.1 (Perelman [103]). Let $g_{i j}(\cdot, t),-\infty<t<T$ with some $T>0$, be a nonflat ancient $\kappa$-solution for some $\kappa>0$. Then there exist a sequence of points $q_{k}$ and a sequence of times $t_{k} \rightarrow-\infty$ such that the scalings of $g_{i j}(\cdot, t)$ around $q_{k}$ with factor $\left|t_{k}\right|^{-1}$ and with the times $t_{k}$ shifting to the new time zero converge to a nonflat gradient shrinking soliton in $C_{\mathrm{loc}}^{\infty}$ topology.

Proof. Clearly, we may assume that the nonflat ancient $\kappa$-solution is not a gradient shrinking soliton. For the arbitrarily fixed $\left(p, t_{0}\right)$, let $q(\tau)\left(\tau=t_{0}-t\right)$ be chosen as in (6.2.1) with $l(q(\tau), \tau) \leq \frac{n}{2}$. We only need to show that the scalings of $g_{i j}(\cdot, t)$ around $q(\tau)$ with factor $\tau^{-1}$ converge along a subsequence of $\tau \rightarrow+\infty$ to a nonflat gradient shrinking soliton in the $C_{\text {loc }}^{\infty}$ topology.

We first claim that for any $A \geq 1$, one can find $B=B(A)<+\infty$ such that for every $\bar{\tau}>1$ there holds

$$
l(q, \tau) \leq B \text { and } \tau R\left(q, t_{0}-\tau\right) \leq B
$$

whenever $\frac{1}{2} \bar{\tau} \leq \tau \leq A \bar{\tau}$ and $d_{t_{0}-\frac{\bar{\tau}}{2}}^{2}\left(q, q\left(\frac{\bar{\tau}}{2}\right)\right) \leq A \bar{\tau}$. 
Indeed, by using (6.2.5) at $\tau=\frac{\bar{\tau}}{2}$, we have

$$
\begin{aligned}
\sqrt{l\left(q, \frac{\bar{\tau}}{2}\right)} & \leq \sqrt{\frac{n}{2}}+\sup \{|\nabla \sqrt{l}|\} \cdot d_{t_{0}-\bar{\tau}}\left(q, q\left(\frac{\bar{\tau}}{2}\right)\right) \\
& \leq \sqrt{\frac{n}{2}}+\sqrt{\frac{3}{2 \bar{\tau}}} \cdot \sqrt{A \bar{\tau}} \\
& =\sqrt{\frac{n}{2}}+\sqrt{\frac{3 A}{2}}
\end{aligned}
$$

and

$$
\begin{aligned}
R\left(q, t_{0}-\frac{\bar{\tau}}{2}\right) & \leq \frac{3 l\left(q, \frac{\bar{\tau}}{2}\right)}{\left(\frac{\bar{\tau}}{2}\right)} \\
& \leq \frac{6}{\bar{\tau}}\left(\sqrt{\frac{n}{2}}+\sqrt{\frac{3 A}{2}}\right)^{2},
\end{aligned}
$$

for $q \in B_{t_{0}-\frac{\bar{\tau}}{2}}\left(q\left(\frac{\bar{\tau}}{2}\right), \sqrt{A \bar{\tau}}\right)$. Recall that the Li-Yau-Hamilton inequality implies that the scalar curvature of the ancient solution is pointwise nondecreasing in time. Thus we know from (6.2.8) that

$$
\tau R\left(q, t_{0}-\tau\right) \leq 6 A\left(\sqrt{\frac{n}{2}}+\sqrt{\frac{3 A}{2}}\right)^{2}
$$

whenever $\frac{1}{2} \bar{\tau} \leq \tau \leq A \bar{\tau}$ and $d_{t_{0}-\frac{\bar{\tau}}{2}}^{2}\left(q, q\left(\frac{\bar{\tau}}{2}\right)\right) \leq A \bar{\tau}$.

By (6.2.2) and (6.2.3) we have

This together with (6.2.9) implies that

$$
\frac{\partial l}{\partial \tau}+\frac{1}{2}|\nabla l|^{2}=-\frac{l}{2 \tau}+\frac{R}{2} .
$$

$$
\frac{\partial l}{\partial \tau} \leq-\frac{l}{2 \tau}+\frac{3 A}{\tau}\left(\sqrt{\frac{n}{2}}+\sqrt{\frac{3 A}{2}}\right)^{2}
$$

i.e.,

$$
\frac{\partial}{\partial \tau}(\sqrt{\tau} l) \leq \frac{3 A}{\sqrt{\tau}}\left(\sqrt{\frac{n}{2}}+\sqrt{\frac{3 A}{2}}\right)^{2}
$$

whenever $\frac{1}{2} \bar{\tau} \leq \tau \leq A \bar{\tau}$ and $d_{t_{0}-\frac{\bar{\tau}}{2}}^{2}\left(q, q\left(\frac{\bar{\tau}}{2}\right)\right) \leq A \bar{\tau}$. Hence by integrating this differential inequality, we obtain

$$
\sqrt{\tau} l(q, \tau)-\sqrt{\frac{\bar{\tau}}{2}} l\left(q, \frac{\bar{\tau}}{2}\right) \leq 6 A\left(\sqrt{\frac{n}{2}}+\sqrt{\frac{3 A}{2}}\right)^{2} \sqrt{\tau}
$$

and then by (6.2.7),

$$
\begin{aligned}
l(q, \tau) & \leq l\left(q, \frac{\bar{\tau}}{2}\right)+6 A\left(\sqrt{\frac{n}{2}}+\sqrt{\frac{3 A}{2}}\right)^{2} \\
& \leq 7 A\left(\sqrt{\frac{n}{2}}+\sqrt{\frac{3 A}{2}}\right)^{2}
\end{aligned}
$$


whenever $\frac{1}{2} \bar{\tau} \leq \tau \leq A \bar{\tau}$ and $d_{t_{0}-\frac{\bar{\tau}}{2}}^{2}\left(q, q\left(\frac{\bar{\tau}}{2}\right)\right) \leq A \bar{\tau}$. So we have proved claim (6.2.6).

Recall that $g_{i j}(\tau)=g_{i j}\left(\cdot, t_{0}-\tau\right)$ satisfies $\left(g_{i j}\right)_{\tau}=2 R_{i j}$. Let us take the scaling of the ancient $\kappa$-solution around $q\left(\frac{\bar{\tau}}{2}\right)$ with factor $\left(\frac{\bar{\tau}}{2}\right)^{-1}$, i.e.,

$$
\tilde{g}_{i j}(s)=\frac{2}{\bar{\tau}} g_{i j}\left(\cdot, t_{0}-s \frac{\bar{\tau}}{2}\right)
$$

where $s \in[0,+\infty)$. Claim (6.2.6) says that for all $s \in[1,2 A]$ and all $q$ such that $\operatorname{dist}_{\tilde{g}_{i j}(1)}^{2}\left(q, q\left(\frac{\bar{\tau}}{2}\right)\right) \leq A$, we have $\tilde{R}(q, s)=\frac{\bar{\tau}}{2} R\left(q, t_{0}-s \frac{\bar{\tau}}{2}\right) \leq B$. Now taking into account the $\kappa$-noncollapsing assumption and Theorem 4.2.2, we can use Hamilton's compactness theorem (Theorem 4.1.5) to obtain a sequence $\bar{\tau}_{k} \rightarrow+\infty$ such that the marked evolving manifolds $\left(M, \tilde{g}_{i j}^{(k)}(s), q\left(\frac{\bar{\tau}_{k}}{2}\right)\right)$, with $\tilde{g}_{i j}^{(k)}(s)=\frac{2}{\bar{\tau}_{k}} g_{i j}\left(\cdot, t_{0}-s \frac{\bar{\tau}_{k}}{2}\right)$ and $s \in[1,+\infty)$, converge to a manifold $\left(\bar{M}, \bar{g}_{i j}(s), \bar{q}\right)$ with $s \in[1,+\infty)$, where $\bar{g}_{i j}(s)$ is also a solution to the Ricci flow on $\bar{M}$.

Denote by $\tilde{l}_{k}$ the corresponding Li-Yau-Perelman distance of $\tilde{g}_{i j}^{(k)}(s)$. It is easy to see that $\tilde{l}_{k}(q, s)=l\left(q, \frac{\bar{\tau}_{k}}{2} s\right)$, for $s \in[1,+\infty)$. From (6.2.5), we also have

$$
\left|\nabla \tilde{l}_{k}\right|_{\tilde{g}_{i j}^{(k)}}^{2}+\tilde{R}^{(k)} \leq 6 \tilde{l}_{k}
$$

where $\tilde{R}^{(k)}$ is the scalar curvature of the metric $\tilde{g}_{i j}^{(k)}$. Claim (6.2.6) says that $\tilde{l}_{k}$ are uniformly bounded on compact subsets of $M \times[1,+\infty)$ (with the corresponding origins $\left.q\left(\frac{\bar{\tau}_{k}}{2}\right)\right)$. Thus the above gradient estimate (6.2.11) implies that the functions $\tilde{l}_{k}$ tend (up to a subsequence) to a function $\bar{l}$ which is a locally Lipschitz function on $\bar{M}$.

We know from (6.2.2)-(6.2.4) that the Li-Yau-Perelman distance $\tilde{l}_{k}$ satisfies the following inequalities:

$$
\begin{gathered}
\left(\tilde{l}_{k}\right)_{s}-\Delta \tilde{l}_{k}+\left|\nabla \tilde{l}_{k}\right|^{2}-\tilde{R}^{(k)}+\frac{n}{2 s} \geq 0, \\
2 \Delta \tilde{l}_{k}-\left|\nabla \tilde{l}_{k}\right|^{2}+\tilde{R}^{(k)}+\frac{\tilde{l}_{k}-n}{s} \leq 0 .
\end{gathered}
$$

We next show that the limit $\bar{l}$ also satisfies the above two inequalities in the sense of distributions. Indeed the above two inequalities can be rewritten as

$$
\begin{gathered}
\left(\frac{\partial}{\partial s}-\triangle+\widetilde{R}^{(k)}\right)\left((4 \pi s)^{-\frac{n}{2}} \exp \left(-\widetilde{l}_{k}\right)\right) \leq 0, \\
-\left(4 \triangle-\widetilde{R}^{(k)}\right) e^{-\frac{\tilde{l}_{k}}{2}}+\frac{\tilde{l}_{k}-n}{s} e^{-\frac{\tilde{\tau}_{k}}{2}} \leq 0,
\end{gathered}
$$

in the sense of distributions. Note that the estimate (6.2.11) implies that $\tilde{l}_{k} \rightarrow \bar{l}$ in the $C_{\text {loc }}^{0, \alpha}$ norm for any $0<\alpha<1$. Thus the inequalities (6.2.14) and (6.2.15) imply that the limit $\bar{l}$ satisfies

$$
\begin{gathered}
\left(\frac{\partial}{\partial s}-\triangle+\bar{R}\right)\left((4 \pi s)^{-\frac{n}{2}} \exp (-\bar{l})\right) \leq 0, \\
-(4 \triangle-\bar{R}) e^{-\frac{\bar{l}}{2}}+\frac{\bar{l}-n}{s} e^{-\frac{\bar{l}}{2}} \leq 0
\end{gathered}
$$


in the sense of distributions.

Denote by $\tilde{V}^{(k)}(s)$ Perelman's reduced volume of the scaled metric $\tilde{g}_{i j}^{(k)}(s)$. Since $\tilde{l}_{k}(q, s)=l\left(q, \frac{\bar{\tau}_{k}}{2} s\right)$, we see that $\tilde{V}^{(k)}(s)=\tilde{V}\left(\frac{\bar{\tau}_{k}}{2} s\right)$ where $\tilde{V}$ is Perelman's reduced volume of the ancient $\kappa$-solution. The monotonicity of Perelman's reduced volume (Theorem 3.2.8) then implies that

$$
\lim _{k \rightarrow \infty} \tilde{V}^{(k)}(s)=\bar{V}, \text { for } s \in[1,2]
$$

for some nonnegative constant $\bar{V}$.

(We remark that by the Jacobian comparison theorem (Theorem 3.2.7), (3.2.18) and (3.2.19), the integrand of $\tilde{V}^{(k)}(s)$ is bounded by

$$
(4 \pi s)^{-\frac{n}{2}} \exp \left(-\tilde{l}_{k}(X, s)\right) \tilde{\mathcal{J}}^{(k)}(s) \leq(4 \pi)^{-\frac{n}{2}} \exp \left(-|X|^{2}\right)
$$

on $T_{p} M$, where $\tilde{\mathcal{J}}^{(k)}(s)$ is the $\mathcal{L}$-Jacobian of the $\mathcal{L}$-exponential map of the metric $\tilde{g}_{i j}^{(k)}(s)$ at $T_{p} M$. Thus we can apply the dominant convergence theorem to get the convergence in (6.2.18). But we are not sure whether the limiting $\bar{V}$ is exactly Perelman's reduced volume of the limiting manifold $\left(\bar{M}, \bar{g}_{i j}(s)\right)$, because the points $q\left(\frac{\bar{\tau}_{k}}{2}\right)$ may diverge to infinity. Nevertheless, we can ensure that $\bar{V}$ is not less than Perelman's reduced volume of the limit.)

Note by (6.2.5) that

$$
\begin{aligned}
& \tilde{V}^{(k)}(2)-\tilde{V}^{(k)}(1) \\
& =\int_{1}^{2} \frac{d}{d s}\left(\tilde{V}^{(k)}(s)\right) d s \\
& =\int_{1}^{2} d s \int_{M}\left(\frac{\partial}{\partial s}-\Delta+\tilde{R}^{(k)}\right)\left((4 \pi s)^{-\frac{n}{2}} \exp \left(-\tilde{l}_{k}\right)\right) d V_{\tilde{g}_{i j}^{(k)}(s)} .
\end{aligned}
$$

Thus we deduce that in the sense of distributions,

$$
\left(\frac{\partial}{\partial s}-\Delta+\bar{R}\right)\left((4 \pi s)^{-\frac{n}{2}} \exp (-\bar{l})\right)=0
$$

and

$$
(4 \Delta-\bar{R}) e^{-\frac{\bar{l}}{2}}=\frac{\bar{l}-n}{s} e^{-\frac{\bar{l}}{2}}
$$

or equivalently,

$$
2 \Delta \bar{l}-|\nabla \bar{l}|^{2}+\bar{R}+\frac{\bar{l}-n}{s}=0,
$$

on $\bar{M} \times[1,2]$. Thus by applying standard parabolic equation theory to $(6.2 .20)$ we find that $\bar{l}$ is actually smooth. Here we used (6.2.2)-(6.2.4) to show that the equality in (6.2.16) implies the equality in (6.2.17).

Set

$$
v=\left[s\left(2 \Delta \bar{l}-|\nabla \bar{l}|^{2}+\bar{R}\right)+\bar{l}-n\right] \cdot(4 \pi s)^{-\frac{n}{2}} e^{-\bar{l}} .
$$

Then by applying Lemma 2.6.1, we have

$$
\left(\frac{\partial}{\partial s}-\Delta+\bar{R}\right) v=-2 s\left|\bar{R}_{i j}+\nabla_{i} \nabla_{j} \bar{l}-\frac{1}{2 s} \bar{g}_{i j}\right|^{2} \cdot(4 \pi s)^{-\frac{n}{2}} e^{-\bar{l}} .
$$


We see from (6.2.21) that the LHS of the equation (6.2.22) is identically zero. Thus the limit metric $\bar{g}_{i j}$ satisfies

$$
\bar{R}_{i j}+\nabla_{i} \nabla_{j} \bar{l}-\frac{1}{2 s} \bar{g}_{i j}=0,
$$

so we have shown the limit is a gradient shrinking soliton.

To show that the limiting gradient shrinking soliton is nonflat, we first show that the constant function $\bar{V}(s)$ is strictly less than 1. Consider Perelman's reduced volume $\tilde{V}(\tau)$ of the ancient $\kappa$-solution. By using Perelman's Jacobian comparison theorem (Theorem 3.2.7), (3.2.18) and (3.2.19) as before, we have

$$
\begin{aligned}
\tilde{V}(\tau) & =\int(4 \pi \tau)^{-\frac{n}{2}} e^{-l(X, \tau)} \mathcal{J}(\tau) d X \\
& \leq \int_{T_{p} M}(4 \pi)^{-\frac{n}{2}} e^{-|X|^{2}} d X \\
& =1 .
\end{aligned}
$$

Recall that we have assumed the nonflat ancient $\kappa$-solution is not a gradient shrinking soliton. Thus for $\tau>0$, we must have $\tilde{V}(\tau)<1$. Since the limiting function $\bar{V}(s)$ is the limit of $\tilde{V}\left(\frac{\bar{\tau}_{k}}{2} s\right)$ with $\bar{\tau}_{k} \rightarrow+\infty$, we deduce that the constant $\bar{V}(s)$ is strictly less than 1 , for $s \in[1,2]$.

We now argue by contradiction. Suppose the limiting gradient shrinking soliton $\bar{g}_{i j}(s)$ is flat. Then by $(6.2 .23)$,

$$
\nabla_{i} \nabla_{j} \bar{l}=\frac{1}{2 s} \bar{g}_{i j} \text { and } \Delta \bar{l}=\frac{n}{2 s} .
$$

Putting these into the identity (6.2.21), we get

$$
|\nabla \bar{l}|^{2}=\frac{\bar{l}}{s}
$$

Since the function $\bar{l}$ is strictly convex, it follows that $\sqrt{4 s l}$ is a distance function (from some point) on the complete flat manifold $\bar{M}$. From the smoothness of the function $\bar{l}$, we conclude that the flat manifold $\bar{M}$ must be $\mathbb{R}^{n}$. In this case we would have its reduced distance to be $\bar{l}$ and its reduced volume to be 1 . Since $\bar{V}$ is not less than the reduced volume of the limit, this is a contradiction. Therefore the limiting gradient shrinking soliton $\bar{g}_{i j}$ is not flat.

To conclude this section, we use the above theorem to derive the classification of all two-dimensional ancient $\kappa$-solutions which was obtained earlier by Hamilton in Section 26 of [63].

THEOREM 6.2.2. The only nonflat ancient $\kappa$-solutions to Ricci flow on twodimensional manifolds are the round sphere $\mathbb{S}^{2}$ and the round real projective plane $\mathbb{R} \mathbb{P}^{2}$.

Proof. Let $g_{i j}(x, t)$ be a nonflat ancient $\kappa$-solution defined on $M \times(-\infty, T)$ (for some $T>0$ ), where $M$ is a two-dimensional manifold. Note that the ancient $\kappa$-solution satisfies the Li-Yau-Hamilton inequality (Corollary 2.5.5). In particular by Corollary 2.5 .8 , the scalar curvature of the ancient $\kappa$-solution is pointwise nondecreasing in 
time. Moreover by the strong maximum principle, the ancient $\kappa$-solution has strictly positive curvature everywhere.

By the above Theorem 6.2.1, we know that the scalings of the ancient $\kappa$-solution along a sequence of points $q_{k}$ in $M$ and a sequence of times $t_{k} \rightarrow-\infty$ converge to a nonflat gradient shrinking soliton $\left(\bar{M}, \bar{g}_{i j}(x, t)\right)$ with $-\infty<t \leq 0$.

We first show that the limiting gradient shrinking soliton $\left(\bar{M}, \bar{g}_{i j}(x, t)\right)$ has uniformly bounded curvature. Clearly, the limiting soliton has nonnegative curvature and is $\kappa$-noncollapsed on all scales, and its scalar curvature is still pointwise nondecreasing in time. Thus we only need to show that the limiting soliton has bounded curvature at $t=0$. We argue by contradiction. Suppose the curvature of the limiting soliton is unbounded at $t=0$. Of course in this case the limiting soliton $\bar{M}$ is noncompact. Then by applying Lemma 6.1 .4 , we can choose a sequence of points $x_{j}, j=1,2, \ldots$, divergent to infinity such that the scalar curvature $\bar{R}$ of the limit satisfies

$$
\bar{R}\left(x_{j}, 0\right) \geq j \text { and } \bar{R}(x, 0) \leq 4 \bar{R}\left(x_{j}, 0\right)
$$

for all $j=1,2, \ldots$, and $x \in B_{0}\left(x_{j}, j / \sqrt{R\left(x_{j}, 0\right)}\right)$. And then by the nondecreasing (in time) of the scalar curvature, we have

$$
\bar{R}(x, t) \leq 4 \bar{R}\left(x_{j}, 0\right),
$$

for all $j=1,2, \ldots, x \in B_{0}\left(x_{j}, j / \sqrt{R\left(x_{j}, 0\right)}\right)$ and $t \leq 0$. By combining with Hamilton's compactness theorem (Theorem 4.1.5) and the $\kappa$-noncollapsing, we know that a subsequence of the rescaling solutions

$$
\left(\bar{M}, \bar{R}\left(x_{j}, 0\right) \bar{g}_{i j}\left(x, t / \bar{R}\left(x_{j}, 0\right)\right), x_{j}\right), \quad j=1,2, \ldots,
$$

converges in the $C_{l o c}^{\infty}$ topology to a nonflat smooth solution of the Ricci flow. Then Proposition 6.1.2 implies that the new (two-dimensional) limit must be flat. This arrives at a contradiction. So we have proved that the limiting gradient shrinking soliton has uniformly bounded curvature.

We next show that the limiting soliton is compact. Suppose the limiting soliton is (complete and) noncompact. By the strong maximum principle we know that the limiting soliton also has strictly positive curvature everywhere. After a shift of the time, we may assume that the limiting soliton satisfies the following equation

$$
\nabla_{i} \nabla_{j} f+\bar{R}_{i j}+\frac{1}{2 t} \bar{g}_{i j}=0, \quad \text { on } \quad-\infty<t<0,
$$

everywhere for some function $f$. Differentiating the equation (6.2.24) and switching the order of differentiations, as in the derivation of (1.1.14), we get

$$
\nabla_{i} \bar{R}=2 \bar{R}_{i j} \nabla_{j} f
$$

Fix some $t<0$, say $t=-1$, and consider a long shortest geodesic $\gamma(s), 0 \leq s \leq \bar{s}$. Let $x_{0}=\gamma(0)$ and $X(s)=\dot{\gamma}(s)$. Let $V(0)$ be any unit vector orthogonal to $\dot{\gamma}(0)$ and translate $V(0)$ along $\gamma(s)$ to get a parallel vector field $V(s), 0 \leq s \leq \bar{s}$ on $\gamma$. Set

$$
\widehat{V}(s)= \begin{cases}s V(s), & \text { for } 0 \leq s \leq 1 \\ V(s), & \text { for } 1 \leq s \leq \bar{s}-1 \\ (\bar{s}-s) V(s), & \text { for } \bar{s}-1 \leq s \leq \bar{s}\end{cases}
$$


It follows from the second variation formula of arclength that

$$
\int_{0}^{\bar{s}}\left(|\dot{\vec{V}}(s)|^{2}-\bar{R}(X, \widehat{V}, X, \widehat{V})\right) d s \geq 0 .
$$

Thus we clearly have

$$
\int_{0}^{\bar{s}} \bar{R}(X, \widehat{V}, X, \widehat{V}) d s \leq \text { const. }
$$

and then

$$
\int_{0}^{\bar{s}} \operatorname{Ric}(X, X) d s \leq \text { const.. }
$$

By integrating the equation (6.2.24) we get

$$
X(f(\gamma(\bar{s})))-X(f(\gamma(0)))+\int_{0}^{\bar{s}} \overline{\operatorname{Ric}}(X, X) d s-\frac{1}{2} \bar{s}=0
$$

and then by (6.2.26), we deduce

$$
\frac{d}{d s}(f \circ \gamma(s)) \geq \frac{s}{2}-\text { const. },
$$

$$
\text { and } f \circ \gamma(s) \geq \frac{s^{2}}{4}-\text { const. } \cdot s-\text { const. }
$$

for $s>0$ large enough. Thus at large distances from the fixed point $x_{0}$ the function $f$ has no critical points and is proper. It then follows from the Morse theory that any two high level sets of $f$ are diffeomorphic via the gradient curves of $f$. Since by (6.2.25),

$$
\begin{aligned}
\frac{d}{d s} \bar{R}(\eta(s),-1) & =\langle\nabla \bar{R}, \dot{\eta}(s)\rangle \\
& =2 \bar{R}_{i j} \nabla_{i} f \nabla_{j} f \\
& \geq 0
\end{aligned}
$$

for any integral curve $\eta(s)$ of $\nabla f$, we conclude that the scalar curvature $\bar{R}(x,-1)$ has a positive lower bound on $\bar{M}$, which contradicts the Bonnet-Myers Theorem. So we have proved that the limiting gradient shrinking soliton is compact.

By Proposition 5.1.10, the compact limiting gradient shrinking soliton has constant curvature. This says that the scalings of the ancient $\kappa$-solution $\left(M, g_{i j}(x, t)\right)$ along a sequence of points $q_{k} \in M$ and a sequence of times $t_{k} \rightarrow-\infty$ converge in the $C^{\infty}$ topology to the round $\mathbb{S}^{2}$ or the round $\mathbb{R} \mathbb{P}^{2}$. In particular, by looking at the time derivative of the volume and the Gauss-Bonnet theorem, we know that the ancient $\kappa$-solution $\left(M, g_{i j}(x, t)\right)$ exists on a maximal time interval $(-\infty, T)$ with $T<+\infty$.

Consider the scaled entropy of Hamilton [60]

$$
E(t)=\int_{M} R \log [R(T-t)] d V_{t}
$$


We compute

$$
\begin{aligned}
\frac{d}{d t} E(t) & =\int_{M} \log [R(T-t)] \Delta R d V_{t}+\int_{M}\left[\Delta R+R^{2}-\frac{R}{(T-t)}\right] d V_{t} \\
& =\int_{M}\left[-\frac{|\nabla R|^{2}}{R}+R^{2}-r R\right] d V_{t} \\
& =\int_{M}\left[-\frac{|\nabla R|^{2}}{R}+(R-r)^{2}\right] d V_{t}
\end{aligned}
$$

where $r=\int_{M} R d V_{t} / \operatorname{Vol}_{t}(M)$ and we have used $\operatorname{Vol}_{t}(M)=\left(\int_{M} R d V_{t}\right) \cdot(T-t)$ (by the Gauss-Bonnet theorem).

For a smooth function $f$ on the surface $M$, one can readily check

$$
\begin{aligned}
\int_{M}(\Delta f)^{2} & =2 \int_{M}\left|\nabla_{i} \nabla_{j} f-\frac{1}{2}(\Delta f) g_{i j}\right|^{2}+\int_{M} R|\nabla f|^{2}, \\
\int_{M} \frac{|\nabla R+R \nabla f|^{2}}{R} & =\int_{M} \frac{|\nabla R|^{2}}{R}-2 \int_{M} R(\Delta f)+\int_{M} R|\nabla f|^{2},
\end{aligned}
$$

and then

$$
\begin{aligned}
& \int_{M} \frac{|\nabla R|^{2}}{R}+\int_{M}(\Delta f)(\Delta f-2 R) \\
& =2 \int_{M}\left|\nabla_{i} \nabla_{j} f-\frac{1}{2}(\Delta f) g_{i j}\right|^{2}+\int_{M} \frac{|\nabla R+R \nabla f|^{2}}{R} .
\end{aligned}
$$

By choosing $\Delta f=R-r$, we get

$$
\begin{aligned}
& \int_{M} \frac{|\nabla R|^{2}}{R}-\int_{M}(R-r)^{2} \\
& =2 \int_{M}\left|\nabla_{i} \nabla_{j} f-\frac{1}{2}(\Delta f) g_{i j}\right|^{2}+\int_{M} \frac{|\nabla R+R \nabla f|^{2}}{R} \geq 0 .
\end{aligned}
$$

If the equality holds, then we have

$$
\nabla_{i} \nabla_{j} f-\frac{1}{2}(\Delta f) g_{i j}=0
$$

i.e., $\nabla f$ is conformal. By the Kazdan-Warner identity [77], it follows that

$$
\int_{M} \nabla R \cdot \nabla f=0
$$

so

$$
\begin{aligned}
0 & =-\int_{M} R \Delta f \\
& =-\int_{M}(R-r)^{2} .
\end{aligned}
$$

Hence we have proved the following inequality due to Chow [37]

$$
\int_{M} \frac{|\nabla R|^{2}}{R} \geq \int_{M}(R-r)^{2}
$$


and the equality holds if and only if $R \equiv r$.

The combination (6.2.27) and (6.2.28) shows that the scaled entropy $E(t)$ is strictly decreasing along the Ricci flow unless we are on the round sphere $\mathbb{S}^{2}$ or its quotient $\mathbb{R P}^{2}$. Moreover the convergence result in Theorem 5.1.11 shows that the scaled entropy $E$ has its minimum value at the constant curvature metric (round $\mathbb{S}^{2}$ or round $\mathbb{R P}^{2}$ ). We had shown that the scalings of the nonflat ancient $\kappa$-solution along a sequence of times $t_{k} \rightarrow-\infty$ converge to the constant curvature metric. Then $E(t)$ has its minimal value at $t=-\infty$, so it was constant all along, hence the ancient $\kappa$-solution must have constant curvature for each $t \in(-\infty, T)$. This proves the theorem.

6.3. Curvature Estimates via Volume Growth. For solutions to the Ricci flow, Perelman's no local collapsing theorems tell us that the local curvature upper bounds imply the local volume lower bounds. Conversely, one would expect to get local curvature upper bounds from local volume lower bounds. If this is the case, one will be able to establish an elliptic type estimate for the curvatures of solutions to the Ricci flow. This will provide the key estimate for the canonical neighborhood structure and thick-thin decomposition of the Ricci flow on three-manifolds. In this section we derive such curvature estimates for nonnegatively curved solutions. In the next chapter we will derive similar estimates for all smooth solutions, as well as surgically modified solutions, of the Ricci flow on three-manifolds.

Let $M$ be an $n$-dimensional complete noncompact Riemannian manifold with nonnegative Ricci curvature. Pick an origin $O \in M$. The well-known Bishop-Gromov volume comparison theorem tells us the ratio $\operatorname{Vol}(B(O, r)) / r^{n}$ is monotone nonincreasing in $r \in[0,+\infty)$. Thus there exists a limit

$$
\nu_{M}=\lim _{r \rightarrow+\infty} \frac{\operatorname{Vol}(B(O, r))}{r^{n}} .
$$

Clearly the number $\nu_{M}$ is invariant under dilation and is independent of the choice of the origin. $\nu_{M}$ is called the asymptotic volume ratio of the Riemannian manifold $M$.

The following result obtained by Perelman in [103] shows that any ancient $\kappa$ solution must have zero asymptotic volume ratio. This result for the Ricci flow on Kähler manifolds was obtained by Chen and the second author in [32] independently. Moreover in the Kähler case, as shown by Chen, Tang and the second author in [29] (for complex two dimension) and by $\mathrm{Ni}$ in [98] (for all dimensions), the condition of nonnegative curvature operator can be replaced by the weaker condition of nonnegative bisectional curvature.

Lemma 6.3.1. Let $M$ be an n-dimensional complete noncompact Riemannian manifold. Suppose $g_{i j}(x, t), x \in M$ and $t \in(-\infty, T)$ with $T>0$, is a nonflat ancient solution of the Ricci flow with nonnegative curvature operator and bounded curvature. Then the asymptotic volume ratio of the solution metric satisfies

$$
\nu_{M}(t)=\lim _{r \rightarrow+\infty} \frac{\operatorname{Vol}_{t}\left(B_{t}(O, r)\right)}{r^{n}}=0
$$

for each $t \in(-\infty, T)$.

Proof. The proof is by induction on the dimension. When the dimension is two, the lemma is valid by Theorem 6.2.2. For dimension $\geq 3$, we argue by contradiction. 
Suppose the lemma is valid for dimensions $\leq n-1$ and suppose $\nu_{M}\left(t_{0}\right)>0$ for some $n$-dimensional nonflat ancient solution with nonnegative curvature operator and bounded curvature at some time $t_{0} \leq 0$. Fixing a point $x_{0} \in M$, we consider the asymptotic scalar curvature ratio

$$
A=\limsup _{d_{t_{0}}\left(x, x_{0}\right) \rightarrow+\infty} R\left(x, t_{0}\right) d_{t_{0}}^{2}\left(x, x_{0}\right) .
$$

We divide the proof into three cases.

Case 1: $A=+\infty$.

By Lemma 6.1.3, there exist sequences of points $x_{k} \in M$ divergent to infinity, of radii $r_{k} \rightarrow+\infty$, and of positive constants $\delta_{k} \rightarrow 0$ such that

(i) $R\left(x, t_{0}\right) \leq\left(1+\delta_{k}\right) R\left(x_{k}, t_{0}\right)$ for all $x$ in the ball $B_{t_{0}}\left(x_{k}, r_{k}\right)$ of radius $r_{k}$ around $x_{k}$

(ii) $r_{k}^{2} R\left(x_{k}, t_{0}\right) \rightarrow+\infty$ as $k \rightarrow+\infty$,

(iii) $d_{t_{0}}\left(x_{k}, x_{0}\right) / r_{k} \rightarrow+\infty$.

By scaling the solution around the points $x_{k}$ with factor $R\left(x_{k}, t_{0}\right)$, and shifting the time $t_{0}$ to the new time zero, we get a sequence of rescaled solutions

$$
g_{k}(s)=R\left(x_{k}, t_{0}\right) g\left(\cdot, t_{0}+\frac{s}{R\left(x_{k}, t_{0}\right)}\right)
$$

to the Ricci flow. Since the ancient solution has nonnegative curvature operator and bounded curvature, there holds the Li-Yau-Hamilton inequality (Corollary 2.5.5). Thus the rescaled solutions satisfy

$$
R_{k}(x, s) \leq\left(1+\delta_{k}\right)
$$

for all $s \leq 0$ and $x \in B_{g_{k}(0)}\left(x_{k}, r_{k} \sqrt{R\left(x_{k}, t_{0}\right)}\right)$. Since $\nu_{M}\left(t_{0}\right)>0$, it follows from the standard volume comparison and Theorem 4.2.2 that the injectivity radii of the rescaled solutions $g_{k}$ at the points $x_{k}$ and the new time zero is uniformly bounded below by a positive number. Then by Hamilton's compactness theorem (Theorem 4.1.5), after passing to a subsequence, $\left(M, g_{k}(s), x_{k}\right)$ will converge to a solution $(\tilde{M}, \tilde{g}(s), O)$ to the Ricci flow with

$$
\tilde{R}(y, s) \leq 1, \text { for all } s \leq 0 \text { and } y \in \tilde{M},
$$

and

$$
\tilde{R}(O, 0)=1 .
$$

Since the metric is shrinking, by (ii) and (iii), we get

$$
R\left(x_{k}, t_{0}\right) d_{g\left(\cdot, t_{0}+\frac{s}{R\left(x_{k}, t_{0}\right)}\right)}^{2}\left(x_{0}, x_{k}\right) \geq R\left(x_{k}, t_{0}\right) d_{g\left(\cdot, t_{0}\right)}^{2}\left(x_{0}, x_{k}\right)
$$

which tends to $+\infty$, as $k \rightarrow+\infty$, for all $s \leq 0$. Thus by Proposition 6.1.2, for each $s \leq 0,(\tilde{M}, \tilde{g}(s))$ splits off a line. We now consider the lifting of the solution $(\tilde{M}, \tilde{g}(s)), s \leq 0$, to its universal cover and denote it by $(\tilde{\tilde{M}}, \tilde{\tilde{g}}(s)), s \leq 0$. Clearly we still have

$$
\nu_{\tilde{M}}(0)>0 \text { and } \nu_{\tilde{M}}(0)>0
$$


By applying Hamilton's strong maximum principle and the de Rham decomposition theorem, the universal cover $\tilde{\tilde{M}}$ splits isometrically as $X \times \mathbb{R}$ for some $(n-1)$ dimensional nonflat (complete) ancient solution $X$ with nonnegative curvature operator and bounded curvature. These imply that $\nu_{X}(0)>0$, which contradicts the induction hypothesis.

Case 2: $0<A<+\infty$.

Take a sequence of points $x_{k}$ divergent to infinity such that

$$
R\left(x_{k}, t_{0}\right) d_{t_{0}}^{2}\left(x_{k}, x_{0}\right) \rightarrow A, \text { as } k \rightarrow+\infty .
$$

Consider the rescaled solutions $\left(M, g_{k}(s)\right)$ (around the fixed point $\left.x_{0}\right)$, where

$$
g_{k}(s)=R\left(x_{k}, t_{0}\right) g\left(\cdot, t_{0}+\frac{s}{R\left(x_{k}, t_{0}\right)}\right), s \in(-\infty, 0] .
$$

Then there is a constant $C>0$ such that

$$
\left\{\begin{array}{l}
R_{k}(x, 0) \leq C / d_{k}^{2}\left(x, x_{0}, 0\right), \\
R_{k}\left(x_{k}, 0\right)=1 \\
d_{k}\left(x_{k}, x_{0}, 0\right) \rightarrow \sqrt{A}>0
\end{array}\right.
$$

where $d_{k}\left(\cdot, x_{0}, 0\right)$ is the distance function from the point $x_{0}$ in the metric $g_{k}(0)$.

Since $\nu_{M}\left(t_{0}\right)>0$, it is a basic result in Alexandrov space theory (see for example Theorem 7.6 of [20]) that a subsequence of $\left(M, g_{k}(s), x_{0}\right)$ converges in the GromovHausdorff sense to an $n$-dimensional metric cone $\left(\tilde{M}, \tilde{g}(0), x_{0}\right)$ with vertex $x_{0}$.

By (6.3.1), the standard volume comparison and Theorem 4.2.2, we know that the injectivity radius of $\left(M, g_{k}(0)\right)$ at $x_{k}$ is uniformly bounded from below by a positive number $\rho_{0}$. After taking a subsequence, we may assume $x_{k}$ converges to a point $x_{\infty}$ in $\tilde{M} \backslash\left\{x_{0}\right\}$. Then by Hamilton's compactness theorem (Theorem 4.1.5), we can take a subsequence such that the metrics $g_{k}(s)$ on the metric balls $B_{0}\left(x_{k}, \frac{1}{2} \rho_{0}\right)(\subset M$ with respect to the metric $\left.g_{k}(0)\right)$ converge in the $C_{l o c}^{\infty}$ topology to a solution of the Ricci flow on a ball $B_{0}\left(x_{\infty}, \frac{1}{2} \rho_{0}\right)$. Clearly the $C_{l o c}^{\infty}$ limit has nonnegative curvature operator and it is a piece of the metric cone at the time $s=0$. By (6.3.1), we have

$$
\tilde{R}\left(x_{\infty}, 0\right)=1 .
$$

Let $x$ be any point in the limiting ball $B_{0}\left(x_{\infty}, \frac{1}{2} \rho_{0}\right)$ and $e_{1}$ be any radial direction at $x$. Clearly $\tilde{\operatorname{Ric}}\left(e_{1}, e_{1}\right)=0$. Recall that the evolution equation of the Ricci tensor in frame coordinates is

$$
\frac{\partial}{\partial t} \tilde{R}_{a b}=\tilde{\triangle} \tilde{R}_{a b}+2 \tilde{R}_{a c b d} \tilde{R}_{c d}
$$

Since the curvature operator is nonnegative, by applying Hamilton's strong maximum principle (Theorem 2.2.1) to the above equation, we deduce that the null space of $\tilde{\text { Ric }}$ is invariant under parallel translation. In particular, all radial directions split off locally and isometrically. While by (6.3.2), the piece of the metric cone is nonflat. This gives a contradiction.

Case 3: $A=0$. 
The gap theorem as was initiated by Mok-Siu-Yau [93] and established by Greene$\mathrm{Wu}[49,50]$, Eschenberg-Shrader-Strake [45], and Drees [44] shows that a complete noncompact $n$-dimensional (except $n=4$ or 8 ) Riemannian manifold with nonnegative sectional curvature and the asymptotic scalar curvature ratio $A=0$ must be flat. So the present case is ruled out except in dimension $n=4$ or 8 . Since in our situation the asymptotic volume ratio is positive and the manifold is the solution of the Ricci flow, we can give an alternative proof for all dimensions as follows.

We claim the sectional curvature of $\left(M, g_{i j}\left(x, t_{0}\right)\right)$ is positive everywhere. Indeed, by Theorem 2.2.2, the image of the curvature operator is just the restricted holonomy algebra $\mathcal{G}$ of the manifold. If the sectional curvature vanishes for some two-plane, then the holonomy algebra $\mathcal{G}$ cannot be $s o(n)$. We observe the manifold is not Einstein since it is noncompact, nonflat and has nonnegative curvature operator. If $\mathcal{G}$ is irreducible, then by Berger's Theorem [7], $\mathcal{G}=u\left(\frac{n}{2}\right)$. Thus the manifold is Kähler with bounded and nonnegative bisectional curvature and with curvature decay faster than quadratic. Then by the gap theorem obtained by Chen and the second author in [31], this Kähler manifold must be flat. This contradicts the assumption. Hence the holonomy algebra $\mathcal{G}$ is reducible and the universal cover of $M$ splits isometrically as $\tilde{M}_{1} \times \tilde{M}_{2}$ nontrivially. Clearly the universal cover of $M$ has positive asymptotic volume ratio. So $\tilde{M}_{1}$ and $\tilde{M}_{2}$ still have positive asymptotic volume ratio and at least one of them is nonflat. By the induction hypothesis, this is also impossible. Thus our claim is proved.

Now we know that the sectional curvature of $\left(M, g_{i j}\left(x, t_{0}\right)\right)$ is positive everywhere. Choose a sequence of points $x_{k}$ divergent to infinity such that

$$
\left\{\begin{array}{l}
R\left(x_{k}, t_{0}\right) d_{t_{0}}^{2}\left(x_{k}, x_{0}\right)=\sup \left\{R\left(x, t_{0}\right) d_{t_{0}}^{2}\left(x, x_{0}\right) \mid d_{t_{0}}\left(x, x_{0}\right) \geq d_{t_{0}}\left(x_{k}, x_{0}\right)\right\}, \\
d_{t_{0}}\left(x_{k}, x_{0}\right) \geq k \\
R\left(x_{k}, t_{0}\right) d_{t_{0}}^{2}\left(x_{k}, x_{0}\right)=\varepsilon_{k} \rightarrow 0 .
\end{array}\right.
$$

Consider the rescaled metric

$$
g_{k}(0)=R\left(x_{k}, t_{0}\right) g\left(\cdot, t_{0}\right)
$$

as before. Then

$$
\left\{\begin{array}{l}
R_{k}(x, 0) \leq \varepsilon_{k} / d_{k}^{2}\left(x, x_{0}, 0\right), \quad \text { for } d_{k}\left(x, x_{0}, 0\right) \geq \sqrt{\varepsilon_{k}} \\
d_{k}\left(x_{k}, x_{0}, 0\right)=\sqrt{\varepsilon_{k}} \rightarrow 0
\end{array}\right.
$$

As in Case 2 , the rescaled marked solutions $\left(M, g_{k}(0), x_{0}\right)$ will converge in the GromovHausdorff sense to a metric cone $\left(\tilde{M}, \tilde{g}(0), x_{0}\right)$. And by the virtue of Hamilton's compactness theorem (Theorem 4.1.5), up to a subsequence, the convergence is in the $C_{l o c}^{\infty}$ topology in $\tilde{M} \backslash\left\{x_{0}\right\}$. We next claim the metric cone $\left(\tilde{M}, \tilde{g}(0), x_{0}\right)$ is isometric to $\mathbb{R}^{n}$.

Indeed, let us write the metric cone $\tilde{M}$ as a warped product $\mathbb{R}_{+} \times_{r} X^{n-1}$ for some $(n-1)$-dimensional manifold $X^{n-1}$. By (6.3.3), the metric cone must be flat and $X^{n-1}$ is isometric to a quotient of the round sphere $\mathbb{S}^{n-1}$ by fixed point free isometries in the standard metric. To show $\tilde{M}$ is isometric to $\mathbb{R}^{n}$, we only need to verify that $X^{n-1}$ is simply connected.

Let $\varphi$ be the Busemann function of $\left(M, g_{i j}\left(\cdot, t_{0}\right)\right)$ with respect to the point $x_{0}$. Since $\left(M, g_{i j}\left(\cdot, t_{0}\right)\right)$ has nonnegative sectional curvature, it is easy to see that for any small $\varepsilon>0$, there is a $r_{0}>0$ such that

$$
(1-\varepsilon) d_{t_{0}}\left(x, x_{0}\right) \leq \varphi(x) \leq d_{t_{0}}\left(x, x_{0}\right)
$$


for all $x \in M \backslash B_{t_{0}}\left(x_{0}, r_{0}\right)$. The strict positivity of the sectional curvature of the manifold $\left(M, g_{i j}\left(\cdot, t_{0}\right)\right)$ implies that the square of the Busemann function is strictly convex (and exhausting). Thus every level set $\varphi^{-1}(a)$, with $a>\inf \{\varphi(x) \mid x \in M\}$, of the Busemann function $\varphi$ is diffeomorphic to the $(n-1)$-sphere $\mathbb{S}^{n-1}$. In particular, $\varphi^{-1}\left(\left[a, \frac{3}{2} a\right]\right)$ is simply connected for $a>\inf \{\varphi(x) \mid x \in M\}$ since $n \geq 3$.

Consider an annulus portion $[1,2] \times X^{n-1}$ of the metric cone $\tilde{M}=\mathbb{R}_{+} \times_{r} X^{n-1}$. It is the limit of $\left(M_{k}, g_{k}(0)\right)$, where

$$
M_{k}=\left\{x \in M \mid \frac{1}{\sqrt{R\left(x_{k}, t_{0}\right)}} \leq d_{t_{0}}\left(x, x_{0}\right) \leq \frac{2}{\sqrt{R\left(x_{k}, t_{0}\right)}}\right\} .
$$

It is clear that

$$
\varphi^{-1}\left(\left[\frac{1}{\sqrt{R\left(x_{k}, t_{0}\right)}}, \frac{2(1-\varepsilon)}{\sqrt{R\left(x_{k}, t_{0}\right)}}\right]\right) \subset M_{k} \subset \varphi^{-1}\left(\left[\frac{1-\varepsilon}{\sqrt{R\left(x_{k}, t_{0}\right)}}, \frac{2}{\sqrt{R\left(x_{k}, t_{0}\right)}}\right]\right)
$$

for $k$ large enough. Thus any closed loop in $\left\{\frac{3}{2}\right\} \times X^{n-1}$ can be shrunk to a point by a homotopy in $[1,2] \times X^{n-1}$. This shows that $X^{n-1}$ is simply connected. Hence we have proven that the metric cone $\left(\tilde{M}, \tilde{g}(0), x_{0}\right)$ is isometric to $\mathbb{R}^{n}$. Consequently,

$$
\lim _{k \rightarrow+\infty} \frac{\operatorname{Vol}_{g\left(t_{0}\right)}\left(B_{g\left(t_{0}\right)}\left(x_{0}, \frac{r}{\sqrt{R\left(x_{k}, t_{0}\right)}}\right) \backslash B_{g\left(t_{0}\right)}\left(x_{0}, \frac{\sigma r}{\sqrt{R\left(x_{k}, t_{0}\right)}}\right)\right)}{\left(\frac{r}{\sqrt{R\left(x_{k}, t_{0}\right)}}\right)^{n}}=\alpha_{n}\left(1-\sigma^{n}\right)
$$

for any $r>0$ and $0<\sigma<1$, where $\alpha_{n}$ is the volume of the unit ball in the Euclidean space $\mathbb{R}^{n}$. Finally, by combining with the monotonicity of the Bishop-Gromov volume comparison, we conclude that the manifold $\left(M, g_{i j}\left(\cdot, t_{0}\right)\right)$ is flat and isometric to $\mathbb{R}^{n}$. This contradicts the assumption.

Therefore, we have proved the lemma.

Finally we would like to include an alternative simpler argument, inspired by $\mathrm{Ni}$ [98], for the above Case 2 and Case 3 to avoid the use of the gap theorem, holonomy groups, and asymptotic cone structure.

Alternative Proof for Case 2 and Case 3. Let us consider the situation of $0 \leq$ $A<+\infty$ in the above proof. Observe that $\nu_{M}(t)$ is nonincreasing in time $t$ by using Lemma 3.4.1(ii) and the fact that the metric is shrinking in $t$. Suppose $\nu_{M}\left(t_{0}\right)>0$, then the solution $g_{i j}(\cdot, t)$ is $\kappa$-noncollapsed for $t \leq t_{0}$ for some uniform $\kappa>0$. By combining with Theorem 6.2.1, there exist a sequence of points $q_{k}$ and a sequence of times $t_{k} \rightarrow-\infty$ such that the scalings of $g_{i j}(\cdot, t)$ around $q_{k}$ with factor $\left|t_{k}\right|^{-1}$ and with the times $t_{k}$ shifting to the new time zero converge to a nonflat gradient shrinking soliton $\bar{M}$ in the $C_{l o c}^{\infty}$ topology. This gradient soliton also has maximal volume growth (i.e. $\nu_{\bar{M}}(t)>0$ ) and satisfies the Li-Yau-Hamilton estimate (Corollary 2.5.5). If the curvature of the shrinking soliton $\bar{M}$ at the time -1 is bounded, then we see from the proof of Theorem 6.2.2 that by using the equations (6.2.24)-(6.2.26), the scalar curvature has a positive lower bound everywhere on $\bar{M}$ at the time -1. In particular, this implies the asymptotic scalar curvature ratio $A=\infty$ for the soliton at the time -1 , which reduces to Case 1 and arrives at a contradiction by the dimension reduction argument. On the other hand, if the scalar curvature is unbounded, then by Lemma 
6.1.4, the Li-Yau-Hamilton estimate (Corollary 2.5.5) and Lemma 6.1.2, we can do the same dimension reduction as in Case 1 to arrive at a contradiction also.

The following lemma is a local and space-time version of Lemma 6.1.4 on picking local (almost) maximum curvature points. We formulate it from Perelman's arguments in the section 10 of [103].

Lemma 6.3.2. For any positive constants $B, C$ with $B>4$ and $C>1000$, there exists $1 \leq A<\min \left\{\frac{1}{4} B, \frac{1}{1000} C\right\}$ which tends to infinity as $B$ and $C$ tend to infinity and satisfies the following property. Suppose we have a (not necessarily complete) solution $g_{i j}(t)$ to the Ricci flow, defined on $M \times\left[-t_{0}, 0\right]$, so that at each time $t \in\left[-t_{0}, 0\right]$ the metric ball $B_{t}\left(x_{0}, 1\right)$ is compactly contained in $M$. Suppose there exists a point $\left(x^{\prime}, t^{\prime}\right) \in M \times\left(-t_{0}, 0\right]$ such that

$$
d_{t^{\prime}}\left(x^{\prime}, x_{0}\right) \leq \frac{1}{4} \text { and }\left|R m\left(x^{\prime}, t^{\prime}\right)\right|>C+B\left(t^{\prime}+t_{0}\right)^{-1}
$$

Then we can find a point $(\bar{x}, \bar{t}) \in M \times\left(-t_{0}, 0\right]$ such that

$$
d_{\bar{t}}\left(\bar{x}, x_{0}\right)<\frac{1}{3} \text { with } Q=|R m(\bar{x}, \bar{t})|>C+B\left(\bar{t}+t_{0}\right)^{-1},
$$

and

$$
|R m(x, t)| \leq 4 Q
$$

for all $\left(-t_{0}<\right) \bar{t}-A Q^{-1} \leq t \leq \bar{t}$ and $d_{t}(x, \bar{x}) \leq \frac{1}{10} A^{\frac{1}{2}} Q^{-\frac{1}{2}}$.

Proof. We first claim that there exists a point $(\bar{x}, \bar{t})$ with $-t_{0}<\bar{t} \leq 0$ and $d_{\bar{t}}\left(\bar{x}, x_{0}\right)<\frac{1}{3}$ such that

$$
Q=|R m(\bar{x}, \bar{t})|>C+B\left(\bar{t}+t_{0}\right)^{-1}
$$

and

$$
|R m(x, t)| \leq 4 Q
$$

wherever $\bar{t}-A Q^{-1} \leq t \leq \bar{t}, \quad d_{t}\left(x, x_{0}\right) \leq d_{\bar{t}}\left(\bar{x}, x_{0}\right)+\left(A Q^{-1}\right)^{\frac{1}{2}}$.

We will construct such $(\bar{x}, \bar{t})$ as a limit of a finite sequence of points. Take an arbitrary $\left(x_{1}, t_{1}\right)$ such that

$$
d_{t_{1}}\left(x_{1}, x_{0}\right) \leq \frac{1}{4}, \quad-t_{0}<t_{1} \leq 0 \text { and }\left|R m\left(x_{1}, t_{1}\right)\right|>C+B\left(t_{1}+t_{0}\right)^{-1} .
$$

Such a point clearly exists by our assumption. Assume we have already constructed $\left(x_{k}, t_{k}\right)$. If we cannot take the point $\left(x_{k}, t_{k}\right)$ to be the desired point $(\bar{x}, \bar{t})$, then there exists a point $\left(x_{k+1}, t_{k+1}\right)$ such that

$$
t_{k}-A\left|R m\left(x_{k}, t_{k}\right)\right|^{-1} \leq t_{k+1} \leq t_{k},
$$

and

$$
d_{t_{k+1}}\left(x_{k+1}, x_{0}\right) \leq d_{t_{k}}\left(x_{k}, x_{0}\right)+\left(A\left|R m\left(x_{k}, t_{k}\right)\right|^{-1}\right)^{\frac{1}{2}},
$$

but

$$
\left|R m\left(x_{k+1}, t_{k+1}\right)\right|>4\left|R m\left(x_{k}, t_{k}\right)\right| .
$$


It then follows that

$$
\begin{aligned}
d_{t_{k+1}}\left(x_{k+1}, x_{0}\right) \leq & d_{t_{1}}\left(x_{1}, x_{0}\right)+A^{\frac{1}{2}}\left(\sum_{i=1}^{k}\left|R m\left(x_{i}, t_{i}\right)\right|^{-\frac{1}{2}}\right) \\
& \leq \frac{1}{4}+A^{\frac{1}{2}}\left(\sum_{i=1}^{k} 2^{-(i-1)}\left|R m\left(x_{1}, t_{1}\right)\right|^{-\frac{1}{2}}\right) \\
& \leq \frac{1}{4}+2\left(A C^{-1}\right)^{\frac{1}{2}} \\
& <\frac{1}{3} \\
t_{k+1}-\left(-t_{0}\right)= & \sum_{i=1}^{k}\left(t_{i+1}-t_{i}\right)+\left(t_{1}-\left(-t_{0}\right)\right) \\
\geq- & \sum_{i=1}^{k} A\left|R m\left(x_{i}, t_{i}\right)\right|^{-1}+\left(t_{1}-\left(-t_{0}\right)\right) \\
\geq & -A \sum_{i=1}^{k} 4^{-(i-1)}\left|R m\left(x_{1}, t_{1}\right)\right|^{-1}+\left(t_{1}-\left(-t_{0}\right)\right) \\
\geq & -\frac{2 A}{B}\left(t_{1}+t_{0}\right)+\left(t_{1}+t_{0}\right) \\
\geq & \frac{1}{2}\left(t_{1}+t_{0}\right)
\end{aligned}
$$

and

$$
\begin{aligned}
\left|R m\left(x_{k+1}, t_{k+1}\right)\right| & >4^{k}\left|R m\left(x_{1}, t_{1}\right)\right| \\
& \geq 4^{k} C \rightarrow+\infty \text { as } k \rightarrow+\infty .
\end{aligned}
$$

Since the solution is smooth, the sequence $\left\{\left(x_{k}, t_{k}\right)\right\}$ is finite and its last element fits. Thus we have proved assertion (6.3.4).

From the above construction we also see that the chosen point $(\bar{x}, \bar{t})$ satisfies

$$
d_{\bar{t}}\left(\bar{x}, x_{0}\right)<\frac{1}{3}
$$

and

$$
Q=|R m(\bar{x}, \bar{t})|>C+B\left(\bar{t}+t_{0}\right)^{-1} .
$$

Clearly, up to some adjustment of the constant $A$, we only need to show that

$$
|R m(x, t)| \leq 4 Q
$$

wherever $\bar{t}-\frac{1}{200 n} A^{\frac{1}{2}} Q^{-1} \leq t \leq \bar{t}$ and $d_{t}(x, \bar{x}) \leq \frac{1}{10} A^{\frac{1}{2}} Q^{-\frac{1}{2}}$.

For any point $(x, \bar{t})$ with $d_{\bar{t}}(x, \bar{x}) \leq \frac{1}{10} A^{\frac{1}{2}} Q^{-\frac{1}{2}}$, we have

$$
\begin{aligned}
d_{\bar{t}}\left(x, x_{0}\right) & \leq d_{\bar{t}}\left(\bar{x}, x_{0}\right)+d_{\bar{t}}(x, \bar{x}) \\
& \leq d_{\bar{t}}\left(\bar{x}, x_{0}\right)+\left(A Q^{-1}\right)^{\frac{1}{2}}
\end{aligned}
$$


and then by (6.3.4)

$$
|R m(x, \bar{t})| \leq 4 Q .
$$

Thus by continuity, there is a minimal $\bar{t}^{\prime} \in\left[\bar{t}-\frac{1}{200 n} A^{\frac{1}{2}} Q^{-1}, \bar{t}\right]$ such that

$$
\sup \left\{|R m(x, t)| \mid \bar{t}^{\prime} \leq t \leq \bar{t}, \quad d_{t}(x, \bar{x}) \leq \frac{1}{10} A^{\frac{1}{2}} Q^{-\frac{1}{2}}\right\} \leq 5 Q .
$$

For any point $(x, t)$ with $\bar{t}^{\prime} \leq t \leq \bar{t}$ and $d_{t}(x, \bar{x}) \leq \frac{1}{10}\left(A Q^{-1}\right)^{\frac{1}{2}}$, we divide the discussion into two cases.

Case (1): $d_{t}\left(\bar{x}, x_{0}\right) \leq \frac{3}{10}\left(A Q^{-1}\right)^{\frac{1}{2}}$.

From assertion (6.3.4) we see that

$$
\sup \left\{|R m(x, t)| \quad \mid \bar{t}^{\prime} \leq t \leq \bar{t}, \quad d_{t}\left(x, x_{0}\right) \leq\left(A Q^{-1}\right)^{\frac{1}{2}}\right\} \leq 4 Q .
$$

Since $d_{t}\left(\bar{x}, x_{0}\right) \leq \frac{3}{10}\left(A Q^{-1}\right)^{\frac{1}{2}}$, we have

$$
\begin{aligned}
d_{t}\left(x, x_{0}\right) & \leq d_{t}(x, \bar{x})+d_{t}\left(\bar{x}, x_{0}\right) \\
& \leq \frac{1}{10}\left(A Q^{-1}\right)^{\frac{1}{2}}+\frac{3}{10}\left(A Q^{-1}\right)^{\frac{1}{2}} \\
& \leq\left(A Q^{-1}\right)^{\frac{1}{2}}
\end{aligned}
$$

which implies the estimate $|R m(x, t)| \leq 4 Q$ from $(6.3 .5)^{\prime}$.

Case $(2): d_{t}\left(\bar{x}, x_{0}\right)>\frac{3}{10}\left(A Q^{-1}\right)^{\frac{1}{2}}$.

From the curvature bounds in (6.3.5) and $(6.3 .5)^{\prime}$, we can apply Lemma 3.4.1 (ii) with $r_{0}=\frac{1}{10} Q^{-\frac{1}{2}}$ to get

$$
\frac{d}{d t}\left(d_{t}\left(\bar{x}, x_{0}\right)\right) \geq-40(n-1) Q^{\frac{1}{2}}
$$

and then

$$
\begin{aligned}
d_{t}\left(\bar{x}, x_{0}\right) & \leq d_{\hat{t}}\left(\bar{x}, x_{0}\right)+40 n\left(Q^{\frac{1}{2}}\right)\left(\frac{1}{200 n} A^{\frac{1}{2}} Q^{-1}\right) \\
& \leq d_{\hat{t}}\left(\bar{x}, x_{0}\right)+\frac{1}{5}\left(A Q^{-1}\right)^{\frac{1}{2}}
\end{aligned}
$$

where $\hat{t} \in(t, \bar{t}]$ satisfies the property that $d_{s}\left(\bar{x}, x_{0}\right) \geq \frac{3}{10}\left(A Q^{-1}\right)^{\frac{1}{2}}$ whenever $s \in[t, \hat{t}]$. So we have either

$$
\begin{aligned}
d_{t}\left(x, x_{0}\right) & \leq d_{t}(x, \bar{x})+d_{t}\left(\bar{x}, x_{0}\right) \\
& \leq \frac{1}{10}\left(A Q^{-1}\right)^{\frac{1}{2}}+\frac{3}{10}\left(A Q^{-1}\right)^{\frac{1}{2}}+\frac{1}{5}\left(A Q^{-1}\right)^{\frac{1}{2}} \\
& \leq\left(A Q^{-1}\right)^{\frac{1}{2}},
\end{aligned}
$$

or

$$
\begin{aligned}
d_{t}\left(x, x_{0}\right) & \leq d_{t}(x, \bar{x})+d_{t}\left(\bar{x}, x_{0}\right) \\
& \leq \frac{1}{10}\left(A Q^{-1}\right)^{\frac{1}{2}}+d_{\bar{t}}\left(\bar{x}, x_{0}\right)+\frac{1}{5}\left(A Q^{-1}\right)^{\frac{1}{2}} \\
& \leq d_{\bar{t}}\left(\bar{x}, x_{0}\right)+\left(A Q^{-1}\right)^{\frac{1}{2}} .
\end{aligned}
$$


It then follows from (6.3.4) that $|R m(x, t)| \leq 4 Q$.

Hence we have proved

$$
|R m(x, t)| \leq 4 Q
$$

for any point $(x, t)$ with $\bar{t}^{\prime} \leq t \leq \bar{t}$ and $d_{t}(x, \bar{x}) \leq \frac{1}{10}\left(A Q^{-1}\right)^{\frac{1}{2}}$. By combining with the choice of $\bar{t}^{\prime}$ in (6.3.5), we must have $\bar{t}^{\prime}=\bar{t}-\frac{1}{200 n} A^{\frac{1}{2}} Q^{-1}$. This proves assertion $(6.3 .4)^{\prime}$.

Therefore we have completed the proof of the lemma.

We now use the volume lower bound assumption to establish the crucial curvature upper bound estimate of Perelman [103] for the Ricci flow. For the Ricci flow on Kähler manifolds, a global version of this estimate (i.e., curvature decaying linear in time and quadratic in space) was independently obtained in [29] and [32]. Note that the volume estimate conclusion in the following Theorem 6.3.3 (ii) was not stated in Corollary 11.6 (b) of Perelman [103]. The estimate will be used later in the proof of Theorem 7.2.2 and Theorem 7.5.2.

Theorem 6.3.3 (Perelman [103]). For every $w>0$ there exist $B=B(w)<$ $+\infty, \quad C=C(w)<+\infty, \quad \tau_{0}=\tau_{0}(w)>0$, and $\xi=\xi(w)>0$ (depending also on the dimension) with the following properties. Suppose we have a (not necessarily complete) solution $g_{i j}(t)$ to the Ricci flow, defined on $M \times\left[-t_{0} r_{0}^{2}, 0\right]$, so that at each time $t \in\left[-t_{0} r_{0}^{2}, 0\right]$ the metric ball $B_{t}\left(x_{0}, r_{0}\right)$ is compactly contained in $M$.

(i) If at each time $t \in\left[-t_{0} r_{0}^{2}, 0\right]$,

$$
\begin{gathered}
R m(., t) \geq-r_{0}^{-2} \text { on } B_{t}\left(x_{0}, r_{0}\right) \\
\text { and } \operatorname{Vol}_{t}\left(B_{t}\left(x_{0}, r_{0}\right)\right) \geq w r_{0}^{n},
\end{gathered}
$$

then we have the estimate

$$
|R m(x, t)| \leq C r_{0}^{-2}+B\left(t+t_{0} r_{0}^{2}\right)^{-1}
$$

whenever $-t_{0} r_{0}^{2}<t \leq 0$ and $d_{t}\left(x, x_{0}\right) \leq \frac{1}{4} r_{0}$.

(ii) If for some $0<\bar{\tau} \leq t_{0}$,

$$
\begin{aligned}
R m(x, t) \geq & -r_{0}^{-2} \text { for } t \in\left[-\bar{\tau} r_{0}^{2}, 0\right], x \in B_{t}\left(x_{0}, r_{0}\right), \\
& \text { and } \operatorname{Vol}_{0}\left(B_{0}\left(x_{0}, r_{0}\right)\right) \geq w r_{0}^{n},
\end{aligned}
$$

then we have the estimates

$$
\operatorname{Vol}_{t}\left(B_{t}\left(x_{0}, r_{0}\right)\right) \geq \xi r_{0}^{n} \text { for all } \max \left\{-\bar{\tau} r_{0}^{2},-\tau_{0} r_{0}^{2}\right\} \leq t \leq 0
$$

and

$$
|R m(x, t)| \leq C r_{0}^{-2}+B\left(t-\max \left\{-\bar{\tau} r_{0}^{2},-\tau_{0} r_{0}^{2}\right\}\right)^{-1}
$$

whenever $\max \left\{-\bar{\tau} r_{0}^{2},-\tau_{0} r_{0}^{2}\right\}<t \leq 0$ and $d_{t}\left(x, x_{0}\right) \leq \frac{1}{4} r_{0}$. 
Proof. By scaling we may assume $r_{0}=1$.

(i) By the standard (relative) volume comparison, we know that there exists some $w^{\prime}>0$, with $w^{\prime} \leq w$, depending only on $w$, such that for each point $(x, t)$ with $-t_{0} \leq t \leq 0$ and $d_{t}\left(x, x_{0}\right) \leq \frac{1}{3}$, and for each $r \leq \frac{1}{3}$, there holds

$$
\operatorname{Vol}_{t}\left(B_{t}(x, r)\right) \geq w^{\prime} r^{n} .
$$

We argue by contradiction. Suppose there are sequences $B, C \rightarrow+\infty$, of solutions $g_{i j}(t)$ and points $\left(x^{\prime}, t^{\prime}\right)$ such that

$$
d_{t^{\prime}}\left(x^{\prime}, x_{0}\right) \leq \frac{1}{4}, \quad-t_{0}<t^{\prime} \leq 0 \text { and }\left|R m\left(x^{\prime}, t^{\prime}\right)\right|>C+B\left(t^{\prime}+t_{0}\right)^{-1} .
$$

Then by Lemma 6.3.2, we can find a sequence of points $(\bar{x}, \bar{t})$ such that

$$
\begin{gathered}
d_{\bar{t}}\left(\bar{x}, x_{0}\right)<\frac{1}{3}, \\
Q=|R m(\bar{x}, \bar{t})|>C+B\left(\bar{t}+t_{0}\right)^{-1},
\end{gathered}
$$

and

$$
|R m(x, t)| \leq 4 Q
$$

wherever $\left(-t_{0}<\right) \bar{t}-A Q^{-1} \leq t \leq \bar{t}, \quad d_{t}(x, \bar{x}) \leq \frac{1}{10} A^{\frac{1}{2}} Q^{-\frac{1}{2}}$, where $A$ tends to infinity with $B, C$. Thus we may take a blow-up limit along the points $(\bar{x}, \bar{t})$ with factors $Q$ and get a non-flat ancient solution $\left(M_{\infty}, g_{i j}^{(\infty)}(t)\right)$ with nonnegative curvature operator and with the asymptotic volume ratio $\nu_{M_{\infty}}(t) \geq w^{\prime}>0$ for each $t \in(-\infty, 0]$ (by (6.3.6)). This contradicts Lemma 6.3.1.

(ii) Let $B(w), C(w)$ be good for the first part of the theorem. By the volume assumption at $t=0$ and the standard (relative) volume comparison, we still have the estimate

$$
\operatorname{Vol}_{0}\left(B_{0}(x, r)\right) \geq w^{\prime} r^{n}
$$

for each $x \in M$ with $d_{0}\left(x, x_{0}\right) \leq \frac{1}{3}$ and $r \leq \frac{1}{3}$. We will show that $\xi=5^{-n} w^{\prime}$, $B=B\left(5^{-n} w^{\prime}\right)$ and $C=C\left(5^{-n} w^{\prime}\right)$ are good for the second part of the theorem.

By continuity and the volume assumption at $t=0$, there is a maximal subinterval $[-\tau, 0]$ of the time interval $[-\bar{\tau}, 0]$ such that

$$
\operatorname{Vol}_{t}\left(B_{t}\left(x_{0}, 1\right)\right) \geq w \geq 5^{-n} w^{\prime} \text { for all } t \in[-\tau, 0] .
$$

This says that the assumptions of (i) hold with $5^{-n} w^{\prime}$ in place of $w$ and with $\tau$ in place of $t_{0}$. Thus the conclusion of the part (i) gives us the estimate

$$
|R m(x, t)| \leq C+B(t+\tau)^{-1}
$$

whenever $t \in(-\tau, 0]$ and $d_{t}\left(x, x_{0}\right) \leq \frac{1}{4}$.

We need to show that one can choose a positive $\tau_{0}$ depending only on $w$ and the dimension such that the maximal $\tau \geq \min \left\{\bar{\tau}, \tau_{0}\right\}$. 
For $t \in(-\tau, 0]$ and $\frac{1}{8} \leq d_{t}\left(x, x_{0}\right) \leq \frac{1}{4}$, we use (6.3.7) and Lemma 3.4.1(ii) to get

$$
\frac{d}{d t} d_{t}\left(x, x_{0}\right) \geq-10(n-1)(\sqrt{C}+(\sqrt{B} / \sqrt{t+\tau}))
$$

which further gives

$$
d_{0}\left(x, x_{0}\right) \geq d_{-\tau}\left(x, x_{0}\right)-10(n-1)(\tau \sqrt{C}+2 \sqrt{B \tau}) .
$$

This means

$$
B_{(-\tau)}\left(x_{0}, \frac{1}{4}\right) \supset B_{0}\left(x_{0}, \frac{1}{4}-10(n-1)(\tau \sqrt{C}+2 \sqrt{B \tau})\right) .
$$

Note that the scalar curvature $R \geq-C(n)$ for some constant $C(n)$ depending only on the dimension since $R m \geq-1$. We have

$$
\begin{aligned}
& \frac{d}{d t} \operatorname{Vol}_{t}\left(B_{0}\left(x_{0}, \frac{1}{4}-10(n-1)(\tau \sqrt{C}+2 \sqrt{B \tau})\right)\right) \\
& =\int_{B_{0}\left(x_{0}, \frac{1}{4}-10(n-1)(\tau \sqrt{C}+2 \sqrt{B \tau})\right)}(-R) d V_{t} \\
& \leq C(n) \operatorname{Vol}_{t}\left(B_{0}\left(x_{0}, \frac{1}{4}-10(n-1)(\tau \sqrt{C}+2 \sqrt{B \tau})\right)\right)
\end{aligned}
$$

and then

$$
\begin{aligned}
& \operatorname{Vol}_{t}\left(B_{0}\left(x_{0}, \frac{1}{4}-10(n-1)(\tau \sqrt{C}+2 \sqrt{B \tau})\right)\right) \\
& \leq e^{C(n) \tau} \operatorname{Vol}_{(-\tau)}\left(B_{0}\left(x_{0}, \frac{1}{4}-10(n-1)(\tau \sqrt{C}+2 \sqrt{B \tau})\right)\right) .
\end{aligned}
$$

Thus by $(6.3 .6)^{\prime},(6.3 .8)$ and $(6.3 .9)$,

$$
\begin{aligned}
& \operatorname{Vol}_{(-\tau)}\left(B_{(-\tau)}\right)\left(x_{0}, 1\right) \\
& \geq \operatorname{Vol}_{(-\tau)}\left(B_{(-\tau)}\right)\left(x_{0}, \frac{1}{4}\right) \\
& \geq \operatorname{Vol}_{(-\tau)}\left(B_{0}\left(x_{0}, \frac{1}{4}-10(n-1)(\tau \sqrt{C}+2 \sqrt{B \tau})\right)\right) \\
& \geq e^{-C(n) \tau} \operatorname{Vol}_{0}\left(B_{0}\left(x_{0}, \frac{1}{4}-10(n-1)(\tau \sqrt{C}+2 \sqrt{B \tau})\right)\right) \\
& \geq e^{-C(n) \tau} w^{\prime}\left(\frac{1}{4}-10(n-1)(\tau \sqrt{C}+2 \sqrt{B \tau})\right)^{n} .
\end{aligned}
$$

So it suffices to choose $\tau_{0}=\tau_{0}(w)$ small enough so that

$$
e^{-C(n) \tau_{0}}\left(\frac{1}{4}-10(n-1)\left(\tau_{0} \sqrt{C}+2 \sqrt{B \tau_{0}}\right)\right)^{n} \geq\left(\frac{1}{5}\right)^{n} .
$$

Therefore we have proved the theorem. $\square$ 
6.4. Ancient $\kappa$-solutions on Three-manifolds. In this section we will determine the structures of ancient $\kappa$-solutions on three-manifolds.

First of all, we consider a special class of ancient solutions - gradient shrinking Ricci solitons. Recall that a solution $g_{i j}(t)$ to the Ricci flow is said to be a gradient shrinking Ricci soliton if there exists a smooth function $f$ such that

$$
\nabla_{i} \nabla_{j} f+R_{i j}+\frac{1}{2 t} g_{i j}=0 \text { for }-\infty<t<0 .
$$

A gradient shrinking Ricci soliton moves by the one parameter group of diffeomorphisms generated by $\nabla f$ and shrinks by a factor at the same time.

The following result of Perelman [103] gives a complete classification for all threedimensional complete $\kappa$-noncollapsed gradient shrinking solitons with bounded and nonnegative sectional curvature.

LEMma 6.4.1 (Classification of three-dimensional shrinking solitons). Let $\left(M, g_{i j}(t)\right)$ be a nonflat gradient shrinking soliton on a three-manifold. Suppose $\left(M, g_{i j}(t)\right)$ has bounded and nonnegative sectional curvature and is $\kappa$-noncollapsed on all scales for some $\kappa>0$. Then $\left(M, g_{i j}(t)\right)$ is one of the following:

(i) the round three-sphere $\mathbb{S}^{3}$, or a metric quotient of $\mathbb{S}^{3}$;

(ii) the round infinite cylinder $\mathbb{S}^{2} \times \mathbb{R}$, or one of its $\mathbb{Z}_{2}$ quotients.

Proof. We first consider the case that the sectional curvature of the nonflat gradient shrinking soliton is not strictly positive. Let us pull back the soliton to its universal cover. Then the pull-back metric is again a nonflat ancient $\kappa$-solution. By Hamilton's strong maximum principle (Theorem 2.2.1), we know that the pull-back solution splits as the metric product of a two-dimensional nonflat ancient $\kappa$-solution and $\mathbb{R}$. Since the two-dimensional nonflat ancient $\kappa$-solution is simply connected, it follows from Theorem 6.2.2 that it must be the round sphere $\mathbb{S}^{2}$. Thus, the gradient shrinking soliton must be $\mathbb{S}^{2} \times \mathbb{R} / \Gamma$, a metric quotient of the round cylinder.

For each $\sigma \in \Gamma$ and $(x, s) \in \mathbb{S}^{2} \times \mathbb{R}$, we write $\sigma(x, s)=\left(\sigma_{1}(x, s), \sigma_{2}(x, s)\right) \in$ $\mathbb{S}^{2} \times \mathbb{R}$. Since $\sigma$ sends lines to lines, and sends cross spheres to cross spheres, we have $\sigma_{2}(x, s)=\sigma_{2}(y, s)$, for all $x, y \in \mathbb{S}^{2}$. This says that $\sigma_{2}$ reduces to a function of $s$ alone on $\mathbb{R}$. Moreover, for any $(x, s),\left(x^{\prime}, s^{\prime}\right) \in \mathbb{S}^{2} \times \mathbb{R}$, since $\sigma$ preserves the distances between cross spheres $\mathbb{S}^{2} \times\{s\}$ and $\mathbb{S}^{2} \times\left\{s^{\prime}\right\}$, we have $\left|\sigma_{2}(x, s)-\sigma_{2}\left(x^{\prime}, s^{\prime}\right)\right|=\left|s-s^{\prime}\right|$. So the projection $\Gamma_{2}$ of $\Gamma$ to the second factor $\mathbb{R}$ is an isometry subgroup of $\mathbb{R}$. If the metric quotient $\mathbb{S}^{2} \times \mathbb{R} / \Gamma$ were compact, it would not be $\kappa$-noncollapsed on sufficiently large scales as $t \rightarrow-\infty$. Thus the metric quotient $\mathbb{S}^{2} \times \mathbb{R} / \Gamma$ is noncompact. It follows that $\Gamma_{2}=\{1\}$ or $\mathbb{Z}_{2}$. In particular, there is a $\Gamma$-invariant cross sphere $\mathbb{S}^{2}$ in the round cylinder $\mathbb{S}^{2} \times \mathbb{R}$. Denote it by $\mathbb{S}^{2} \times\{0\}$. Then $\Gamma$ acts on the round two-sphere $\mathbb{S}^{2} \times\{0\}$ isometrically without fixed points. This implies $\Gamma$ is either $\{1\}$ or $\mathbb{Z}_{2}$. Hence we conclude that the gradient shrinking soliton is either the round cylinder $\mathbb{S}^{2} \times \mathbb{R}$, or $\mathbb{R} \mathbb{P}^{2} \times \mathbb{R}$, or the twisted product $\mathbb{S}^{2} \tilde{\times} \mathbb{R}$ where $\mathbb{Z}_{2}$ flips both $\mathbb{S}^{2}$ and $\mathbb{R}$.

We next consider the case that the gradient shrinking soliton is compact and has strictly positive sectional curvature everywhere. By the proof of Theorem 5.2.1 (see also Remark 5.2.8) we see that the compact gradient shrinking soliton is getting round and tends to a space form (with positive constant curvature) as the time approaches the maximal time $t=0$. Since the shape of a gradient shrinking Ricci soliton does not change up to reparametrizations and homothetical scalings, the gradient shrinking soliton has to be the round three-sphere $\mathbb{S}^{3}$ or a metric quotient of $\mathbb{S}^{3}$.

Finally we want to exclude the case that the gradient shrinking soliton is noncompact and has strictly positive sectional curvature everywhere. 
Suppose there is a (complete three-dimensional) noncompact $\kappa$-noncollapsed gradient shrinking soliton $g_{i j}(t),-\infty<t<0$, with bounded and positive sectional curvature at each $t \in(-\infty, 0)$ and satisfying the equation (6.4.1). Then as in (6.2.25), we have

$$
\nabla_{i} R=2 R_{i j} \nabla_{j} f
$$

Fix some $t<0$, say $t=-1$, and consider a long shortest geodesic $\gamma(s), 0 \leq s \leq \bar{s}$. Let $x_{0}=\gamma(0)$ and $X(s)=\dot{\gamma}(s)$. Let $U(0)$ be any unit vector orthogonal to $\dot{\gamma}(0)$ and translate $U(0)$ along $\gamma(s)$ to get a parallel vector field $U(s), 0 \leq s \leq \bar{s}$, on $\gamma$. Set

$$
\widetilde{U}(s)= \begin{cases}s U(s), & \text { for } \quad 0 \leq s \leq 1 \\ U(s), & \text { for } \quad 1 \leq s \leq \bar{s}-1 \\ (\bar{s}-s) U(s), & \text { for } \quad \bar{s}-1 \leq s \leq \bar{s}\end{cases}
$$

It follows from the second variation formula of arclength that

$$
\int_{0}^{\bar{s}}\left(|\dot{\widetilde{U}}(s)|^{2}-R(X, \widetilde{U}, X, \widetilde{U})\right) d s \geq 0 .
$$

Since the curvature of the metric $g_{i j}(-1)$ is bounded, we clearly have

$$
\int_{0}^{\bar{s}} R(X, U, X, U) d s \leq \text { const. }
$$

and then

$$
\int_{0}^{\bar{s}} \operatorname{Ric}(X, X) d s \leq \text { const.. }
$$

Moreover, since the curvature of the metric $g_{i j}(-1)$ is positive, it follows from the Cauchy-Schwarz inequality that for any unit vector field $Y$ along $\gamma$ and orthogonal to $X(=\dot{\gamma}(s))$, we have

$$
\begin{aligned}
\int_{0}^{\bar{s}}|\operatorname{Ric}(X, Y)|^{2} d s & \leq \int_{0}^{\bar{s}} \operatorname{Ric}(X, X) \operatorname{Ric}(Y, Y) d s \\
& \leq \text { const. } \cdot \int_{0}^{\bar{s}} \operatorname{Ric}(X, X) d s \\
& \leq \text { const. }
\end{aligned}
$$

and then

$$
\int_{0}^{\bar{s}}|\operatorname{Ric}(X, Y)| d s \leq \text { const. } \cdot(\sqrt{\bar{s}}+1) .
$$

From (6.4.1) we know

$$
\nabla_{X} \nabla_{X} f+\operatorname{Ric}(X, X)-\frac{1}{2}=0
$$

and by integrating this equation we get

$$
X(f(\gamma(\bar{s})))-X(f(\gamma(0)))+\int_{0}^{\bar{s}} \operatorname{Ric}(X, X) d s-\frac{1}{2} \bar{s}=0 .
$$


Thus by (6.4.3) we deduce

$$
\frac{\bar{s}}{2}-\text { const. } \leq\langle X, \nabla f(\gamma(\bar{s}))\rangle \leq \frac{\bar{s}}{2}+\text { const.. }
$$

Similarly by integrating (6.4.1) and using (6.4.4) we can deduce

$$
|\langle Y, \nabla f(\gamma(\bar{s}))\rangle| \leq \text { const. } \cdot(\sqrt{\bar{s}}+1) .
$$

These two inequalities tell us that at large distances from the fixed point $x_{0}$ the function $f$ has no critical point, and its gradient makes a small angle with the gradient of the distance function from $x_{0}$.

Now from (6.4.2) we see that at large distances from $x_{0}, R$ is strictly increasing along the gradient curves of $f$, in particular

$$
\bar{R}=\limsup _{d_{(-1)}\left(x, x_{0}\right) \rightarrow+\infty} R(x,-1)>0 .
$$

Let us choose a sequence of points $\left(x_{k},-1\right)$ where $R\left(x_{k},-1\right) \rightarrow \bar{R}$. By the noncollapsing assumption we can take a limit along this sequence of points of the gradient soliton and get an ancient $\kappa$-solution defined on $-\infty<t<0$. By Proposition 6.1.2, we deduce that the limiting ancient $\kappa$-solution splits off a line. Since the soliton has positive sectional curvature, we know from Gromoll-Meyer [52] that it is orientable. Then it follows from Theorem 6.2.2 that the limiting solution is the shrinking round infinite cylinder with scalar curvature $\bar{R}$ at time $t=-1$. Since the limiting solution exists on $(-\infty, 0)$, we conclude that $\bar{R} \leq 1$. Hence

$$
R(x,-1)<1
$$

when the distance from $x$ to the fixed $x_{0}$ is large enough on the gradient shrinking soliton.

Let us consider the level surface $\{f=a\}$ of $f$. The second fundamental form of the level surface is given by

$$
\begin{aligned}
h_{i j} & =\left\langle\nabla_{i}\left(\frac{\nabla f}{|\nabla f|}\right), e_{j}\right\rangle \\
& =\nabla_{i} \nabla_{j} f /|\nabla f|, \quad i, j=1,2,
\end{aligned}
$$

where $\left\{e_{1}, e_{2}\right\}$ is an orthonormal basis of the level surface. By (6.4.1), we have

$$
\nabla_{e_{i}} \nabla_{e_{i}} f=\frac{1}{2}-\operatorname{Ric}\left(e_{i}, e_{i}\right) \geq \frac{1}{2}-\frac{R}{2}>0, \quad i=1,2,
$$

since for a three-manifold the positivity of sectional curvature is equivalent to $R \geq$ 2 Ric. It then follows from the first variation formula that

$$
\begin{aligned}
\frac{d}{d a} \text { Area }\{f=a\} & =\int_{\{f=a\}} \operatorname{div}\left(\frac{\nabla f}{|\nabla f|}\right) \\
& \geq \int_{\{f=a\}} \frac{1}{|\nabla f|}(1-R) \\
& >\int_{\{f=a\}} \frac{1}{|\nabla f|}(1-\bar{R}) \\
& \geq 0
\end{aligned}
$$


for $a$ large enough. We conclude that Area $\{f=a\}$ strictly increases as $a$ increases. From (6.4.5) we see that for $s$ large enough

$$
\left|\frac{d f}{d s}-\frac{s}{2}\right| \leq \text { const. }
$$

and then

$$
\left|f-\frac{s^{2}}{4}\right| \leq \text { const. } \cdot(s+1)
$$

Thus we get from (6.4.7)

$$
\frac{d}{d a} \text { Area }\{f=a\}>\frac{1-\bar{R}}{2 \sqrt{a}} \text { Area }\{f=a\}
$$

for $a$ large enough. This implies that

$$
\log \text { Area }\{f=a\}>(1-\bar{R}) \sqrt{a}-\text { const. }
$$

for $a$ large enough. But it is clear from (6.4.7) that Area $\{f=a\}$ is uniformly bounded from above by the area of the round sphere of scalar curvature $\bar{R}$ for all large $a$. Thus we deduce that $\bar{R}=1$. So

$$
\text { Area }\{f=a\}<8 \pi
$$

for $a$ large enough.

Denote by $X$ the unit normal vector to the level surface $\{f=a\}$. By using the Gauss equation and (6.4.1), the intrinsic curvature of the level surface $\{f=a\}$ can be computed as

$$
\begin{aligned}
& \text { intrinsic curvature } \\
& =R_{1212}+\operatorname{det}\left(h_{i j}\right) \\
& =\frac{1}{2}(R-2 \operatorname{Ric}(X, X))+\frac{\operatorname{det}(\operatorname{Hess}(f))}{|\nabla f|^{2}} \\
& \leq \frac{1}{2}(R-2 \operatorname{Ric}(X, X))+\frac{1}{4|\nabla f|^{2}}(\operatorname{tr}(\operatorname{Hess}(f)))^{2} \\
& =\frac{1}{2}(R-2 \operatorname{Ric}(X, X))+\frac{1}{4|\nabla f|^{2}}(1-(R-\operatorname{Ric}(X, X)))^{2} \\
& =\frac{1}{2}\left[1-\operatorname{Ric}(X, X)-(1-R+\operatorname{Ric}(X, X))+\frac{(1-R+\operatorname{Ric}(X, X))^{2}}{2|\nabla f|^{2}}\right] \\
& <\frac{1}{2}
\end{aligned}
$$

for sufficiently large $a$, since $(1-R+\operatorname{Ric}(X, X))>0$ and $|\nabla f|$ is large when $a$ is large. Thus the combination of (6.4.8) and (6.4.9) gives a contradiction to the Gauss-Bonnet formula.

Therefore we have proved the lemma. $\mathrm{Q}$

As a direct consequence, there is a universal positive constant $\kappa_{0}$ such that any nonflat three-dimensional gradient shrinking soliton, which is also an ancient 
$\kappa$-solution, to the Ricci flow must be $\kappa_{0}$-noncollapsed on all scales unless it is a metric quotient of round three-sphere. The following result, claimed by Perelman in the section 1.5 of [104], shows that this property actually holds for all nonflat threedimensional ancient $\kappa$-solutions.

Proposition 6.4.2 (Universal noncollapsing). There exists a positive constant $\kappa_{0}$ with the following property. Suppose we have a nonflat three-dimensional ancient $\kappa$-solution for some $\kappa>0$. Then either the solution is $\kappa_{0}$-noncollapsed on all scales, or it is a metric quotient of the round three-sphere.

Proof. Let $g_{i j}(x, t), x \in M$ and $t \in(-\infty, 0]$, be a nonflat ancient $\kappa$-solution for some $\kappa>0$. For an arbitrary point $\left(p, t_{0}\right) \in M \times(-\infty, 0]$, we define as in Chapter 3 that

$$
\begin{aligned}
& \tau=t_{0}-t, \quad \text { for } t<t_{0}, \\
& l(q, \tau)=\frac{1}{2 \sqrt{\tau}} \inf \left\{\int_{0}^{\tau} \sqrt{s}\left(R\left(\gamma(s), t_{0}-s\right)+|\dot{\gamma}(s)|_{g_{i j}\left(t_{0}-s\right)}^{2}\right) d s \mid\right. \\
& \gamma:[0, \tau] \rightarrow M \text { with } \gamma(0)=p, \gamma(\tau)=q\}, \\
& \text { and } \widetilde{V}(\tau)=\int_{M}(4 \pi \tau)^{-\frac{3}{2}} \exp (-l(q, \tau)) d V_{t_{0}-\tau}(q) \text {. }
\end{aligned}
$$

Recall from (6.2.1) that for each $\tau>0$ we can find $q=q(\tau)$ such that $l(q, \tau) \leq \frac{3}{2}$. In view of Lemma 6.4.1, we may assume that the ancient $\kappa$-solution is not a gradient shrinking Ricci soliton. Thus by (the proof of) Theorem 6.2.1, the scalings of $g_{i j}\left(t_{0}-\tau\right)$ at $q(\tau)$ with factor $\tau^{-1}$ converge along a subsequence of $\tau \rightarrow+\infty$ to a nonflat gradient shrinking soliton with nonnegative curvature operator which is $\kappa$-noncollapsed on all scales. We now show that the limit has bounded curvature.

Denote the limiting nonflat gradient shrinking soliton by $\left(\bar{M}, \bar{g}_{i j}(x, t)\right)$ with $-\infty<$ $t \leq 0$. Note that there holds the Li-Yau-Hamilton inequality (Theorem 2.5.4) on any ancient $\kappa$-solution and in particular, the scalar curvature of the ancient $\kappa$-solution is pointwise nondecreasing in time. This implies that the scalar curvature of the limiting soliton $\left(\bar{M}, \bar{g}_{i j}(x, t)\right)$ is still pointwise nondecreasing in time. Thus we only need to show that the limiting soliton has bounded curvature at $t=0$.

We argue by contradiction. By lifting to its orientable cover, we may assume that $\bar{M}$ is orientable. Suppose the curvature of the limiting soliton is unbounded at $t=0$. Of course in this case the limiting soliton $\bar{M}$ is noncompact. Then by applying Lemma 6.1 .4 , we can choose a sequence of points $x_{j}, j=1,2, \ldots$, divergent to infinity such that the scalar curvature $\bar{R}$ of the limit satisfies

$$
\bar{R}\left(x_{j}, 0\right) \geq j \text { and } \bar{R}(x, 0) \leq 4 \bar{R}\left(x_{j}, 0\right)
$$

for all $x \in B_{0}\left(x_{j}, j / \sqrt{R\left(x_{j}, 0\right)}\right)$ and $j=1,2, \ldots$ Since the scalar curvature is nondecreasing in time, we have

$$
\bar{R}(x, t) \leq 4 \bar{R}\left(x_{j}, 0\right),
$$

for all $x \in B_{0}\left(x_{j}, j / \sqrt{R\left(x_{j}, 0\right)}\right)$, all $t \leq 0$ and $j=1,2, \ldots$ By combining with Hamilton's compactness theorem (Theorem 4.1.5) and the $\kappa$-noncollapsing, we know that a subsequence of the rescaled solutions

$$
\left(\bar{M}, \bar{R}\left(x_{j}, 0\right) \bar{g}_{i j}\left(x, t / \bar{R}\left(x_{j}, 0\right)\right), x_{j}\right), \quad j=1,2, \ldots,
$$


converges in the $C_{\mathrm{loc}}^{\infty}$ topology to a nonflat smooth solution of the Ricci flow. Then Proposition 6.1.2 implies that the new limit at the new time $\{t=0\}$ must split off a line. By pulling back the new limit to its universal cover and applying Hamilton's strong maximum principle, we deduce that the pull-back of the new limit on the universal cover splits off a line for all time $t \leq 0$. Thus by combining with Theorem 6.2.2 and the argument in the proof of Lemma 6.4.1, we further deduce that the new limit is either the round cylinder $\mathbb{S}^{2} \times \mathbb{R}$ or the round $\mathbb{R P}^{2} \times \mathbb{R}$. Since $\bar{M}$ is orientable, the new limit must be $\mathbb{S}^{2} \times \mathbb{R}$. Since $\left(\bar{M}, \bar{g}_{i j}(x, 0)\right)$ has nonnegative curvature operator and the points $\left\{x_{j}\right\}$ going to infinity and $\bar{R}\left(x_{j}, 0\right) \rightarrow+\infty$, this gives a contradiction to Proposition 6.1.1. So we have proved that the limiting gradient shrinking soliton has bounded curvature at each time.

Hence by Lemma 6.4.1, the limiting gradient shrinking soliton is either the round three-sphere $\mathbb{S}^{3}$ or its metric quotients, or the infinite cylinder $\mathbb{S}^{2} \times \mathbb{R}$ or one of its $\mathbb{Z}_{2}$ quotients. If the asymptotic gradient shrinking soliton is the round three-sphere $\mathbb{S}^{3}$ or its metric quotients, it follows from Lemma 5.2.4 and Proposition 5.2.5 that the ancient $\kappa$-solution must be round. Thus in the following we may assume the asymptotic gradient shrinking soliton is the infinite cylinder $\mathbb{S}^{2} \times \mathbb{R}$ or a $\mathbb{Z}_{2}$ quotient of $\mathbb{S}^{2} \times \mathbb{R}$.

We now come back to consider the original ancient $\kappa$-solution $\left(M, g_{i j}(x, t)\right)$. By rescaling, we can assume that $R(x, t) \leq 1$ for all $(x, t)$ satisfying $d_{t_{0}}(x, p) \leq 2$ and $t \in\left[t_{0}-1, t_{0}\right]$. We will argue as in the proof of Theorem 3.3.2 (Perelman's no local collapsing theorem I) to obtain a positive lower bound for $\operatorname{Vol}_{t_{0}}\left(B_{t_{0}}(p, 1)\right)$.

Denote by $\xi=\operatorname{Vol}_{t_{0}}\left(B_{t_{0}}(p, 1)\right)^{\frac{1}{3}}$. For any $v \in T_{p} M$ we can find an $\mathcal{L}$-geodesic $\gamma(\tau)$, starting at $p$, with $\lim _{\tau \rightarrow 0^{+}} \sqrt{\tau} \dot{\gamma}(\tau)=v$. It follows from the $\mathcal{L}$-geodesic equation (3.2.1) that

$$
\frac{d}{d \tau}(\sqrt{\tau} \dot{\gamma})-\frac{1}{2} \sqrt{\tau} \nabla R+2 \operatorname{Ric}(\sqrt{\tau} \dot{\gamma}, \cdot)=0
$$

By integrating as before we see that for $\tau \leq \xi$ with the property $\gamma(\sigma) \in B_{t_{0}}(p, 1)$ as long as $\sigma<\tau$, there holds

$$
|\sqrt{\tau} \dot{\gamma}(\tau)-v| \leq C \xi(|v|+1)
$$

where $C$ is some positive constant depending only on the dimension. Without loss of generality, we may assume $C \xi \leq \frac{1}{4}$ and $\xi \leq \frac{1}{100}$. Then for $v \in T_{p} M$ with $|v| \leq \frac{1}{4} \xi^{-\frac{1}{2}}$ and for $\tau \leq \xi$ with the property $\gamma(\sigma) \in B_{t_{0}}(p, 1)$ as long as $\sigma<\tau$, we have

$$
\begin{aligned}
d_{t_{0}}(p, \gamma(\tau)) & \leq \int_{0}^{\tau}|\dot{\gamma}(\sigma)| d \sigma \\
& <\frac{1}{2} \xi^{-\frac{1}{2}} \int_{0}^{\tau} \frac{d \sigma}{\sqrt{\sigma}} \\
& =1 .
\end{aligned}
$$

This shows

$$
\mathcal{L} \exp \left\{|v| \leq \frac{1}{4} \xi^{-\frac{1}{2}}\right\}(\xi) \subset B_{t_{0}}(p, 1)
$$


We decompose Perelman's reduced volume $\widetilde{V}(\xi)$ as

$$
\begin{aligned}
\widetilde{V}(\xi)= & \int_{\mathcal{L} \exp \left\{|v| \leq \frac{1}{4} \xi^{-\frac{1}{2}}\right\}(\xi)} \\
& +\int_{M \backslash \mathcal{L} \exp \left\{|v| \leq \frac{1}{4} \xi^{-\frac{1}{2}}\right\}(\xi)}(4 \pi \xi)^{-\frac{3}{2}} \exp (-l(q, \xi)) d V_{t_{0}-\xi}(q) .
\end{aligned}
$$

By using (6.4.11) and the metric evolution equation of the Ricci flow, the first term on the RHS of (6.4.12) can be estimated by

$$
\begin{aligned}
& \int_{\mathcal{L} \exp \left\{|v| \leq \frac{1}{4} \xi^{-\frac{1}{2}}\right\}(\xi)}(4 \pi \xi)^{-\frac{3}{2}} \exp (-l(q, \xi)) d V_{t_{0}-\xi}(q) \\
& \leq \int_{B_{t_{0}}(p, 1)}(4 \pi \xi)^{-\frac{3}{2}} e^{3 \xi} d V_{t_{0}}(q) \\
& =(4 \pi)^{-\frac{3}{2}} e^{3 \xi} \xi^{\frac{3}{2}} \\
& <\xi^{\frac{3}{2}}
\end{aligned}
$$

while by using Theorem 3.2.7 (Perelman's Jacobian comparison theorem), the second term on the RHS of (6.4.12) can be estimated by

$$
\begin{aligned}
& \int_{M \backslash \mathcal{L} \exp \left\{|v| \leq \frac{1}{4} \xi^{-\frac{1}{2}}\right\}(\xi)}(4 \pi \xi)^{-\frac{3}{2}} \exp (-l(q, \xi)) d V_{t_{0}-\xi}(q) \\
& \leq\left.\int_{\left\{|v|>\frac{1}{4} \xi^{-\frac{1}{2}}\right\}}(4 \pi \tau)^{-\frac{3}{2}} \exp (-l(\tau)) \mathcal{J}(\tau)\right|_{\tau=0} d v \\
& =(4 \pi)^{-\frac{3}{2}} \int_{\left\{|v|>\frac{1}{4} \xi^{-\frac{1}{2}}\right\}} \exp \left(-|v|^{2}\right) d v \\
& <\xi^{\frac{3}{2}}
\end{aligned}
$$

since $\lim _{\tau \rightarrow 0^{+}} \tau^{-\frac{3}{2}} \mathcal{J}(\tau)=1$ and $\lim _{\tau \rightarrow 0^{+}} l(\tau)=|v|^{2}$ by (3.2.18) and (3.2.19) respectively. Thus we obtain

$$
\widetilde{V}(\xi)<2 \xi^{\frac{3}{2}}
$$

On the other hand, we recall that there exist a sequence $\tau_{k} \rightarrow+\infty$ and a sequence of points $q\left(\tau_{k}\right) \in M$ with $l\left(q\left(\tau_{k}\right), \tau_{k}\right) \leq \frac{3}{2}$ so that the scalings of the ancient $\kappa$-solution at $q\left(\tau_{k}\right)$ with factor $\tau_{k}^{-1}$ converge to either round $\mathbb{S}^{2} \times \mathbb{R}$ or one of its $\mathbb{Z}_{2}$ quotients. For sufficiently large $k$, we construct a path $\gamma: \quad\left[0,2 \tau_{k}\right] \rightarrow M$, connecting $p$ to any given point $q \in M$, as follows: the first half path $\left.\gamma\right|_{\left[0, \tau_{k}\right]}$ connects $p$ to $q\left(\tau_{k}\right)$ such that

$$
l\left(q\left(\tau_{k}\right), \tau_{k}\right)=\frac{1}{2 \sqrt{\tau_{k}}} \int_{0}^{\tau_{k}} \sqrt{\tau}\left(R+|\dot{\gamma}(\tau)|^{2}\right) d \tau \leq 2,
$$

and the second half path $\left.\gamma\right|_{\left[\tau_{k}, 2 \tau_{k}\right]}$ is a shortest geodesic connecting $q\left(\tau_{k}\right)$ to $q$ with respect to the metric $g_{i j}\left(t_{0}-\tau_{k}\right)$. Note that the rescaled metric $\tau_{k}^{-1} g_{i j}\left(t_{0}-\tau\right)$ over the domain $B_{t_{0}-\tau_{k}}\left(q\left(\tau_{k}\right), \sqrt{\tau_{k}}\right) \times\left[t_{0}-2 \tau_{k}, t_{0}-\tau_{k}\right]$ is sufficiently close to the round 
$\mathbb{S}^{2} \times \mathbb{R}$ or its $\mathbb{Z}_{2}$ quotients. Then there is a universal positive constant $\beta$ such that

$$
\begin{aligned}
l\left(q, 2 \tau_{k}\right) & \leq \frac{1}{2 \sqrt{2 \tau_{k}}}\left(\int_{0}^{\tau_{k}}+\int_{\tau_{k}}^{2 \tau_{k}}\right) \sqrt{\tau}\left(R+|\dot{\gamma}(\tau)|^{2}\right) d \tau \\
& \leq \sqrt{2}+\frac{1}{2 \sqrt{2 \tau_{k}}} \int_{\tau_{k}}^{2 \tau_{k}} \sqrt{\tau}\left(R+|\dot{\gamma}(\tau)|^{2}\right) d \tau \\
& \leq \beta
\end{aligned}
$$

for all $q \in B_{t_{0}-\tau_{k}}\left(q\left(\tau_{k}\right), \sqrt{\tau_{k}}\right)$. Thus

$$
\begin{aligned}
\tilde{V}\left(2 \tau_{k}\right) & =\int_{M}\left(4 \pi\left(2 \tau_{k}\right)\right)^{-\frac{3}{2}} \exp \left(-l\left(q, 2 \tau_{k}\right)\right) d V_{t_{0}-2 \tau_{k}}(q) \\
& \geq e^{-\beta} \int_{B_{t_{0}-\tau_{k}}\left(q\left(\tau_{k}\right), \sqrt{\tau_{k}}\right)}\left(4 \pi\left(2 \tau_{k}\right)\right)^{-\frac{3}{2}} d V_{t_{0}-2 \tau_{k}}(q) \\
& \geq \tilde{\beta}
\end{aligned}
$$

for some universal positive constant $\tilde{\beta}$. Here we have used the curvature estimate (6.2.6). By combining with the monotonicity of Perelman's reduced volume (Theorem 3.2.8) and (6.4.14), we deduce that

$$
\tilde{\beta} \leq \tilde{V}\left(2 \tau_{k}\right) \leq \tilde{V}(\xi)<2 \xi^{\frac{3}{2}} .
$$

This proves

$$
\operatorname{Vol}_{t_{0}}\left(B_{t_{0}}(p, 1)\right) \geq \kappa_{0}>0
$$

for some universal positive constant $\kappa_{0}$. So we have proved that the ancient $\kappa$-solution is also an ancient $\kappa_{0}$-solution.

The important Li-Yau-Hamilton inequality gives rise to a parabolic Harnack estimate (Corollary 2.5.7) for solutions of the Ricci flow with bounded and nonnegative curvature operator. As explained in the previous section, the no local collapsing theorem of Perelman implies a volume lower bound from a curvature upper bound, while the estimate in the previous section implies a curvature upper bound from a volume lower bound. The combination of these two estimates as well as the Li-Yau-Hamilton inequality will give an important elliptic type property for three-dimensional ancient $\kappa$-solutions. This elliptic type property was first implicitly given by Perelman in [103] and it will play a crucial role in the analysis of singularities.

THEOREM 6.4 .3 (Elliptic type estimate). There exist a positive constant $\eta$ and a positive increasing function $\omega: \quad[0,+\infty) \rightarrow(0,+\infty)$ with the following properties. Suppose we have a three-dimensional ancient $\kappa$-solution $\left(M, g_{i j}(t)\right),-\infty<t \leq 0$, for some $\kappa>0$. Then

(i) for every $x, y \in M$ and $t \in(-\infty, 0]$, there holds

$$
R(x, t) \leq R(y, t) \cdot \omega\left(R(y, t) d_{t}^{2}(x, y)\right) ;
$$

(ii) for all $x \in M$ and $t \in(-\infty, 0]$, there hold

$$
|\nabla R|(x, t) \leq \eta R^{\frac{3}{2}}(x, t) \text { and } \quad\left|\frac{\partial R}{\partial t}\right|(x, t) \leq \eta R^{2}(x, t) .
$$


Proof. (i) Consider a three-dimensional nonflat ancient $\kappa$-solution $g_{i j}(x, t)$ on $M \times(-\infty, 0]$. In view of Proposition 6.4.2, we may assume that the ancient solution is universal $\kappa_{0}$-noncollapsed. Obviously we only need to establish the estimate at $t=0$. Let $y$ be an arbitrarily fixed point in $M$. By rescaling, we can assume $R(y, 0)=1$.

Let us first consider the case that $\sup \left\{R(x, 0) d_{0}^{2}(x, y) \mid x \in M\right\}>1$. Define $z$ to be the closest point to $y$ (at time $t=0$ ) satisfying $R(z, 0) d_{0}^{2}(z, y)=1$. We want to bound $R(x, 0) / R(z, 0)$ from above for $x \in B_{0}\left(z, 2 R(z, 0)^{-\frac{1}{2}}\right)$.

Connect $y$ and $z$ by a shortest geodesic and choose a point $\tilde{z}$ lying on the geodesic satisfying $d_{0}(\tilde{z}, z)=\frac{1}{4} R(z, 0)^{-\frac{1}{2}}$. Denote by $B$ the ball centered at $\tilde{z}$ and with radius $\frac{1}{4} R(z, 0)^{-\frac{1}{2}}$ (with respect to the metric at $t=0$ ). Clearly the ball $B$ lies in $B_{0}\left(y, R(z, 0)^{-\frac{1}{2}}\right)$ and lies outside $B_{0}\left(y, \frac{1}{2} R(z, 0)^{-\frac{1}{2}}\right)$. Thus for $x \in B$, we have

$$
R(x, 0) d_{0}^{2}(x, y) \leq 1 \quad \text { and } \quad d_{0}(x, y) \geq \frac{1}{2} R(z, 0)^{-\frac{1}{2}}
$$

and hence

$$
R(x, 0) \leq \frac{1}{\left(\frac{1}{2} R(z, 0)^{-\frac{1}{2}}\right)^{2}} \quad \text { for all } x \in B .
$$

Then by the Li-Yau-Hamilton inequality and the $\kappa_{0}$-noncollapsing, we have

$$
\operatorname{Vol}_{0}(B) \geq \kappa_{0}\left(\frac{1}{4} R(z, 0)^{-\frac{1}{2}}\right)^{3}
$$

and then

$$
\operatorname{Vol}_{0}\left(B_{0}\left(z, 8 R(z, 0)^{-\frac{1}{2}}\right)\right) \geq \frac{\kappa_{0}}{2^{15}}\left(8 R(z, 0)^{-\frac{1}{2}}\right)^{3} .
$$

So by Theorem 6.3.3(ii), there exist positive constants $B\left(\kappa_{0}\right), C\left(\kappa_{0}\right)$, and $\tau_{0}\left(\kappa_{0}\right)$ such that

$$
R(x, 0) \leq\left(C\left(\kappa_{0}\right)+\frac{B\left(\kappa_{0}\right)}{\tau_{0}\left(\kappa_{0}\right)}\right) R(z, 0)
$$

for all $x \in B_{0}\left(z, 2 R(z, 0)^{-\frac{1}{2}}\right)$.

We now consider the remaining case. If $R(x, 0) d_{0}^{2}(x, y) \leq 1$ everywhere, we choose a point $z$ satisfying $\sup \{R(x, 0) \mid x \in M\} \leq 2 R(z, 0)$. Obviously we also have the estimate (6.4.15) in this case.

We next want to bound $R(z, 0)$ for the chosen $z \in M$. By (6.4.15) and the Li-Yau-Hamilton inequality, we have

$$
R(x, t) \leq\left(C\left(\kappa_{0}\right)+\frac{B\left(\kappa_{0}\right)}{\tau_{0}\left(\kappa_{0}\right)}\right) R(z, 0)
$$

for all $x \in B_{0}\left(z, 2 R(z, 0)^{-\frac{1}{2}}\right)$ and all $t \leq 0$. It then follows from the local derivative estimates of Shi that

$$
\frac{\partial R}{\partial t}(z, t) \leq \widetilde{C}\left(\kappa_{0}\right) R(z, 0)^{2}, \quad \text { for all }-R^{-1}(z, 0) \leq t \leq 0
$$

which implies

$$
R\left(z,-c R^{-1}(z, 0)\right) \geq c R(z, 0)
$$


for some small positive constant $c$ depending only on $\kappa_{0}$. On the other hand, by using the Harnack estimate in Corollary 2.5.7, we have

$$
1=R(y, 0) \geq \widetilde{c} R\left(z,-c R^{-1}(z, 0)\right)
$$

for some small positive constant $\widetilde{c}$ depending only on $\kappa_{0}$, since $d_{0}(y, z) \leq R(z, 0)^{-\frac{1}{2}}$ and the metric $g_{i j}(t)$ is equivalent on

$$
B_{0}\left(z, 2 R(z, 0)^{-\frac{1}{2}}\right) \times\left[-c R^{-1}(z, 0), 0\right]
$$

with $c>0$ small enough. Thus we get from (6.4.16) and (6.4.17) that

$$
R(z, 0) \leq \widetilde{A}
$$

for some positive constant $\widetilde{A}$ depending only on $\kappa_{0}$.

Since $B_{0}\left(z, 2 R(z, 0)^{-\frac{1}{2}}\right) \supset B_{0}\left(y, R(z, 0)^{-\frac{1}{2}}\right)$ and $R(z, 0)^{-\frac{1}{2}} \geq(\widetilde{A})^{-\frac{1}{2}}$, the combination of (6.4.15) and (6.4.18) gives

$$
R(x, 0) \leq\left(C\left(\kappa_{0}\right)+\frac{B\left(\kappa_{0}\right)}{\tau_{0}\left(\kappa_{0}\right)}\right) \widetilde{A}
$$

whenever $x \in B_{0}\left(y,(\widetilde{A})^{-\frac{1}{2}}\right)$. Then by the $\kappa_{0}$-noncollapsing there exists a positive constant $r_{0}$ depending only on $\kappa_{0}$ such that

$$
\operatorname{Vol}_{0}\left(B_{0}\left(y, r_{0}\right)\right) \geq \kappa_{0} r_{0}^{3} .
$$

For any fixed $R_{0} \geq r_{0}$, we then have

$$
\operatorname{Vol}_{0}\left(B_{0}\left(y, R_{0}\right)\right) \geq \kappa_{0} r_{0}^{3}=\kappa_{0}\left(\frac{r_{0}}{R_{0}}\right)^{3} \cdot R_{0}^{3} .
$$

By applying Theorem 6.3.3 (ii) again and noting that the constant $\kappa_{0}$ is universal, there exists a positive constant $\omega\left(R_{0}\right)$ depending only on $R_{0}$ such that

$$
R(x, 0) \leq \omega\left(R_{0}^{2}\right) \quad \text { for all } x \in B_{0}\left(y, \frac{1}{4} R_{0}\right) .
$$

This gives the desired estimate.

(ii) This follows immediately from conclusion (i), the Li-Yau-Hamilton inequality and the local derivative estimate of Shi.

As a consequence, we have the following compactness result due to Perelman [103].

Corollary 6.4.4 (Compactness of ancient $\kappa_{0}$-solutions). The set of nonflat three-dimensional ancient $\kappa_{0}$-solutions is compact modulo scaling in the sense that for any sequence of such solutions and marking points $\left(x_{k}, 0\right)$ with $R\left(x_{k}, 0\right)=1$, we can extract a $C_{\text {loc }}^{\infty}$ converging subsequence whose limit is also an ancient $\kappa_{0}$-solution.

Proof. Consider any sequence of three-dimensional ancient $\kappa_{0}$-solutions and marking points $\left(x_{k}, 0\right)$ with $R\left(x_{k}, 0\right)=1$. By Theorem 6.4.3(i), the Li-Yau-Hamilton inequality and Hamilton's compactness theorem (Theorem 4.1.5), we can extract a $C_{l o c}^{\infty}$ converging subsequence such that the limit $\left(\bar{M}, \bar{g}_{i j}(x, t)\right)$, with $-\infty<t \leq 0$, is an ancient solution to the Ricci flow with nonnegative curvature operator and $\kappa_{0^{-}}$ noncollapsed on all scales. Since any ancient $\kappa_{0}$-solution satisfies the Li-Yau-Hamilton 
inequality, it implies that the scalar curvature $\bar{R}(x, t)$ of the limit $\left(\bar{M}, \bar{g}_{i j}(x, t)\right)$ is pointwise nondecreasing in time. Thus it remains to show that the limit solution has bounded curvature at $t=0$.

Obviously we may assume the limiting manifold $\bar{M}$ is noncompact. By pulling back the limiting solution to its orientable cover, we can assume that the limiting manifold $\bar{M}$ is orientable. We now argue by contradiction. Suppose the scalar curvature $\bar{R}$ of the limit at $t=0$ is unbounded.

By applying Lemma 6.1.4, we can choose a sequence of points $x_{j} \in \bar{M}, j=$ $1,2, \ldots$, divergent to infinity such that the scalar curvature $\bar{R}$ of the limit satisfies

$$
\bar{R}\left(x_{j}, 0\right) \geq j \text { and } \bar{R}(x, 0) \leq 4 \bar{R}\left(x_{j}, 0\right)
$$

for all $j=1,2, \ldots$, and $x \in B_{0}\left(x_{j}, j / \sqrt{\bar{R}\left(x_{j}, 0\right)}\right)$. Then from the fact that the limiting scalar curvature $\bar{R}(x, t)$ is pointwise nondecreasing in time, we have

$$
\bar{R}(x, t) \leq 4 \bar{R}\left(x_{j}, 0\right)
$$

for all $j=1,2, \ldots, x \in B_{0}\left(x_{j}, j / \sqrt{R\left(x_{j}, 0\right)}\right)$ and $t \leq 0$. By combining with Hamilton's compactness theorem (Theorem 4.1.5) and the $\kappa_{0}$-noncollapsing, we know that a subsequence of the rescaled solutions

$$
\left(\bar{M}, \bar{R}\left(x_{j}, 0\right) \bar{g}_{i j}\left(x, t / \bar{R}\left(x_{j}, 0\right)\right), x_{j}\right), \quad j=1,2, \ldots,
$$

converges in the $C_{l o c}^{\infty}$ topology to a nonflat smooth solution of the Ricci flow. Then Proposition 6.1.2 implies that the new limit at the new time $\{t=0\}$ must split off a line. By pulling back the new limit to its universal cover and applying Hamilton's strong maximum principle, we deduce that the pull-back of the new limit on the universal cover splits off a line for all time $t \leq 0$. Thus by combining with Theorem 6.2.2 and the argument in the proof of Lemma 6.4.1, we further deduce that the new limit is either the round cylinder $\mathbb{S}^{2} \times \mathbb{R}$ or the round $\mathbb{R P}^{2} \times \mathbb{R}$. Since $\bar{M}$ is orientable, the new limit must be $\mathbb{S}^{2} \times \mathbb{R}$. Moreover, since $\left(\bar{M}, \bar{g}_{i j}(x, 0)\right)$ has nonnegative curvature operator and the points $\left\{x_{j}\right\}$ are going to infinity and $\bar{R}\left(x_{j}, 0\right) \rightarrow+\infty$, this gives a contradiction to Proposition 6.1.1. So we have proved that the limit $\left(\bar{M}, \bar{g}_{i j}(x, t)\right)$ has uniformly bounded curvature.

Arbitrarily fix $\varepsilon>0$. Let $g_{i j}(x, t)$ be a nonflat ancient $\kappa$-solution on a threemanifold $M$ for some $\kappa>0$. We say that a point $x_{0} \in M$ is the center of an evolving $\varepsilon$-neck at $t=0$, if the solution $g_{i j}(x, t)$ in the set $\left\{(x, t) \mid-\varepsilon^{-2} Q^{-1}<t \leq\right.$ $\left.0, d_{t}^{2}\left(x, x_{0}\right)<\varepsilon^{-2} Q^{-1}\right\}$, where $Q=R\left(x_{0}, 0\right)$, is, after scaling with factor $Q$, $\varepsilon$-close (in the $C^{\left[\varepsilon^{-1}\right]}$ topology) to the corresponding set of the evolving round cylinder having scalar curvature one at $t=0$.

As another consequence of the elliptic type estimate, we have the following global structure result obtained by Perelman in [103] for noncompact ancient $\kappa$-solutions.

Corollary 6.4.5. For any $\varepsilon>0$ there exists $C=C(\varepsilon)>0$, such that if $g_{i j}(t)$ is a nonflat ancient $\kappa$-solution on a noncompact three-manifold $M$ for some $\kappa>0$, and $M_{\varepsilon}$ denotes the set of points in $M$ which are not centers of evolving $\varepsilon$-necks at $t=0$, then at $t=0$, either the whole manifold $M$ is the round cylinder $\mathbb{S}^{2} \times \mathbb{R}$ or its $\mathbb{Z}_{2}$ metric quotients, or $M_{\varepsilon}$ satisfies the following

(i) $M_{\varepsilon}$ is compact,

(ii) diam $M_{\varepsilon} \leq C Q^{-\frac{1}{2}}$ and $C^{-1} Q \leq R(x, 0) \leq C Q$, whenever $x \in M_{\varepsilon}$, where $Q=R\left(x_{0}, 0\right)$ for some $x_{0} \in \partial M_{\varepsilon}$. 
Proof. We first consider the easy case that the curvature operator of the ancient $\kappa$-solution has a nontrivial null vector somewhere at some time. Let us pull back the solution to its universal cover. By applying Hamilton's strong maximum principle and Theorem 6.2.2, we see that the universal cover is the evolving round cylinder $\mathbb{S}^{2} \times \mathbb{R}$. Thus in this case, by the argument in the proof of Lemma 6.4.1, we conclude that the ancient $\kappa$-solution is either isometric to the round cylinder $\mathbb{S}^{2} \times \mathbb{R}$ or one of its $\mathbb{Z}_{2}$ metric quotients (i.e., $\mathbb{R}^{2} \times \mathbb{R}$, or the twisted product $\mathbb{S}^{2} \tilde{\times} \mathbb{R}$ where $\mathbb{Z}_{2}$ flips both $\mathbb{S}^{2}$, or $\mathbb{R})$.

We then assume that the curvature operator of the nonflat ancient $\kappa$-solution is positive everywhere. Firstly we want to show $M_{\varepsilon}$ is compact. We argue by contradiction. Suppose there exists a sequence of points $z_{k}, k=1,2, \ldots$, going to infinity (with respect to the metric $g_{i j}(0)$ ) such that each $z_{k}$ is not the center of any evolving $\varepsilon$-neck. For an arbitrarily fixed point $z_{0} \in M$, it follows from Theorem 6.4.3(i) that

$$
0<R\left(z_{0}, 0\right) \leq R\left(z_{k}, 0\right) \cdot \omega\left(R\left(z_{k}, 0\right) d_{0}^{2}\left(z_{k}, z_{0}\right)\right)
$$

which implies that

$$
\lim _{k \rightarrow \infty} R\left(z_{k}, 0\right) d_{0}^{2}\left(z_{k}, z_{0}\right)=+\infty
$$

Since the sectional curvature of the ancient $\kappa$-solution is positive everywhere, the underlying manifold is diffeomorphic to $\mathbb{R}^{3}$, and in particular, orientable. Then as before, by Proposition 6.1.2, Theorem 6.2.2 and Corollary 6.4.4, we conclude that $z_{k}$ is the center of an evolving $\varepsilon$-neck for $k$ sufficiently large. This is a contradiction, so we have proved that $M_{\varepsilon}$ is compact.

Again, we notice that $M$ is diffeomorphic to $\mathbb{R}^{3}$ since the curvature operator is positive. According to the resolution of the Schoenflies conjecture in three-dimensions, every approximately round two-sphere cross-section through the center of an evolving $\varepsilon$-neck divides $M$ into two parts such that one of them is diffeomorphic to the three-ball $\mathbb{B}^{3}$. Let $\varphi$ be the Busemann function on $M$, it is a standard fact that $\varphi$ is convex and proper. Since $M_{\varepsilon}$ is compact, $M_{\varepsilon}$ is contained in a compact set $K=\varphi^{-1}((-\infty, A])$ for some large $A$. We note that each point $x \in M \backslash M_{\varepsilon}$ is the center of an $\varepsilon$-neck. It is clear that there is an $\varepsilon$-neck $N$ lying entirely outside $K$. Consider a point $x$ on one of the boundary components of the $\varepsilon$-neck $N$. Since $x \in M \backslash M_{\varepsilon}$, there is an $\varepsilon$-neck adjacent to the initial $\varepsilon$-neck, producing a longer neck. We then take a point on the boundary of the second $\varepsilon$-neck and continue. This procedure can either terminate when we get into $M_{\varepsilon}$ or go on infinitely to produce a semi-infinite (topological) cylinder. The same procedure can be repeated for the other boundary component of the initial $\varepsilon$-neck. This procedure will give a maximal extended neck $\tilde{N}$. If $\tilde{N}$ never touches $M_{\varepsilon}$, the manifold will be diffeomorphic to the standard infinite cylinder, which is a contradiction. If both ends of $\tilde{N}$ touch $M_{\varepsilon}$, then there is a geodesic connecting two points of $M_{\varepsilon}$ and passing through $N$. This is impossible since the function $\varphi$ is convex. So we conclude that one end of $\tilde{N}$ will touch $M_{\varepsilon}$ and the other end will tend to infinity to produce a semi-infinite (topological) cylinder. Thus we can find an approximately round two-sphere cross-section which encloses the whole set $M_{\varepsilon}$ and touches some point $x_{0} \in \partial M_{\varepsilon}$. We next want to show that $R\left(x_{0}, 0\right)^{\frac{1}{2}} \cdot \operatorname{diam}\left(M_{\varepsilon}\right)$ is bounded from above by some positive constant $C=C(\varepsilon)$ depending only on $\varepsilon$.

Suppose not; then there exists a sequence of nonflat noncompact threedimensional ancient $\kappa$-solutions with positive curvature operator such that for the above chosen points $x_{0} \in \partial M_{\varepsilon}$ there would hold

$$
R\left(x_{0}, 0\right)^{\frac{1}{2}} \cdot \operatorname{diam}\left(M_{\varepsilon}\right) \rightarrow+\infty .
$$


By Proposition 6.4.2, we know that the ancient solutions are $\kappa_{0}$-noncollapsed on all scales for some universal positive constant $\kappa_{0}$. Let us dilate the ancient solutions around the points $x_{0}$ with the factors $R\left(x_{0}, 0\right)$. By Corollary 6.4.4, we can extract a convergent subsequence. From the choice of the points $x_{0}$ and (6.4.21), the limit has at least two ends. Then by Toponogov's splitting theorem the limit is isometric to $X \times \mathbb{R}$ for some nonflat two-dimensional ancient $\kappa_{0}$-solution $X$. Since $M$ is orientable, we conclude from Theorem 6.2.2 that limit must be the evolving round cylinder $\mathbb{S}^{2} \times \mathbb{R}$. This contradicts the fact that each chosen point $x_{0}$ is not the center of any evolving $\varepsilon$-neck. Therefore we have proved

$$
\operatorname{diam}\left(M_{\varepsilon}\right) \leq C Q^{-\frac{1}{2}}
$$

for some positive constant $C=C(\varepsilon)$ depending only on $\varepsilon$, where $Q=R\left(x_{0}, 0\right)$.

Finally by combining this diameter estimate with Theorem 6.4.3(i), we immediately deduce

$$
\widetilde{C}^{-1} Q \leq R(x, 0) \leq \widetilde{C} Q, \text { whenever } x \in M_{\varepsilon},
$$

for some positive constant $\widetilde{C}$ depending only on $\varepsilon$. $\square$

We now can describe the canonical structures for three-dimensional nonflat (compact or noncompact) ancient $\kappa$-solutions. The following theorem was given by Perelman in the section 1.5 of [104]. Recently in [34], this canonical neighborhood result has been extended to four-dimensional ancient $\kappa$-solutions with isotropic curvature pinching.

Theorem 6.4.6 (Canonical neighborhood theorem). For any $\varepsilon>0$ one can find positive constants $C_{1}=C_{1}(\varepsilon)$ and $C_{2}=C_{2}(\varepsilon)$ with the following property. Suppose we have a three-dimensional nonflat (compact or noncompact) ancient $\kappa$ solution $\left(M, g_{i j}(x, t)\right)$. Then either the ancient solution is the round $\mathbb{R P}^{2} \times \mathbb{R}$, or every point $(x, t)$ has an open neighborhood $B$, with $B_{t}(x, r) \subset B \subset B_{t}(x, 2 r)$ for some $0<r<C_{1} R(x, t)^{-\frac{1}{2}}$, which falls into one of the following three categories:

(a) $B$ is an evolving $\varepsilon$-neck (in the sense that it is the slice at the time $t$ of the parabolic region $\left\{\left(x^{\prime}, t^{\prime}\right) \mid x^{\prime} \in B, t^{\prime} \in\left[t-\varepsilon^{-2} R(x, t)^{-1}, t\right]\right\}$ which is, after scaling with factor $R(x, t)$ and shifting the time $t$ to zero, $\varepsilon$-close (in the $C^{\left[\varepsilon^{-1}\right]}$ topology) to the subset $\left(\mathbb{S}^{2} \times \mathbb{I}\right) \times\left[-\varepsilon^{-2}, 0\right]$ of the evolving standard round cylinder with scalar curvature 1 and length $2 \varepsilon^{-1}$ to $\mathbb{I}$ at the time zero), or

(b) $B$ is an evolving $\varepsilon$-cap (in the sense that it is the time slice at the time $t$ of an evolving metric on $\mathbb{B}^{3}$ or $\mathbb{R P}^{3} \backslash \mathbb{B}^{3}$ such that the region outside some suitable compact subset of $\mathbb{B}^{3}$ or $\mathbb{R P}^{3} \backslash \overline{\mathbb{B}}^{3}$ is an evolving $\varepsilon$-neck), or

(c) $B$ is a compact manifold (without boundary) with positive sectional curvature (thus it is diffeomorphic to the round three-sphere $\mathbb{S}^{3}$ or a metric quotient of $\left.\mathbb{S}^{3}\right)$

furthermore, the scalar curvature of the ancient $\kappa$-solution on $B$ at time $t$ is between $C_{2}^{-1} R(x, t)$ and $C_{2} R(x, t)$, and the volume of $B$ in case (a) and case (b) satisfies

$$
\left(C_{2} R(x, t)\right)^{-\frac{3}{2}} \leq \operatorname{Vol}_{t}(B) \leq \varepsilon r^{3} .
$$

Proof. As before, we first consider the easy case that the curvature operator has a nontrivial null vector somewhere at some time. By pulling back the solution to 
its universal cover and applying Hamilton's strong maximum principle and Theorem 6.2 .2 , we deduce that the universal cover is the evolving round cylinder $\mathbb{S}^{2} \times \mathbb{R}$. Then exactly as before, by the argument in the proof of Lemma 6.4.1, we conclude that the ancient $\kappa$-solution is isometric to the round $\mathbb{S}^{2} \times \mathbb{R}, \mathbb{R} \mathbb{P}^{2} \times \mathbb{R}$, or the twisted product $\mathbb{S}^{2} \tilde{\times} \mathbb{R}$ where $\mathbb{Z}_{2}$ flips both $\mathbb{S}^{2}$ and $\mathbb{R}$. Clearly each point of the round cylinder $\mathbb{S}^{2} \times \mathbb{R}$ or the twisted product $\mathbb{S}^{2} \tilde{\times} \mathbb{R}$ has a neighborhood falling into the category (a) or (b) (over $\mathbb{R} \mathbb{P}^{3} \backslash \mathbb{B}^{3}$ ).

We now assume that the curvature operator of the nonflat ancient $\kappa$-solution is positive everywhere. Then the manifold is orientable by the Cheeger-Gromoll theorem [23] for the noncompact case or the Synge theorem [22] for the compact case.

Without loss of generality, we may assume $\varepsilon$ is suitably small, say $0<\varepsilon<\frac{1}{100}$. If the nonflat ancient $\kappa$-solution is noncompact, the conclusions follow immediately from the combination of Corollary 6.4.5 and Theorem 6.4.3(i). Thus we may assume the nonflat ancient $\kappa$-solution is compact. By Proposition 6.4.2, either the compact ancient $\kappa$-solution is isometric to a metric quotient of the round $\mathbb{S}^{3}$, or it is $\kappa_{0^{-}}$ noncollapsed on all scales for the universal positive constant $\kappa_{0}$. Clearly each point of a metric quotient of the round $\mathbb{S}^{3}$ has a neighborhood falling into category (c). Thus we may further assume the ancient $\kappa$-solution is also $\kappa_{0}$-noncollapsing.

We argue by contradiction. Suppose that for some $\varepsilon \in\left(0, \frac{1}{100}\right)$, there exist a sequence of compact orientable ancient $\kappa_{0}$-solutions $\left(M_{k}, g_{k}\right)$ with positive curvature operator, a sequence of points $\left(x_{k}, 0\right)$ with $x_{k} \in M_{k}$ and sequences of positive constants $C_{1 k} \rightarrow \infty$ and $C_{2 k}=\omega\left(4 C_{1 k}^{2}\right)$, with the function $\omega$ given in Theorem 6.4.3, such that for every radius $r, 0<r<C_{1 k} R\left(x_{k}, 0\right)^{-\frac{1}{2}}$, any open neighborhood $B$, with $B_{0}\left(x_{k}, r\right) \subset B \subset B_{0}\left(x_{k}, 2 r\right)$, does not fall into one of the three categories (a), (b) and (c), where in the case (a) and case (b), we require the neighborhood $B$ to satisfy the volume estimate

$$
\left(C_{2 k} R\left(x_{k}, 0\right)\right)^{-\frac{3}{2}} \leq \operatorname{Vol}_{0}(B) \leq \varepsilon r^{3} .
$$

By Theorem 6.4.3(i) and the choice of the constants $C_{2 k}$ we see that the diameter of each $M_{k}$ at $t=0$ is at least $C_{1 k} R\left(x_{k}, 0\right)^{-\frac{1}{2}}$; otherwise we can choose suitable $r \in\left(0, C_{1 k} R\left(x_{k}, 0\right)^{-\frac{1}{2}}\right)$ and $B=M_{k}$, which falls into the category (c) with the scalar curvature between $C_{2 k}^{-1} R(x, 0)$ and $C_{2 k} R(x, 0)$ on $B$. Now by scaling the ancient $\kappa_{0}$-solutions along the points $\left(x_{k}, 0\right)$ with factors $R\left(x_{k}, 0\right)$, it follows from Corollary 6.4.4 that a sequence of the ancient $\kappa_{0}$-solutions converge in the $C_{l o c}^{\infty}$ topology to a noncompact orientable ancient $\kappa_{0}$-solution.

If the curvature operator of the noncompact limit has a nontrivial null vector somewhere at some time, it follows exactly as before by using the argument in the proof of Lemma 6.4.1 that the orientable limit is isometric to the round $\mathbb{S}^{2} \times \mathbb{R}$, or the twisted product $\mathbb{S}^{2} \tilde{x} \mathbb{R}$ where $\mathbb{Z}_{2}$ flips both $\mathbb{S}^{2}$ and $\mathbb{R}$. Then for $k$ large enough, a suitable neighborhood $B$ (for suitable $r$ ) of the point $\left(x_{k}, 0\right)$ would fall into the category (a) or (b) (over $\mathbb{R P}^{3} \backslash \overline{\mathbb{B}^{3}}$ ) with the desired volume estimate. This is a contradiction.

If the noncompact limit has positive sectional curvature everywhere, then by using Corollary 6.4.5 and Theorem 6.4.3(i) for the noncompact limit we see that for $k$ large enough, a suitable neighborhood $B$ (for suitable $r$ ) of the point $\left(x_{k}, 0\right)$ would fall into category (a) or (b) (over $\mathbb{B}^{3}$ ) with the desired volume estimate. This is also a contradiction.

Finally, the statement on the curvature estimate in the neighborhood $B$ follows directly from Theorem 6.4.3(i). 
7. Ricci Flow on Three-manifolds. We will use the Ricci flow to study the topology of compact orientable three-manifolds. Let $M$ be a compact threedimensional orientable manifold. Arbitrarily given a Riemannian metric on the manifold, we evolve it by the Ricci flow. The basic idea is to understand the topology of the underlying manifold by studying long-time behavior of the solution of the Ricci flow. We have seen in Chapter 5 that for a compact three-manifold with positive Ricci curvature as initial data, the solution to the Ricci flow tends, up to scalings, to a metric of positive constant curvature. Consequently, a compact three-manifold with positive Ricci curvature is diffeomorphic to the round three-sphere or a metric quotient of it.

However, for general initial metrics, the Ricci flow may develop singularities in some parts while it keeps smooth in other parts. Naturally one would like to cut off the singularities and continue to run the Ricci flow. If the Ricci flow still develops singularities after a while, one can do the surgeries and run the Ricci flow again. By repeating this procedure, one will get a kind of "weak" solution to the Ricci flow. Furthermore, if the "weak" solution has only a finite number of surgeries at any finite time interval and one can remember what had been cut during the surgeries, and if the "weak" solution has a well-understood long-time behavior, then one will also get the topology structure of the initial manifold. This theory of surgically modified Ricci flow was first developed by Hamilton [64] for compact four-manifolds and further developed more recently by Perelman [104] for compact orientable three-manifolds.

The main purpose of this chapter is to give a complete and detailed discussion of the Ricci flow with surgery on three-manifolds.

7.1. Canonical Neighborhood Structures. Let us call a Riemannian metric on a compact orientable three-dimensional manifold normalized if the eigenvalues of its curvature operator at every point are bounded by $\frac{1}{10} \geq \lambda \geq \mu \geq \nu \geq-\frac{1}{10}$, and every geodesic ball of radius one has volume at least one. By the evolution equation of the curvature and the maximum principle, it is easy to see that any solution to the Ricci flow with (compact and three-dimensional) normalized initial metric exists on a maximal time interval $\left[0, t_{\max }\right)$ with $t_{\max }>1$.

Consider a smooth solution $g_{i j}(x, t)$ to the Ricci flow on $M \times[0, T)$, where $M$ is a compact orientable three-manifold and $T<+\infty$. After rescaling, we may always assume the initial metric $g_{i j}(\cdot, 0)$ is normalized. By Theorem 5.3.2, the solution $g_{i j}(\cdot, t)$ then satisfies the pinching estimate

$$
R \geq(-\nu)[\log (-\nu)+\log (1+t)-3]
$$

whenever $\nu<0$ on $M \times[0, T)$. Recall the function

$$
y=f(x)=x(\log x-3), \text { for } e^{2} \leq x<+\infty,
$$

is increasing and convex with range $-e^{2} \leq y<+\infty$, and its inverse function is also increasing and satisfies

$$
\lim _{y \rightarrow+\infty} f^{-1}(y) / y=0 .
$$

We can rewrite the pinching estimate (7.1.1) as

$$
R m(x, t) \geq-\left[f^{-1}(R(x, t)(1+t)) /(R(x, t)(1+t))\right] R(x, t)
$$

on $M \times[0, T)$. 
Suppose that the solution $g_{i j}(\cdot, t)$ becomes singular as $t \rightarrow T$. Let us take a sequence of times $t_{k} \rightarrow T$, and a sequence of points $p_{k} \in M$ such that for some positive constant $C,|R m|(x, t) \leq C Q_{k}$ with $Q_{k}=\left|R m\left(p_{k}, t_{k}\right)\right|$ for all $x \in M$ and $t \in\left[0, t_{k}\right]$. Thus, $\left(p_{k}, t_{k}\right)$ is a sequence of (almost) maximum points. By applying Hamilton's compactness theorem and Perelman's no local collapsing theorem I as well as the pinching estimate (7.1.2), a sequence of the scalings of the solution $g_{i j}(x, t)$ around the points $p_{k}$ with factors $Q_{k}$ converges to a nonflat complete three-dimensional orientable ancient $\kappa$-solution (for some $\kappa>0$ ). For an arbitrarily given $\varepsilon>0$, the canonical neighborhood theorem (Theorem 6.4.6) in the previous chapter implies that each point in the ancient $\kappa$-solution has a neighborhood which is either an evolving $\varepsilon$-neck, or an evolving $\varepsilon$-cap, or a compact (without boundary) positively curved manifold. This gives the structure of singularities coming from a sequence of (almost) maximum points.

However the above argument does not work for singularities coming from a sequence of points $\left(y_{k}, \tau_{k}\right)$ with $\tau_{k} \rightarrow T$ and $\left|R m\left(y_{k}, \tau_{k}\right)\right| \rightarrow+\infty$ when $\left|R m\left(y_{k}, \tau_{k}\right)\right|$ is not comparable with the maximum of the curvature at the time $\tau_{k}$, since we cannot take a limit directly. In [103], Perelman developed a refined rescaling argument to obtain the following singularity structure theorem. We remark that our statement of the singularity structure theorem below is slightly different from Perelman's original statement (cf. Theorem 12.1 of [103]). While Perelman assumed the condition of $\kappa$-noncollapsing on scales less than $r_{0}$, we assume that the initial metric is normalized so that from the rescaling argument one can get the $\kappa$-noncollapsing on all scales for the limit solutions.

TheOREM 7.1.1 (Singularity structure theorem). Given $\varepsilon>0$ and $T_{0}>1$, one can find $r_{0}>0$ with the following property. If $g_{i j}(x, t), x \in M$ and $t \in[0, T)$ with $1<T \leq T_{0}$, is a solution to the Ricci flow on a compact orientable three-manifold $M$ with normalized initial metric, then for any point $\left(x_{0}, t_{0}\right)$ with $t_{0} \geq 1$ and $Q=$ $R\left(x_{0}, t_{0}\right) \geq r_{0}^{-2}$, the solution in $\left\{(x, t) \mid d_{t_{0}}^{2}\left(x, x_{0}\right)<\varepsilon^{-2} Q^{-1}, t_{0}-\varepsilon^{-2} Q^{-1} \leq t \leq t_{0}\right\}$ is, after scaling by the factor $Q$, $\varepsilon$-close (in the $C^{\left[\varepsilon^{-1}\right]}$-topology) to the corresponding subset of some orientable ancient $\kappa$-solution (for some $\kappa>0$ ).

Proof. Since the initial metric is normalized, it follows from the no local collapsing theorem I or I' (and their proofs) that there is a positive constant $\kappa$, depending only on $T_{0}$, such that the solution in Theorem 7.1.1 is $\kappa$-noncollapsed on all scales less than $\sqrt{T_{0}}$. Let $C(\varepsilon)$ be a positive constant larger than or equal to $\varepsilon^{-2}$. It suffices to prove that there exists $r_{0}>0$ such that for any point $\left(x_{0}, t_{0}\right)$ with $t_{0} \geq 1$ and $Q=$ $R\left(x_{0}, t_{0}\right) \geq r_{0}^{-2}$, the solution in the parabolic region $\left\{(x, t) \in M \times[0, T) \mid d_{t_{0}}^{2}\left(x, x_{0}\right)<\right.$ $\left.C(\varepsilon) Q^{-1}, t_{0}-C(\varepsilon) Q^{-1} \leq t \leq t_{0}\right\}$ is, after scaling by the factor $Q, \varepsilon$-close to the corresponding subset of some orientable ancient $\kappa$-solution. The constant $C(\varepsilon)$ will be determined later.

We argue by contradiction. Suppose for some $\varepsilon>0$, there exist a sequence of solutions $\left(M_{k}, g_{k}(\cdot, t)\right)$ to the Ricci flow on compact orientable three-manifolds with normalized initial metrics, defined on the time intervals $\left[0, T_{k}\right)$ with $1<T_{k} \leq T_{0}$, a sequence of positive numbers $r_{k} \rightarrow 0$, and a sequence of points $x_{k} \in M_{k}$ and times $t_{k} \geq 1$ with $Q_{k}=R_{k}\left(x_{k}, t_{k}\right) \geq r_{k}^{-2}$ such that each solution $\left(M_{k}, g_{k}(\cdot, t)\right)$ in the parabolic region $\left\{(x, t) \in M_{k} \times\left[0, T_{k}\right) \mid d_{t_{k}}^{2}\left(x, x_{k}\right)<C(\varepsilon) Q_{k}^{-1}, t_{k}-C(\varepsilon) Q_{k}^{-1} \leq t \leq t_{k}\right\}$ is not, after scaling by the factor $Q_{k}, \varepsilon$-close to the corresponding subset of any orientable ancient $\kappa$-solution, where $R_{k}$ denotes the scalar curvature of $\left(M_{k}, g_{k}\right)$.

For each solution $\left(M_{k}, g_{k}(\cdot, t)\right)$, we may adjust the point $\left(x_{k}, t_{k}\right)$ with $t_{k} \geq \frac{1}{2}$ 
and with $Q_{k}=R_{k}\left(x_{k}, t_{k}\right)$ to be as large as possible so that the conclusion of the theorem fails at $\left(x_{k}, t_{k}\right)$, but holds for any $(x, t) \in M_{k} \times\left[t_{k}-H_{k} Q_{k}^{-1}, t_{k}\right]$ satisfying $R_{k}(x, t) \geq 2 Q_{k}$, where $H_{k}=\frac{1}{4} r_{k}^{-2} \rightarrow+\infty$ as $k \rightarrow+\infty$. Indeed, suppose not, by setting $\left(x_{k_{1}}, t_{k_{1}}\right)=\left(x_{k}, t_{k}\right)$, we can choose a sequence of points $\left(x_{k_{l}}, t_{k_{l}}\right) \in M_{k} \times$ $\left[t_{k_{(l-1)}}-H_{k} R_{k}\left(x_{k_{(l-1)}}, t_{k_{(l-1)}}\right)^{-1}, t_{k_{(l-1)}}\right]$ such that $R_{k}\left(x_{k_{l}}, t_{k_{l}}\right) \geq 2 R_{k}\left(x_{k_{(l-1)}}, t_{k_{(l-1)}}\right)$ and the conclusion of the theorem fails at $\left(x_{k_{l}}, t_{k_{l}}\right)$ for each $l=2,3, \ldots$. Since the solution is smooth, but

$$
R_{k}\left(x_{k_{l}}, t_{k_{l}}\right) \geq 2 R_{k}\left(x_{k_{(l-1)}}, t_{k_{(l-1)}}\right) \geq \cdots \geq 2^{l-1} R_{k}\left(x_{k}, t_{k}\right),
$$

and

$$
\begin{aligned}
t_{k_{l}} & \geq t_{k_{(l-1)}}-H_{k} R_{k}\left(x_{k_{(l-1)}}, t_{k_{(l-1)}}\right)^{-1} \\
& \geq t_{k}-H_{k} \sum_{i=1}^{l-1} \frac{1}{2^{i-1}} R_{k}\left(x_{k}, t_{k}\right)^{-1} \\
& \geq \frac{1}{2},
\end{aligned}
$$

this process must terminate after a finite number of steps and the last element fits.

Let $\left(M_{k}, \tilde{g}_{k}(\cdot, t), x_{k}\right)$ be the rescaled solutions obtained by rescaling $\left(M_{k}, g_{k}(\cdot, t)\right)$ around $x_{k}$ with the factors $Q_{k}=R_{k}\left(x_{k}, t_{k}\right)$ and shifting the time $t_{k}$ to the new time zero. Denote by $\tilde{R}_{k}$ the rescaled scalar curvature. We will show that a subsequence of the orientable rescaled solutions $\left(M_{k}, \tilde{g}_{k}(\cdot, t), x_{k}\right)$ converges in the $C_{l o c}^{\infty}$ topology to an orientable ancient $\kappa$-solution, which is a contradiction. In the following we divide the argument into four steps.

Step 1. First of all, we need a local bound on curvatures.

Lemma 7.1.2. For each $(\bar{x}, \bar{t})$ with $t_{k}-\frac{1}{2} H_{k} Q_{k}^{-1} \leq \bar{t} \leq t_{k}$, we have $R_{k}(x, t) \leq 4 \bar{Q}_{k}$ whenever $\bar{t}-c \bar{Q}_{k}^{-1} \leq t \leq \bar{t}$ and $d_{\bar{t}}^{2}(x, \bar{x}) \leq c \bar{Q}_{k}^{-1}$, where $\bar{Q}_{k}=Q_{k}+R_{k}(\bar{x}, \bar{t})$ and $c>0$ is a small universal constant.

Proof. Consider any point $(x, t) \in B_{\bar{t}}\left(\bar{x},\left(c \bar{Q}_{k}^{-1}\right)^{\frac{1}{2}}\right) \times\left[\bar{t}-c \bar{Q}_{k}^{-1}, \bar{t}\right]$ with $c>0$ to be determined. If $R_{k}(x, t) \leq 2 Q_{k}$, there is nothing to show. If $R_{k}(x, t)>2 Q_{k}$, consider a space-time curve $\gamma$ from $(x, t)$ to $(\bar{x}, \bar{t})$ that goes straight from $(x, t)$ to $(x, \bar{t})$ and goes from $(x, \bar{t})$ to $(\bar{x}, \bar{t})$ along a minimizing geodesic (with respect to the metric $g_{k}(\cdot, \bar{t})$ ). If there is a point on $\gamma$ with the scalar curvature $2 Q_{k}$, let $y_{0}$ be the nearest such point to $(x, t)$. If not, put $y_{0}=(\bar{x}, \bar{t})$. On the segment of $\gamma$ from $(x, t)$ to $y_{0}$, the scalar curvature is at least $2 Q_{k}$. According to the choice of the point $\left(x_{k}, t_{k}\right)$, the solution along the segment is $\varepsilon$-close to that of some ancient $\kappa$-solution. It follows from Theorem 6.4.3 (ii) that

$$
\left|\nabla\left(R_{k}^{-\frac{1}{2}}\right)\right| \leq 2 \eta \text { and }\left|\frac{\partial}{\partial t}\left(R_{k}^{-1}\right)\right| \leq 2 \eta
$$

on the segment. (Here, without loss of generality, we may assume $\varepsilon$ is suitably small). Then by choosing $c>0$ (depending only on $\eta$ ) small enough we get the desired curvature bound by integrating the above derivative estimates along the segment. This proves the lemma.

Step 2. Next we want to show that for each $A<+\infty$, there exist a positive constant $C(A)$ (independent of $k$ ) such that the curvatures of the rescaled solutions 
$\tilde{g}_{k}(\cdot, t)$ at the new time $t=0$ (corresponding to the original times $t_{k}$ ) satisfy the estimate

$$
\left|\widetilde{R m}_{k}\right|(y, 0) \leq C(A)
$$

whenever $d_{\tilde{g}_{k}(\cdot, 0)}\left(y, x_{k}\right) \leq A$ and $k \geq 1$.

For each $\rho \geq 0$, set

$$
M(\rho)=\sup \left\{\tilde{R}_{k}(x, 0) \mid k \geq 1, x \in M_{k} \text { with } d_{0}\left(x, x_{k}\right) \leq \rho\right\}
$$

and

$$
\rho_{0}=\sup \{\rho \geq 0 \mid M(\rho)<+\infty\} .
$$

By the pinching estimate (7.1.1), it suffices to show $\rho_{0}=+\infty$.

Note that $\rho_{0}>0$ by applying Lemma 7.1 .2 with $(\bar{x}, \bar{t})=\left(x_{k}, t_{k}\right)$. We now argue by contradiction to show $\rho_{0}=+\infty$. Suppose not, we may find (after passing to a subsequence if necessary) a sequence of points $y_{k} \in M_{k}$ with $d_{0}\left(x_{k}, y_{k}\right) \rightarrow \rho_{0}<+\infty$ and $\tilde{R}_{k}\left(y_{k}, 0\right) \rightarrow+\infty$. Let $\gamma_{k}\left(\subset M_{k}\right)$ be a minimizing geodesic segment from $x_{k}$ to $y_{k}$. Let $z_{k} \in \gamma_{k}$ be the point on $\gamma_{k}$ closest to $y_{k}$ with $\tilde{R}_{k}\left(z_{k}, 0\right)=2$, and let $\beta_{k}$ be the subsegment of $\gamma_{k}$ running from $z_{k}$ to $y_{k}$. By Lemma 7.1.2 the length of $\beta_{k}$ is bounded away from zero independent of $k$. By the pinching estimate (7.1.1), for each $\rho<\rho_{0}$, we have a uniform bound on the curvatures on the open balls $B_{0}\left(x_{k}, \rho\right) \subset\left(M_{k}, \tilde{g}_{k}\right)$. The injectivity radii of the rescaled solutions $\tilde{g}_{k}$ at the points $x_{k}$ and the time $t=0$ are also uniformly bounded from below by the $\kappa$-noncollapsing property. Therefore by Lemma 7.1.2 and Hamilton's compactness theorem (Theorem 4.1.5), after passing to a subsequence, we can assume that the marked sequence $\left(B_{0}\left(x_{k}, \rho_{0}\right), \tilde{g}_{k}(\cdot, 0), x_{k}\right)$ converges in the $C_{\text {loc }}^{\infty}$ topology to a marked (noncomplete) manifold $\left(B_{\infty}, \tilde{g}_{\infty}, x_{\infty}\right)$, the segments $\gamma_{k}$ converge to a geodesic segment (missing an endpoint) $\gamma_{\infty} \subset B_{\infty}$ emanating from $x_{\infty}$, and $\beta_{k}$ converges to a subsegment $\beta_{\infty}$ of $\gamma_{\infty}$. Let $\bar{B}_{\infty}$ denote the completion of $\left(B_{\infty}, \tilde{g}_{\infty}\right)$, and $y_{\infty} \in \bar{B}_{\infty}$ the limit point of $\gamma_{\infty}$.

Denote by $\tilde{R}_{\infty}$ the scalar curvature of $\left(B_{\infty}, \tilde{g}_{\infty}\right)$. Since the rescaled scalar curvatures $\tilde{R}_{k}$ along $\beta_{k}$ are at least 2 , it follows from the choice of the points $\left(x_{k}, 0\right)$ that for any $q_{0} \in \beta_{\infty}$, the manifold $\left(B_{\infty}, \tilde{g}_{\infty}\right)$ in $\left\{q \in B_{\infty} \mid \operatorname{dist}_{\tilde{g}_{\infty}}^{2}\left(q, q_{0}\right)<C(\varepsilon)\left(\tilde{R}_{\infty}\left(q_{0}\right)\right)^{-1}\right\}$ is $2 \varepsilon$-close to the corresponding subset of (a time slice of) some orientable ancient $\kappa$-solution. Then by Theorem 6.4.6, we know that the orientable ancient $\kappa$-solution at each point $(x, t)$ has a radius $r, 0<r<C_{1}(2 \varepsilon) R(x, t)^{-\frac{1}{2}}$, such that its canonical neighborhood $B$, with $B_{t}(x, r) \subset B \subset B_{t}(x, 2 r)$, is either an evolving $2 \varepsilon$-neck, or an evolving $2 \varepsilon$-cap, or a compact manifold (without boundary) diffeomorphic to a metric quotient of the round three-sphere $\mathbb{S}^{3}$, and moreover the scalar curvature is between $\left(C_{2}(2 \varepsilon)\right)^{-1} R(x, t)$ and $C_{2}(2 \varepsilon) R(x, t)$, where $C_{1}(2 \varepsilon)$ and $C_{2}(2 \varepsilon)$ are the positive constants in Theorem 6.4.6.

We now choose $C(\varepsilon)=\max \left\{2 C_{1}^{2}(2 \varepsilon), \varepsilon^{-2}\right\}$. By the local curvature estimate in Lemma 7.1.2, we see that the scalar curvature $\tilde{R}_{\infty}$ becomes unbounded when approaching $y_{\infty}$ along $\gamma_{\infty}$. This implies that the canonical neighborhood around $q_{0}$ cannot be a compact manifold (without boundary) diffeomorphic to a metric quotient of the round three-sphere $\mathbb{S}^{3}$. Note that $\gamma_{\infty}$ is shortest since it is the limit of a sequence of shortest geodesics. Without loss of generality, we may assume $\varepsilon$ is suitably small (say, $\varepsilon \leq \frac{1}{100}$ ). These imply that as $q_{0}$ gets sufficiently close to $y_{\infty}$, the canonical neighborhood around $q_{0}$ cannot be an evolving $2 \varepsilon$-cap. Thus we conclude that each $q_{0} \in \gamma_{\infty}$ sufficiently close to $y_{\infty}$ is the center of an evolving $2 \varepsilon$-neck. 
Let

$$
U=\bigcup_{q_{0} \in \gamma_{\infty}} B\left(q_{0}, 4 \pi\left(\tilde{R}_{\infty}\left(q_{0}\right)\right)^{-\frac{1}{2}}\right)\left(\subset\left(B_{\infty}, \tilde{g}_{\infty}\right)\right)
$$

where $B\left(q_{0}, 4 \pi\left(\tilde{R}_{\infty}\left(q_{0}\right)\right)^{-\frac{1}{2}}\right)$ is the ball centered at $q_{0} \in B_{\infty}$ with the radius $4 \pi\left(\tilde{R}_{\infty}\left(q_{0}\right)\right)^{-\frac{1}{2}}$. Clearly $U$ has nonnegative sectional curvature by the pinching estimate (7.1.1). Since the metric $\tilde{g}_{\infty}$ is cylindrical at any point $q_{0} \in \gamma_{\infty}$ which is sufficiently close to $y_{\infty}$, we see that the metric space $\bar{U}=U \cup\left\{y_{\infty}\right\}$ by adding in the point $y_{\infty}$, is locally complete and intrinsic near $y_{\infty}$. Furthermore $y_{\infty}$ cannot be an interior point of any geodesic segment in $\bar{U}$. This implies the curvature of $\bar{U}$ at $y_{\infty}$ is nonnegative in the Alexandrov sense. It is a basic result in Alexandrov space theory (see for example Theorem 10.9.3 and Corollary 10.9.5 of [9]) that there exists a three-dimensional tangent cone $C_{y_{\infty}} \bar{U}$ at $y_{\infty}$ which is a metric cone. It is clear that its aperture is $\leq 10 \varepsilon$, thus the tangent cone is nonflat.

Pick a point $p \in C_{y_{\infty}} \bar{U}$ such that the distance from the vertex $y_{\infty}$ to $p$ is one and it is nonflat around $p$. Then the ball $B\left(p, \frac{1}{2}\right) \subset C_{y_{\infty}} \bar{U}$ is the Gromov-Hausdorff limit of the scalings of a sequence of balls $B_{0}\left(p_{k}, s_{k}\right) \subset\left(M_{k}, \tilde{g}_{k}(\cdot, 0)\right)$ by some factors $a_{k}$, where $s_{k} \rightarrow 0^{+}$. Since the tangent cone is three-dimensional and nonflat around $p$, the factors $a_{k}$ must be comparable with $\tilde{R}_{k}\left(p_{k}, 0\right)$. By using the local curvature estimate in Lemma 7.1.2, we actually have the convergence in the $C_{\mathrm{loc}}^{\infty}$ topology for the solutions $\tilde{g}_{k}(\cdot, t)$ on the balls $B_{0}\left(p_{k}, s_{k}\right)$ and over some time interval $t \in[-\delta, 0]$ for some sufficiently small $\delta>0$. The limiting ball $B\left(p, \frac{1}{2}\right) \subset C_{y_{\infty}} \bar{U}$ is a piece of the nonnegative curved and nonflat metric cone whose radial directions are all Ricci flat. On the other hand, by applying Hamilton's strong maximum principle to the evolution equation of the Ricci curvature tensor as in the proof of Lemma 6.3.1, the limiting ball $B\left(p, \frac{1}{2}\right)$ would split off all radial directions isometrically (and locally). Since the limit is nonflat around $p$, this is impossible. Therefore we have proved that the curvatures of the rescaled solutions $\tilde{g}_{k}(\cdot, t)$ at the new times $t=0$ (corresponding to the original times $t_{k}$ ) stay uniformly bounded at bounded distances from $x_{k}$ for all $k$.

We have proved that for each $A<+\infty$, the curvature of the marked manifold $\left(M_{k}, \tilde{g}_{k}(\cdot, 0), x_{k}\right)$ at each point $y \in M_{k}$ with distance from $x_{k}$ at most $A$ is bounded by $C(A)$. Lemma 7.1.2 extends this curvature control to a backward parabolic neighborhood centered at $y$ whose radius depends only on the distance from $y$ to $x_{k}$. Thus by Shi's local derivative estimates (Theorem 1.4.2) we can control all derivatives of the curvature in such backward parabolic neighborhoods. Then by using the $\kappa$-noncollapsing and Hamilton's compactness theorem (Theorem 4.1.5), we can take a $C_{\text {loc }}^{\infty}$ subsequent limit to obtain $\left(M_{\infty}, \tilde{g}_{\infty}(\cdot, t), x_{\infty}\right)$, which is $\kappa$-noncollapsed on all scales and is defined on a space-time open subset of $M_{\infty} \times(-\infty, 0]$ containing the time slice $M_{\infty} \times\{0\}$. Clearly it follows from the pinching estimate (7.1.1) that the limit $\left(M_{\infty}, \tilde{g}_{\infty}(\cdot, 0), x_{\infty}\right)$ has nonnegative curvature operator (and hence nonnegative sectional curvature).

Step 3. We further claim that the limit $\left(M_{\infty}, \tilde{g}_{\infty}(\cdot, 0), x_{\infty}\right)$ at the time slice $\{t=0\}$ has bounded curvature.

We know that the sectional curvature of the limit $\left(M_{\infty}, \tilde{g}_{\infty}(\cdot, 0), x_{\infty}\right)$ is nonnegative everywhere. Argue by contradiction. Suppose the curvature of $\left(M_{\infty}, \tilde{g}_{\infty}(\cdot, 0), x_{\infty}\right)$ is not bounded, then by Lemma 6.1.4, there exists a sequence of points $q_{j} \in M_{\infty}$ diverging to infinity such that their scalar curvatures $\tilde{R}_{\infty}\left(q_{j}, 0\right) \rightarrow+\infty$ as $j \rightarrow+\infty$ 
and

$$
\tilde{R}_{\infty}(x, 0) \leq 4 \tilde{R}_{\infty}\left(q_{j}, 0\right)
$$

for $x \in B\left(q_{j}, j / \sqrt{\tilde{R}_{\infty}\left(q_{j}, 0\right)}\right) \subset\left(M_{\infty}, \tilde{g}_{\infty}(\cdot, 0)\right)$. By combining with Lemma 7.1.2 and the $\kappa$-noncollapsing, a subsequence of the rescaled and marked manifolds $\left(M_{\infty}, \tilde{R}_{\infty}\left(q_{j}, 0\right) \tilde{g}_{\infty}(\cdot, 0), q_{j}\right)$ converges in the $C_{\text {loc }}^{\infty}$ topology to a smooth nonflat limit $Y$. By Proposition 6.1.2, the new limit $Y$ is isometric to a metric product $N \times \mathbb{R}$ for some two-dimensional manifold $N$. On the other hand, in view of the choice of the points $\left(x_{k}, t_{k}\right)$, the original limit $\left(M_{\infty}, \tilde{g}_{\infty}(\cdot, 0), x_{\infty}\right)$ at the point $q_{j}$ has a canonical neighborhood which is either a $2 \varepsilon$-neck, a $2 \varepsilon$-cap, or a compact manifold (without boundary) diffeomorphic to a metric quotient of the round $\mathbb{S}^{3}$. It follows that for $j$ large enough, $q_{j}$ is the center of a $2 \varepsilon$-neck of radius $\left(\tilde{R}_{\infty}\left(q_{j}, 0\right)\right)^{-\frac{1}{2}}$. Without loss of generality, we may further assume that $2 \varepsilon<\varepsilon_{0}$, where $\varepsilon_{0}$ is the positive constant given in Proposition 6.1.1. Since $\left(\tilde{R}_{\infty}\left(q_{j}, 0\right)\right)^{-\frac{1}{2}} \rightarrow 0$ as $j \rightarrow+\infty$, this contradicts Proposition 6.1.1. So the curvature of $\left(M_{\infty}, \tilde{g}_{\infty}(\cdot, 0)\right)$ is bounded.

Step 4 . Finally we want to extend the limit backwards in time to $-\infty$.

By Lemma 7.1.2 again, we now know that the limiting solution $\left(M_{\infty}, \tilde{g}_{\infty}(\cdot, t)\right)$ is defined on a backward time interval $[-a, 0]$ for some $a>0$.

Denote by

$$
\begin{gathered}
t^{\prime}=\inf \{\tilde{t} \mid \text { we can take a smooth limit on }(\tilde{t}, 0] \text { (with bounded } \\
\text { curvature at each time slice) from a subsequence } \\
\text { of the convergent rescaled solutions } \left.\tilde{g}_{k}\right\} .
\end{gathered}
$$

We first claim that there is a subsequence of the rescaled solutions $\tilde{g}_{k}$ which converges in the $C_{\mathrm{loc}}^{\infty}$ topology to a smooth limit $\left(M_{\infty}, \tilde{g}_{\infty}(\cdot, t)\right)$ on the maximal time interval $\left(t^{\prime}, 0\right]$.

Indeed, let $t_{k}^{\prime}$ be a sequence of negative numbers such that $t_{k}^{\prime} \rightarrow t^{\prime}$ and there exist smooth limits $\left(M_{\infty}, \tilde{g}_{\infty}^{k}(\cdot, t)\right)$ defined on $\left(t_{k}^{\prime}, 0\right]$. For each $k$, the limit has nonnegative sectional curvature and has bounded curvature at each time slice. Moreover by Lemma 7.1.2, the limit has bounded curvature on each subinterval $[-b, 0] \subset\left(t_{\tilde{Q}}^{\prime}, 0\right]$. Denote by $\tilde{Q}$ the scalar curvature upper bound of the limit at time zero (where $\tilde{Q}$ is the same for all $k$ ). Then we can apply the Li-Yau-Hamilton estimate (Corollary 2.5.7) to get

$$
\tilde{R}_{\infty}^{k}(x, t) \leq \tilde{Q}\left(\frac{-t_{k}^{\prime}}{t-t_{k}^{\prime}}\right)
$$

where $\tilde{R}_{\infty}^{k}(x, t)$ are the scalar curvatures of the limits $\left(M_{\infty}, \tilde{g}_{\infty}^{k}(\cdot, t)\right)$. Hence by the definition of convergence and the above curvature estimates, we can find a subsequence of the above convergent rescaled solutions $\tilde{g}_{k}$ which converges in the $C_{\mathrm{loc}}^{\infty}$ topology to a smooth limit $\left(M_{\infty}, \tilde{g}_{\infty}(\cdot, t)\right)$ on the maximal time interval $\left(t^{\prime}, 0\right]$.

We next claim that $t^{\prime}=-\infty$.

Suppose not, then by Lemma 7.1.2, the curvature of the limit $\left(M_{\infty}, \tilde{g}_{\infty}(\cdot, t)\right)$ becomes unbounded as $t \rightarrow t^{\prime}>-\infty$. By applying the maximum principle to the evolution equation of the scalar curvature, we see that the infimum of the scalar curvature is nondecreasing in time. Note that $\tilde{R}_{\infty}\left(x_{\infty}, 0\right)=1$. Thus there exists some point $y_{\infty} \in M_{\infty}$ such that

$$
\tilde{R}_{\infty}\left(y_{\infty}, t^{\prime}+\frac{c}{10}\right)<\frac{3}{2}
$$


where $c>0$ is the universal constant in Lemma 7.1.2. By using Lemma 7.1.2 again we see that the limit $\left(M_{\infty}, \tilde{g}_{\infty}(\cdot, t)\right)$ in a small neighborhood of the point $\left(y_{\infty}, t^{\prime}+\frac{c}{10}\right)$ extends backwards to the time interval $\left[t^{\prime}-\frac{c}{10}, t^{\prime}+\frac{c}{10}\right]$. We remark that the distances at time $t$ and time 0 are roughly equivalent in the following sense

$$
d_{t}(x, y) \geq d_{0}(x, y) \geq d_{t}(x, y)-\text { const. }
$$

for any $x, y \in M_{\infty}$ and $t \in\left(t^{\prime}, 0\right]$. Indeed from the Li-Yau-Hamilton inequality (Corollary 2.5.7) we have the estimate

$$
\tilde{R}_{\infty}(x, t) \leq \tilde{Q}\left(\frac{-t^{\prime}}{t-t^{\prime}}\right), \quad \text { on } M_{\infty} \times\left(t^{\prime}, 0\right] .
$$

By applying Lemma 3.4.1 (ii), we have

$$
d_{t}(x, y) \leq d_{0}(x, y)+30\left(-t^{\prime}\right) \sqrt{\tilde{Q}}
$$

for any $x, y \in M_{\infty}$ and $t \in\left(t^{\prime}, 0\right]$. On the other hand, since the curvature of the limit metric $\tilde{g}_{\infty}(\cdot, t)$ is nonnegative, we have

$$
d_{t}(x, y) \geq d_{0}(x, y)
$$

for any $x, y \in M_{\infty}$ and $t \in\left(t^{\prime}, 0\right]$. Thus we obtain the estimate (7.1.3).

Let us still denote by $\left(M_{k}, \tilde{g}_{k}(\cdot, t)\right)$ the subsequence which converges on the maximal time interval $\left(t^{\prime}, 0\right]$. Consider the rescaled sequence $\left(M_{k}, \tilde{g}_{k}(\cdot, t)\right)$ with the marked points $x_{k}$ replaced by the associated sequence of points $y_{k} \rightarrow y_{\infty}$ and the (original unshifted) times $t_{k}$ replaced by any $s_{k} \in\left[t_{k}+\left(t^{\prime}-\frac{c}{20}\right) Q_{k}^{-1}, t_{k}+\left(t^{\prime}+\frac{c}{20}\right) Q_{k}^{-1}\right]$. It follows from Lemma 7.1.2 that for $k$ large enough, the rescaled solutions $\left(M_{k}, \tilde{g}_{k}(\cdot, t)\right)$ at $y_{k}$ satisfy

$$
\tilde{R}_{k}\left(y_{k}, t\right) \leq 10
$$

for all $t \in\left[t^{\prime}-\frac{c}{10}, t^{\prime}+\frac{c}{10}\right]$. By applying the same arguments as in the above Step 2, we conclude that for any $A>0$, there is a positive constant $C(A)<+\infty$ such that

$$
\tilde{R}_{k}(x, t) \leq C(A)
$$

for all $(x, t)$ with $d_{t}\left(x, y_{k}\right) \leq A$ and $t \in\left[t^{\prime}-\frac{c}{20}, t^{\prime}+\frac{c}{20}\right]$. The estimate (7.1.3) implies that there is a positive constant $A_{0}$ such that for arbitrarily given small $\epsilon^{\prime} \in\left(0, \frac{c}{100}\right)$, for $k$ large enough, there hold

$$
d_{t}\left(x_{k}, y_{k}\right) \leq A_{0}
$$

for all $t \in\left[t^{\prime}+\epsilon^{\prime}, 0\right]$. By combining with Lemma 7.1.2, we then conclude that for any $A>0$, there is a positive constant $\tilde{C}(A)$ such that for $k$ large enough, the rescaled solutions $\left(M_{k}, \tilde{g}_{k}(\cdot, t)\right)$ satisfy

$$
\tilde{R}_{k}(x, t) \leq \tilde{C}(A)
$$

for all $x \in \tilde{B}_{0}\left(x_{k}, A\right)$ and $t \in\left[t^{\prime}-\frac{c}{100}(C(A))^{-1}, 0\right]$.

Now, by taking convergent subsequences from the (original) rescaled solutions $\left(M_{k}, \tilde{g}_{k}(\cdot, t), x_{k}\right)$, we see that the limiting solution $\left(M_{\infty}, \tilde{g}_{\infty}(\cdot, t)\right)$ is defined on a spacetime open subset of $M_{\infty} \times(-\infty, 0]$ containing $M_{\infty} \times\left[t^{\prime}, 0\right]$. By repeating the argument 
of Step 3 and using Lemma 7.1.2, we further conclude the limit $\left(M_{\infty}, \tilde{g}_{\infty}(\cdot, t)\right)$ has uniformly bounded curvature on $M_{\infty} \times\left[t^{\prime}, 0\right]$. This is a contradiction.

Therefore we have proved a subsequence of the rescaled solutions $\left(M_{k}, \tilde{g}_{k}(\cdot, t), x_{k}\right)$ converges to an orientable ancient $\kappa$-solution, which gives the desired contradiction. This completes the proof of the theorem.

We remark that this singularity structure theorem has been extended by Chen and the second author in [34] to the Ricci flow on compact four-manifolds with positive isotropic curvature.

7.2. Curvature Estimates for Smooth Solutions. Let us consider solutions to the Ricci flow on compact orientable three-manifolds with normalized initial metrics. The above singularity structure theorem (Theorem 7.1.1) tells us that the solutions around high curvature points are sufficiently close to ancient $\kappa$-solutions. It is thus reasonable to expect that the elliptic type estimate (Theorem 6.4.3) and the curvature estimate via volume growth (Theorem 6.3.3) for ancient $\kappa$-solutions are heritable to general solutions of the Ricci flow on three-manifolds. The main purpose of this section is to establish such curvature estimates. In the fifth section of this chapter, we will further extend these estimates to surgically modified solutions.

The first result of this section is an extension of the elliptic type estimate (Theorem 6.4.3). This result is reminiscent of the second step in the proof of Theorem 7.1.1.

Theorem 7.2.1 (Perelman [103]). For any $A<+\infty$, there exist $K=K(A)<$ $+\infty$ and $\alpha=\alpha(A)>0$ with the following property. Suppose we have a solution to the Ricci flow on a three-dimensional, compact and orientable manifold $M$ with normalized initial metric. Suppose that for some $x_{0} \in M$ and some $r_{0}>0$ with $r_{0}<\alpha$, the solution is defined for $0 \leq t \leq r_{0}^{2}$ and satisfies

$$
|R m|(x, t) \leq r_{0}^{-2}, \quad \text { for } 0 \leq t \leq r_{0}^{2}, d_{0}\left(x, x_{0}\right) \leq r_{0},
$$

and

$$
\operatorname{Vol}_{0}\left(B_{0}\left(x_{0}, r_{0}\right)\right) \geq A^{-1} r_{0}^{3} .
$$

Then $R\left(x, r_{0}^{2}\right) \leq K r_{0}^{-2}$ whenever $d_{r_{0}^{2}}\left(x, x_{0}\right)<A r_{0}$.

Proof. Given any large $A>0$ and letting $\alpha>0$ be chosen later, by Perelman's no local collapsing theorem II (Theorem 3.4.2), there exists a positive constant $\kappa=\kappa(A)$ (independent of $\alpha$ ) such that any complete solution satisfying the assumptions of the theorem is $\kappa$-noncollapsed on scales $\leq r_{0}$ over the region $\left\{(x, t) \mid \frac{1}{5} r_{0}^{2} \leq t \leq\right.$ $\left.r_{0}^{2}, d_{t}\left(x, x_{0}\right) \leq 5 A r_{0}\right\}$. Set

$$
\varepsilon=\min \left\{\frac{1}{4} \varepsilon_{0}, \frac{1}{100}\right\}
$$

where $\varepsilon_{0}$ is the positive constant in Proposition 6.1.1. We first prove the following assertion.

Claim. For the above fixed $\varepsilon>0$, one can find $K=K(A, \varepsilon)<+\infty$ such that if we have a three-dimensional complete orientable solution with normalized initial metric and satisfying

$$
|R m|(x, t) \leq r_{0}^{-2} \quad \text { for } 0 \leq t \leq r_{0}^{2}, d_{0}\left(x, x_{0}\right) \leq r_{0},
$$


and

$$
\operatorname{Vol}_{0}\left(B_{0}\left(x_{0}, r_{0}\right)\right) \geq A^{-1} r_{0}^{3}
$$

for some $x_{0} \in M$ and some $r_{0}>0$, then for any point $x \in M$ with $d_{r_{0}^{2}}\left(x, x_{0}\right)<3 A r_{0}$, either

$$
R\left(x, r_{0}^{2}\right)<K r_{0}^{-2}
$$

or the subset $\left\{(y, t) \mid d_{r_{0}^{2}}^{2}(y, x) \leq \varepsilon^{-2} R\left(x, r_{0}^{2}\right)^{-1}, r_{0}^{2}-\varepsilon^{-2} R\left(x, r_{0}^{2}\right)^{-1} \leq t \leq r_{0}^{2}\right\}$ around the point $\left(x, r_{0}^{2}\right)$ is $\varepsilon$-close to the corresponding subset of an orientable ancient $\kappa$ solution.

Notice that in this assertion we don't impose the restriction of $r_{0}<\alpha$, so we can consider for the moment $r_{0}>0$ to be arbitrary in proving the above claim. Note that the assumption on the normalization of the initial metric is just to ensure the pinching estimate. By scaling, we may assume $r_{0}=1$. The proof of the claim is essentially adapted from that of Theorem 7.1.1. But we will meet the difficulties of adjusting points and verifying a local curvature estimate.

Suppose that the claim is not true. Then there exist a sequence of solutions $\left(M_{k}, g_{k}(\cdot, t)\right)$ to the Ricci flow satisfying the assumptions of the claim with the origins $x_{0_{k}}$, and a sequence of positive numbers $K_{k} \rightarrow \infty$, times $t_{k}=1$ and points $x_{k} \in$ $M_{k}$ with $d_{t_{k}}\left(x_{k}, x_{0_{k}}\right)<3 A$ such that $Q_{k}=R_{k}\left(x_{k}, t_{k}\right) \geq K_{k}$ and the solution in $\left\{(x, t) \mid t_{k}-C(\varepsilon) Q_{k}^{-1} \leq t \leq t_{k}, d_{t_{k}}^{2}\left(x, x_{k}\right) \leq C(\varepsilon) Q_{k}^{-1}\right\}$ is not, after scaling by the factor $Q_{k}, \varepsilon$-close to the corresponding subset of any orientable ancient $\kappa$-solution, where $R_{k}$ denotes the scalar curvature of $\left(M_{k}, g_{k}(\cdot, t)\right)$ and $C(\varepsilon)\left(\geq \varepsilon^{-2}\right)$ is the constant defined in the proof of Theorem 7.1.1. As before we need to first adjust the point $\left(x_{k}, t_{k}\right)$ with $t_{k} \geq \frac{1}{2}$ and $d_{t_{k}}\left(x_{k}, x_{0_{k}}\right)<4 A$ so that $Q_{k}=R_{k}\left(x_{k}, t_{k}\right) \geq K_{k}$ and the conclusion of the claim fails at $\left(x_{k}, t_{k}\right)$, but holds for any $(x, t)$ satisfying $R_{k}(x, t) \geq$ $2 Q_{k}, t_{k}-H_{k} Q_{k}^{-1} \leq t \leq t_{k}$ and $d_{t}\left(x, x_{0_{k}}\right)<d_{t_{k}}\left(x_{k}, x_{0_{k}}\right)+H_{k}^{\frac{1}{2}} Q_{k}^{-\frac{1}{2}}$, where $H_{k}=$ $\frac{1}{4} K_{k} \rightarrow \infty$, as $k \rightarrow+\infty$.

Indeed, by starting with $\left(x_{k_{1}}, t_{k_{1}}\right)=\left(x_{k}, 1\right)$ we can choose $\left(x_{k_{2}}, t_{k_{2}}\right) \in M_{k} \times$ $(0,1]$ with $t_{k_{1}}-H_{k} R_{k}\left(x_{k_{1}}, t_{k_{1}}\right)^{-1} \leq t_{k_{2}} \leq t_{k_{1}}$, and $d_{t_{k_{2}}}\left(x_{k_{2}}, x_{0_{k}}\right)<d_{t_{k_{1}}}\left(x_{k_{1}}, x_{0_{k}}\right)+$ $H_{k}^{\frac{1}{2}} R_{k}\left(x_{k_{1}}, t_{k_{1}}\right)^{-\frac{1}{2}}$ such that $R_{k}\left(x_{k_{2}}, t_{k_{2}}\right) \geq 2 R_{k}\left(x_{k_{1}}, t_{k_{1}}\right)$ and the conclusion of the claim fails at $\left(x_{k_{2}}, t_{k_{2}}\right)$; otherwise we have the desired point. Repeating this process, we can choose points $\left(x_{k_{i}}, t_{k_{i}}\right), i=2, \ldots, j$, such that

$$
\begin{gathered}
R_{k}\left(x_{k_{i}}, t_{k_{i}}\right) \geq 2 R_{k}\left(x_{k_{i-1}}, t_{k_{i-1}}\right) \\
t_{k_{i-1}}-H_{k} R_{k}\left(x_{k_{i-1}}, t_{k_{i-1}}\right)^{-1} \leq t_{k_{i}} \leq t_{k_{i-1}}, \\
d_{t_{k_{i}}}\left(x_{k_{i}}, x_{0_{k}}\right)<d_{t_{k_{i-1}}}\left(x_{k_{i-1}}, x_{0_{k}}\right)+H_{k}^{\frac{1}{2}} R_{k}\left(x_{k_{i-1}}, t_{k_{i-1}}\right)^{-\frac{1}{2}},
\end{gathered}
$$

and the conclusion of the claim fails at the points $\left(x_{k_{i}}, t_{k_{i}}\right), i=2, \ldots, j$. These inequalities imply

$$
\begin{gathered}
R_{k}\left(x_{k_{j}}, t_{k_{j}}\right) \geq 2^{j-1} R_{k}\left(x_{k_{1}}, t_{k_{1}}\right) \geq 2^{j-1} K_{k}, \\
1 \geq t_{k_{j}} \geq t_{k_{1}}-H_{k} \sum_{i=0}^{j-2} \frac{1}{2^{i}} R_{k}\left(x_{k_{1}}, t_{k_{1}}\right)^{-1} \geq \frac{1}{2},
\end{gathered}
$$


and

$$
d_{t_{k_{j}}}\left(x_{k_{j}}, x_{0_{k}}\right)<d_{t_{k_{1}}}\left(x_{k_{1}}, x_{0_{k}}\right)+H_{k}^{\frac{1}{2}} \sum_{i=0}^{j-2} \frac{1}{(\sqrt{2})^{i}} R_{k}\left(x_{k_{1}}, t_{k_{1}}\right)^{-\frac{1}{2}}<4 A .
$$

Since the solutions are smooth, this process must terminate after a finite number of steps to give the desired point, still denoted by $\left(x_{k}, t_{k}\right)$.

For each adjusted $\left(x_{k}, t_{k}\right)$, let $\left[t^{\prime}, t_{k}\right]$ be the maximal subinterval of $\left[t_{k}-\right.$ $\left.\frac{1}{2} \varepsilon^{-2} Q_{k}^{-1}, t_{k}\right]$ so that the conclusion of the claim with $K=2 Q_{k}$ holds on

$$
\begin{aligned}
& P\left(x_{k}, t_{k}, \frac{1}{10} H_{k}^{\frac{1}{2}} Q_{k}^{-\frac{1}{2}}, t^{\prime}-t_{k}\right) \\
& =\left\{(x, t) \mid x \in B_{t}\left(x_{k}, \frac{1}{10} H_{k}^{\frac{1}{2}} Q_{k}^{-\frac{1}{2}}\right), t \in\left[t^{\prime}, t_{k}\right]\right\}
\end{aligned}
$$

for all sufficiently large $k$. We now want to show $t^{\prime}=t_{k}-\frac{1}{2} \varepsilon^{-2} Q_{k}^{-1}$.

Consider the scalar curvature $R_{k}$ at the point $x_{k}$ over the time interval $\left[t^{\prime}, t_{k}\right]$. If there is a time $\tilde{t} \in\left[t^{\prime}, t_{k}\right]$ satisfying $R_{k}\left(x_{k}, \tilde{t}\right) \geq 2 Q_{k}$, we let $\tilde{t}$ be the first of such time from $t_{k}$. Then the solution $\left(M_{k}, g_{k}(\cdot, t)\right)$ around the point $x_{k}$ over the time interval $\left[\tilde{t}-\frac{1}{2} \varepsilon^{-2} Q_{k}^{-1}, \tilde{t}\right]$ is $\varepsilon$-close to some orientable ancient $\kappa$-solution. Note from the Li-Yau-Hamilton inequality that the scalar curvature of any ancient $\kappa$-solution is pointwise nondecreasing in time. Consequently, we have the following curvature estimate

$$
R_{k}\left(x_{k}, t\right) \leq 2(1+\varepsilon) Q_{k}
$$

for $t \in\left[\tilde{t}-\frac{1}{2} \varepsilon^{-2} Q_{k}^{-1}, t_{k}\right]$ (or $t \in\left[t^{\prime}, t_{k}\right]$ if there is no such time $\tilde{t}$ ). By combining with the elliptic type estimate for ancient $\kappa$-solutions (Theorem 6.4.3) and the HamiltonIvey pinching estimate, we further have

$$
|R m(x, t)| \leq 5 \omega(1) Q_{k}
$$

for all $x \in B_{t}\left(x_{k},\left(3 Q_{k}\right)^{-\frac{1}{2}}\right)$ and $t \in\left[\tilde{t}-\frac{1}{2} \varepsilon^{-2} Q_{k}^{-1}, t_{k}\right]$ (or $t \in\left[t^{\prime}, t_{k}\right]$ ) and all sufficiently large $k$, where $\omega$ is the positive function in Theorem 6.4.3.

For any point $(x, t)$ with $\tilde{t}-\frac{1}{2} \varepsilon^{-2} Q_{k}^{-1} \leq t \leq t_{k}$ (or $\left.t \in\left[t^{\prime}, t_{k}\right]\right)$ and $d_{t}\left(x, x_{k}\right) \leq$ $\frac{1}{10} H_{k}^{\frac{1}{2}} Q_{k}^{-\frac{1}{2}}$, we divide the discussion into two cases.

Case (1): $d_{t}\left(x_{k}, x_{0_{k}}\right) \leq \frac{3}{10} H_{k}^{\frac{1}{2}} Q_{k}^{-\frac{1}{2}}$.

$$
\begin{aligned}
d_{t}\left(x, x_{0_{k}}\right) & \leq d_{t}\left(x, x_{k}\right)+d_{t}\left(x_{k}, x_{0_{k}}\right) \\
& \leq \frac{1}{10} H_{k}^{\frac{1}{2}} Q_{k}^{-\frac{1}{2}}+\frac{3}{10} H_{k}^{\frac{1}{2}} Q_{k}^{-\frac{1}{2}} \\
& \leq \frac{1}{2} H_{k}^{\frac{1}{2}} Q_{k}^{-\frac{1}{2}} .
\end{aligned}
$$

Case (2): $d_{t}\left(x_{k}, x_{0_{k}}\right)>\frac{3}{10} H_{k}^{\frac{1}{2}} Q_{k}^{-\frac{1}{2}}$.

From the curvature bound (7.2.1) and the assumption, we apply Lemma 3.4.1(ii) with $r_{0}=Q_{k}^{-\frac{1}{2}}$ to get

$$
\frac{d}{d t}\left(d_{t}\left(x_{k}, x_{0_{k}}\right)\right) \geq-20(\omega(1)+1) Q_{k}^{\frac{1}{2}},
$$


and then for $k$ large enough,

$$
\begin{aligned}
d_{t}\left(x_{k}, x_{0_{k}}\right) & \leq d_{\hat{t}}\left(x_{k}, x_{0_{k}}\right)+20(\omega(1)+1) \varepsilon^{-2} Q_{k}^{-\frac{1}{2}} \\
& \leq d_{\hat{t}}\left(x_{k}, x_{0_{k}}\right)+\frac{1}{10} H_{k}^{\frac{1}{2}} Q_{k}^{-\frac{1}{2}}
\end{aligned}
$$

where $\hat{t} \in\left(t, t_{k}\right]$ satisfies the property that $d_{s}\left(x_{k}, x_{0_{k}}\right) \geq \frac{3}{10} H_{k}^{\frac{1}{2}} Q_{k}^{-\frac{1}{2}}$ whenever $s \in[t, \hat{t}]$. So we have

$$
\begin{aligned}
d_{t}\left(x, x_{0_{k}}\right) & \leq d_{t}\left(x, x_{k}\right)+d_{t}\left(x_{k}, x_{0_{k}}\right) \\
& \leq \frac{1}{10} H_{k}^{\frac{1}{2}} Q_{k}^{-\frac{1}{2}}+d_{\hat{t}}\left(x_{k}, x_{0_{k}}\right)+\frac{1}{10} H_{k}^{\frac{1}{2}} Q_{k}^{-\frac{1}{2}} \\
& \leq d_{t_{k}}\left(x_{k}, x_{0_{k}}\right)+\frac{1}{2} H_{k}^{\frac{1}{2}} Q_{k}^{-\frac{1}{2}},
\end{aligned}
$$

for all sufficiently large $k$. Then the combination of (7.2.2), (7.2.3) and the choice of the points $\left(x_{k}, t_{k}\right)$ implies $t^{\prime}=t_{k}-\frac{1}{2} \varepsilon^{-2} Q_{k}^{-1}$ for all sufficiently large $k$. (Here we also used the maximality of the subinterval $\left[t^{\prime}, t_{k}\right]$ in the case that there is no time in $\left[t^{\prime}, t_{k}\right]$ with $R_{k}\left(x_{k}, \cdot\right) \geq 2 Q_{k}$.)

Now we rescale the solutions $\left(M_{k}, g_{k}(\cdot, t)\right)$ into $\left(M_{k}, \tilde{g}_{k}(\cdot, t)\right)$ around the points $x_{k}$ by the factors $Q_{k}=R_{k}\left(x_{k}, t_{k}\right)$ and shift the times $t_{k}$ to the new times zero. Then the same arguments from Step 1 to Step 3 in the proof of Theorem 7.1.1 prove that a subsequence of the rescaled solutions $\left(M_{k}, \tilde{g}_{k}(\cdot, t)\right)$ converges in the $C_{\text {loc }}^{\infty}$ topology to a limiting (complete) solution $\left(M_{\infty}, \tilde{g}_{\infty}(\cdot, t)\right)$, which is defined on a backward time interval $[-a, 0]$ for some $a>0$. (The only modification is in Lemma 7.1.2 of Step 1 by further requiring $\left.t_{k}-\frac{1}{4} \varepsilon^{-2} Q_{k}^{-1} \leq \bar{t} \leq t_{k}\right)$.

We next study how to adapt the argument of Step 4 in the proof of Theorem 7.1.1. As before, we have a maximal time interval $\left(t_{\infty}, 0\right]$ for which we can take a smooth limit $\left(M_{\infty}, \tilde{g}_{\infty}(\cdot, t), x_{\infty}\right)$ from a subsequence of the rescaled solutions $\left(M_{k}, \tilde{g}_{k}(\cdot, t), x_{k}\right)$. We want to show $t_{\infty}=-\infty$.

Suppose not; then $t_{\infty}>-\infty$. Let $c>0$ be a positive constant much smaller than $\frac{1}{10} \varepsilon^{-2}$. Note that the infimum of the scalar curvature is nondecreasing in time. Then we can find some point $y_{\infty} \in M_{\infty}$ and some time $t=t_{\infty}+\theta$ with $0<\theta<\frac{c}{3}$ such that $\tilde{R}_{\infty}\left(y_{\infty}, t_{\infty}+\theta\right) \leq \frac{3}{2}$.

Consider the (unrescaled) scalar curvature $R_{k}$ of $\left(M_{k}, g_{k}(\cdot, t)\right)$ at the point $x_{k}$ over the time interval $\left[t_{k}+\left(t_{\infty}+\frac{\theta}{2}\right) Q_{k}^{-1}, t_{k}\right]$. Since the scalar curvature $\tilde{R}_{\infty}$ of the limit on $M_{\infty} \times\left[t_{\infty}+\frac{\theta}{3}, 0\right]$ is uniformly bounded by some positive constant $C$, we have the curvature estimate

$$
R_{k}\left(x_{k}, t\right) \leq 2 C Q_{k}
$$

for all $t \in\left[t_{k}+\left(t_{\infty}+\frac{\theta}{2}\right) Q_{k}^{-1}, t_{k}\right]$ and all sufficiently large $k$. Then by repeating the same arguments as in deriving (7.2.1), (7.2.2) and (7.2.3), we deduce that the conclusion of the claim with $K=2 Q_{k}$ holds on the parabolic neighborhood $P\left(x_{k}, t_{k}, \frac{1}{10} H_{k}^{\frac{1}{2}} Q_{k}^{-\frac{1}{2}},\left(t_{\infty}+\frac{\theta}{2}\right) Q_{k}^{-1}\right)$ for all sufficiently large $k$.

Let $\left(y_{k}, t_{k}+\left(t_{\infty}+\theta_{k}\right) Q_{k}^{-1}\right)$ be a sequence of associated points and times in the (unrescaled) solutions $\left(M_{k}, g_{k}(\cdot, t)\right)$ so that after rescaling, the sequence converges to the $\left(y_{\infty}, t_{\infty}+\theta\right)$ in the limit. Clearly $\frac{\theta}{2} \leq \theta_{k} \leq 2 \theta$ for all sufficiently large $k$. Then, by considering the scalar curvature $R_{k}$ at the point $y_{k}$ over the time interval $\left[t_{k}+\left(t_{\infty}-\frac{c}{3}\right) Q_{k}^{-1}, t_{k}+\left(t_{\infty}+\theta_{k}\right) Q_{k}^{-1}\right]$, the above argument (as in deriving the similar 
estimates (7.2.1)-(7.2.3)) implies that the conclusion of the claim with $K=2 Q_{k}$ holds on the parabolic neighborhood $P\left(y_{k}, t_{k}, \frac{1}{10} H_{k}^{\frac{1}{2}} Q_{k}^{-\frac{1}{2}},\left(t_{\infty}-\frac{c}{3}\right) Q_{k}^{-1}\right)$ for all sufficiently large $k$. In particular, we have the curvature estimate

$$
R_{k}\left(y_{k}, t\right) \leq 4(1+\varepsilon) Q_{k}
$$

for $t \in\left[t_{k}+\left(t_{\infty}-\frac{c}{3}\right) Q_{k}^{-1}, t_{k}+\left(t_{\infty}+\theta_{k}\right) Q_{k}^{-1}\right]$ for all sufficiently large $k$.

We now consider the rescaled sequence $\left(M_{k}, \tilde{g}_{k}(\cdot, t)\right)$ with the marked points replaced by $y_{k}$ and the times replaced by $s_{k} \in\left[t_{k}+\left(t_{\infty}-\frac{c}{4}\right) Q_{k}^{-1}, t_{k}+\left(t_{\infty}+\frac{c}{4}\right) Q_{k}^{-1}\right]$. By applying the same arguments from Step 1 to Step 3 in the proof of Theorem 7.1.1 and the Li-Yau-Hamilton inequality as in Step 4 of Theorem 7.1.1, we conclude that there is some small constant $a^{\prime}>0$ such that the original limit $\left(M_{\infty}, \tilde{g}_{\infty}(\cdot, t)\right)$ is actually well defined on $M_{\infty} \times\left[t_{\infty}-a^{\prime}, 0\right]$ with uniformly bounded curvature. This is a contradiction. Therefore we have checked the claim.

To finish the proof, we next argue by contradiction. Suppose there exist sequences of positive numbers $K_{k} \rightarrow+\infty, \alpha_{k} \rightarrow 0$, as $k \rightarrow+\infty$, and a sequence of solutions $\left(M_{k}, g_{k}(\cdot, t)\right)$ to the Ricci flow satisfying the assumptions of the theorem with origins $x_{0_{k}}$ and with radii $r_{0_{k}}$ satisfying $r_{0_{k}}<\alpha_{k}$ such that for some points $x_{k} \in M_{k}$ with $d_{r_{0_{k}}^{2}}\left(x_{k}, x_{0_{k}}\right)<A r_{0_{k}}$ we have

$$
R\left(x_{k}, r_{0_{k}}^{2}\right)>K_{k} r_{0_{k}}^{-2}
$$

for all $k$. Let $\left(M_{k}, \hat{g}_{k}(\cdot, t), x_{0_{k}}\right)$ be the rescaled solutions of $\left(M_{k}, g_{k}(\cdot, t)\right)$ around the origins $x_{0_{k}}$ by the factors $r_{0_{k}}^{-2}$ and shifting the times $r_{0_{k}}^{2}$ to the new times zero. The above claim tells us that for $k$ large, any point $(y, 0) \in\left(M_{k}, \hat{g}_{k}(\cdot, 0), x_{0_{k}}\right)$ with $d_{\hat{g}_{k}(\cdot, 0)}\left(y, x_{0_{k}}\right)<3 A$ and with the rescaled scalar curvature $\hat{R}_{k}(y, 0)>K_{k}$ has a canonical neighborhood which is either a $2 \varepsilon$-neck, or a $2 \varepsilon$-cap, or a compact manifold (without boundary) diffeomorphic to a metric quotient of the round three-sphere. Note that the pinching estimate (7.1.1) and the condition $\alpha_{k} \rightarrow 0$ imply any subsequential limit of the rescaled solutions $\left(M_{k}, \hat{g}_{k}(\cdot, t), x_{0_{k}}\right)$ must have nonnegative sectional curvature. Thus the same argument as in Step 2 of the proof of Theorem 7.1.1 shows that for all sufficiently large $k$, the curvatures of the rescaled solutions at the time zero stay uniformly bounded at those points whose distances from the origins $x_{0_{k}}$ do not exceed $2 A$. This contradicts (7.2.4) for $k$ large enough.

Therefore we have completed the proof of the theorem.

The next result is a generalization of the curvature estimate via volume growth in Theorem 6.3.3 (ii) where the condition on the curvature lower bound over a time interval is replaced by that at a time slice only.

Theorem 7.2.2 (Perelman [103]). For any $w>0$ there exist $\tau=\tau(w)>0$, $K=K(w)<+\infty, \alpha=\alpha(w)>0$ with the following property. Suppose we have a three-dimensional, compact and orientable solution to the Ricci flow defined on $M \times[0, T)$ with normalized initial metric. Suppose that for some radius $r_{0}>0$ with $r_{0}<\alpha$ and a point $\left(x_{0}, t_{0}\right) \in M \times[0, T)$ with $T>t_{0} \geq 4 \tau r_{0}^{2}$, the solution on the ball $B_{t_{0}}\left(x_{0}, r_{0}\right)$ satisfies

$$
\begin{gathered}
R m\left(x, t_{0}\right) \geq-r_{0}^{-2} \quad \text { on } B_{t_{0}}\left(x_{0}, r_{0}\right) \\
\text { and } \operatorname{Vol}_{t_{0}}\left(B_{t_{0}}\left(x_{0}, r_{0}\right)\right) \geq w r_{0}^{3}
\end{gathered}
$$


Then $R(x, t) \leq K r_{0}^{-2}$ whenever $t \in\left[t_{0}-\tau r_{0}^{2}, t_{0}\right]$ and $d_{t}\left(x, x_{0}\right) \leq \frac{1}{4} r_{0}$.

Proof. If we knew that

$$
R m(x, t) \geq-r_{0}^{-2}
$$

for all $t \in\left[0, t_{0}\right]$ and $d_{t}\left(x, x_{0}\right) \leq r_{0}$, then we could just apply Theorem 6.3 .3 (ii) and take $\tau(w)=\tau_{0}(w) / 2, K(w)=C(w)+2 B(w) / \tau_{0}(w)$. Now fix these values of $\tau$ and $K$.

We argue by contradiction. Consider a three-dimensional, compact and orientable solution $g_{i j}(t)$ to the Ricci flow with normalized initial metric, a point $\left(x_{0}, t_{0}\right)$ and some radius $r_{0}>0$ with $r_{0}<\alpha$, for $\alpha>0$ a sufficiently small constant to be determined later, such that the assumptions of the theorem do hold whereas the conclusion does not. We first claim that we may assume that any other point $\left(x^{\prime}, t^{\prime}\right)$ and radius $r^{\prime}>0$ with the same property has either $t^{\prime}>t_{0}$ or $t^{\prime}<t_{0}-2 \tau r_{0}^{2}$, or $2 r^{\prime}>$ $r_{0}$. Indeed, suppose otherwise. Then there exist $\left(x_{0}^{\prime}, t_{0}^{\prime}\right)$ and $r_{0}^{\prime}$ with $t_{0}^{\prime} \in\left[t_{0}-2 \tau r_{0}^{2}, t_{0}\right]$ and $r_{0}^{\prime} \leq \frac{1}{2} r_{0}$, for which the assumptions of the theorem hold but the conclusion does not. Thus, there is a point $(x, t)$ such that

$$
\begin{gathered}
t \in\left[t_{0}^{\prime}-\tau\left(r_{0}^{\prime}\right)^{2}, t_{0}^{\prime}\right] \subset\left[t_{0}-2 \tau r_{0}^{2}-\frac{\tau}{4} r_{0}^{2}, t_{0}\right] \\
\text { and } R(x, t)>K\left(r_{0}^{\prime}\right)^{-2} \geq 4 K r_{0}^{-2} .
\end{gathered}
$$

If the point $\left(x_{0}^{\prime}, t_{0}^{\prime}\right)$ and the radius $r_{0}^{\prime}$ satisfy the claim then we stop, and otherwise we iterate the procedure. Since $t_{0} \geq 4 \tau r_{0}^{2}$ and the solution is smooth, the iteration must terminate in a finite number of steps, which provides the desired point and the desired radius.

Let $\tau^{\prime} \geq 0$ be the largest number such that

$$
R m(x, t) \geq-r_{0}^{-2}
$$

whenever $t \in\left[t_{0}-\tau^{\prime} r_{0}^{2}, t_{0}\right]$ and $d_{t}\left(x, x_{0}\right) \leq r_{0}$. If $\tau^{\prime} \geq 2 \tau$, we are done by Theorem 6.3 .3 (ii). Thus we may assume $\tau^{\prime}<2 \tau$. By applying Theorem 6.3.3(ii), we know that at time $t^{\prime}=t_{0}-\tau^{\prime} r_{0}^{2}$, the ball $B_{t^{\prime}}\left(x_{0}, r_{0}\right)$ has

$$
\operatorname{Vol}_{t^{\prime}}\left(B_{t^{\prime}}\left(x_{0}, r_{0}\right)\right) \geq \xi(w) r_{0}^{3}
$$

for some positive constant $\xi(w)$ depending only on $w$. We next claim that there exists a ball (at time $\left.t^{\prime}=t_{0}-\tau^{\prime} r_{0}^{2}\right) B_{t^{\prime}}\left(x^{\prime}, r^{\prime}\right) \subset B_{t^{\prime}}\left(x_{0}, r_{0}\right)$ with

$$
\operatorname{Vol}_{t^{\prime}}\left(B_{t^{\prime}}\left(x^{\prime}, r^{\prime}\right)\right) \geq \frac{1}{2} \alpha_{3}\left(r^{\prime}\right)^{3}
$$

and with

$$
\frac{r_{0}}{2}>r^{\prime} \geq c(w) r_{0}
$$

for some small positive constant $c(w)$ depending only on $w$, where $\alpha_{3}$ is the volume of the unit ball $\mathbb{B}^{3}$ in the Euclidean space $\mathbb{R}^{3}$.

Indeed, suppose that it is not true. Then after rescaling, there is a sequence of Riemannian manifolds $M_{i}, i=1,2, \ldots$, with balls $B\left(x_{i}, 1\right) \subset M_{i}$ so that

$$
R m \geq-1 \quad \text { on } B\left(x_{i}, 1\right)
$$


and

$$
\operatorname{Vol}\left(B\left(x_{i}, 1\right)\right) \geq \xi(w)
$$

for all $i$, but all balls $B\left(x_{i}^{\prime}, r_{i}^{\prime}\right) \subset B\left(x_{i}, 1\right)$ with $\frac{1}{2}>r_{i}^{\prime} \geq \frac{1}{i}$ satisfy

$$
\operatorname{Vol}\left(B\left(x_{i}^{\prime}, r_{i}^{\prime}\right)\right)<\frac{1}{2} \alpha_{3}\left(r_{i}^{\prime}\right)^{3} .
$$

It follows from basic results in Alexandrov space theory (see for example Theorem 10.7.2 and Theorem 10.10.10 of [9]) that, after taking a subsequence, the marked balls $\left(B\left(x_{i}, 1\right), x_{i}\right)$ converge in the Gromov-Hausdorff topology to a marked length space $\left(B_{\infty}, x_{\infty}\right)$ with curvature bounded from below by -1 in the Alexandrov space sense, and the associated Riemannian volume forms $d \operatorname{Vol}_{M_{i}}$ over $\left(B\left(x_{i}, 1\right), x_{i}\right)$ converge weakly to the Hausdorff measure $\mu$ of $B_{\infty}$. It is well-known that the Hausdorff dimension of any Alexandrov space is either an integer or infinity (see for example Theorem 10.8.2 of [9]). Then by $(7.2 .6)^{\prime}$, we know the limit $\left(B_{\infty}, x_{\infty}\right)$ is a threedimensional Alexandrov space of curvature $\geq-1$. In the Alexandrov space theory, a point $p \in B_{\infty}$ is said to be regular if the tangent cone of $B_{\infty}$ at $p$ is isometric to $\mathbb{R}^{3}$. It is also a basic result in Alexandrov space theory (see for example Corollary 10.9.13 of [9]) that the set of regular points in $B_{\infty}$ is dense and for each regular point there is a small neighborhood which is almost isometric to an open set of the Euclidean space $\mathbb{R}^{3}$. Thus for any $\varepsilon>0$, there are balls $B\left(x_{\infty}^{\prime}, r_{\infty}^{\prime}\right) \subset B_{\infty}$ with $0<r_{\infty}^{\prime}<\frac{1}{3}$ and satisfying

$$
\mu\left(B\left(x_{\infty}^{\prime}, r_{\infty}^{\prime}\right)\right) \geq(1-\varepsilon) \alpha_{3}\left(r_{\infty}^{\prime}\right)^{3} .
$$

This is a contradiction with (7.2.9).

Without loss of generality, we may assume $w \leq \frac{1}{4} \alpha_{3}$. Since $\tau^{\prime}<2 \tau$, it follows from the choice of the point $\left(x_{0}, t_{0}\right)$ and the radius $r_{0}$ and (7.2.5), (7.2.7), (7.2.8) that the conclusion of the theorem holds for $\left(x^{\prime}, t^{\prime}\right)$ and $r^{\prime}$. Thus we have the estimate

$$
R(x, t) \leq K\left(r^{\prime}\right)^{-2}
$$

whenever $t \in\left[t^{\prime}-\tau\left(r^{\prime}\right)^{2}, t^{\prime}\right]$ and $d_{t}\left(x, x^{\prime}\right) \leq \frac{1}{4} r^{\prime}$. For $\alpha>0$ small, by combining with the pinching estimate (7.1.1), we have

$$
|R m(x, t)| \leq K^{\prime}\left(r^{\prime}\right)^{-2}
$$

whenever $t \in\left[t^{\prime}-\tau\left(r^{\prime}\right)^{2}, t^{\prime}\right]$ and $d_{t}\left(x, x^{\prime}\right) \leq \frac{1}{4} r^{\prime}$, where $K^{\prime}$ is some positive constant depending only on $K$. Note that this curvature estimate implies the evolving metrics are equivalent over a suitable subregion of $\left\{(x, t) \mid t \in\left[t^{\prime}-\tau\left(r^{\prime}\right)^{2}, t^{\prime}\right]\right.$ and $d_{t}\left(x, x^{\prime}\right) \leq$ $\left.\frac{1}{4} r^{\prime}\right\}$. Now we can apply Theorem 7.2.1 to choose $\alpha=\alpha(w)>0$ so small that

$$
R(x, t) \leq \tilde{K}(w)\left(r^{\prime}\right)^{-2} \leq \tilde{K}(w) c(w)^{-2} r_{0}^{-2}
$$

whenever $t \in\left[t^{\prime}-\frac{\tau}{2}\left(r^{\prime}\right)^{2}, t^{\prime}\right]$ and $d_{t}\left(x, x^{\prime}\right) \leq 10 r_{0}$. Then the combination of (7.2.10) with the pinching estimate (7.1.2) would imply

$$
\begin{aligned}
R m(x, t) & \geq-\left[f^{-1}(R(x, t)(1+t)) /(R(x, t)(1+t))\right] R(x, t) \\
& \geq-\frac{1}{2} r_{0}^{-2}
\end{aligned}
$$


on the region $\left\{(x, t) \mid t \in\left[t^{\prime}-\frac{\tau}{2}\left(r^{\prime}\right)^{2}, t^{\prime}\right]\right.$ and $\left.d_{t}\left(x, x_{0}\right) \leq r_{0}\right\}$ when $\alpha=\alpha(w)>r_{0}$ small enough. This contradicts the choice of $\tau^{\prime}$. Therefore we have proved the theorem.

The combination of the above two theorems immediately gives the following consequence.

Corollary 7.2.3. For any $w>0$ and $A<+\infty$, there exist $\tau=\tau(w, A)>0$, $K=K(w, A)<+\infty$, and $\alpha=\alpha(w, A)>0$ with the following property. Suppose we have a three-dimensional, compact and orientable solution to the Ricci flow defined on $M \times[0, T)$ with normalized initial metric. Suppose that for some radius $r_{0}>0$ with $r_{0}<\alpha$ and a point $\left(x_{0}, t_{0}\right) \in M \times[0, T)$ with $T>t_{0} \geq 4 \tau r_{0}^{2}$, the solution on the ball $B_{t_{0}}\left(x_{0}, r_{0}\right)$ satisfies

$$
\begin{gathered}
R m\left(x, t_{0}\right) \geq-r_{0}^{-2} \quad \text { on } B_{t_{0}}\left(x_{0}, r_{0}\right), \\
\text { and } \operatorname{Vol}_{t_{0}}\left(B_{t_{0}}\left(x_{0}, r_{0}\right)\right) \geq w r_{0}^{3} .
\end{gathered}
$$

Then $R(x, t) \leq K r_{0}^{-2}$ whenever $t \in\left[t_{0}-\tau r_{0}^{2}, t_{0}\right]$ and $d_{t}\left(x, x_{0}\right) \leq A r_{0}$.

We can also state the previous corollary in the following version.

Corollary 7.2.4 (Perelman [103]). For any $w>0$ one can find $\rho=\rho(w)>0$ such that if $g_{i j}(t)$ is a complete solution to the Ricci flow defined on $M \times[0, T)$ with $T>1$ and with normalized initial metric, where $M$ is a three-dimensional, compact and orientable manifold, and if $B_{t_{0}}\left(x_{0}, r_{0}\right)$ is a metric ball at time $t_{0} \geq 1$, with $r_{0}<\rho$, such that

$$
\min \left\{R m\left(x, t_{0}\right) \mid x \in B_{t_{0}}\left(x_{0}, r_{0}\right)\right\}=-r_{0}^{-2},
$$

then

$$
\operatorname{Vol}_{t_{0}}\left(B_{t_{0}}\left(x_{0}, r_{0}\right)\right) \leq w r_{0}^{3}
$$

Proof. We argue by contradiction. Suppose for any $\rho>0$, there is a solution and a ball $B_{t_{0}}\left(x_{0}, r_{0}\right)$ satisfying the assumption of the corollary with $r_{0}<\rho, t_{0} \geq 1$, and with

$$
\min \left\{R m\left(x, t_{0}\right) \mid x \in B_{t_{0}}\left(x_{0}, r_{0}\right)\right\}=-r_{0}^{-2},
$$

but

$$
\operatorname{Vol}_{t_{0}}\left(B_{t_{0}}\left(x_{0}, r_{0}\right)\right)>w r_{0}^{3} .
$$

We can apply Corollary 7.2.3 to get

$$
R(x, t) \leq K r_{0}^{-2}
$$

whenever $t \in\left[t_{0}-\tau r_{0}^{2}, t_{0}\right]$ and $d_{t}\left(x, x_{0}\right) \leq 2 r_{0}$, provided $\rho>0$ is so small that $4 \tau \rho^{2} \leq 1$ and $\rho<\alpha$, where $\tau, \alpha$ and $K$ are the positive constants obtained in Corollary 7.2.3. Then for $r_{0}<\rho$ and $\rho>0$ sufficiently small, it follows from the pinching estimate (7.1.2) that

$$
\begin{aligned}
R m(x, t) & \geq-\left[f^{-1}(R(x, t)(1+t)) /(R(x, t)(1+t))\right] R(x, t) \\
& \geq-\frac{1}{2} r_{0}^{-2}
\end{aligned}
$$


in the region $\left\{(x, t) \mid t \in\left[t_{0}-\tau\left(r_{0}\right)^{2}, t_{0}\right]\right.$ and $\left.d_{t}\left(x, x_{0}\right) \leq 2 r_{0}\right\}$. In particular, this would imply

$$
\min \left\{R m\left(x, t_{0}\right) \mid x \in B_{t_{0}}\left(x_{0}, r_{0}\right)\right\}>-r_{0}^{-2} .
$$

This contradicts the assumption.

7.3. Ricci Flow with Surgery. One of the central themes of the Ricci flow theory is to give a classification of all compact orientable three-manifolds. As we mentioned before, the basic idea is to obtain long-time behavior of solutions to the Ricci flow. However the solutions will in general become singular in finite time. Fortunately, we now understand the precise structures of the solutions around the singularities, thanks to Theorem 7.1.1. When a solution develops singularities, one can perform geometric surgeries by cutting off the canonical neighborhoods around the singularities and gluing back some known pieces, and then continue running the Ricci flow. By repeating this procedure, one hopes to get a kind of "weak" solution. In this section we will give a detailed description of this surgery procedure and define a global "weak" solution to the Ricci flow.

Given any $\varepsilon>0$, based on the singularity structure theorem (Theorem 7.1.1), we can get a clear picture of the solution near the singular time as follows.

Let $\left(M, g_{i j}(\cdot, t)\right)$ be a maximal solution to the Ricci flow on the maximal time interval $[0, T)$ with $T<+\infty$, where $M$ is a connected compact orientable threemanifold and the initial metric is normalized. For the given $\varepsilon>0$ and the solution $\left(M, g_{i j}(\cdot, t)\right)$, we can find $r_{0}>0$ such that each point $(x, t)$ with $R(x, t) \geq r_{0}^{-2}$ satisfies the derivative estimates

$$
|\nabla R(x, t)|<\eta R^{\frac{3}{2}}(x, t) \text { and }\left|\frac{\partial}{\partial t} R(x, t)\right|<\eta R^{2}(x, t),
$$

where $\eta>0$ is a universal constant, and has a canonical neighborhood which is either an evolving $\varepsilon$-neck, or an evolving $\varepsilon$-cap, or a compact positively curved manifold (without boundary). In the last case the solution becomes extinct at the maximal time $T$ and the manifold $M$ is diffeomorphic to the round three-sphere $\mathbb{S}^{3}$ or a metric quotient of $\mathbb{S}^{3}$ by Theorem 5.2.1.

Let $\Omega$ denote the set of all points in $M$ where the curvature stays bounded as $t \rightarrow T$. The gradient estimates in (7.3.1) imply that $\Omega$ is open and that $R(x, t) \rightarrow \infty$ as $t \rightarrow T$ for each $x \in M \backslash \Omega$.

If $\Omega$ is empty, then the solution becomes extinct at time $T$. In this case, either the manifold $M$ is compact and positively curved, or it is entirely covered by evolving $\varepsilon$-necks and evolving $\varepsilon$-caps shortly before the maximal time $T$. So the manifold $M$ is diffeomorphic to either $\mathbb{S}^{3}$, or a metric quotient of the round $\mathbb{S}^{3}$, or $\mathbb{S}^{2} \times \mathbb{S}^{1}$, or $\mathbb{R P}^{3} \# \mathbb{R P}^{3}$. The reason is as follows. Clearly, we only need to consider the situation that the manifold $M$ is entirely covered by evolving $\varepsilon$-necks and evolving $\varepsilon$-caps shortly before the maximal time $T$. If $M$ contains a cap $C$, then there is a cap or a neck adjacent to the neck-like end of $C$. The former case implies that $M$ is diffeomorphic to $\mathbb{S}^{3}, \mathbb{R P}^{3}$, or $\mathbb{R} \mathbb{P}^{3} \# \mathbb{R P}^{3}$. In the latter case, we get a new longer cap and continue. Finally, we must end up with a cap, producing a $\mathbb{S}^{3}, \mathbb{R P}^{3}$, or $\mathbb{R} \mathbb{P}^{3} \# \mathbb{R} \mathbb{P}^{3}$. If $M$ contains no caps, we start with a neck $N$. By connecting with the necks that are adjacent to the boundary of $N$, we get a longer neck and continue. After a finite number of steps, the resulting neck must repeat itself. Since $M$ is orientable, we conclude that $M$ is diffeomorphic to $\mathbb{S}^{2} \times \mathbb{S}^{1}$. 
We can now assume that $\Omega$ is nonempty. By using the local derivative estimates of Shi (Theorem 1.4.2), we see that as $t \rightarrow T$ the solution $g_{i j}(\cdot, t)$ has a smooth limit $\bar{g}_{i j}(\cdot)$ on $\Omega$. Let $\bar{R}(x)$ denote the scalar curvature of $\bar{g}_{i j}$. For any $\rho<r_{0}$, let us consider the set

$$
\Omega_{\rho}=\left\{x \in \Omega \mid \bar{R}(x) \leq \rho^{-2}\right\} .
$$

By the evolution equation of the Ricci flow, we see that the initial metric $g_{i j}(\cdot, 0)$ and the limit metric $\bar{g}_{i j}(\cdot)$ are equivalent over any fixed region where the curvature remains uniformly bounded. Note that for any fixed $x \in \partial \Omega$, and any sequence of points $x_{j} \in \Omega$ with $x_{j} \rightarrow x$ with respect to the initial metric $g_{i j}(\cdot, 0)$, we have $\bar{R}\left(x_{j}\right) \rightarrow+\infty$. In fact, if there were a subsequence $x_{j_{k}}$ so that $\lim _{k \rightarrow \infty} \bar{R}\left(x_{j_{k}}\right)$ exists and is finite, then it would follow from the gradient estimates (7.3.1) that $\bar{R}$ is uniformly bounded in some small neighborhood of $x \in \partial \Omega$ (with respect to the induced topology of the initial metric $\left.g_{i j}(\cdot, 0)\right)$; this is a contradiction. From this observation and the compactness of the initial manifold, we see that $\Omega_{\rho}$ is compact (with respect to the metric $\bar{g}_{i j}(\cdot)$ ).

For further discussions, let us introduce the following terminologies. Denote by $\mathbb{I}$ an interval.

Recall that an $\varepsilon$-neck (of radius $r$ ) is an open set with a Riemannian metric, which is, after scaling the metric with factor $r^{-2}, \varepsilon$-close to the standard neck $\mathbb{S}^{2} \times \mathbb{I}$ with the product metric, where $\mathbb{S}^{2}$ has constant scalar curvature one and $\mathbb{I}$ has length $2 \varepsilon^{-1}$ and the $\varepsilon$-closeness refers to the $C^{\left[\varepsilon^{-1}\right]}$ topology.

A metric on $\mathbb{S}^{2} \times \mathbb{I}$, such that each point is contained in some $\varepsilon$-neck, is called an $\varepsilon$-tube, or an $\varepsilon$-horn, or a double $\varepsilon$-horn, if the scalar curvature stays bounded on both ends, or stays bounded on one end and tends to infinity on the other, or tends to infinity on both ends, respectively.

A metric on $\mathbb{B}^{3}$ or $\mathbb{R P}^{3} \backslash \bar{B}^{3}$ is called a $\varepsilon$-cap if the region outside some suitable compact subset is an $\varepsilon$-neck. A metric on $\mathbb{B}^{3}$ or $\mathbb{R P}^{3} \backslash \overline{\mathbb{B}}^{3}$ is called an capped $\varepsilon$-horn if each point outside some compact subset is contained in an $\varepsilon$-neck and the scalar curvature tends to infinity on the end.

Now take any $\varepsilon$-neck in $\left(\Omega, \bar{g}_{i j}\right)$ and consider a point $x$ on one of its boundary components. If $x \in \Omega \backslash \Omega_{\rho}$, then there is either an $\varepsilon$-cap or an $\varepsilon$-neck, adjacent to the initial $\varepsilon$-neck. In the latter case we can take a point on the boundary of the second $\varepsilon$-neck and continue. This procedure can either terminate when we get into $\Omega_{\rho}$ or an $\varepsilon$-cap, or go on indefinitely, producing an $\varepsilon$-horn. The same procedure can be repeated for the other boundary component of the initial $\varepsilon$-neck. Therefore, taking into account that $\Omega$ has no compact components, we conclude that each $\varepsilon$-neck of $\left(\Omega, \bar{g}_{i j}\right)$ is contained in a subset of $\Omega$ of one of the following types:

(a) an $\varepsilon$-tube with boundary components in $\Omega_{\rho}$, or

(b) an $\varepsilon$-cap with boundary in $\Omega_{\rho}$, or

(c) an $\varepsilon$-horn with boundary in $\Omega_{\rho}$, or

(d) a capped $\varepsilon$-horn, or

(e) a double $\varepsilon$-horn.

Similarly, each $\varepsilon$-cap of $\left(\Omega, \bar{g}_{i j}\right)$ is contained in a subset of $\Omega$ of either type (b) or type (d).

It is clear that there is a definite lower bound (depending on $\rho$ ) for the volume of subsets of types (a), (b) and (c), so there can be only a finite number of them. Thus 
we conclude that there is only a finite number of components of $\Omega$ containing points of $\Omega_{\rho}$, and every such component has a finite number of ends, each being an $\varepsilon$-horn. On the other hand, every component of $\Omega$, containing no points of $\Omega_{\rho}$, is either a capped $\varepsilon$-horn, or a double $\varepsilon$-horn. If we look at the solution at a slightly earlier time, the above argument shows each $\varepsilon$-neck or $\varepsilon$-cap of $\left(M, g_{i j}(\cdot, t)\right)$ is contained in a subset of types (a) or (b), while the $\varepsilon$-horns, capped $\varepsilon$-horns and double $\varepsilon$-horns (at the maximal time $\mathrm{T}$ ) are connected together to form $\varepsilon$-tubes and $\varepsilon$-caps at the times $t$ shortly before $T$.

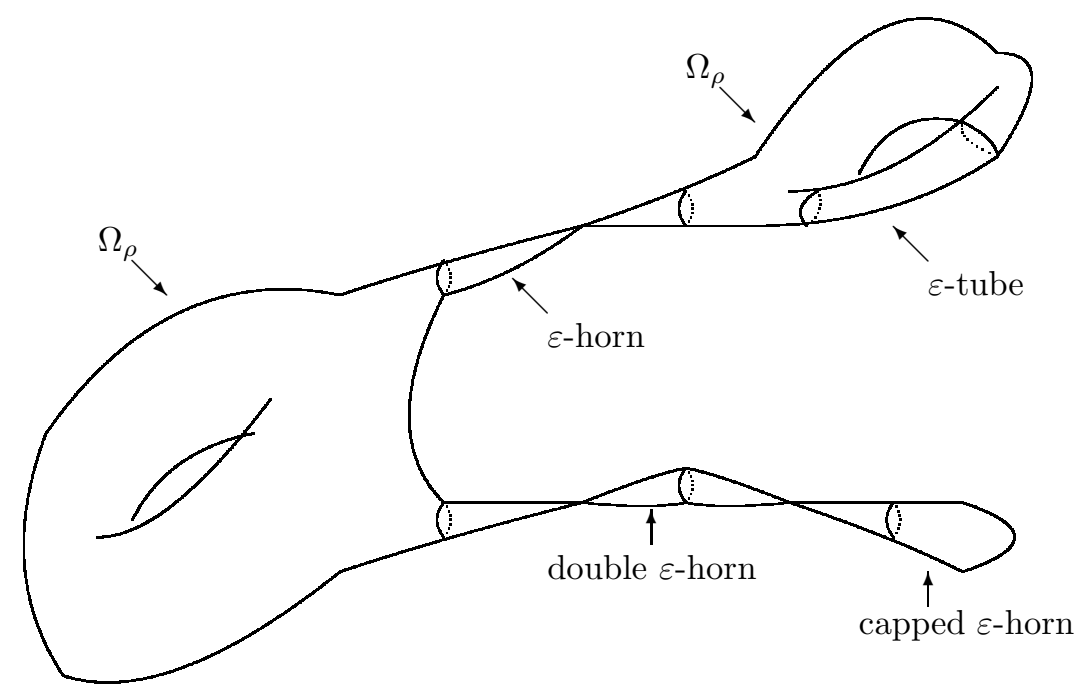

Hence, by looking at the solution at times shortly before $T$, we see that the topology of $M$ can be reconstructed as follows: take the components $\Omega_{j}, 1 \leq j \leq k$, of $\Omega$ which contain points of $\Omega_{\rho}$, truncate their $\varepsilon$-horns, and glue to the boundary components of truncated $\Omega_{j}$ a finite collection of tubes $\mathbb{S}^{2} \times \mathbb{I}$ and caps $\mathbb{B}^{3}$ or $\mathbb{R P}^{3} \backslash \overline{\mathbb{B}}^{3}$. Thus, $M$ is diffeomorphic to a connected sum of $\bar{\Omega}_{j}, 1 \leq j \leq k$, with a finite number of copies of $\mathbb{S}^{2} \times \mathbb{S}^{1}$ (which correspond to gluing a tube to two boundary components of the same $\Omega_{j}$ ), and a finite number of copies of $\mathbb{R P}^{3}$. Here $\bar{\Omega}_{j}$ denotes $\Omega_{j}$ with each $\varepsilon$-horn one point compactified. More geometrically, one can get $\bar{\Omega}_{j}$ in the following way: in every $\varepsilon$-horn of $\Omega_{j}$ one can find an $\varepsilon$-neck, cut it along the middle two-sphere, remove the horn-shaped end, and glue back a cap (or more precisely, a differentiable three-ball). Thus to understand the topology of $M$, one only needs to understand the topologies of the compact orientable three-manifolds $\bar{\Omega}_{j}, 1 \leq j \leq k$.

Naturally one can evolve each $\bar{\Omega}_{j}$ by the Ricci flow again and, when singularities develop again, perform the above surgery for each $\varepsilon$-horn to get new compact orientable three-manifolds. By repeating this procedure indefinitely, it will likely give a long-time "weak" solution to the Ricci flow. The following abstract definition for this kind of "weak" solution was introduced by Perelman in [104] .

Definition 7.3.1. Suppose we are given a (finite or countably infinite) collection of three-dimensional smooth solutions $g_{i j}^{k}(t)$ to the Ricci flow defined on $M_{k} \times\left[t_{k}^{-}, t_{k}^{+}\right)$ 
and go singular as $t \rightarrow t_{k}^{+}$, where each manifold $M_{k}$ is compact and orientable, possibly disconnected with only a finite number of connected components. Let $\left(\Omega_{k}, \bar{g}_{i j}^{k}\right)$ be the limits of the corresponding solutions $g_{i j}^{k}(t)$ as $t \rightarrow t_{k}^{+}$, as above. Suppose also that for each $k$ we have $t_{k}^{-}=t_{k-1}^{+}$, and that $\left(\Omega_{k-1}, \bar{g}_{i j}^{k-1}\right)$ and $\left(M_{k}, g_{i j}^{k}\left(t_{k}^{-}\right)\right)$contain compact (possibly disconnected) three-dimensional submanifolds with smooth boundary which are isometric. Then by identifying these isometric submanifolds, we say the collection of solutions $g_{i j}^{k}(t)$ is a solution to the Ricci flow with surgery (or a surgically modified solution to the Ricci flow) on the time interval which is the union of all $\left[t_{k}^{-}, t_{k}^{+}\right)$, and say the times $t_{k}^{+}$are surgery times.

To get the topology of the initial manifold from the solution to the Ricci flow with surgery, one has to overcome the following two difficulties:

(i) how to prevent the surgery times from accumulating?

(ii) how to obtain the long time behavior of the solution to the Ricci flow with surgery?

Thus it is natural to consider those solutions having "good" properties. For any arbitrarily fixed positive number $\varepsilon$, we will only consider those solutions to the Ricci flow with surgery which satisfy the following a priori assumptions (with accuracy $\varepsilon$ ).

Pinching assumption. The eigenvalues $\lambda \geq \mu \geq \nu$ of the curvature operator of the solution to the Ricci flow with surgery at each point and each time satisfy

$$
R \geq(-\nu)[\log (-\nu)+\log (1+t)-3]
$$

whenever $\nu<0$.

Canonical neighborhood assumption (with accuracy $\varepsilon$ ). For any given $\varepsilon>0$, there exist positive constants $C_{1}$ and $C_{2}$ depending only on $\varepsilon$, and a nonincreasing positive function $r:[0,+\infty) \rightarrow(0,+\infty)$ such that at each time $t>0$, every point $x$ where scalar curvature $R(x, t)$ is at least $r^{-2}(t)$ has a neighborhood $B$, with $B_{t}(x, \sigma) \subset B \subset B_{t}(x, 2 \sigma)$ for some $0<\sigma<C_{1} R^{-\frac{1}{2}}(x, t)$, which falls into one of the following three categories:

(a) $B$ is a strong $\varepsilon$-neck (in the sense $B$ is the slice at time $t$ of the parabolic neighborhood $\left\{\left(x^{\prime}, t^{\prime}\right) \mid x^{\prime} \in B, t^{\prime} \in\left[t-R(x, t)^{-1}, t\right]\right\}$, where the solution is well defined on the whole parabolic neighborhood and is, after scaling with factor $R(x, t)$ and shifting the time to zero, $\varepsilon$-close (in the $C^{\left[\varepsilon^{-1}\right]}$ topology) to the subset $\left(\mathbb{S}^{2} \times \mathbb{I}\right) \times[-1,0]$ of the evolving standard round cylinder with scalar curvature 1 to $\mathbb{S}^{2}$ and length $2 \varepsilon^{-1}$ to $\mathbb{I}$ at time zero), or

(b) $B$ is an $\varepsilon$-cap, or

(c) $B$ is a compact manifold (without boundary) of positive sectional curvature. Furthermore, the scalar curvature in $B$ at time $t$ is between $C_{2}^{-1} R(x, t)$ and $C_{2} R(x, t)$, satisfies the gradient estimates

$$
|\nabla R|<\eta R^{\frac{3}{2}} \text { and }\left|\frac{\partial R}{\partial t}\right|<\eta R^{2},
$$

and the volume of $B$ in case (a) and case (b) satisfies

$$
\left(C_{2} R(x, t)\right)^{-\frac{3}{2}} \leq \operatorname{Vol}_{t}(B) \leq \varepsilon \sigma^{3} .
$$

Here $\eta$ is a universal positive constant. 
Without loss of generality, we always assume the above constants $C_{1}$ and $C_{2}$ are twice bigger than the corresponding constants $C_{1}\left(\frac{\varepsilon}{2}\right)$ and $C_{2}\left(\frac{\varepsilon}{2}\right)$ in Theorem 6.4.6 with the accuracy $\frac{\varepsilon}{2}$.

We remark that the above definition of the canonical neighborhood assumption is slightly different from that of Perelman in [104] in two aspects: (1) it allows the parameter $r$ to depend on time; (2) it also includes an volume upper bound for the canonical neighborhoods of types (a) and (b).

Arbitrarily given a compact orientable three-manifold with a Riemannian metric, by scaling, we may assume the Riemannian metric is normalized. In the rest of this section and the next section, we will show the Ricci flow with surgery, with the normalized metric as initial data, has a long-time solution which satisfies the above a priori assumptions and has only a finite number of surgery times at each finite time interval. The construction of the long-time solution will be given by an induction argument.

First, for the arbitrarily given compact orientable normalized three-dimensional Riemannian manifold $\left(M, g_{i j}(x)\right)$, the Ricci flow with it as initial data has a maximal solution $g_{i j}(x, t)$ on a maximal time interval $[0, T)$ with $T>1$. It follows from Theorem 5.3.2 and Theorem 7.1.1 that the a priori assumptions (with accuracy $\varepsilon$ ) hold for the smooth solution on $[0, T)$. If $T=+\infty$, we have the desired long time solution. Thus, without loss of generality, we may assume the maximal time $T<+\infty$ so that the solution goes singular at time $T$.

Suppose that we have a solution to the Ricci flow with surgery, with the normalized metric as initial data, satisfying the a priori assumptions (with accuracy $\varepsilon$ ), defined on $[0, T)$ with $T<+\infty$, going singular at time $T$ and having only a finite number of surgery times on $[0, T)$. Let $\Omega$ denote the set of all points where the curvature stays bounded as $t \rightarrow T$. As we have seen before, the canonical neighborhood assumption implies that $\Omega$ is open and that $R(x, t) \rightarrow \infty$ as $t \rightarrow T$ for all $x$ lying outside $\Omega$. Moreover, as $t \rightarrow T$, the solution $g_{i j}(x, t)$ has a smooth limit $\bar{g}_{i j}(x)$ on $\Omega$.

For some $\delta>0$ to be chosen much smaller than $\varepsilon$, we let $\rho=\delta r(T)$ where $r(t)$ is the positive nonincreasing function in the definition of the canonical neighborhood assumption. We consider the corresponding compact set

$$
\Omega_{\rho}=\left\{x \in \Omega \mid \bar{R}(x) \leq \rho^{-2}\right\},
$$

where $\bar{R}(x)$ is the scalar curvature of $\bar{g}_{i j}$. If $\Omega_{\rho}$ is empty, the manifold (near the maximal time $T$ ) is entirely covered by $\varepsilon$-tubes, $\varepsilon$-caps and compact components with positive curvature. Clearly, the number of the compact components is finite. Then in this case the manifold (near the maximal time $T$ ) is diffeomorphic to the union of a finite number of copies of $\mathbb{S}^{3}$, or metric quotients of the round $\mathbb{S}^{3}$, or $\mathbb{S}^{2} \times \mathbb{S}^{1}$, or a connected sum of them. Thus when $\Omega_{\rho}$ is empty, the procedure stops here, and we say the solution becomes extinct. We now assume $\Omega_{\rho}$ is not empty. Then we know that every point $x \in \Omega \backslash \Omega_{\rho}$ lies in one of the subsets of $\Omega$ listed in (7.3.2), or in a compact component with positive curvature, or in a compact component which is contained in $\Omega \backslash \Omega_{\rho}$ and is diffeomorphic to either $\mathbb{S}^{3}$, or $\mathbb{S}^{2} \times \mathbb{S}^{1}$ or $\mathbb{R} \mathbb{P}^{3} \# \mathbb{R} \mathbb{P}^{3}$. Note again that the number of the compact components is finite. Let us throw away all the compact components lying in $\Omega \backslash \Omega_{\rho}$ and all the compact components with positive curvature, and then consider those components $\Omega_{j}, 1 \leq j \leq k$, of $\Omega$ which contain points of $\Omega_{\rho}$. (We will consider those components of $\Omega \backslash \Omega_{\rho}$ consisting of capped $\varepsilon$-horns and double $\varepsilon$-horns later). We will perform surgical procedures, as we roughly described before, by finding an $\varepsilon$-neck in every horn of $\Omega_{j}, 1 \leq j \leq k$, 
and then cutting it along the middle two-sphere, removing the horn-shaped end, and gluing back a cap.

In order to maintain the a priori assumptions with the same accuracy after the surgery, we will need to find sufficient "fine" necks in the $\varepsilon$-horns and to glue sufficient "fine" caps. Note that $\delta>0$ will be chosen much smaller than $\varepsilon>0$. The following lemma due to Perelman [104] gives us the "fine" necks in the $\varepsilon$-horns. (At the first sight, we should also cut off all those $\varepsilon$-tubes and $\varepsilon$-caps in the surgery procedure. However, in general we are not able to find a "fine" neck in an $\varepsilon$-tube or in an $\varepsilon$-cap, and surgeries at "rough" $\varepsilon$-necks will certainly lose some accuracy. If we perform surgeries at the necks with some fixed accuracy $\varepsilon$ in the high curvature region at each surgery time, then it is possible that the errors of surgeries may accumulate to a certain amount so that at some later time we cannot recognize the structure of very high curvature regions. This prevents us from carrying out the whole process in finite time with a finite number of steps. This is the reason why we will only perform the surgeries at the $\varepsilon$-horns.)

Lemma 7.3.2 (Perelman [104]). Given $0<\varepsilon \leq \frac{1}{100}, 0<\delta<\varepsilon$ and $0<T<+\infty$, there exists a radius $0<h<\delta \rho$, depending only on $\delta$ and $r(T)$, such that if we have a solution to the Ricci flow with surgery, with a normalized metric as initial data, satisfying the a priori assumptions (with accuracy $\varepsilon$ ), defined on $[0, T)$, going singular at time $T$ and having only a finite number of surgery times on $[0, T)$, then for each point $x$ with $h(x)=\bar{R}^{-\frac{1}{2}}(x) \leq h$ in an $\varepsilon$-horn of $\left(\Omega, \bar{g}_{i j}\right)$ with boundary in $\Omega_{\rho}$, the neighborhood $B_{T}\left(x, \delta^{-1} h(x)\right) \triangleq\left\{y \in \Omega \mid d_{T}(y, x) \leq \delta^{-1} h(x)\right\}$ is a strong $\delta$-neck (i.e., $B_{T}\left(x, \delta^{-1} h(x)\right) \times\left[T-h^{2}(x), T\right]$ is, after scaling with factor $h^{-2}(x)$, $\delta$-close (in the $C^{\left[\delta^{-1}\right]}$ topology) to the corresponding subset of the evolving standard round cylinder $\mathbb{S}^{2} \times \mathbb{R}$ over the time interval $[-1,0]$ with scalar curvature 1 at time zero).

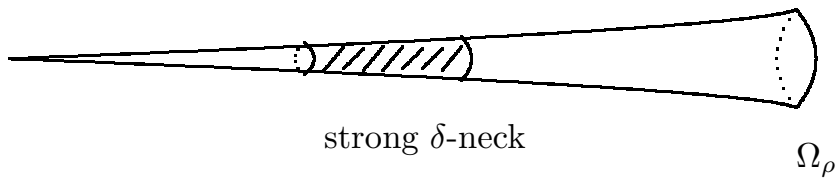

Proof. We argue by contradiction. Suppose that there exists a sequence of solutions $g_{i j}^{k}(\cdot, t), k=1,2, \ldots$, to the Ricci flow with surgery, satisfying the a priori assumptions (with accuracy $\varepsilon$ ), defined on $[0, T)$ with limit metrics $\left(\Omega^{k}, \bar{g}_{i j}^{k}\right), k=1,2, \ldots$, and points $x^{k}$, lying inside an $\varepsilon$-horn of $\Omega^{k}$ with boundary in $\Omega_{\rho}^{k}$, and having $h\left(x^{k}\right) \rightarrow 0$ such that the neighborhoods $B_{T}\left(x^{k}, \delta^{-1} h\left(x^{k}\right)\right)=\left\{y \in \Omega^{k} \mid d_{T}\left(y, x^{k}\right) \leq \delta^{-1} h\left(x^{k}\right)\right\}$ are not strong $\delta$-necks.

Let $\widetilde{g}_{i j}^{k}(\cdot, t)$ be the solutions obtained by rescaling by the factor $\bar{R}\left(x^{k}\right)=h^{-2}\left(x^{k}\right)$ around $x^{k}$ and shifting the time $T$ to the new time zero. We now want to show that a subsequence of $\widetilde{g}_{i j}^{k}(\cdot, t), k=1,2, \ldots$, converges to the evolving round cylinder, which will give a contradiction.

Note that $\widetilde{g}_{i j}^{k}(\cdot, t), k=1,2, \ldots$, are solutions modified by surgery. So, we cannot apply Hamilton's compactness theorem directly since it is stated only for smooth solutions. For each (unrescaled) surgical solution $\bar{g}_{i j}^{k}(\cdot, t)$, we pick a point $z^{k}$, with $\bar{R}\left(z^{k}\right)=2 C_{2}^{2}(\varepsilon) \rho^{-2}$, in the $\varepsilon$-horn of $\left(\Omega^{k}, \bar{g}_{i j}^{k}\right)$ with boundary in $\Omega_{\rho}^{k}$, where $C_{2}(\varepsilon)$ is the positive constant in the canonical neighborhood assumption. From the definition 
of $\varepsilon$-horn and the canonical neighborhood assumption, we know that each point $x$ lying inside the $\varepsilon$-horn of $\left(\Omega^{k}, \bar{g}_{i j}^{k}\right)$ with $d_{\bar{g}_{i j}^{k}}\left(x, \Omega_{\rho}^{k}\right) \geq d_{\bar{g}_{i j}^{k}}\left(z^{k}, \Omega_{\rho}^{k}\right)$ has a strong $\varepsilon$-neck as its canonical neighborhood. Since $h\left(x^{k}\right) \rightarrow 0$, each $x^{k}$ lies deeply inside an $\varepsilon$-horn. Thus for each positive $A<+\infty$, the rescaled (surgical) solutions $\widetilde{g}_{i j}^{k}(\cdot, t)$ with the marked origins $x^{k}$ over the geodesic balls $B_{\widetilde{g}_{i j}^{k}(\cdot, 0)}\left(x^{k}, A\right)$, centered at $x^{k}$ of radii $A$ (with respect to the metrics $\widetilde{g}_{i j}^{k}(\cdot, 0)$ ), will be smooth on some uniform (size) small time intervals for all sufficiently large $k$, if the curvatures of the rescaled solutions $\widetilde{g}_{i j}^{k}$ at $t=0$ in $B_{\widetilde{g}_{i j}^{k}(\cdot, 0)}\left(x^{k}, A\right)$ are uniformly bounded. In such a situation, Hamilton's compactness theorem is applicable. Then we can apply the same argument as in the second step of the proof of Theorem 7.1.1 to conclude that for each $A<+\infty$, there exists a positive constant $C(A)$ such that the curvatures of the rescaled solutions $\widetilde{g}_{i j}^{k}(\cdot, t)$ at the new time 0 satisfy the estimate

$$
\left|\widetilde{R} m_{k}\right|(y, 0) \leq C(A)
$$

whenever $d_{\widetilde{g}_{i j}^{k}(\cdot, 0)}\left(y, x^{k}\right) \leq A$ and $k \geq 1$; otherwise we would get a piece of a non-flat nonnegatively curved metric cone as a blow-up limit, which contradicts Hamilton's strong maximum principle. Moreover, by Hamilton's compactness theorem (Theorem 4.1.5), a subsequence of the rescaled solutions $\widetilde{g}_{i j}^{k}(\cdot, t)$ converges to a $C_{l o c}^{\infty} \operatorname{limit} \widetilde{g}_{i j}^{\infty}(\cdot, t)$, defined on a spacetime set which is relatively open in the half spacetime $\{t \leq 0\}$ and contains the time slice $t=0$.

By the pinching assumption, the limit is a complete manifold with nonnegative sectional curvature. Since $x^{k}$ was contained in an $\varepsilon$-horn with boundary in $\Omega_{\rho}^{k}$ and $h\left(x^{k}\right) / \rho \rightarrow 0$, the limiting manifold has two ends. Thus, by Toponogov's splitting theorem, the limiting manifold admits a metric splitting $\Sigma^{2} \times \mathbb{R}$, where $\Sigma^{2}$ is diffeomorphic to the two-sphere $\mathbb{S}^{2}$ because $x^{k}$ was the center of a strong $\varepsilon$-neck.

By combining with the canonical neighborhood assumption (with accuracy $\varepsilon$ ), we see that the limit is defined on the time interval $[-1,0]$ and is $\varepsilon$-close to the evolving standard round cylinder. In particular, the scalar curvature of the limit at time $t=-1$ is $\varepsilon$-close to $1 / 2$.

Since $h\left(x^{k}\right) / \rho \rightarrow 0$, each point in the limiting manifold at time $t=-1$ also has a strong $\varepsilon$-neck as its canonical neighborhood. Thus the limit is defined at least on the time interval $[-2,0]$ and the limiting manifold at time $t=-2$ is, after rescaling, $\varepsilon$-close to the standard round cylinder.

By using the canonical neighborhood assumption again, every point in the limiting manifold at time $t=-2$ still has a strong $\varepsilon$-neck as its canonical neighborhood. Also note that the scalar curvature of the limit at $t=-2$ is not bigger than $1 / 2+\varepsilon$. Thus the limit is defined at least on the time interval $[-3,0]$ and the limiting manifold at time $t=-3$ is, after rescaling, $\varepsilon$-close to the standard round cylinder. By repeating this argument we prove that the limit exists on the ancient time interval $(-\infty, 0]$.

The above argument also shows that at every time, each point of the limit has a strong $\varepsilon$-neck as its canonical neighborhood. This implies that the limit is $\kappa$ noncollaped on all scales for some $\kappa>0$. Therefore, by Theorem 6.2.2, the limit is the evolving round cylinder $\mathbb{S}^{2} \times \mathbb{R}$, which gives the desired contradiction.

In the above lemma, the property that the radius $h$ depends only on $\delta$ and the time $T$ but is independent of the surgical solution is crucial; otherwise we will not be able to cut off enough volume at each surgery to guarantee the number of surgeries being finite in each finite time interval. We also remark that the above proof actually 
proves a stronger result: the parabolic region $\left\{(y, t) \mid y \in B_{T}\left(x, \delta^{-1} h(x)\right), t \in[T-\right.$ $\left.\left.\delta^{-2} h^{2}(x), T\right]\right\}$ is, after scaling with factor $h^{-2}(x), \delta$-close (in the $C^{\left[\delta^{-1}\right]}$ topology) to the corresponding subset of the evolving standard round cylinder $\mathbb{S}^{2} \times \mathbb{R}$ over the time interval $\left[-\delta^{-2}, 0\right]$ with scalar curvature 1 at the time zero. This fact will be used later in the proof of Proposition 7.4.1.

We next want to construct "fine" caps. Take a rotationally symmetric metric on $\mathbb{R}^{3}$ with nonnegative sectional curvature and positive scalar curvature such that outside some compact set it is a semi-infinite standard round cylinder (i.e. the metric product of a ray with the round two-sphere of scalar curvature 1). We call such a metric on $\mathbb{R}^{3}$ a standard capped infinite cylinder. By the short-time existence theorem of Shi (Theorem 1.2.3), the Ricci flow with a standard capped infinite cylinder as initial data has a complete solution on a maximal time interval $[0, T)$ such that the curvature of the solution is bounded on $\mathbb{R}^{3} \times\left[0, T^{\prime}\right]$ for each $0<T^{\prime}<T$. Such a solution is called a standard solution by Perelman [104].

The following result proved by Chen and the second author in [34] gives the curvature estimate for standard solutions.

Proposition 7.3.3. Let $g_{i j}$ be a complete Riemannian metric on $\mathbb{R}^{n}(n \geq 3)$ with nonnegative curvature operator and positive scalar curvature which is asymptotic to a round cylinder of scalar curvature 1 at infinity. Then there is a complete solution $g_{i j}(\cdot, t)$ to the Ricci flow, with $g_{i j}$ as initial metric, which exists on the time interval $\left[0, \frac{n-1}{2}\right)$, has bounded curvature at each time $t \in\left[0, \frac{n-1}{2}\right)$, and satisfies the estimate

$$
R(x, t) \geq \frac{C^{-1}}{\frac{n-1}{2}-t}
$$

for some $C$ depending only on the initial metric $g_{i j}$.

Proof. Since the initial metric has bounded curvature operator and a positive lower bound on its scalar curvature, the Ricci flow has a solution $g_{i j}(\cdot, t)$ defined on a maximal time interval $[0, T)$ with $T<\infty$ which has bounded curvature on $\mathbb{R}^{n} \times\left[0, T^{\prime}\right]$ for each $0<T^{\prime}<T$. By Proposition 2.1.4, the solution $g_{i j}(\cdot, t)$ has nonnegative curvature operator for all $t \in[0, T)$. Note that the injectivity radius of the initial metric has a positive lower bound. As we remarked at the beginning of Section 3.4, the same proof of Perelman's no local collapsing theorem I concludes that $g_{i j}(\cdot, t)$ is $\kappa$-noncollapsed on all scales less than $\sqrt{T}$ for some $\kappa>0$ depending only on the initial metric.

We will first prove the following assertion.

Claim 1. There is a positive function $\omega:[0, \infty) \rightarrow[0, \infty)$ depending only on the initial metric and $\kappa$ such that

$$
R(x, t) \leq R(y, t) \omega\left(R(y, t) d_{t}^{2}(x, y)\right)
$$

for all $x, y \in \mathbb{R}^{n}, t \in[0, T)$.

The proof is similar to that of Theorem 6.4.3. Notice that the initial metric has nonnegative curvature operator and its scalar curvature satisfies the bounds

$$
C^{-1} \leqslant R(x) \leqslant C
$$

for some positive constant $C$. By the maximum principle, we know $T \geq \frac{1}{2 n C}$ and $R(x, t) \leq 2 C$ for $t \in\left[0, \frac{1}{4 n C}\right]$. The assertion is clearly true for $t \in\left[0, \frac{1}{4 n C}\right]$. 
Now fix $\left(y, t_{0}\right) \in \mathbb{R}^{n} \times[0, T)$ with $t_{0} \geq \frac{1}{4 n C}$. Let $z$ be the closest point to $y$ with the property $R\left(z, t_{0}\right) d_{t_{0}}^{2}(z, y)=1$ (at time $t_{0}$ ). Draw a shortest geodesic from $y$ to $z$ and choose a point $\tilde{z}$ on the geodesic satisfying $d_{t_{0}}(z, \tilde{z})=\frac{1}{4} R\left(z, t_{0}\right)^{-\frac{1}{2}}$, then we have

$$
R\left(x, t_{0}\right) \leq \frac{1}{\left(\frac{1}{2} R\left(z, t_{0}\right)^{-\frac{1}{2}}\right)^{2}} \quad \text { on } \quad B_{t_{0}}\left(\tilde{z}, \frac{1}{4} R\left(z, t_{0}\right)^{-\frac{1}{2}}\right) .
$$

Note that $R(x, t) \geqslant C^{-1}$ everywhere by the evolution equation of the scalar curvature. Then by the Li-Yau-Hamilton inequality (Corollary 2.5.5), for all $(x, t) \in$ $B_{t_{0}}\left(\tilde{z}, \frac{1}{8 n C} R\left(z, t_{0}\right)^{-\frac{1}{2}}\right) \times\left[t_{0}-\left(\frac{1}{8 n C} R\left(z, t_{0}\right)^{-\frac{1}{2}}\right)^{2}, t_{0}\right]$, we have

$$
\begin{aligned}
R(x, t) & \leq\left(\frac{t_{0}}{t_{0}-\left(\frac{1}{8 n \sqrt{C}}\right)^{2}}\right) \frac{1}{\left(\frac{1}{2} R\left(z, t_{0}\right)^{-\frac{1}{2}}\right)^{2}} \\
& \leq\left[\frac{1}{8 n C} R\left(z, t_{0}\right)^{-\frac{1}{2}}\right]^{-2} .
\end{aligned}
$$

Combining this with the $\kappa$-noncollapsing property, we have

$$
\operatorname{Vol}\left(B_{t_{0}}\left(\tilde{z}, \frac{1}{8 n C} R\left(z, t_{0}\right)^{-\frac{1}{2}}\right)\right) \geq \kappa\left(\frac{1}{8 n C} R\left(z, t_{0}\right)^{-\frac{1}{2}}\right)^{n}
$$

and then

$$
\operatorname{Vol}\left(B_{t_{0}}\left(z, 8 R\left(z, t_{0}\right)^{-\frac{1}{2}}\right)\right) \geq \kappa\left(\frac{1}{64 n C}\right)^{n}\left(8 R\left(z, t_{0}\right)^{-\frac{1}{2}}\right)^{n} .
$$

So by Theorem 6.3 .3 (ii), we have

$$
R\left(x, t_{0}\right) \leq C(\kappa) R\left(z, t_{0}\right) \text { for all } x \in B_{t_{0}}\left(z, 4 R\left(z, t_{0}\right)^{-\frac{1}{2}}\right) .
$$

Here and in the following we denote by $C(\kappa)$ various positive constants depending only on $\kappa, n$ and the initial metric.

Now by the Li-Yau-Hamilton inequality (Corollary 2.5.5) and local gradient estimate of Shi (Theorem 1.4.2), we obtain

$$
R(x, t) \leq C(\kappa) R\left(z, t_{0}\right) \text { and }\left|\frac{\partial}{\partial t} R\right|(x, t) \leq C(\kappa)\left(R\left(z, t_{0}\right)\right)^{2}
$$

for all $\left.(x, t) \in B_{t_{0}}\left(z, 2 R\left(z, t_{0}\right)^{-\frac{1}{2}}\right)\right) \times\left[t_{0}-\left(\frac{1}{8 n C} R\left(z, t_{0}\right)^{-\frac{1}{2}}\right)^{2}, t_{0}\right]$. Therefore by combining with the Harnack estimate (Corollary 2.5.7), we obtain

$$
\begin{aligned}
R\left(y, t_{0}\right) & \geq C(\kappa)^{-1} R\left(z, t_{0}-C(\kappa)^{-1} R\left(z, t_{0}\right)^{-1}\right) \\
& \geq C(\kappa)^{-2} R\left(z, t_{0}\right)
\end{aligned}
$$

Consequently, we have showed that there is a constant $C(\kappa)$ such that

$$
\text { Vol }\left(B_{t_{0}}\left(y, R\left(y, t_{0}\right)^{-\frac{1}{2}}\right)\right) \geq C(\kappa)^{-1}\left(R\left(y, t_{0}\right)^{-\frac{1}{2}}\right)^{n}
$$

and

$$
R\left(x, t_{0}\right) \leq C(\kappa) R\left(y, t_{0}\right) \quad \text { for all } x \in B_{t_{0}}\left(y, R\left(y, t_{0}\right)^{-\frac{1}{2}}\right) .
$$


In general, for any $r \geq R\left(y, t_{0}\right)^{-\frac{1}{2}}$, we have

$$
\operatorname{Vol}\left(B_{t_{0}}(y, r)\right) \geq C(\kappa)^{-1}\left(r^{2} R\left(y, t_{0}\right)\right)^{-\frac{n}{2}} r^{n} .
$$

By applying Theorem 6.3.3(ii) again, there exists a positive constant $\omega\left(r^{2} R\left(y, t_{0}\right)\right)$ depending only on the constant $r^{2} R\left(y, t_{0}\right)$ and $\kappa$ such that

$$
R\left(x, t_{0}\right) \leq R\left(y, t_{0}\right) \omega\left(r^{2} R\left(y, t_{0}\right)\right) \quad \text { for all } x \in B_{t_{0}}\left(y, \frac{1}{4} r\right) .
$$

This proves the desired Claim 1.

Now we study the asymptotic behavior of the solution at infinity. For any $0<$ $t_{0}<T$, we know that the metrics $g_{i j}(x, t)$ with $t \in\left[0, t_{0}\right]$ has uniformly bounded curvature. Let $x_{k}$ be a sequence of points with $d_{0}\left(x_{0}, x_{k}\right) \rightarrow \infty$. After passing to a subsequence, $g_{i j}(x, t)$ around $x_{k}$ will converge to a solution to the Ricci flow on $\mathbb{R} \times \mathbb{S}^{n-1}$ with round cylinder metric of scalar curvature 1 as initial data. Denote the limit by $\tilde{g}_{i j}$. Then by the uniqueness theorem (Theorem 1.2.4), we have

$$
\tilde{R}(x, t)=\frac{\frac{n-1}{2}}{\frac{n-1}{2}-t} \text { for all } t \in\left[0, t_{0}\right] .
$$

It follows that $T \leq \frac{n-1}{2}$. In order to show $T=\frac{n-1}{2}$, it suffices to prove the following assertion.

Claim 2. Suppose $T<\frac{n-1}{2}$. Fix a point $x_{0} \in \mathbb{R}^{n}$, then there is a $\delta>0$, such that for any $x \in M$ with $d_{0}\left(x, x_{0}\right) \geq \delta^{-1}$, we have

$$
R(x, t) \leq 2 C+\frac{n-1}{\frac{n-1}{2}-t} \quad \text { for all } t \in[0, T),
$$

where $C$ is the constant in (7.3.5).

In view of Claim 1, if Claim 2 holds, then

$$
\begin{aligned}
\sup _{M^{n} \times[0, T)} R(y, t) & \leq \omega\left(\delta^{-2}\left(2 C+\frac{n-1}{\frac{n-1}{2}-T}\right)\right)\left(2 C+\frac{n-1}{\frac{n-1}{2}-T}\right) \\
& <\infty
\end{aligned}
$$

which will contradict the definition of $T$.

To show Claim 2, we argue by contradiction. Suppose for each $\delta>0$, there is a point $\left(x_{\delta}, t_{\delta}\right)$ with $0<t_{\delta}<T$ such that

$$
R\left(x_{\delta}, t_{\delta}\right)>2 C+\frac{n-1}{\frac{n-1}{2}-t_{\delta}} \text { and } d_{0}\left(x_{\delta}, x_{0}\right) \geq \delta^{-1} .
$$

Let

$$
\bar{t}_{\delta}=\sup \left\{t \mid \sup _{M^{n} \backslash B_{0}\left(x_{0}, \delta^{-1}\right)} R(y, t)<2 C+\frac{n-1}{\frac{n-1}{2}-t}\right\} .
$$

Since $\lim _{d_{0}\left(y, x_{0}\right) \rightarrow \infty} R(y, t)=\frac{\frac{n-1}{2}}{\frac{n-1}{2}-t}$ and $\sup _{M \times\left[0, \frac{1}{4 n C}\right]} R(y, t) \leq 2 C$, we know $\frac{1}{4 n C} \leq \bar{t}_{\delta} \leq$ $t_{\delta}$ and there is a $\bar{x}_{\delta}$ such that $d_{0}\left(x_{0}, \bar{x}_{\delta}\right) \geq \delta^{-1}$ and $R\left(\bar{x}_{\delta}, \bar{t}_{\delta}\right)=2 C+\frac{n-1}{\frac{n-1}{2}-\bar{t}_{\delta}}$. By Claim 
1 and Hamilton's compactness theorem (Theorem 4.1.5), for $\delta \rightarrow 0$ and after taking a subsequence, the metrics $g_{i j}(x, t)$ on $B_{0}\left(\bar{x}_{\delta}, \frac{\delta^{-1}}{2}\right)$ over the time interval $\left[0, \bar{t}_{\delta}\right]$ will converge to a solution $\tilde{g}_{i j}$ on $\mathbb{R} \times \mathbb{S}^{n-1}$ with the standard metric of scalar curvature 1 as initial data over the time interval $\left[0, \bar{t}_{\infty}\right]$, and its scalar curvature satisfies

$$
\begin{aligned}
\tilde{R}\left(\bar{x}_{\infty}, \bar{t}_{\infty}\right) & =2 C+\frac{n-1}{\frac{n-1}{2}-\bar{t}_{\infty}}, \\
\tilde{R}(x, t) & \leqslant 2 C+\frac{n-1}{\frac{n-1}{2}-\bar{t}_{\infty}}, \text { for all } t \in\left[0, \bar{t}_{\infty}\right],
\end{aligned}
$$

where $\left(\bar{x}_{\infty}, \bar{t}_{\infty}\right)$ is the limit of $\left(\bar{x}_{\delta}, \bar{t}_{\delta}\right)$. On the other hand, by the uniqueness theorem (Theorem 1.2.4) again, we know

$$
\tilde{R}\left(\bar{x}_{\infty}, \bar{t}_{\infty}\right)=\frac{\frac{n-1}{2}}{\frac{n-1}{2}-\bar{t}_{\infty}}
$$

which is a contradiction. Hence we have proved Claim 2 and then have verified $T=\frac{n-1}{2}$.

Now we are ready to show

$$
R(x, t) \geq \frac{\tilde{C}^{-1}}{\frac{n-1}{2}-t}, \quad \text { for all }(x, t) \in \mathbb{R}^{n} \times\left[0, \frac{n-1}{2}\right),
$$

for some positive constant $\tilde{C}$ depending only on the initial metric.

For any $(x, t) \in \mathbb{R}^{n} \times\left[0, \frac{n-1}{2}\right)$, by Claim 1 and $\kappa$-noncollapsing, there is a constant $C(\kappa)>0$ such that

$$
\operatorname{Vol}_{t}\left(B_{t}\left(x, R(x, t)^{-\frac{1}{2}}\right)\right) \geq C(\kappa)^{-1}\left(R(x, t)^{-\frac{1}{2}}\right)^{n} .
$$

Then by the well-known volume estimate of Calabi-Yau (see for example [128] or [112]) for complete manifolds with Ric $\geq 0$, for any $a \geq 1$, we have

$$
\operatorname{Vol}_{t}\left(B_{t}\left(x, a R(x, t)^{-\frac{1}{2}}\right)\right) \geq C(\kappa)^{-1} \frac{a}{8 n}\left(R(x, t)^{-\frac{1}{2}}\right)^{n} .
$$

On the other hand, since $\left(\mathbb{R}^{n}, g_{i j}(\cdot, t)\right)$ is asymptotic to a cylinder of scalar curvature $\left(\frac{n-1}{2}\right) /\left(\frac{n-1}{2}-t\right)$, for sufficiently large $a>0$, we have

$$
\mathrm{Vol}_{t}\left(B_{t}\left(x, a \sqrt{\frac{n-1}{2}-t}\right)\right) \leq C(n) a\left(\frac{n-1}{2}-t\right)^{\frac{n}{2}} .
$$

Combining the two inequalities, for all sufficiently large $a$, we have:

$$
\begin{aligned}
C(n) a\left(\frac{n-1}{2}-t\right)^{\frac{n}{2}} & \geq \operatorname{Vol}_{t}\left(B_{t}\left(x, a\left(\frac{\sqrt{\frac{n-1}{2}-t}}{R(x, t)^{-\frac{1}{2}}}\right) R(x, t)^{-\frac{1}{2}}\right)\right) \\
& \geq C(\kappa)^{-1} \frac{a}{8 n}\left(\frac{\sqrt{\frac{n-1}{2}-t}}{R(x, t)^{-\frac{1}{2}}}\right)\left(R(x, t)^{-\frac{1}{2}}\right)^{n},
\end{aligned}
$$

which gives the desired estimate (7.3.6). Therefore the proof of the proposition is complete. 
We now fix a standard capped infinite cylinder for dimension $n=3$ as follows. Consider the semi-infinite standard round cylinder $N_{0}=\mathbb{S}^{2} \times(-\infty, 4)$ with the metric $g_{0}$ of scalar curvature 1. Denote by $z$ the coordinate of the second factor $(-\infty, 4)$. Let $f$ be a smooth nondecreasing convex function on $(-\infty, 4)$ defined by

$$
\left\{\begin{array}{l}
f(z)=0, \quad z \leq 0, \\
f(z)=c e^{-\frac{P}{z}}, \quad z \in(0,3], \\
f(z) \text { is strictly convex on } z \in[3,3.9], \\
f(z)=-\frac{1}{2} \log \left(16-z^{2}\right), \quad z \in[3.9,4),
\end{array}\right.
$$

where the (small) constant $c>0$ and (big) constant $P>0$ will be determined later. Let us replace the standard metric $g_{0}$ on the portion $\mathbb{S}^{2} \times[0,4)$ of the semi-infinite cylinder by $\hat{g}=e^{-2 f} g_{0}$. Then the resulting metric $\hat{g}$ will be smooth on $\mathbb{R}^{3}$ obtained by adding a point to $\mathbb{S}^{2} \times(-\infty, 4)$ at $z=4$. We denote by $C(c, P)=\left(\mathbb{R}^{3}, \hat{g}\right)$. Clearly, $C(c, P)$ is a standard capped infinite cylinder.

We next use a compact portion of the standard capped infinite cylinder $C(c, P)$ and the $\delta$-neck obtained in Lemma 7.3.2 to perform the following surgery procedure due to Hamilton [64].

Consider the metric $\bar{g}$ at the maximal time $T<+\infty$. Take an $\varepsilon$-horn with boundary in $\Omega_{\rho}$. By Lemma 7.3.2, there exists a $\delta$-neck $N$ of radius $0<h<\delta \rho$ in the $\varepsilon$-horn. By definition, $\left(N, h^{-2} \bar{g}\right)$ is $\delta$-close (in the $C^{\left[\delta^{-1}\right]}$ topology) to the standard round neck $\mathbb{S}^{2} \times \mathbb{I}$ of scalar curvature 1 with $\mathbb{I}=\left(-\delta^{-1}, \delta^{-1}\right)$. Using the parameter $z \in \mathbb{I}$, we see the above function $f$ is defined on the $\delta$-neck $N$.

Let us cut the $\delta$-neck $N$ along the middle (topological) two-sphere $N \cap\{z=$ $0\}$. Without loss of generality, we may assume that the right hand half portion $N \bigcap\{z \geq 0\}$ is contained in the horn-shaped end. Let $\varphi$ be a smooth bump function with $\varphi=1$ for $z \leq 2$, and $\varphi=0$ for $z \geq 3$. Construct a new metric $\tilde{g}$ on a (topological) three-ball $\mathbb{B}^{3}$ as follows

$$
\tilde{g}= \begin{cases}\bar{g}, & z=0, \\ e^{-2 f} \bar{g}, & z \in[0,2], \\ \varphi e^{-2 f} \bar{g}+(1-\varphi) e^{-2 f} h^{2} g_{0}, & z \in[2,3], \\ h^{2} e^{-2 f} g_{0}, & z \in[3,4] .\end{cases}
$$

The surgery is to replace the horn-shaped end by the cap $\left(\mathbb{B}^{3}, \tilde{g}\right)$. We call such surgery procedure a $\delta$-cutoff surgery.

The following lemma determines the constants $c$ and $P$ in the $\delta$-cutoff surgery so that the pinching assumption is preserved under the surgery.

LEMma 7.3.4 (Justification of the pinching assumption). There are universal positive constants $\delta_{0}, c_{0}$ and $P_{0}$ such that if we take a $\delta$-cutoff surgery at a $\delta$-neck of radius $h$ at time $T$ with $\delta \leq \delta_{0}$ and $h^{-2} \geq 2 e^{2} \log (1+T)$, then we can choose $c=c_{0}$ and $P=P_{0}$ in the definition of $f(z)$ such that after the surgery, there still holds the pinching condition

$$
\tilde{R} \geq(-\tilde{\nu})[\log (-\tilde{\nu})+\log (1+T)-3]
$$

whenever $\tilde{\nu}<0$, where $\tilde{R}$ is the scalar curvature of the metric $\tilde{g}$ and $\tilde{\nu}$ is the least eigenvalue of the curvature operator of $\tilde{g}$. Moreover, after the surgery, any metric ball 
of radius $\delta^{-\frac{1}{2}} h$ with center near the tip (i.e., the origin of the attached cap) is, after scaling with factor $h^{-2}, \delta^{\frac{1}{2}}$-close (in the $C^{\left[\delta^{-\frac{1}{2}}\right]}$ topology) to the corresponding ball of the standard capped infinite cylinder $C\left(c_{0}, P_{0}\right)$.

Proof. First, we consider the metric $\tilde{g}$ on the portion $\{0 \leq z \leq 2\}$. Under the conformal change $\tilde{g}=e^{-2 f} \bar{g}$, the curvature tensor $\tilde{R}_{i j k l}$ is given by

$$
\begin{aligned}
\tilde{R}_{i j k l}=e^{-2 f}\left[\bar{R}_{i j k l}+|\bar{\nabla} f|^{2}\left(\bar{g}_{i l} \bar{g}_{j k}-\bar{g}_{i k} \bar{g}_{j l}\right)+\left(f_{i k}+f_{i} f_{k}\right) \bar{g}_{j l}\right. \\
\left.+\left(f_{j l}+f_{j} f_{l}\right) \bar{g}_{i k}-\left(f_{i l}+f_{i} f_{l}\right) \bar{g}_{j k}-\left(f_{j k}+f_{j} f_{k}\right) \bar{g}_{i l}\right] .
\end{aligned}
$$

If $\left\{\bar{F}_{a}=\bar{F}_{a}^{i} \frac{\partial}{\partial x^{i}}\right\}$ is an orthonormal frame for $\bar{g}_{i j}$, then $\left\{\tilde{F}_{a}=e^{f} \bar{F}_{a}=\tilde{F}_{a}^{i} \frac{\partial}{\partial x^{i}}\right\}$ is an orthonormal frame for $\tilde{g}_{i j}$. Let

$$
\begin{aligned}
\bar{R}_{a b c d} & =\bar{R}_{i j k l} \bar{F}_{a}^{i} \bar{F}_{b}^{j} \bar{F}_{c}^{k} \bar{F}_{d}^{l}, \\
\tilde{R}_{a b c d} & =\tilde{R}_{i j k l} \tilde{F}_{a}^{i} \tilde{F}_{b}^{j} \tilde{F}_{c}^{k} \tilde{F}_{d}^{l},
\end{aligned}
$$

then

$$
\begin{aligned}
\tilde{R}_{a b c d}=e^{2 f}\left[\bar{R}_{a b c d}+|\bar{\nabla} f|^{2}\left(\delta_{a d} \delta_{b c}-\delta_{a c} \delta_{b d}\right)+\left(f_{a c}+f_{a} f_{c}\right) \delta_{b d}\right. \\
\left.+\left(f_{b d}+f_{b} f_{d}\right) \delta_{a c}-\left(f_{a d}+f_{a} f_{d}\right) \delta_{b c}-\left(f_{b c}+f_{b} f_{c}\right) \delta_{a d}\right]
\end{aligned}
$$

and

$$
\tilde{R}=e^{2 f}\left(\bar{R}+4 \bar{\triangle} f-2|\bar{\nabla} f|^{2}\right) .
$$

Since

$$
\frac{d f}{d z}=c e^{-\frac{P}{z}} \frac{P}{z^{2}}, \quad \frac{d^{2} f}{d z^{2}}=c e^{-\frac{P}{z}}\left(\frac{P^{2}}{z^{4}}-\frac{2 P}{z^{3}}\right),
$$

then for any small $\theta>0$, we may choose $c>0$ small and $P>0$ large such that for $z \in[0,3]$, we have

$$
\left|e^{2 f}-1\right|+\left|\frac{d f}{d z}\right|+\left|\left(\frac{d f}{d z}\right)^{2}\right|<\theta \frac{d^{2} f}{d z^{2}}, \frac{d^{2} f}{d z^{2}}<\theta
$$

On the other hand, by the definition of $\delta$-neck of radius $h$, we have

$$
\begin{gathered}
\left|\bar{g}-h^{2} g_{0}\right|_{g_{0}}<\delta h^{2}, \\
\left|\nabla^{j} \bar{g}\right|_{g_{0}}<\delta h^{2}, \text { for } 1 \leq j \leq\left[\delta^{-1}\right],
\end{gathered}
$$

where $g_{0}$ is the standard metric of the round cylinder $\mathbb{S}^{2} \times \mathbb{R}$. Note that in three dimensions, we can choose the orthonormal frame $\left\{\bar{F}_{1}, \bar{F}_{2}, \bar{F}_{3}\right\}$ for the metric $\bar{g}$ so that its curvature operator is diagonal in the orthonormal frame $\left\{\sqrt{2} \bar{F}_{2} \wedge \bar{F}_{3}, \sqrt{2} \bar{F}_{3} \wedge\right.$ $\left.\bar{F}_{1}, \sqrt{2} \bar{F}_{1} \wedge \bar{F}_{2}\right\}$ with eigenvalues $\bar{\nu} \leq \bar{\mu} \leq \bar{\lambda}$ and

$$
\bar{\nu}=2 \bar{R}_{2323}, \quad \bar{\mu}=2 \bar{R}_{3131}, \quad \bar{\lambda}=2 \bar{R}_{1212} .
$$


Since $h^{-2} \bar{g}$ is $\delta$-close to the standard round cylinder metric $g_{0}$ on the $\delta$-neck, we have

$$
\left\{\begin{array}{l}
\left|\bar{R}_{3131}\right|+\left|\bar{R}_{2323}\right|<\delta^{\frac{7}{8}} h^{-2}, \\
\left|\bar{R}_{1212}-\frac{1}{2} h^{-2}\right|<\delta^{\frac{7}{8}} h^{-2}, \\
\left|\bar{F}_{3}-h^{-1} \frac{\partial}{\partial z}\right|_{g_{0}}<\delta^{\frac{7}{8}} h^{-1},
\end{array}\right.
$$

for suitably small $\delta>0$. Since $\bar{\nabla}_{a} z=\bar{\nabla} z\left(\bar{F}_{a}\right)$ and $\bar{\nabla}_{a} \bar{\nabla}_{b} z=\bar{\nabla}^{2} z\left(\bar{F}_{a}, \bar{F}_{b}\right)$, it follows that

$$
\begin{aligned}
\left|\bar{\nabla}_{3} z-h^{-1}\right| & <\delta^{\frac{7}{8}} h^{-1}, \\
\left|\bar{\nabla}_{1} z\right|+\left|\bar{\nabla}_{2} z\right| & <\delta^{\frac{7}{8}} h^{-1},
\end{aligned}
$$

and

$$
\left|\bar{\nabla}_{a} \bar{\nabla}_{b} z\right|<\delta^{\frac{7}{8}} h^{-2}, \text { for } 1 \leq a, b \leq 3 .
$$

By combining with

$$
\bar{\nabla}_{a} f=\frac{d f}{d z} \bar{\nabla}_{a} z, \quad \bar{\nabla}_{a} \bar{\nabla}_{b} f=\frac{d f}{d z} \bar{\nabla}_{a} \bar{\nabla}_{b} z+\frac{d^{2} f}{d z^{2}} \bar{\nabla}_{a} z \bar{\nabla}_{b} z
$$

and (7.3.12), we get

$$
\begin{cases}\left|\bar{\nabla}_{a} f\right|<2 \theta h^{-1} \frac{d^{2} f}{d z^{2}}, & \text { for } 1 \leq a \leq 3, \\ \left|\bar{\nabla}_{a} \bar{\nabla}_{b} f\right|<\delta^{\frac{3}{4}} h^{-2} \frac{d^{2} f}{d z^{2}}, & \text { unless } a=b=3, \\ \left|\bar{\nabla}_{3} \bar{\nabla}_{3} f-h^{-2} \frac{d^{2} f}{d z^{2}}\right|<\delta^{\frac{3}{4}} h^{-2} \frac{d^{2} f}{d z^{2}} . & \end{cases}
$$

By combining (7.3.10) and (7.3.14), we have

$$
\left\{\begin{array}{l}
\tilde{R}_{1212} \geq \bar{R}_{1212}-\left(\theta^{\frac{1}{2}}+\delta^{\frac{5}{8}}\right) h^{-2} \frac{d^{2} f}{d z^{2}} \\
\tilde{R}_{3131} \geq \bar{R}_{3131}+\left(1-\theta^{\frac{1}{2}}-\delta^{\frac{5}{8}}\right) h^{-2} \frac{d^{2} f}{d z^{2}} \\
\tilde{R}_{2323} \geq \bar{R}_{2323}+\left(1-\theta^{\frac{1}{2}}-\delta^{\frac{5}{8}}\right) h^{-2} \frac{d^{2} f}{d z^{2}} \\
\left|\tilde{R}_{a b c d}\right| \leq\left(\theta^{\frac{1}{2}}+\delta^{\frac{5}{8}}\right) h^{-2} \frac{d^{2} f}{d z^{2}}, \quad \text { otherwise }
\end{array}\right.
$$

where $\theta$ and $\delta$ are suitably small. Then it follows that

$$
\begin{gathered}
\tilde{R} \geq \bar{R}+\left[4-6\left(\theta^{\frac{1}{3}}+\delta^{\frac{1}{2}}\right)\right] h^{-2} \frac{d^{2} f}{d z^{2}}, \\
-\tilde{\nu} \leq-\bar{\nu}-\left[2-2\left(\theta^{\frac{1}{3}}+\delta^{\frac{1}{2}}\right)\right] h^{-2} \frac{d^{2} f}{d z^{2}},
\end{gathered}
$$

for suitably small $\theta$ and $\delta$.

If $0<-\tilde{\nu} \leq e^{2}$, then by the assumption that $h^{-2} \geq 2 e^{2} \log (1+T)$, we have

$$
\begin{aligned}
\tilde{R} & \geq \bar{R} \\
& \geq \frac{1}{2} h^{-2} \\
& \geq e^{2} \log (1+T) \\
& \geq(-\tilde{\nu})[\log (-\tilde{\nu})+\log (1+T)-3] .
\end{aligned}
$$


While if $-\tilde{\nu}>e^{2}$, then by the pinching estimate of $\bar{g}$, we have

$$
\begin{aligned}
\tilde{R} & \geq \bar{R} \\
& \geq(-\bar{\nu})[\log (-\bar{\nu})+\log (1+T)-3] \\
& \geq(-\tilde{\nu})[\log (-\tilde{\nu})+\log (1+T)-3] .
\end{aligned}
$$

So we have verified the pinching condition on the portion $\{0 \leq z \leq 2\}$.

Next, we consider the metric $\tilde{g}$ on the portion $\{2 \leq z \leq 4\}$. Let $\theta$ be a fixed suitably small positive number. Then the constant $c=c_{0}$ and $P=P_{0}$ are fixed. So $\zeta=\min _{z \in[1,4]} \frac{d^{2} f}{d z^{2}}>0$ is also fixed. By the same argument as in the derivation of (7.3.15) from (7.3.10), we see that the curvature of the metric $\hat{g}=e^{-2 f} g_{0}$ of the standard capped infinite cylinder $C\left(c_{0}, P_{0}\right)$ on the portion $\{1 \leq z \leq 4\}$ is bounded from below by $\frac{2}{3} \zeta>0$. Since $h^{-2} \bar{g}$ is $\delta$-close to the standard round metric $g_{0}$, the metric $h^{-2} \tilde{g}$ defined by (7.3.8) is clearly $\delta^{\frac{3}{4}}$-close to the metric $\hat{g}=e^{-2 f} g_{0}$ of the standard capped infinite cylinder on the portion $\{1 \leq z \leq 4\}$. Thus as $\delta$ is sufficiently small, the curvature operator of $\tilde{g}$ on the portion $\{2 \leq z \leq 4\}$ is positive. Hence the pinching condition (7.3.9) holds trivially on the portion $\{2 \leq z \leq 4\}$.

The last statement in Lemma 7.3.4 is obvious from the definition (7.3.8).

Recall from Lemma 7.3.2 that the $\delta$-necks at a time $t>0$, where we performed Hamilton's surgeries, have their radii $0<h<\delta \rho=\delta^{2} r(t)$. Without loss of generality, we may assume the positive nonincreasing function $r(t)$ in the definition of the canonical neighborhood assumption is less than 1 and the universal constant $\delta_{0}$ in Lemma 7.3.4 is also less than 1. We define a positive function $\bar{\delta}(t)$ by

$$
\bar{\delta}(t)=\min \left\{\frac{1}{2 e^{2} \log (1+t)}, \delta_{0}\right\} \quad \text { for } t \in[0,+\infty) .
$$

From now on, we always assume $0<\delta<\bar{\delta}(t)$ for any $\delta$-cutoff surgery at time $t>0$ and assume $c=c_{0}$ and $P=P_{0}$. As a result, the standard capped infinite cylinder and the standard solution are also fixed. The following lemma, which will be used in the next section, gives the canonical neighborhood structure for the fixed standard solution.

LEMma 7.3.5. Let $g_{i j}(x, t)$ be the above fixed standard solution to the Ricci flow on $\mathbb{R}^{3} \times[0,1)$. Then for any $\varepsilon>0$, there is a positive constant $C(\varepsilon)$ such that each point $(x, t) \in \mathbb{R}^{3} \times[0,1)$ has an open neighborhood $B$, with $B_{t}(x, r) \subset B \subset B_{t}(x, 2 r)$ for some $0<r<C(\varepsilon) R(x, t)^{-\frac{1}{2}}$, which falls into one of the following two categories: either

(a) $B$ is an $\varepsilon$-cap, or

(b) $B$ is an $\varepsilon$-neck and it is the slice at the time $t$ of the parabolic neighborhood $B_{t}\left(x, \varepsilon^{-1} R(x, t)^{-\frac{1}{2}}\right) \times\left[t-\min \left\{R(x, t)^{-1}, t\right\}, t\right]$, on which the standard solution is, after scaling with the factor $R(x, t)$ and shifting the time $t$ to zero, $\varepsilon$-close (in the $C^{\left[\varepsilon^{-1}\right]}$ topology) to the corresponding subset of the evolving standard cylinder $\mathbb{S}^{2} \times \mathbb{R}$ over the time interval $[-\min \{t R(x, t), 1\}, 0]$ with scalar curvature 1 at the time zero.

Proof. The proof of the lemma is reduced to two assertions. We now state and prove the first assertion which takes care of those points with times close to 1 . 
Assertion 1. For any $\varepsilon>0$, there is a positive number $\theta=\theta(\varepsilon)$ with $0<\theta<1$ such that for any $\left(x_{0}, t_{0}\right) \in \mathbb{R}^{3} \times[\theta, 1)$, the standard solution on the parabolic neighborhood $B_{t_{0}}\left(x, \varepsilon^{-1} R\left(x_{0}, t_{0}\right)^{-\frac{1}{2}}\right) \times\left[t_{0}-\varepsilon^{-2} R\left(x_{0}, t_{0}\right)^{-1}, t_{0}\right]$ is well-defined and is, after scaling with the factor $R\left(x_{0}, t_{0}\right), \varepsilon$-close (in the $C^{\left[\varepsilon^{-1}\right]}$ topology) to the corresponding subset of some orientable ancient $\kappa$-solution.

We argue by contradiction. Suppose Assertion 1 is not true, then there exist $\bar{\varepsilon}>0$ and a sequence of points $\left(x_{k}, t_{k}\right)$ with $t_{k} \rightarrow 1$, such that for each $k$, the standard solution on the parabolic neighborhood

$$
B_{t_{k}}\left(x_{k}, \bar{\varepsilon}^{-1} R\left(x_{k}, t_{k}\right)^{-\frac{1}{2}}\right) \times\left[t_{k}-\bar{\varepsilon}^{-2} R\left(x_{k}, t_{k}\right)^{-1}, t_{k}\right]
$$

is not, after scaling by the factor $R\left(x_{k}, t_{k}\right), \bar{\varepsilon}$-close to the corresponding subset of any ancient $\kappa$-solution. Note that by Proposition 7.3.3, there is a constant $C>0$ (depending only on the initial metric, hence it is universal ) such that $R(x, t) \geq$ $C^{-1} /(1-t)$. This implies

$$
\bar{\varepsilon}^{-2} R\left(x_{k}, t_{k}\right)^{-1} \leq C \bar{\varepsilon}^{-2}\left(1-t_{k}\right)<t_{k},
$$

and then the standard solution on the parabolic neighborhood $B_{t_{k}}\left(x_{k}\right.$, $\left.\bar{\varepsilon}^{-1} R\left(x_{k}, t_{k}\right)^{-\frac{1}{2}}\right) \times\left[t_{k}-\bar{\varepsilon}^{-2} R\left(x_{k}, t_{k}\right)^{-1}, t_{k}\right]$ is well-defined for $k$ large. By Claim 1 in the proof of Proposition 7.3.3, there is a positive function $\omega:[0, \infty) \rightarrow[0, \infty)$ such that

$$
R\left(x, t_{k}\right) \leq R\left(x_{k}, t_{k}\right) \omega\left(R\left(x_{k}, t_{k}\right) d_{t_{k}}^{2}\left(x, x_{k}\right)\right)
$$

for all $x \in \mathbb{R}^{3}$. Now by scaling the standard solution $g_{i j}(\cdot, t)$ around $x_{k}$ with the factor $R\left(x_{k}, t_{k}\right)$ and shifting the time $t_{k}$ to zero, we get a sequence of the rescaled solutions $\tilde{g}_{i j}^{k}(x, \tilde{t})=R\left(x_{k}, t_{k}\right) g_{i j}\left(x, t_{k}+\tilde{t} / R\left(x_{k}, t_{k}\right)\right)$ to the Ricci flow defined on $\mathbb{R}^{3}$ with $\tilde{t} \in\left[-R\left(x_{k}, t_{k}\right) t_{k}, 0\right]$. We denote the scalar curvature and the distance of the rescaled metric $\tilde{g}_{i j}^{k}$ by $\tilde{R}^{k}$ and $\tilde{d}$. By combining with Claim 1 in the proof of Proposition 7.3.3 and the Li-Yau-Hamilton inequality, we get

$$
\begin{aligned}
\tilde{R}^{k}(x, 0) & \leq \omega\left(\tilde{d}_{0}^{2}\left(x, x_{k}\right)\right) \\
\tilde{R}^{k}(x, \tilde{t}) & \leq \frac{R\left(x_{k}, t_{k}\right) t_{k}}{\tilde{t}+R\left(x_{k}, t_{k}\right) t_{k}} \omega\left(\tilde{d}_{0}^{2}\left(x, x_{k}\right)\right)
\end{aligned}
$$

for any $x \in \mathbb{R}^{3}$ and $\tilde{t} \in\left(-R\left(x_{k}, t_{k}\right) t_{k}, 0\right]$. Note that $R\left(x_{k}, t_{k}\right) t_{k} \rightarrow \infty$ by Proposition 7.3.3. We have shown in the proof of Proposition 7.3.3 that the standard solution is $\kappa$ noncollapsed on all scales less than 1 for some $\kappa>0$. Then from the $\kappa$-noncollapsing property, the above curvature estimates and Hamilton's compactness theorem, we know $\tilde{g}_{i j}^{k}(x, \tilde{t})$ has a convergent subsequence (as $k \rightarrow \infty$ ) whose limit is an ancient, $\kappa$ noncollapsed, complete and orientable solution with nonnegative curvature operator. This limit must have bounded curvature by the same proof of Step 3 in the proof of Theorem 7.1.1. This gives a contradiction. Hence Assertion 1 is proved.

We now fix the constant $\theta(\varepsilon)$ obtained in Assertion 1. Let $O$ be the tip of the standard capped infinite cylinder $\mathbb{R}^{3}$ (it is rotationally symmetric about $O$ at time 0 , and it remains so as $t>0$ by the uniqueness Theorem 1.2.4).

Assertion 2. There are constants $B_{1}(\varepsilon)$ and $B_{2}(\varepsilon)$ depending only on $\varepsilon$, such that if $\left(x_{0}, t_{0}\right) \in \mathbb{R}^{3} \times[0, \theta)$ with $d_{t_{0}}\left(x_{0}, O\right) \leq B_{1}(\varepsilon)$, then there is a $0<r<B_{2}(\varepsilon)$ 
such that $B_{t_{0}}\left(x_{0}, r\right)$ is an $\varepsilon$-cap; if $\left(x_{0}, t_{0}\right) \in \mathbb{R}^{3} \times[0, \theta)$ with $d_{t_{0}}\left(x_{0}, O\right) \geq B_{1}(\varepsilon)$, then the parabolic neighborhood $B_{t_{0}}\left(x_{0}, \varepsilon^{-1} R\left(x_{0}, t_{0}\right)^{-\frac{1}{2}}\right) \times\left[t_{0}-\min \left\{R\left(x_{0}, t_{0}\right)^{-1}, t_{0}\right\}, t_{0}\right]$ is after scaling with the factor $R\left(x_{0}, t_{0}\right)$ and shifting the time $t_{0}$ to zero, $\varepsilon$-close (in the $C^{\left[\varepsilon^{-1}\right]}$ topology) to the corresponding subset of the evolving standard cylinder $\mathbb{S}^{2} \times \mathbb{R}$ over the time interval $\left[-\min \left\{t_{0} R\left(x_{0}, t_{0}\right), 1\right\}, 0\right]$ with scalar curvature 1 at time zero.

Since the standard solution exists on the time interval $[0,1)$, there is a constant $B_{0}(\varepsilon)$ such that the curvatures on $[0, \theta(\varepsilon)]$ are uniformly bounded by $B_{0}(\varepsilon)$. This implies that the metrics in $[0, \theta(\varepsilon)]$ are equivalent. Note that the initial metric is asymptotic to the standard capped infinite cylinder. For any sequence of points $x_{k}$ with $d_{0}\left(O, x_{k}\right) \rightarrow \infty$, after passing to a subsequence, $g_{i j}(x, t)$ around $x_{k}$ will converge to a solution to the Ricci flow on $\mathbb{R} \times \mathbb{S}^{2}$ with round cylinder metric of scalar curvature 1 as initial data. By the uniqueness theorem (Theorem 1.2.4), the limit solution must be the standard evolving round cylinder. This implies that there is a constant $B_{1}(\varepsilon)>0$ depending on $\varepsilon$ such that for any $\left(x_{0}, t_{0}\right)$ with $t_{0} \leq \theta(\varepsilon)$ and $d_{t_{0}}\left(x_{0}, O\right) \geq B_{1}(\varepsilon)$, the standard solution on the parabolic neighborhood $B_{t_{0}}\left(x_{0}, \varepsilon^{-1} R\left(x_{0}, t_{0}\right)^{-\frac{1}{2}}\right) \times\left[t_{0}-\min \left\{R\left(x_{0}, t_{0}\right)^{-1}, t_{0}\right\}, t_{0}\right]$ is, after scaling with the factor $R\left(x_{0}, t_{0}\right)$, $\varepsilon$-close to the corresponding subset of the evolving round cylinder. Since the solution is rotationally symmetric around $O$, the cap neighborhood structures of those points $x_{0}$ with $d_{t_{0}}\left(x_{0}, O\right) \leq B_{1}(\varepsilon)$ follow directly. Hence Assertion 2 is proved.

The combination of these two assertions proves the lemma.

Since there are only a finite number of horns with the other end connected to $\Omega_{\rho}$, we perform only a finite number of such $\delta$-cutoff surgeries at time $T$. Besides those horns, there could be capped horns and double horns which lie in $\Omega \backslash \Omega_{\rho}$. As explained before, they are connected to form tubes or capped tubes at any time slightly before $T$. So we can regard the capped horns and double horns (of $\Omega \backslash \Omega_{\rho}$ ) to be extinct and throw them away at time $T$. We only need to remember that the connected sums were broken there. Remember that we have thrown away all compact components, either lying in $\Omega \backslash \Omega_{\rho}$ or with positive sectional curvature, each of which is diffeomorphic to either $\mathbb{S}^{3}$, or a metric quotient of $\mathbb{S}^{3}$, or $\mathbb{S}^{2} \times \mathbb{S}^{1}$ or $\mathbb{R} \mathbb{P}^{3} \# \mathbb{R} \mathbb{P}^{3}$. So we have also removed a finite number of copies of $\mathbb{S}^{3}$, or metric quotients of $\mathbb{S}^{3}$, or $\mathbb{S}^{2} \times \mathbb{S}^{1}$ or $\mathbb{R} \mathbb{P}^{3} \# \mathbb{R} \mathbb{P}^{3}$ at the time $T$. Let us agree to declare extinct every compact component either with positive sectional curvature or lying in $\Omega \backslash \Omega_{\rho}$; in particular, this allows us to exclude the components with positive sectional curvature from the list of canonical neighborhoods.

In summary, our surgery at time $T$ consists of the following four procedures: $\Omega_{\rho}$;

(1) perform $\delta$-cutoff surgeries for all $\varepsilon$-horns, whose other ends are connected to

(2) declare extinct every compact component which has positive sectional curvature;

(3) throw away all capped horns and double horns lying in $\Omega \backslash \Omega_{\rho}$;

(4) declare extinct all compact components lying in $\Omega \backslash \Omega_{\rho}$.

(In Sections 7.6 and 7.7, we will add one more procedure by declaring extinct every compact component which has nonnegative scalar curvature.)

By Lemma 7.3.4, after performing surgeries at time $T$, the pinching assumption (7.3.3) still holds for the surgically modified manifold. With this surgically modified manifold (possibly disconnected) as initial data, we now continue our solution under 
the Ricci flow until it becomes singular again at some time $T^{\prime}(>T)$. Therefore, we have extended the solution to the Ricci flow with surgery, originally defined on $[0, T)$ with $T<+\infty$, to the new time interval $\left[0, T^{\prime}\right)$ with $T^{\prime}>T$. By the proof of Theorem 5.3 .2 , we see that the solution to the Ricci flow with surgery also satisfies the pinching assumption on $\left[0, T^{\prime}\right)$. It remains to verify the canonical neighborhood assumption (with accuracy $\varepsilon$ ) for the solution on the time interval $\left[T, T^{\prime}\right.$ ) and to prove that this extension procedure works indefinitely (unless it becomes extinct at some finite time) and that there exists at most a finite number of surgeries at every finite time interval. We leave these arguments to the next section.

Before we end this section, we check the following two assertions of Perelman in [104] which will be used in the next section to estimate the Li-Yau-Perelman distance of space-time curves which stretch to surgery regions.

Lemma 7.3.6 (Perelman [104]). For any $0<\varepsilon \leq 1 / 100,1<A<+\infty$ and $0<\theta<1$, one can find $\bar{\delta}=\bar{\delta}(A, \theta, \varepsilon)$ with the following property. Suppose we have a solution to the Ricci flow with surgery which satisfies the a priori assumptions (with accuracy $\varepsilon)$ on $[0, T]$ and is obtained from a compact orientable three-manifold by a finite number of $\delta$-cutoff surgeries with each $\delta<\bar{\delta}$. Suppose we have a cutoff surgery at time $T_{0} \in(0, T)$, let $x_{0}$ be any fixed point on the gluing caps (i.e., the regions affected by the cutoff surgeries at time $\left.T_{0}\right)$, and let $T_{1}=\min \left\{T, T_{0}+\theta h^{2}\right\}$, where $h$ is the cutoff radius around $x_{0}$ obtained in Lemma 7.3.2. Then either

(i) the solution is defined on $P\left(x_{0}, T_{0}, A h, T_{1}-T_{0}\right) \triangleq\left\{(x, t) \mid x \in B_{t}\left(x_{0}, A h\right), t \in\right.$ $\left.\left[T_{0}, T_{1}\right]\right\}$ and is, after scaling with factor $h^{-2}$ and shifting time $T_{0}$ to zero, $A^{-1}$-close to a corresponding subset of the standard solution, or

(ii) the assertion (i) holds with $T_{1}$ replaced by some time $t^{+} \in\left(T_{0}, T_{1}\right)$, where $t^{+}$is a surgery time; moreover, for each point in $B_{T_{0}}\left(x_{0}, A h\right)$, the solution is defined for $t \in\left[T_{0}, t^{+}\right)$but is not defined past $t^{+}$(i.e., the whole ball $B_{T_{0}}\left(x_{0}, A h\right)$ is cut off at the time $\left.t^{+}\right)$.

Proof. Let $Q$ be the maximum of the scalar curvature of the standard solution in the time interval $[0, \theta]$ and choose a large positive integer $N$ so that $\Delta t=\frac{\left(T_{1}-T_{0}\right)}{N}<$ $\varepsilon \eta^{-1} Q^{-1} h^{2}$, where the positive constant $\eta$ is given in the canonical neighborhood assumption. Set $t_{k}=T_{0}+k \Delta t, k=0,1, \ldots, N$.

From Lemma 7.3.4, the geodesic ball $B_{T_{0}}\left(x_{0}, A_{0} h\right)$ at time $T_{0}$, with $A_{0}=\delta^{-\frac{1}{2}}$ is, after scaling with factor $h^{-2}, \delta^{\frac{1}{2}}$-close to the corresponding ball in the standard capped infinite cylinder with the center near the tip. Assume first that for each point in $B_{T_{0}}\left(x_{0}, A_{0} h\right)$, the solution is defined on $\left[T_{0}, t_{1}\right]$. By the gradient estimates (7.3.4) in the canonical neighborhood assumption and the choice of $\Delta t$ we have a uniform curvature bound on this set for $h^{-2}$-scaled metric. Then by the uniqueness theorem (Theorem 1.2.4), if $\delta^{\frac{1}{2}} \rightarrow 0$ (i.e. $A_{0}=\delta^{-\frac{1}{2}} \rightarrow+\infty$ ), the solution with $h^{-2}$-scaled metric will converge to the standard solution in the $C_{\text {loc }}^{\infty}$ topology. Therefore we can define $A_{1}$, depending only on $A_{0}$ and tending to infinity with $A_{0}$, such that the solution in the parabolic region $P\left(x_{0}, T_{0}, A_{1} h, t_{1}-T_{0}\right) \triangleq\left\{(x, t) \mid x \in B_{t}\left(x_{0}, A_{1} h\right), t \in\right.$ $\left.\left[T_{0}, T_{0}+\left(t_{1}-T_{0}\right)\right]\right\}$ is, after scaling with factor $h^{-2}$ and shifting time $T_{0}$ to zero, $A_{1}^{-1}$-close to the corresponding subset in the standard solution. In particular, the scalar curvature on this subset does not exceed $2 Q h^{-2}$. Now if for each point in $B_{T_{0}}\left(x_{0}, A_{1} h\right)$ the solution is defined on $\left[T_{0}, t_{2}\right]$, then we can repeat the procedure, defining $A_{2}$, such that the solution in the parabolic region $P\left(x_{0}, T_{0}, A_{2} h, t_{2}-T_{0}\right) \triangleq$ $\left\{(x, t) \mid x \in B_{t}\left(x_{0}, A_{2} h\right), t \in\left[T_{0}, T_{0}+\left(t_{2}-T_{0}\right)\right]\right\}$ is, after scaling with factor $h^{-2}$ and shifting time $T_{0}$ to zero, $A_{2}^{-1}$-close to the corresponding subset in the standard 
solution. Again, the scalar curvature on this subset still does not exceed $2 Q h^{-2}$. Continuing this way, we eventually define $A_{N}$. Note that $N$ is depends only on $\theta$ and $\varepsilon$. Thus there exists a positive $\bar{\delta}=\bar{\delta}(A, \theta, \varepsilon)$ such that for $\delta<\bar{\delta}$, we have $A_{0}>A_{1}>\cdots>A_{N}>A$, and assertion (i) holds when the solution is defined on $B_{T_{0}}\left(x_{0}, A_{(N-1)} h\right) \times\left[T_{0}, T_{1}\right]$.

The above argument shows that either assertion (i) holds, or there exists some $k(0 \leq k \leq N-1)$ and a surgery time $t^{+} \in\left(t_{k}, t_{k+1}\right]$ such that the solution on $B_{T_{0}}\left(x_{0}, A_{k} h\right)$ is defined on $\left[T_{0}, t^{+}\right)$, but for some point of this set it is not defined past $t^{+}$. Now we consider the latter case. Clearly the above argument also shows that the parabolic region $P\left(x_{0}, T_{0}, A_{k+1} h, t^{+}-T_{0}\right) \triangleq\left\{(x, t) \mid x \in B_{t}\left(x, A_{k+1} h\right), t \in\left[T_{0}, t^{+}\right)\right\}$ is, after scaling with factor $h^{-2}$ and shifting time $T_{0}$ to zero, $A_{k+1}^{-1}$-close to the corresponding subset in the standard solution. In particular, as time tends to $t^{+}$, the ball $B_{T_{0}}\left(x_{0}, A_{k+1} h\right)$ keeps on looking like a cap. Since the scalar curvature on $B_{T_{0}}\left(x_{0}, A_{k} h\right) \times\left[T_{0}, t_{k}\right]$ does not exceed $2 Q h^{-2}$, it follows from the pinching assumption, the gradient estimates in the canonical neighborhood assumption and the evolution equation of the metric that the diameter of the set $B_{T_{0}}\left(x_{0}, A_{k} h\right)$ at any time $t \in\left[T_{0}, t^{+}\right)$is bounded from above by $4 \delta^{-\frac{1}{2}} h$. These imply that no point of the ball $B_{T_{0}}\left(x_{0}, A_{k} h\right)$ at any time near $t^{+}$can be the center of a $\delta$-neck for any $0<\delta<\bar{\delta}(A, \theta, \varepsilon)$ with $\bar{\delta}(A, \theta, \varepsilon)>0$ small enough, since $4 \delta^{-\frac{1}{2}} h$ is much smaller than $\delta^{-1} h$. However the solution disappears somewhere in the set $B_{T_{0}}\left(x_{0}, A_{k} h\right)$ at time $t^{+}$by a cutoff surgery and the surgery is always done along the middle two-sphere of a $\delta$-neck. So the set $B_{T_{0}}\left(x_{0}, A_{k} h\right)$ at time $t^{+}$is a part of a capped horn. (Recall that we have declared extinct every compact component with positive curvature and every compact component lying in $\left.\Omega \backslash \Omega_{\rho}\right)$. Hence for each point of $B_{T_{0}}\left(x_{0}, A_{k} h\right)$ the solution terminates at $t^{+}$. This proves assertion (ii).

Corollary 7.3.7 (Perelman [104]). For any $l<\infty$ one can find $A=A(l)<$ $\infty$ and $\theta=\theta(l), 0<\theta<1$, with the following property. Suppose we are in the situation of the lemma above, with $\delta<\bar{\delta}(A, \theta, \varepsilon)$. Consider smooth curves $\gamma$ in the set $B_{T_{0}}\left(x_{0}, A h\right)$, parametrized by $t \in\left[T_{0}, T_{\gamma}\right]$, such that $\gamma\left(T_{0}\right) \in B_{T_{0}}\left(x_{0}, \frac{A h}{2}\right)$ and either $T_{\gamma}=T_{1}<T$, or $T_{\gamma}<T_{1}$ and $\gamma\left(T_{\gamma}\right) \in \partial B_{T_{0}}\left(x_{0}, A h\right)$, where $x_{0}$ is any fixed point on a gluing cap at $T_{0}$ and $T_{1}=\min \left\{T, T_{0}+\theta h^{2}\right\}$. Then

$$
\int_{T_{0}}^{T_{\gamma}}\left(R(\gamma(t), t)+|\dot{\gamma}(t)|^{2}\right) d t>l .
$$

Proof. We know from Proposition 7.3.3 that on the standard solution,

$$
\begin{aligned}
\int_{0}^{\theta} R d t & \geq \text { const. } \int_{0}^{\theta}(1-t)^{-1} d t \\
& =- \text { const. } \cdot \log (1-\theta) .
\end{aligned}
$$

By choosing $\theta=\theta(l)$ sufficiently close to 1 we have the desired estimate for the standard solution.

Let us consider the first case: $T_{\gamma}=T_{1}<T$. For $\theta=\theta(l)$ fixed above, by Lemma 7.3.6, our solution in the subset $B_{T_{0}}\left(x_{0}, A h\right)$ and in the time interval $\left[T_{0}, T_{\gamma}\right]$ is, after scaling with factor $h^{-2}$ and shifting time $T_{0}$ to zero, $A^{-1}$-close to the corresponding 
subset in the standard solution for any sufficiently large $A$. So we have

$$
\begin{aligned}
\int_{T_{0}}^{T_{\gamma}}\left(R(\gamma(t), t)+|\dot{\gamma}(t)|^{2}\right) d t & \geq \text { const. } \int_{0}^{\theta}(1-t)^{-1} d t \\
& =- \text { const. } \cdot \log (1-\theta) .
\end{aligned}
$$

Hence we have obtained the desired estimate in the first case.

We now consider the second case: $T_{\gamma}<T_{1}$ and $\gamma\left(T_{\gamma}\right) \in \partial B_{T_{0}}\left(x_{0}, A h\right)$. Let $\theta=\theta(l)$ be chosen above and let $Q=Q(l)$ be the maximum of the scalar curvature on the standard solution in the time interval $[0, \theta]$.

On the standard solution, we can choose $A=A(l)$ so large that for each $t \in[0, \theta]$,

$$
\begin{aligned}
\operatorname{dist}_{t}\left(x_{0}, \partial B_{0}\left(x_{0}, A\right)\right) & \geq \operatorname{dist}_{0}\left(x_{0}, \partial B_{0}\left(x_{0}, A\right)\right)-4(Q+1) t \\
& \geq A-4(Q+1) \theta \\
& \geq \frac{4}{5} A
\end{aligned}
$$

and

$$
\operatorname{dist}_{t}\left(x_{0}, \partial B_{0}\left(x_{0}, \frac{A}{2}\right)\right) \leq \frac{A}{2}
$$

where we have used Lemma 3.4.1(ii) in the first inequality. Now our solution in the subset $B_{T_{0}}\left(x_{0}, A h\right)$ and in the time interval $\left[T_{0}, T_{\gamma}\right]$ is, after scaling with factor $h^{-2}$ and shifting time $T_{0}$ to zero, $A^{-1}$-close to the corresponding subset in the standard solution. This implies that for $A=A(l)$ large enough

$$
\frac{1}{5} A h \leq \int_{T_{0}}^{T_{\gamma}}|\dot{\gamma}(t)| d t \leq\left(\int_{T_{0}}^{T_{\gamma}}|\dot{\gamma}(t)|^{2} d t\right)^{\frac{1}{2}} \cdot\left(T_{\gamma}-T_{0}\right)^{\frac{1}{2}},
$$

Hence

$$
\int_{T_{0}}^{T_{\gamma}}\left(R(\gamma(t), t)+|\dot{\gamma}(t)|^{2}\right) d t \geq \frac{A^{2}}{25 \theta}>l
$$

This proves the desired estimate.

7.4. Justification of the Canonical Neighborhood Assumptions. We continue the induction argument for the construction of a long-time solution to the Ricci flow with surgery. Let us recall what we have done in the previous section. Let $\varepsilon$ be an arbitrarily given positive constant satisfying $0<\varepsilon \leq 1 / 100$. For an arbitrarily given compact orientable normalized three-manifold, we evolve it by the Ricci flow. We may assume that the solution goes singular at some time $0<t_{1}^{+}<+\infty$ and know that the solution satisfies the a priori assumptions (with accuracy $\varepsilon$ ) on $\left[0, t_{1}^{+}\right)$for a nonincreasing positive function $r=r_{1}(t)$ (defined on $[0,+\infty)$ ). Suppose that we have a solution to the Ricci flow with surgery, defined on $\left[0, t_{k}^{+}\right)$with $0<t_{1}^{+}<t_{2}^{+}<\cdots<t_{k}^{+}<+\infty$, satisfying the a priori assumptions (with accuracy $\varepsilon$ ) for some nonincreasing positive function $r=r_{k}(t)$ (defined on $[0,+\infty)$ ), going singular at time $t_{k}^{+}$and having $\delta_{i}$-cutoff surgeries at each time $t_{i}^{+}, 1 \leq i \leq k-1$, where $\delta_{i}<\bar{\delta}\left(t_{i}^{+}\right)$for each $1 \leq i \leq k-1$. Then for any $0<\delta_{k}<\bar{\delta}\left(t_{k}^{+}\right)$, we can perform $\delta_{k}$-cutoff surgeries at the time $t_{k}^{+}$and extend the solution to the interval $\left[0, t_{k+1}^{+}\right)$with $t_{k+1}^{+}>t_{k}^{+}$. Here $\bar{\delta}(t)$ is the positive function 
defined in (7.3.16). We have already shown in Lemma 7.3.4 that the extended solution still satisfies the pinching assumption on $\left[0, t_{k+1}^{+}\right)$.

In view of Theorem 7.1.1, there always is a nonincreasing positive function $r=$ $r_{k+1}(t)$, defined on $[0,+\infty)$, such that the canonical neighborhood assumption (with accuracy $\varepsilon$ ) holds on the extended time interval $\left[0, t_{k+1}^{+}\right)$with the positive function $r=$ $r_{k+1}(t)$. Nevertheless, in order to prevent the surgery times from accumulating, the key is to choose the nonincreasing positive functions $r=r_{i}(t), i=1,2, \ldots$, uniformly. That is, to justify the canonical neighborhood assumption (with accuracy $\varepsilon$ ) for the indefinitely extending solution, we need to show that there exists a nonincreasing positive function $\widetilde{r}(t)$, defined on $[0,+\infty)$, which is independent of $k$, such that the above chosen nonincreasing positive functions satisfy

$$
r_{i}(t) \geq \widetilde{r}(t), \quad \text { on } \quad[0,+\infty),
$$

for all $i=1,2, \ldots, k+1$.

By a further restriction on the positive function $\bar{\delta}(t)$, we can verify this after proving the following assertion which was stated by Perelman in [104].

Proposition 7.4.1 (Justification of the canonical neighborhood assumption). Given any small $\varepsilon>0$, there exist decreasing sequences $0<\widetilde{r}_{j}<\varepsilon, \kappa_{j}>0$, and $0<\widetilde{\delta}_{j}<\varepsilon^{2}, j=1,2, \cdots$, with the following property. Define the positive function $\widetilde{\delta}(t)$ on $[0,+\infty)$ by $\widetilde{\delta}(t)=\widetilde{\delta}_{j}$ for $t \in\left[(j-1) \varepsilon^{2}, j \varepsilon^{2}\right)$. Suppose there is a surgically modified solution, defined on $[0, T)$ with $T<+\infty$, to the Ricci flow which satisfies the following:

(1) it starts on a compact orientable three-manifold with normalized initial metric, and

(2) it has only a finite number of surgeries such that each surgery at a time $t \in(0, T)$ is a $\delta(t)$-cutoff surgery with

$$
0<\delta(t) \leq \min \{\widetilde{\delta}(t), \bar{\delta}(t)\}
$$

Then on each time interval $\left[(j-1) \varepsilon^{2}, j \varepsilon^{2}\right] \bigcap[0, T), j=1,2, \cdots$, the solution satisfies the $\kappa_{j}$-noncollapsing condition on all scales less than $\varepsilon$ and the canonical neighborhood assumption (with accuracy $\varepsilon$ ) with $r=\widetilde{r}_{j}$.

Here and in the following, we call a (three-dimensional) surgically modified solution $g_{i j}(t), 0 \leq t<T, \kappa$-noncollapsed at $\left(x_{0}, t_{0}\right)$ on the scales less than $\rho$ (for some $\kappa>0, \rho>0)$ if it satisfies the following property: whenever $r<\rho$ and

$$
|R m(x, t)| \leq r^{-2}
$$

for all those $(x, t) \in P\left(x_{0}, t_{0}, r,-r^{2}\right)=\left\{\left(x^{\prime}, t^{\prime}\right) \mid x^{\prime} \in B_{t^{\prime}}\left(x_{0}, r\right), t^{\prime} \in\left[t_{0}-r^{2}, t_{0}\right]\right\}$, for which the solution is defined, we have

$$
\operatorname{Vol}_{t_{0}}\left(B_{t_{0}}\left(x_{0}, r\right)\right) \geq \kappa r^{3} .
$$

Before we give the proof of the proposition, we need to verify a $\kappa$-noncollapsing estimate which was given by Perelman in [104].

Lemma 7.4.2. Given any $0<\varepsilon \leq \bar{\varepsilon}_{0}$ (for some sufficiently small universal constant $\bar{\varepsilon}_{0}$ ), suppose we have constructed the sequences satisfying the proposition for $1 \leq j \leq m$ (for some positive integer $m$ ). Then there exists $\kappa>0$, such that for any 
$r, 0<r<\varepsilon$, one can find $\widetilde{\delta}=\widetilde{\delta}(r, \varepsilon), 0<\widetilde{\delta}<\varepsilon^{2}$, which may also depend on the already constructed sequences, with the following property. Suppose we have a solution with a compact orientable normalized three-manifold as initial data, to the Ricci flow with finite number of surgeries on a time interval $[0, \bar{T}]$ with $m \varepsilon^{2} \leq \bar{T}<(m+1) \varepsilon^{2}$, satisfying the assumptions and the conclusions of Proposition 7.4 .1 on $\left[0, m \varepsilon^{2}\right)$, and the canonical neighborhood assumption (with accuracy $\varepsilon$ ) with $r$ on $\left[m \varepsilon^{2}, \bar{T}\right]$, as well as $0<\delta(t) \leq \min \{\widetilde{\delta}, \bar{\delta}(t)\}$ for any $\delta$-cutoff surgery with $\delta=\delta(t)$ at a time $t \in$ $\left[(m-1) \varepsilon^{2}, \bar{T}\right]$. Then the solution is $\kappa$-noncollapsed on $[0, \bar{T}]$ for all scales less than $\varepsilon$.

Proof. Consider a parabolic neighborhood

$$
P\left(x_{0}, t_{0}, r_{0},-r_{0}^{2}\right) \triangleq\left\{(x, t) \mid x \in B_{t}\left(x_{0}, r_{0}\right), t \in\left[t_{0}-r_{0}^{2}, t_{0}\right]\right\}
$$

with $m \varepsilon^{2} \leq t_{0} \leq \bar{T}$ and $0<r_{0}<\varepsilon$, where the solution satisfies $|R m| \leq r_{0}^{-2}$, whenever it is defined. We will use an argument analogous to the proof of Theorem 3.3.2 (no local collapsing theorem I) to prove

$$
\mathrm{Vol}_{t_{0}}\left(B_{t_{0}}\left(x_{0}, r_{0}\right)\right) \geq \kappa r_{0}^{3} .
$$

Let $\eta$ be the universal positive constant in the definition of the canonical neighborhood assumption. Without loss of generality, we always assume $\eta \geq 10$. Firstly, we want to show that one may assume $r_{0} \geq \frac{1}{2 \eta} r$.

Obviously, the curvature satisfies the estimate

$$
|R m(x, t)| \leq 20 r_{0}^{-2},
$$

for those $(x, t) \in P\left(x_{0}, t_{0}, \frac{1}{2 \eta} r_{0},-\frac{1}{8 \eta} r_{0}^{2}\right)=\left\{(x, t) \mid x \in B_{t}\left(x_{0}, \frac{1}{2 \eta} r_{0}\right), t \in\left[t_{0}-\frac{1}{8 \eta} r_{0}^{2}, t_{0}\right]\right\}$, for which the solution is defined. When $r_{0}<\frac{1}{2 \eta} r$, we can enlarge $r_{0}$ to some $r_{0}^{\prime} \in\left[r_{0}, r\right]$ so that

$$
|R m| \leq 20 r_{0}^{\prime-2}
$$

on $P\left(x_{0}, t_{0}, \frac{1}{2 \eta} r_{0}^{\prime},-\frac{1}{8 \eta} r_{0}^{\prime 2}\right)$ (whenever it is defined), and either the equality holds somewhere in $P\left(x_{0}, t_{0}, \frac{1}{2 \eta} r_{0}^{\prime},-\left(\frac{1}{8 \eta} r_{0}^{\prime 2}+\epsilon^{\prime}\right)\right)$ for any arbitrarily small $\epsilon^{\prime}>0$ or $r_{0}^{\prime}=r$.

In the case that the equality holds somewhere, it follows from the pinching assumption that we have

$$
R>10 r_{0}^{\prime-2}
$$

somewhere in $P\left(x_{0}, t_{0}, \frac{1}{2 \eta} r_{0}^{\prime},-\left(\frac{1}{8 \eta} r_{0}^{\prime 2}+\epsilon^{\prime}\right)\right)$ for any arbitrarily small $\epsilon^{\prime}>0$. Here, without loss of generality, we have assumed $r$ is suitably small. Then by the gradient estimates in the definition of the canonical neighborhood assumption, we know

$$
R\left(x_{0}, t_{0}\right)>r_{0}^{\prime-2} \geq r^{-2} .
$$

Hence the desired noncollapsing estimate (7.4.1) in this case follows directly from the canonical neighborhood assumption. (Recall that we have excluded every compact component which has positive sectional curvature in the surgery procedure and then we have excluded them from the list of canonical neighborhoods. Here we also used the standard volume comparison when the canonical neighborhood is an $\varepsilon$-cap.) 
While in the case that $r_{0}^{\prime}=r$, we have the curvature bound

$$
|R m(x, t)| \leq\left(\frac{1}{2 \eta} r\right)^{-2}
$$

for those $(x, t) \in P\left(x_{0}, t_{0}, \frac{1}{2 \eta} r,-\left(\frac{1}{2 \eta} r\right)^{2}\right)=\left\{(x, t) \mid x \in B_{t}\left(x_{0}, \frac{1}{2 \eta} r\right), t \in\left[t_{0}-\right.\right.$ $\left.\left.\left(\frac{1}{2 \eta} r\right)^{2}, t_{0}\right]\right\}$, for which the solution is defined. It follows from the standard volume comparison that we only need to verify the noncollapsing estimate (7.4.1) for $r_{0}=\frac{1}{2 \eta} r$. Thus we have reduced the proof to the case $r_{0} \geq \frac{1}{2 \eta} r$.

Recall from Theorem 3.3.2 that if a solution is smooth everywhere, we can get a lower bound for the volume of the ball $B_{t_{0}}\left(x_{0}, r_{0}\right)$ as follows: define $\tau(t)=t_{0}-t$ and consider Perelman's reduced volume function and the Li-Yau-Perelman distance associated to the point $x_{0}$; take a point $\bar{x}$ at the time $t=\varepsilon^{2}$ so that the Li-YauPerelman distance $l$ attains its minimum $l_{\min }(\tau)=l(\bar{x}, \tau) \leq \frac{3}{2}$ for $\tau=t_{0}-\varepsilon^{2}$; use it to obtain an upper bound for the Li-Yau-Perelman distance from $x_{0}$ to each point of $B_{0}(\bar{x}, 1)$, thus getting a lower bound for Perelman's reduced volume at $\tau=t_{0}$; apply the monotonicity of Perelman's reduced volume to deduce a lower bound for Perelman's reduced volume at $\tau$ near 0 , and then get the desired estimate for the volume of the ball $B_{t_{0}}\left(x_{0}, r_{0}\right)$. Now since our solution has undergone surgeries, we need to localize this argument to the region which is unaffected by surgery.

We call a space-time curve in the solution track admissible if it stays in the spacetime region unaffected by surgery, and we call a space-time curve in the solution track a barely admissible curve if it is on the boundary of the set of admissible curves.

First of all, we want to estimate the $\mathcal{L}$-length of a barely admissible curve.

Claim. For any $L<\infty$ one can find $\bar{\delta}=\bar{\delta}\left(L, r, \widetilde{r}_{m}, \varepsilon\right)>0$ with the following property. Suppose that we have a curve $\gamma$, parametrized by $t \in\left[T_{0}, t_{0}\right],(m-1) \varepsilon^{2} \leq$ $T_{0}<t_{0}$, such that $\gamma\left(t_{0}\right)=x_{0}, T_{0}$ is a surgery time, and $\gamma\left(T_{0}\right)$ lies in the gluing cap. Suppose also each $\delta$-cutoff surgery at a time in $\left[(m-1) \varepsilon^{2}, \bar{T}\right]$ has $\delta \leq \bar{\delta}$. Then we have an estimate

$$
\int_{T_{0}}^{t_{0}} \sqrt{t_{0}-t}\left(R_{+}(\gamma(t), t)+|\dot{\gamma}(t)|^{2}\right) d t \geq L
$$

where $R_{+}=\max \{R, 0\}$.

Since $r_{0} \geq \frac{1}{2 \eta} r$ and $|R m| \leq r_{0}^{-2}$ on $P\left(x_{0}, t_{0}, r_{0},-r_{0}^{2}\right)$ (whenever it is defined), we can require $\bar{\delta}>0$, depending on $r$ and $\widetilde{r}_{m}$, to be so small that $\gamma\left(T_{0}\right)$ does not lie in the region $P\left(x_{0}, t_{0}, r_{0},-r_{0}^{2}\right)$. Let $\Delta t$ be the maximal number such that $\left.\gamma\right|_{\left[t_{0}-\Delta t, t_{0}\right]} \subset$ $P\left(x_{0}, t_{0}, r_{0},-\Delta t\right)$ (i.e., $t_{0}-\Delta t$ is the first time when $\gamma$ escapes the parabolic region $\left.P\left(x_{0}, t_{0}, r_{0},-r_{0}^{2}\right)\right)$. Obviously we only need to consider the case:

$$
\int_{t_{0}-\Delta t}^{t_{0}} \sqrt{t_{0}-t}\left(R_{+}(\gamma(t), t)+|\dot{\gamma}(t)|^{2}\right) d t<L .
$$

We observe that $\Delta t$ can be bounded from below in terms of $L$ and $r_{0}$. Indeed, if $\Delta t \geq r_{0}^{2}$, there is nothing to prove. Thus we may assume $\Delta t<r_{0}^{2}$. By the curvature bound $|R m| \leq r_{0}^{-2}$ on $P\left(x_{0}, t_{0}, r_{0},-r_{0}^{2}\right)$ and the Ricci flow equation we see

$$
\int_{t_{0}-\Delta t}^{t_{0}}|\dot{\gamma}(t)| d t \geq c r_{0}
$$


for some universal positive constant $c$. On the other hand, by the Cauchy-Schwarz inequality, we have

$$
\begin{aligned}
\int_{t_{0}-\Delta t}^{t_{0}}|\dot{\gamma}(t)| d t & \leq\left(\int_{t_{0}-\Delta t}^{t_{0}} \sqrt{t_{0}-t}\left(R_{+}+|\dot{\gamma}|^{2}\right) d t\right)^{\frac{1}{2}} \cdot\left(\int_{t_{0}-\Delta t}^{t_{0}} \frac{1}{\sqrt{t_{0}-t}} d t\right)^{\frac{1}{2}} \\
& \leq(2 L)^{\frac{1}{2}}(\Delta t)^{\frac{1}{4}}
\end{aligned}
$$

which implies

$$
(\Delta t)^{\frac{1}{2}} \geq \frac{c^{2} r_{0}^{2}}{2 L}
$$

Thus

$$
\begin{aligned}
\int_{T_{0}}^{t_{0}} \sqrt{t_{0}-t}\left(R_{+}+|\dot{\gamma}|^{2}\right) d t & \geq \int_{T_{0}}^{t_{0}-\Delta t} \sqrt{t_{0}-t}\left(R_{+}+|\dot{\gamma}|^{2}\right) d t \\
& \geq(\Delta t)^{\frac{1}{2}} \int_{T_{0}}^{t_{0}-\Delta t}\left(R_{+}+|\dot{\gamma}|^{2}\right) d t \\
& \geq\left(\min \left\{\frac{c^{2} r_{0}^{2}}{2 L}, r_{0}\right\}\right) \int_{T_{0}}^{t_{0}-\Delta t}\left(R_{+}+|\dot{\gamma}|^{2}\right) d t
\end{aligned}
$$

while by Corollary 7.3.7, we can find $\bar{\delta}=\bar{\delta}\left(L, r, \widetilde{r}_{m}, \varepsilon\right)>0$ so small that

$$
\int_{T_{0}}^{t_{0}-\Delta t}\left(R_{+}+|\dot{\gamma}|^{2}\right) d t \geq L\left(\min \left\{\frac{c^{2} r_{0}^{2}}{2 L}, r_{0}\right\}\right)^{-1} .
$$

Then we have proved the desired assertion (7.4.2).

Recall that for a curve $\gamma$, parametrized by $\tau=t_{0}-t \in[0, \bar{\tau}]$, with $\gamma(0)=x_{0}$ and $\bar{\tau} \leq t_{0}-(m-1) \varepsilon^{2}$, we have $L(\gamma)=\int_{0}^{\bar{\tau}} \sqrt{\tau}\left(R+|\dot{\gamma}|^{2}\right) d \tau$. We can also define $L_{+}(\gamma)$ by replacing $R$ with $R_{+}$in the previous formula. Recall that $R \geq-1$ at the initial time $t=0$ for the normalized initial manifold. Recall that the surgeries occur at the parts where the scalar curvatures are very large. Thus we can apply the maximum principle to conclude that the solution with surgery still satisfies $R \geq-1$ everywhere in space-time. This implies

$$
L_{+}(\gamma) \leq L(\gamma)+\left(2 \varepsilon^{2}\right)^{\frac{3}{2}}
$$

By applying the assertion (7.4.2), we now choose $\tilde{\delta}>0$ (depending on $r, \varepsilon$ and $\widetilde{r}_{m}$ ) such that as each $\delta$-cutoff surgery at the time interval $t \in\left[(m-1) \varepsilon^{2}, T\right]$ has $\delta \leq \tilde{\delta}$, every barely admissible curve $\gamma$ from $\left(x_{0}, t_{0}\right)$ to a point $(x, t)\left(\right.$ with $\left.t \in\left[(m-1) \varepsilon^{2}, t_{0}\right)\right)$ has

$$
L_{+}(\gamma) \geq 22 \sqrt{2}
$$

Thus if the Li-Yau-Perelman distance from $\left(x_{0}, t_{0}\right)$ to a point $(x, t)$ (with $t \in[(m-$ 1) $\left.\left.\varepsilon^{2}, t_{0}\right)\right)$ is achieved by a space-time curve which is not admissible, then its Li-YauPerelman distance has

$$
l \geq \frac{L_{+}-\left(2 \varepsilon^{2}\right)^{\frac{3}{2}}}{2 \sqrt{2} \varepsilon}>10 \varepsilon^{-1}
$$


We also observe that the absolute value of $l\left(x_{0}, \tau\right)$ is very small as $\tau$ closes to zere. Thus the maximum principle argument in Corollary 3.2.6 still works for our solutions with surgery because barely admissible curves do not attain the minimum. So we conclude that

$$
l_{\min }(\bar{\tau})=\min \left\{l(x, \bar{\tau}) \mid x \text { lies in the solution manifold at } t_{0}-\bar{\tau}\right\} \leq \frac{3}{2}
$$

for $\bar{\tau} \in\left(0, t_{0}-(m-1) \varepsilon^{2}\right]$. In particular, there exists a minimizing curve $\gamma$ of $l_{\min }\left(t_{0}-\right.$ $\left.(m-1) \varepsilon^{2}\right)$, defined on $\tau \in\left[0, t_{0}-(m-1) \varepsilon^{2}\right]$ with $\gamma(0)=x_{0}$, such that

$$
\begin{aligned}
L_{+}(\gamma) & \leq \frac{3}{2} \cdot 2 \sqrt{2} \varepsilon+2 \sqrt{2} \varepsilon^{3} \\
& \leq 5 \varepsilon
\end{aligned}
$$

since $0<\varepsilon \leq \bar{\varepsilon}_{0}$ with $\bar{\varepsilon}_{0}$ sufficiently small (to be further determined). Consequently, there exists a point $(\bar{x}, \bar{t})$ on the minimizing curve $\gamma$ with $\bar{t} \in\left[(m-1) \varepsilon^{2}+\frac{1}{4} \varepsilon^{2},(m-\right.$ 1) $\left.\varepsilon^{2}+\frac{3}{4} \varepsilon^{2}\right]$ (i.e., $\left.\tau \in\left[t_{0}-(m-1) \varepsilon^{2}-\frac{3}{4} \varepsilon^{2}, t_{0}-(m-1) \varepsilon^{2}-\frac{1}{4} \varepsilon^{2}\right]\right)$ such that

$$
R(\bar{x}, \bar{t}) \leq 25 \widetilde{r}_{m}^{-2} .
$$

Otherwise, we have

$$
\begin{aligned}
L_{+}(\gamma) & \geq \int_{t_{0}-(m-1) \varepsilon^{2}-\frac{3}{4} \varepsilon^{2}}^{t_{0}-(m-1) \varepsilon^{2}-\frac{1}{4} \varepsilon^{2}} \sqrt{\tau} R\left(\gamma(\tau), t_{0}-\tau\right) d \tau \\
& >25 \widetilde{r}_{m}^{-2} \sqrt{\frac{1}{4} \varepsilon^{2}}\left(\frac{1}{2} \varepsilon^{2}\right) \\
& >5 \varepsilon
\end{aligned}
$$

since $0<\widetilde{r}_{m}<\varepsilon$. This contradicts (7.4.6).

Next we want to get a lower bound for Perelman's reduced volume of a ball around $\bar{x}$ of radius about $\widetilde{r}_{m}$ at some time slightly before $\bar{t}$.

Denote by $\theta_{1}=\frac{1}{16} \eta^{-1}$ and $\theta_{2}=\frac{1}{64} \eta^{-1}$, where $\eta$ is the universal positive constant in the gradient estimates (7.3.4). Since the solution satisfies the canonical neighborhood assumption on the time interval $\left[(m-1) \varepsilon^{2}, m \varepsilon^{2}\right)$, it follows from the gradient estimates (7.3.4) that

$$
R(x, t) \leq 400 \widetilde{r}_{m}^{-2}
$$

for those $(x, t) \in P\left(\bar{x}, \bar{t}, \theta_{1} \widetilde{r}_{m},-\theta_{2} \widetilde{r}_{m}^{2}\right) \triangleq\left\{\left(x^{\prime}, t^{\prime}\right) \mid x^{\prime} \in B_{t^{\prime}}\left(\bar{x}, \theta_{1} \widetilde{r}_{m}\right), t^{\prime} \in\left[\bar{t}-\theta_{2} \widetilde{r}_{m}^{2}, \bar{t}\right]\right\}$, for which the solution is defined. And since the scalar curvature at the points where the $\delta$-cutoff surgeries occur in the time interval $\left[(m-1) \varepsilon^{2}, m \varepsilon^{2}\right)$ is at least $(\widetilde{\delta})^{-2} \widetilde{r}_{m}^{-2}$, the solution is well-defined on the whole parabolic region $P\left(\bar{x}, \bar{t}, \theta_{1} \widetilde{r}_{m},-\theta_{2} \widetilde{r}_{m}^{2}\right)$ (i.e., this parabolic region is unaffected by surgery). Thus by combining (7.4.6) and (7.4.8), we know that the Li-Yau-Perelman distance from $\left(x_{0}, t_{0}\right)$ to each point of the ball $B_{\bar{t}-\theta_{2} \widetilde{r}_{m}^{2}}\left(\bar{x}, \theta_{1} \widetilde{r}_{m}\right)$ is uniformly bounded by some universal constant. Let us define Perelman's reduced volume of the ball $B_{\bar{t}-\theta_{2} \widetilde{r}_{m}^{2}}\left(\bar{x}, \theta_{1} \widetilde{r}_{m}\right)$, by

$$
\begin{aligned}
& \widetilde{V}_{t_{0}-\bar{t}+\theta_{2} \widetilde{r}_{m}^{2}}\left(B_{\bar{t}-\theta_{2} \widetilde{r}_{m}^{2}}\left(\bar{x}, \theta_{1} \widetilde{r}_{m}\right)\right) \\
& =\int_{B_{\bar{t}-\theta_{2} \hat{r}_{m}^{2}}\left(\bar{x}, \theta_{1} \widetilde{r}_{m}\right)}\left(4 \pi\left(t_{0}-\bar{t}+\theta_{2} \widetilde{r}_{m}^{2}\right)\right)^{-\frac{3}{2}} \\
& \quad \cdot \exp \left(-l\left(q, t_{0}-\bar{t}+\theta_{2} \widetilde{r}_{m}^{2}\right)\right) d V_{\bar{t}-\theta_{2} \widetilde{r}_{m}^{2}}(q),
\end{aligned}
$$


where $l(q, \tau)$ is the Li-Yau-Perelman distance from $\left(x_{0}, t_{0}\right)$. Hence by the $\kappa_{m^{-}}$ noncollapsing assumption on the time interval $\left[(m-1) \varepsilon^{2}, m \varepsilon^{2}\right)$, we conclude that Perelman's reduced volume of the ball $B_{\bar{t}-\theta_{2} \widetilde{r}_{m}^{2}}\left(\bar{x}, \theta_{1} \widetilde{r}_{m}\right)$ is bounded from below by a positive constant depending only on $\kappa_{m}$ and $\widetilde{r}_{m}$.

Finally we want to apply a local version of the monotonicity of Perelman's reduced volume to get a lower bound estimate for the volume of the ball $B_{t_{0}}\left(x_{0}, r_{0}\right)$.

We have seen that the Li-Yau-Perelman distance from $\left(x_{0}, t_{0}\right)$ to each point of the ball $B_{\bar{t}-\theta_{2} \widetilde{r}_{m}^{2}}\left(\bar{x}, \theta_{1} \widetilde{r}_{m}\right)$ is uniformly bounded by some universal constant. Now we can choose a sufficiently small (universal) positive constant $\bar{\varepsilon}_{0}$ such that when $0<\varepsilon \leq \bar{\varepsilon}_{0}$, by (7.4.5), all the points in the ball $B_{\bar{t}-\theta_{2} \widetilde{r}_{m}^{2}}\left(\bar{x}, \theta_{1} \widetilde{r}_{m}\right)$ can be connected to $\left(x_{0}, t_{0}\right)$ by shortest $\mathcal{L}$-geodesics, and all of these $\mathcal{L}$-geodesics are admissible (i.e., they stay in the region unaffected by surgery). The union of all shortest $\mathcal{L}$-geodesics from $\left(x_{0}, t_{0}\right)$ to the ball $B_{\bar{t}-\theta_{2} \widetilde{r}_{m}^{2}}\left(\bar{x}, \theta_{1} \widetilde{r}_{m}\right)$ defined by $C B_{\bar{t}-\theta_{2} \widetilde{r}_{m}^{2}}\left(\bar{x}, \theta_{1} \widetilde{r}_{m}\right)=\{(x, t) \mid(x, t)$ lies in a shortest $\mathcal{L}$-geodesic from $\left(x_{0}, t_{0}\right)$ to a point in $\left.B_{\bar{t}-\theta_{2} \widetilde{r}_{m}^{2}}\left(\bar{x}, \theta_{1} \widetilde{r}_{m}\right)\right\}$, forms a conelike subset in space-time with the vertex $\left(x_{0}, t_{0}\right)$. Denote $B(t)$ by the intersection of the cone-like subset $C B_{\bar{t}-\theta_{2} \widetilde{r}_{m}^{2}}\left(\bar{x}, \theta_{1} \widetilde{r}_{m}\right)$ with the time-slice at $t$. Perelman's reduced volume of the subset $B(t)$ is given by

$$
\widetilde{V}_{t_{0}-t}(B(t))=\int_{B(t)}\left(4 \pi\left(t_{0}-t\right)\right)^{-\frac{3}{2}} \exp \left(-l\left(q, t_{0}-t\right)\right) d V_{t}(q)
$$

Since the cone-like subset $C B_{\bar{t}-\theta_{2} \widetilde{r}_{m}^{2}}\left(\bar{x}, \theta_{1} \widetilde{r}_{m}\right)$ lies entirely in the region unaffected by surgery, we can apply Perelman's Jacobian comparison theorem (Theorem 3.2.7) to conclude that

$$
\begin{aligned}
\widetilde{V}_{t_{0}-t}(B(t)) & \geq \widetilde{V}_{t_{0}-\bar{t}+\theta_{2} \widetilde{r}_{m}^{2}}\left(B_{\bar{t}-\theta_{2} \widetilde{r}_{m}^{2}}\left(\bar{x}, \theta_{1} \widetilde{r}_{m}\right)\right) \\
& \geq c\left(\kappa_{m}, \widetilde{r}_{m}\right)
\end{aligned}
$$

for all $t \in\left[\bar{t}-\theta_{2} \widetilde{r}_{m}^{2}, t_{0}\right]$, where $c\left(\kappa_{m}, \widetilde{r}_{m}\right)$ is some positive constant depending only on $\kappa_{m}$ and $\widetilde{r}_{m}$.

Set $\xi=r_{0}^{-1} \operatorname{Vol}_{t_{0}}\left(B_{t_{0}}\left(x_{0}, r_{0}\right)\right)^{\frac{1}{3}}$. Our purpose is to give a positive lower bound for $\xi$. Without loss of generality, we may assume $\xi<\frac{1}{4}$, thus $0<\xi r_{0}^{2}<t_{0}-\bar{t}+\theta_{2} \widetilde{r}_{m}^{2}$. Denote by $\widetilde{B}\left(t_{0}-\xi r_{0}^{2}\right)$ the subset of the time-slice $\left\{t=t_{0}-\xi r_{0}^{2}\right\}$ of which every point can be connected to $\left(x_{0}, t_{0}\right)$ by an admissible shortest $\mathcal{L}$-geodesic. Clearly, $B\left(t_{0}-\xi r_{0}^{2}\right) \subset \widetilde{B}\left(t_{0}-\xi r_{0}^{2}\right)$. We now argue as in the proof of Theorem 3.3.2 to bound Perelman's reduced volume of $\widetilde{B}\left(t_{0}-\xi r_{0}^{2}\right)$ from above.

Since $r_{0} \geq \frac{1}{2 \eta} r$ and $\tilde{\delta}=\tilde{\delta}\left(r, \varepsilon, \widetilde{r}_{m}\right)$ sufficiently small, the whole region $P\left(x_{0}, t_{0}, r_{0}\right.$, $\left.-r_{0}^{2}\right)$ is unaffected by surgery. Then by exactly the same argument as in deriving (3.3.5), we see that there exists a universal positive constant $\xi_{0}$ such that when $0<$ $\xi \leq \xi_{0}$, there holds

$$
\mathcal{L} \exp _{\left\{|v| \leq \frac{1}{4} \xi^{-\frac{1}{2}}\right\}}\left(\xi r_{0}^{2}\right) \subset B_{t_{0}}\left(x_{0}, r_{0}\right)
$$


Perelman's reduced volume of $\widetilde{B}\left(t_{0}-\xi r_{0}^{2}\right)$ is given by

$$
\begin{aligned}
& \widetilde{V}_{\xi r_{0}^{2}}\left(\widetilde{B}\left(t_{0}-\xi r_{0}^{2}\right)\right) \\
& =\int_{\widetilde{B}\left(t_{0}-\xi r_{0}^{2}\right)}\left(4 \pi \xi r_{0}^{2}\right)^{-\frac{3}{2}} \exp \left(-l\left(q, \xi r_{0}^{2}\right)\right) d V_{t_{0}-\xi r_{0}^{2}}(q) \\
& =\int_{\widetilde{B}\left(t_{0}-\xi r_{0}^{2}\right) \cap \mathcal{L} \exp }\left(4 \pi \xi r_{0}^{2}\right)^{-\frac{3}{2}} \exp \left(-l\left(q, \xi r_{0}^{2}\right)\right) d V_{t_{0}-\xi r_{0}^{2}}(q) \xi^{\left.-\frac{1}{2}\right\}}\left(\xi r_{0}^{2}\right) \\
& \quad+\int_{\widetilde{B}\left(t_{0}-\xi r_{0}^{2}\right) \backslash \mathcal{L} \exp _{\left\{|v| \leq \frac{1}{4} \xi^{\left.-\frac{1}{2}\right\}}\right.}\left(\xi r_{0}^{2}\right)}\left(4 \pi \xi r_{0}^{2}\right)^{-\frac{3}{2}} \exp \left(-l\left(q, \xi r_{0}^{2}\right)\right) d V_{t_{0}-\xi r_{0}^{2}}(q) .
\end{aligned}
$$

The first term on the RHS of (7.4.11) can be estimated by

$$
\begin{aligned}
& \int_{\widetilde{B}\left(t_{0}-\xi r_{0}^{2}\right) \cap \mathcal{L} \exp }\left(4 \pi \xi r_{0}^{2}\right)^{-\frac{3}{2}} \exp \left(-l\left(q, \xi r_{0}^{2}\right)\right) d V_{t_{0}-\xi r_{0}^{2}}(q) \\
& \leq e^{C \xi}(4 \pi)^{-\frac{3}{2}} \cdot \xi^{\frac{3}{2}}\left(\xi r_{0}^{2}\right)
\end{aligned}
$$

for some universal constant $C$, as in deriving (3.3.7). While as in deriving (3.3.8), the second term on the RHS of (7.4.11) can be estimated by

$$
\begin{aligned}
& \int_{\widetilde{B}\left(t_{0}-\xi r_{0}^{2}\right) \backslash \mathcal{L} \exp }\left\{\begin{array}{l}
\left\{|v| \leq \frac{1}{4} \xi^{-\frac{1}{2}}\right\} \\
\leq
\end{array}\left(\xi r_{0}^{2}\right)\right. \\
& \left.\leq\left.\int_{\left\{|v|>\frac{1}{4} \xi^{-\frac{1}{2}}\right\}}(4 \pi \tau)^{-\frac{3}{2}} \exp (-l(\tau)) \mathcal{J}(\tau)\right|_{\tau=0} ^{2}\right)^{-\frac{3}{2}} \exp \left(-l\left(q, \xi r_{0}^{2}\right)\right) d V_{t_{0}-\xi r_{0}^{2}}(q) \\
& =(4 \pi)^{-\frac{3}{2}} \int_{\left\{|v|>\frac{1}{4} \xi^{-\frac{1}{2}}\right\}} \exp \left(-|v|^{2}\right) d v
\end{aligned}
$$

where we have used Perelman's Jacobian comparison theorem (Theorem 3.2.7) in the first inequality. Hence the combination of (7.4.9), (7.4.11), (7.4.12) and (7.4.13) bounds $\xi$ from below by a positive constant depending only on $\kappa_{m}$ and $\widetilde{r}_{m}$. Therefore we have completed the proof of the lemma.

Now we can prove the proposition.

Proof of Proposition 7.4.1. The proof of the proposition is by induction: having constructed our sequences for $1 \leq j \leq m$, we make one more step, defining $\widetilde{r}_{m+1}$, $\kappa_{m+1}, \widetilde{\delta}_{m+1}$, and redefining $\widetilde{\delta}_{m}=\widetilde{\delta}_{m+1}$. In view of the previous lemma, we only need to define $\widetilde{r}_{m+1}$ and $\widetilde{\delta}_{m+1}$.

In Theorem 7.1.1 we have obtained the canonical neighborhood structure for smooth solutions. When adapting the arguments in the proof of Theorem 7.1.1 to the present surgical solutions, we will encounter the new difficulty of how to take a limit for the surgically modified solutions. The idea to overcome the difficulty consists of two parts. The first part, due to Perelman [104], is to choose $\widetilde{\delta}_{m}$ and $\widetilde{\delta}_{m+1}$ small enough to push the surgical regions to infinity in space. (This is the reason why we need to redefine $\widetilde{\delta}_{m}=\widetilde{\delta}_{m+1}$.) The second part is to show that solutions are smooth on some small, but uniform, time intervals (on compact subsets) so that we can apply Hamilton's compactness theorem, since we only have curvature bounds; 
otherwise Shi's interior derivative estimate may not be applicable. In fact, the second part is more crucial. That is just concerned with the question of whether the surgery times accumulate or not. Our argument will use the canonical neighborhood characterization of the standard solution in Lemma 7.3.5.

We now start to prove the proposition by contradiction. Suppose for sequence of positive numbers $r^{\alpha}$ and $\widetilde{\delta}^{\alpha \beta}$, satisfying $r^{\alpha} \rightarrow 0$ as $\alpha \rightarrow \infty$ and $\widetilde{\delta}^{\alpha \beta} \leq \frac{1}{\alpha \cdot \beta}(\rightarrow 0)$, there exist sequences of solutions $g_{i j}^{\alpha \beta}$ to the Ricci flow with surgery, where each of them has only a finite number of cutoff surgeries and has a compact orientable normalized three-manifold as initial data, so that the following two assertions hold:

(i) each $\delta$-cutoff at a time $t \in\left[(m-1) \varepsilon^{2},(m+1) \varepsilon^{2}\right]$ satisfies $\delta \leq \widetilde{\delta}^{\alpha \beta}$; and

(ii) the solutions satisfy the statement of the proposition on $\left[0, m \varepsilon^{2}\right]$, but violate the canonical neighborhood assumption (with accuracy $\varepsilon$ ) with $r=r^{\alpha}$ on $\left[m \varepsilon^{2},(m+1) \varepsilon^{2}\right]$.

For each solution $g_{i j}^{\alpha \beta}$, we choose $\bar{t}$ (depending on $\alpha$ and $\beta$ ) to be the nearly first time for which the canonical neighborhood assumption (with accuracy $\varepsilon$ ) is violated. More precisely, we choose $\bar{t} \in\left[m \varepsilon^{2},(m+1) \varepsilon^{2}\right]$ so that the canonical neighborhood assumption with $r=r^{\alpha}$ and with accuracy parameter $\varepsilon$ is violated at some $(\bar{x}, \bar{t})$, however the canonical neighborhood assumption with accuracy parameter $2 \varepsilon$ holds on $t \in\left[m \varepsilon^{2}, \bar{t}\right]$. After passing to subsequences, we may assume each $\widetilde{\delta}^{\alpha \beta}$ is less than the $\widetilde{\delta}$ in Lemma 7.4.2 with $r=r^{\alpha}$ when $\alpha$ is fixed. Then by Lemma 7.4.2 we have uniform $\kappa$-noncollapsing on all scales less than $\varepsilon$ on $[0, \bar{t}]$ with some $\kappa>0$ independent of $\alpha, \beta$.

Slightly abusing notation, we will often drop the indices $\alpha$ and $\beta$.

Let $\widetilde{g}_{i j}^{\alpha \beta}$ be the rescaled solutions around $(\bar{x}, \bar{t})$ with factors $R(\bar{x}, \bar{t})\left(\geq r^{-2} \rightarrow+\infty\right)$ and shift the times $\bar{t}$ to zero. We hope to take a limit of the rescaled solutions for subsequences of $\alpha, \beta \rightarrow \infty$ and show the limit is an orientable ancient $\kappa$-solution, which will give the desired contradiction. We divide our arguments into the following six steps.

Step 1. Let $(y, \hat{t})$ be a point on the rescaled solution $\widetilde{g}_{i j}^{\alpha \beta}$ with $\widetilde{R}(y, \hat{t}) \leq A$ (for some $A \geq 1)$ and $\hat{t} \in\left[-\left(\bar{t}-(m-1) \varepsilon^{2}\right) R(\bar{x}, \bar{t}), 0\right]$. Then we have estimate

$$
\widetilde{R}(x, t) \leq 10 A
$$

for those $(x, t)$ in the parabolic neighborhood $P\left(y, \hat{t}, \frac{1}{2} \eta^{-1} A^{-\frac{1}{2}},-\frac{1}{8} \eta^{-1} A^{-1}\right) \triangleq$ $\left\{\left(x^{\prime}, t^{\prime}\right) \mid x^{\prime} \in \widetilde{B}_{t^{\prime}}\left(y, \frac{1}{2} \eta^{-1} A^{-\frac{1}{2}}\right), t^{\prime} \in\left[\hat{t}-\frac{1}{8} \eta^{-1} A^{-1}, \hat{t}\right]\right\}$, for which the rescaled solution is defined.

Indeed, as in the first step of the proof of Theorem 7.1.1, this follows directly from the gradient estimates (7.3.4) in the canonical neighborhood assumption with parameter $2 \varepsilon$.

Step 2. In this step, we will prove three time extension results.

Assertion 1. For arbitrarily fixed $\alpha, 0<A<+\infty, 1 \leq C<+\infty$ and $0 \leq B<\frac{1}{2} \varepsilon^{2}\left(r^{\alpha}\right)^{-2}-\frac{1}{8} \eta^{-1} C^{-1}$, there is a $\beta_{0}=\beta_{0}(\varepsilon, A, B, C$ ) (independent of $\alpha$ ) such that if $\beta \geq \beta_{0}$ and the rescaled solution $\widetilde{g}_{i j}^{\alpha \beta}$ on the ball $\widetilde{B}_{0}(\bar{x}, A)$ is defined on a time interval $[-b, 0]$ with $0 \leq b \leq B$ and the scalar curvature satisfies

$$
\widetilde{R}(x, t) \leq C, \quad \text { on } \widetilde{B}_{0}(\bar{x}, A) \times[-b, 0],
$$

then the rescaled solution $\widetilde{g}_{i j}^{\alpha \beta}$ on the ball $\widetilde{B}_{0}(\bar{x}, A)$ is also defined on the extended time interval $\left[-b-\frac{1}{8} \eta^{-1} C^{-1}, 0\right]$. 
Before giving the proof, we make a simple observation: once a space point in the Ricci flow with surgery is removed by surgery at some time, then it never appears for later time; if a space point at some time $t$ cannot be defined before the time $t$, then either the point lies in a gluing cap of the surgery at time $t$ or the time $t$ is the initial time of the Ricci flow.

Proof of Assertion 1. Firstly we claim that there exists $\beta_{0}=\beta_{0}(\varepsilon, A, B, C)$ such that when $\beta \geq \beta_{0}$, the rescaled solution $\widetilde{g}_{i j}^{\alpha \beta}$ on the ball $\widetilde{B}_{0}(\bar{x}, A)$ can be defined before the time $-b$ (i.e., there are no surgeries interfering in $\widetilde{B}_{0}(\bar{x}, A) \times\left[-b-\epsilon^{\prime},-b\right]$ for some $\left.\epsilon^{\prime}>0\right)$.

We argue by contradiction. Suppose not, then there is some point $\tilde{x} \in \widetilde{B}_{0}(\bar{x}, A)$ such that the rescaled solution $\widetilde{g}_{i j}^{\alpha \beta}$ at $\tilde{x}$ cannot be defined before the time $-b$. By the above observation, there is a surgery at the time $-b$ such that the point $\tilde{x}$ lies in the instant gluing cap.

Let $\tilde{h}\left(=R(\bar{x}, \bar{t})^{\frac{1}{2}} h\right)$ be the cut-off radius at the time $-b$ for the rescaled solution. Clearly, there is a universal constant $D$ such that $D^{-1} \tilde{h} \leq \widetilde{R}(\tilde{x},-b)^{-\frac{1}{2}} \leq D \tilde{h}$.

By Lemma 7.3.4 and looking at the rescaled solution at the time $-b$, the gluing cap and the adjacent $\delta$-neck, of radius $\tilde{h}$, constitute a $\left(\widetilde{\delta}^{\alpha \beta}\right)^{\frac{1}{2}}$-cap $\mathcal{K}$. For any fixed small positive constant $\delta^{\prime}$ (much smaller than $\varepsilon$ ), we see that

$$
\widetilde{B}_{(-b)}\left(\tilde{x},\left(\delta^{\prime}\right)^{-1} \widetilde{R}(\tilde{x},-b)^{-\frac{1}{2}}\right) \subset \mathcal{K}
$$

when $\beta$ large enough. We first verify the following

Claim 1. For any small constants $0<\tilde{\theta}<1, \delta^{\prime}>0$, there exists a $\beta\left(\delta^{\prime}, \varepsilon, \tilde{\theta}\right)>0$ such that when $\beta \geq \beta\left(\delta^{\prime}, \varepsilon, \tilde{\theta}\right)$, we have

(i) the rescaled solution $\widetilde{g}_{i j}^{\alpha \beta}$ over $\widetilde{B}_{(-b)}\left(\tilde{x},\left(\delta^{\prime}\right)^{-1} \tilde{h}\right)$ is defined on the time interval $[-b, 0] \cap\left[-b,-b+(1-\tilde{\theta}) \tilde{h}^{2}\right] ;$

(ii) the ball $\widetilde{B}_{(-b)}\left(\tilde{x},\left(\delta^{\prime}\right)^{-1} \tilde{h}\right)$ in the $\left(\widetilde{\delta}^{\alpha \beta}\right)^{\frac{1}{2}}$-cap $\mathcal{K}$ evolved by the Ricci flow on the time interval $[-b, 0] \cap\left[-b,-b+(1-\tilde{\theta}) \tilde{h}^{2}\right]$ is, after scaling with factor $\tilde{h}^{-2}$, $\delta^{\prime}$-close (in the $C^{\left[\delta^{\prime-1}\right]}$ topology) to the corresponding subset of the standard solution.

This claim essentially follows from Lemma 7.3.6. Indeed, suppose there is a surgery at some time $\tilde{\tilde{t}} \in[-b, 0] \cap\left(-b,-b+(1-\tilde{\theta}) \tilde{h}^{2}\right]$ which removes some point $\tilde{\tilde{x}} \in \widetilde{B}_{(-b)}\left(\tilde{x},\left(\delta^{\prime}\right)^{-1} \tilde{h}\right)$. We assume $\tilde{\tilde{t}} \in(-b, 0]$ is the first time with that property.

Then by Lemma 7.3.6, there is a $\bar{\delta}=\bar{\delta}\left(\delta^{\prime}, \varepsilon, \tilde{\theta}\right)$ such that if $\widetilde{\delta}^{\alpha \beta}<\bar{\delta}$, then the ball $\widetilde{B}_{(-b)}\left(\tilde{x},\left(\delta^{\prime}\right)^{-1} \tilde{h}\right)$ in the $\left(\widetilde{\delta}^{\alpha \beta}\right)^{\frac{1}{2}}$-cap $\mathcal{K}$ evolved by the Ricci flow on the time interval $[-b, \tilde{\tilde{t}})$ is, after scaling with factor $\tilde{h}^{-2}, \delta^{\prime}$-close to the corresponding subset of the standard solution. Note that the metrics for times in $[-b, \tilde{\tilde{t}})$ on $\widetilde{B}_{(-b)}\left(\tilde{x},\left(\delta^{\prime}\right)^{-1} \tilde{h}\right)$ are equivalent. By Lemma 7.3.6, the solution on $\widetilde{B}_{(-b)}\left(\tilde{x},\left(\delta^{\prime}\right)^{-1} \tilde{h}\right)$ keeps looking like a cap for $t \in[-b, \tilde{\tilde{t}})$. On the other hand, by the definition, the surgery is always done along the middle two-sphere of a $\delta$-neck with $\delta<\widetilde{\delta}^{\alpha \beta}$. Then for $\beta$ large, all the points in $\widetilde{B}_{(-b)}\left(\tilde{x},\left(\delta^{\prime}\right)^{-1} \tilde{h}\right)$ are removed (as a part of a capped horn) at the time $\tilde{\tilde{t}}$. But $\tilde{x}$ (near the tip of the cap) exists past the time $\tilde{\tilde{t}}$. This is a contradiction. Hence we have proved that $\widetilde{B}_{(-b)}\left(\tilde{x},\left(\delta^{\prime}\right)^{-1} \tilde{h}\right)$ is defined on the time interval $[-b, 0] \cap\left[-b,-b+(1-\tilde{\theta}) \tilde{h}^{2}\right]$. 
The $\delta^{\prime}$-closeness of the solution on $\widetilde{B}_{(-b)}\left(\tilde{x},\left(\delta^{\prime}\right)^{-1} h\right) \times\left([-b, 0] \cap\left[-b,-b+(1-\tilde{\theta}) \tilde{h}^{2}\right]\right)$ with the corresponding subset of the standard solution follows from Lemma 7.3.6. Then we have proved Claim 1.

We next verify the following

Claim 2. There is $\tilde{\theta}=\tilde{\theta}(C B), 0<\tilde{\theta}<1$, such that $b \leq(1-\tilde{\theta}) \tilde{h}^{2}$ when $\beta$ large.

Note from Proposition 7.3.3, there is a universal constant $D^{\prime}>0$ such that the standard solution satisfies the following curvature estimate

$$
R(y, s) \geq \frac{2 D^{\prime}}{1-s}
$$

We choose $\tilde{\theta}=D^{\prime} / 2\left(D^{\prime}+C B\right)$. Then for $\beta$ large enough, the rescaled solution satisfies

$$
\widetilde{R}(x, t) \geq \frac{D^{\prime}}{1-(t+b) \tilde{h}^{-2}} \tilde{h}^{-2}
$$

on $\widetilde{B}_{(-b)}\left(\tilde{x},\left(\delta^{\prime}\right)^{-1} \tilde{h}\right) \times\left([-b, 0] \cap\left[-b,-b+(1-\tilde{\theta}) \tilde{h}^{2}\right]\right)$.

Suppose $b \geq(1-\tilde{\theta}) \tilde{h}^{2}$. Then by combining with the assumption $\widetilde{R}(\tilde{x}, t) \leq C$ for $t=(1-\tilde{\theta}) \tilde{h}^{2}-b$, we have

$$
C \geq \frac{D^{\prime}}{1-(t+b) \tilde{h}^{-2}} \tilde{h}^{-2}
$$

and then

$$
1 \geq(1-\tilde{\theta})\left(1+\frac{D^{\prime}}{C B}\right)
$$

This is a contradiction. Hence we have proved Claim 2.

The combination of the above two claims shows that there is a positive constant $0<\tilde{\theta}=\tilde{\theta}(C B)<1$ such that for any small $\delta^{\prime}>0$, there is a positive $\beta\left(\delta^{\prime}, \varepsilon, \tilde{\theta}\right)$ such that when $\beta \geq \beta\left(\delta^{\prime}, \varepsilon, \tilde{\theta}\right)$, we have $b \leq(1-\tilde{\theta}) \tilde{h}^{2}$ and the rescaled solution in the ball $\widetilde{B}_{(-b)}\left(\tilde{x},\left(\delta^{\prime}\right)^{-1} \tilde{h}\right)$ on the time interval $[-b, 0]$ is, after scaling with factor $\tilde{h}^{-2}, \delta^{\prime}$-close ( in the $C^{\left[\left(\delta^{\prime}\right)^{-1}\right]}$ topology) to the corresponding subset of the standard solution.

By (7.4.15) and the assumption $\widetilde{R} \leq C$ on $\widetilde{B}_{0}(\bar{x}, A) \times[-b, 0]$, we know that the cut-off radius $\tilde{h}$ at the time $-b$ for the rescaled solution satisfies

$$
\tilde{h} \geq \sqrt{\frac{D^{\prime}}{C}} .
$$

Let $\delta^{\prime}>0$ be much smaller than $\varepsilon$ and $\min \left\{A^{-1}, A\right\}$. Since $\tilde{d}_{0}(\tilde{x}, \bar{x}) \leq A$, it follows that there is constant $C(\tilde{\theta})$ depending only on $\tilde{\theta}$ such that $\tilde{d}_{(-b)}(\tilde{x}, \bar{x}) \leq C(\tilde{\theta}) A \ll$ $\left(\delta^{\prime}\right)^{-1} \tilde{h}$. We now apply Lemma 7.3 .5 with the accuracy parameter $\varepsilon / 2$. Let $C(\varepsilon / 2)$ be the positive constant in Lemma 7.3.5. Without loss of generality, we may assume the positive constant $C_{1}(\varepsilon)$ in the canonical neighborhood assumption is larger than $4 C(\varepsilon / 2)$. When $\delta^{\prime}(>0)$ is much smaller than $\varepsilon$ and $\min \left\{A^{-1}, A\right\}$, the point $\bar{x}$ at the time $\bar{t}$ has a neighborhood which is either a $\frac{3}{4} \varepsilon$-cap or a $\frac{3}{4} \varepsilon$-neck.

Since the canonical neighborhood assumption with accuracy parameter $\varepsilon$ is violated at $(\bar{x}, \bar{t})$, the neighborhood of the point $\bar{x}$ at the new time zero for the rescaled 
solution must be a $\frac{3}{4} \varepsilon$-neck. By Lemma 7.3.5 (b), we know the neighborhood is the slice at the time zero of the parabolic neighborhood

$$
P\left(\bar{x}, 0, \frac{4}{3} \varepsilon^{-1} \widetilde{R}(\bar{x}, 0)^{-\frac{1}{2}},-\min \left\{\widetilde{R}(\bar{x}, 0)^{-1}, b\right\}\right)
$$

(with $\widetilde{R}(\bar{x}, 0)=1$ ) which is $\frac{3}{4} \varepsilon$-close (in the $C^{\left[\frac{4}{3} \varepsilon^{-1}\right]}$ topology) to the corresponding subset of the evolving standard cylinder $\mathbb{S}^{2} \times \mathbb{R}$ over the time interval $[-\min \{b, 1\}, 0]$ with scalar curvature 1 at the time zero. If $b \geq 1$, the $\frac{3}{4} \varepsilon$-neck is strong, which is a contradiction. While if $b<1$, the $\frac{3}{4} \varepsilon$-neck at time $-b$ is contained in the union of the gluing cap and the adjacent $\delta$-neck where the $\delta$-cutoff surgery took place. Since $\varepsilon$ is small (say $\varepsilon<1 / 100$ ), it is clear that the point $\bar{x}$ at time $-b$ is the center of an $\varepsilon$-neck which is entirely contained in the adjacent $\delta$-neck. By the proof of Lemma 7.3.2, the adjacent $\delta$-neck approximates an ancient $\kappa$-solution. This implies the point $\bar{x}$ at the time $\bar{t}$ has a strong $\varepsilon$-neck, which is also a contradiction.

Hence we have proved that there exists $\beta_{0}=\beta_{0}(\varepsilon, A, B, C)$ such that when $\beta \geq \beta_{0}$, the rescaled solution on the ball $\widetilde{B}_{0}(\bar{x}, A)$ can be defined before the time $-b$.

Let $\left[t_{A}^{\alpha \beta}, 0\right] \supset[-b, 0]$ be the largest time interval so that the rescaled solution $\widetilde{g}_{i j}^{\alpha \beta}$ can be defined on $\widetilde{B}_{0}(\bar{x}, A) \times\left[t_{A}^{\alpha \beta}, 0\right]$. We finally claim that $t_{A}^{\alpha \beta} \leq-b-\frac{1}{8} \eta^{-1} C^{-1}$ for $\beta$ large enough.

Indeed, suppose not, by the gradient estimates as in Step 1, we have the curvature estimate

$$
\widetilde{R}(x, t) \leq 10 C
$$

on $\widetilde{B}_{0}(\bar{x}, A) \times\left[t_{A}^{\alpha \beta},-b\right]$. Hence we have the curvature estimate

$$
\widetilde{R}(x, t) \leq 10 C
$$

on $\widetilde{B}_{0}(\bar{x}, A) \times\left[t_{A}^{\alpha \beta}, 0\right]$. By the above argument there is a $\beta_{0}=\beta_{0}(\varepsilon, A, B+$ $\left.\frac{1}{8} \eta^{-1} C^{-1}, 10 C\right)$ such that for $\beta \geq \beta_{0}$, the solution in the ball $\widetilde{B}_{0}(\bar{x}, A)$ can be defined before the time $t_{A}^{\alpha \beta}$. This is a contradiction.

Therefore we have proved Assertion 1.

Assertion 2. For arbitrarily fixed $\alpha, 0<A<+\infty, 1 \leq C<+\infty$ and $0<B<\frac{1}{2} \varepsilon^{2}\left(r^{\alpha}\right)^{-2}-\frac{1}{50} \eta^{-1}$, there is a $\beta_{0}=\beta_{0}(\varepsilon, A, B, C)$ (independent of $\alpha$ ) such that if $\beta \geq \beta_{0}$ and the rescaled solution $\widetilde{g}_{i j}^{\alpha \beta}$ on the ball $\widetilde{B}_{0}(\bar{x}, A)$ is defined on a time interval $\left[-b+\epsilon^{\prime}, 0\right]$ with $0<b \leq B$ and $0<\epsilon^{\prime}<\frac{1}{50} \eta^{-1}$ and the scalar curvature satisfies

$$
\widetilde{R}(x, t) \leq C \quad \text { on } \quad \widetilde{B}_{0}(\bar{x}, A) \times\left[-b+\epsilon^{\prime}, 0\right],
$$

and there is a point $y \in \widetilde{B}_{0}(\bar{x}, A)$ such that $\widetilde{R}\left(y,-b+\epsilon^{\prime}\right) \leq \frac{3}{2}$, then the rescaled solution $\widetilde{g}_{i j}^{\alpha \beta}$ at $y$ is also defined on the extended time interval $\left[-b-\frac{1}{50} \eta^{-1}, 0\right]$ and satisfies the estimate

$$
\widetilde{R}(y, t) \leq 15
$$

for $t \in\left[-b-\frac{1}{50} \eta^{-1},-b+\epsilon^{\prime}\right]$.

Proof of Assertion 2. We imitate the proof of Assertion 1. If the rescaled solution $\widetilde{g}_{i j}^{\alpha \beta}$ at $y$ cannot be defined for some time in $\left[-b-\frac{1}{50} \eta^{-1},-b+\epsilon^{\prime}\right)$, then there is a 
surgery at some time $\tilde{\tilde{t}} \in\left[-b-\frac{1}{50} \eta^{-1},-b+\epsilon^{\prime}\right]$ such that $y$ lies in the instant gluing cap. Let $\tilde{h}\left(=R(\bar{x}, \bar{t})^{\frac{1}{2}} h\right)$ be the cutoff radius at the time $\tilde{\tilde{t}}$ for the rescaled solution. Clearly, there is a universal constant $D>1$ such that $D^{-1} \tilde{h} \leq \widetilde{R}(y, \tilde{\tilde{t}})^{-\frac{1}{2}} \leq D \tilde{h}$. By the gradient estimates as in Step 1, the cutoff radius satisfies

$$
\tilde{h} \geq D^{-1} 15^{-\frac{1}{2}} .
$$

As in Claim 1 (i) in the proof of Assertion 1, for any small constants $0<\tilde{\theta}<\frac{1}{2}$, $\delta^{\prime}>0$, there exists a $\beta\left(\delta^{\prime}, \varepsilon, \tilde{\theta}\right)>0$ such that for $\beta \geq \beta\left(\delta^{\prime}, \varepsilon, \tilde{\theta}\right)$, there is no surgery interfering in $\widetilde{B}_{\tilde{t}}\left(y,\left(\delta^{\prime}\right)^{-1} \tilde{h}\right) \times\left(\left[\tilde{\tilde{t}},(1-\tilde{\theta}) \tilde{h}^{2}+\tilde{\tilde{t}}\right] \cap(\tilde{\tilde{t}}, 0]\right)$. Without loss of generality, we may assume that the universal constant $\eta$ is much larger than $D$. Then we have $(1-\tilde{\theta}) \tilde{h}^{2}+\tilde{\tilde{t}}>-b+\frac{1}{50} \eta^{-1}$. As in Claim 2 in the proof of Assertion 1, we can use the curvature bound assumption to choose $\tilde{\theta}=\tilde{\theta}(B, C)$ such that $(1-\tilde{\theta}) \tilde{h}^{2}+\tilde{\tilde{t}} \geq 0$; otherwise

$$
C \geq \frac{D^{\prime}}{\tilde{\theta} \tilde{h}^{2}}
$$

for some universal constant $D^{\prime}>1$, and

$$
|\tilde{\tilde{t}}+b| \leq \frac{1}{50} \eta^{-1}
$$

which implies

$$
1 \geq(1-\tilde{\theta})\left(1+\frac{D^{\prime}}{C\left(B+\frac{1}{50} \eta^{-1}\right)}\right) .
$$

This is a contradiction if we choose $\tilde{\theta}=D^{\prime} / 2\left(D^{\prime}+C\left(B+\frac{1}{50} \eta^{-1}\right)\right)$.

So there is a positive constant $0<\tilde{\theta}=\tilde{\theta}(B, C)<1$ such that for any $\delta^{\prime}>0$, there is a positive $\beta\left(\delta^{\prime}, \varepsilon, \tilde{\theta}\right)$ such that when $\beta \geq \beta\left(\delta^{\prime}, \varepsilon, \tilde{\theta}\right)$, we have $-\tilde{\tilde{t}} \leq(1-\tilde{\theta}) \tilde{h}^{2}$ and the solution in the ball $\widetilde{B}_{\tilde{t}}\left(\tilde{x},\left(\delta^{\prime}\right)^{-1} \tilde{h}\right)$ on the time interval $[\tilde{\tilde{t}}, 0]$ is, after scaling with factor $\tilde{h}^{-2}, \delta^{\prime}$-close (in the $C^{\left[\delta^{\prime-1}\right]}$ topology) to the corresponding subset of the standard solution.

Then exactly as in the proof of Assertion 1, by using the canonical neighborhood structure of the standard solution in Lemma 7.3.5, this gives the desired contradiction with the hypothesis that the canonical neighborhood assumption with accuracy parameter $\varepsilon$ is violated at $(\bar{x}, \bar{t})$, for $\beta$ sufficiently large.

The curvature estimate at the point $y$ follows from Step 1. Therefore the proof of Assertion 2 is complete.

Note that the standard solution satisfies $R\left(x_{1}, t\right) \leq D^{\prime \prime} R\left(x_{2}, t\right)$ for any $t \in\left[0, \frac{1}{2}\right]$ and any two points $x_{1}, x_{2}$, where $D^{\prime \prime} \geq 1$ is a universal constant.

Assertion 3. For arbitrarily fixed $\alpha, 0<A<+\infty, 1 \leq C<+\infty$, there is a $\beta_{0}=\beta_{0}\left(\varepsilon, A C^{\frac{1}{2}}\right)$ such that if any point $\left(y_{0}, t_{0}\right)$ with $0 \leq-t_{0}<\frac{1}{2} \varepsilon^{2}\left(r^{\alpha}\right)^{-2}-\frac{1}{8} \eta^{-1} C^{-1}$ of the rescaled solution $\widetilde{g}_{i j}^{\alpha \beta}$ for $\beta \geq \beta_{0}$ satisfies $\widetilde{R}\left(y_{0}, t_{0}\right) \leq C$, then either the rescaled solution at $y_{0}$ can be defined at least on $\left[t_{0}-\frac{1}{16} \eta^{-1} C^{-1}, t_{0}\right]$ and the rescaled scalar curvature satisfies

$$
\widetilde{R}\left(y_{0}, t\right) \leq 10 C \text { for } t \in\left[t_{0}-\frac{1}{16} \eta^{-1} C^{-1}, t_{0}\right],
$$


or we have

$$
\widetilde{R}\left(x_{1}, t_{0}\right) \leq 2 D^{\prime \prime} \widetilde{R}\left(x_{2}, t_{0}\right)
$$

for any two points $x_{1}, x_{2} \in \widetilde{B}_{t_{0}}\left(y_{0}, A\right)$, where $D^{\prime \prime}$ is the above universal constant.

Proof of Assertion 3. Suppose the rescaled solution $\widetilde{g}_{i j}^{\alpha \beta}$ at $y_{0}$ cannot be defined for some $t \in\left[t_{0}-\frac{1}{16} \eta^{-1} C^{-1}, t_{0}\right)$; then there is a surgery at some time $\tilde{t} \in\left[t_{0}-\right.$ $\left.\frac{1}{16} \eta^{-1} C^{-1}, t_{0}\right]$ such that $y_{0}$ lies in the instant gluing cap. Let $\tilde{h}\left(=R(\bar{x}, \bar{t})^{\frac{1}{2}} h\right)$ be the cutoff radius at the time $\tilde{t}$ for the rescaled solution $\widetilde{g}_{i j}^{\alpha \beta}$. By the gradient estimates as in Step 1, the cutoff radius satisfies

$$
\tilde{h} \geq D^{-1} 10^{-\frac{1}{2}} C^{-\frac{1}{2}},
$$

where $D$ is the universal constant in the proof of the Assertion 1. Since we assume $\eta$ is suitably larger than $D$ as before, we have $\frac{1}{2} \tilde{h}^{2}+\tilde{t}>t_{0}$. As in Claim 1 (ii) in the proof of Assertion 1, for arbitrarily small $\delta^{\prime}>0$, we know that for $\beta$ large enough the rescaled solution on $\widetilde{B}_{\tilde{t}}\left(y_{0},\left(\delta^{\prime}\right)^{-1} \tilde{h}\right) \times\left[\tilde{t}, t_{0}\right]$ is, after scaling with factor $\tilde{h}^{-2}, \delta^{\prime}$-close (in the $C^{\left[\left(\delta^{\prime}\right)^{-1}\right]}$ topology) to the corresponding subset of the standard solution. Since $\left(\delta^{\prime}\right)^{-1} \tilde{h} \gg A$ for $\beta$ large enough, Assertion 3 follows from the curvature estimate of standard solution in the time interval $\left[0, \frac{1}{2}\right]$.

Step 3. For any subsequence $\left(\alpha_{k}, \beta_{k}\right)$ of $(\alpha, \beta)$ with $r^{\alpha_{k}} \rightarrow 0$ and $\delta^{\alpha_{k} \beta_{k}} \rightarrow 0$ as $k \rightarrow \infty$, we next argue as in the second step of the proof of Theorem 7.1.1 to show that the curvatures of the rescaled solutions $\tilde{g}_{i j}^{\alpha_{k} \beta_{k}}$ at the new times zero (after shifting) stay uniformly bounded at bounded distances from $\bar{x}$ for all sufficiently large $k$. More precisely, we will prove the following assertion:

Assertion 4. Given any subsequence of the rescaled solutions $\tilde{g}_{i j}^{\alpha_{k} \beta_{k}}$ with $r^{\alpha_{k}} \rightarrow$ 0 and $\delta^{\alpha_{k} \beta_{k}} \rightarrow 0$ as $k \rightarrow \infty$, then for any $L>0$, there are constants $C(L)>0$ and $k(L)$ such that the rescaled solutions $\tilde{g}_{i j}^{\alpha_{k} \beta_{k}}$ satisfy

(i) $\tilde{R}(x, 0) \leq C(L)$ for all points $x$ with $\tilde{d}_{0}(x, \bar{x}) \leq L$ and all $k \geq 1$;

(ii) the rescaled solutions over the ball $\tilde{B}_{0}(\bar{x}, L)$ are defined at least on the time interval $\left[-\frac{1}{16} \eta^{-1} C(L)^{-1}, 0\right]$ for all $k \geq k(L)$.

Proof of Assertion 4. For each $\rho>0$, set

$$
M(\rho)=\sup \left\{\tilde{R}(x, 0) \mid k \geq 1 \text { and } \tilde{d}_{0}(x, \bar{x}) \leq \rho \text { in the rescaled solutions } \tilde{g}_{i j}^{\alpha_{k} \beta_{k}}\right\}
$$

and

$$
\rho_{0}=\sup \{\rho>0 \mid M(\rho)<+\infty\} .
$$

Note that the estimate (7.4.14) implies that $\rho_{0}>0$. For (i), it suffices to prove $\rho_{0}=+\infty$.

We argue by contradiction. Suppose $\rho_{0}<+\infty$. Then there is a sequence of points $y$ in the rescaled solutions $\tilde{g}_{i j}^{\alpha_{k} \beta_{k}}$ with $\tilde{d}_{0}(\bar{x}, y) \rightarrow \rho_{0}<+\infty$ and $\tilde{R}(y, 0) \rightarrow+\infty$. Denote by $\gamma$ a minimizing geodesic segment from $\bar{x}$ to $y$ and denote by $\tilde{B}_{0}\left(\bar{x}, \rho_{0}\right)$ the open geodesic ball centered at $\bar{x}$ of radius $\rho_{0}$ on the rescaled solution $\tilde{g}_{i j}^{\alpha_{k} \beta_{k}}$.

First, we claim that for any $0<\rho<\rho_{0}$ with $\rho$ near $\rho_{0}$, the rescaled solutions on the balls $\tilde{B}_{0}(\bar{x}, \rho)$ are defined on the time interval $\left[-\frac{1}{16} \eta^{-1} M(\rho)^{-1}, 0\right]$ for all large 
$k$. Indeed, this follows from Assertion 3 or Assertion 1. For the later purpose in Step 6, we now present an argument by using Assertion 3. If the claim is not true, then there is a surgery at some time $\tilde{t} \in\left[-\frac{1}{16} \eta^{-1} M(\rho)^{-1}, 0\right]$ such that some point $\tilde{y} \in \tilde{B}_{0}(\bar{x}, \rho)$ lies in the instant gluing cap. We can choose sufficiently small $\delta^{\prime}>0$ such that $2 \rho_{0}<\left(\delta^{\prime}\right)^{-\frac{1}{2}} \tilde{h}$, where $\tilde{h} \geq D^{-1} 20^{-\frac{1}{2}} M(\rho)^{-\frac{1}{2}}$ is the cutoff radius of the rescaled solutions at $\tilde{t}$. By applying Assertion 3 with $(\tilde{y}, 0)=\left(y_{0}, t_{0}\right)$, we see that there is a $k\left(\rho_{0}, M(\rho)\right)>0$ such that when $k \geq k\left(\rho_{0}, M(\rho)\right)$,

$$
\widetilde{R}(x, 0) \leq 2 D^{\prime \prime}
$$

for all $x \in \widetilde{B}_{0}(\bar{x}, \rho)$. This is a contradiction as $\rho \rightarrow \rho_{0}$.

Since for each fixed $0<\rho<\rho_{0}$ with $\rho$ near $\rho_{0}$, the rescaled solutions are defined on $\tilde{B}_{0}(\bar{x}, \rho) \times\left[-\frac{1}{16} \eta^{-1} M(\rho)^{-1}, 0\right]$ for all large $k$, by Step 1 and Shi's derivative estimate, we know that the covariant derivatives and higher order derivatives of the curvatures on $\tilde{B}_{0}\left(\bar{x}, \rho-\frac{\left(\rho_{0}-\rho\right)}{2}\right) \times\left[-\frac{1}{32} \eta^{-1} M(\rho)^{-1}, 0\right]$ are also uniformly bounded.

By the uniform $\kappa$-noncollapsing property and Hamilton's compactness theorem (Theorem 4.1.5), after passing to a subsequence, we can assume that the marked sequence $\left(\tilde{B}_{0}\left(\bar{x}, \rho_{0}\right), \widetilde{g}_{i j}^{\alpha_{k} \beta_{k}}, \bar{x}\right)$ converges in the $C_{l o c}^{\infty}$ topology to a marked (noncomplete) manifold $\left(B_{\infty}, \widetilde{g}_{i j}^{\infty}, \bar{x}\right)$ and the geodesic segments $\gamma$ converge to a geodesic segment (missing an endpoint) $\gamma_{\infty} \subset B_{\infty}$ emanating from $\bar{x}$.

Clearly, the limit has nonnegative sectional curvature by the pinching assumption. Consider a tubular neighborhood along $\gamma_{\infty}$ defined by

$$
V=\bigcup_{q_{0} \in \gamma_{\infty}} B_{\infty}\left(q_{0}, 4 \pi\left(\widetilde{R}_{\infty}\left(q_{0}\right)\right)^{-\frac{1}{2}}\right),
$$

where $\widetilde{R}_{\infty}$ denotes the scalar curvature of the limit and

$$
B_{\infty}\left(q_{0}, 4 \pi\left(\widetilde{R}_{\infty}\left(q_{0}\right)\right)^{-\frac{1}{2}}\right)
$$

is the ball centered at $q_{0} \in B_{\infty}$ with the radius $4 \pi\left(\widetilde{R}_{\infty}\left(q_{0}\right)\right)^{-\frac{1}{2}}$. Let $\bar{B}_{\infty}$ denote the completion of $\left(B_{\infty}, \widetilde{g}_{i j}^{\infty}\right)$, and $y_{\infty} \in \bar{B}_{\infty}$ the limit point of $\gamma_{\infty}$. Exactly as in the second step of the proof of Theorem 7.1.1, it follows from the canonical neighborhood assumption with accuracy parameter $2 \varepsilon$ that the limiting metric $\widetilde{g}_{i j}^{\infty}$ is cylindrical at any point $q_{0} \in \gamma_{\infty}$ which is sufficiently close to $y_{\infty}$ and then the metric space $\bar{V}=V \cup\left\{y_{\infty}\right\}$ by adding the point $y_{\infty}$ has nonnegative curvature in the Alexandrov sense. Consequently we have a three-dimensional non-flat tangent cone $C_{y_{\infty}} \bar{V}$ at $y_{\infty}$ which is a metric cone with aperture $\leq 20 \varepsilon$.

On the other hand, note that by the canonical neighborhood assumption, the canonical $2 \varepsilon$-neck neighborhoods are strong. Thus at each point $q \in V$ near $y_{\infty}$, the limiting metric $\widetilde{g}_{i j}^{\infty}$ actually exists on the whole parabolic neighborhood

$$
V \bigcap P\left(q, 0, \frac{1}{3} \eta^{-1}\left(\widetilde{R}_{\infty}(q)\right)^{-\frac{1}{2}},-\frac{1}{10} \eta^{-1}\left(\widetilde{R}_{\infty}(q)\right)^{-1}\right)
$$

and is a smooth solution of the Ricci flow there. Pick $z \in C_{y_{\infty}} \bar{V}$ with distance one from the vertex $y_{\infty}$ and it is nonflat around $z$. By definition the ball $B\left(z, \frac{1}{2}\right) \subset$ $C_{y_{\infty}} \bar{V}$ is the Gromov-Hausdorff convergent limit of the scalings of a sequence of balls $B_{\infty}\left(z_{\ell}, \sigma_{\ell}\right)\left(\subset\left(V, \widetilde{g}_{i j}^{\infty}\right)\right)$ where $\sigma_{\ell} \rightarrow 0$. Since the estimate (7.4.14) survives on $\left(V, \widetilde{g}_{i j}^{\infty}\right)$ for all $A<+\infty$, and the tangent cone is three-dimensional and nonflat around $z$, 
we see that this convergence is actually in the $C_{\text {loc }}^{\infty}$ topology and over some ancient time interval. Since the limiting $B_{\infty}\left(z, \frac{1}{2}\right)\left(\subset C_{y_{\infty}} \bar{V}\right)$ is a piece of nonnegatively curved nonflat metric cone, we get a contradiction with Hamilton's strong maximum principle (Theorem 2.2.1) as before. So we have proved $\rho_{0}=\infty$. This proves (i).

By the same proof of Assertion 1 in Step 2, we can further show that for any $L$, the rescaled solutions on the balls $\tilde{B}_{0}(\bar{x}, L)$ are defined at least on the time interval $\left[-\frac{1}{16} \eta^{-1} C(L)^{-1}, 0\right]$ for all sufficiently large $k$. This proves (ii).

Step 4. For any subsequence $\left(\alpha_{k}, \beta_{k}\right)$ of $(\alpha, \beta)$ with $r^{\alpha_{k}} \rightarrow 0$ and $\widetilde{\delta}^{\alpha_{k} \beta_{k}} \rightarrow 0$ as $k \rightarrow \infty$, by Step 3, the $\kappa$-noncollapsing property and Hamilton's compactness theorem, we can extract a $C_{l o c}^{\infty}$ convergent subsequence of $\tilde{g}_{i j}^{\alpha_{k} \beta_{k}}$ over some space-time open subsets containing the slice $\{t=0\}$. We now want to show any such limit has bounded curvature at $t=0$. We prove by contradiction. Suppose not, then there is a sequence of points $z_{\ell}$ divergent to infinity in the limiting metric at time zero with curvature divergent to infinity. Since the curvature at $z_{\ell}$ is large (comparable to one), $z_{\ell}$ has a canonical neighborhood which is a $2 \varepsilon$-cap or strong $2 \varepsilon$-neck. Note that the boundary of $2 \varepsilon$-cap lies in some $2 \varepsilon$-neck. So we get a sequence of $2 \varepsilon$-necks with radius going to zero. Note also that the limit has nonnegative sectional curvature. Without loss of generality, we may assume $2 \varepsilon<\varepsilon_{0}$, where $\varepsilon_{0}$ is the positive constant in Proposition 6.1.1. Thus this arrives at a contradiction with Proposition 6.1.1.

Step 5. In this step, we will choose some subsequence $\left(\alpha_{k}, \beta_{k}\right)$ of $(\alpha, \beta)$ so that we can extract a complete smooth limit of the rescaled solutions $\widetilde{g}_{i j}^{\alpha_{k} \beta_{k}}$ to the Ricci flow with surgery on a time interval $[-a, 0]$ for some $a>0$.

Choose $\alpha_{k}, \beta_{k} \rightarrow \infty$ so that $r^{\alpha_{k}} \rightarrow 0, \widetilde{\delta}^{\alpha_{k} \beta_{k}} \rightarrow 0$, and Assertion 1, 2, 3 hold with $\alpha=\alpha_{k}, \beta=\beta_{k}$ for all $A \in\{p / q \mid p, q=1,2, \ldots, k\}$, and $B, C \in\{1,2, \ldots, k\}$. By Step 3 , we may assume the rescaled solutions $\widetilde{g}_{i j}^{\alpha_{k} \beta_{k}}$ converge in the $C_{\text {loc }}^{\infty}$ topology at the time $t=0$. Since the curvature of the limit at $t=0$ is bounded by Step 4 , it follows from Assertion 1 in Step 2 and the choice of the sequence $\left(\alpha_{k}, \beta_{k}\right)$ that the limiting $\left(M_{\infty}, \widetilde{g}_{i j}^{\infty}(\cdot, t)\right)$ is defined at least on a backward time interval $[-a, 0]$ for some positive constant $a$ and is a smooth solution to the Ricci flow there.

Step 6. We further want to extend the limit in Step 5 backwards in time to infinity to get an ancient $\kappa$-solution. Let $\widetilde{g}_{i j}^{\alpha_{k} \beta_{k}}$ be the convergent sequence obtained in the above Step 5.

Denote by

$$
\begin{gathered}
t_{\max }=\sup \left\{t^{\prime} \mid \text { we can take a smooth limit on }\left(-t^{\prime}, 0\right]\right. \text { (with bounded } \\
\text { curvature at each time slice) from a subsequence of } \\
\text { the rescaled solutions } \left.\widetilde{g}_{i j}^{\alpha_{k} \beta_{k}}\right\} .
\end{gathered}
$$

We first claim that there is a subsequence of the rescaled solutions $\widetilde{g}_{i j}^{\alpha_{k} \beta_{k}}$ which converges in the $C_{\mathrm{loc}}^{\infty}$ topology to a smooth limit $\left(M_{\infty}, \widetilde{g}_{i j}^{\infty}(\cdot, t)\right)$ on the maximal time interval $\left(-t_{\max }, 0\right]$.

Indeed, let $t_{\ell}$ be a sequence of positive numbers such that $t_{\ell} \rightarrow t_{\max }$ and there exist smooth limits $\left(M_{\infty}, \widetilde{g}_{\ell}^{\infty}(\cdot, t)\right)$ defined on $\left(-t_{\ell}, 0\right]$. For each $\ell$, the limit has nonnegative sectional curvature and has bounded curvature at each time slice. Moreover by the gradient estimate in canonical neighborhood assumption with accuracy parameter $2 \varepsilon$, the limit has bounded curvature on each subinterval $[-b, 0] \subset\left(-t_{\ell}, 0\right]$. Denote by $\widetilde{Q}$ 
the scalar curvature upper bound of the limit at time zero $(\widetilde{Q}$ is independent of $\ell$ ). Then we can apply Li-Yau-Hamilton inequality (Corollary 2.5.5) to get

$$
\widetilde{R}_{\ell}^{\infty}(x, t) \leq \frac{t_{\ell}}{t+t_{\ell}} \widetilde{Q}
$$

where $\widetilde{R}_{\ell}^{\infty}(x, t)$ are the scalar curvatures of the limits $\left(M_{\infty}, \widetilde{g}_{\ell}^{\infty}(\cdot, t)\right)$. Hence by the definition of convergence and the above curvature estimates, we can find a subsequence of the rescaled solutions $\widetilde{g}_{i j}^{\alpha_{k} \beta_{k}}$ which converges in the $C_{l o c}^{\infty}$ topology to a smooth limit $\left(M_{\infty}, \widetilde{g}_{i j}^{\infty}(\cdot, t)\right)$ on the maximal time interval $\left(-t_{\max }, 0\right]$.

We need to show $-t_{\max }=-\infty$. Suppose $-t_{\max }>-\infty$, there are only the following two possibilities: either

(1) The curvature of the limiting solution $\left(M_{\infty}, \widetilde{g}_{i j}^{\infty}(\cdot, t)\right)$ becomes unbounded as $t \searrow-t_{\max }$; or

(2) For each small constant $\theta>0$ and each large integer $k_{0}>0$, there is some $k \geq$ $k_{0}$ such that the rescaled solution $\widetilde{g}_{i j}^{\alpha_{k} \beta_{k}}$ has a surgery time $T_{k} \in\left[-t_{\max }-\theta, 0\right]$ and a surgery point $x_{k}$ lying in a gluing cap at the times $T_{k}$ so that $d_{T_{k}}^{2}\left(x_{k}, \bar{x}\right)$ is uniformly bounded from above by a constant independent of $\theta$ and $k_{0}$.

We next claim that the possibility (1) always occurs. Suppose not; then the curvature of the limiting solution $\left(M_{\infty}, \widetilde{g}_{i j}^{\infty}(\cdot, t)\right)$ is bounded on $M_{\infty} \times\left(-t_{\max }, 0\right]$ by some positive constant $\hat{C}$. In particular, for any $A>0$, there is a sufficiently large integer $k_{1}>0$ such that any rescaled solution $\widetilde{g}_{i j}^{\alpha_{k} \beta_{k}}$ with $k \geq k_{1}$ on the geodesic ball $\widetilde{B}_{0}(\bar{x}, A)$ is defined on the time interval $\left[-t_{\max }+\frac{1}{50} \eta^{-1} \hat{C}^{-1}, 0\right]$ and its scalar curvature is bounded by $2 \hat{C}$ there. (Here, without loss of generality, we may assume that the upper bound $\hat{C}$ is so large that $-t_{\max }+\frac{1}{50} \eta^{-1} \hat{C}^{-1}<0$.) By Assertion 1 in Step 2, for $k$ large enough, the rescaled solution $\widetilde{g}_{i j}^{\alpha_{k} \beta_{k}}$ over $\widetilde{B}_{0}(\bar{x}, A)$ can be defined on the extended time interval $\left[-t_{\max }-\frac{1}{50} \eta^{-1} \hat{C}^{-1}, 0\right]$ and has the scalar curvature $\widetilde{R} \leq 10 \hat{C}$ on $\widetilde{B}_{0}(\bar{x}, A) \times\left[-t_{\max }-\frac{1}{50} \eta^{-1} \hat{C}^{-1}, 0\right]$. So we can extract a smooth limit from the sequence to get the limiting solution which is defined on a larger time interval $\left[-t_{\max }-\frac{1}{50} \eta^{-1} \hat{C}^{-1}, 0\right]$. This contradicts the definition of the maximal time $-t_{\max }$.

It remains to exclude the possibility (1).

By using Li-Yau-Hamilton inequality (Corollary 2.5.5) again, we have

$$
\widetilde{R}_{\infty}(x, t) \leq \frac{t_{\max }}{t+t_{\max }} \widetilde{Q} .
$$

So we only need to control the curvature near $-t_{\max }$. Exactly as in Step 4 in the proof of Theorem 7.1.1, it follows from Li-Yau-Hamilton inequality that

$$
\tilde{d}_{0}(x, y) \leq \tilde{d}_{t}(x, y) \leq \tilde{d}_{0}(x, y)+30 t_{\max } \sqrt{\widetilde{Q}}
$$

for any $x, y \in M_{\infty}$ and $t \in\left(-t_{\max }, 0\right]$.

Since the infimum of the scalar curvature is nondecreasing in time, we have some point $y_{\infty} \in M_{\infty}$ and some time $-t_{\max }<t_{\infty}<-t_{\max }+\frac{1}{50} \eta^{-1}$ such that $\widetilde{R}_{\infty}\left(y_{\infty}, t_{\infty}\right)<$ $5 / 4$. By (7.4.16), there is a constant $\widetilde{A}_{0}>0$ such that $\tilde{d}_{t}\left(\bar{x}, y_{\infty}\right) \leq \widetilde{A}_{0} / 2$ for all $t \in\left(-t_{\max }, 0\right]$.

Now we come back to the rescaled solution $\widetilde{g}_{i j}^{\alpha_{k} \beta_{k}}$. Clearly, for arbitrarily given small $\epsilon^{\prime}>0$, when $k$ large enough, there is a point $y_{k}$ in the underlying manifold of $\widetilde{g}_{i j}^{\alpha_{k} \beta_{k}}$ at time 0 satisfying the following properties

$$
\widetilde{R}\left(y_{k}, t_{\infty}\right)<\frac{3}{2}, \quad \widetilde{d}_{t}\left(\bar{x}, y_{k}\right) \leq \widetilde{A}_{0}
$$


for $t \in\left[-t_{\max }+\epsilon^{\prime}, 0\right]$. By the definition of convergence, we know that for any fixed $A_{0} \geq 2 \widetilde{A}_{0}$, for $k$ large enough, the rescaled solution over $\widetilde{B}_{0}\left(\bar{x}, A_{0}\right)$ is defined on the time interval $\left[t_{\infty}, 0\right]$ and satisfies

$$
\widetilde{R}(x, t) \leq \frac{2 t_{\max }}{t+t_{\max }} \widetilde{Q}
$$

on $\widetilde{B}_{0}\left(\bar{x}, A_{0}\right) \times\left[t_{\infty}, 0\right]$. Then by Assertion 2 of Step 2, we have proved that there is a sufficiently large integer $\bar{k}_{0}$ such that when $k \geq \bar{k}_{0}$, the rescaled solutions $\widetilde{g}_{i j}^{\alpha_{k} \beta_{k}}$ at $y_{k}$ can be defined on $\left[-t_{\max }-\frac{1}{50} \eta^{-1}, 0\right]$, and satisfy

$$
\widetilde{R}\left(y_{k}, t\right) \leq 15
$$

for $t \in\left[-t_{\max }-\frac{1}{50} \eta^{-1}, t_{\infty}\right]$.

We now prove a statement analogous to Assertion 4 (i) of Step 3.

Assertion 5. For the above rescaled solutions $\widetilde{g}_{i j}^{\alpha_{k} \beta_{k}}$ and $\bar{k}_{0}$, we have that for any $L>0$, there is a positive constant $\omega(L)$ such that the rescaled solutions $\widetilde{g}_{i j}^{\alpha_{k} \beta_{k}}$ satisfy

$$
\widetilde{R}(x, t) \leq \omega(L)
$$

for all $(x, t)$ with $\tilde{d}_{t}\left(x, y_{k}\right) \leq L$ and $t \in\left[-t_{\max }-\frac{1}{50} \eta^{-1}, t_{\infty}\right]$, and for all $k \geq \bar{k}_{0}$.

Proof of Assertion 5. We slightly modify the argument in the proof of Assertion 4 (i). Let

$$
\begin{gathered}
M(\rho)=\sup \left\{\widetilde{R}(x, t) \mid \tilde{d}_{t}\left(x, y_{k}\right) \leq \rho \text { and } t \in\left[-t_{\max }-\frac{1}{50} \eta^{-1}, t_{\infty}\right]\right. \\
\text { in the rescaled solutions } \left.\widetilde{g}_{i j}^{\alpha_{k} \beta_{k}}, k \geq \bar{k}_{0}\right\}
\end{gathered}
$$

and

$$
\rho_{0}=\sup \{\rho>0 \mid M(\rho)<+\infty\} .
$$

Note that the estimate (7.4.14) implies that $\rho_{0}>0$. We only need to show $\rho_{0}=+\infty$.

We argue by contradiction. Suppose $\rho_{0}<+\infty$. Then, after passing to a subsequence, there is a sequence $\left(\tilde{y}_{k}, t_{k}\right)$ in the rescaled solutions $\widetilde{g}_{i j}^{\alpha_{k} \beta_{k}}$ with $t_{k} \in\left[-t_{\max }-\frac{1}{50} \eta^{-1}, t_{\infty}\right]$ and $\tilde{d}_{t_{k}}\left(y_{k}, \tilde{y}_{k}\right) \rightarrow \rho_{0}<+\infty$ such that $\widetilde{R}\left(\tilde{y}_{k}, t_{k}\right) \rightarrow+\infty$. Denote by $\gamma_{k}$ a minimizing geodesic segment from $y_{k}$ to $\tilde{y}_{k}$ at the time $t_{k}$ and denote by $\widetilde{B}_{t_{k}}\left(y_{k}, \rho_{0}\right)$ the open geodesic ball centered at $y_{k}$ of radius $\rho_{0}$ on the rescaled solution $\widetilde{g}_{i j}^{\alpha_{k} \beta_{k}}\left(\cdot, t_{k}\right)$.

For any $0<\rho<\rho_{0}$ with $\rho$ near $\rho_{0}$, by applying Assertion 3 as before, we get that the rescaled solutions on the balls $\widetilde{B}_{t_{k}}\left(y_{k}, \rho\right)$ are defined on the time interval $\left[t_{k}-\frac{1}{16} \eta^{-1} M(\rho)^{-1}, t_{k}\right]$ for all large $k$. By Step 1 and Shi's derivative estimate, we further know that the covariant derivatives and higher order derivatives of the curvatures on $\widetilde{B}_{t_{k}}\left(y_{k}, \rho-\frac{\left(\rho_{0}-\rho\right)}{2}\right) \times\left[t_{k}-\frac{1}{32} \eta^{-1} M(\rho)^{-1}, t_{k}\right]$ are also uniformly bounded. Then by the uniform $\kappa$-noncollapsing property and Hamilton's compactness theorem (Theorem 4.1.5), after passing to a subsequence, we can assume that the marked sequence $\left(\tilde{B}_{t_{k}}\left(y_{k}, \rho_{0}\right), \widetilde{g}_{i j}^{\alpha_{k} \beta_{k}}\left(\cdot, t_{k}\right), y_{k}\right)$ converges in the $C_{l o c}^{\infty}$ topology to a marked (noncomplete) 
manifold $\left(B_{\infty}, \widetilde{g}_{i j}^{\infty}, y_{\infty}\right)$ and the geodesic segments $\gamma_{k}$ converge to a geodesic segment (missing an endpoint) $\gamma_{\infty} \subset B_{\infty}$ emanating from $y_{\infty}$.

Clearly, the limit also has nonnegative sectional curvature by the pinching assumption. Then by repeating the same argument as in the proof of Assertion 4 (i) in the rest, we derive a contradiction with Hamilton's strong maximum principle. This proves Assertion 5.

We then apply the second estimate of (7.4.17) and Assertion 5 to conclude that for any large constant $0<A<+\infty$, there is a positive constant $C(A)$ such that for any small $\epsilon^{\prime}>0$, the rescaled solutions $\widetilde{g}_{i j}^{\alpha_{k} \beta_{k}}$ satisfy

$$
\widetilde{R}(x, t) \leq C(A)
$$

for all $x \in \widetilde{B}_{0}(\bar{x}, A)$ and $t \in\left[-t_{\max }+\epsilon^{\prime}, 0\right]$, and for all sufficiently large $k$. Then by applying Assertion 1 in Step 2, we conclude that the rescaled solutions $\widetilde{g}_{i j}^{\alpha_{k} \beta_{k}}$ on the geodesic balls $\widetilde{B}_{0}(\bar{x}, A)$ are also defined on the extended time interval $\left[-t_{\max }+\epsilon^{\prime}-\right.$ $\left.\frac{1}{8} \eta^{-1} C(A)^{-1}, 0\right]$ for all sufficiently large $k$. Furthermore, by the gradient estimates as in Step 1, we have

$$
\widetilde{R}(x, t) \leq 10 C(A),
$$

for $x \in \widetilde{B}_{0}(\bar{x}, A)$ and $t \in\left[-t_{\max }+\epsilon^{\prime}-\frac{1}{8} \eta^{-1} C(A)^{-1}, 0\right]$. Since $\epsilon^{\prime}>0$ is arbitrarily small and the positive constant $C(A)$ is independent of $\epsilon^{\prime}$, we conclude that the rescaled solutions $\widetilde{g}_{i j}^{\alpha_{k} \beta_{k}}$ on $\widetilde{B}_{0}(\bar{x}, A)$ are defined on the extended time interval $\left[-t_{\max }-\right.$ $\left.\frac{1}{16} \eta^{-1} C(A)^{-1}, 0\right]$ and satisfy

$$
\widetilde{R}(x, t) \leq 10 C(A),
$$

for $x \in \widetilde{B}_{0}(\bar{x}, A)$ and $t \in\left[-t_{\max }-\frac{1}{16} \eta^{-1} C(A)^{-1}, 0\right]$, and for all sufficiently large $k$.

Now, by taking convergent subsequences from the rescaled solutions $\widetilde{g}_{i j}^{\alpha_{k} \beta_{k}}$, we see that the limit solution is defined smoothly on a space-time open subset of $M_{\infty} \times$ $(-\infty, 0]$ containing $M_{\infty} \times\left[-t_{\max }, 0\right]$. By Step 4, we see that the limiting metric $\widetilde{g}_{i j}^{\infty}\left(\cdot,-t_{\max }\right)$ at time $-t_{\max }$ has bounded curvature. Then by combining with the canonical neighborhood assumption of accuracy $2 \varepsilon$, we conclude that the curvature of the limit is uniformly bounded on the time interval $\left[-t_{\max }, 0\right]$. So we have excluded the possibility (1).

Hence we have proved a subsequence of the rescaled solutions converges to an orientable ancient $\kappa$-solution.

Finally by combining with the canonical neighborhood theorem (Theorem 6.4.6), we see that $(\bar{x}, \bar{t})$ has a canonical neighborhood with parameter $\varepsilon$, which is a contradiction. Therefore we have completed the proof of the proposition.

Summing up, we have proved that for any $\varepsilon>0$, (without loss of generality, we may assume $\varepsilon \leq \bar{\varepsilon}_{0}$ ), there exist nonincreasing (continuous) positive functions $\widetilde{\delta}(t)$ and $\widetilde{r}(t)$, defined on $[0,+\infty)$ with

$$
\widetilde{\delta}(t) \leq \bar{\delta}(t)=\min \left\{\frac{1}{2 e^{2} \log (1+t)}, \delta_{0}\right\},
$$

such that for arbitrarily given (continuous) positive function $\delta(t)$ with $\delta(t)<\widetilde{\delta}(t)$ on $[0,+\infty)$, and arbitrarily given a compact orientable normalized three-manifold as 
initial data, the Ricci flow with surgery has a solution on $[0, T)$ obtained by evolving the Ricci flow and by performing $\delta$-cutoff surgeries at a sequence of times $0<t_{1}<$ $t_{2}<\cdots<t_{i}<\cdots<T$, with $\delta\left(t_{i}\right) \leq \delta \leq \widetilde{\delta}\left(t_{i}\right)$ at each time $t_{i}$, so that the pinching assumption and the canonical neighborhood assumption (with accuracy $\varepsilon$ ) with $r=$ $\tilde{r}(t)$ are satisfied. (At this moment we still do not know whether the surgery times $t_{i}$ are discrete.)

Since the $\delta$-cutoff surgeries occur at the points lying deeply in the $\varepsilon$-horns, the minimum of the scalar curvature $R_{\min }(t)$ of the solution to the Ricci flow with surgery at each time-slice is achieved in the region unaffected by the surgeries. Thus we know from the evolution equation of the scalar curvature that

$$
\frac{d}{d t} R_{\min }(t) \geq \frac{2}{3} R_{\min }^{2}(t)
$$

In particular, the minimum of the scalar curvature $R_{\min }(t)$ is nondecreasing in time. Also note that each $\delta$-cutoff surgery decreases volume. Then the upper derivative of the volume in time satisfies

$$
\begin{aligned}
\left(\frac{\bar{d}}{d t}\right) V(t) & \triangleq \lim \sup _{\triangle t \rightarrow 0} \frac{V(t+\triangle t)-V(t)}{\triangle t} \\
& \leq-R_{\min }(0) V(t)
\end{aligned}
$$

which implies that

$$
V(t) \leq V(0) e^{-R_{\min }(0) t}
$$

On the other hand, by Lemma 7.3.2 and the $\delta$-cutoff procedure given in the previous section, we know that at each time $t_{i}$, each $\delta$-cutoff surgery cuts down the volume at least at an amount of $h^{3}\left(t_{i}\right)$ with $h\left(t_{i}\right)$ depending only on $\delta\left(t_{i}\right)$ and $\widetilde{r}\left(t_{i}\right)$. Thus the surgery times $t_{i}$ cannot accumulate in any finite interval. When the solution becomes extinct at some finite time $T$, the solution at time near $T$ is entirely covered by canonical neighborhoods and then the initial manifold is diffeomorphic to a connected sum of a finite copies of $\mathbb{S}^{2} \times \mathbb{S}^{1}$ and $\mathbb{S}^{3} / \Gamma$ (the metric quotients of round three-sphere). So we have proved the following long-time existence result which was proposed by Perelman in [104].

THEOREM 7.4 .3 (Long-time existence theorem). For any fixed constant $\varepsilon>0$, there exist nonincreasing (continuous) positive functions $\widetilde{\delta}(t)$ and $\widetilde{r}(t)$, defined on $[0,+\infty)$, such that for an arbitrarily given (continuous) positive function $\delta(t)$ with $\delta(t) \leq \widetilde{\delta}(t)$ on $[0,+\infty)$, and arbitrarily given a compact orientable normalized threemanifold as initial data, the Ricci flow with surgery has a solution with the following properties: either

(i) it is defined on a finite interval $[0, T)$ and obtained by evolving the Ricci flow and by performing a finite number of cutoff surgeries, with each $\delta$-cutoff at a time $t \in(0, T)$ having $\delta=\delta(t)$, so that the solution becomes extinct at the finite time $T$, and the initial manifold is diffeomorphic to a connected sum of a finite copies of $\mathbb{S}^{2} \times \mathbb{S}^{1}$ and $\mathbb{S}^{3} / \Gamma$ (the metric quotients of round three-sphere) ; or

(ii) it is defined on $[0,+\infty)$ and obtained by evolving the Ricci flow and by performing at most countably many cutoff surgeries, with each $\delta$-cutoff at a time $t \in[0,+\infty)$ having $\delta=\delta(t)$, so that the pinching assumption and the canonical neighborhood assumption (with accuracy $\varepsilon$ ) with $r=\widetilde{r}(t)$ are satisfied, and there exist at most a finite number of surgeries on every finite time interval. 
In particular, if the initial manifold has positive scalar curvature, say $R \geq a>0$, then by (7.4.20), the solution becomes extinct at $T \leq \frac{3}{2} a$. Hence we have the following topological description of compact three-manifolds with nonnegative scalar curvature which improves the well-known work of Schoen-Yau [109], [110].

Corollary 7.4.4 (Perelman [104]). Let $M$ be a compact orientable threemanifold with nonnegative scalar curvature. Then either $M$ is flat or it is diffeomorphic to a connected sum of a finite copies of $\mathbb{S}^{2} \times \mathbb{S}^{1}$ and $\mathbb{S}^{3} / \Gamma$ (the metric quotients of the round three-sphere).

The famous Poincaré conjecture states that every compact three-manifold with trivial fundamental group is diffeomorphic to $\mathbb{S}^{3}$. Developing tools to attack the conjecture formed the basis for much of the works in three-dimensional topology over the last one hundred years. Now we use the Ricci flow to discuss the Poincaré conjecture.

Let $M$ be a compact three-manifold with trivial fundamental group. In particular, the three-manifold $M$ is orientable. Arbitrarily given a Riemannian metric on $M$, by scaling we may assume the metric is normalized. With this normalized metric as initial data, we consider the solution to the Ricci flow with surgery. If one can show the solution becomes extinct in finite time, it will follow from Theorem 7.4.3 (i) that the three-manifold $M$ is diffeomorphic to the three-sphere $\mathbb{S}^{3}$. Such finite extinction time result was first proposed by Perelman in [105], and recently, Colding-Minicozzi has published a proof to it in [42]. So the combination of Theorem 7.4.3 (i) and Colding-Minicozzi's finite extinction result gives a complete proof of the Poincaré conjecture.

We also remark that the above long-time existence result has been extended to compact four-manifolds with positive isotropic curvature by Chen and the second author in [34]. As a consequence it gave a complete proof of the following classification theorem of compact four-manifolds, with no essential incompressible space-form and with a metric of positive isotropic curvature. The theorem was first proved by Hamilton in ([64]), though it was later found that the proof contains some gaps (see for example the comment of Perelman in Page 1, the second paragraph, of [104]).

THEOREM 7.4.5. A compact four-manifold with no essential incompressible spaceform and with a metric of positive isotropic curvature is diffeomorphic to $\mathbb{S}^{4}$, or $\mathbb{R} \mathbb{P}^{4}$, or $\mathbb{S}^{3} \times \mathbb{S}^{1}$, or $\mathbb{S}^{3} \widetilde{\times} \mathbb{S}^{1}$ (the $\mathbb{Z}_{2}$ quotient of $\mathbb{S}^{3} \times \mathbb{S}^{1}$ where $\mathbb{Z}_{2}$ flips $\mathbb{S}^{3}$ antipodally and rotates $\mathbb{S}^{1}$ by $\left.180^{0}\right)$, or a connected sum of them.

7.5. Curvature Estimates for Surgically Modified Solutions. In this section we will generalize the curvature estimates for smooth solutions in Section 7.2 to that of solutions with cutoff surgeries. We first state and prove a version of Theorem 7.2.1.

Theorem 7.5.1 (Perelman [104]). For any $\varepsilon>0$ and $1 \leq A<+\infty$, one can find $\kappa=\kappa(A, \varepsilon)>0, K_{1}=K_{1}(A, \varepsilon)<+\infty, K_{2}=K_{2}(A, \varepsilon)<+\infty$ and $\bar{r}=$ $\bar{r}(A, \varepsilon)>0$ such that for any $t_{0}<+\infty$ there exists $\bar{\delta}_{A}=\bar{\delta}_{A}\left(t_{0}\right)>0$ (depending also on $\varepsilon$ ), nonincreasing in $t_{0}$, with the following property. Suppose we have a solution, constructed by Theorem 7.4.3 with the nonincreasing (continuous) positive functions $\widetilde{\delta}(t)$ and $\widetilde{r}(t)$, to the Ricci flow with $\delta$-cutoff surgeries on time interval $[0, T]$ and with a compact orientable normalized three-manifold as initial data, where each $\delta$-cutoff at a time $t$ satisfies $\delta=\delta(t) \leq \widetilde{\delta}(t)$ on $[0, T]$ and $\delta=\delta(t) \leq \bar{\delta}_{A}$ on $\left[\frac{t_{0}}{2}, t_{0}\right]$; assume 
that the solution is defined on the whole parabolic neighborhood $P\left(x_{0}, t_{0}, r_{0},-r_{0}^{2}\right) \triangleq$ $\left\{(x, t) \mid x \in B_{t}\left(x_{0}, r_{0}\right), t \in\left[t_{0}-r_{0}^{2}, t_{0}\right]\right\}, 2 r_{0}^{2}<t_{0}$, and satisfies

$$
\begin{aligned}
& |R m| \leq r_{0}^{-2} \text { on } P\left(x_{0}, t_{0}, r_{0},-r_{0}^{2}\right), \\
& \text { and } \quad \operatorname{Vol}_{t_{0}}\left(B_{t_{0}}\left(x_{0}, r_{0}\right)\right) \geq A^{-1} r_{0}^{3} \text {. }
\end{aligned}
$$

Then

(i) the solution is $\kappa$-noncollapsed on all scales less than $r_{0}$ in the ball $B_{t_{0}}\left(x_{0}, A r_{0}\right)$;

(ii) every point $x \in B_{t_{0}}\left(x_{0}, A r_{0}\right)$ with $R\left(x, t_{0}\right) \geq K_{1} r_{0}^{-2}$ has a canonical neighborhood $B$, with $B_{t_{0}}(x, \sigma) \subset B \subset B_{t_{0}}(x, 2 \sigma)$ for some $0<\sigma<C_{1}(\varepsilon) R^{-\frac{1}{2}}\left(x, t_{0}\right)$, which is either a strong $\varepsilon$-neck or an $\varepsilon$-cap;

(iii) if $r_{0} \leq \bar{r} \sqrt{t_{0}}$ then $R \leq K_{2} r_{0}^{-2}$ in $B_{t_{0}}\left(x_{0}, A r_{0}\right)$.

Here $C_{1}(\varepsilon)$ is the positive constant in the canonical neighborhood assumption.

Proof. Without loss of generality, we may assume $0<\varepsilon \leq \bar{\varepsilon}_{0}$, where $\bar{\varepsilon}_{0}$ is the sufficiently small (universal) positive constant in Lemma 7.4.2.

(i) This is analog of no local collapsing theorem II (Theorem 3.4.2). In comparison with the no local collapsing theorem II, this statement gives $\kappa$-noncollapsing property no matter how big the time is and it also allows the solution to be modified by surgery.

Let $\eta(\geq 10)$ be the universal constant in the definition of the canonical neighborhood assumption. Recall that we had removed every component which has positive sectional curvature in our surgery procedure. By the same argument as in the first part of the proof of Lemma 7.4.2, the canonical neighborhood assumption of the solution implies the $\kappa$-noncollapsing on the scales less than $\frac{1}{2 \eta} \widetilde{r}\left(t_{0}\right)$ for some positive constant $\kappa$ depending only on $C_{1}(\varepsilon)$ and $C_{2}(\varepsilon)$ (in the definition of the canonical neighborhood assumption). So we may assume $\frac{1}{2 \eta} \widetilde{r}\left(t_{0}\right) \leq r_{0} \leq \sqrt{\frac{t_{0}}{2}}$, and study the scales $\rho, \frac{1}{2 \eta} \widetilde{r}\left(t_{0}\right) \leq \rho \leq r_{0}$. Let $x \in B_{t_{0}}\left(x_{0}, A r_{0}\right)$ and assume that the solution satisfies

$$
|R m| \leq \rho^{-2}
$$

for those points in $P\left(x, t_{0}, \rho,-\rho^{2}\right) \triangleq\left\{(y, t) \mid y \in B_{t}(x, \rho), t \in\left[t_{0}-\rho^{2}, t_{0}\right]\right\}$ for which the solution is defined. We want to bound the ratio $\operatorname{Vol}_{t_{0}}\left(B_{t_{0}}(x, \rho)\right) / \rho^{3}$ from below.

Recall that a space-time curve is called admissible if it stays in the region unaffected by surgery, and a space-time curve on the boundary of the set of admissible curves is called a barely admissible curve. Consider any barely admissible curve $\gamma$, parametrized by $t \in\left[t_{\gamma}, t_{0}\right], t_{0}-r_{0}^{2} \leq t_{\gamma} \leq t_{0}$, with $\gamma\left(t_{0}\right)=x$. The same proof for the assertion (7.4.2) (in the proof of Lemma 7.4.2) shows that for arbitrarily large $L>0$ (to be determined later), one can find a sufficiently small $\bar{\delta}\left(L, t_{0}, \widetilde{r}\left(t_{0}\right), \widetilde{r}\left(\frac{t_{0}}{2}\right), \varepsilon\right)>0$ such that when each $\delta$-cutoff in $\left[\frac{t_{0}}{2}, t_{0}\right]$ satisfies $\delta \leq \bar{\delta}\left(L, t_{0}, \widetilde{r}\left(t_{0}\right), \widetilde{r}\left(\frac{t_{0}}{2}\right), \varepsilon\right)$, there holds

$$
\int_{t_{\gamma}}^{t_{0}} \sqrt{t_{0}-t}\left(R_{+}(\gamma(t), t)+|\dot{\gamma}(t)|^{2}\right) d t \geq L r_{0} .
$$

From now on, we assume that each $\delta$-cutoff of the solution in the time interval $\left[\frac{t_{0}}{2}, t_{0}\right]$ satisfies $\delta \leq \bar{\delta}\left(L, t_{0}, \widetilde{r}\left(t_{0}\right), \widetilde{r}\left(\frac{t_{0}}{2}\right), \varepsilon\right)$. 
Let us scale the solution, still denoted by $g_{i j}(\cdot, t)$, to make $r_{0}=1$ and the time as $t_{0}=1$. By the maximum principle, it is easy to see that the (rescaled) scalar curvature satisfies

$$
R \geq-\frac{3}{2 t}
$$

on $(0,1]$. Let us consider the time interval $\left[\frac{1}{2}, 1\right]$ and define a function of the form

$$
h(y, t)=\phi\left(d_{t}\left(x_{0}, y\right)-A(2 t-1)\right)(\bar{L}(y, \tau)+2 \sqrt{\tau})
$$

where $\tau=1-t, \phi$ is the function of one variable chosen in the proof of Theorem 3.4 .2 which is equal to one on $\left(-\infty, \frac{1}{20}\right)$, rapidly increasing to infinity on $\left(\frac{1}{20}, \frac{1}{10}\right)$, and satisfies $2 \frac{\left(\phi^{\prime}\right)^{2}}{\phi}-\phi^{\prime \prime} \geq(2 A+300) \phi^{\prime}-C(A) \phi$ for some constant $C(A)<+\infty$, and $\bar{L}$ is the function defined by

$$
\bar{L}(q, \tau)=\inf \left\{2 \sqrt{\tau} \int_{0}^{\tau} \sqrt{s}\left(R+|\dot{\gamma}|^{2}\right) d s \mid(\gamma(s), s), s \in[0, \tau]\right.
$$

is a space-time curve with $\gamma(0)=x$ and $\gamma(\tau)=q\}$.

Note that

$$
\begin{aligned}
\bar{L}(y, \tau) & \geq 2 \sqrt{\tau} \int_{0}^{\tau} \sqrt{s} R d s \\
& \geq-4 \tau^{2} \\
& >-2 \sqrt{\tau}
\end{aligned}
$$

since $R \geq-3$ and $0<\tau \leq \frac{1}{2}$. This says $h$ is positive for $t \in\left[\frac{1}{2}, 1\right]$. Also note that

$$
\frac{\partial}{\partial \tau} \bar{L}+\triangle \bar{L} \leq 6
$$

as long as $\bar{L}$ is achieved by admissible curves. Then as long as the shortest $\mathcal{L}$-geodesics from $\left(x_{0}, 0\right)$ to $(y, \tau)$ are admissible, there holds at $y$ and $t=1-\tau$,

$$
\begin{aligned}
\left(\frac{\partial}{\partial t}-\triangle\right) h \geq & \left(\phi^{\prime}\left[\left(\frac{\partial}{\partial t}-\triangle\right) d_{t}-2 A\right]-\phi^{\prime \prime}\right) \cdot(\bar{L}+2 \sqrt{\tau}) \\
& -\left(6+\frac{1}{\sqrt{\tau}}\right) \phi-2\langle\nabla \phi, \nabla \bar{L}\rangle .
\end{aligned}
$$

Firstly, we may assume the constant $L$ in (7.5.1) is not less than $2 \exp (C(A)+100)$. We claim that Lemma 3.4.1(i) is applicable for $d=d_{t}\left(\cdot, x_{0}\right)$ at $y$ and $t=1-\tau$ (with $\tau \in\left[0, \frac{1}{2}\right]$ ) whenever $\bar{L}(y, \tau)$ is achieved by admissible curves and satisfies the estimate

$$
\bar{L}(y, \tau) \leq 3 \sqrt{\tau} \exp (C(A)+100) .
$$

Indeed, since the solution is defined on the whole neighborhood $P\left(x_{0}, t_{0}, r_{0},-r_{0}^{2}\right)$ with $r_{0}=1$ and $t_{0}=1$, the point $x_{0}$ at the time $t=1-\tau$ lies on the region unaffected by surgery. Note that $R \geq-3$ for $t \in\left[\frac{1}{2}, 1\right]$. When $\bar{L}(y, \tau)$ is achieved by admissible curves and satisfies $\bar{L}(y, \tau) \leq 3 \sqrt{\tau} \exp (C(A)+100)$, the estimate (7.5.1) implies that 
the point $y$ at the time $t=1-\tau$ does not lie in the collars of the gluing caps. Thus any minimal geodesic (with respect to the metric $g_{i j}(\cdot, t)$ with $t=1-\tau$ ) connecting $x_{0}$ and $y$ also lies in the region unaffected by surgery; otherwise the geodesic is not minimal. Then from the proof of Lemma 3.4.1(i), we see that it is applicable.

Assuming the minimum of $h$ at a time, say $t=1-\tau$, is achieved at a point, say $y$, and assuming $\bar{L}(y, \tau)$ is achieved by admissible curves and satisfies $\bar{L}(y, \tau) \leq$ $3 \sqrt{\tau} \exp (C(A)+100)$, we have

$$
(\bar{L}+2 \sqrt{\tau}) \nabla \phi=-\phi \nabla \bar{L},
$$

and then by the computations and estimates in the proof of Theorem 3.4.2,

$$
\begin{aligned}
& \left(\frac{\partial}{\partial t}-\triangle\right) h \\
& \geq\left(\phi^{\prime}\left[\left(\frac{\partial}{\partial t}-\triangle\right) d_{t}-2 A\right]-\phi^{\prime \prime}+2 \frac{\left(\phi^{\prime}\right)^{2}}{\phi}\right) \cdot(\bar{L}+2 \sqrt{\tau})-\left(6+\frac{1}{\sqrt{\tau}}\right) \phi \\
& \geq-C(A) h-\left(6+\frac{1}{\sqrt{\tau}}\right) \frac{h}{\left(2 \sqrt{\tau}-4 \tau^{2}\right)}
\end{aligned}
$$

at $y$ and $t=1-\tau$. Here we used (7.5.2) and Lemma 3.4.1(i).

As before, denoting by $h_{\min }(\tau)=\min _{z} h(z, 1-\tau)$, we obtain

$$
\begin{aligned}
\frac{d}{d \tau}\left(\log \left(\frac{h_{\min }(\tau)}{\sqrt{\tau}}\right)\right) & \leq C(A)+\frac{6 \sqrt{\tau}+1}{2 \tau-4 \tau^{2} \sqrt{\tau}}-\frac{1}{2 \tau} \\
& \leq C(A)+\frac{50}{\sqrt{\tau}}
\end{aligned}
$$

as long as the associated shortest $\mathcal{L}$-geodesics are admissible with $\bar{L} \leq 3 \sqrt{\tau} \exp (C(A)+$ 100). On the other hand, by definition, we have

$$
\lim _{\tau \rightarrow 0^{+}} \frac{h_{\min }(\tau)}{\sqrt{\tau}} \leq \phi\left(d_{1}\left(x_{0}, x\right)-A\right) \cdot 2=2 .
$$

The combination of (7.5.4) and (7.5.5) gives the following assertion:

Let $\tau \in\left[0, \frac{1}{2}\right]$. If for each $s \in[0, \tau], \inf \left\{\bar{L}(y, s) \mid d_{t}\left(x_{0}, y\right) \leq A(2 t-1)+\right.$ $\frac{1}{10}$ with $\left.s=1-t\right\}$ is achieved by admissible curves, then we have

$$
\begin{aligned}
& \inf \left\{\bar{L}(y, \tau) \mid d_{t}\left(x_{0}, y\right) \leq A(2 t-1)+\frac{1}{10} \text { with } \tau=1-t\right\} \\
& \leq 2 \sqrt{\tau} \exp (C(A)+100) .
\end{aligned}
$$

Note again that $R \geq-3$ for $t \in\left[\frac{1}{2}, 1\right]$. By combining with (7.5.1), we know that any barely admissible curve $\gamma$, parametrized by $s \in[0, \tau], 0 \leq \tau \leq \frac{1}{2}$, with $\gamma(0)=x$, satisfies

$$
\int_{0}^{\tau} \sqrt{s}\left(R+|\dot{\gamma}|^{2}\right) d s \geq \frac{7}{4} \exp (C(A)+100)
$$

by assuming $L \geq 2 \exp (C(A)+100)$. 
Since $|R m| \leq \rho^{-2}$ on $P\left(x, t_{0}, \rho,-\rho^{2}\right)$ with $\rho \geq \frac{1}{2 \eta} \widetilde{r}\left(t_{0}\right)$ (and $t_{0}=1$ ) and $\bar{\delta}\left(L, t_{0}, \widetilde{r}\left(t_{0}\right), \widetilde{r}\left(\frac{t_{0}}{2}\right), \varepsilon\right)>0$ is sufficiently small, the parabolic neighborhood $P\left(x, 1, \rho,-\rho^{2}\right)$ around the point $(x, 1)$ is contained in the region unaffected by the surgery. Thus as $\tau=1-t$ is sufficiently close to zero, $\frac{1}{2 \sqrt{\tau}} \inf \bar{L}$ can be bounded from above by a small positive constant and then the infimum $\inf \left\{\bar{L}(y, \tau) \mid d_{t}\left(x_{0}, y\right) \leq\right.$ $A(2 t-1)+\frac{1}{10}$ with $\left.\tau=1-t\right\}$ is achieved by admissible curves.

Hence we conclude that for each $\tau \in\left[0, \frac{1}{2}\right]$, any minimizing curve $\gamma_{\tau}$ of $\inf \left\{\bar{L}(y, \tau) \mid d_{t}\left(x_{0}, y\right) \leq A(2 t-1)+\frac{1}{10}\right.$ with $\left.\tau=1-t\right\}$ is admissible and satisfies

$$
\int_{0}^{\tau} \sqrt{s}\left(R+\left|\dot{\gamma}_{\tau}\right|^{2}\right) d s \leq \exp (C(A)+100) .
$$

Now we come back to the unrescaled solution. It then follows that the Li-YauPerelman distance $l$ from $\left(x, t_{0}\right)$ satisfies the following estimate

$$
\min \left\{l\left(y, t_{0}-\frac{1}{2} r_{0}^{2}\right) \mid y \in B_{t_{0}-\frac{1}{2} r_{0}^{2}}\left(x_{0}, \frac{1}{10} r_{0}\right)\right\} \leq \exp (C(A)+100),
$$

by noting the (parabolic) scaling invariance of the Li-Yau-Perelman distance.

By the assumption that $|R m| \leq r_{0}^{-2}$ on $P\left(x_{0}, t_{0}, r_{0},-r_{0}^{2}\right)$, exactly as before, for any $q \in B_{t_{0}-r_{0}^{2}}\left(x_{0}, r_{0}\right)$, we can choose a path $\gamma$ parametrized by $\tau \in\left[0, r_{0}^{2}\right]$ with $\gamma(0)=x, \gamma\left(r_{0}^{2}\right)=q$, and $\gamma\left(\frac{1}{2} r_{0}^{2}\right)=y \in B_{t_{0}-\frac{1}{2} r_{0}^{2}}\left(x_{0}, \frac{1}{10} r_{0}\right)$, where $\left.\gamma\right|_{\left[0, \frac{1}{2} r_{0}^{2}\right]}$ achieves the minimum $\min \left\{l\left(y, t_{0}-\frac{1}{2} r_{0}^{2}\right) \mid y \in B_{t_{0}-\frac{1}{2} r_{0}^{2}}\left(x_{0}, \frac{1}{10} r_{0}\right)\right\}$ and $\left.\gamma\right|_{\left[\frac{1}{2} r_{0}^{2}, r_{0}^{2}\right]}$ is a suitable curve satisfying $\left.\gamma\right|_{\left[\frac{1}{2} r_{0}^{2}, r_{0}^{2}\right]}(\tau) \in B_{t_{0}-\tau}\left(x_{0}, r_{0}\right)$, for each $\tau \in\left[\frac{1}{2} r_{0}^{2}, r_{0}^{2}\right]$, so that the $\mathcal{L}$-length of $\gamma$ is uniformly bounded from above by a positive constant (depending only on $A$ ) multiplying $r_{0}$. This implies that the Li-Yau-Perelman distance from $\left(x, t_{0}\right)$ to the ball $B_{t_{0}-r_{0}^{2}}\left(x_{0}, r_{0}\right)$ is uniformly bounded by a positive constant $L(A)$ (depending only on $A$ ). Now we can choose the constant $L$ in (7.5.1) by

$$
L=\max \{2 L(A), 2 \exp (C(A)+100)\} .
$$

Thus every shortest $\mathcal{L}$-geodesic from $\left(x, t_{0}\right)$ to the ball $B_{t_{0}-r_{0}^{2}}\left(x_{0}, r_{0}\right)$ is necessarily admissible. By combining with the assumption that $\operatorname{Vol}_{t_{0}}\left(B_{t_{0}}\left(x_{0}, r_{0}\right)\right) \geq A^{-1} r_{0}^{3}$, we conclude that Perelman's reduced volume of the ball $B_{t_{0}-r_{0}^{2}}\left(x_{0}, r_{0}\right)$ satisfies the estimate

$$
\begin{aligned}
\widetilde{V}_{r_{0}^{2}}\left(B_{t_{0}-r_{0}^{2}}\left(x_{0}, r_{0}\right)\right) & =\int_{B_{t_{0}-r_{0}^{2}}\left(x_{0}, r_{0}\right)}\left(4 \pi r_{0}^{2}\right)^{-\frac{3}{2}} \exp \left(-l\left(q, r_{0}^{2}\right)\right) d V_{t_{0}-r_{0}^{2}}(q) \\
& \geq c(A)
\end{aligned}
$$

for some positive constant $c(A)$ depending only on $A$.

We can now argue as in the last part of the proof of Lemma 7.4.2 to get a lower bound estimate for the volume of the ball $B_{t_{0}}(x, \rho)$. The union of all shortest $\mathcal{L}$ geodesics from $\left(x, t_{0}\right)$ to the ball $B_{t_{0}-r_{0}^{2}}\left(x_{0}, r_{0}\right)$, defined by

$$
\begin{aligned}
& C B_{t_{0}-r_{0}^{2}}\left(x_{0}, r_{0}\right)=\{(y, t) \mid(y, t) \text { lies in a shortest } \mathcal{L} \text {-geodesic from } \\
& \left.\qquad\left(x, t_{0}\right) \text { to a point in } B_{t_{0}-r_{0}^{2}}\left(x_{0}, r_{0}\right)\right\},
\end{aligned}
$$

forms a cone-like subset in space-time with vertex $\left(x, t_{0}\right)$. Denote by $B(t)$ the intersection of the cone-like subset $C B_{t_{0}-r_{0}^{2}}\left(x_{0}, r_{0}\right)$ with the time-slice at $t$. Perelman's 
reduced volume of the subset $B(t)$ is given by

$$
\widetilde{V}_{t_{0}-t}(B(t))=\int_{B(t)}\left(4 \pi\left(t_{0}-t\right)\right)^{-\frac{3}{2}} \exp \left(-l\left(q, t_{0}-t\right)\right) d V_{t}(q)
$$

Since the cone-like subset $C B_{t_{0}-r_{0}^{2}}\left(x_{0}, r_{0}\right)$ lies entirely in the region unaffected by surgery, we can apply Perelman's Jacobian comparison Theorem 3.2.7 and the estimate (7.5.8) to conclude that

$$
\begin{aligned}
\widetilde{V}_{t_{0}-t}(B(t)) & \geq \widetilde{V}_{r_{0}^{2}}\left(B_{t_{0}-r_{0}^{2}}\left(x_{0}, r_{0}\right)\right) \\
& \geq c(A)
\end{aligned}
$$

for all $t \in\left[t_{0}-r_{0}^{2}, t_{0}\right]$.

As before, denoting by $\xi=\rho^{-1} \operatorname{Vol}_{t_{0}}\left(B_{t_{0}}(x, \rho)\right)^{\frac{1}{3}}$, we only need to get a positive lower bound for $\xi$. Of course we may assume $\xi<1$. Consider $\widetilde{B}\left(t_{0}-\xi \rho^{2}\right)$, the subset at the time-slice $\left\{t=t_{0}-\xi \rho^{2}\right\}$ where every point can be connected to $\left(x, t_{0}\right)$ by an admissible shortest $\mathcal{L}$-geodesic. Perelman's reduced volume of $\widetilde{B}\left(t_{0}-\xi \rho^{2}\right)$ is given by

$$
\begin{aligned}
& \widetilde{V}_{\xi \rho^{2}}\left(\widetilde{B}\left(t_{0}-\xi \rho^{2}\right)\right) \\
& =\int_{\widetilde{B}\left(t_{0}-\xi \rho^{2}\right)}\left(4 \pi \xi \rho^{2}\right)^{-\frac{3}{2}} \exp \left(-l\left(q, \xi \rho^{2}\right)\right) d V_{t_{0}-\xi \rho^{2}}(q) \\
& =\int_{\widetilde{B}\left(t_{0}-\xi \rho^{2}\right) \cap \mathcal{L} \exp }\left(4 \pi \xi \rho^{2}\right)^{-\frac{3}{2}} \exp \left(-l\left(q, \xi \rho^{2}\right)\right) d V_{t_{0}-\xi \rho^{2}}(q) \\
& \quad+\int_{\widetilde{B}\left(t_{0}-\xi \rho^{2}\right) \backslash \mathcal{L} \exp _{\left\{|v| \leq \frac{1}{4} \xi^{\left.-\frac{1}{2}\right\}}\right.}\left(\xi \rho^{2}\right)}\left(4 \pi \xi \rho^{2}\right)^{-\frac{3}{2}} \exp \left(-l\left(q, \xi \rho^{2}\right)\right) d V_{t_{0}-\xi \rho^{2}}(q) .
\end{aligned}
$$

Note that the whole region $P\left(x, t_{0}, \rho,-\rho^{2}\right)$ is unaffected by surgery because $\rho \geq$ $\frac{1}{2 \eta} \widetilde{r}\left(t_{0}\right)$ and $\bar{\delta}\left(L, t_{0}, \widetilde{r}\left(t_{0}\right), \widetilde{r}\left(\frac{t_{0}}{2}\right), \varepsilon\right)>0$ is sufficiently small. Then exactly as before, there is a universal positive constant $\xi_{0}$ such that when $0<\xi \leq \xi_{0}$, there holds

$$
\mathcal{L} \exp _{\left\{|v| \leq \frac{1}{4} \xi^{-\frac{1}{2}}\right\}}\left(\xi \rho^{2}\right) \subset B_{t_{0}}(x, \rho)
$$

and the first term on RHS of (7.5.10) can be estimated by

$$
\begin{aligned}
& \int_{\widetilde{B}\left(t_{0}-\xi \rho^{2}\right) \cap \mathcal{L} \exp }\left(4 \pi \xi \rho^{2}\right)^{-\frac{3}{2}} \exp \left(-l\left(q, \xi \rho^{2}\right)\right) d V_{t_{0}-\xi \rho^{2}}(q) \\
& \leq e^{C \xi}(4 \pi)^{-\frac{3}{2}} \xi^{\frac{3}{2}}
\end{aligned}
$$

for some universal constant $C$; while the second term on RHS of (7.5.10) can be estimated by

$$
\begin{aligned}
& \int_{\widetilde{B}\left(t_{0}-\xi \rho^{2}\right) \backslash \mathcal{L} \exp }\left\{\begin{array}{l}
\left\{|v| \leq \frac{1}{4} \xi^{-\frac{1}{2}}\right\} \\
\leq\left(\xi \rho^{2}\right)
\end{array}\left(4 \pi \xi \rho^{2}\right)^{-\frac{3}{2}} \exp \left(-l\left(q, \xi \rho^{2}\right)\right) d V_{t_{0}-\xi \rho^{2}}(q)\right. \\
& \leq(4 \pi)^{-\frac{3}{2}} \int_{\left\{|v|>\frac{1}{4} \xi^{-\frac{1}{2}}\right\}} \exp \left(-|v|^{2}\right) d v .
\end{aligned}
$$

Since $B\left(t_{0}-\xi \rho^{2}\right) \subset \widetilde{B}\left(t_{0}-\xi \rho^{2}\right)$, the combination of (7.5.9)-(7.5.12) bounds $\xi$ from below by a positive constant depending only on $A$. This proves the statement (i). 
(ii) This is analogous to the claim in the proof of Theorem 7.2.1. We argue by contradiction. Suppose that for some $A<+\infty$ and a sequence $K_{1}^{\alpha} \rightarrow \infty$, there exists a sequence $t_{0}^{\alpha}$ such that for any sequences $\bar{\delta}^{\alpha \beta}>0$ with $\bar{\delta}^{\alpha \beta} \rightarrow 0$ for fixed $\alpha$, we have sequences of solutions $g_{i j}^{\alpha \beta}$ to the Ricci flow with surgery and sequences of points $x_{0}^{\alpha \beta}$, of radii $r_{0}^{\alpha \beta}$, which satisfy the assumptions but violate the statement (ii) at some $x^{\alpha \beta} \in B_{t_{0}^{\alpha}}\left(x_{0}^{\alpha \beta}, A r_{0}^{\alpha \beta}\right)$ with $R\left(x^{\alpha \beta}, t_{0}^{\alpha}\right) \geq K_{1}^{\alpha}\left(r_{0}^{\alpha \beta}\right)^{-2}$. Slightly abusing notation, we will often drop the indices $\alpha, \beta$ in the following argument.

Exactly as in the proof of Theorem 7.2.1, we need to adjust the point $\left(x, t_{0}\right)$. More precisely, we claim that there exists a point $(\bar{x}, \bar{t}) \in B_{\bar{t}}\left(x_{0}, 2 A r_{0}\right) \times\left[t_{0}-\frac{r_{0}^{2}}{2}, t_{0}\right]$ with $\bar{Q} \triangleq$ $R(\bar{x}, \bar{t}) \geq K_{1} r_{0}^{-2}$ such that the point $(\bar{x}, \bar{t})$ does not satisfy the canonical neighborhood statement, but each point $(y, t) \in \bar{P}$ with $R(y, t) \geq 4 \bar{Q}$ does, where $\bar{P}$ is the set of all $\left(x^{\prime}, t^{\prime}\right)$ satisfying $\bar{t}-\frac{1}{4} K_{1} \bar{Q}^{-1} \leq t^{\prime} \leq \bar{t}, d_{t^{\prime}}\left(x_{0}, x^{\prime}\right) \leq d_{\bar{t}}\left(x_{0}, \bar{x}\right)+K_{1}^{\frac{1}{2}} \bar{Q}^{-\frac{1}{2}}$. Indeed as before, the point $(\bar{x}, \bar{t})$ is chosen by an induction argument. We first choose $\left(x_{1}, t_{1}\right)=$ $\left(x, t_{0}\right)$ which satisfies $d_{t_{1}}\left(x_{0}, x_{1}\right) \leq A r_{0}$ and $R\left(x_{1}, t_{1}\right) \geq K_{1} r_{0}^{-2}$, but does not satisfy the canonical neighborhood statement. Now if $\left(x_{k}, t_{k}\right)$ is already chosen and is not the desired $(\bar{x}, \bar{t})$, then some point $\left(x_{k+1}, t_{k+1}\right)$ satisfies $t_{k}-\frac{1}{4} K_{1} R\left(x_{k}, t_{k}\right)^{-1} \leq t_{k+1} \leq t_{k}$, $d_{t_{k+1}}\left(x_{0}, x_{k+1}\right) \leq d_{t_{k}}\left(x_{0}, x_{k}\right)+K_{1}^{\frac{1}{2}} R\left(x_{k}, t_{k}\right)^{-\frac{1}{2}}$, and $R\left(x_{k+1}, t_{k+1}\right) \geq 4 R\left(x_{k}, t_{k}\right)$, but $\left(x_{k+1}, t_{k+1}\right)$ does not satisfy the canonical neighborhood statement. Then we have

$$
\begin{aligned}
R\left(x_{k+1}, t_{k+1}\right) & \geq 4^{k} R\left(x_{1}, t_{1}\right) \geq 4^{k} K_{1} r_{0}^{-2}, \\
d_{t_{k+1}}\left(x_{0}, x_{k+1}\right) & \leq d_{t_{1}}\left(x_{0}, x_{1}\right)+K_{1}^{\frac{1}{2}} \sum_{i=1}^{k} R\left(x_{i}, t_{i}\right)^{-\frac{1}{2}} \leq A r_{0}+2 r_{0},
\end{aligned}
$$

and

$$
t_{0} \geq t_{k+1} \geq t_{0}-\frac{1}{4} K_{1} \sum_{i=1}^{k} R\left(x_{i}, t_{i}\right)^{-1} \geq t_{0}-\frac{1}{2} r_{0}^{2} .
$$

So the sequence must be finite and its last element is the desired $(\bar{x}, \bar{t})$.

Rescale the solutions along $(\bar{x}, \bar{t})$ with factor $R(\bar{x}, \bar{t})\left(\geq K_{1} r_{0}^{-2}\right)$ and shift the times $\bar{t}$ to zero. We will adapt both the proof of Proposition 7.4.1 and that of Theorem 7.2.1 to show that a sequence of the rescaled solutions $\widetilde{g}_{i j}^{\alpha \beta}$ converges to an ancient $\kappa$-solution, which will give the desired contradiction. Since we only need to consider the scale of the curvature less than $\widetilde{r}(\bar{t})^{-2}$, the present situation is much easier than that of Proposition 7.4.1.

Firstly as before, we need to get a local curvature estimate.

For each adjusted $(\bar{x}, \bar{t})$, let $\left[t^{\prime}, \bar{t}\right]$ be the maximal subinterval of $\left[\bar{t}-\frac{1}{20} \eta^{-1} \bar{Q}^{-1}, \bar{t}\right]$ so that for each sufficiently large $\alpha$ and then sufficiently large $\beta$, the canonical neighborhood statement holds for any $(y, t)$ in $P\left(\bar{x}, \bar{t}, \frac{1}{10} K_{1}^{\frac{1}{2}} \bar{Q}^{-\frac{1}{2}}, t^{\prime}-\bar{t}\right)=\{(x, t) \mid x \in$ $\left.B_{t}\left(\bar{x}, \frac{1}{10} K_{1}^{\frac{1}{2}} \bar{Q}^{-\frac{1}{2}}\right), t \in\left[t^{\prime}, \bar{t}\right]\right\}$ with $R(y, t) \geq 4 \bar{Q}$, where $\eta$ is the universal positive constant in the definition of canonical neighborhood assumption. We want to show

$$
t^{\prime}=\bar{t}-\frac{1}{20} \eta^{-1} \bar{Q}^{-1}
$$

Consider the scalar curvature $R$ at the point $\bar{x}$ over the time interval $\left[t^{\prime}, \bar{t}\right]$. If there is a time $\tilde{t} \in\left[t^{\prime}, \bar{t}\right]$ satisfying $R(\bar{x}, \tilde{t}) \geq 4 \bar{Q}$, we let $\tilde{t}$ be the first of such time from $\bar{t}$. Since the chosen point $(\bar{x}, \bar{t})$ does not satisfy the canonical neighborhood statement, 
we know $R(\bar{x}, \bar{t}) \leq \widetilde{r}(\bar{t})^{-2}$. Recall from our designed surgery procedure that if there is a cutoff surgery at a point $x$ at a time $t$, the scalar curvature at $(x, t)$ is at least $\left(\bar{\delta}^{\alpha \beta}\right)^{-2} \widetilde{r}(t)^{-2}$. Then for each fixed $\alpha$, for $\beta$ large enough, the solution $g_{i j}^{\alpha \beta}(\cdot, t)$ around the point $\bar{x}$ over the time interval $\left[\tilde{t}-\frac{1}{20} \eta^{-1} \bar{Q}^{-1}, \tilde{t}\right]$ is well defined and satisfies the following curvature estimate

$$
R(\bar{x}, t) \leq 8 \bar{Q},
$$

for $t \in\left[\tilde{t}-\frac{1}{20} \eta^{-1} \bar{Q}^{-1}, \bar{t}\right]$ (or $t \in\left[t^{\prime}, \bar{t}\right]$ if there is no such time $\tilde{t}$ ). By the assumption that $t_{0}>2 r_{0}^{2}$, we have

$$
\begin{aligned}
\bar{t} R(\bar{x}, \bar{t}) & \geq \frac{t_{0}}{2} R(\bar{x}, \bar{t}) \\
& \geq r_{0}^{2}\left(K_{1} r_{0}^{-2}\right) \\
& =K_{1} \rightarrow+\infty .
\end{aligned}
$$

Thus by using the pinching assumption and the gradient estimates in the canonical neighborhood assumption, we further have

$$
|R m(x, t)| \leq 30 \bar{Q},
$$

for all $x \in B_{t}\left(\bar{x}, \frac{1}{10} \eta^{-1} \bar{Q}^{-\frac{1}{2}}\right.$ ) and $t \in\left[\tilde{t}-\frac{1}{20} \eta^{-1} \bar{Q}^{-1}, \bar{t}\right]$ (or $t \in\left[t^{\prime}, \bar{t}\right]$ ) and all sufficiently large $\alpha$ and $\beta$. Observe that Lemma 3.4.1 (ii) is applicable for $d_{t}\left(x_{0}, \bar{x}\right)$ with $t \in$ $\left[\tilde{t}-\frac{1}{20} \eta^{-1} \bar{Q}^{-1}, \bar{t}\right]$ (or $t \in\left[t^{\prime}, \bar{t}\right]$ ) since any minimal geodesic, with respect to the metric $g_{i j}(\cdot, t)$, connecting $x_{0}$ and $\bar{x}$ lies in the region unaffected by surgery; otherwise the geodesic is not minimal. After having obtained the above curvature estimate, we can argue as deriving (7.2.2) and (7.2.3) in the proof of Theorem 7.2.1 to conclude that any point $(x, t)$, with $\tilde{t}-\frac{1}{20} \eta^{-1} \bar{Q}^{-1} \leq t \leq \bar{t}$ (or $t \in\left[t^{\prime}, \bar{t}\right]$ ) and $d_{t}(x, \bar{x}) \leq \frac{1}{10} K_{1}^{\frac{1}{2}} \bar{Q}^{-\frac{1}{2}}$, satisfies

$$
d_{t}\left(x, x_{0}\right) \leq d_{\bar{t}}\left(\bar{x}, x_{0}\right)+\frac{1}{2} K_{1}^{\frac{1}{2}} \bar{Q}^{-\frac{1}{2}}
$$

for all sufficiently large $\alpha$ and $\beta$. Then by combining with the choice of the points $(\bar{x}, \bar{t})$, we prove $t^{\prime}=\bar{t}-\frac{1}{20} \eta^{-1} \bar{Q}^{-1}$ (i.e., the canonical neighborhood statement holds for any point $(y, t)$ in the parabolic neighborhood $P\left(\bar{x}, \bar{t}, \frac{1}{10} K_{1}^{\frac{1}{2}} \bar{Q}^{-\frac{1}{2}},-\frac{1}{20} \eta^{-1} \bar{Q}^{-1}\right)$ with $R(y, t) \geq 4 \bar{Q})$ for all sufficiently large $\alpha$ and then sufficiently large $\beta$.

Now it follows from the gradient estimates in the canonical neighborhood assumption that the scalar curvatures of the rescaled solutions $\widetilde{g}_{i j}^{\alpha \beta}$ satisfy

$$
\widetilde{R}(x, t) \leq 40
$$

for those $(x, t) \in P\left(\bar{x}, 0, \frac{1}{10} \eta^{-1},-\frac{1}{20} \eta^{-1}\right) \triangleq\left\{\left(x^{\prime}, t^{\prime}\right) \mid x^{\prime} \in \widetilde{B}_{t^{\prime}}\left(\bar{x}, \frac{1}{10} \eta^{-1}\right), t^{\prime} \in\right.$ $\left.\left[-\frac{1}{20} \eta^{-1}, 0\right]\right\}$, for which the rescaled solution is defined. (Here $\widetilde{B}_{t^{\prime}}$ denotes the geodesic ball in the rescaled solution at time $\left.t^{\prime}\right)$. Note again that $R(\bar{x}, \bar{t}) \leq \widetilde{r}(\bar{t})^{-2}$ and recall from our designed surgery procedure that if there is a cutoff surgery at a point $x$ at a time $t$, the scalar curvature at $(x, t)$ is at least $\left(\bar{\delta}^{\alpha \beta}\right)^{-2} \widetilde{r}(t)^{-2}$. Then for each fixed sufficiently large $\alpha$, for $\beta$ large enough, the rescaled solution $\widetilde{g}_{i j}^{\alpha \beta}$ is defined on the whole parabolic neighborhood $P\left(\bar{x}, 0, \frac{1}{10} \eta^{-1},-\frac{1}{20} \eta^{-1}\right)$. More generally, for arbitrarily fixed $0<\widetilde{K}<+\infty$, there is a positive integer $\alpha_{0}$ so that for each $\alpha \geq \alpha_{0}$ we can 
find $\beta_{0}>0$ (depending on $\alpha$ ) such that if $\beta \geq \beta_{0}$ and $(y, 0)$ is a point on the rescaled solution $\widetilde{g}_{i j}^{\alpha \beta}$ with $\widetilde{R}(y, 0) \leq \widetilde{K}$ and $\widetilde{d}_{0}(y, \bar{x}) \leq \widetilde{K}$, we have estimate

$$
\widetilde{R}(x, t) \leq 40 \widetilde{K}
$$

for $(x, t) \in P\left(y, 0, \frac{1}{10} \eta^{-1} \widetilde{K}^{-\frac{1}{2}},-\frac{1}{20} \eta^{-1} \widetilde{K}^{-1}\right) \triangleq\left\{\left(x^{\prime}, t^{\prime}\right) \mid x^{\prime} \in \widetilde{B}_{t^{\prime}}(y\right.$, $\left.\left.\frac{1}{10} \eta^{-1} \widetilde{K}^{-\frac{1}{2}}\right), t^{\prime} \in\left[-\frac{1}{20} \eta^{-1} \widetilde{K}^{-1}, 0\right]\right\}$. In particular, the rescaled solution is defined on the whole parabolic neighborhood $P\left(y, 0, \frac{1}{10} \eta^{-1} \widetilde{K}^{-\frac{1}{2}},-\frac{1}{20} \eta^{-1} \widetilde{K}^{-1}\right)$.

Next, we want to show the curvature of the rescaled solutions at the new times zero (after shifting) stay uniformly bounded at bounded distances from $\bar{x}$ for some subsequences of $\alpha$ and $\beta$. Let $\alpha_{m}, \beta_{m} \rightarrow+\infty$ be chosen so that the estimate (7.5.14) holds with $\widetilde{K}=m$. For all $\rho \geq 0$, set

$$
M(\rho)=\sup \left\{\widetilde{R}(x, 0) \mid m \geq 1, d_{0}(x, \bar{x}) \leq \rho \text { in the rescaled solutions } \widetilde{g}_{i j}^{\alpha_{m} \beta_{m}}\right\}
$$

and

$$
\rho_{0}=\sup \{\rho \geq 0 \mid M(\rho)<+\infty\} .
$$

Clearly the estimate (7.5.14) yields $\rho_{0}>0$. As we consider the unshifted time $\bar{t}$, by combining with the assumption that $t_{0}>2 r_{0}^{2}$, we have

$$
\begin{aligned}
\bar{t} R(\bar{x}, \bar{t}) & \geq \frac{t_{0}}{2} R(\bar{x}, \bar{t}) \\
& \geq r_{0}^{2}\left(K_{1} r_{0}^{-2}\right) \\
& =K_{1} \rightarrow+\infty .
\end{aligned}
$$

It then follows from the pinching assumption that we only need to show $\rho_{0}=+\infty$. As before, we argue by contradiction. Suppose we have a sequence of points $y_{m}$ in the rescaled solutions $\widetilde{g}_{i j}^{\alpha_{m} \beta_{m}}$ with $\widetilde{d}_{0}\left(\bar{x}, y_{m}\right) \rightarrow \rho_{0}<+\infty$ and $\widetilde{R}\left(y_{m}, 0\right) \rightarrow+\infty$. Denote by $\gamma_{m}$ a minimizing geodesic segment from $\bar{x}$ to $y_{m}$ and denote by $\widetilde{B}_{0}\left(\bar{x}, \rho_{0}\right)$ the open geodesic balls centered at $\bar{x}$ of radius $\rho_{0}$ of the rescaled solutions. By applying the assertion in statement (i), we have uniform $\kappa$-noncollapsing at the points $(\bar{x}, \bar{t})$. By combining with the local curvature estimate (7.5.14) and Hamilton's compactness theorem, we can assume that, after passing to a subsequence, the marked sequence $\left(\widetilde{B}_{0}\left(\bar{x}, \rho_{0}\right), \widetilde{g}_{i j}^{\alpha_{m} \beta_{m}}, \bar{x}\right)$ converges in the $C_{\text {loc }}^{\infty}$ topology to a marked (noncomplete) manifold $\left(B_{\infty}, \widetilde{g}_{i j}^{\infty}, x_{\infty}\right)$ and the geodesic segments $\gamma_{m}$ converge to a geodesic segment (missing an endpoint) $\gamma_{\infty} \subset B_{\infty}$ emanating from $x_{\infty}$. Moreover, by the pinching assumption and the estimate (7.5.15), the limit has nonnegative sectional curvature.

Then exactly as before, we consider the tubular neighborhood along $\gamma_{\infty}$

$$
V=\bigcup_{q_{0} \in \gamma_{\infty}} B_{\infty}\left(q_{0}, 4 \pi\left(\widetilde{R}_{\infty}\left(q_{0}\right)\right)^{-\frac{1}{2}}\right)
$$

and the completion $\bar{B}_{\infty}$ of $\left(B_{\infty}, \widetilde{g}_{i j}^{\infty}\right)$ with $y_{\infty} \in \bar{B}_{\infty}$ the limit point of $\gamma_{\infty}$. As before, by the choice of the points $(\bar{x}, \bar{t})$, we know that the limiting metric $\widetilde{g}_{i j}^{\infty}$ is cylindrical at any point $q_{0} \in \gamma_{\infty}$ which is sufficiently close to $y_{\infty}$. Then by the same reason as before the metric space $\bar{V}=V \cup\left\{y_{\infty}\right\}$ has nonnegative curvature in Alexandrov sense, and we have a three-dimensional nonflat tangent cone $C_{y_{\infty}} \bar{V}$ at $y_{\infty}$. Pick $z \in C_{y_{\infty}} \bar{V}$ with distance one from the vertex and it is nonflat around $z$. By 
definition $B\left(z, \frac{1}{2}\right)\left(\subset C_{y_{\infty}} \bar{V}\right)$ is the Gromov-Hausdoff convergent limit of the scalings of a sequence of balls $B_{\infty}\left(z_{k}, \sigma_{k}\right)\left(\subset\left(V, \widetilde{g}_{i j}^{\infty}\right)\right)$ with $\sigma_{k} \rightarrow 0$. Since the estimate (7.5.14) survives on $\left(V, \widetilde{g}_{i j}^{\infty}\right)$ for all $\widetilde{K}<+\infty$, we know that this convergence is actually in the $C_{\text {loc }}^{\infty}$ topology and over some time interval. Since the limit $B\left(z, \frac{1}{2}\right)\left(\subset C_{y_{\infty}} \bar{V}\right)$ is a piece of a nonnegatively curved nonflat metric cone, we get a contradiction with Hamilton's strong maximum Principle (Theorem 2.2.1) as before. Hence we have proved that a subsequence of the rescaled solution $\widetilde{g}_{i j}^{\alpha_{m} \beta_{m}}$ has uniformly bounded curvatures at bounded distance from $\bar{x}$ at the new times zero.

Further, by the uniform $\kappa$-noncollapsing at the points $(\bar{x}, \bar{t})$ and the estimate (7.5.14) again, we can take a $C_{\text {loc }}^{\infty} \operatorname{limit}\left(M_{\infty}, \widetilde{g}_{i j}^{\infty}, x_{\infty}\right)$, defined on a space-time subset which contains the time slice $\{t=0\}$ and is relatively open in $M_{\infty} \times(-\infty, 0]$, for the subsequence of the rescaled solutions. The limit is a smooth solution to the Ricci flow, and is complete at $t=0$, as well as has nonnegative sectional curvature by the pinching assumption and the estimate (7.5.15). Thus by repeating the same argument as in the Step 4 of the proof Proposition 7.4.1, we conclude that the curvature of the limit at $t=0$ is bounded.

Finally we try to extend the limit backwards in time to get an ancient $\kappa$-solution. Since the curvature of the limit is bounded at $t=0$, it follows from the estimate (7.5.14) that the limit is a smooth solution to the Ricci flow defined at least on a backward time interval $[-a, 0]$ for some positive constant $a$. Let $\left(t_{\infty}, 0\right]$ be the maximal time interval over which we can extract a smooth limiting solution. It suffices to show $t_{\infty}=-\infty$. If $t_{\infty}>-\infty$, there are only two possibilities: either there exist surgeries in finite distance around the time $t_{\infty}$ or the curvature of the limiting solution becomes unbounded as $t \searrow t_{\infty}$.

Let $c>0$ be a positive constant much smaller than $\frac{1}{100} \eta^{-1}$. Note again that the infimum of the scalar curvature is nondecreasing in time. Then we can find some point $y_{\infty} \in M_{\infty}$ and some time $t=t_{\infty}+\theta$ with $0<\theta<\frac{c}{3}$ such that $\widetilde{R}_{\infty}\left(y_{\infty}, t_{\infty}+\theta\right) \leq 2$.

Consider the (unrescaled) scalar curvature $R$ of $g_{i j}^{\alpha_{m} \beta_{m}}(\cdot, t)$ at the point $\bar{x}$ over the time interval $\left[\bar{t}+\left(t_{\infty}+\frac{\theta}{2}\right) \bar{Q}^{-1}, \bar{t}\right]$. Since the scalar curvature $R_{\infty}$ of the limit on $M_{\infty} \times\left[t_{\infty}+\frac{\theta}{3}, 0\right]$ is uniformly bounded by some positive constant $C$, we have the curvature estimate

$$
R(\bar{x}, t) \leq 2 C \bar{Q}
$$

for all $t \in\left[\bar{t}+\left(t_{\infty}+\frac{\theta}{2}\right) \bar{Q}^{-1}, \bar{t}\right]$ and all sufficiently large $m$. For each fixed $m$ and $\alpha_{m}$, we may require the chosen $\beta_{m}$ to satisfy

$$
\left(\bar{\delta}^{\alpha_{m} \beta_{m}}\right)^{-2}\left(\widetilde{r}\left(\frac{t_{0}^{\alpha_{m}}}{2}\right)\right)^{-2} \geq m \widetilde{r}\left(t_{0}^{\alpha_{m}}\right)^{-2} \geq m \bar{Q} .
$$

When $m$ is large enough, we observe again that Lemma 3.4.1 (ii) is applicable for $d_{t}\left(x_{0}, \bar{x}\right)$ with $t \in\left[\bar{t}+\left(t_{\infty}+\frac{\theta}{2}\right) \bar{Q}^{-1}, \bar{t}\right]$. Then by repeating the argument as in the derivation of (7.2.1), (7.2.2) and (7.2.3), we deduce that for all sufficiently large $m$, the canonical neighborhood statement holds for any $(y, t)$ in the parabolic neighborhood $P\left(\bar{x}, \bar{t}, \frac{1}{10} K_{1}^{\frac{1}{2}} \bar{Q}^{-\frac{1}{2}},\left(t_{\infty}+\frac{\theta}{2}\right) \bar{Q}^{-1}\right)$.

Let $\left(y_{m}, \bar{t}+\left(t_{\infty}+\theta_{m}\right) \bar{Q}^{-1}\right)$ be a sequence of associated points and times in the (unrescaled) solutions $g_{i j}^{\alpha_{m} \beta_{m}}(\cdot, t)$ so that after rescaling, the sequence converges to $\left(y_{\infty}, t_{\infty}+\theta\right)$ in the limit. Clearly $\frac{\theta}{2} \leq \theta_{m} \leq 2 \theta$ for all sufficiently large $m$. Then by the argument as in the derivation of (7.5.13), we know that for all sufficiently large 
$m$, the solution $g_{i j}^{\alpha_{m} \beta_{m}}(\cdot, t)$ at $y_{m}$ is defined on the whole time interval $\left[\bar{t}+\left(t_{\infty}+\theta_{m}-\right.\right.$ $\left.\left.\frac{1}{20} \eta^{-1}\right) \bar{Q}^{-1}, \bar{t}+\left(t_{\infty}+\theta_{m}\right) \bar{Q}^{-1}\right]$ and satisfies the curvature estimate

$$
R\left(y_{m}, t\right) \leq 8 \bar{Q}
$$

there; moreover the canonical neighborhood statement holds for any $(y, t)$ with $R(y, t) \geq 4 \bar{Q}$ in the parabolic neighborhood $P\left(y_{m}, \bar{t}, \frac{1}{10} K_{1}^{\frac{1}{2}} \bar{Q}^{-\frac{1}{2}},\left(t_{\infty}-\frac{c}{3}\right) \bar{Q}^{-1}\right)$.

We now consider the rescaled sequence $\widetilde{g}_{i j}^{\alpha_{m} \beta_{m}}(\cdot, t)$ with the marked points replaced by $y_{m}$ and the times replaced by $s_{m} \in\left[\bar{t}+\left(t_{\infty}-\frac{c}{4}\right) \bar{Q}^{-1}, \bar{t}+\left(t_{\infty}+\frac{c}{4}\right) \bar{Q}^{-1}\right]$. As before the Li-Yau-Hamilton inequality implies the rescaling limit around $\left(y_{m}, s_{m}\right)$ agrees with the original one. Then the arguments in previous paragraphs imply the limit is well-defined and smooth on a space-time open neighborhood of the maximal time slice $\left\{t=t_{\infty}\right\}$. Particularly this excludes the possibility of existing surgeries in finite distance around the time $t_{\infty}$. Moreover, the limit at $t=t_{\infty}$ also has bounded curvature. By using the gradient estimates in the canonical neighborhood assumption on the parabolic neighborhood $P\left(y_{m}, \bar{t}, \frac{1}{10} K_{1}^{\frac{1}{2}} \bar{Q}^{-\frac{1}{2}},\left(t_{\infty}-\frac{c}{3}\right) \bar{Q}^{-1}\right)$, we see that the second possibility is also impossible. Hence we have proved a subsequence of the rescaled solutions converges to an ancient $\kappa$-solution.

Therefore we have proved the canonical neighborhood statement (ii).

(iii) This is analogous to Theorem 7.2.1. We also argue by contradiction. Suppose for some $A<+\infty$ and sequences of positive numbers $K_{2}^{\alpha} \rightarrow+\infty, \bar{r}^{\alpha} \rightarrow 0$ there exists a sequence of times $t_{0}^{\alpha}$ such that for any sequences $\bar{\delta}^{\alpha \beta}>0$ with $\bar{\delta}^{\alpha \beta} \rightarrow 0$ for fixed $\alpha$, we have sequences of solutions $g_{i j}^{\alpha \beta}$ to the Ricci flow with surgery and sequences of points $x_{0}^{\alpha \beta}$, of positive constants $r_{0}^{\alpha \beta}$ with $r_{0}^{\alpha \beta} \leq \bar{r}^{\alpha} \sqrt{t_{0}^{\alpha}}$ which satisfy the assumptions, but for all $\alpha, \beta$ there hold

$$
R\left(x^{\alpha \beta}, t_{0}^{\alpha}\right)>K_{2}^{\alpha}\left(r_{0}^{\alpha \beta}\right)^{-2}, \text { for some } x^{\alpha \beta} \in B_{t_{0}^{\alpha}}\left(x_{0}^{\alpha \beta}, A r_{0}^{\alpha \beta}\right) .
$$

We may assume that $\bar{\delta}^{\alpha \beta} \leq \bar{\delta}_{4 A}\left(t_{0}^{\alpha}\right)$ for all $\alpha, \beta$, where $\bar{\delta}_{4 A}\left(t_{0}^{\alpha}\right)$ is chosen so that the statements (i) and (ii) hold on $B_{t_{0}^{\alpha}}\left(x_{0}^{\alpha \beta}, 4 A r_{0}^{\alpha \beta}\right)$. Let $\hat{g}_{i j}^{\alpha \beta}$ be the rescaled solutions of $g_{i j}^{\alpha \beta}$ around the origins $x_{0}^{\alpha \beta}$ with factor $\left(r_{0}^{\alpha \beta}\right)^{-2}$ and shift the times $t_{0}^{\alpha}$ to zero. Then by applying the statement (ii), we know that the regions, where the scalar curvature of the rescaled solutions $\hat{g}_{i j}^{\alpha \beta}$ is at least $K_{1}\left(=K_{1}(4 A)\right)$, are canonical neighborhood regions. Note that canonical $\varepsilon$-neck neighborhoods are strong. Also note that the pinching assumption and the assertion

$$
t_{0}^{\alpha}\left(r_{0}^{\alpha \beta}\right)^{-2} \geq\left(\bar{r}^{\alpha}\right)^{-2} \rightarrow+\infty, \text { as } \alpha \rightarrow+\infty,
$$

imply that any subsequent limit of the rescaled solutions $\hat{g}_{i j}^{\alpha \beta}$ must have nonnegative sectional curvature. Thus by the above argument in the proof of the statement (ii) (or the argument in Step 2 of the proof of Theorem 7.1.1), we conclude that there exist subsequences $\alpha=\alpha_{m}, \beta=\beta_{m}$ such that the curvatures of the rescaled solutions $\hat{g}_{i j}^{\alpha_{m} \beta_{m}}$ stay uniformly bounded at distances from the origins $x_{0}^{\alpha_{m} \beta_{m}}$ not exceeding $2 A$. This contradicts (7.5.16) for $m$ sufficiently large. This proves the statements (iii).

Clearly for fixed $A$, after defining the $\bar{\delta}_{A}\left(t_{0}\right)$ for each $t_{0}$, one can adjust the $\bar{\delta}_{A}\left(t_{0}\right)$ so that it is nonincreasing in $t_{0}$.

Therefore we have completed the proof of the theorem.

From now on we redefine the function $\widetilde{\delta}(t)$ so that it is also less than $\bar{\delta}_{2(t+1)}(2 t)$ and then the above theorem always holds for $A \in\left[1,2\left(t_{0}+1\right)\right]$. Particularly, we still 
have

$$
\widetilde{\delta}(t) \leq \bar{\delta}(t)=\min \left\{\frac{1}{2 e^{2} \log (1+t)}, \delta_{0}\right\},
$$

which tends to zero as $t \rightarrow+\infty$. We may also require that $\widetilde{r}(t)$ tends to zero as $t \rightarrow+\infty$.

The next result is a version of Theorem 7.2.2 for solutions with surgery.

Theorem 7.5.2 (Perelman [104]). For any $\varepsilon>0$ and $w>0$, there exist $\tau=$ $\tau(w, \varepsilon)>0, K=K(w, \varepsilon)<+\infty, \bar{r}=\bar{r}(w, \varepsilon)>0, \theta=\theta(w, \varepsilon)>0$ and $T=$ $T(w)<+\infty$ with the following property. Suppose we have a solution, constructed by Theorem 7.4.3 with the nonincreasing (continuous) positive functions $\widetilde{\delta}(t)$ and $\widetilde{r}(t)$, to the Ricci flow with surgery on the time interval $\left[0, t_{0}\right]$ with a compact orientable normalized three-manifold as initial data, where each $\delta$-cutoff at a time $t \in\left[0, t_{0}\right]$ has $\delta=\delta(t) \leq \min \{\widetilde{\delta}(t), \widetilde{r}(2 t)\}$. Let $r_{0}, t_{0}$ satisfy $\theta^{-1} h \leq r_{0} \leq \bar{r} \sqrt{t_{0}}$ and $t_{0} \geq T$, where $h$ is the maximal cutoff radius for surgeries in $\left[\frac{t_{0}}{2}, t_{0}\right]$ (if there is no surgery in the time interval $\left[\frac{t_{0}}{2}, t_{0}\right]$, we take $\left.h=0\right)$, and assume that the solution on the ball $B_{t_{0}}\left(x_{0}, r_{0}\right)$ satisfies

$$
\begin{gathered}
R m\left(x, t_{0}\right) \geq-r_{0}^{-2}, \text { on } B_{t_{0}}\left(x_{0}, r_{0}\right), \\
\text { and } \operatorname{Vol}_{t_{0}}\left(B_{t_{0}}\left(x_{0}, r_{0}\right)\right) \geq w r_{0}^{3}
\end{gathered}
$$

Then the solution is well defined and satisfies

$$
R(x, t)<K r_{0}^{-2}
$$

in the whole parabolic neighborhood

$$
P\left(x_{0}, t_{0}, \frac{r_{0}}{4},-\tau r_{0}^{2}\right)=\left\{(x, t) \mid x \in B_{t}\left(x_{0}, \frac{r_{0}}{4}\right), t \in\left[t_{0}-\tau r_{0}^{2}, t_{0}\right]\right\} .
$$

Proof. We are given that $R m\left(x, t_{0}\right) \geq-r_{0}^{-2}$ for $x \in B_{t_{0}}\left(x_{0}, r_{0}\right)$, and $\operatorname{Vol}_{t_{0}}\left(B_{t_{0}}\left(x_{0}, r_{0}\right)\right) \geq w r_{0}^{3}$. The same argument in the derivation of (7.2.7) and (7.2.8) (by using the Alexandrov space theory) implies that there exists a ball $B_{t_{0}}\left(x^{\prime}, r^{\prime}\right) \subset B_{t_{0}}\left(x_{0}, r_{0}\right)$ with

$$
\operatorname{Vol}_{t_{0}}\left(B_{t_{0}}\left(x^{\prime}, r^{\prime}\right)\right) \geq \frac{1}{2} \alpha_{3}\left(r^{\prime}\right)^{3}
$$

and with

$$
r^{\prime} \geq c(w) r_{0}
$$

for some small positive constant $c(w)$ depending only on $w$, where $\alpha_{3}$ is the volume of the unit ball in $\mathbb{R}^{3}$.

As in (7.1.2), we can rewrite the pinching assumption (7.3.3) as

$$
R m \geq-\left[f^{-1}(R(1+t)) /(R(1+t))\right] R,
$$

where

$$
y=f(x)=x(\log x-3), \quad \text { for } e^{2} \leq x<+\infty,
$$


is increasing and convex with range $-e^{2} \leq y<+\infty$, and its inverse function is also increasing and satisfies

$$
\lim _{y \rightarrow+\infty} f^{-1}(y) / y=0 .
$$

Note that $t_{0} r_{0}^{-2} \geq \bar{r}^{-2}$ by the hypotheses. We may require $T(w) \geq 8 c(w)^{-1}$. Then by applying Theorem 7.5.1 (iii) with $A=8 c(w)^{-1}$ and combining with the pinching assumption, we can reduce the proof of the theorem to the special case $w=\frac{1}{2} \alpha_{3}$. In the following we simply assume $w=\frac{1}{2} \alpha_{3}$.

Let us first consider the case $r_{0}<\widetilde{r}\left(t_{0}\right)$. We claim that $R\left(x, t_{0}\right) \leq C_{0}^{2} r_{0}^{-2}$ on $B_{t_{0}}\left(x_{0}, \frac{r_{0}}{3}\right)$, for some sufficiently large positive constant $C_{0}$ depending only on $\varepsilon$. If not, then there is a canonical neighborhood around $\left(x, t_{0}\right)$. Note that the type (c) canonical neighborhood has already been ruled out by our design of cutoff surgeries. Thus $\left(x, t_{0}\right)$ belongs to an $\varepsilon$-neck or an $\varepsilon$-cap. This tells us that there is a nearby point $y$, with $R\left(y, t_{0}\right) \geq C_{2}^{-1} R\left(x, t_{0}\right)>C_{2}^{-1} C_{0}^{2} r_{0}^{-2}$ and $d_{t_{0}}(y, x) \leq C_{1} R\left(x, t_{0}\right)^{-\frac{1}{2}} \leq C_{1} C_{0}^{-1} r_{0}$, which is the center of the $\varepsilon$-neck $B_{t_{0}}\left(y, \varepsilon^{-1} R\left(y, t_{0}\right)^{-\frac{1}{2}}\right)$. (Here $C_{1}, C_{2}$ are the positive constants in the definition of canonical neighborhood assumption). Clearly, when we choose $C_{0}$ to be much larger than $C_{1}, C_{2}$ and $\varepsilon^{-1}$, the whole $\varepsilon$-neck $B_{t_{0}}\left(y, \varepsilon^{-1} R\left(y, t_{0}\right)^{-\frac{1}{2}}\right)$ is contained in $B_{t_{0}}\left(x_{0}, \frac{r_{0}}{2}\right)$ and we have

$$
\frac{\operatorname{Vol}_{t_{0}}\left(B_{t_{0}}\left(y, \varepsilon^{-1} R\left(y, t_{0}\right)^{-\frac{1}{2}}\right)\right)}{\left(\varepsilon^{-1} R\left(y, t_{0}\right)^{-\frac{1}{2}}\right)^{3}} \leq 8 \pi \varepsilon^{2} .
$$

Without loss of generality, we may assume $\varepsilon>0$ is very small. Since we have assumed that $R m \geq-r_{0}^{-2}$ on $B_{t_{0}}\left(x_{0}, r_{0}\right)$ and $\operatorname{Vol}_{t_{0}}\left(B_{t_{0}}\left(x_{0}, r_{0}\right)\right) \geq \frac{1}{2} \alpha_{3} r_{0}^{3}$, we then get a contradiction by applying the standard Bishop-Gromov volume comparison. Thus we have the desired curvature estimate $R\left(x, t_{0}\right) \leq C_{0}^{2} r_{0}^{-2}$ on $B_{t_{0}}\left(x_{0}, \frac{r_{0}}{3}\right)$.

Furthermore, by using the gradient estimates in the definition of canonical neighborhood assumption, we can take $K=10 C_{0}^{2}, \tau=\frac{1}{100} \eta^{-1} C_{0}^{-2}$ and $\theta=\frac{1}{5} C_{0}^{-1}$ in this case. And since $r_{0} \geq \theta^{-1} h$, we have $R<10 C_{0}^{2} r_{0}^{-2} \leq \frac{1}{2} h^{-2}$ and the surgeries do not interfere in $P\left(x_{0}, t_{0}, \frac{r_{0}}{4},-\tau r_{0}^{2}\right)$.

We now consider the remaining case $\widetilde{r}\left(t_{0}\right) \leq r_{0} \leq \bar{r} \sqrt{t_{0}}$. Let us redefine

$$
\begin{aligned}
\tau & =\min \left\{\frac{\bar{\tau}_{0}}{2}, \frac{1}{100} \eta^{-1} C_{0}^{-2}\right\}, \\
K & =\max \left\{2\left(\bar{C}+\frac{2 \bar{B}}{\bar{\tau}_{0}}\right), 25 C_{0}^{2}\right\},
\end{aligned}
$$

and

$$
\theta=\frac{1}{2} K^{-\frac{1}{2}}
$$

where $\bar{\tau}_{0}=\tau_{0}(w), \bar{B}=B(w)$ and $\bar{C}=C(w)$ are the positive constants in Theorem 6.3.3(ii) with $w=\frac{1}{2} \alpha_{3}$, and $C_{0}$ is the positive constant chosen above. We will show there is a sufficiently small $\bar{r}>0$ such that the conclusion of the theorem for $w=\frac{1}{2} \alpha_{3}$ holds for the chosen $\tau, K$ and $\theta$.

Argue by contradiction. Suppose not, then there exist a sequence of $\bar{r}^{\alpha} \rightarrow 0$, and a sequence of solutions $g_{i j}^{\alpha}$ with points $\left(x_{0}^{\alpha}, t_{0}^{\alpha}\right)$ and radii $r_{0}^{\alpha}$ such that the assumptions of the theorem do hold with $\widetilde{r}\left(t_{0}^{\alpha}\right) \leq r_{0}^{\alpha} \leq \bar{r}^{\alpha} \sqrt{t_{0}^{\alpha}}$ whereas the conclusion 
does not. Similarly as in the proof of Theorem 7.2.2, we claim that we may assume that for all sufficiently large $\alpha$, any other point $\left(x^{\alpha}, t^{\alpha}\right)$ and radius $r^{\alpha}>0$ with that property has either $t^{\alpha}>t_{0}^{\alpha}$ or $t^{\alpha}=t_{0}^{\alpha}$ with $r^{\alpha} \geq r_{0}^{\alpha}$; moreover $t^{\alpha}$ tends to $+\infty$ as $\alpha \rightarrow+\infty$. Indeed, for fixed $\alpha$ and the solution $g_{i j}^{\alpha}$, let $t_{\min }^{\alpha}$ be the infimum of all possible times $t^{\alpha}$ with some point $x^{\alpha}$ and some radius $r^{\alpha}$ having that property. Since each such $t^{\alpha}$ satisfies $\bar{r}^{\alpha} \sqrt{t^{\alpha}} \geq r^{\alpha} \geq \widetilde{r}\left(t^{\alpha}\right)$, it follows that when $\alpha$ is large, $t_{\min }^{\alpha}$ must be positive and very large. Clearly for each fixed sufficiently large $\alpha$, by passing to a limit, there exist some point $x_{\min }^{\alpha}$ and some radius $r_{\min }^{\alpha}\left(\geq \widetilde{r}\left(t_{\min }^{\alpha}\right)>0\right)$ so that all assumptions of the theorem still hold for $\left(x_{\min }^{\alpha}, t_{\min }^{\alpha}\right)$ and $r_{\min }^{\alpha}$, whereas the conclusion of the theorem does not hold with $R \geq K\left(r_{\min }^{\alpha}\right)^{-2}$ somewhere in $P\left(x_{\min }^{\alpha}, t_{\min }^{\alpha}, \frac{1}{4} r_{\min }^{\alpha},-\tau\left(r_{\min }^{\alpha}\right)^{2}\right)$ for all sufficiently large $\alpha$. Here we used the fact that if $R<K\left(r_{\min }^{\alpha}\right)^{-2}$ on $P\left(x_{\min }^{\alpha}, t_{\min }^{\alpha}, \frac{1}{4} r_{\min }^{\alpha},-\tau\left(r_{\min }^{\alpha}\right)^{2}\right)$, then there is no $\delta$-cutoff surgery there; otherwise there must be a point there with scalar curvature at least $\frac{1}{2} \delta^{-2}\left(\frac{t_{\min }^{\alpha}}{2}\right)\left(\widetilde{r}\left(\frac{t_{\min }^{\alpha}}{2}\right)\right)^{-2} \geq \frac{1}{2}\left(\widetilde{r}\left(t_{\min }^{\alpha}\right)\right)^{-2}\left(\widetilde{r}\left(\frac{t_{\min }^{\alpha}}{2}\right)\right)^{-2} \gg K\left(r_{\min }^{\alpha}\right)^{-2}$ since $\bar{r}^{\alpha} \sqrt{t_{\min }^{\alpha}} \geq r_{\min }^{\alpha} \geq \widetilde{r}\left(t_{\min }^{\alpha}\right)$ and $\bar{r}^{\alpha} \rightarrow 0$, which is a contradiction.

After choosing the first time $t_{\min }^{\alpha}$, by passing to a limit again, we can then choose $r_{\min }^{\alpha}$ to be the smallest radius for all possible $\left(x_{\min }^{\alpha}, t_{\min }^{\alpha}\right)$ 's and $r_{\min }^{\alpha}$ 's with that property. Thus we have verified the claim.

For simplicity, we will drop the index $\alpha$ in the following arguments. By the assumption and the standard volume comparison, we have

$$
\operatorname{Vol}_{t_{0}}\left(B_{t_{0}}\left(x_{0}, \frac{1}{2} r_{0}\right)\right) \geq \xi_{0} r_{0}^{3}
$$

for some universal positive $\xi_{0}$. As in deriving (7.2.7) and (7.2.8), we can find a ball $B_{t_{0}}\left(x_{0}^{\prime}, r_{0}^{\prime}\right) \subset B_{t_{0}}\left(x_{0}, \frac{r_{0}}{2}\right)$ with

$$
\operatorname{Vol}_{t_{0}}\left(B_{t_{0}}\left(x_{0}^{\prime}, r_{0}^{\prime}\right)\right) \geq \frac{1}{2} \alpha_{3}\left(r_{0}^{\prime}\right)^{3} \text { and } \frac{1}{2} r_{0} \geq r_{0}^{\prime} \geq \xi_{0}^{\prime} r_{0}
$$

for some universal positive constant $\xi_{0}^{\prime}$. Then by what we had proved in the previous case and by the choice of the first time $t_{0}$ and the smallest radius $r_{0}$, we know that the solution is defined in $P\left(x_{0}^{\prime}, t_{0}, \frac{r_{0}^{\prime}}{4},-\tau\left(r_{0}^{\prime}\right)^{2}\right)$ with the curvature bound

$$
R<K\left(r_{0}^{\prime}\right)^{-2} \leq K\left(\xi_{0}^{\prime}\right)^{-2} r_{0}^{-2} .
$$

Since $\bar{r} \sqrt{t_{0}} \geq r_{0} \geq \widetilde{r}\left(t_{0}\right)$ and $\bar{r} \rightarrow 0$ as $\alpha \rightarrow \infty$, we see that $t_{0} \rightarrow+\infty$ and $t_{0} r_{0}^{-2} \rightarrow+\infty$ as $\alpha \rightarrow+\infty$. Define $T(w)=8 c(w)^{-1}+\bar{\xi}$, for some suitable large universal positive constant $\bar{\xi}$. Then for $\alpha$ sufficiently large, we can apply Theorem 7.5.1(iii) and the pinching assumption to conclude that

$$
R \leq K^{\prime} r_{0}^{-2}, \quad \text { on } P\left(x_{0}, t_{0}, 4 r_{0},-\frac{\tau}{2}\left(\xi_{0}^{\prime}\right)^{2} r_{0}^{2}\right),
$$

for some positive constant $K^{\prime}$ depending only on $K$ and $\xi_{0}^{\prime}$.

Furthermore, by combining with the pinching assumption, we deduce that when $\alpha$ sufficiently large,

$$
\begin{aligned}
R m & \geq-\left[f^{-1}(R(1+t)) /(R(1+t))\right] R \\
& \geq-r_{0}^{-2}
\end{aligned}
$$


on $P\left(x_{0}, t_{0}, r_{0},-\frac{\tau}{2}\left(\xi_{0}^{\prime}\right)^{2} r_{0}^{2}\right)$. So by applying Theorem 6.3 .3 (ii) with $w=\frac{1}{2} \alpha_{3}$, we have that when $\alpha$ sufficiently large,

$$
\operatorname{Vol}_{t}\left(B_{t}\left(x_{0}, r_{0}\right)\right) \geq \xi_{1} r_{0}^{3}
$$

for all $t \in\left[t_{0}-\frac{\tau}{2}\left(\xi_{0}^{\prime}\right)^{2} r_{0}^{2}, t_{0}\right]$, and

$$
R \leq\left(\bar{C}+\frac{2 \bar{B}}{\bar{\tau}_{0}}\right) r_{0}^{-2} \leq \frac{1}{2} K r_{0}^{-2}
$$

on $P\left(x_{0}, t_{0}, \frac{r_{0}}{4},-\frac{\tau}{2}\left(\xi_{0}^{\prime}\right)^{2} r_{0}^{2}\right)$, where $\xi_{1}$ is some universal positive constant.

Next we want to extend the estimate (7.5.23) backwards in time. Denote by $t_{1}=t_{0}-\frac{\tau}{2}\left(\xi_{0}^{\prime}\right)^{2} r_{0}^{2}$. The estimate $(7.5 .22)$ gives

$$
\operatorname{Vol}_{t_{1}}\left(B_{t_{1}}\left(x_{0}, r_{0}\right)\right) \geq \xi_{1} r_{0}^{3} .
$$

By the same argument in the derivation of (7.2.7) and (7.2.8) again, we can find a ball $B_{t_{1}}\left(x_{1}, r_{1}\right) \subset B_{t_{1}}\left(x_{0}, r_{0}\right)$ with

$$
\operatorname{Vol}_{t_{1}}\left(B_{t_{1}}\left(x_{1}, r_{1}\right)\right) \geq \frac{1}{2} \alpha_{3} r_{1}^{3}
$$

and with

$$
r_{1} \geq \xi_{1}^{\prime} r_{0}
$$

for some universal positive constant $\xi_{1}^{\prime}$. Then by what we had proved in the previous case and by the lower bound (7.5.21) at $t_{1}$ and the choice of the first time $t_{0}$, we know that the solution is defined on $P\left(x_{1}, t_{1}, \frac{r_{1}}{4},-\tau r_{1}^{2}\right)$ with the curvature bound $R<K r_{1}^{-2}$. By applying Theorem 7.5.1(iii) and the pinching assumption again we get that for $\alpha$ sufficiently large,

$$
R \leq K^{\prime \prime} r_{0}^{-2}
$$

on $P\left(x_{0}, t_{1}, 4 r_{0},-\frac{\tau}{2}\left(\xi_{1}^{\prime}\right)^{2} r_{0}^{2}\right)$, for some positive constant $K^{\prime \prime}$ depending only on $K$ and $\xi_{1}^{\prime}$. Moreover, by combining with the pinching assumption, we have

$$
\begin{aligned}
R m & \geq-\left[f^{-1}(R(1+t)) /(R(1+t))\right] R \\
& \geq-r_{0}^{-2}
\end{aligned}
$$

on $P\left(x_{0}, t_{1}, 4 r_{0},-\frac{\tau}{2}\left(\xi_{1}^{\prime}\right)^{2} r_{0}^{2}\right)$, for $\alpha$ sufficiently large. So by applying Theorem 6.3 .3 (ii) with $w=\frac{1}{2} \alpha_{3}$ again, we have that for $\alpha$ sufficiently large,

$$
\operatorname{Vol}_{t}\left(B_{t}\left(x_{0}, r_{0}\right)\right) \geq \xi_{1} r_{0}^{3},
$$

for all $t \in\left[t_{0}-\frac{\tau}{2}\left(\xi_{0}^{\prime}\right)^{2} r_{0}^{2}-\frac{\tau}{2}\left(\xi_{1}^{\prime}\right)^{2} r_{0}^{2}, t_{0}\right]$, and

$$
R \leq\left(\bar{C}+\frac{2 \bar{B}}{\bar{\tau}_{0}}\right) r_{0}^{-2} \leq \frac{1}{2} K r_{0}^{-2}
$$

on $P\left(x_{0}, t_{0}, \frac{r_{0}}{4},-\frac{\tau}{2}\left(\xi_{0}^{\prime}\right)^{2} r_{0}^{2}-\frac{\tau}{2}\left(\xi_{1}^{\prime}\right)^{2} r_{0}^{2}\right)$. 
Note that the constants $\xi_{0}, \xi_{0}^{\prime}, \xi_{1}$ and $\xi_{1}^{\prime}$ are universal, independent of the time $t_{1}$ and the choice of the ball $B_{t_{1}}\left(x_{1}, r_{1}\right)$. Then we can repeat the above procedure as many times as we like, until we reach the time $t_{0}-\tau r_{0}^{2}$. Hence we obtain the estimate

$$
R \leq\left(\bar{C}+\frac{2 \bar{B}}{\bar{\tau}_{0}}\right) r_{0}^{-2} \leq \frac{1}{2} K r_{0}^{-2}
$$

on $P\left(x_{0}, t_{0}, \frac{r_{0}}{4},-\tau r_{0}^{2}\right)$, for sufficiently large $\alpha$. This contradicts the choice of the point $\left(x_{0}, t_{0}\right)$ and the radius $r_{0}$ which make $R \geq K r_{0}^{-2}$ somewhere in $P\left(x_{0}, t_{0}, \frac{r_{0}}{4},-\tau r_{0}^{2}\right)$.

Therefore we have completed the proof of the theorem. $\square$

Consequently, we have the following result which is analog of Corollary 7.2.4.

Corollary 7.5.3. For any $\varepsilon>0$ and $w>0$, there exist $\bar{r}=\bar{r}(w, \varepsilon)>0$, $\theta=\theta(w, \varepsilon)>0$ and $T=T(w)$ with the following property. Suppose we have a solution, constructed by Theorem 7.4.3 with the positive functions $\widetilde{\delta}(t)$ and $\widetilde{r}(t)$, to the Ricci flow with surgery with a compact orientable normalized three-manifold as initial data, where each $\delta$-cutoff at a time $t$ has $\delta=\delta(t) \leq \min \{\widetilde{\delta}(t), \widetilde{r}(2 t)\}$. If $B_{t_{0}}\left(x_{0}, r_{0}\right)$ is a geodesic ball at time $t_{0}$, with $\theta^{-1} h \leq r_{0} \leq \bar{r} \sqrt{t_{0}}$ and $t_{0} \geq T$, where $h$ is the maximal cutoff radii for surgeries in $\left[\frac{t_{0}}{2}, t_{0}\right]$ (if there is no surgery in the time interval $\left[\frac{t_{0}}{2}, t_{0}\right]$, we take $h=0$ ), and satisfies

$$
\min \left\{\operatorname{Rm}\left(x, t_{0}\right) \mid x \in B_{t_{0}}\left(x_{0}, r_{0}\right)\right\}=-r_{0}^{-2},
$$

then

$$
\operatorname{Vol}_{t_{0}}\left(B_{t_{0}}\left(x_{0}, r_{0}\right)\right)<w r_{0}^{3}
$$

Proof. We argue by contradiction. Let $\theta=\theta(w, \varepsilon)$ and $T=2 T(w)$, where $\theta(w, \varepsilon)$ and $T(w)$ are the positive constant in Theorem 7.5.2. Suppose for any $\bar{r}>0$ there is a solution and a geodesic ball $B_{t_{0}}\left(x_{0}, r_{0}\right)$ satisfying the assumptions of the corollary with $\theta^{-1} h \leq r_{0} \leq \bar{r} \sqrt{t_{0}}$ and $t_{0} \geq T$, and with

$$
\min \left\{\operatorname{Rm}\left(x, t_{0}\right) \mid x \in B_{t_{0}}\left(x_{0}, r_{0}\right)\right\}=-r_{0}^{-2},
$$

but

$$
\operatorname{Vol}_{t_{0}}\left(B_{t_{0}}\left(x_{0}, r_{0}\right)\right) \geq w r_{0}^{3} .
$$

Without loss of generality, we may assume that $\bar{r}$ is less than the corresponding constant in Theorem 7.5.2. We can then apply Theorem 7.5.2 to get

$$
R(x, t) \leq K r_{0}^{-2}
$$

whenever $t \in\left[t_{0}-\tau r_{0}^{2}, t_{0}\right]$ and $d_{t}\left(x, x_{0}\right) \leq \frac{r_{0}}{4}$, where $\tau$ and $K$ are the positive constants in Theorem 7.5.2. Note that $t_{0} r_{0}^{-2} \geq \bar{r}^{-2} \rightarrow+\infty$ as $\bar{r} \rightarrow 0$. By combining with the pinching assumption we have

$$
\begin{aligned}
R m & \geq-\left[f^{-1}(R(1+t)) /(R(1+t))\right] R \\
& \geq-\frac{1}{2} r_{0}^{-2}
\end{aligned}
$$


in the region $P\left(x_{0}, t_{0}, \frac{r_{0}}{4},-\tau r_{0}^{2}\right)=\left\{(x, t) \mid x \in B_{t}\left(x_{0}, \frac{r_{0}}{4}\right), t \in\left[t_{0}-\tau r_{0}^{2}, t_{0}\right]\right\}$, provided $\bar{r}>0$ is sufficiently small. Thus we get the estimate

$$
|R m| \leq K^{\prime} r_{0}^{-2}
$$

in $P\left(x_{0}, t_{0}, \frac{r_{0}}{4},-\tau r_{0}^{2}\right)$, where $K^{\prime}$ is a positive constant depending only on $w$ and $\varepsilon$.

We can now apply Theorem 7.5.1 (iii) to conclude that

$$
R(x, t) \leq \widetilde{K} r_{0}^{-2}
$$

whenever $t \in\left[t_{0}-\frac{\tau}{2} r_{0}^{2}, t_{0}\right]$ and $d_{t}\left(x, x_{0}\right) \leq r_{0}$, where $\widetilde{K}$ is a positive constant depending only on $w$ and $\varepsilon$. By using the pinching assumption again we further have

$$
R m(x, t) \geq-\frac{1}{2} r_{0}^{-2}
$$

in the region $P\left(x_{0}, t_{0}, r_{0},-\frac{\tau}{2} r_{0}^{2}\right)=\left\{(x, t) \mid x \in B_{t}\left(x_{0}, r_{0}\right), t \in\left[t_{0}-\frac{\tau}{2} r_{0}^{2}, t_{0}\right]\right\}$, as long as $\bar{r}$ is sufficiently small. In particular, this would imply

$$
\min \left\{\operatorname{Rm}\left(x, t_{0}\right) \mid x \in B_{t_{0}}\left(x_{0}, r_{0}\right)\right\}>-r_{0}^{-2},
$$

which is a contradiction. $\square$

Remark 7.5.4. In section 7.3 of [104], Perelman claimed a stronger statement than the above Corollary 7.5.3 that allows $r_{0}<\theta^{-1} h$ in the assumptions. Nevertheless, the above weaker statement is sufficient to deduce the geometrization result.

7.6. Long Time Behavior. In Section 5.3, we obtained the long time behavior for smooth (compact) solutions to the three-dimensional Ricci flow with bounded normalized curvature. The purpose of this section is to adapt the arguments there to solutions of the Ricci flow with surgery and to drop the bounded normalized curvature assumption.

Recall from Corollary 7.4.4 that we have completely understood the topological structure of a compact, orientable three-manifold with nonnegative scalar curvature. From now on we assume that our initial manifold does not admit any metric with nonnegative scalar curvature, and that once we get a compact component with nonnegative scalar curvature, it is immediately removed. Furthermore, if a solution to the Ricci flow with surgery becomes extinct in a finite time, we have also obtained the topological structure of the initial manifold. So in the following we only consider those solutions to the Ricci flow with surgery which exist for all times $t \geq 0$.

Let $g_{i j}(t), 0 \leq t<+\infty$, be a solution to the Ricci flow with $\delta$-cutoff surgeries, constructed by Theorem 7.4.3 with normalized initial data. Let $0<t_{1}<t_{2}<\cdots<$ $t_{k}<\cdots$ be the surgery times, where each $\delta$-cutoff at a time $t_{k}$ has $\delta=\delta\left(t_{k}\right) \leq$ $\min \left\{\widetilde{\delta}\left(t_{k}\right), \widetilde{r}\left(2 t_{k}\right)\right\}$. On each time interval $\left(t_{k-1}, t_{k}\right)$ (denote by $t_{0}=0$ ), the scalar curvature satisfies the evolution equation

$$
\frac{\partial}{\partial t} R=\Delta R+2|\stackrel{\text { Ric }}{2}|^{2}+\frac{2}{3} R^{2}
$$

where Ric is the trace-free part of Ric. Then $R_{\min }(t)$, the minimum of the scalar curvature at the time $t$, satisfies

$$
\frac{d}{d t} R_{\min }(t) \geq \frac{2}{3} R_{\min }^{2}(t)
$$


for $t \in\left(t_{k-1}, t_{k}\right)$, for each $k=1,2, \ldots$. Since our surgery procedure had removed all components with nonnegative scalar curvature, the minimum $R_{\min }(t)$ is negative for all $t \in[0,+\infty)$. Also recall that the cutoff surgeries were performed only on $\delta$-necks. Thus the surgeries do not occur at the parts where $R_{\min }(t)$ are achieved. So the differential inequality

$$
\frac{d}{d t} R_{\min }(t) \geq \frac{2}{3} R_{\min }^{2}(t)
$$

holds for all $t \geq 0$, and then by normalization, $R_{\min }(0) \geq-1$, we have

$$
R_{\min }(t) \geq-\frac{3}{2} \cdot \frac{1}{t+\frac{3}{2}}, \text { for all } t \geq 0 .
$$

Meanwhile, on each time interval $\left(t_{k-1}, t_{k}\right)$, the volume satisfies the evolution equation

$$
\frac{d}{d t} V=-\int R d V
$$

and then by (7.6.2),

$$
\frac{d}{d t} V \leq \frac{3}{2} \cdot \frac{1}{\left(t+\frac{3}{2}\right)} V
$$

Since the cutoff surgeries do not increase volume, we thus have

$$
\frac{d}{d t} \log \left(V(t)\left(t+\frac{3}{2}\right)^{-\frac{3}{2}}\right) \leq 0
$$

for all $t \geq 0$. Equivalently, the function $V(t)\left(t+\frac{3}{2}\right)^{-\frac{3}{2}}$ is nonincreasing on $[0,+\infty)$.

We can now use the monotonicity of the function $V(t)\left(t+\frac{3}{2}\right)^{-\frac{3}{2}}$ to extract the information of the solution at large times. On each time interval $\left(t_{k-1}, t_{k}\right)$, we have

$$
\begin{aligned}
& \frac{d}{d t} \log \left(V(t)\left(t+\frac{3}{2}\right)^{-\frac{3}{2}}\right) \\
& =-\left(R_{\min }(t)+\frac{3}{2\left(t+\frac{3}{2}\right)}\right)+\frac{1}{V} \int_{M}\left(R_{\min }(t)-R\right) d V .
\end{aligned}
$$

Then by noting that the cutoff surgeries do not increase volume, we get

$$
\begin{gathered}
\frac{V(t)}{\left(t+\frac{3}{2}\right)^{\frac{3}{2}}} \leq \frac{V(0)}{\left(\frac{3}{2}\right)^{\frac{3}{2}}} \exp \left\{-\int_{0}^{t}\left(R_{\min }(t)+\frac{3}{2\left(t+\frac{3}{2}\right)}\right) d t\right. \\
\left.-\int_{0}^{t} \frac{1}{V} \int_{M}\left(R-R_{\min }(t)\right) d V d t\right\}
\end{gathered}
$$

for all $t>0$. Now by this inequality and the equation (7.6.1), we obtain the following consequence.

Lemma 7.6.1. Let $_{i j}(t)$ be a solution to the Ricci flow with surgery, constructed by Theorem 7.4.3 with normalized initial data. If for a fixed $0<r<1$ and a sequence of times $t^{\alpha} \rightarrow \infty$, the rescalings of the solution on the parabolic neighborhoods 
$P\left(x^{\alpha}, t^{\alpha}, r \sqrt{t^{\alpha}},-r^{2} t^{\alpha}\right)=\left\{(x, t) \mid x \in B_{t}\left(x^{\alpha}, r \sqrt{t^{\alpha}}\right), t \in\left[t^{\alpha}-r^{2} t^{\alpha}, t^{\alpha}\right]\right\}$, with factor $\left(t^{\alpha}\right)^{-1}$ and shifting the times $t^{\alpha}$ to 1 , converge in the $C^{\infty}$ topology to some smooth limiting solution, defined in an abstract parabolic neighborhood $P\left(\bar{x}, 1, r,-r^{2}\right)$, then this limiting solution has constant sectional curvature $-1 / 4 t$ at any time $t \in\left[1-r^{2}, 1\right]$.

In the previous section we obtained several curvature estimates for the solutions to the Ricci flow with surgery. Now we combine the curvature estimates with the above lemma to derive the following asymptotic result for the curvature.

Lemma 7.6.2 (Perelman [104]). For any $\varepsilon>0$, let $g_{i j}(t), 0 \leq t<+\infty$, be a solution to the Ricci flow with surgery, constructed by Theorem 7.4 .3 with normalized initial data.

(i) Given $w>0, r>0, \xi>0$, one can find $T=T(w, r, \xi, \varepsilon)<+\infty$ such that if the geodesic ball $B_{t_{0}}\left(x_{0}, r \sqrt{t_{0}}\right)$ at some time $t_{0} \geq T$ has volume at least $w r^{3} t_{0}^{\frac{3}{2}}$ and the sectional curvature at least $-r^{-2} t_{0}^{-1}$, then the curvature at $x_{0}$ at time $t=t_{0}$ satisfies

$$
\left|2 t R_{i j}+g_{i j}\right|<\xi
$$

(ii) Given in addition $1 \leq A<\infty$ and allowing $T$ to depend on $A$, we can ensure (7.6.5) for all points in $B_{t_{0}}\left(x_{0}, A r \sqrt{t_{0}}\right)$.

(iii) The same is true for all points in the forward parabolic neighborhood $P\left(x_{0}, t_{0}, A r \sqrt{t_{0}}, A r^{2} t_{0}\right) \triangleq\left\{(x, t) \mid x \in B_{t}\left(x_{0}, A r \sqrt{t_{0}}\right), t \in\left[t_{0}, t_{0}+A r^{2} t_{0}\right]\right\}$.

Proof. (i) By the assumptions and the standard volume comparison, we have

$$
\operatorname{Vol}_{t_{0}}\left(B_{t_{0}}\left(x_{0}, \rho\right)\right) \geq c w \rho^{3}
$$

for all $0<\rho \leq r \sqrt{t_{0}}$, where $c$ is a universal positive constant. Let $\bar{r}=\bar{r}(c w, \varepsilon)$ be the positive constant in Theorem 7.5.2 and set $r_{0}=\min \{r, \bar{r}\}$. On $B_{t_{0}}\left(x_{0}, r_{0} \sqrt{t_{0}}\right)(\subset$ $\left.B_{t_{0}}\left(x_{0}, r \sqrt{t_{0}}\right)\right)$, we have

$$
\begin{gathered}
R m \geq-\left(r_{0} \sqrt{t_{0}}\right)^{-2} \\
\text { and } \quad \operatorname{Vol}_{t_{0}}\left(B_{t_{0}}\left(x_{0}, r_{0} \sqrt{t_{0}}\right)\right) \geq c w\left(r_{0} \sqrt{t_{0}}\right)^{3} .
\end{gathered}
$$

Obviously, there holds $\theta^{-1} h \leq r_{0} \sqrt{t_{0}} \leq \bar{r} \sqrt{t_{0}}$ when $t_{0}$ is large enough, where $\theta=$ $\theta(c w, \varepsilon)$ is the positive constant in Theorem 7.5.2 and $h$ is the maximal cutoff radius for surgeries in $\left[\frac{t_{0}}{2}, t_{0}\right]$ (if there is no surgery in the time interval $\left[\frac{t_{0}}{2}, t_{0}\right]$, we take $h=0$ ). Then it follows from Theorem 7.5.2 that the solution is defined and satisfies

$$
R<K\left(r_{0} \sqrt{t_{0}}\right)^{-2}
$$

on whole parabolic neighborhood $P\left(x_{0}, t_{0}, \frac{r_{0} \sqrt{t_{0}}}{4},-\tau\left(r_{0} \sqrt{t_{0}}\right)^{2}\right)$. Here $\tau=\tau(c w, \varepsilon)$ and $K=K(c w, \varepsilon)$ are the positive constants in Theorem 7.5.2. By combining with the pinching assumption we have

$$
\begin{aligned}
R m & \geq-\left[f^{-1}(R(1+t)) /(R(1+t))\right] R \\
& \geq- \text { const. } K\left(r_{0} \sqrt{t_{0}}\right)^{-2}
\end{aligned}
$$

in the region $P\left(x_{0}, t_{0}, \frac{r_{0} \sqrt{t_{0}}}{4},-\tau\left(r_{0} \sqrt{t_{0}}\right)^{2}\right)$. Thus we get the estimate

$$
|R m| \leq K^{\prime}\left(r_{0} \sqrt{t_{0}}\right)^{-2}
$$


on $P\left(x_{0}, t_{0}, \frac{r_{0} \sqrt{t_{0}}}{4},-\tau\left(r_{0} \sqrt{t_{0}}\right)^{2}\right)$, for some positive constant $K^{\prime}=K^{\prime}(w, \varepsilon)$ depending only on $w$ and $\varepsilon$.

The curvature estimate (7.6.7) and the volume estimate (7.6.6) ensure that as $t_{0} \rightarrow+\infty$ we can take smooth (subsequent) limits for the rescalings of the solution with factor $\left(t_{0}\right)^{-1}$ on parabolic neighborhoods $P\left(x_{0}, t_{0}, \frac{r_{0} \sqrt{t_{0}}}{4},-\tau\left(r_{0} \sqrt{t_{0}}\right)^{2}\right)$. Then by applying Lemma 7.6.1, we can find $T=T(w, r, \xi, \varepsilon)<+\infty$ such that when $t_{0} \geq T$, there holds

$$
\left|2 t R_{i j}+g_{i j}\right|<\xi
$$

on $P\left(x_{0}, t_{0}, \frac{r_{0} \sqrt{t_{0}}}{4},-\tau\left(r_{0} \sqrt{t_{0}}\right)^{2}\right)$, in particular,

$$
\left|2 t R_{i j}+g_{i j}\right|\left(x_{0}, t_{0}\right)<\xi
$$

This proves the assertion (i).

(ii) In view of the above argument, to get the estimate (7.6.5) for all points in $B_{t_{0}}\left(x_{0}, A r \sqrt{t_{0}}\right)$, the key point is to get a upper bound for the scalar curvature on the parabolic neighborhood $P\left(x_{0}, t_{0}, A r \sqrt{t_{0}},-\tau\left(r_{0} \sqrt{t_{0}}\right)^{2}\right)$. After having the estimates (7.6.6) and (7.6.7), one would like to use Theorem 7.5.1(iii) to obtain the desired scalar curvature estimate. Unfortunately it does not work since our $r_{0}$ may be much larger than the constant $\bar{r}(A, \varepsilon)$ there when $A$ is very large. In the following we will use Theorem 7.5.1(ii) to overcome the difficulty.

Given $1 \leq A<+\infty$, based on (7.6.6) and (7.6.7), we can use Theorem 7.5.1(ii) to find a positive constant $K_{1}=K_{1}(w, r, A, \varepsilon)$ such that each point in $B_{t_{0}}\left(x_{0}, 2 A r \sqrt{t_{0}}\right)$ with its scalar curvature at least $K_{1}\left(r \sqrt{t_{0}}\right)^{-2}$ has a canonical neighborhood. We claim that there exists $T=T(w, r, A, \varepsilon)<+\infty$ so that when $t_{0} \geq T$, we have

$$
R<K_{1}\left(r \sqrt{t_{0}}\right)^{-2} \text {, on } B_{t_{0}}\left(x_{0}, 2 A r \sqrt{t_{0}}\right) \text {. }
$$

Argue by contradiction. Suppose not; then there exist a sequence of times $t_{0}^{\alpha} \rightarrow$ $+\infty$ and sequences of points $x_{0}^{\alpha}, x^{\alpha}$ with $x^{\alpha} \in B_{t_{0}^{\alpha}}\left(x_{0}^{\alpha}, 2 A r \sqrt{t_{0}^{\alpha}}\right)$ and $R\left(x^{\alpha}, t_{0}^{\alpha}\right)=$ $K_{1}\left(r \sqrt{t_{0}^{\alpha}}\right)^{-2}$. Since there exist canonical neighborhoods ( $\varepsilon$-necks or $\varepsilon$-caps) around the points $\left(x^{\alpha}, t_{0}^{\alpha}\right)$, there exist positive constants $c_{1}, C_{2}$ depending only on $\varepsilon$ such that

$$
\operatorname{Vol}_{t_{0}^{\alpha}}\left(B_{t_{0}^{\alpha}}\left(x^{\alpha}, K_{1}^{-\frac{1}{2}}\left(r \sqrt{t_{0}^{\alpha}}\right)\right)\right) \geq c_{1}\left(K_{1}^{-\frac{1}{2}}\left(r \sqrt{t_{0}^{\alpha}}\right)\right)^{3}
$$

and

$$
C_{2}^{-1} K_{1}\left(r \sqrt{t_{0}^{\alpha}}\right)^{-2} \leq R\left(x, t_{0}^{\alpha}\right) \leq C_{2} K_{1}\left(r \sqrt{t_{0}^{\alpha}}\right)^{-2},
$$

on $B_{t_{0}^{\alpha}}\left(x^{\alpha}, K_{1}^{-\frac{1}{2}}\left(r \sqrt{t_{0}^{\alpha}}\right)\right)$, for all $\alpha$. By combining with the pinching assumption we have

$$
\begin{aligned}
R m & \geq-\left[f^{-1}(R(1+t)) /(R(1+t))\right] R \\
& \geq- \text { const. } C_{2} K_{1}\left(r \sqrt{t_{0}^{\alpha}}\right)^{-2},
\end{aligned}
$$

on $B_{t_{0}^{\alpha}}\left(x^{\alpha}, K_{1}^{-\frac{1}{2}}\left(r \sqrt{t_{0}^{\alpha}}\right)\right)$, for all $\alpha$. It then follows from the assertion (i) we just proved that

$$
\lim _{\alpha \rightarrow+\infty}\left|2 t R_{i j}+g_{i j}\right|\left(x^{\alpha}, t_{0}^{\alpha}\right)=0 .
$$


In particular, we have

$$
t_{0}^{\alpha} R\left(x^{\alpha}, t_{0}^{\alpha}\right)<-1
$$

for $\alpha$ sufficiently large. This contradicts our assumption that $R\left(x^{\alpha}, t_{0}^{\alpha}\right)=$ $K_{1}\left(r \sqrt{t_{0}^{\alpha}}\right)^{-2}$. So we have proved assertion (7.6.9).

Now by combining (7.6.9) with the pinching assumption as before, we have

$$
R m \geq-K_{2}\left(r \sqrt{t_{0}}\right)^{-2}
$$

on $B_{t_{0}}\left(x_{0}, 2 A r \sqrt{t_{0}}\right)$, where $K_{2}=K_{2}(w, r, A, \varepsilon)$ is some positive constant depending only on $w, r, A$ and $\varepsilon$. Thus by (7.6.9) and (7.6.10) we have

$$
|R m| \leq K_{1}^{\prime}\left(r \sqrt{t_{0}}\right)^{-2} \text {, on } B_{t_{0}}\left(x_{0}, 2 A r \sqrt{t_{0}}\right),
$$

for some positive constant $K_{1}^{\prime}=K_{1}^{\prime}(w, r, A, \varepsilon)$ depending only on $w, r, A$ and $\varepsilon$. This gives us the curvature estimate on $B_{t_{0}}\left(x_{0}, 2 A r \sqrt{t_{0}}\right)$ for all $t_{0} \geq T(w, r, A, \varepsilon)$.

From the arguments in proving the above assertion (i), we have the estimates (7.6.6) and (7.6.7) and the solution is well-defined on the whole parabolic neighborhood $P\left(x_{0}, t_{0}, \frac{r_{0} \sqrt{t_{0}}}{4},-\tau\left(r_{0} \sqrt{t_{0}}\right)^{2}\right)$ for all $t_{0} \geq T(w, r, A, \varepsilon)$. Clearly we may assume that $\left(K_{1}^{\prime}\right)^{-\frac{1}{2}} r<\min \left\{\frac{r_{0}}{4}, \sqrt{\tau} r_{0}\right\}$. Thus by combining with the curvature estimate (7.6.11), we can apply Theorem 7.5.1(i) to get the following volume control

$$
\operatorname{Vol}_{t_{0}}\left(B_{t_{0}}\left(x,\left(K_{1}^{\prime}\right)^{-\frac{1}{2}} r \sqrt{t_{0}}\right)\right) \geq \kappa\left(\left(K_{1}^{\prime}\right)^{-\frac{1}{2}} r \sqrt{t_{0}}\right)^{3}
$$

for any $x \in B_{t_{0}}\left(x_{0}, A r \sqrt{t_{0}}\right)$, where $\kappa=\kappa(w, r, A, \varepsilon)$ is some positive constant depending only on $w, r, A$ and $\varepsilon$. So by using the assertion (i), we see that for $t_{0} \geq T$ with $T=T(w, r, \xi, A, \varepsilon)$ large enough, the curvature estimate (7.6.5) holds for all points in $B_{t_{0}}\left(x_{0}, A r \sqrt{t_{0}}\right)$.

(iii) We next want to extend the curvature estimate (7.6.5) to all points in the forward parabolic neighborhood $P\left(x_{0}, t_{0}, A r \sqrt{t_{0}}, A r^{2} t_{0}\right)$. Consider the time interval $\left[t_{0}, t_{0}+A r^{2} t_{0}\right]$ in the parabolic neighborhood. In assertion (ii), we have obtained the desired estimate (7.6.5) at $t=t_{0}$. Suppose estimate (7.6.5) holds on a maximal time interval $\left[t_{0}, t^{\prime}\right)$ with $t^{\prime} \leq t_{0}+A r^{2} t_{0}$. This says that we have

$$
\left|2 t R_{i j}+g_{i j}\right|<\xi
$$

on $P\left(x_{0}, t_{0}, A r \sqrt{t_{0}}, t^{\prime}-t_{0}\right) \triangleq\left\{(x, t) \mid x \in B_{t}\left(x_{0}, A r \sqrt{t_{0}}\right), t \in\left[t_{0}, t^{\prime}\right)\right\}$ so that either there exists a surgery in the ball $B_{t^{\prime}}\left(x_{0}, A r \sqrt{t_{0}}\right)$ at $t=t^{\prime}$, or there holds $\left|2 t R_{i j}+g_{i j}\right|=$ $\xi$ somewhere in $B_{t^{\prime}}\left(x_{0}, A r \sqrt{t_{0}}\right)$ at $t=t^{\prime}$. Since the Ricci curvature is near $-\frac{1}{2 t^{\prime}}$ in the geodesic ball, the surgeries cannot occur there. Thus we only need to consider the latter possibility.

Recall that the evolution of the length of a curve $\gamma$ and the volume of a domain $\Omega$ are given by

$$
\begin{aligned}
\frac{d}{d t} L_{t}(\gamma) & =-\int_{\gamma} \operatorname{Ric}(\dot{\gamma}, \dot{\gamma}) d s_{t} \\
\text { and } \quad \frac{d}{d t} \operatorname{Vol}_{t}(\Omega) & =-\int_{\Omega} R d V_{t} .
\end{aligned}
$$


By substituting the curvature estimate (7.6.13) into the above two evolution equations and using the volume lower bound (7.6.6), it is not hard to see

$$
\operatorname{Vol}_{t^{\prime}}\left(B_{t^{\prime}}\left(x_{0}, \sqrt{t^{\prime}}\right)\right) \geq \kappa^{\prime}\left(t^{\prime}\right)^{\frac{3}{2}}
$$

for some positive constant $\kappa^{\prime}=\kappa^{\prime}(w, r, \xi, A, \varepsilon)$ depending only on $w, r, \xi, A$ and $\varepsilon$. Then by the above assertion (ii), the combination of the curvature estimate (7.6.13) and the volume lower bound (7.6.14) implies that the curvature estimate (7.6.5) still holds for all points in $B_{t^{\prime}}\left(x_{0}, A r \sqrt{t_{0}}\right)$ provided $T=T(w, r, \xi, A, \varepsilon)$ is chosen large enough. This is a contradiction. Therefore we have proved assertion (iii).

We now state and prove the important Thick-thin decomposition theorem. A more general version (without the restriction on $\varepsilon$ ) was implicitly claimed by Perelman in [103] and [104].

TheOREm 7.6.3 (The Thick-thin decomposition theorem). For any $w>0$ and $0<\varepsilon \leq \frac{1}{2} w$, there exists a positive constant $\rho=\rho(w, \varepsilon) \leq 1$ with the following property. Suppose $g_{i j}(t)(t \in[0,+\infty))$ is a solution, constructed by Theorem 7.4.3 with the nonincreasing (continuous) positive functions $\widetilde{\delta}(t)$ and $\widetilde{r}(t)$, to the Ricci flow with surgery and with a compact orientable normalized three-manifold as initial data, where each $\delta$-cutoff at a time $t$ has $\delta=\delta(t) \leq \min \{\widetilde{\delta}(t), \widetilde{r}(2 t)\}$. Then for any arbitrarily fixed $\xi>0$, for $t$ large enough, the manifold $M_{t}$ at time $t$ admits a decomposition $M_{t}=M_{\text {thin }}(w, t) \cup M_{\text {thick }}(w, t)$ with the following properties:

(a) For every $x \in M_{\text {thin }}(w, t)$, there exists some $r=r(x, t)>0$, with $0<r \sqrt{t}<$ $\rho \sqrt{t}$, such that

$$
\begin{gathered}
R m \geq-(r \sqrt{t})^{-2} \text { on } B_{t}(x, r \sqrt{t}), \quad \text { and } \\
\operatorname{Vol}_{t}\left(B_{t}(x, r \sqrt{t})\right)<w(r \sqrt{t})^{3} .
\end{gathered}
$$

(b) For every $x \in M_{\mathrm{thick}}(w, t)$, we have

$$
\begin{gathered}
\left|2 t R_{i j}+g_{i j}\right|<\xi \text { on } B_{t}(x, \rho \sqrt{t}), \quad \text { and } \\
\operatorname{Vol}_{t}\left(B_{t}(x, \rho \sqrt{t})\right) \geq \frac{1}{10} w(\rho \sqrt{t})^{3} .
\end{gathered}
$$

Moreover, if we take any sequence of points $x^{\alpha} \in M_{\mathrm{thick}}\left(w, t^{\alpha}\right), t^{\alpha} \rightarrow+\infty$, then the scalings of $g_{i j}\left(t^{\alpha}\right)$ around $x^{\alpha}$ with factor $\left(t^{\alpha}\right)^{-1}$ converge smoothly, along a subsequence of $\alpha \rightarrow+\infty$, to a complete hyperbolic manifold of finite volume with constant sectional curvature $-\frac{1}{4}$.

Proof. Let $\bar{r}=\bar{r}(w, \varepsilon), \theta=\theta(w, \varepsilon)$ and $h$ be the positive constants obtained in Corollary 7.5.3. We may assume $\rho \leq \bar{r} \leq e^{-3}$. For any point $x \in M_{t}$, there are two cases: either

(i) $\min \left\{R m \mid B_{t}(x, \rho \sqrt{t})\right\} \geq-(\rho \sqrt{t})^{-2}$,

or

(ii) $\min \left\{R m \mid B_{t}(x, \rho \sqrt{t})\right\}<-(\rho \sqrt{t})^{-2}$. 
Let us first consider Case (i). If $\operatorname{Vol}_{t}\left(B_{t}(x, \rho \sqrt{t})\right)<\frac{1}{10} w(\rho \sqrt{t})^{3}$, then we can choose $r$ slightly less than $\rho$ so that

$$
R m \geq-(\rho \sqrt{t})^{-2} \geq-(r \sqrt{t})^{-2}
$$

on $B_{t}(x, r \sqrt{t})\left(\subset B_{t}(x, \rho \sqrt{t})\right)$, and

$$
\operatorname{Vol}_{t}\left(B_{t}(x, r \sqrt{t})\right)<\frac{1}{10} w(\rho \sqrt{t})^{3}<w(r \sqrt{t})^{3} ;
$$

thus $x \in M_{\text {thin }}(w, t)$. If $\operatorname{Vol}_{t}\left(B_{t}(x, \rho \sqrt{t})\right) \geq \frac{1}{10} w(\rho \sqrt{t})^{3}$, we can apply Lemma 7.6.2(ii) to conclude that for $t$ large enough,

$$
\left|2 t R_{i j}+g_{i j}\right|<\xi \quad \text { on } B_{t}(x, \rho \sqrt{t})
$$

thus $x \in M_{\text {thick }}(w, t)$.

Next we consider Case (ii). By continuity, there exists $0<r=r(x, t)<\rho$ such that

$$
\min \left\{R m \mid B_{t}(x, r \sqrt{t})\right\}=-(r \sqrt{t})^{-2} .
$$

If $\theta^{-1} h \leq r \sqrt{t}(\leq \bar{r} \sqrt{t})$, we can apply Corollary 7.5.3 to conclude

$$
\operatorname{Vol}_{t}\left(B_{t}(x, r \sqrt{t})\right)<w(r \sqrt{t})^{3} ;
$$

thus $x \in M_{\text {thin }}(w, t)$.

We now consider the difficult subcase $r \sqrt{t}<\theta^{-1} h$. By the pinching assumption, we have

$$
\begin{aligned}
R & \geq(r \sqrt{t})^{-2}\left(\log \left[(r \sqrt{t})^{-2}(1+t)\right]-3\right) \\
& \geq\left(\log r^{-2}-3\right)(r \sqrt{t})^{-2} \\
& \geq 2(r \sqrt{t})^{-2} \\
& \geq 2 \theta^{2} h^{-2}
\end{aligned}
$$

somewhere in $B_{t}(x, r \sqrt{t})$. Since $h$ is the maximal cutoff radius for surgeries in $\left[\frac{t}{2}, t\right]$, by the design of the $\delta$-cutoff surgery, we have

$$
\begin{aligned}
h & \leq \sup \left\{\delta^{2}(s) \widetilde{r}(s) \mid s \in\left[\frac{t}{2}, t\right]\right\} \\
& \leq \widetilde{\delta}\left(\frac{t}{2}\right) \widetilde{r}(t) \widetilde{r}\left(\frac{t}{2}\right) .
\end{aligned}
$$

Note also $\widetilde{\delta}\left(\frac{t}{2}\right) \rightarrow 0$ as $t \rightarrow+\infty$. Thus from the canonical neighborhood assumption, we see that for $t$ large enough, there exists a point in the ball $B_{t}(x, r \sqrt{t})$ which has a canonical neighborhood.

We claim that for $t$ sufficiently large, the point $x$ satisfies

$$
R(x, t) \geq \frac{1}{2}(r \sqrt{t})^{-2},
$$

and then the above argument shows that the point $x$ also has a canonical $\varepsilon$-neck or $\varepsilon$-cap neighborhood. Otherwise, by continuity, we can choose a point $x^{*} \in B_{t}(x, r \sqrt{t})$ 
with $R\left(x^{*}, t\right)=\frac{1}{2}(r \sqrt{t})^{-2}$. Clearly the new point $x^{*}$ has a canonical neighborhood $B^{*}$ by the above argument. In particular, there holds

$$
C_{2}^{-1}(\varepsilon) R \leq \frac{1}{2}(r \sqrt{t})^{-2} \leq C_{2}(\varepsilon) R
$$

on the canonical neighborhood $B^{*}$. By the definition of canonical neighborhood assumption, we have

$$
B_{t}\left(x^{*}, \sigma^{*}\right) \subset B^{*} \subset B_{t}\left(x^{*}, 2 \sigma^{*}\right)
$$

for some $\sigma^{*} \in\left(0, C_{1}(\varepsilon) R^{-\frac{1}{2}}\left(x^{*}, t\right)\right)$. Clearly, without loss of generality, we may assume (in the definition of canonical neighborhood assumption) that $\sigma^{*}>2 R^{-\frac{1}{2}}\left(x^{*}, t\right)$. Then

$$
\begin{aligned}
R(1+t) & \geq \frac{1}{2} C_{2}^{-1}(\varepsilon) r^{-2} \\
& \geq \frac{1}{2} C_{2}^{-1}(\varepsilon) \rho^{-2}
\end{aligned}
$$

on $B_{t}\left(x^{*}, 2 r \sqrt{t}\right)$. Thus when we choose $\rho=\rho(w, \varepsilon)$ small enough, it follows from the pinching assumption that

$$
\begin{aligned}
R m & \geq-\left[f^{-1}(R(1+t)) /(R(1+t))\right] R \\
& \geq-\frac{1}{2}(r \sqrt{t})^{-2},
\end{aligned}
$$

on $B_{t}\left(x^{*}, 2 r \sqrt{t}\right)$. This is a contradiction with (7.6.15).

We have seen that $t R(x, t) \geq \frac{1}{2} r^{-2}\left(\geq \frac{1}{2} \rho^{-2}\right)$. Since $r^{-2}>\theta^{2} h^{-2} t$ in this subcase, we conclude that for arbitrarily given $A<+\infty$,

$$
t R(x, t)>A^{2} \rho^{-2}
$$

as long as $t$ is large enough.

Let $B$, with $B_{t}(x, \sigma) \subset B \subset B_{t}(x, 2 \sigma)$, be the canonical $\varepsilon$-neck or $\varepsilon$-cap neighborhood of $(x, t)$. By the definition of the canonical neighborhood assumption, we have

$$
\begin{gathered}
0<\sigma<C_{1}(\varepsilon) R^{-\frac{1}{2}}(x, t), \\
C_{2}^{-1}(\varepsilon) R \leq R(x, t) \leq C_{2}(\varepsilon) R, \text { on } B,
\end{gathered}
$$

and

$$
\operatorname{Vol}_{t}(B) \leq \varepsilon \sigma^{3} \leq \frac{1}{2} w \sigma^{3} .
$$

Choose $0<A<C_{1}(\varepsilon)$ so that $\sigma=A R^{-\frac{1}{2}}(x, t)$. For sufficiently large $t$, since

$$
\begin{aligned}
R(1+t) & \geq C_{2}^{-1}(\varepsilon)(t R(x, t)) \\
& \geq \frac{1}{2} C_{2}^{-1}(\varepsilon) \rho^{-2},
\end{aligned}
$$


on $B$, we can require $\rho=\rho(w, \varepsilon)$ to be smaller still, and use the pinching assumption to conclude

$$
\begin{aligned}
R m & \geq-\left[f^{-1}(R(1+t)) /(R(1+t))\right] R \\
& \geq-\left(A R^{-\frac{1}{2}}(x, t)\right)^{-2} \\
& =-\sigma^{-2},
\end{aligned}
$$

on $B$. For sufficiently large $t$, we adjust

$$
\begin{aligned}
r & =\sigma(\sqrt{t})^{-1} \\
& =\left(A R^{-\frac{1}{2}}(x, t)\right)(\sqrt{t})^{-1} \\
& <\rho,
\end{aligned}
$$

by (7.6.16). Then the combination of (7.6.17), (7.6.18) and (7.6.19) implies that $x \in M_{\text {thin }}(w, t)$.

The last statement in (b) follows directly from Lemma 7.6.2. (Here we also used Bishop-Gromov volume comparison, Theorem 7.5.2 and Hamilton's compactness theorem to take a subsequent limit.)

Therefore we have completed the proof of the theorem.

To state the long-time behavior of a solution to the Ricci flow with surgery, we first recall some basic terminology in three-dimensional topology. A three-manifold $M$ is called irreducible if every smooth two-sphere embedded in $M$ bounds a threeball in $M$. If we have a solution $\left(M_{t}, g_{i j}(t)\right)$ obtained by Theorem 7.4.3 with a compact, orientable and irreducible three-manifold $\left(M, g_{i j}\right)$ as initial data, then at each time $t>0$, by the cutoff surgery procedure, the solution manifold $M_{t}$ consists of a finite number of components where one of the components, called the essential component and denoted by $M_{t}^{(1)}$, is diffeomorphic to the initial manifold $M$ while the rest are diffeomorphic to the three-sphere $\mathbb{S}^{3}$.

The main result of this section is the following generalization of Theorem 5.3.4. A more general version of the result (without the restriction on $\varepsilon$ ) was implicitly claimed by Perelman in [104].

TheOrem 7.6.4 (Long-time behavior of the Ricci flow with surgery). Let $w>$ 0 and $0<\varepsilon \leq \frac{1}{2} w$ be any small positive constants and let $\left(M_{t}, g_{i j}(t)\right), 0<t<$ $+\infty$, be a solution to the Ricci flow with surgery, constructed by Theorem 7.4.3 with the nonincreasing (continuous) positive functions $\widetilde{\delta}(t)$ and $\widetilde{r}(t)$ and with a compact, orientable, irreducible and normalized three-manifold $M$ as initial data, where each $\delta$-cutoff at a time $t$ has $\delta=\delta(t) \leq \min \{\widetilde{\delta}(t), \widetilde{r}(2 t)\}$. Then one of the following holds: either

(i) for all sufficiently large $t$, we have $M_{t}=M_{\text {thin }}(w, t)$; or

(ii) there exists a sequence of times $t^{\alpha} \rightarrow+\infty$ such that the scalings of $g_{i j}\left(t^{\alpha}\right)$ on the essential component $M_{t^{\alpha}}^{(1)}$, with factor $\left(t^{\alpha}\right)^{-1}$, converge in the $C^{\infty}$ topology to a hyperbolic metric on the initial compact manifold $M$ with constant sectional curvature $-\frac{1}{4}$; or

(iii) we can find a finite collection of complete noncompact hyperbolic threemanifolds $\mathcal{H}_{1}, \ldots, \mathcal{H}_{m}$, with finite volume, and compact subsets $K_{1}, \ldots, K_{m}$ of $\mathcal{H}_{1}, \ldots, \mathcal{H}_{m}$ respectively obtained by truncating each cusp of the hyperbolic manifolds along constant mean curvature torus of small area, and for all $t$ beyond some time $T<+\infty$ we can find diffeomorphisms $\varphi_{l}, 1 \leq l \leq m$, of $K_{l}$ 
into $M_{t}$ so that as long as $t$ is sufficiently large, the metric $t^{-1} \varphi_{l}^{*}(t) g_{i j}(t)$ is as close to the hyperbolic metric as we like on the compact sets $K_{1}, \ldots, K_{m}$; moreover, the complement $M_{t} \backslash\left(\varphi_{1}\left(K_{1}\right) \cup \cdots \cup \varphi_{m}\left(K_{m}\right)\right)$ is contained in the thin part $M_{\mathrm{thin}}(w, t)$, and the boundary tori of each $K_{l}$ are incompressible in the sense that each $\varphi_{l}$ injects $\pi_{1}\left(\partial K_{l}\right)$ into $\pi_{1}\left(M_{t}\right)$.

Proof. The proof of the theorem follows, with some modifications, essentially from the same argument of Hamilton as in the proof of Theorem 5.3.4.

Clearly we may assume that the thick part $M_{\text {thick }}(w, t)$ is not empty for a sequence $t^{\alpha} \rightarrow+\infty$, since otherwise we have case (i). If we take a sequence of points $x^{\alpha} \in$ $M_{\text {thick }}\left(w, t^{\alpha}\right)$, then by Theorem 7.6.3(b) the scalings of $g_{i j}\left(t^{\alpha}\right)$ around $x^{\alpha}$ with factor $\left(t^{\alpha}\right)^{-1}$ converge smoothly, along a subsequence of $\alpha \rightarrow+\infty$, to a complete hyperbolic manifold of finite volume with constant sectional curvature $-\frac{1}{4}$. The limits may be different for different choices of $\left(x^{\alpha}, t^{\alpha}\right)$. If a limit is compact, we have case (ii). Thus we assume that all limits are noncompact.

Consider all the possible hyperbolic limits of the solution, and among them choose one such complete noncompact hyperbolic three-manifold $\mathcal{H}$ with the least possible number of cusps. Denote by $h_{i j}$ the hyperbolic metric of $\mathcal{H}$. For all small $a>0$ we can truncate each cusp of $\mathcal{H}$ along a constant mean curvature torus of area $a$ which is uniquely determined; we denote the remainder by $\mathcal{H}_{a}$. Fix $a>0$ so small that Lemma 5.3.7 is applicable for the compact set $\mathcal{K}=\mathcal{H}_{a}$. Pick an integer $l_{0}$ sufficiently large and an $\epsilon_{0}$ sufficiently small to guarantee from Lemma 5.3.8 that the identity map $I d$ is the only harmonic map $F$ from $\mathcal{H}_{a}$ to itself with taking $\partial \mathcal{H}_{a}$ to itself, with the normal derivative of $F$ at the boundary of the domain normal to the boundary of the target, and with $d_{C^{l_{0}\left(\mathcal{H}_{a}\right)}}(F, I d)<\epsilon_{0}$. Then choose a positive integer $q_{0}$ and a small number $\delta_{0}>0$ from Lemma 5.3.7 such that if $\widetilde{F}$ is a diffeomorphism of $\mathcal{H}_{a}$ into another complete noncompact hyperbolic three-manifold $\left(\widetilde{\mathcal{H}}, \widetilde{h}_{i j}\right)$ with no fewer cusps (than $\mathcal{H}$ ), of finite volume and satisfying

$$
\left\|\widetilde{F}^{*} \widetilde{h}_{i j}-h_{i j}\right\|_{C^{q_{0}}\left(\mathcal{H}_{a}\right)} \leq \delta_{0}
$$

then there exists an isometry $I$ of $\mathcal{H}$ to $\widetilde{\mathcal{H}}$ such that

$$
d_{C^{l_{0}\left(\mathcal{H}_{a}\right)}}(\widetilde{F}, I)<\epsilon_{0} .
$$

By Lemma 5.3.8 we further require $q_{0}$ and $\delta_{0}$ to guarantee the existence of a harmonic diffeomorphism from $\left(\mathcal{H}_{a}, \widetilde{g}_{i j}\right)$ to $\left(\mathcal{H}_{a}, h_{i j}\right)$ for any metric $\widetilde{g}_{i j}$ on $\mathcal{H}_{a}$ with $\left\|\widetilde{g}_{i j}-h_{i j}\right\|_{C^{q_{0}}\left(\mathcal{H}_{a}\right)} \leq \delta_{0}$.

Let $x^{\alpha} \in M_{\text {thick }}\left(w, t^{\alpha}\right), t^{\alpha} \rightarrow+\infty$, be a sequence of points such that the scalings of $g_{i j}\left(t^{\alpha}\right)$ around $x^{\alpha}$ with factor $\left(t^{\alpha}\right)^{-1}$ converge to $h_{i j}$. Then there exist a marked point $x^{\infty} \in \mathcal{H}_{a}$ and a sequence of diffeomorphisms $F_{\alpha}$ from $\mathcal{H}_{a}$ into $M_{t^{\alpha}}$ such that $F_{\alpha}\left(x^{\infty}\right)=x^{\alpha}$ and

$$
\left\|\left(t^{\alpha}\right)^{-1} F_{\alpha}^{*} g_{i j}\left(t^{\alpha}\right)-h_{i j}\right\|_{C^{m}\left(\mathcal{H}_{a}\right)} \rightarrow 0
$$

as $\alpha \rightarrow \infty$ for all positive integers $m$. By applying Lemma 5.3 .8 and the implicit function theorem, we can change $F_{\alpha}$ by an amount which goes to zero as $\alpha \rightarrow \infty$ so as to make $F_{\alpha}$ a harmonic diffeomorphism taking $\partial \mathcal{H}_{a}$ to a constant mean curvature hypersurface $F_{\alpha}\left(\partial \mathcal{H}_{a}\right)$ of $\left(M_{t^{\alpha}},\left(t^{\alpha}\right)^{-1} g_{i j}\left(t^{\alpha}\right)\right)$ with the area $a$ and satisfying the free boundary condition that the normal derivative of $F_{\alpha}$ at the boundary of the domain is normal to the boundary of the target; and by combining with Lemma 7.6.2 (iii), 
we can smoothly continue each harmonic diffeomorphism $F_{\alpha}$ forward in time a little to a family of harmonic diffeomorphisms $F_{\alpha}(t)$ from $\mathcal{H}_{a}$ into $M_{t}$ with the metric $t^{-1} g_{i j}(t)$, with $F_{\alpha}\left(t^{\alpha}\right)=F_{\alpha}$ and with the time $t$ slightly larger than $t^{\alpha}$, where $F_{\alpha}(t)$ takes $\partial \mathcal{H}_{a}$ into a constant mean curvature hypersurface of $\left(M_{t}, t^{-1} g_{i j}(t)\right)$ with the area $a$ and also satisfies the free boundary condition. Moreover, since the surgeries do not take place at the points where the scalar curvature is negative, by the same argument as in Theorem 5.3.4 for an arbitrarily given positive integer $q \geq q_{0}$, positive number $\delta<\delta_{0}$, and sufficiently large $\alpha$, we can ensure the extension $F_{\alpha}(t)$ satisfies $\left\|t^{-1} F_{\alpha}^{*}(t) g_{i j}(t)-h_{i j}\right\|_{C^{q}\left(\mathcal{H}_{a}\right)} \leq \delta$ on a maximal time interval $t^{\alpha} \leq t \leq \omega^{\alpha}$ (or $t^{\alpha} \leq$ $t<\omega^{\alpha}$ when $\left.\omega^{\alpha}=+\infty\right)$, and with $\left\|\left(\omega^{\alpha}\right)^{-1} F_{\alpha}^{*}\left(\omega^{\alpha}\right) g_{i j}\left(\omega^{\alpha}\right)-h_{i j}\right\|_{C^{q}\left(\mathcal{H}_{a}\right)}=\delta$, when $\omega^{\alpha}<+\infty$. Here we have implicitly used the fact that $F_{\alpha}\left(\omega^{\alpha}\right)\left(\partial \mathcal{H}_{a}\right)$ is still strictly concave to ensure the map $F_{\alpha}\left(\omega^{\alpha}\right)$ is diffeomorphic.

We further claim that there must be some $\alpha$ such that $\omega^{\alpha}=+\infty$; in other words, at least one hyperbolic piece persists. Indeed, suppose that for each large enough $\alpha$ we can only continue the family $F_{\alpha}(t)$ on a finite interval $t^{\alpha} \leq t \leq \omega^{\alpha}<+\infty$ with

$$
\left\|\left(\omega^{\alpha}\right)^{-1} F_{\alpha}^{*}\left(\omega^{\alpha}\right) g_{i j}\left(\omega^{\alpha}\right)-h_{i j}\right\|_{C^{q}\left(\mathcal{H}_{a}\right)}=\delta .
$$

Consider the new sequence of manifolds $\left(M_{\omega^{\alpha}}, g_{i j}\left(\omega^{\alpha}\right)\right)$. Clearly by Lemma 7.6.1, the scalings of $g_{i j}\left(\omega^{\alpha}\right)$ around the new origins $F_{\alpha}\left(\omega^{\alpha}\right)\left(x^{\infty}\right)$ with factor $\left(\omega^{\alpha}\right)^{-1}$ converge smoothly (by passing to a subsequence) to a complete noncompact hyperbolic threemanifold $\widetilde{\mathcal{H}}$ with the metric $\widetilde{h}_{i j}$ and the origin $\widetilde{x}^{\infty}$ and with finite volume. By the choice of the old limit $\mathcal{H}$, the new limit $\widetilde{\mathcal{H}}$ has at least as many cusps as $\mathcal{H}$. By the definition of convergence, we can find a sequence of compact subsets $\widetilde{U}_{\alpha}$ exhausting $\widetilde{\mathcal{H}}$ and containing $\widetilde{x}^{\infty}$, and a sequence of diffeomorphisms $\widetilde{F}_{\alpha}$ of neighborhood of $\widetilde{U}_{\alpha}$ into $M_{\omega^{\alpha}}$ with $\widetilde{F}_{\alpha}\left(\widetilde{x}^{\infty}\right)=F_{\alpha}\left(\omega^{\alpha}\right)\left(x^{\infty}\right)$ such that for each compact subset $\widetilde{U}$ of $\widetilde{\mathcal{H}}$ and each integer $m$,

$$
\left\|\left(\omega^{\alpha}\right)^{-1} \widetilde{F}_{\alpha}^{*}\left(g_{i j}\left(\omega^{\alpha}\right)\right)-\widetilde{h}_{i j}\right\|_{C^{m}(\widetilde{U})} \rightarrow 0
$$

as $\alpha \rightarrow+\infty$. Thus for sufficiently large $\alpha$, we have the map

$$
G_{\alpha}=\widetilde{F}_{\alpha}^{-1} \circ F_{\alpha}\left(\omega^{\alpha}\right): \mathcal{H}_{a} \rightarrow \widetilde{\mathcal{H}}
$$

such that

$$
\left\|G_{\alpha}^{*} \widetilde{h}_{i j}-h_{i j}\right\|_{C^{q}\left(\mathcal{H}_{a}\right)}<\widetilde{\delta}
$$

for any fixed $\widetilde{\delta}>\delta$. Then a subsequence of $G_{\alpha}$ converges at least in the $C^{q-1}\left(\mathcal{H}_{a}\right)$ topology to a map $G_{\infty}$ of $\mathcal{H}_{a}$ into $\widetilde{\mathcal{H}}$ which is a harmonic map from $\mathcal{H}_{a}$ into $\widetilde{\mathcal{H}}$ and takes $\partial \mathcal{H}_{a}$ to a constant mean curvature hypersurface $G_{\infty}\left(\partial \mathcal{H}_{a}\right)$ of $\left(\widetilde{\mathcal{H}}, \widetilde{h}_{i j}\right)$ with the area $a$, as well as satisfies the free boundary condition. Clearly, $G_{\infty}$ is at least a local diffeomorphism. Since $G_{\infty}$ is the limit of diffeomorphisms, the only possibility of overlap is at the boundary. Note that $G_{\infty}\left(\partial \mathcal{H}_{a}\right)$ is still strictly concave. So $G_{\infty}$ is still a diffeomorphism. Moreover by using the standard regularity result of elliptic partial differential equations (see for example [48]), we also have

$$
\left\|G_{\infty}^{*} \widetilde{h}_{i j}-h_{i j}\right\|_{C^{q}\left(\mathcal{H}_{a}\right)}=\delta .
$$

Now by Lemma 5.3.7 we deduce that there exists an isometry $I$ of $\mathcal{H}$ to $\widetilde{\mathcal{H}}$ with

$$
d_{C^{l_{0}\left(\mathcal{H}_{a}\right)}}\left(G_{\infty}, I\right)<\epsilon_{0} .
$$


Thus $I^{-1} \circ G_{\infty}$ is a harmonic diffeomorphism of $\mathcal{H}_{a}$ to itself which satisfies the free boundary condition and

$$
d_{C^{l_{0}\left(\mathcal{H}_{a}\right)}}\left(I^{-1} \circ G_{\infty}, I d\right)<\epsilon_{0} .
$$

However the uniqueness in Lemma 5.3.8 concludes that $I^{-1} \circ G_{\infty}=I d$ which contradicts (7.6.20). So we have shown that at least one hyperbolic piece persists and the metric $t^{-1} F_{\alpha}^{*}(t) g_{i j}(t)$, for $\omega^{\alpha} \leq t<\infty$, is as close to the hyperbolic metric $h_{i j}$ as we like.

We can continue to form other persistent hyperbolic pieces in the same way as long as there is a sequence of points $y^{\beta} \in M_{\text {thick }}\left(w, t^{\beta}\right), t^{\beta} \rightarrow+\infty$, lying outside the chosen pieces. Note that $V(t)\left(t+\frac{3}{2}\right)^{-\frac{3}{2}}$ is nonincreasing on $[0,+\infty)$. Therefore by combining with Margulis lemma (see for example [55] or [76]), we have proved that there exists a finite collection of complete noncompact hyperbolic three-manifolds $\mathcal{H}_{1}, \ldots, \mathcal{H}_{m}$ with finite volume, a small number $a>0$ and a time $T<+\infty$ such that for all $t$ beyond $T$ we can find diffeomorphisms $\varphi_{l}(t)$ of $\left(\mathcal{H}_{l}\right)_{a}$ into $M_{t}, 1 \leq l \leq m$, so that as long as $t$ is sufficiently large, the metric $t^{-1} \varphi_{l}^{*}(t) g_{i j}(t)$ is as close to the hyperbolic metrics as we like and the complement $M_{t} \backslash\left(\varphi_{1}(t)\left(\left(\mathcal{H}_{1}\right)_{a}\right) \cup \cdots \cup \varphi_{m}(t)\left(\left(\mathcal{H}_{m}\right)_{a}\right)\right)$ is contained in the thin part $M_{\text {thin }}(w, t)$.

It remains to show the boundary tori of any persistent hyperbolic piece are incompressible. Let $B$ be a small positive number and assume the above positive number $a$ is much smaller than $B$. Let $M_{a}(t)=\varphi_{l}(t)\left(\left(\mathcal{H}_{l}\right)_{a}\right)(1 \leq l \leq m)$ be such a persistent hyperbolic piece of the manifold $M_{t}$ truncated by boundary tori of area at with constant mean curvature, and denote by $M_{a}^{c}(t)=M_{t} \backslash \stackrel{\circ}{M_{a}}(t)$ the part of $M_{t}$ exterior to $M_{a}(t)$. Thus there exists a family of subsets $M_{B}(t) \subset M_{a}(t)$ which is a persistent hyperbolic piece of the manifold $M_{t}$ truncated by boundary tori of area $B t$ with constant mean curvature. We also denote by $M_{B}^{c}(t)=M_{t} \backslash \stackrel{\circ}{M_{B}}(t)$. By Van Kampen's Theorem, if $\pi_{1}\left(\partial M_{B}(t)\right)$ injects into $\pi_{1}\left(M_{B}^{c}(t)\right)$ then it injects into $\pi_{1}\left(M_{t}\right)$ also. Thus we only need to show $\pi_{1}\left(\partial M_{B}(t)\right)$ injects into $\pi_{1}\left(M_{B}^{c}(t)\right)$.

As before we will use a contradiction argument to show $\pi_{1}\left(\partial M_{B}(t)\right)$ injects into $\pi_{1}\left(M_{B}^{c}(t)\right)$. Let $T$ be a torus in $\partial M_{B}(t)$ and suppose $\pi_{1}(T)$ does not inject into $\pi_{1}\left(M_{B}^{c}(t)\right)$. By Dehn's Lemma we know that the kernel is a cyclic subgroup of $\pi_{1}(T)$ generated by a primitive element. Consider the normalized metric $\widetilde{g}_{i j}(t)=t^{-1} g_{i j}(t)$ on $M_{t}$. Then by the work of Meeks-Yau [86] or Meeks-Simon-Yau [87], we know that among all disks in $M_{B}^{c}(t)$ whose boundary curve lies in $T$ and generates the kernel of $\pi_{1}(T)$, there is a smooth embedded disk normal to the boundary which has the least possible area (with respect to the normalized metric $\widetilde{g}_{i j}(t)$ ). Denote by $D$ the minimal disk and $\widetilde{A}=\widetilde{A}(t)$ its area. We will show that $\widetilde{A}(t)$ decreases at a certain rate which will arrive at a contradiction.

We first consider the case that there exist no surgeries at the time $t$. Exactly as in Part III of the proof of Theorem 5.3.4, the change of the area $\widetilde{A}(t)$ comes from the change in the metric and the change in the boundary. For the change in the metric, we choose an orthonormal frame $X, Y, Z$ at a point $x$ in the disk $D$ so that $X$ and $Y$ are tangent to the disk $D$ while $Z$ is normal. Since the normalized metric $\widetilde{g}_{i j}$ evolves by

$$
\frac{\partial}{\partial t} \widetilde{g}_{i j}=-t^{-1}\left(\widetilde{g}_{i j}+2 \widetilde{R}_{i j}\right)
$$


the (normalized) area element $d \widetilde{\sigma}$ of the disk $D$ around $x$ satisfies

$$
\frac{\partial}{\partial t} d \widetilde{\sigma}=-t^{-1}(1+\widetilde{\operatorname{Ric}}(X, X)+\widetilde{\operatorname{Ric}}(Y, Y)) d \widetilde{\sigma}
$$

For the change in the boundary, we notice that the tensor $\widetilde{g}_{i j}+2 \widetilde{R}_{i j}$ is very small for the persistent hyperbolic piece. Then by using the Gauss-Bonnet theorem as before, we obtain the rate of change of the area

$$
\frac{d \widetilde{A}}{d t} \leq-\int_{D}\left(\frac{1}{t}+\frac{\widetilde{R}}{2 t}\right) d \widetilde{\sigma}+\frac{1}{t} \int_{\partial D} \widetilde{k} d \widetilde{s}-\frac{2 \pi}{t}+o\left(\frac{1}{t}\right) \widetilde{L},
$$

where $\widetilde{k}$ is the geodesic curvature of the boundary and $\widetilde{L}$ is the length of the boundary curve $\partial D$ (with respect to the normalized metric $\widetilde{g}_{i j}(t)$ ). Since $\widetilde{R} \geq-3 t / 2\left(t+\frac{3}{2}\right)$ for all $t \geq 0$ by (7.6.2), the first term on the RHS of (7.6.21) is bounded above by

$$
-\int_{D}\left(\frac{1}{t}+\frac{\widetilde{R}}{2 t}\right) d \widetilde{\sigma} \leq-\frac{1}{t}\left(\frac{1}{4}-o(1)\right) \widetilde{A}
$$

while the second term on the RHS of (7.6.21) can be estimated exactly as before by

$$
\frac{1}{t} \int_{\partial D} \widetilde{k} d \widetilde{s} \leq \frac{1}{t}\left(\frac{1}{4}+o(1)\right) \widetilde{L}
$$

Thus we obtain

$$
\frac{d \widetilde{A}}{d t} \leq \frac{1}{t}\left[\left(\frac{1}{4}+o(1)\right) \widetilde{L}-\left(\frac{1}{4}-o(1)\right) \widetilde{A}-2 \pi\right] .
$$

Next we show that these arguments also work for the case that there exist surgeries at the time $t$. To this end, we only need to check that the embedded minimal disk $D$ lies in the region which is unaffected by surgery. Our surgeries for the irreducible three-manifold took place on $\delta$-necks in $\varepsilon$-horns, where the scalar curvatures are at least $\delta^{-2}(\widetilde{r}(t))^{-1}$, and the components with nonnegative scalar curvature have been removed. So the hyperbolic piece is not affected by the surgeries. In particular, the boundary $\partial D$ is unaffected by the surgeries. Thus if surgeries occur on the minimal disk, the minimal disk has to pass through a long thin neck before it reaches the surgery regions. Look at the intersections of the embedding minimal disk with a generic center two-sphere $\mathbb{S}^{2}$ of the long thin neck; these are circles. Since the twosphere $\mathbb{S}^{2}$ is simply connected, we can replace the components of the minimal disk $D$ outside the center two-sphere $\mathbb{S}^{2}$ by some corresponding components on the center two-sphere $\mathbb{S}^{2}$ to form a new disk which also has $\partial D$ as its boundary. Since the metric on the long thin neck is nearly a product metric, we could choose the generic center two-sphere $\mathbb{S}^{2}$ properly so that the area of the new disk is strictly less than the area of the original disk $D$. This contradiction proves the minimal disk lies entirely in the region unaffected by surgery.

Since $a$ is much smaller than $B$, the region within a long distance from $\partial M_{B}(t)$ into $M_{B}^{c}(t)$ will look nearly like a hyperbolic cusplike collar and is unaffected by the surgeries. So we can repeat the arguments in the last part of the proof of Theorem 5.3.4 to bound the length $\widetilde{L}$ by the area $\widetilde{A}$ and to conclude

$$
\frac{d \widetilde{A}}{d t} \leq-\frac{\pi}{t}
$$


for all sufficiently large times $t$, which is impossible because the RHS is not integrable. This proves that the boundary tori of any persistent hyperbolic piece are incompressible.

Therefore we have proved the theorem. $\mathrm{Q}$

7.7. Geometrization of Three-manifolds. In the late 70's and early 80's, Thurston [122], [123] [124] proved a number of remarkable results on the existence of geometric structures on a class of three-manifolds: Haken manifolds (i.e. each of them contains an incompressible surface of genus $\geq 1$ ). These results motivated him to formulate a profound conjecture which roughly says every compact three-manifold admits a canonical decomposition into domains, each of which has a canonical geometric structure. To give a detailed description of the conjecture, we recall some terminology as follows.

An $n$-dimensional complete Riemannian manifold $(M, g)$ is called a homogeneous manifold if its group of isometries acts transitively on the manifold. This means that the homogeneous manifold looks the same metrically at everypoint. For example, the round $n$-sphere $\mathbb{S}^{n}$, the Euclidean space $\mathbb{R}^{n}$ and the standard hyperbolic space $\mathbb{H}^{n}$ are homogeneous manifolds. A Riemannian manifold is said to be modeled on a given homogeneous manifold $(M, g)$ if every point of the manifold has a neighborhood isometric to an open set of $(M, g)$. And an $n$-dimensional Riemannian manifold is called a locally homogeneous manifold if it is complete and is modeled on a homogeneous manifold. By a theorem of Singer [119], the universal cover of a locally homogeneous manifold (with the pull-back metric) is a homogeneous manifold.

In dimension three, every locally homogeneous manifold with finite volume is modeled on one of the following eight homogeneous manifolds (see for example Theorem 3.8.4 of [125]):

(1) $\mathbb{S}^{3}$, the round three-sphere;

(2) $\mathbb{R}^{3}$, the Euclidean space ;

(3) $\mathbb{H}^{3}$, the standard hyperbolic space;

(4) $\mathbb{S}^{2} \times \mathbb{R}$

(5) $\mathbb{H}^{2} \times \mathbb{R}$;

(6) Nil, the three-dimensional nilpotent Heisenberg group (consisting of upper triangular $3 \times 3$ matrices with diagonal entries 1 );

(7) $\widetilde{P S L}(2, \mathbb{R})$, the universal cover of the unit sphere bundle of $\mathbb{H}^{2}$;

(8) Sol, the three-dimensional solvable Lie group.

A three-manifold $M$ is called prime if it is not diffeomorphic to $\mathbb{S}^{3}$ and if every (topological) $\mathbb{S}^{2} \subset M$, which separates $M$ into two pieces, has the property that one of the two pieces is diffeomorphic to a three-ball. Recall that a three-manifold is irreducible if every embedded two-sphere bounds a three-ball in the manifold. Clearly an irreducible three-manifold is either prime or is diffeomorphic to $\mathbb{S}^{3}$. Conversely, an orientable prime three-manifold is either irreducible or is diffeomorphic to $\mathbb{S}^{2} \times \mathbb{S}^{1}$ (see for example [69]). One of the first results in three-manifold topology is the following prime decomposition theorem obtained by Kneser [79] in 1929 (see also Theorem 3.15 of [69]).

Prime Decomposition Theorem. Every compact orientable three-manifold admits a decomposition as a finite connected sum of orientable prime three-manifolds.

In [90], Milnor showed that the factors involved in the above Prime Decomposition are unique. Based on the prime decomposition, the question about topology 
of compact orientable three-manifolds is reduced to the question about prime threemanifolds. Thurston's Geometrization Conjecture is about prime three-manifolds.

Thurston's Geometrization Conjecture. Let $M$ be a compact, orientable and prime three-manifold. Then there is an embedding of a finite number of disjoint unions, possibly empty, of incompressible two-tori $\bigsqcup_{i} T_{i}^{2} \subset M$ such that every component of the complement admits a locally homogeneous Riemannian metric of finite volume.

We remark that the existence of a torus decomposition, also called JSJdecomposition, was already obtained by Jaco-Shalen [74] and Johannsen [75]. The JSJ-decomposition states that any compact, orientable, and prime three-manifold has a finite collection, possibly empty, of disjoint incompressible embedding two-tori $\left\{T_{i}^{2}\right\}$ which separate the manifold into a finite collection of compact three-manifolds (with toral boundary), each of which is either a graph manifold or is atoroidal in the sense that any subgroup of its fundamental group isomorphic to $\mathbb{Z} \times \mathbb{Z}$ is conjugate into the fundamental group of some component of its boundary. A compact three-manifold $X$, possibly with boundary, is called a graph manifold if there is a finite collection of disjoint embedded tori $T_{i} \subset X$ such that each component of $X \backslash \bigcup T_{i}$ is an $\mathbb{S}^{1}$ bundle over a surface. Thus the point of the conjecture is that the components should all be geometric.

The geometrization conjecture for a general compact orientable 3-manifold is the statement that each of its prime factors satisfies the above conjecture. We say a compact orientable three-manifold is geometrizable if it satisfies the geometric conjecture.

We also remark that the Poincaré conjecture can be deduced from Thurston's geometrization conjecture. Indeed, suppose that we have a compact simply connected three-manifold that satisfies the conclusion of the geometrization conjecture. If it were not diffeomorphic to the three-sphere $\mathbb{S}^{3}$, there would be a prime factor in the prime decomposition of the manifold. Since the prime factor still has vanishing fundamental group, the (torus) decomposition of the prime factor in the geometrization conjecture must be trivial. Thus the prime factor is a compact homogeneous manifold model. From the list of above eight models, we see that the only compact three-dimensional model is $\mathbb{S}^{3}$. This is a contradiction. Consequently, the compact simply connected three-manifold is diffeomorphic to $\mathbb{S}^{3}$.

Now we apply the Ricci flow to discuss Thurston's geometrization conjecture. Let $M$ be a compact, orientable and prime three-manifold. Since a prime orientable three-manifold is either irreducible or is diffeomorphic to $\mathbb{S}^{2} \times \mathbb{S}^{1}$, we may thus assume the manifold $M$ is irreducible also. Arbitrarily given a (normalized) Riemannian metric for the manifold $M$, we use it as initial data to evolve the metric by the Ricci flow with surgery. From Theorem 7.4.3, we know that the Ricci flow with surgery has a long-time solution on a maximal time interval $[0, T)$ which satisfies the a priori assumptions and has a finite number of surgeries on each finite time interval. Furthermore, from the long-time behavior theorem (Theorem 7.6.4), we have wellunderstood geometric structures on the thick part. Whereas, to understand the thin part, Perelman announced the following assertion in [104].

Perelman's Claim ([104]). Suppose $\left(M^{\alpha}, g_{i j}^{\alpha}\right)$ is a sequence of compact orientable three-manifolds, closed or with convex boundary, and $w^{\alpha} \rightarrow 0$. Assume that

(1) for each point $x \in M^{\alpha}$ there exists a radius $\rho=\rho^{\alpha}(x), 0<\rho<1$, not exceeding the diameter of the manifold, such that the ball $B(x, \rho)$ in the 
metric $g_{i j}^{\alpha}$ has volume at most $w^{\alpha} \rho^{3}$ and sectional curvatures at least $-\rho^{-2}$;

(2) each component of the boundary of $M^{\alpha}$ has diameter at most $w^{\alpha}$, and has a (topologically trivial) collar of length one, where the sectional curvatures are between $-1 / 4-\epsilon$ and $-1 / 4+\epsilon$.

Then $M^{\alpha}$ for sufficiently large $\alpha$ are diffeomorphic to graph manifolds.

The topology of graph manifolds is well understood; in particular, every graph manifold is geometrizable (see [126]).

The proof of Perelman's Claim promised in [104] is still not available in literature. Nevertheless, recently in [118], Shioya and Yamaguchi provided a proof of Perelman's Claim for the special case that all the manifolds $\left(M^{\alpha}, g_{i j}^{\alpha}\right)$ are closed. That is, they proposed a proof for the following weaker assertion.

Weaker Assertion (Theorem 8.1 of Shioya-Yamaguchi [118]). Suppose $\left(M^{\alpha}, g_{i j}^{\alpha}\right)$ is a sequence of compact orientable three-manifolds without boundary, and $w^{\alpha} \rightarrow 0$. Assume that for each point $x \in M^{\alpha}$ there exists a radius $\rho=\rho^{\alpha}(x)$, not exceeding the diameter of the manifold, such that the ball $B(x, \rho)$ in the metric $g_{i j}^{\alpha}$ has volume at most $w^{\alpha} \rho^{3}$ and sectional curvatures at least $-\rho^{-2}$. Then $M^{\alpha}$ for sufficiently large $\alpha$ are diffeomorphic to graph manifolds.

Based on the the long-time behavior theorem (Theorem 7.6.4) and assuming the above Weaker Assertion, we can now give a proof for Thurston's geometrization conjecture. We remark that if we assume the above Perelman's Claim, then we does not need to use Thurston's theorem for Haken manifolds in the proof of Theorem 7.7.1.

THEOREM 7.7.1. Thurston's geometrization conjecture is true.

Proof. Let $M$ be a compact, orientable, and prime three-manifold (without boundary). Without loss of generality, we may assume that the manifold $M$ is irreducible also.

Recall that the theorem of Thurston (see for example Theorem A and Theorem B in Section 3 of [94], see also [85] and [102]) says that any compact, orientable, and irreducible Haken three-manifold (with or without boundary) is geometrizable. Thus in the following, we may assume that the compact three-manifold $M$ (without boundary) is atoroidal, and then the fundamental group $\pi_{1}(M)$ contains no noncyclic, abelian subgroup.

Arbitrarily given a (normalized) Riemannian metric on the manifold $M$, we use it as initial data for the Ricci flow. Arbitrarily take a sequence of small positive constants $w^{\alpha} \rightarrow 0$ as $\alpha \rightarrow+\infty$. For each fixed $\alpha$, we set $\varepsilon=w^{\alpha} / 2>0$. Then by Theorem 7.4 .3 , the Ricci flow with surgery has a long-time solution $\left(M_{t}^{\alpha}, g_{i j}^{\alpha}(t)\right)$ on a maximal time interval $\left[0, T^{\alpha}\right.$ ), which satisfies the a priori assumptions (with the accuracy parameter $\varepsilon=w^{\alpha} / 2$ ) and has a finite number of surgeries on each finite time interval. Since the initial manifold is irreducible, by the surgery procedure, we know that for each $\alpha$ and each $t>0$ the solution manifold $M_{t}^{\alpha}$ consists of a finite number of components where the essential component $\left(M_{t}^{\alpha}\right)^{(1)}$ is diffeomorphic to the initial manifold $M$ and the others are diffeomorphic to the three-sphere $\mathbb{S}^{3}$.

If for some $\alpha=\alpha_{0}$ the maximal time $T^{\alpha_{0}}$ is finite, then the solution $\left(M_{t}^{\alpha_{0}}, g_{i j}^{\alpha_{0}}(t)\right)$ becomes extinct at $T^{\alpha_{0}}$ and the (irreducible) initial manifold $M$ is diffeomorphic to $\mathbb{S}^{3} / \Gamma$ (the metric quotients of round three-sphere); in particular, the manifold $M$ is geometrizable. Thus we may assume that the maximal time $T^{\alpha}=+\infty$ for all $\alpha$.

We now apply the long-time behavior theorem (Theorem 7.6.4). If there is some $\alpha$ such that case (ii) of Theorem 7.6.4 occurs, then for some sufficiently large time 
$t$, the essential component $\left(M_{t}^{\alpha}\right)^{(1)}$ of the solution manifold $M_{t}^{\alpha}$ is diffeomorphic to a compact hyperbolic space, so the initial manifold $M$ is geometrizable. Whereas if there is some sufficiently large $\alpha$ such that case (iii) of Theorem 7.6.4 occurs, then it follows that for all sufficiently large $t$, there is an embedding of a (nonempty) finite number of disjoint unions of incompressible two-tori $\coprod_{i} T_{i}^{2}$ in the essential component $\left(M_{t}^{\alpha}\right)^{(1)}$ of $M_{t}^{\alpha}$. This is a contradiction since we have assumed the initial manifold $M$ is atoroidal.

It remains to deal with the situation that there is a sequence of positive $\alpha_{k} \rightarrow$ $+\infty$ such that the solutions $\left(M_{t}^{\alpha_{k}}, g_{i j}^{\alpha_{k}}(t)\right)$ always satisfy case (i) of Theorem 7.6.4. That is, for each $\alpha_{k}, M_{t}^{\alpha_{k}}=M_{\text {thin }}\left(w^{\alpha_{k}}, t\right)$ when the time $t$ is sufficiently large. By the Thick-thin decomposition theorem (Theorem 7.6.3), there is a positive constant, $0<\rho\left(w^{\alpha_{k}}\right) \leq 1$, such that as long as $t$ is sufficiently large, for every $x \in M_{t}^{\alpha_{k}}=$ $M_{\text {thin }}\left(w^{\alpha_{k}}, t\right)$, we have some $r=r(x, t)$, with $0<r \sqrt{t}<\rho\left(w^{\alpha_{k}}\right) \sqrt{t}$, such that

$$
R m \geq-(r \sqrt{t})^{-2} \quad \text { on } B_{t}(x, r \sqrt{t}),
$$

and

$$
\operatorname{Vol}_{t}\left(B_{t}(x, r \sqrt{t})\right)<w^{\alpha_{k}}(r \sqrt{t})^{3} .
$$

Clearly we only need to consider the essential component $\left(M_{t}^{\alpha_{k}}\right)^{(1)}$. We divide the discussion into the following two cases:

(1) there is a positive constant $1<C<+\infty$ such that for each $\alpha_{k}$ there is a sufficiently large time $t_{k}>0$ such that

$$
r\left(x, t_{k}\right) \sqrt{t_{k}}<C \cdot \operatorname{diam}\left(\left(M_{t_{k}}^{\alpha_{k}}\right)^{(1)}\right)
$$

for all $x \in\left(M_{t_{k}}^{\alpha_{k}}\right)^{(1)} \subset M_{\text {thin }}\left(w^{\alpha_{k}}, t_{k}\right)$;

(2) there are a subsequence $\alpha_{k}$ (still denoted by $\alpha_{k}$ ), and sequences of positive constants $C_{k} \rightarrow+\infty$ and times $T_{k}<+\infty$ such that for each $t \geq T_{k}$, we have

$$
r(x(t), t) \sqrt{t} \geq C_{k} \cdot \operatorname{diam}\left(\left(M_{t}^{\alpha_{k}}\right)^{(1)}\right)
$$

for some $x(t) \in\left(M_{t}^{\alpha_{k}}\right)^{(1)}, k=1,2, \ldots$ Here we denote by diam $\left(\left(M_{t}^{\alpha}\right)^{(1)}\right)$ the diameter of the essential component $\left(M_{t}^{\alpha}\right)^{(1)}$ with the metric $g_{i j}^{\alpha}(t)$ at the time $t$.

Let us first consider case (1). For each point $x \in\left(M_{t_{k}}^{\alpha_{k}}\right)^{(1)} \subset M_{\text {thin }}\left(w^{\alpha_{k}}, t_{k}\right)$, we denote by $\rho_{k}(x)=C^{-1} r\left(x, t_{k}\right) \sqrt{t_{k}}$. Then by (7.7.1), (7.7.2) and (7.7.3), we have

$$
\begin{gathered}
\rho_{k}(x)<\operatorname{diam}\left(\left(M_{t_{k}}^{\alpha_{k}}\right)^{(1)}\right), \\
\operatorname{Vol}_{t_{k}}\left(B_{t_{k}}\left(x, \rho_{k}(x)\right)\right) \leq \operatorname{Vol}_{t_{k}}\left(B_{t_{k}}\left(x, r\left(x, t_{k}\right) \sqrt{t_{k}}\right)\right)<C^{3} w^{\alpha_{k}}\left(\rho_{k}(x)\right)^{3},
\end{gathered}
$$

and

$$
R m \geq-\left(r\left(x, t_{k}\right) \sqrt{t_{k}}\right)^{-2} \geq-\left(\rho_{k}(x)\right)^{-2}
$$

on $B_{t_{k}}\left(x, \rho_{k}(x)\right)$. Then it follows from the above Weaker Assertion that $\left(M_{t_{k}}^{\alpha_{k}}\right)^{(1)}$, for sufficiently large $k$, are diffeomorphic to graph manifolds. This implies that the (irreducible) initial manifold $M$ is diffeomorphic to a graph manifold. So the manifold $M$ is geometrizable in case (1). 
We next consider case (2). Clearly, for each $\alpha_{k}$ and the chosen $T_{k}$, we may assume that the estimates (7.7.1) and (7.7.2) hold for all $t \geq T_{k}$ and $x \in\left(M_{t}^{\alpha_{k}}\right)^{(1)}$. The combination of (7.7.1) and (7.7.4) gives

$$
R m \geq-C_{k}^{-2}\left(\operatorname{diam}\left(\left(M_{t}^{\alpha_{k}}\right)^{(1)}\right)\right)^{-2} \quad \text { on }\left(M_{t}^{\alpha_{k}}\right)^{(1)},
$$

for all $t \geq T_{k}$. If there are a subsequence $\alpha_{k}$ (still denoted by $\alpha_{k}$ ) and a sequence of times $t_{k} \in\left(T_{k},+\infty\right)$ such that

$$
\operatorname{Vol}_{t_{k}}\left(\left(M_{t_{k}}^{\alpha_{k}}\right)^{(1)}\right)<w_{k}^{\prime}\left(\operatorname{diam}\left(\left(M_{t_{k}}^{\alpha_{k}}\right)^{(1)}\right)\right)^{3}
$$

for some sequence $w_{k}^{\prime} \rightarrow 0$, then it follows from the Weaker Assertion that $\left(M_{t_{k}}^{\alpha_{k}}\right)^{(1)}$, for sufficiently large $k$, are diffeomorphic to graph manifolds which implies the initial manifold $M$ is geometrizable. Thus we may assume that there is a positive constant $w^{\prime}$ such that

$$
\operatorname{Vol}_{t}\left(\left(M_{t}^{\alpha_{k}}\right)^{(1)}\right) \geq w^{\prime}\left(\operatorname{diam}\left(\left(M_{t}^{\alpha_{k}}\right)^{(1)}\right)\right)^{3}
$$

for each $k$ and all $t \geq T_{k}$.

In view of the estimates (7.7.5) and (7.7.7), we now want to use Theorem 7.5.2 to get a uniform upper bound for the curvatures of the essential components $\left(\left(M_{t}^{\alpha_{k}}\right)^{(1)}, g_{i j}^{\alpha_{k}}(t)\right)$ with sufficiently large time $t$. Note that the estimate in Theorem 7.5.2 depends on the parameter $\varepsilon$ and our $\varepsilon$ 's depend on $w^{\alpha_{k}}$ with $0<\varepsilon=w^{\alpha_{k}} / 2$; so it does not work in the present situation. Fortunately we notice that the curvature estimate for smooth solutions in Corollary 7.2.3 is independent of $\varepsilon$. In the following we try to use Corollary 7.2.3 to obtain the desired curvature estimate.

We first claim that for each $k$, there is a sufficiently large $T_{k}^{\prime} \in\left(T_{k},+\infty\right)$ such that the solution, when restricted to the essential component $\left(\left(M_{t}^{\alpha_{k}}\right)^{(1)}, g_{i j}^{\alpha_{k}}(t)\right)$, has no surgery for all $t \geq T_{k}^{\prime}$. Indeed, for each fixed $k$, if there is a $\delta(t)$-cutoff surgery at a sufficiently large time $t$, then the manifold $\left(\left(M_{t}^{\alpha_{k}}\right)^{(1)}, g_{i j}^{\alpha_{k}}(t)\right)$ would contain a $\delta(t)$-neck $B_{t}\left(y, \delta(t)^{-1} R(y, t)^{-\frac{1}{2}}\right)$ for some $y \in\left(M_{t}^{\alpha_{k}}\right)^{(1)}$ with the volume ratio

$$
\frac{\operatorname{Vol}_{t}\left(B_{t}\left(y, \delta(t)^{-1} R(y, t)^{-\frac{1}{2}}\right)\right)}{\left(\delta(t)^{-1} R(y, t)^{-\frac{1}{2}}\right)^{3}} \leq 8 \pi \delta(t)^{2} .
$$

On the other hand, by (7.7.5) and (7.7.7), the standard Bishop-Gromov volume comparison implies that

$$
\frac{\operatorname{Vol}_{t}\left(B_{t}\left(y, \delta(t)^{-1} R(y, t)^{-\frac{1}{2}}\right)\right)}{\left(\delta(t)^{-1} R(y, t)^{-\frac{1}{2}}\right)^{3}} \geq c\left(w^{\prime}\right)
$$

for some positive constant $c\left(w^{\prime}\right)$ depending only on $w^{\prime}$. Since $\delta(t)$ is very small when $t$ is large, this arrives at a contradiction with (7.7.8). So for each $k$, the essential component $\left(\left(M_{t}^{\alpha_{k}}\right)^{(1)}, g_{i j}^{\alpha_{k}}(t)\right)$ has no surgery for all sufficiently large $t$.

For each $k$, we consider any fixed time $\tilde{t}_{k}>3 T_{k}^{\prime}$. Let us scale the solution $g_{i j}^{\alpha_{k}}(t)$ on the essential component $\left(M_{t}^{\alpha_{k}}\right)^{(1)}$ by

$$
\tilde{g}_{i j}^{\alpha_{k}}(\cdot, s)=\left(\tilde{t}_{k}\right)^{-1} g_{i j}^{\alpha_{k}}\left(\cdot, \tilde{t}_{k} s\right) .
$$

Note that $\left(M_{t}^{\alpha_{k}}\right)^{(1)}$ is diffeomorphic to $M$ for all $t$. By the above claim, we see that the rescaled solution $\left(M, \tilde{g}_{i j}^{\alpha_{k}}(\cdot, s)\right)$ is a smooth solution to the Ricci flow on the time interval $s \in\left[\frac{1}{2}, 1\right]$. Set

$$
\tilde{r}_{k}=\left(\sqrt{\tilde{t}_{k}}\right)^{-1} \operatorname{diam}\left(\left(M_{\tilde{t}_{k}}^{\alpha_{k}}\right)^{(1)}\right) .
$$


Then by (7.7.4), (7.7.5) and (7.7.7), we have

$$
\begin{gathered}
\tilde{r}_{k} \leq C_{k}^{-1} \rightarrow 0, \quad \text { as } k \rightarrow+\infty, \\
\widetilde{R m} \geq-C_{k}^{-2}\left(\tilde{r}_{k}\right)^{-2}, \quad \text { on } B_{1}\left(x\left(\tilde{t}_{k}\right), \tilde{r}_{k}\right),
\end{gathered}
$$

and

$$
\operatorname{Vol}_{1}\left(B_{1}\left(x\left(\tilde{t}_{k}\right), \tilde{r}_{k}\right)\right) \geq w^{\prime}\left(\tilde{r}_{k}\right)^{3},
$$

where $\widetilde{R m}$ is the rescaled curvature, $x\left(\tilde{t}_{k}\right)$ is the point given by $(7.7 .4)$ and $B_{1}\left(x\left(\tilde{t}_{k}\right), \tilde{r}_{k}\right)$ is the geodesic ball of rescaled solution at the time $s=1$. Moreover, the closure of $B_{1}\left(x\left(\tilde{t}_{k}\right), \tilde{r}_{k}\right)$ is the whole manifold $\left(M, \tilde{g}_{i j}^{\alpha_{k}}(\cdot, 1)\right)$.

Note that in Theorem 7.2.1, Theorem 7.2.2 and Corollary 7.2.3, the condition about normalized initial metrics is just to ensure that the solutions satisfy the Hamilton-Ivey pinching estimate. Since our solutions $\left(M_{t}^{\alpha_{k}}, g_{i j}^{\alpha_{k}}(t)\right)$ have already satisfied the pinching assumption, we can then apply Corollary 7.2.3 to conclude

$$
|\widetilde{R m}(x, s)| \leq K\left(w^{\prime}\right)\left(\tilde{r}_{k}\right)^{-2},
$$

whenever $s \in\left[1-\tau\left(w^{\prime}\right)\left(\tilde{r}_{k}\right)^{2}, 1\right], x \in\left(M, \tilde{g}_{i j}^{\alpha_{k}}(\cdot, s)\right)$ and $k$ is sufficiently large. Here $K\left(w^{\prime}\right)$ and $\tau\left(w^{\prime}\right)$ are positive constants depending only on $w^{\prime}$. Equivalently, we have the curvature estimates

$$
|R m(\cdot, t)| \leq K\left(w^{\prime}\right)\left(\operatorname{diam}\left(\left(M_{\tilde{t}_{k}}^{\alpha_{k}}\right)^{(1)}\right)\right)^{-2}, \quad \text { on } M,
$$

whenever $t \in\left[\tilde{t}_{k}-\tau\left(w^{\prime}\right)\left(\operatorname{diam}\left(\left(M_{\tilde{t}_{k}}^{\alpha_{k}}\right)^{(1)}\right)\right)^{2}, \tilde{t}_{k}\right]$ and $k$ is sufficiently large.

For each $k$, let us scale $\left(\left(M_{t}^{\alpha_{k}}\right)^{(1)}, g_{i j}^{\alpha_{k}}(t)\right)$ with the factor $\left(\operatorname{diam}\left(\left(M_{\tilde{t}_{k}}^{\alpha_{k}}\right)^{(1)}\right)\right)^{-2}$ and shift the time $\tilde{t}_{k}$ to the new time zero. By the curvature estimate (7.7.9) and Hamilton's compactness theorem (Theorem 4.1.5), we can take a subsequential limit (in the $C^{\infty}$ topology) and get a smooth solution to the Ricci flow on $M \times\left(-\tau\left(w^{\prime}\right), 0\right]$. Moreover, by (7.7.5), the limit has nonnegative sectional curvature on $M \times\left(-\tau\left(w^{\prime}\right), 0\right]$. Recall that we have removed all compact components with nonnegative scalar curvature. By combining this with the strong maximum principle, we conclude that the limit is a flat metric. Hence in case (2), $M$ is diffeomorphic to a flat manifold and then it is also geometrizable.

Therefore we have completed the proof of the theorem.

\section{REFERENCES}

[1] U. Abresch And W.T. Meyer, Injectivity radius estimates and sphere theorems, Comparison Geometry, MSRI Publications, 30 (1997), pp. 1-48.

[2] S. Aloff and N. Wallach, An infinite family of distinct 7-manifolds admitting positively curved Riemannian structure, Bull. Amer. Math. Soc., 81 (1975), pp. 93-97.

[3] W. Ambrose And I.M. Singer, A theorem on holonomy, Trans. Amer. Math. Soc., 75 (1953), pp. $428-443$.

[4] M. Anderson, Geometrization of 3-manifolds via the Ricci flow, Notices Amer. Math. Soc., 51:2 (2004), pp. 184-193.

[5] S. BANDo, On the classification of three-dimensional compact Kähler manifolds of nonnegative bisectional curvature, J. Differential Geom., 19:2 (1984), pp. 283-297.

[6] J. Bartz, M. Struwe, and R. Ye, A new approach to the Ricci flow on $S^{2}$, Ann. Scuola Norm. Sup. Pisa Cl. Sci. (4), 21:3 (1994), pp. 475-482. 
[7] M. BERGER, Sur les groupes d'holonomie des variétés à connexion affine et les variétés riemanniennes, Bull. Soc. Math. France, 83 (1955), pp. 279-330.

[8] Y. Burago, M. Gromov, and G. Perelman, A.D. Alexandrov spaces with curvatures bounded below, Russian Math. Surveys, 47 (1992), pp. 1-58.

[9] D. Burago, Y. Burago, and S. Ivanov, A Course in Metric Geometry, Graduate Studies in Mathematics, 33, Amer. Math. Soc. Providence, Rhode Island.

[10] R. BRYANT, Local existence of gradient Ricci solitons, preprint.

[11] H.-D. CAO, Deformation of Kähler metrics to Kähler-Einstein metrics on compact Kähler manifolds, Invent. Math., 81:2 (1985), pp. 359-372.

[12] _ On Harnack's inequalities for the Kähler-Ricci flow, Invent. Math., 109:2 (1992), pp. $247-263$.

[13] __ Existence of gradient Kähler-Ricci solitons, Elliptic and Parabolic Methods in Geometry (Minneapolis, MN, 1994), pp. 1-16, A K Peters, Wellesley, MA, 1996.

[14] _ Limits of Solutions to the Kähler-Ricci flow, J. Differential Geom., 45 (1997), pp. 257272.

[15] H.-D. CaO, B.L. Chen, And X.P. Zhu, Ricci flow on compact Kähler manifolds of positive bisectional curvature, C.R. Math. Acad. Sci. Paris, 337:12 (2003), pp. 781-784.

[16] H.-D. CaO And B. Chow, Recent developments on the Ricci flow, Bull. Amer. Math. Soc (N.S.), 36:1 (1999), pp. 59-74.

[17] H.-D. CaO, B. Chow, S.C. Chu, and S.-T. Yau, Collected Papers on Ricci Flow, Series in Geometry and Topology, 37, International Press, 2003.

[18] H.-D. Cao, R.S. Hamilton, and T. Ilmanen, Gaussian densities and stability for some Ricci solitons, arXiv:DG.math.DG/0404165.

[19] J. Cheeger, Finiteness theorems for Riemannian manifolds, Amer. J. Math., 92 (1970), pp. $61-74$.

[20] J. Cheeger And T.H. Colding, Lower bounds on Ricci curvature and the almost rigidity of warped products, Ann. of Math. (2), 144:1 (1996), pp. 189-237.

[21] On the structure of the spaces with Ricci curvature bounded below, I, J. Differential Geom., 46 (1997), pp. 406-480.

[22] J. Cheeger And D. EBIn, Comparison theorems in Riemannian geometry, North-Holland, 1975.

[23] J. Cheeger And D. Gromoll, On the structure of complete manifolds of nonnegative curvature, Ann. of Math., 46 (1972), pp. 413-433.

[24] J. Cheeger And M. Gromov, Collapsing Riemannian manifolds while keeping their curvature bounded, I, J. Differential Geom., 23:3 (1986), pp. 309-346.

[25] _ Collapsing Riemannian manifolds while keeping their curvature bounded, II, J. Differential Geom., 32:1 (1990), pp. 269-298.

[26] J. Cheeger, M. Gromov, and K. Fukaya, Mikhael Nilpotent structures and invariant metrics on collapsed manifolds, J. Amer. Math. Soc., 5:2 (1992), pp. 327-372.

[27] J. Cheeger, M. Gromov, And M. TAylor, Finite propagation speed, kernel estimates for functions of the Laplace operator, and the geometry of complete Riemannian manifolds, J. Differential Geom., 17 (1982), pp. 15-53.

[28] J. Cheeger and S.-T. Yau, A lower bound for the heat kernel, Comm. on Pure and Appl. Math., 34 (1981), pp. 465-480.

[29] B.L. Chen, S.H. TANG, And X.P. ZhU, A uniformization theorem of complete noncompact Kähler surfaces with positive bisectional curvature, J. Differential Geometry, 67 (2004), pp. 519-570.

[30] B.L. Chen And X.P. Zhu, Complete Riemannian manifolds with pointwise pinched curvature, Invent. Math., 140:2 (2000), pp. 423-452.

[31] - On complete noncompact Kähler manifolds with positive bisectional curvature, Math. Ann., 327 (2003), pp. 1-23.

[32] _ Volume growth and curvature decay of positively curved Kähler manifolds, Quarterly Journal of Pure and Applied Mathematics, 1:1 (2005), pp. 68-108.

[33] _ Uniqueness of the Ricci Flow on Complete Noncompact Manifolds, arXiv:DG (AP) math. DG/0505447, May 2005.

[34] _ Ricci Flow with Surgery on Four-manifolds with Positive Isotropic Curvature, arXiv:math.DG/0504478, April 2005.

[35] S.Y. Cheng, P. LI, And S.-T. YAu, On the upper estimate of the heat kernel of complete Riemannian manifold, Amer. J. Math., 103:5 (1981), pp. 1021-1063.

[36] B. CHow, The Ricci flow on 2-sphere, J. Differential Geom., 33 (1991), pp. 325-334.

[37] Geom., 33 (1991), pp. 597-600. 
[38] B. Chow And S.C. ChU, A geometric interpretation of Hamilton's Harnack inequality for the Ricci flow, Math. Res. Lett., 2 (1995), pp. 701-718.

[39] B. Chow And D. Knopf, The Ricci flow: An introduction, Mathematical Surveys and Monographs, Amer. Math. Soc., Providence, RI, 2004.

[40] B. Chow AND P. Lu, The maximum principle for systems of parabolic equations subject to an avoidance set, Pacific J. Math., 214:2 (2004), pp. 201-222.

[41] B. Chow, P. Lu, And L. Ni, Hamilton's Ricci flow, American Mathematical Society and Science Press, to appear.

[42] T.H. Colding And W.P. Minicozzi, II, Estimates for the extinction time for the Ricci flow on certain 3-manifolds and a question of Perelman, J. Amer. Math. Soc., 18:3 (2005), pp. 561-569.

[43] D. De Turck, Deforming metrics in the direction of their Ricci tensors, J. Differential Geom., 18 (1983), pp. 157-162.

[44] G. Drees, Asymptotically flat manifold of nonnegative curvature, Diff. Geom. Appl., 4 (1994), pp. $77-90$.

[45] J. Eschenburg, V. Schroeder, and M. Strake, Curvature at infinity of open nonnegatively curved manifold, J. Differential Geom., 30 (1989), pp. 155-166.

[46] M. Feldman, T. Ilmanen, And D. Knopf, Rotationally symmetric shrinking and expanding Kähler-Ricci solitons, J. Differential Geom., 65 (2003), pp. 169-209.

[47] T. Frankel, Manifolds with positive curvature, Pacific J. Math., 11 (1961), pp. 165-174.

[48] D. Gilbarg and N.S. Trudinger, Elliptic partial differential equations of second order, Reprint of the 1998 edition, Classics in Mathematics, Springer-Verlag, Berlin, 2001.

[49] R.E. Greene and H. Wu, On a new gap phenomonon in Riemannian geometry, Proc. Nat. Acad. Sci. U.S.A., 79:2 (1982), pp. 714-715.

[50] _ Gap theorems for noncompact Riemannian manifolds, Duke Math. J., 49 (1982), pp. 731-756.

[51] — Lipschitz convergence of Riemannian manifolds, Pacific J. Math., 131 (1988), pp. 119-141.

[52] D. Gromoll And W. Meyer, On complete open manifolds of positive curvature, Ann. of Math., 90 (1969), pp. 75-90.

[53] M. Gromov, Metric Structures for Riemannian and Non-Riemannian Spaces, Edited by J. LaFontaine and P. Pansu, English translation by Sean Michael Bates, Birkhäuser, 1998.

[54] _ Almost flat manifolds, J. Differential Geom., 13 (1978), pp. 231-241.

[55] _ Hyperbolic manifolds (according to Thurston and J $\phi$ rgensen), Bourbaki Seminar, Vol. 1979/80, pp. 40-53, Lecture Notes in Math., 842, Springer, Berlin-New York, 1981.

[56] M. Gromov And H.B. Lawson, JR., The classification of simply connected manifolds of positive scalar curvature, Ann. of Math. (2), 111:3 (1980), pp. 423-434.

[57] - Positive scalar curvature and the Dirac operator on complete Riemannian manifolds, Inst. Hautes Études Sci. Publ. Math., 58 (1983), pp. 83-196.

[58] R.S. Hamilton, Three manifolds with positive Ricci curvature, J. Differential Geom., 17 (1982), pp. 255-306.

[59] _ Four-manifolds with positive curvature operator, J. Differential Geom., 24 (1986), pp. 153-179.

[60] 는 The Ricci flow on surfaces, Contemporary Mathematics, 71 (1988), pp. 237-261.

[61] _ The Harnack estimate for the Ricci flow, J. Differential Geom., 37 (1993), pp. 225243.

[62] — A compactness property for solution of the Ricci flow, Amer. J. Math., 117 (1995), pp. $545-572$.

[63] - The formation of singularities in the Ricci flow, Surveys in Differential Geometry, 2, pp. 7-136, International Press, 1995.

[64] _ Four manifolds with positive isotropic curvature, Comm. in Analysis and Geometry, 5 (1997), pp. 1-92.

[65] , Non-singular solutions to the Ricci flow on three manifolds, Comm. Anal. Geom., 7 (1999), pp. 695-729.

[66] _ Eternal solutions to the Ricci flow, J. Differential Geom., 38:1 (1993), pp. 1-11.

[67] _ Lectures on the Ricci flow at Columbia University, Fall, 2003.

[68] S. Helgason, Differential Geometry and Symmetric Spaces, Academic Press, 1962 (Second edition, 1978).

[69] J. Hempel, 3-manifolds, Reprint of the 1976 original, AMS Chelsea Publishing, Providence, RI, 2004.

[70] M.W. Hirsch, Differential Topology, Springer-Verlag, 1976. 
[71] G. Huisken, Ricci deformation of the metric on a Riemanian manifold, J. Differential Geometry, 21 (1985), pp. 47-62.

[72] Asymptotic behavior for singularities of the mean curvature flow. J. Differential Geometry, 31 (1990), pp. 285-299.

[73] T. Ivey, Ricci solitons on compact three-manifolds, Diff. Geom. Appl., 3 (1993), pp. 301-307.

[74] W. Jaco And P. Shalen, Seifert fibered spaces in 3-manifolds, Men. Amer. Math. Soc., 220 (1979).

[75] K. Johannson, Homotopy equivalences of 3-manifolds with boundaries, Lecture Notes in Math., 761 (1979).

[76] D.A. Kazdan and G. Margulis, A simple proof of Selberg's hypothesis, Math. Sb. (117), 75 (1968), pp. 163-168.

[77] J.L. KAZDAN AND F.W. WARner, Curvature functions for compact 2-manifolds, Ann. of Math. (2), 99 (1974), pp. 14-47.

[78] B. Kleiner and J. LotT, Note on Perelman's paper, http://www.math.lsa.umich.edu /research /ricciflow /perelman.html.

[79] H. Kneser, Geschlossene Flächen in dreidmensionalen mannigfaltigkeiten, Jahresbericht der Deut. Math. Verein., 38 (1929), pp. 248-260.

[80] N. KoIso, On rotationally symmmetric Hamilton's equation for Kähler-Einstein metrics, Recent Topics in Diff. Anal. Geom. Adv. Studies Pure Math., 18-I (1990), pp. 327-337.

[81] O.A. Ladyzenskaja, V.A. Solonnikov, and N.N. Uralceva, Linear and quasilinear equations of parabolic type, Transl. Amer. Math. Soc., 23 (1968).

[82] P. Li AND S.-T. YAU, On the parabolic kernel of the Schrödinger operator, Acta Math., 156:3-4 (1986), pp. 153-201.

[83] C. MARGERIN, Une caractérisation optimale de la structure différentielle standard de la sphère en terme de courbure pour (presque) toutes les dimensions. I. Les énoncés, (French) [A sharp curvature-type characterization of the smooth sphere in all but a few dimensions. I. Statement of the theorems] C.R. Acad. Sci. Paris Sér. I Math., 319:6 (1994), pp. 605-607.

[84] A sharp characterization of the smooth 4-sphere in curvature terms, Comm. Anal. Geom., 6:1 (1998), pp. 21-65.

[85] C. McMullen, Renormalization and 3-manifolds which fiber over the circle, Annals of Mathematics Studies 12, Princeton U. Press, 1996.

[86] W.W. Meeks, III AND S.-T. YAu, The existence of embedded minimal surfaces and the problem of uniqueness, Math. Z., 179:2 (1982), pp. 151-168.

[87] W.W. MeEKs, III, L. Simon, AND S.-T. YAU, Embedded minimal surfaces, exotic spheres, and manifolds with positive Ricci curvature, Ann. of Math., 116 (1982), pp. 621-659.

[88] M. Micallef AND J.D. Moore, J Minimal two-spheres and the topology of manifolds with positive curvature on totally isotropic two-planes, Ann. of Math. (2), 127 (1988), pp. 199227.

[89] A.D. Milka, Metric structure of some class of spaces containing straight lines, Ukrain. Geometrical. Sbornik, 4 (1967), pp. 43-48.

[90] J. Milnor, A unique factorization theorem for 3-manifolds, Amer. J. Math., 84 (1962), pp. 17.

[91] _ Towards the Poincaré conjecture and the classification of 3-manifolds, Notices Amer. Math. Soc., 50:10 (2003), pp. 1226-1233.

[92] N. MoK, The uniformization theorem for compact Kähler manifolds of nonnegative holomorphic bisectional curvature, J. Differential Geom., 27 (1988), pp. 179-214.

[93] N. MoK, Y.T. SiU, AND S.-T. YAu, The Poincaré-Lelong equation on complete Kähler manifolds, Compositio Math., 44 (1981), pp. 183-218.

[94] J.W. Morgan, On Thurston's uniformization theorem for three-dimensional manifolds, The Smith Conjecture (Bass and Morgan, eds.), Pure Appl. Math., 112, Academic Press, (1984), pp. 37-125.

[95] - Recent progress on the Poincaré conjecture and the classification of 3-manifolds, Bull.of the A.M.S., 42:1 (2005), pp. 57-78.

[96] S. Mori, Projective manifolds with ample tangent bundles, Ann. of Math., 100 (1979), pp. 593606.

[97] G.D. Mostow, Strong rigidity of locally symmetric spaces, Annals of Mathematics Studies, 78, Princeton University Press, Princeton, N.J.; University of Tokyo Press, Tokyo, 1973.

[98] L. NI, Ancient solution to Kahler-Ricci flow, arXiv:math.DG.0502494, February 2005, preprint.

[99] _ A note on Perelman's LYH inequality, arXiv:math.DG.0602337, February 2006, preprint.

[100] L. Ni AND L.F. TAm, Plurisubharmonic functions and the structure of complete Kähler man- 
ifolds with nonnegative curvature, J. Differential Geom., 64:3 (2003), pp. 457-524.

[101] S. NishikaWA, Deformation of Riemannian metrics and manifolds with bounded curvature ratios, Geometric measure theory and the calculus of variations (Arcata, Calif., 1984), pp. 343-352, Proc. Sympos. Pure Math., 44, Amer. Math. Soc., Providence, RI, 1986.

[102] J.-P. OtaL, Le théoréme d'hyperbolisation pir les variétés fibrées de dimension 3, Atérisque 235, 1996.

[103] G Perelmann, The entropy formula for the Ricci flow and its geometric applications, arXiv:math. DG/0211159 v1 November 11, 2002, preprint.

[104] _ Ricci flow with surgery on three manifolds, arXiv:math.DG/0303109 v1 March 10, 2003, preprint.

[105] - Finite extinction time to the solutions to the Ricci flow on certain three manifolds, arXiv:math.DG/0307245 July 17, 2003, preprint.

[106] S. Peters, Convergence of Riemannian mainfolds, Comp. Math., 62 (1987), pp. 3-16.

[107] G. Prasad, Strong rigidity of Q-rank 1 lattices, Invent. Math., 21 (1973), pp. 255-286.

[108] O.S. Rothaus, Logarithmic Sobolev inequalities and the spectrum of Schrödinger opreators, J. Funct. Anal., 42:1 (1981), pp. 110-120.

[109] R. Schoen And S.-T. YAU, On the structure of manifolds with positive scalar curvature, Manuscripta math., 28 (1979), pp. 159-183.

[110] _ Existence of incompressible minimal surfaces and the topology of three-dimensional manifolds with nonnegative scalar curvature, Ann. of Math. (2), 110:1 (1979), pp. 127142.

[111] Complete three dimensional manifolds with positive Ricci curvature and scalar curvature, Seminor on Differential Geometry, Princeton University Press, 1982, pp. 209-228.

[112] _ Lectures on differential geometry, in 'Conference Proceedings and Lecture Notes in Geometry and Topology', 1, International Press Publications, 1994.

[113] N. Sesum, Limiting behaviour of the Ricci flow, arXiv:math.DG/0402194, February 2004.

[114] W.X. SHI, Deforming the metric on complete Riemannian manifold, J. Differential Geometry, 30 (1989), pp. 223-301.

[115] _ Ricci deformation of the metric on complete noncompact Kähler manifolds, Ph.D. Thesis, Harvard University, 1990.

[116] _ Complete noncompact Kähler manifolds with positive holomorphic bisectional curvature, Bull. Amer Math. Soc., 23 (1990), pp. 437-440.

[117] _ Ricci flow and the uniformization on complete noncompact Kähler manifolds, J. Differential Geom., 45:1 (1997), pp. 94-220.

[118] T. Shioya And T. YAmaguchi, Volume collapsed three-manifolds with a lower curvature bound, Math. Ann., 333 (2005), pp. 131-155.

[119] I.M. Singer, Infinitesimallly homogeneous spaces, Comm. Pure Appl. Math., 13 (1960), pp. 685-697.

[120] Y.T. SiU AND S.-T. YAU, Complete Kähler manifolds with non-positive curvature of faster than quadratic decay, Ann. of Math., 105 (1977), pp. 225-264.

[121] M. Struwe, Curvature flows on surfaces, Ann. Sc. Norm. Super. Pisa Cl. Sci. (5), 1:2 (2002), pp. $247-274$.

[122] W.P. Thurston, Three-dimensional manifolds, Kleinian groups and hyperbolic geometry, Bull. Amer. Math. Soc. (N.S.), 6:3 (1982), pp. 357-381.

[123] — Hyperbolic structures on 3-manifolds. I: Deformation of acylindrical manifolds, Ann. of Math. (2), 124:2 (1986), pp. 203-246.

[124] Hyperbolic structures on 3-manifolds II: Surface groups and 3-manifolds which fiber over the circle, preprint, 1988.

[125] — Three-dimensional geometry and topology, Vol. 1, Edited by Silvio Levy, Princeton Mathematical Series, 35, Princeton University Press, Princeton, NJ, 1997.

[126] F. Waldhausen, Eine Klasse von 3-dimensionalen Mannigfaltigkeiten I, II, Invent. Math., 3 (1967), pp. 308-333; 4 (1967), pp. 87-117.

[127] X.J. WANG, AND X.H. Zhu, Kähler-Ricci solitons on toric manifolds with positive first Chern class, Adv. Math., 188:1 (2004), pp. 87-103.

[128] S.-T. YAU, Some function-theoretic properties of complete Riemannian manifold and their applications to geometry, Indiana Univ. Math. J., 25 (1976), pp. 659-670.

[129] the International Congress of Mathematicians', Helsinki, 1978, pp. 237-250.

[130] Perspectives on geometric analysis, to appear in Survey in Differential Geometry, Vol. X. (arXiv: math.DG/0602363, February, 2006) 


\section{INDEX}

$T_{i}$ converges to a tensor $T, 267$

$\delta$-cutoff surgery, 424

$\kappa$-noncollapsed, 255, 433

$\lambda$-remote, 360

$\mathcal{L}$-Jacobian, 252

$\mathcal{L}$-Jacobian field, 248

$\mathcal{L}$-distance function, 245

$\mathcal{L}$-exponential map with parameter $\bar{\tau}, 252$

$\mathcal{L}$-geodesic, 244

curve, 244

equation, 244

$\mathcal{L}$-length, 244

$\varepsilon$-cap, 414

$\varepsilon$-horn, 414

$\varepsilon$-neck, 414

strong, 416

$\varepsilon$-neck of radius $r, 357$

$\varepsilon$-tube, 414

$k$-jet

distance, 343

space, 343

(almost) maximum points, 291

a priori assumptions (with accuracy $\varepsilon$ ), 416

admissible curve, 435

ancient

$\kappa$-solution, 357

solution, 233

asymptotic scalar curvature ratio, 303, 362

asymptotic volume ratio, 373

atoroidal, 482

barely admissible curve, 435

be modeled, 481

Berger's rigidity theorem, 321

breather, 199

expanding, 199

shrinking, 199

steady, 199

canonical neighborhood assumption (with accuracy $\varepsilon$ ), 416

canonical neighborhood theorem, 396

capped $\varepsilon$-horn, 414

center of an evolving $\varepsilon$-neck, 394
Cheeger's lemma, 288

classical sphere theorems, 321

classification of three-dimensional shrinking solitons, 384

collapsed, 338

compactness of ancient $\kappa_{0}$-solutions, 393

conjugate heat equation, 235

converges to a marked manifold, 267

converges to an evolving marked manifold, 282

curvature $\beta$-bump, 360

degree, 308

double $\varepsilon$-horn, 414

Einstein

manifold, 174

metric, 174

elliptic type estimate, 391

essential component, 476

evolving $\varepsilon$-cap, 396

evolving $\varepsilon$-neck, 396

exceptional part, 338

finite bump theorem, 360

free boundary condition, 345

geometrizable, 482

gradient shrinking Ricci soliton, 384

graph manifold, 482

Gromoll-Meyer injectivity radius estimate, 286

Haken, 481

Hamilton's advanced maximum principle, 218

Hamilton's compactness theorem, 285

Hamilton's strong maximum principle, 213

Hamilton-Ivey pinching estimate, 224, 336

homogeneous manifold, 481

locally, 481

incompressible, 338

injectivity radius, 286

condition, 291 
irreducible, 476

Jacobian comparison theorem, 253

justification of the canonical neighborhood assumption, 433

justification of the pinching assumption, 424

Kähler-Ricci flow, 176

Kähler-Ricci soliton

expanding, 176

shrinking, 176

steady, 176

Klingenberg's lemma, 286

Li-Yau-Hamilton estimate, 226, 230

Li-Yau-Hamilton quadratic, 230

Li-Yau-Perelman distance, 238, 250

Little Loop Lemma, 290

marked Riemannian manifold, 267

marking, 267

maximal solution, 291

Mostow type rigidity, 343

no local collapsing theorem I, 255, 256

no local collapsing theorem I', 259

no local collapsing theorem II, 263

noncollapsing limit, 338

normalized, 398

normalized Ricci flow, 307

origin, 267

Perelman's claim, 482

Perelman's reduced volume, 243, 252 element, 254

pinching assumption, 416

Poincaré conjecture, 452

prime, 481 decomposition theorem, 481

regular, 411

Ricci flow, 173

Ricci flow with surgery, 416

Ricci soliton

expanding, 175

shrinking, 175

gradient, 175

steady, 175

Shi's derivative estimate, 192 singularity model, 292

singularity structure theorem, 399

soliton

cigar, 177

steady, 175

solution

ancient, 233

nonsingular, 336

standard, 420

solution becomes extinct, 417

solution develops a singularity, 267

standard capped infinite cylinder, 420

support function, 220

surgery times, 416

surgically modified solution, 416

tangent cone, 219

thick-thin decomposition theorem, 473

Thurston's geometrization conjecture, 482,483

type I, 291-293

type II, 293

(a), 291, 294

(b), 292, 295

type III, 293

(a), 292, 296

(b), 292

universal noncollapsing, 388

Weaker Assertion, 483 